a Ph.D. dissertation on

Nonlinear Acoustic Waves

in Complex Media

Noé Jiménez

dissertation directors

F. Camarena

J. Redondo

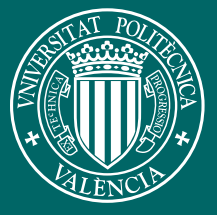

UNIVERSITAT POLITĖCNICA DE VALÈNCIA

June 2015 


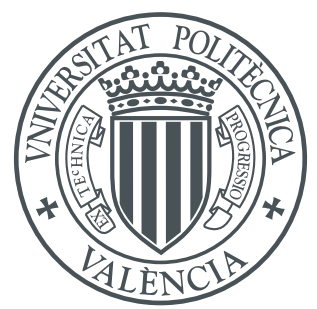

\title{
Nonlinear Acoustic Waves in Complex Media
}

\author{
Noé Jiménez \\ Instituto para la Gestión Integrada de Zonas Costeras \\ Universitat Politècnica de València
}

A thesis submitted in partial fulfillment of the requirements of the degree of

Doctor of Philosophy

June 23, 2015

\section{Dissertation directors:}

Francisco Camarena Javier Redondo

\section{Dissertation jury:}

Georgios L. Theocharis

Carlos Fritsch

Enrique Riera

Yiu Way Lam

Germán J. de Valcárcel
Universitat Politècnica de València, Spain Universitat Politècnica de València, Spain
Centre National de la Recherche Scientifique (CNRS), France Consejo Superior de Investigaciones Cientificas (CSIC), Spain Consejo Superior de Investigaciones Cientificas (CSIC), Spain University of Salford, UK

Universidad de Valencia, Spain 


Le savant n'étudie pas la nature parce que cela est utile; il l'étudie parce qu'il y prend plaisir et il y prend plaisir parce qu'elle est belle.

Henri Poincaré 
El científico no estudia la naturaleza por la utilidad que le pueda reportar; la estudia por el gozo que le proporciona, y este gozo se debe a la belleza que hay en ella. 



\section{Acknowledgements}

While finishing my MSc studies on acoustics, Javier Redondo and Paco Camarena gave me the opportunity to collaborate with them in Gandía, firstly to investigate the ultrasonic dynamics of orange fruits. When I started my $\mathrm{PhD}$, founded by a grant from the Polytechnic University of Valencia (UPV), I continued with their studies on nonlinear ultrasound applying them to nonlinear propagation in biological media. Building a lab from scratch is a very challenging task, and almost impossible under the actual founding conditions. I acknowledge the outstanding work of Paco and Javi, and his efforts for maintaining student founding as a priority. With them, I learned all I know about simulations and nonlinear ultrasound propagation in soft-tissues. As a result of his effort, I moved to the Columbia University in New York City for an astonishing stay in the UEIL laboratory directed by Elisa E. Konofagou, leading to the results of Part III.

I appreciate my $\mathrm{PhD}$ directors for their patience and for giving me freedom to explore other areas, that has enriched my work with a global view of many other acoustic topics. The interchange of ideas in the lab and the curiosity for test unknown things, triggered my collaboration with many other people. I want to acknowledge to Víctor SánchezMorcillo, not only for the opportunity to work in other interesting areas, but also for his contagious passion for science and its pleasure of understanding the nature. Because of him, I worked closely to many other researchers that have contributed to the present work, as Juan F.R. Archilla (Universidad de Sevilla, Spain), Lluis M. García-Raffi (UPV) and Yuriy Kosevich (Moscow University, Russia). With they, we developed the work summarized in Chapter 1, we organized a conference (Quodons in mica 2013) in Altea, Spain, and we are currently editing a book, among other publications.

Many other people has deeply contributed also to the contents of this thesis as Vicent Romero-García (LAUM, France), Rubén Picó (UPV) and El Mokhtar Hamham (Abdelmalek Essaadi University, Morocco) who is the major author of Chapter 3. Also, always inspiring were the collaborations with the people of Terrasa (Universitat Politénica de Catalunya, Spain) and specially with Kestutis Staliunas (iCREA) who deeply contributed to the contents of this Thesis. Even, I'm pleased also with the enlightening collaborations in other subjects that are not included in this document, as our works in microbubbles and nonlinear coupled oscillator rings with Serge Dos Santos and Ayache Bouakaz (Université Francois Rabelais, France), and their students Jennifer Chaline and Loïc Maurin.

Beyond these exciting professional collaborations, working in Gandía was strongly gratifying due to friendships there, not only with the seniors, but also with the oompa loompas of science, Jandri, Patri, Vicent, Silvia, Ester, María, and also in the last years Pablo, Ahmed and Luis.

También agradezco a mis padres y a mi familia su apoyo incondicional, incluso durante mis primeros años rebeldes.

A Mariajo, por todo el tiempo que le he usurpado. 



\section{Abstract}

Nature is nonlinear. The linear description of physical phenomena is useful for explain observations with the simplest mathematical models, but they are only accurate for a limited range of input values. In the case of intense acoustics waves, linear models obviate a wide range of physical phenomena that are necessary for accurately describe such high-amplitude waves, indispensable for explain other exotic acoustic waves and mandatory for developing new applied techniques based on nonlinear processes. In this Thesis we study the interactions between nonlinearity and other basic wave phenomena such as non-classical attenuation, anisotropic dispersion and periodicity, and diffraction in specific configurations.

First, we present intense strain waves in a chain of cations coupled by realistic interatomic potentials. Here, the nonlinear ionic interactions and lattice dispersion lead to the formation of supersonic kinks. These intrinsically-nonlinear localized dislocations travel long distances without changing its properties and explain the formation of dark traces in mica crystals. Then, we analyze nonlinear wave processes in a system composed of multilayered acoustic media. The rich nonlinear dynamics of this system is characterized by its strong dispersion. Here, harmonic generation processes and the relation with its band structure are presented, showing that the nonlinear processes can be enhanced, strongly minimized or simply modified by tuning the layer parameters. In this way, we show how the dynamics of intense monochromatic waves and acoustic solitons can be controlled by artificial layered materials.

In a second part, we include diffraction and analyze four types of singular beams. First, we study nonlinear beams in two dimensional sonic crystals. In this system, the inclusion of anisotropic dispersion is tuned for obtain simultaneous self-collimation for fundamental and second harmonic beams. The conditions for optimal second harmonic generation are presented. Secondly, we present limited diffraction beam generation using equispaced axisymmetric diffraction gratings. The obtained beams are truncated version of zero-th order Bessel beams. Third, the grating spacing can be modified to achieve focusing, where the generated nonlinear beams presents high gain, around $30 \mathrm{~dB}$, with a focal width which is between the diffraction limit and the sub-wavelength regime, but with its characteristic high amplitude side lobes strongly reduced. Finally, we observe that waves diffracted by spiral-shaped gratings generate high-order Bessel beams, conforming nonlinear acoustic vortex. The conditions to obtain arbitrary-order Bessel beams by these passive elements are presented.

Finally, the interplay of nonlinearity and attenuation in biological media is studied in the context of medical ultrasound. First, a numerical method is developed. The method solves the constitutive relations for nonlinear acoustics and the frequency power law attenuation of biological media is modeled as a sum of relaxation processes. A new technique for reducing numerical dispersion based on 
artificial relaxation is included. Second, this method is used to study the harmonic balance as a function of the power law, showing the role of weak dispersion and its impact on the efficiency of the harmonic generation in soft-tissues. Finally, the study concerns the nonlinear behavior of acoustic radiation forces in frequency power law attenuation media. We present how the interplay between nonlinearity and the specific frequency power law of biological media can modify the value for acoustic radiation forces. The relation of the nonlinear acoustic radiation force with thermal effects are also discussed.

The broad range of nonlinear processes analyzed in this Thesis contributes to understanding the behavior of intense acoustic waves traveling trough complex media, while its implications for enhancing existent applied acoustics techniques are presented. 


\section{Resumen}

La Naturaleza es no lineal. La descripción lineal de los fenómenos físicos es de gran utilidad para explicar nuestras observaciones con modelos matemáticos simples, pero éstos sólo son precisos en un limitado rango de validez. En el caso de onda acústica de alta intensidad, los modelos lineales obvian un amplio rango de fenómenos físicos que son necesarios para describir con precisión las ondas de gran amplitud, pero además son necesarios para explicar otros procesos más exóticos e indispensables para desarrollar nuevas aplicaciones basadas en propagación no lineal. En esta Tesis, estudiamos las interacciones entre no linealidad y otros procesos complejos como atenuación no-clásica, dispersión anisotrópica y periodicidad, y difracción en configuraciones específicas.

En primer lugar, presentamos ondas de deformación en una cadena de cationes acoplados por potenciales realísticas. Aquí, las interacciones no lineales entre iones, producen la conformación de kinks supersónicos. Estas dislocaciones localizadas intrínsecamente no lineales viajan por la red largas distancias sin variar sus propiedades, y pueden explicar la formación de trazas en minerales como la mica. Aumentando la escala del problema, estudiamos los procesos acústicos no lineales en medios multicapa. La rica dinámica de estos medios está caracterizada por la fuerte dispersión debido a la periodicidad del sistema. Aquí, estudiamos los procesos de generación de harmónicos, mostrando como modificando la estructura podemos potenciar, minimizar, o simplemente modificar artificialmente la transferencia de energía entre las componentes espectrales, y de esta manera controlar la dinámica de las ondas y solitones en el interior de la estructura.

En la segunda parte, incluimos difracción y analizamos cuatro tipos de haces singulares. En primer lugar, analizamos haces ultrasónicos no lineales en cristales de sonido bidimensionales. En este sistema, las propiedades de anisotropía del medio son ajustadas para obtener la auto-colimación simultánea del primer y segundo harmónico. Así, se obtiene la propagación no difractiva para las dos componentes. En segundo lugar, presentamos haces de difracción limitada empleando rejillas de difracción axisimétricas. Por último, demostramos la generación de haces de Bessel de orden superior mediante estructuras en espiral.

En la última parte, estudiamos la competición entre no linealidad y la atenuación y dispersión observable en medios biológicos en el contexto de las aplicaciones de biomédicas de los ultrasonidos. En primer lugar desarrollamos un nuevo método computacional para la dependencia frecuencial en forma de ley de potencia de la absorción característica de los tejidos. Este método en dominio temporal es usado posteriormente para revisar los procesos básicos no lineales prestando especial interés en el paper de la dispersión del tejido. Por último, la resolución de las ecuaciones constitutivas nos permite abordar la descripción no lineal de la fuerza de radiación acústica producida en tejidos biológicos, y las implicaciones existentes con la deposición de energía y transferencia de momento para ondas 
ultrasónicas de alta intensidad.

El amplio abanico de procesos no lineales analizados en esta tesis contribuye a una mejor comprensión de la dinámica de las ondas acústicas de alta intensidad en medios complejos, donde las implicaciones existentes en cuanto a la mejora de sus aplicaciones prácticas son puestas de manifiesto. 


\section{Resum}

La Naturalesa és no lineal. La descripció lineal dels fenòmens físics és de gran utilitat per a explicar les nostres observacions amb models matemàtics simples, però aquests sol són precisos en un limitat rang de validesa. En el cas d'ona acústica d'alta intensitat, els models lineals obvien un ampli rang de fenòmens físics que són necessaris per a descriure amb precisió les ones de gran amplitud, però a més són necessaris per a explicar altres processos més exòtics i indispensables per a desenvolupar noves aplicacions basades en propagació no lineal. En aquesta Tesi, estudiem les interaccions entre no-linealitat $\mathrm{i}$ altres processos complexos com atenuació no-clàssica, dispersió anisotròpica i periodicitat, i difracció en configuracions específiques.

En primer lloc, presentem ones de deformació en una cadena de cations acoblats per potencials realistes. Ací, les interaccions no lineals entre ions, produeixen la conformació de kinks supersònics. Aquestes dislocacions localitzades intrínsecament no lineals viatgen per la xarxa llargues distàncies sense variar les seues propietats, i poden explicar la formació de traces en minerals com la mica. Augmentant l'escala del problema, estudiem els processos acústics no lineals en mitjans multicapa. La rica dinàmica d'aquests mitjans es caracteritza per la forta dispersió a causa de la periodicitat del sistema. Ací, estudiem els processos de generació d'harmònics, mostrant com modificant l'estructura podem potenciar, minimitzar, o simplement modificar artificialment la transferència d'energia entre les components espectrals, i d'aquesta manera controlar la dinàmica de les ones i solitons a l'interior de l'estructura.

En la segona part, incloem difracció i analitzem quatre tipus de feixos singulars. En primer lloc, analitzem feixos ultrasònics no lineals en cristalls de so bidimensionals. En aquest sistema, les propietats d'anisotropia del medi són ajustades per a obtenir l'acte-col-limació simultània del primer i segon harmònic. Així, s'obté la propagació no difractiva per a les dues components. En segon lloc, presentem feixos de difracció limitada emprant reixetes de difracció axisimètriques. Per últim, vam demostrar la generació de feixos de Bessel d'ordre superior mitjançant estructures en espiral. En l'última part, estudiem la competició entre no linealitat i l'atenuació i dispersió observable en medis biològics en el context de les aplicacions biomèdiques dels ultrasons. En primer lloc desenvolupem un nou mètode computacional per a la dependència freqüencial en forma de llei de potència de l'absorció característica dels teixits biològics. Aquest mètode en domini temporal és usat posteriorment per a revisar els processos bàsics no lineals prestant especial interés en el paper de la dispersió del teixit. Per últim, la resolució de les equacions constitutives ens permet abordar la descripció no lineal de la força de radiació acústica produïda en teixits biològics, i les implicacions existents amb la deposició d'energia i transferència de moment per a ones ultrasòniques d'alta intensitat. 
L'ampli ventall de processos no lineals analitzats en aquesta tesi contribueix a una millor comprensió de la dinàmica de les ones acústiques d'alta intensitat en medis complexos, on les implicacions existents quant a la millora de les seues aplicacions practiques són posades de manifest. 



\section{Contents}

Page

Part I Nonlinear Waves in Lattices

Chapter 1 Localized nonlinear waves in lattices 3

1.1 Motivation: dynamics of potassium ions in layered silicates . . . . . . . 4

1.2 Minimal model with realistic potentials $\ldots \ldots \ldots \ldots \ldots \ldots \ldots .7$

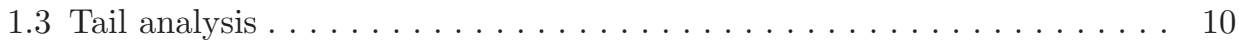

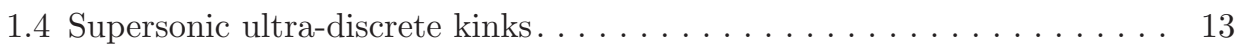

1.5 Interaction with several neighbours $\ldots \ldots \ldots \ldots \ldots \ldots \ldots \ldots .23$

1.6 The effect of nuclear repulsion: kinks with short-range ZBL potential . . 26

1.7 The effect of the substrate potential: lattice kinks or crowdions . . . . . 28

1.8 Some numerical simulations with ultradiscrete kinks or crowdions . . . . 37

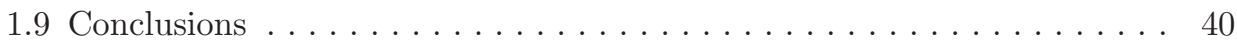

Chapter 2 Nonlinear acoustic waves in layered media 45

2.1 Introduction . . . . . . . . . . . . . . . . . . . . . 46

2.2 Dispersion relations. . . . . . . . . . . . . . . . . 47

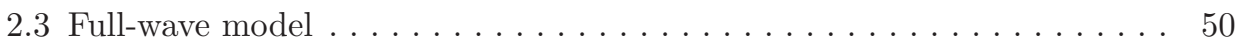

2.4 Harmonic generation in layered media $\ldots \ldots \ldots \ldots \ldots \ldots \ldots .57$

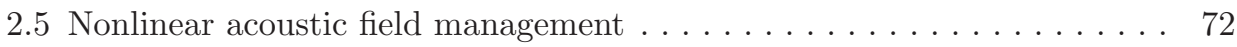

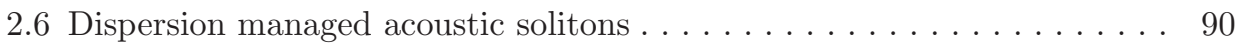

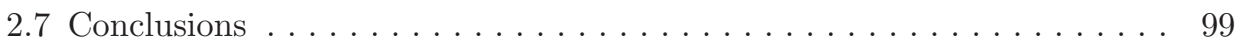

Part II Nonlinear Singular Beams _ 103

Chapter 3 Nonlinear self-collimated sound beams in periodic media 105

3.1 Introduction . . . . . . . . . . . . . . . . . 106

3.2 Nonlinear sound beam propagation model . . . . . . . . . . . . 108

3.3 Self-collimation of intense acoustic beams . . . . . . . . . . . . 110

3.4 Numerical simulation. . . . . . . . . . . . . . . . . . . . . . . . . 114

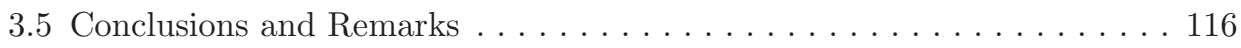


Chapter 4 Acoustic Bessel beams generated by axisymmetric gratings

4.1 Introduction . . . . . . . . . . . . . . . . . . . . . . . . . . . . . . 122

4.2 Generation of Zero th-order Bessel beams by axisymmetric gratings. . . . 123

4.3 Experimental validation. . . . . . . . . . . . . . . . . . 126

4.4 Conclusions . . . . . . . . . . . . . . . . . . . . . . . . . . . 129

Chapter 5 Nonlinear focused beams by axisymmetric gratings

5.1 Introduction . . . . . . . . . . . . . . . . . . . . . . 134

5.2 Axisymmetric diffraction grating design . . . . . . . . . . . . . 137

5.3 Small amplitude focusing. . . . . . . . . . . . . . . . . . . . . . . . . . . . . . . . . . . . . .

5.4 Transition from linear-to-nonlinear focusing . . . . . . . . . . . . . . . 144

5.5 High intensity regime . . . . . . . . . . . . . . . . . . . . . 145

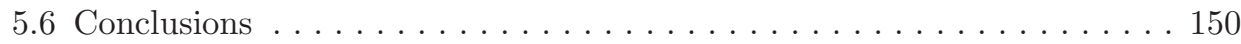

Chapter 6 Chiral beams generated by spiral gratings 153

6.1 Introduction . . . . . . . . . . . . . . . . . . . . . 154

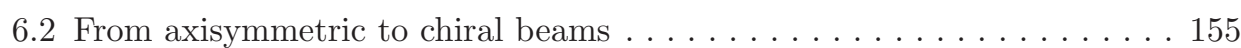

6.3 Chiral beams generated by spiral gratings. . . . . . . . . . . . . . . . 158

6.4 High order Bessel beams generated by $n$-arm spirals . . . . . . . . . . . . . . . . . . . . . . . . . . . . . . . . .

6.5 Weakly nonlinear chiral beams. . . . . . . . . . . . . . . . . 180

6.6 Conclusions . . . . . . . . . . . . . . . . . . . . . . . . . 184

Part III Nonlinear Acoustic Waves in Biological Media 189

Chapter 7 Time-domain nonlinear acoustic wave mod$\begin{array}{ll}\text { elling of soft-tissue media } & 191\end{array}$

7.1 Introduction . . . . . . . . . . . . . . . . . . . . . . . . . 192

7.2 Generalized nonlinear acoustics model for multiple relaxation media . . . 197

7.3 Numerical solution by finite differences in time domain . . . . . . . . . . . 200

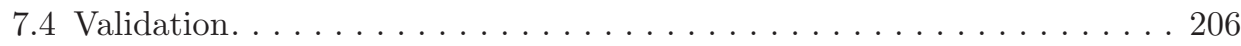

7.5 Results. . . . . . . . . . . . . . . . . . . . . . . . 210

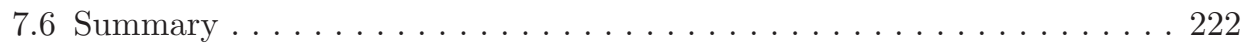

Chapter 8 Nonlinear plane waves in frequency power law attenuation media $\quad 229$

8.1 Introduction . . . . . . . . . . . . . . . . . . . . . . . . . . . . . . . . . . . . . . . . . . . . .

8.2 Nonlinear coherence length . . . . . . . . . . . . . . . . . . . 233

8.3 Competition between nonlinearity and power law attenuation and dis-

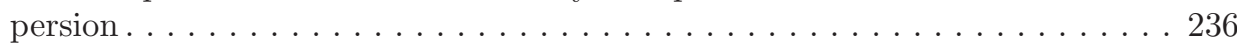

8.4 Plane wave simulations in power law media . . . . . . . . . . . . . 238

8.5 Nonlinear efficiency . . . . . . . . . . . . . . . . . . . . . . . . . 242 
8.6 Phase changes induced by dispersion . . . . . . . . . . . . . . . . . . . . . . . . . . . . . . . . . . . . . . . . . . . . . . .

8.7 Conclusions . . . . . . . . . . . . . . . . . . . . . . 247

Chapter 9 Nonlinear acoustic radiation forces in biological media 251

9.1 Introduction . . . . . . . . . . . . . . . . . . . . . . . . . . 252

9.2 One dimensional acoustic radiation force . . . . . . . . . . . . . . . . . . . . . 254

9.3 Nonlinear radiation force in tissue-like media . . . . . . . . . . . . . . . 258

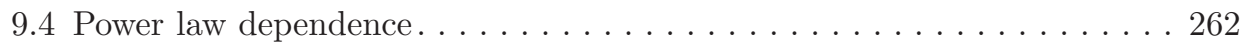

9.5 Relation of nonlinear absorption and tissue heating rate . . . . . . . . 263

9.6 Conclusions . . . . . . . . . . . . . . . . . . . . . . 269

Index of terms $\quad 272$ 


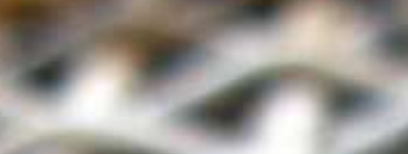
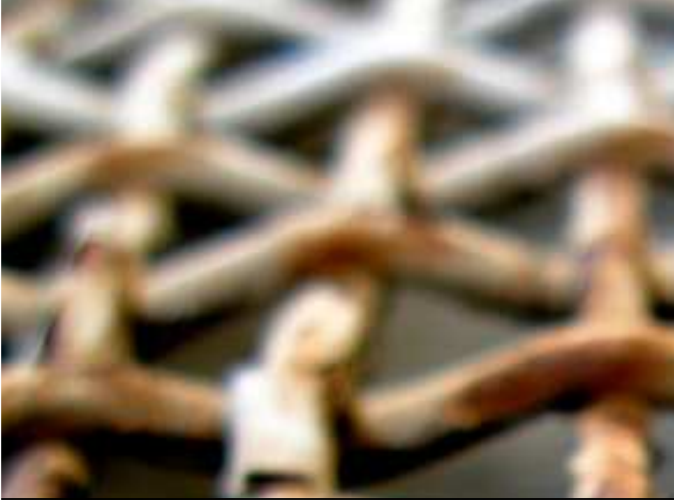

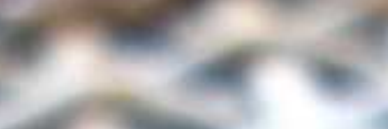
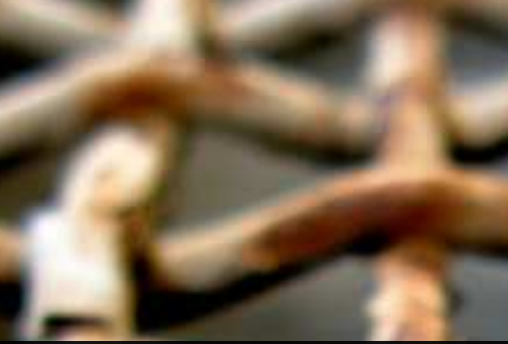

Part I

\section{Nonlinear Waves in Lattices}

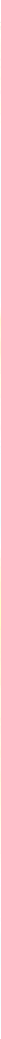





\title{
Chapter 1
}

\section{Localized Nonlinear Waves in Lattices}

\begin{abstract}
In this chapter we will study the nonlinear behavior of atomic lattices coupled by realistic potentials. Essentially, in this conservative system the dispersion is caused by lattice periodicity, where the nonlinearity appears at the coupling between oscillators following a Coulomb type interaction. Thus, strongly supersonic localized waves can be generated and propagates along the lattice in the form of kinks. With the aim of study a more realistic physical system, we include a model accounting for nuclear repulsion effects that modifies the nonlinear interaction for short interatomic distances. In addition, we propose a substrate potential that modifies the lattice dispersion relation. The physical parameters are those of the $\mathrm{K}^{+}$ion lattice in a mica muscovite layered silicate.
\end{abstract}




\subsection{Motivation: dynamics of potassium ions in layered silicates}

Many minerals are known by their capability of recording the tracks of charged particles, and are often used as solid state nuclear track detectors (SSNTDs); a review of the subject can be found in Refs. (Durrani, 2001; Durrani, 2008).

Among them, mica muscovite has been relevant as it was the second material and the first natural one where tracks from fission fragments were found (Silk et al., 1959). Very soon later fossil tracks were also found in mica (Price et al., 1962). It seems to be one of the most sensitive of natural SSNTD (Fleischer, 2011). Due to its resistance to heat, it can be used inside a nuclear reactor core for particle detection. It has also been used in geochronology and to probe the existence of dark matter (Snowden-Ifft et al., 1995), to find exotic nuclear reactions, decays of superheavy elements and weakly interacting massive particles (WIMPs) (Durrani, 2001; Durrani, 2008). Micas have also important technological applications due their dielectric and heat shielding properties. Other uses are as matrixes for films, monochromators and dispersive crystals.

Silicates are also among the materials considered as engineered barriers for nuclear waste storage due to its high rate of reaction with heavy ions in low temperature reconstructive transformations (Alba et al., 2001). This property can be of importance for the development of advanced structural ceramics (Hong et al., 2002) and has been related to the existence of nonlinear localized vibrations related with the ones described in this work (Archilla et al., 2006; Dubinko et al., 2011).

Tracks of positrons, muons and other particles have been reported (Russell, 1967b; Russell, 1967a; Russell, 1988b; Russell, 1988a) in mica muscovite. Some of these tracks were identified as produced by positrons resulting from the $\beta+$ decay of ${ }^{40} \mathrm{~K}$. This isotope is relatively abundant in the minerals and can also experience $\beta-$, electron capture and other kind of decays. Most of the tracks, however, cannot be explained as being produced by charged particles but could have been produced by some kind of vibrational excitation because, among other properties, the tracks are along the close-packed lines of the $\mathrm{K}^{+}$hexagonal layer shown in Fig. 1.1. One interpretation of these tracks is that they are formed by the localized nonlinear excitations, sometimes called quodons (Schlößer et al., 1994), whose exact nature is still not known.

A likely source for the vibrational energy required to initiate a quodon is the recoil energy of the ${ }^{40} \mathrm{~K}$ after $\beta$ decay, which can be up to $52 \mathrm{eV}$. In muscovite, there are about 3 decays per second and $\mathrm{cm}^{3}$, so after many years of the sensitive period, when tracks recording is possible, there are many possibilities to initiate a quodon. An experiment was done to shed some light into the relationship between tracks and quodons (Russell et al., 2007). A mica specimen was irradiated with alpha 


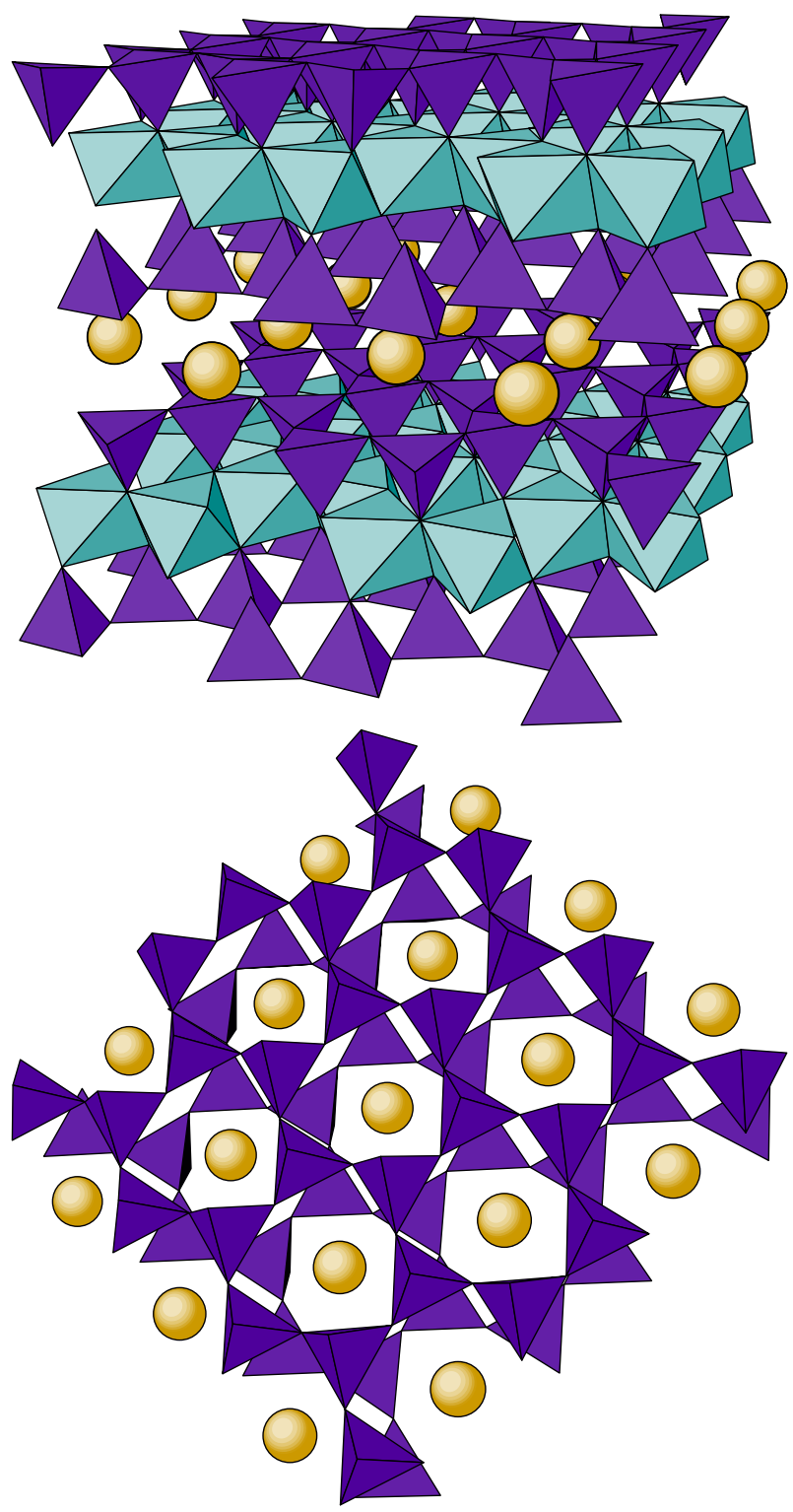

Figure 1.1: Representation of the mica structure from the point of view that emphasizes the close-packed lines of the $\mathrm{K}^{+}$hexagonal layer represented by yellow balls. Crystal structure of muscovite. The circles represent the potassium ions forming the interlayer sheet. The unit cell includes two silicate layers and two $\mathrm{K}^{+}$ions with parameters $a=5.19 \AA, b=9.02 \AA ; c=20.0 \AA$; and $\beta=95.7^{\circ}$. For a view from the top, see the later Fig. 1.16 
particles and the ejection of atoms was detected on the other side, along closed packed lines. The ejected atoms could not been identified and also sputtering energies are not known exactly, however from experimental and theoretical studies, they are known to be in the range of $4-8 \mathrm{eV}$ (Kudriavtsev et al., 2005).

In an attempt to understand this phenomenon, numerical simulations were conducted in an idealized 1D model where the particles of increasing energy hit the boundary of the lattice (Dou et al., 2011). This study reports the formation of a breather and kinks and the ejection of atoms at the opposite boundary of the sample. However, the model did not intend to use realistic values of the mica muscovite parameters.

A minimal model of the cation lattice with realistic parameters was proposed recently (Archilla et al., 2013; Archilla et al., 2014). Only $\mathrm{K}^{+}$ions were considered with the actual potassium mass and Coulomb interaction between them. The interaction with the rest of the lattice was implicitly considered as the force keeping the ions inside the crystal with the known interatomic distance in muscovite. In those publications, it was reported the existence of supersonic kinks, which were easily generated within a wide range of energies and velocities.

However such model is too simplistic because for relatively high ion energies it results in unrealistically small distances between the ions, of tenths of angstrom.

In this chapter, we develop a dynamical model in which we use the ZieglerBiersack-Littmark (ZBL) short-range repulsive potential, introduced in particle bombardment studies (Biersack et al., 2008). ZBL potential describes the Coulomb repulsion between nuclei, which is partially screened by the atom electrons and rapidly decays in few $\AA$. The results for the moving energy-carrying objects in such potential are similar to those obtained with only pure Coulomb interaction (Archilla et al., 2013; Archilla et al., 2014), namely the supersonic kinks, traveling without attenuation for long distances, can be produced with arbitrary energies. But the inter-particle distances in the kinks are limited by physically reasonable values in our dynamical model. Note that our dynamical model allows for bond dissociation, which is a necessary condition for the realistic modeling of the normal energy transport in low-dimensional systems (Savin et al., 2014).

As a next step towards a more realistic description, we construct explicitly the interaction with the surrounding atoms, using standard empiric potentials introduced in molecular dynamics, which give rise to a periodic non-sinusoidal substrate potential. Supersonic lattice kinks, also called crowdions (Kosevich et al., 1973), are also easily obtained. They propagate in such potential with a velocity which is independent of the input and is determined only by the lattice potential parameters. For the smaller energies, the kink dissipates in phonons, while for larger energies the excess energy is radiated until reaching the value of crowdion energy.

The final crowdion energy is approximately $26 \mathrm{eV}$, which can be provided by 
the recoil of isotopes of potassium after radioactive decay and is larger than the sputtering energy. This ratio of characteristic energies allows to assume that the tracks found in mica muscovite can be related with the lattice kinks or crowdions.

The literature about kink propagation in lattices with different inter-particle and on-site potentials is extensive. The most studied and generic model is the Frenkel-Kontorova (FK) model(Frenkel et al., 1938), see reviews in Refs. (Chaikin et al., 1995; Braun et al., 1998; Braun et al., 2004). However, most of the kinks considered in this model are subsonic ones. Supersonic kinks in the systems with substrate have been found in models with anharmonic intersite coupling (Kosevich et al., 1973; Milchev, 1990; Savin, 1995; Zolotaryuk et al., 1997). They have the property than only a discrete set of velocities allows the propagation of kinks without attenuation. They can be described as multi-kinks or lattice N-solitons depending on whether the description is done in terms of coordinates, strains or velocities. The kink with the unique supersonic velocity, propagating in our dynamical model of the cation layer on a substrate, is a double-kink.

The FK model has also been considered in layered materials, to model, for example, the in-plane dynamics of a few-layer graphene, in order to explain molecular dynamics simulation results for the cross-plane thermal conductance (Ni et al., 2014). One particular characteristic, found in the kinks studied in the present work, is extreme discreteness of the kinks, namely only two particles, and for higher energies, practically only one particle is in motion at a given time. This discreteness allows the complete understanding in physical terms of the mechanism that brings about a single velocity of the kink and why the kink is a double-kink.

The Chapter is organized as follows. First we review and extend the results obtained with only the Coulomb interaction, using the sinusoidal waveform proposed for supersonic kinks in the Fermi-Pasta-Ulam (FPU) lattice (Kosevich, 1993; Kosevich et al., 2004). The sinusoidal waveform is a good description for $\lambda \simeq 3 a$, where $\lambda$ is a characteristic wavelength of the sinusoidal waveform and $a$ is a lattice constant, but fails for $\lambda \simeq 2 a$, been replaced by an almost triangular waveform, corresponding to nearly hard-sphere collisions. Afterward we consider the effects of long-range interactions, with several neighbours, and introduce a short-range nearest-neighbor ZBL potential. Thereafter, the substrate potential is constructed and the properties of the single-velocity lattice kinks in the cation layer on the substrate are analyzed with details.

\subsection{Minimal model with realistic potentials}

\subsubsection{Model}

We consider as a starting point a one-dimensional (1D) model for the dynamics of $\mathrm{K}^{+}$ions. Thus, considering nearest-neighbour Coulomb interaction the position, 
$x_{n}$, of each $n$-th atom is given by

$$
m \frac{\partial^{2} x_{n}}{\partial t^{2}}=-\frac{\mathrm{K}_{\mathrm{C}} \mathrm{e}^{2}}{\left(x_{n+1}-x_{n}\right)^{2}}+\frac{\mathrm{K}_{\mathrm{C}} \mathrm{e}^{2}}{\left(x_{n}-x_{n-1}\right)^{2}},
$$

where $m$ is the $\mathrm{K}^{+}$mass, $\mathrm{K}_{\mathrm{C}} \mathrm{e}^{2}$ is the inter-ion Coulomb electrostatic constant. With the aim of explore solutions from a more general approach, we can write the equation in dimensionless form by choosing as unit of distance, $a=5.19 \AA$ (i.e. the lattice constant or the equilibrium distance between $\mathrm{K}^{+}$ions; for masses, the mass of a $\mathrm{K}^{+}$ion, $m_{\mathrm{K}^{+}}=39.1 \mathrm{amu}$; for time, $\tau=\sqrt{m_{K} a^{3} / \mathrm{k}_{\mathrm{e}} \mathrm{e}^{2}} \simeq 0.2 \mathrm{ps}$, where $\mathrm{k}_{\mathrm{e}}$ is the Coulomb constant and $e$ is the elementary unit of charge. Other physical units in the system are velocity $u_{V}=2600 \mathrm{~m} / \mathrm{s}$, energy $u_{E}=2.77 \mathrm{eV}$ and frequency $5 \mathrm{THz}$. Dimensionless speed of sound in this system is $c_{0}=\sqrt{2}$, or about $3.700 \mathrm{~km} / \mathrm{s}$ in physical units.

Thus, defining $u_{n}$ as the displacement with respect to the equilibrium position normalized to the lattice constant, witch can be expressed as $x_{n}=a\left(n+u_{n}\right)$, the dimensionless motion equations become

$$
\frac{\partial^{2} u_{n}}{\partial t^{2}}=-\frac{1}{\left(1+u_{n+1}-u_{n}\right)^{2}}+\frac{1}{\left(1+u_{n}-u_{n-1}\right)^{2}},
$$

which describes the chain of ions coupled to their nearest neighbours by electrostatic Coulomb potential. Note hereinafter $t$ will be dimensionless time.

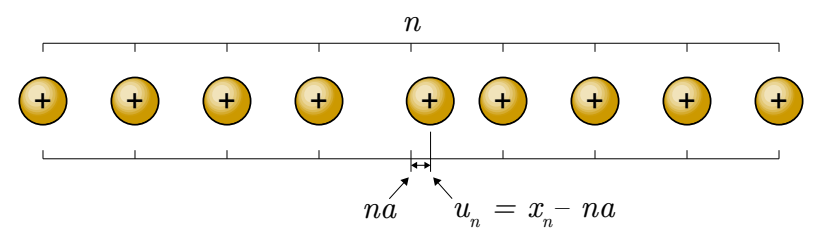

Figure 1.2: Scheme of the one dimensional model of the $\mathrm{K}^{+}$ion layer

\subsubsection{FPU equivalent lattice}

For small amplitudes, the potentials in Eq. (1.2) can be expanded in a series, using that $1 /(1+y)^{2} \simeq 1-2 y+3 y^{2}-4 y^{3} \ldots$ Retaining cubic and smaller terms, we obtain

$$
\begin{aligned}
\frac{\partial^{2} u_{n}}{\partial t^{2}}= & c_{0}^{2}\left[\left(u_{n+1}+u_{n-1}-2 u_{n}\right)\right. \\
& -3 / 2\left(u_{n+1}-u_{n}\right)^{2}+3 / 2\left(u_{n}-u_{n-1}\right)^{2} \\
& \left.+2\left(u_{n+1}-u_{n}\right)^{3}-2\left(u_{n}-u_{n-1}\right)^{3}+\ldots\right],
\end{aligned}
$$


which are the $\alpha-\beta$ FPU equations of motion. We would like to emphasize that the $\alpha$ - $\beta$ FPU Eq. (1.3) describes the Coulomb lattice (1.2) only in the smalland intermediate-amplitude limit and is not applicable to this lattice in the largeamplitude limit, see Fig. 1.9 and the subsection devoted to the triangular waveform below.

\subsubsection{Dispersion relations}

Linearization Eq. (1.2), i.e. setting $\alpha=\beta=0$ in Eq. (1.3), the equation of motion is reduced to the well-known discrete linear wave equation

$$
\frac{\partial^{2} u_{n}}{\partial t^{2}}=c_{0}^{2}\left(u_{n+1}+u_{n-1}-2 u_{n}\right),
$$

where $c_{0}$, the speed of sound, is the long wavelength phonon velocity. Note that in our scaling $c_{0}=\sqrt{2}$. A phonon corresponds to small amplitude particle displacements of the form $u_{n}=\exp (i(q n-\omega t))$, with $q$ and $\omega$ being the phonon wave number and frequency respectively. The dispersion relation and velocities are well known, we include them here for comparison with. Thus, the the phonon dispersion equation in normalized units reads

$$
\omega^{2}=4 c_{0}^{2} \sin ^{2} \frac{q}{2} \quad \text { with } \quad q=\frac{2 \pi m}{N} \quad \text { and } \quad m=1,2, \ldots, N .
$$

Then, phase and group velocity are expressed for the lattice as

$$
\begin{aligned}
& c_{p}=\frac{\omega}{q}=2 c_{0}\left|\frac{\sin \frac{q}{2}}{q}\right|, \\
& c_{g}=\frac{\partial \omega}{\partial q}=c_{0}\left|\cos \frac{q}{2}\right| .
\end{aligned}
$$

Thus, those well known relations dispersion of the linear lattice and its group and phase velocity velocity are plotted for the positive first Brillouin zone in Fig. 1.3. The maximum frequency corresponding to the mode with the wave number $q=\pi$ is $\omega_{M}=2 c_{0}$. In the limit of long wavelengths, both velocities are equal to the speed of sound $c_{0}$, which is also the maximum value. Note that the group velocity becomes zero at the top of the phonon band $q=\pi$.

The maximum frequency in physical units is about $2.2 \mathrm{THz}$ which is larger than the one obtained with molecular dynamics and neutron spectroscopy of about 1.6 THz (Collins et al., 1993; Wada et al., 1991; Chaplot et al., 2002). This is understandable given the simplicity of our model taking into account just one type of atom compared with the complexity of real mica, but it is within the same range of values. Note that the predicted speed of sound in physical units, $c_{0}=3690.2 \mathrm{~m} / \mathrm{s}$, is similar to that found in Ref. (Brudeylins et al., 1995) of $3400-3700 \mathrm{~m} / \mathrm{s}$. 

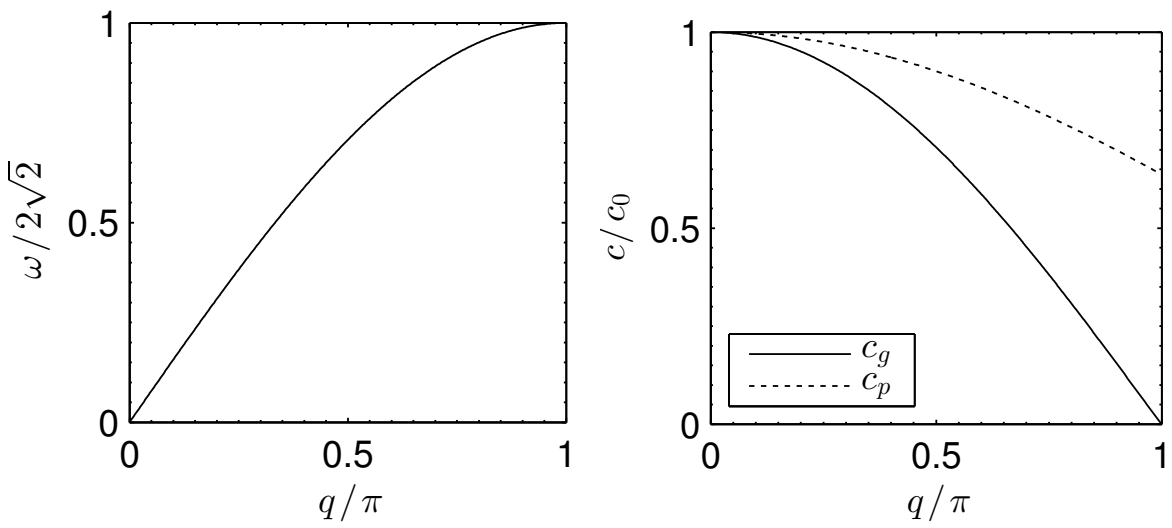

Figure 1.3: Left: Dispersion relation for the linearized Coulomb lattice. Right: Phase speed and group velocity for linearized Coulomb lattice. In the limit of long wavelengths, both give the sound velocity, which can be compared with the values of $3.4-3.7 \mathrm{~km} / \mathrm{s}$ obtained in Ref. (Brudeylins et al., 1995)

Also note that, in contrast with the repulsive character of the full Coulomb interaction, this linearized equation is attractive, i.e. if the distance $u_{n+1}-u_{n}$ increases, the force on the particle $u_{n}$ is positive. The reason for it is the decreasing nature of the Coulomb force: when the distance with the particle in front increases the repulsion weakens and the repulsion from the particle behind becomes larger pushing the particle $n$ forward.

\subsection{Tail analysis}

Different types of nonlinear localized solutions are supported by the Coulomb lattice. Although these solutions are large enough to be intrinsically nonlinear, they also have tails of small amplitude which abide the linear equation. Tails cannot exist by themselves but depends on the existence of the nonlinear solution, however its study gives information about the parent excitation. We obtain first the tail properties and later we consider the fully nonlinear solutions. Tails are obtained with the linear system, therefore their properties are common to any system with the same linearization. However, they only give the conditions that the tails have to fulfill. The properties of existence of the nonlinear excitation, its velocity, amplitude and other characteristics have to be worked out with the whole nonlinear system.

Consider a nonlinear localized moving excitation, travelling with velocity $V$. We also assume that its amplitude decays exponentially far away enough from the 

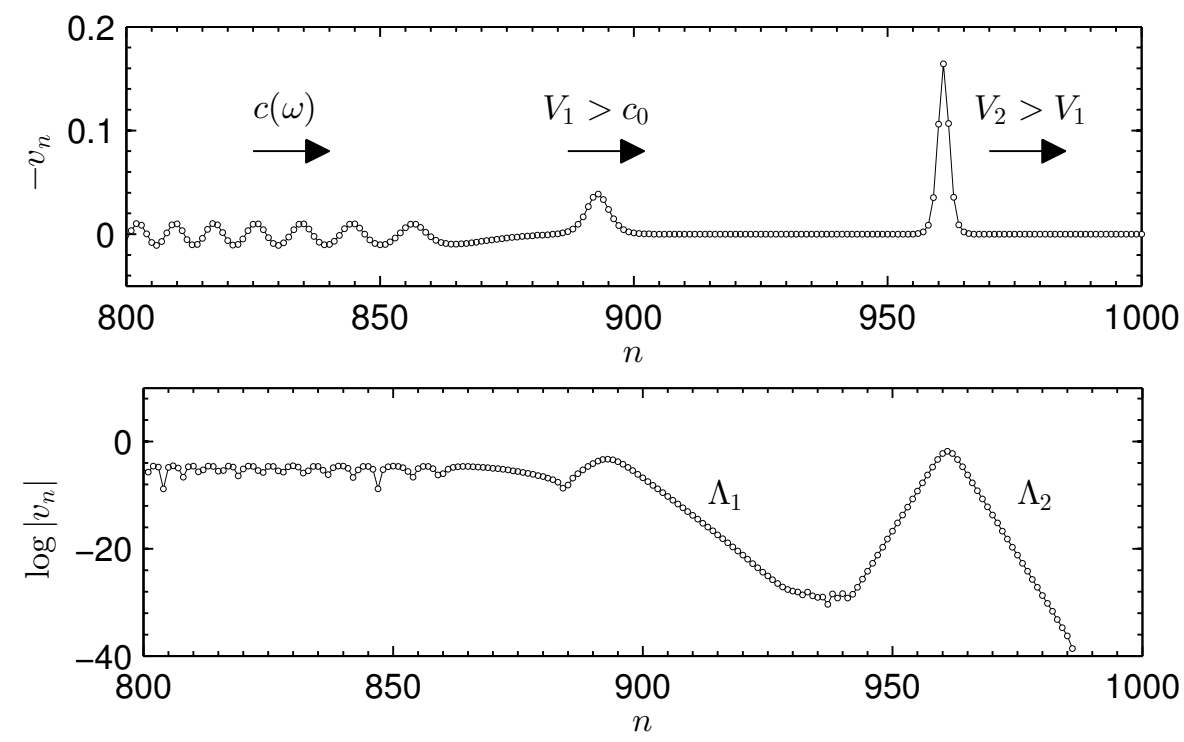

Figure 1.4: Numerical example of two nonlinear localized waves travelling at different velocities and leaving a phonon tail. (Top) profiles obtained at fixed time. (Bottom) logarithmic representation showing that front and back tails exist with different decay length. Despite the type of the excitation and its nonlinear features, the tail analysis predict the relation between the parent velocity and its localization, i.e. $V_{i}\left(\Lambda_{i}\right)$.

center. Tails are well described by the expression

$$
u_{n}=\exp (-\xi(n-V t)) \exp (\mathrm{i}(q n-\omega t))
$$

where $|\xi|$ is the localization parameter and its inverse $\Lambda=1 /|\xi|$ the decay length. This equation is valid for $n>V t$ if $\xi>0$ and it corresponds to the front tail of the structure, where for $n<V t$ if $\xi<0$ corresponding to the back tail. The limit case $\xi=0$ corresponds to extended phonons. Figure 1.4 shows an example were two localized waves are numerically obtained presenting different tails and velocities.

Since the tail amplitude is by definition small, it satisfies the linear equation Eq. (1.4). Using that $u_{n+1}=\exp (-\xi+\mathrm{i} q) u_{n}, u_{n-1}=\exp (\xi-\mathrm{i} q) u_{n}$ and $\dot{u}_{n}=$ $(\xi V-\mathrm{i} \omega) u_{n}$, substitution of (1.7) in (1.4) yields the following two equations:

$$
\begin{aligned}
\xi^{2} V^{2}-\omega^{2} & =2 c_{0}^{2}(\cosh (\xi) \cos (q)-1) \\
\xi V \omega & =c_{0}^{2} \sinh (\xi) \sin (q)
\end{aligned}
$$

As there are two equation with four unknowns, we can express two of them as $\omega$ and $V$ as functions of the other two $\xi$ and $q$. First, the frequency $\omega$ is given by:

$$
\omega=\cosh (\xi / 2) 2 c_{0} \sin (q / 2)=\cosh (\xi / 2) \omega_{\text {phonons }} .
$$




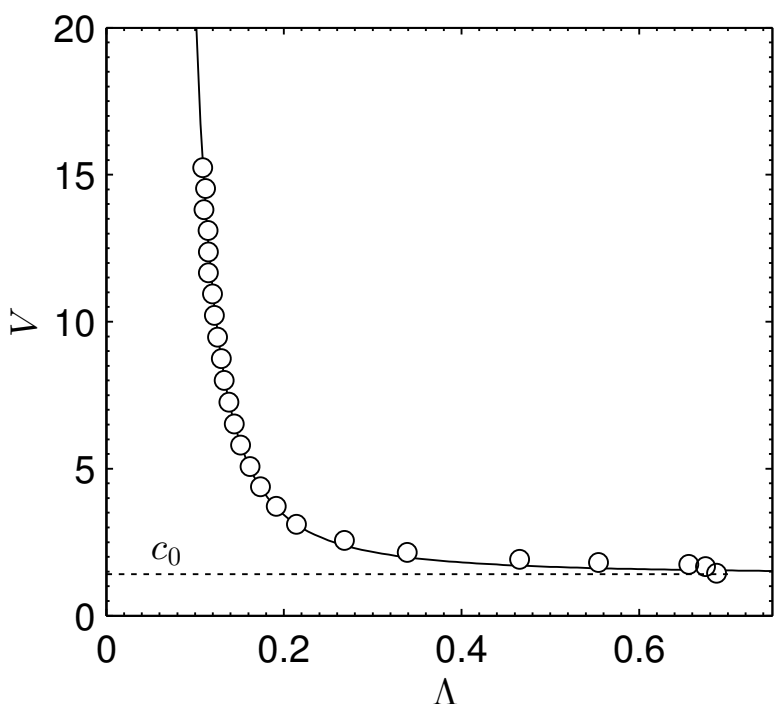

Figure 1.5: Comparison of the numerical (circles) and theoretical (continuous line) decay lengths as a function of the velocity for a localized wave (kink). Dimensionless units

Note that Eq. (1.9) reduces to the phonon dispersion equation when there is no localization, i.e. $\xi \rightarrow 0$.

The velocity of the tail of the hypothetical localized wave is given by:

$$
V=\frac{\sinh (\xi / 2)}{\xi / 2} c_{0} \cos (q / 2)=\frac{\sinh (\xi / 2)}{\xi / 2} c_{g}
$$

The relationship between the decay length $\Lambda=1 /|\xi|$ and $V$ given by the equation above is in excellent agreement with the numerical results, as can be seen in Fig. 1.5. Note that both $\sinh (\xi / 2) /(\xi / 2)$ and $\cosh (\xi / 2)$ are larger than the unity and also monotonically increasing functions in $|\xi|$, which means that the tails oscillate and move faster than the phonons. Except for the degenerate case $\xi=0$, which corresponds to no localization and no tail, i.e., to phonons. Essentially, the conclusion that arise for the tail analysis states that localized travelling waves, either solitons or kinks, with tails following Eq. (1.7) are supersonic, where its velocity depends on the localization. Also note this analysis was developed with the linearized discrete wave equation and does not depend of the specific nonlinearity of the coupling. Is thus generic for many systems of coupled oscillators. However, for studying the specific features of localized nonlinear travelling waves in the Coulomb lattice the nonlinear terms can not be neglected. 
(a)

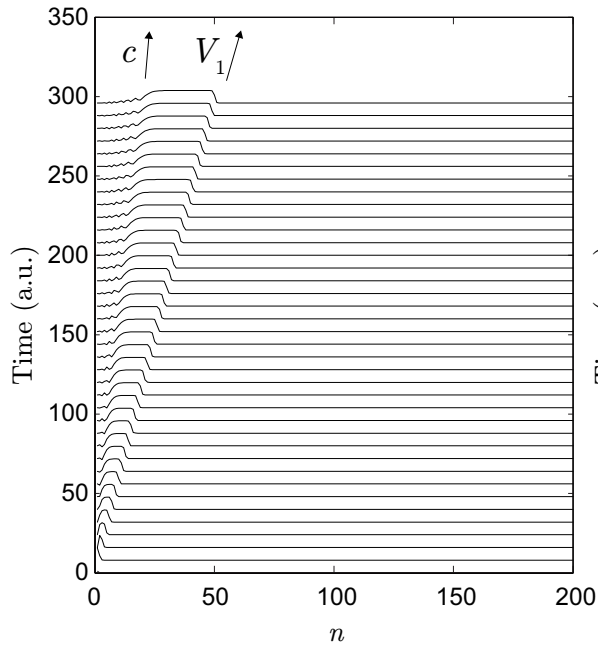

(b)

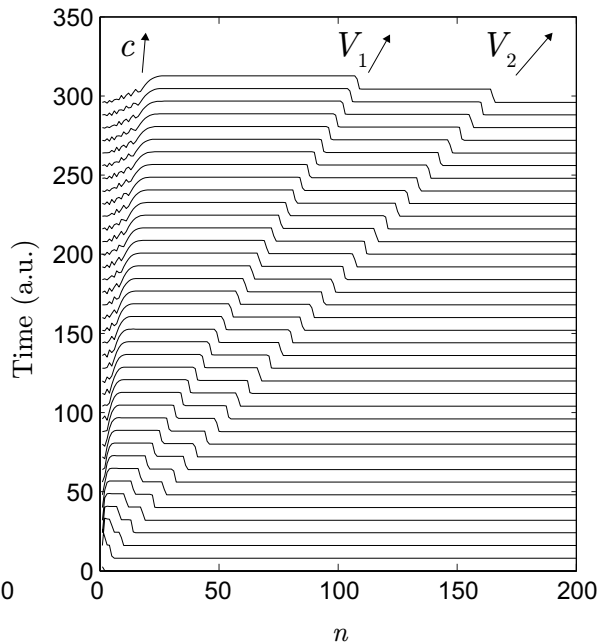

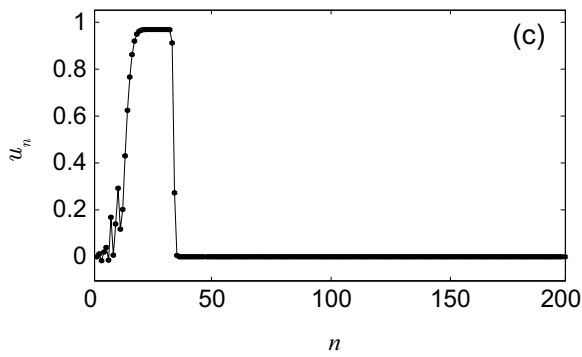

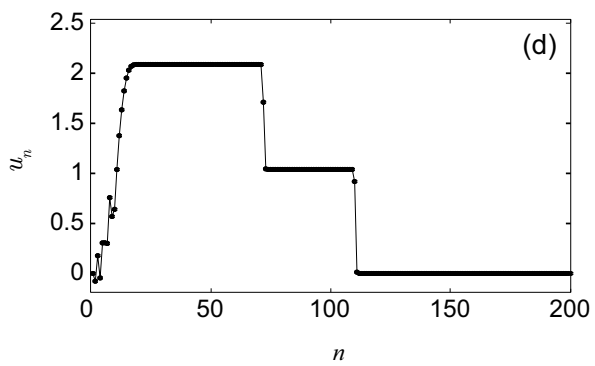

Figure 1.6: (a) Profile and evolution of a single kink for initial amplitude $A_{0}=1.05$. (b) Excitation of multikinks for initial amplitude $A_{0}=2.5$ where two kinks with different velocities are observed. (c,d) Detail of the corresponding displacements at $t=200$ scaled units. A tail of phonons is left behind the localized travelling nonlinear waves.

\subsection{Supersonic ultra-discrete kinks}

\subsubsection{First supersonic kinks and multi-kinks}

We propose the study of kink solutions in the lattice. Essentially, a kink is a nonlinear localized wave that connects and divide two phases of the domain. Kinks can be stationary or propagating. We find that the simplified proposed model for the repulsive lattice is extremely well suited for propagation ultra-discrete supersonic kinks and multi-kinks. Due to the analytical analysis of the kink characteristics will be somehow restricted, a numerical integration of the complete set of equations of motion has been used to explore the full nonlinear features of the lattice. 
The numerical evaluation of the Eq. (1.2) retaining the exact form of the Coulomb type interaction is trivial using centered finite differences with a "leapfrog" time marching scheme. Thus, for one-dimensional lattices of $N<1000$ elements and the integration times considered on this Chapter, the temporal step required can be small enough to achieve convergence and the error due to numerical dispersion can be controlled for all simulations.

Thus, the boundary conditions for the simulations are set to fix at rest the first and last atoms of the chain, i.e. those conditions implicitly includes the effects of the other atoms of the mica crystal in the simplest way, forcing the central part of the chain to remain at rest. Then, a excitation is forced in one extreme of the chain. As a first example, as in Ref. (Kosevich et al., 2004), the first particle in the chain is forced to move sinusoidally during half a period, with an amplitude $A_{0}$, so that the initial and final positions are both zero (more details of this waveform will be given in following sections). Increasing the amplitude of the perturbation $A_{0}$ the velocity of the kink increases, and eventually, two, three, and any number of kinks are produced, travelling one after the other. The dependency of the velocity and number of kinks with the initial amplitude is shown in Fig. (1.6) for one (a, c) and for two (b, d) kinks.

\subsubsection{Test solution}

In order to obtain the properties of the full nonlinear system it is convenient to introduce a new variable, the deformation from the equilibrium position or strain $v_{n}=u_{n}-u_{n-1}$, the equations above can be written as:

$$
\frac{\partial^{2} v_{n}}{\partial t^{2}}=2 F_{n}-F_{n+1}-F_{n-1} ; \quad \text { with } \quad F_{n}=\frac{1}{\left(1+v_{n}\right)^{2}},
$$

where $v_{n}=0$ corresponds to the unperturbed lattice. The boundary conditions assume fixed particles at the ends of the lattice. Kinks are produced numerically by applying at the chain boundary a half-wave perturbation, which is a sinusoidal displacement during half of a period, starting and finishing at the equilibrium position (Archilla et al., 2013; Kosevich et al., 2004). In order to describe a kink traveling to the right, the following ansatz was introduced as in Refs. (Kosevich, 1993; Kosevich et al., 2004):

$$
v_{n}=-\frac{A}{2}(1+\cos (q n-\omega t)) \quad \text { if } \quad-\pi \leq q n-\omega t<\pi,
$$

and $v_{n}=0$ otherwise, where $A$ is the kink strain amplitude. The bonds are always compressed so $v_{n}$ is negative with a minimum value of $-A$, corresponding to the maximum compression of the bond. For analogy, we use the usual wave terminology, so $\phi_{n}=\omega t-q n$ is the phase; $q$ is the wave number; $\omega$ is the angular 

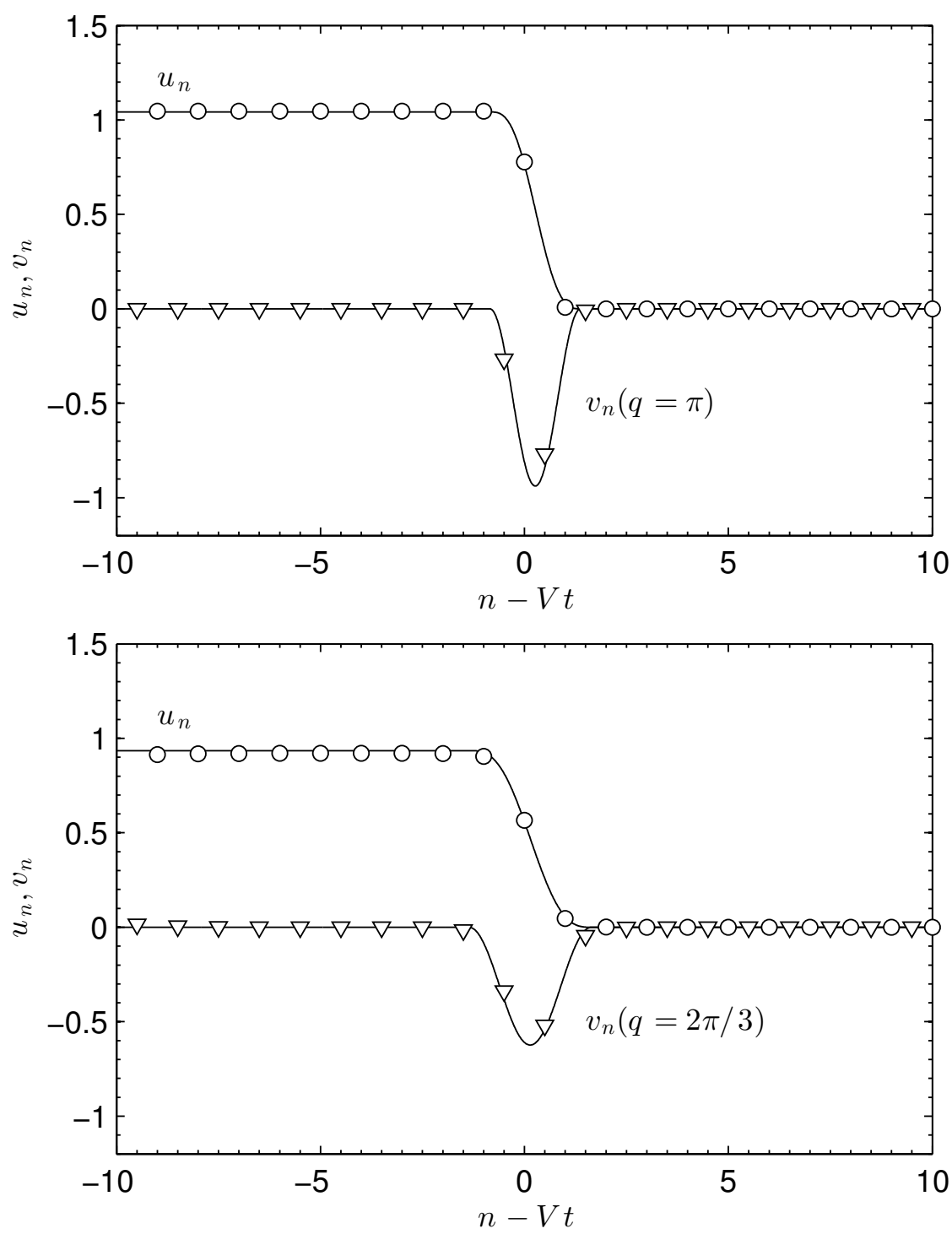

Figure 1.7: Spatial profile at fixed time for kinks represented in displacement $\left(u_{n}\right)$ and strains $v_{n}=u_{n}-u_{n-1}$. Circles and triangles are numerical results and the continuous lines are obtained from Eq. (1.12) with magic wave number $q \simeq 2 \pi / 3$ (top), when basically two particles are moving at a given time, and with $q \simeq \pi$ (bottom) for the amplitude $A$ close to 1 , when basically one particle is moving at a given time. Scaled units are equal to the lattice unit. 
frequency; $T$ is the period; $\lambda=2 \pi / q$ is the wavelength. The sinusoidal waveform with $q=2 \pi / 3$, being an exact solution for the FPU lattice(Kosevich, 1993; Kosevich et al., 2004), is not an exact solution for the Coulomb lattice but is a good approximation for intermediate values of the amplitude $A$ and provides very useful framework for understanding the relative phases of the particles and the behavior of the kink.

Figure 1.7 (top) compares the result of the numerical simulation for displacements and strains, with the corresponding analytical expressions derived from Eq. (1.12), for the intermediate value of the amplitude $A=0.55$ and wave number $q \simeq 2 \pi / 3$. The analytical expression fits very well the numerical data. Figure 1.7 (bottom) pictures a kink with wavenumber $q=\pi$. There are not enough particles to compare with the analytical form, but the main properties of the latter are valid.

For the dimensionless wave number $q=2 \pi / \lambda$, with $\lambda$ an integer, Eq. (1.12) represents a solution where basically $\lambda$ bonds and $\lambda-1$ particles (the kink core) are in motion, while the others remain at rest.

We will use the term active to describe related states of the different magnitudes. The active particles or coordinates at a given time or time interval (or phase or phase interval) are those for which $u_{n}$ is changing, the active bonds are those for which $v_{n} \neq 0$, i.e., they are the compressed ones. For a particle, the time interval is active when it is moving, and for a bond - when it is compressed.

If the dimensionless wavelength $\lambda$ is between two integers $m_{1}$ and $m_{2}$, the number of active bonds oscillates between $m_{1}$ and $m_{2}$ and the number of active oscillating particles is between $m_{1}-1$ and $m_{2}-1$.

Of particular interest in this work will be $\lambda=3$, with $q=2 \pi / 3$, which is called the magic wave number (Kosevich et al., 2004), and $\lambda=2$, with $q=\pi$, which will be referred to as the $\pi$-mode. These two values are extreme cases of localization, with $q=\pi$ being the limit when only one particle is moving at a given time.

\subsubsection{Rotating wave approximation}

The velocities of the kinks can be analytically obtained using the rotating wave approximation (RWA), which consists in an approximation of the Fourier series up to the first harmonic (Archilla et al., 2013). When we substitute (1.12) in the equation of motion (1.11), nonlinearity generates multiple frequencies. The RWA approximation states that nonlinear contributions higher than second harmonics can be neglected. This implies that the force terms can be approximated by their first-order Fourier expansions, as $F_{n}=a_{0}+a_{1} \cos (q n-\omega t)$. On the other hand, the left hand side of Eq. (1.11) gives, after substitution of Eq. (1.12), $\ddot{v}_{n}=(A / 2) \omega^{2} \cos (q n-\omega t)$ which is equal to the first harmonic term of the right hand side. The zero-harmonic cancels out due to the dependency of the right hand 


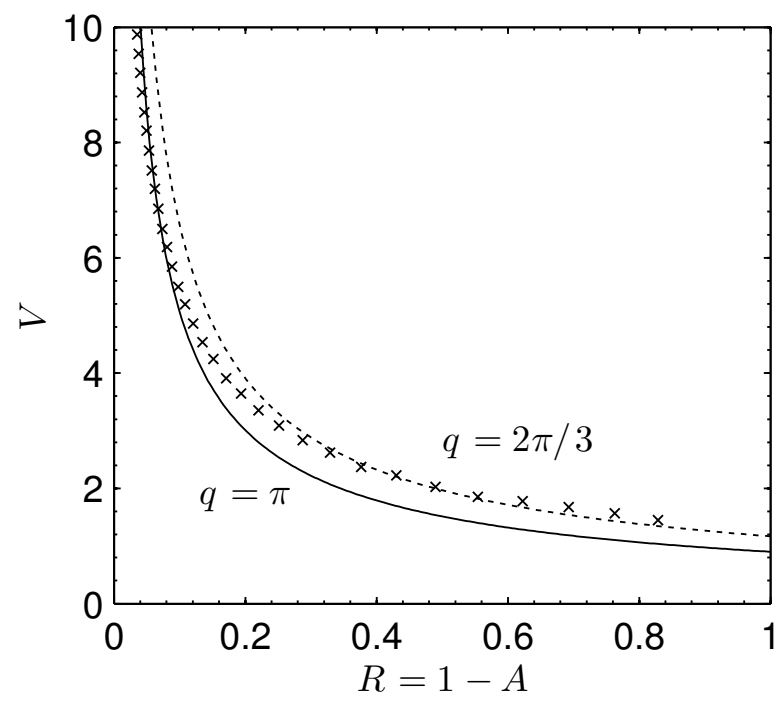

Figure 1.8: Kink velocity versus minimal interparticle distance $R=1-A$, calculated numerically (crosses) and analytically from Eq.(1.15) with wave number $q=2 \pi / 3$ (upper dotted-dashed line) and $q=\pi$ (lower dotted-dashed line). Scaled units are the lattice constant for distances and $2.6 \mathrm{~km} / \mathrm{s}$ for velocities.

side on $F_{n}$. The first harmonic of $F_{n}$ is given by:

$$
a_{1}=\frac{1}{\pi} \int_{-\pi}^{\pi} \frac{\cos (\theta) \mathrm{d} \theta}{\left(1-\frac{A}{2}-\frac{A}{2} \cos (\theta)\right)^{2}} .
$$

For $A<1$ we obtain

$$
a_{1}=\frac{A}{(1-A)^{3 / 2}}
$$

Therefore, substituting $\ddot{v}_{n}$ and $F_{n}=a_{0}+a_{1} \cos (q n-\omega t)$ in Eq. (1.11), is straightforward to obtain the frequency as a function of the amplitude $A$ :

$$
\omega=\frac{1}{(1-A)^{3 / 4}} 2 c_{0} \sin (q / 2)
$$

and the velocity as:

$$
V=\frac{1}{(1-A)^{3 / 4}} c_{0} \frac{\sin (q / 2)}{(q / 2)} .
$$

Kinks are therefore supersonic. These magnitudes are equal to the corresponding ones for phonons, multiplied by a amplitude factor $1 /(1-A)^{3 / 4}$ : in the small 


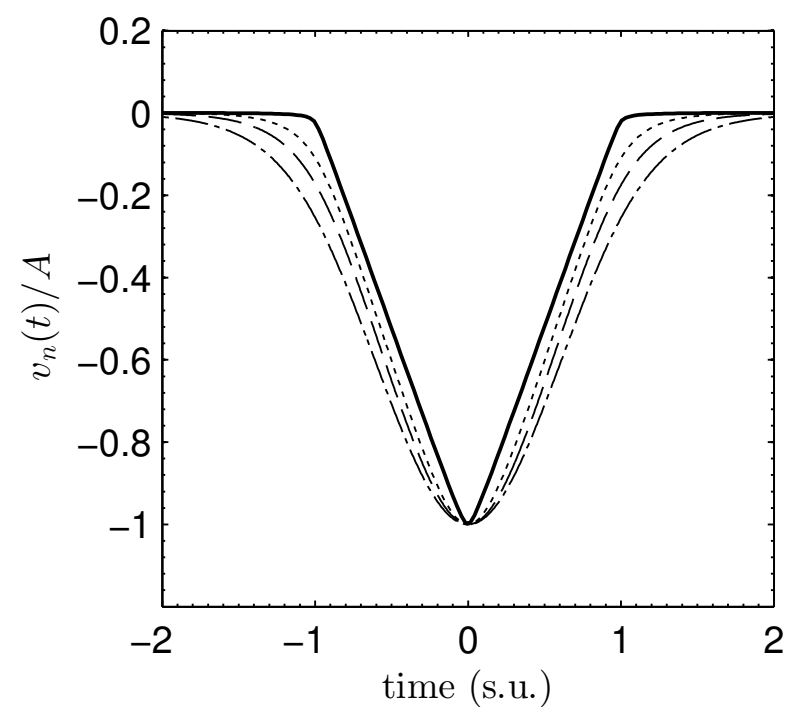

Figure 1.9: Temporal variation of the strain $v_{n}=u_{n}-u_{n-1}$ for different amplitudes. As the amplitude $A$ increases, the shape becomes almost triangular, corresponding to almost hardspheres collisions. The amplitude $A$ is given in lattice units and the scaled time unit is 2 ps.

amplitude $(A \rightarrow 0)$ and long wavelength $(q \rightarrow 0)$ limit, $V$ tends to the sound speed $c_{0}$.

In Ref. (Archilla et al., 2013) it was shown that the magic wave number represents a good agreement between the ansatz and simulations. However this agreement fails at the kink amplitudes $A$ approaching unity, when the minimal interparticle distance in the kink (in lattice units) $R=1-A$ diminishes and the $q=\pi$ brings about a much better fit as can be seen in Fig. 1.8. The conclusion is that the magic wave number is a suitable approximation for an intermediate range of amplitudes in the Coulomb lattice, in contrast to the FPU chain where it is valid for all amplitudes(Kosevich, 1993; Kosevich et al., 2004), and that $q$ actually changes continuously with the amplitude in the Coulomb lattice. The complementary approach is to use Eq. (1.15) to find the values of the wave number $q$, with respect to the amplitude $A$ or the velocity $V$. This will be shown in Fig. 1.14, where it can be appreciated that the magic wave number is a satisfactory solution only for the intermediate values of kink velocity in the Coulomb lattice.

\subsubsection{Triangular waveform}

For the higher amplitudes, with $A$ close to 1 , the waveform deviates from the sinusoidal one given by Eq. (1.12) and approaches instead the triangular waveform, which is shown in Fig. 1.9. The almost straight lines in such waveform mean that 
the velocity is almost constant except during a very short interaction time. The system behavior is very similar to that of the hard-spheres model.

This waveform is also another way of looking at the mode $q=\pi$. In this mode only one particle is moving at a given time, which means that there are no forces on the particle acting from its neighbours (because it moves at constant velocity and those forces would accelerate it). Strictly speaking, the mode $q=\pi$ and an exact triangular waveform are unattainable because of the electrostatic Coulomb forces acting on the particles. However, if the energy of the particle is large with respect to the change in the potential during a large part of the path between collisions, the particle will move almost free during most of the time between collisions.

The triangular waveform has been described as the high-energy limit for the Lennard-Jones interatomic potential (Friesecke et al., 2002) and has been observed experimentally in a system of repelling magnets (Molerón et al., 2014). It is worth mentioning that the triangular waveform can also be related with strongly-stretched bonds in the high-energy limit in the potentials allowing for bond dissociation, like the Lennard-Jones potential. The strongly-stretched bonds result in finite (normal) thermal conductivity in one-dimensional systems with such interatomic potentials (Savin et al., 2014).

\subsubsection{Analytical results}

\section{Sinusoidal waveform and mode with $q=2 \pi / 3$}

Some analytical results can be obtained in this model, see also (Kosevich et al., 2004). Let us consider the wavenumber $q=2 \pi / 3$ and choose $t=0$ for the time for which $\phi_{n}=q n-\omega t=0$ at $n=0$ after a change of the origin of $n$ and $t$, but keep the notation $n$ for generality. If we consider the time interval $\Delta t:-T / 6 \leq t<T / 6$, there are three active strains: $v_{n+1}, v_{n}$ and $v_{n-1}$. At the end of the interval, that is at $t=T / 6, v_{n-1}$ becomes zero and $v_{n+2}$ starts being perturbed, so all the indexes $n$ of the particles will change in a unity.

During $\Delta t, u_{n^{\prime}}=0$ for $n^{\prime} \geq n+1, u_{n}$ and $u_{n+1}$ are changing but $u_{n-1}$ has already attained the value $3 A / 2$, its final constant value as can be checked by direct sum of 1.12 for $q=2 \pi / 3$. Also, $u_{n^{\prime}}=3 A / 2$ for $n^{\prime}<n-1$, their final value after the kink has passed over them as seen in Fig. 1.7. The active coordinates are therefore $u_{n}=-v_{n+1}$ and $u_{n-1}=-v_{n}+v_{n+1}$, i.e. the mode $q=2 \pi / 3$ states that essentially two particles are moving at same time and there are tree active bounds. 
After some algebra we get

$$
\begin{aligned}
u_{n} & =\frac{A}{2}+\frac{A}{2} \cos \left(\phi_{n}+2 \pi / 3\right) \\
u_{n-1} & =A-\frac{A}{2} \cos \left(\phi_{n}-2 \pi / 3\right) .
\end{aligned}
$$

We can obtain immediately the kink kinetic energy as $K=\frac{1}{2} \dot{u}_{n}^{2}+\frac{1}{2} \dot{u}_{n-1}^{2}$, which results in

$$
K=\frac{\omega^{2} A^{2}}{8}\left(1+\frac{1}{2} \cos \left(2 \phi_{n}\right)\right),
$$

with maximum value

$$
K_{M}=\frac{\pi^{2}}{12} V^{2} A^{2}
$$

The potential energy, with respect to the equilibrium state, is given by:

$$
U=\frac{1}{1+v_{n-1}}+\frac{1}{1+v_{n}}+\frac{1}{1+v_{n+1}}-3 .
$$

By substitution, it can be seen that the maximum potential energy corresponds to the bond $n$ at its maximum compression, i.e., with $\phi_{n}=0$, while the bonds $n-1, n+1$ have a phase difference of $\pm 2 \pi / 3$ with a strain $u_{n-1, n+1}=$ $-A / 2(1+\cos ( \pm 2 \pi / 3))=-A / 4$. Therefore the maximum energy becomes:

$$
U_{M}=\frac{1}{1-A}+\frac{2}{1-A / 4}-3 .
$$

There is also a minimum potential energy which corresponds to the limit, in which only two bonds are different from zero with phases $\pm \pi / 3$ and strain $v_{n}=-3 A / 4$. The minimum energy becomes

$$
U_{m}=\frac{2 A}{1-3 A / 4}-2 .
$$

Therefore the kink for the mode $q=2 \pi / 3$ has always some compression energy $U_{m}$ above the ground state. 


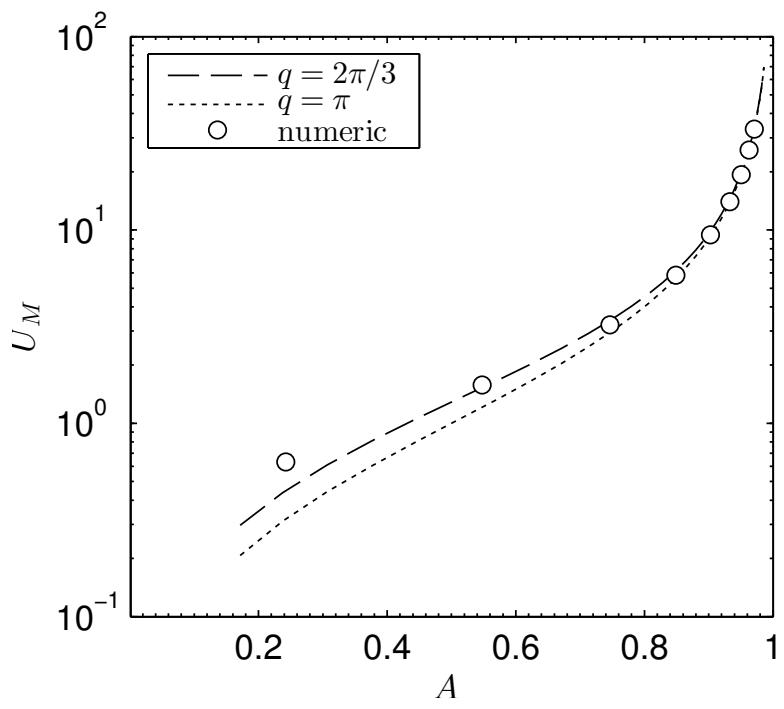

Figure 1.10: Maximum potential energies of the kinks in the Coulomb potential versus minimal interparticle distance in lattice units $R=1-A$ (for amplitude $A$ close to 1 ). The analytical results are very similar for the waveforms with $q=\pi$ and $q=2 \pi / 3$, as the maximum potential energy of the kink depends mainly on the minimal separation between particles $R$. The values of the dimension units are the lattice unit and $2.77 \mathrm{eV}$ respectively.

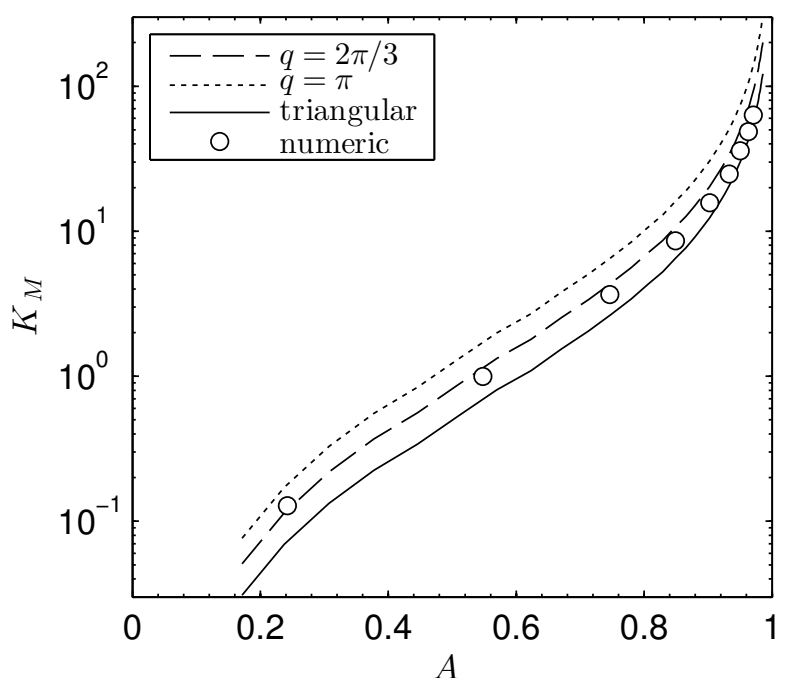

Figure 1.11: Maximum kinetic energies of the kinks in the Coulomb potential versus minimal interparticle distance in lattice units $R=1-A$ (for amplitude $A$ close to 1 ). It can be seen that the kink with wavenumber $\pi$ is better described by a triangular waveform than by a sinusoidal one. The values of the dimension units are the lattice unit and $2.77 \mathrm{eV}$ respectively. 


\section{Sinusoidal waveform and mode with $q=\pi$}

The properties of sinusoidal kinks with mode $q=\pi$ are easy to obtain as there are only two active strain variables and one coordinate, which during the interval $\Delta_{\pi} t, 0 \leq t<T / 2$, are:

$$
\begin{aligned}
v_{n}= & -\frac{A}{2}(1+\cos (q n-\omega t)) ; \\
v_{n+1}= & -\frac{A}{2}(1+\cos (q[n+1]-\omega t))= \\
& -\frac{A}{2}(1-\cos (q n-\omega t)) ; \\
u_{n}= & -v_{n+1} ; \quad \dot{u}_{n}=\frac{A}{2} \omega \sin (q n-\omega t) .
\end{aligned}
$$

The kinetic and potential energies, $K=\frac{1}{2} \dot{u}_{n}^{2}$ and $U=\frac{1}{1+v_{n}}+\frac{1}{1+v_{n+1}}-2=$ $\frac{1}{1+v_{n}}+\frac{1}{1-v_{n}}-2$, can be obtained. The maximum kinetic, maximum and minimum potential energies are given by

$$
K_{M}=\frac{\pi^{2}}{8} A^{2} V^{2} ; U_{M}=\frac{1}{1-A}-1 ; U_{m}=\frac{2}{1-A / 2}-2 .
$$

\section{Triangular waveform and mode with $q=\pi$}

The potential energy fits very well the numerical values, unlike the kinetic energy, as can be seen in Figs. 1.10 and 1.11. This is because the $\pi$ kink is better described by a triangular waveform in the limit of large energies, as Fig. (1.9) shows. Let us suppose that $t=0$ is the time for which $v_{n}=-A$, as it takes half a period to change from $-A$ to 0 , then $V T / 2=2$. Therefore, for the interval $0 \leq t<T / 2$ the active variables for a ideal triangular waveform are:

$$
\begin{array}{cl}
v_{n}=-A+A V t ; & v_{n+1}=-A V t ; \\
u_{n}=-v_{n+1}=A V t ; & \dot{u}_{n}=A V .
\end{array}
$$

The maximum and minimum potential energies are identical to that of the $q=\pi$ sinusoidal waveform given by Eq. (1.24).

On the other hand, the kinetic energy is a constant:

$$
K=K_{M}=\frac{1}{2} A^{2} V^{2} .
$$

In the numerical simulations, there are short time intervals when $K$ changes, separated by a larger interval when $K$ is almost constant. 
In the high energy limit, $A \rightarrow 1$, the numerical solution converges to the triangular waveform: at fixed time only one particle is in movement, and its velocity is constant. During $0<t<T / 2$ the potential energy in this limit is negligible compared with the kink kinetic energy. Only when $t \rightarrow 0$ and $t \rightarrow T / 2$ the moving particle see the Coulomb potential of the adjacent particles at rest. For $t \rightarrow T / 2$ the particle experiment a deceleration, that in the limit $A \rightarrow 1$ can be considered as a rigid collision and therefore the kinetic energy must vanish for $t \rightarrow 0$ and for $t \rightarrow T / 2$. Here a sharp peak on the potential energy is observed, where in the high amplitude limit a Dirac $\delta(t)$ is expected for energy conservation considerations.

Also, this result has implications in the interpretation of the kink as a particlelike excitation for high energies. In the limit $A \rightarrow 1$ the kink travels trough the lattice at constant velocity and energy, $E$, fixed by Eq. (1.26) as $E=\frac{1}{2} V^{2}$, which means that the kink behaves itself as a quasiparticle of unit mass (or a $\mathrm{K}^{+}$ion mass in physical units).

\subsection{Interaction with several neighbours}

All the previous results apply to the case of nearest-neighbor coupling. However, for a more realistic description the long range interaction must be taken into account. With multiple neighbours, $M$, the dynamical equations become:

$$
\frac{\partial^{2} u_{n}}{\partial t^{2}}=\sum_{m=1}^{M}\left(-\frac{1}{\left(m+u_{n+m}-u_{n}\right)^{2}}+\frac{1}{\left(m+u_{n}-u_{n-m}\right)^{2}}\right),
$$

which linearized become:

$$
\frac{\partial^{2} u_{n}}{\partial t^{2}}=c_{0}^{2} \sum_{m=1}^{M} \frac{1}{m^{3}}\left(u_{n-m}-2 u_{n}+u_{n+m}\right) .
$$

Substitution of $u_{n}=\exp (\mathrm{i}(q n-\omega t))$, leads to:

$$
-\omega^{2} u_{n}=c_{0}^{2} \sum_{m=1}^{M} \frac{1}{m^{3}}(\exp (-\mathrm{i} q m)-2+\exp (\mathrm{i} q m)) u_{n}
$$

with $c_{0}=\sqrt{2}$, or:

$$
\omega^{2}=4 c_{0}^{2} \sum_{m=1}^{M} \frac{1}{m^{3}} \sin ^{2}\left(\frac{q m}{2}\right)
$$

The function $\omega=\omega(q)$ is quite similar to the one with a single neighbour, but with a slope at $q=0$ that increases with the number of neighbours. The phase 
and group velocity are plotted in Fig. for the positive first Brillouin zone (1.12) for $M=1,3,8,50$. It is worth noting here that close to the continuous limit, i.e. $q \rightarrow 0, \sin (q m / 2) \simeq q m / 2$ and we obtain:

$$
\omega^{2}=q^{2} c_{0}^{2} \sum_{m=1}^{M} \frac{1}{M} .
$$

Which leads to the group velocity at $q \rightarrow 0$ and $\lambda \rightarrow \infty c_{M}=\partial \omega / \partial q$

$$
c_{M}=c_{0} \sqrt{\sum_{m=1}^{M} \frac{1}{m}} .
$$

For a finite number of neighbours, the speed of sound is larger than with a single one. For example $c_{3}=1.354 c_{0}$ and $c_{8}=1.6486 c_{0}$. Note if causality is not imposed $\lim _{M \rightarrow+\infty} c_{M}=\infty$, although the convergence of is slow, e.g. for $M=10^{6}$ neighbours $c_{M}=3.7938 c_{0}$.

Simulation results show that kinks still exist and propagate supersonically when the Coulomb interaction extends beyond the nearest neighbours. Kinks have similar velocity and wave number if we normalize kink velocity to the sound speed in the Coulomb chain with interactions between $M$ nearest neighbours, i.e. $V / c_{M}$.

Figure 1.13 shows the dependence of the relative velocity $V / c_{p}$ on the minimum interparticle distance and Fig. 1.14 shows the dependence of the numerical best fit for the wave number as a function of the relative velocity, emphasizing the essential identity of the phenomenon. Other aspects of these figures will be commented below. For clarity, only the cases of three and eight neighbours are represented although up to 30 neighbours have been tested.
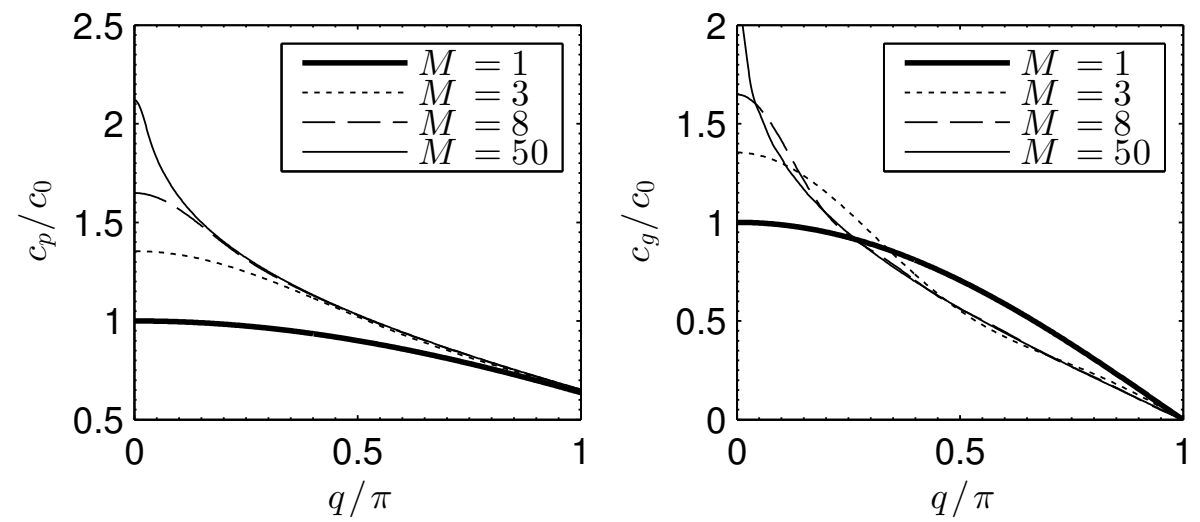

Figure 1.12: (left) Phase and (right) group velocity for the linearized Coulomb lattice including $M=[1,3,8$ and 50$]$ neighbours. 


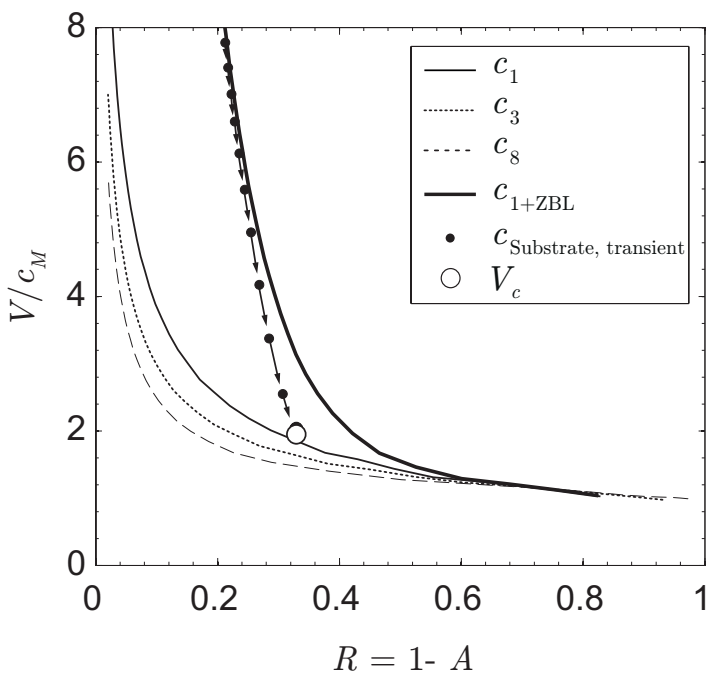

Figure 1.13: Kink velocities as a function of minimal interatomic distance in lattice units $R=1-A$ ( $A$ is kink's amplitude) for several potentials. Notations in the legend: $c_{m}, m=1,3,8$, refers to the Coulomb interaction between the first $m$ neighbours. Velocities are normalized to the sound speed $c_{M}$ for a system with interaction between $M$ neighbours, except for the system with a substrate which is normalized to $c_{1}=c_{s}$. It can be seen that the increase of the number of interacting neighbours slows the normalized velocity $V / c_{M}$ of the kink (but not its absolute velocity). Also, it can be seen that in the system with a substrate the normalized velocity $V / c_{M}$ deviates from the curve $c_{1+\mathrm{ZBL}}$ to some specific velocity $V_{c}$ on the $c_{1}$ curve. The values of the sound velocities are $c_{1}=c_{s}=3.7 \mathrm{~km} / \mathrm{s}, c_{3}=5.0 \mathrm{~km} / \mathrm{s}$ and $c_{8}=6.1 \mathrm{~km} / \mathrm{s}$.

It is worth commenting the problem that arises when the interaction with an infinite number of neighbours is taken into account. If the pair potential depends on the interparticle distance $|r|$ as $1 /|r|^{s}$, the long-wave phonon velocity is finite for $s>1$, but it diverges with the number of particles $N$ as $v_{\mathrm{ph}} \propto \sqrt{\ln (N / 2)}$ for the unscreened Coulomb potential $(s=1)$. However, this divergence occurs only in the electrostatic limit when the electromagnetic wave retardation is neglected. With an account of the retardation, the long-wave group velocity tends to the speed of light in vacuum. If the particles are in a material medium, there is a rearrangement of the electron density that can be described as a screening of the Coulomb interaction with some characteristic length $l_{\mathrm{scr}}$. The screening brings about a finite long-wave phonon velocity $v_{\mathrm{ph}} \propto \sqrt{\ln \left(l_{\mathrm{scr}} / a\right)}$, where $a$ is the lattice constant. As it has been mentioned, we do not study in depth this problem here and have considered only a few neighbours for simplicity. 


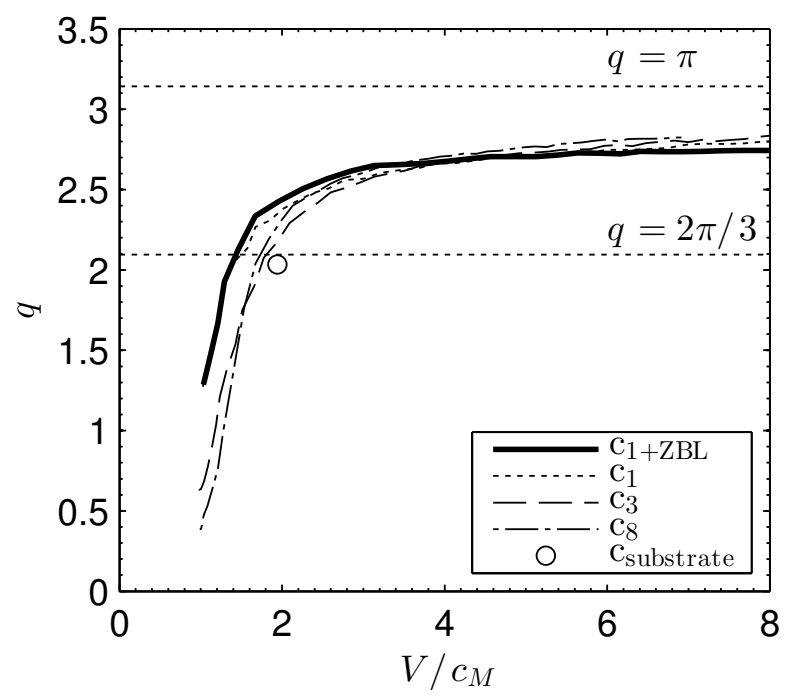

Figure 1.14: Best fit for the wavenumber $q$ of the kinks as a function of the velocities normalized to the sound speed. Notations in the legend: $C_{i}$ is the Coulomb interaction between the first $i$ neighbours. Note that the magic wave number $q=2 \pi / 3$ is also chosen in the system with a substrate. The values of the sound velocities are $c_{1}=c_{s}=3.7 \mathrm{~km} / \mathrm{s}, c_{3}=5.0 \mathrm{~km} / \mathrm{s}$ and $c_{8}=6.1 \mathrm{~km} / \mathrm{s}$.

\subsection{The effect of nuclear repulsion: kinks with short-range ZBL potential}

The minimal interatomic distance in lattice units $R=1-A$, obtained for fast large-amplitude kinks, is clearly impossible in realistic systems. At short distances short-range forces appear, which are produced by the overlapping electronic shells of the two close atoms. A large number of different repulsive potentials and screening functions have been proposed over the years, some determined semi-empirically, others from theoretical calculations. Much used repulsive potential is the one given by Ziegler, Biersack and Littmark, the so-called ZBL repulsive potential. It has been constructed by fitting a universal screening function to theoretically obtained potentials calculated for a large variety of atom pairs (Biersack et al., 2008). The ZBL potential has the form

$$
U_{\mathrm{ZBL}}(r)=\mathrm{k}_{\mathrm{e}} \frac{Z_{1} Z_{2} \mathrm{e}^{2}}{r} \phi\left(\frac{r}{\rho}\right)
$$

with $\mathrm{k}_{\mathrm{e}}$ being the Coulomb constant, $Z_{1}$ and $Z_{2}$ are the atomic numbers of the involved atoms, and $r$ the distance between them, $\phi(x)$ is the universal screening 


\begin{tabular}{lllll}
\hline Constant & $i=1$ & $i=2$ & $i=3$ & $i=4$ \\
\hline$A_{i}$ & 0.1818 & 0.5099 & 0.2802 & 0.02817 \\
$b_{i}$ & 3.2 & 0.9423 & 0.4029 & 0.2016 \\
\hline
\end{tabular}

Table 1.1: Constants used for the Ziegler-Biersack-Littmark (ZBL) screening potential of Eq. (1.34)

function:

$$
\phi(x)=\sum_{i=1}^{4} A_{i} \exp \left(-b_{i} x\right)
$$

where the constants $A_{i}$ and $b_{i}$ are listed in Table (1.1).

The screening length is $\rho=0.8854 a_{0} /\left(Z_{1}^{0.23}+Z_{2}^{0.23}\right)$, with $a_{0}=0.529 \AA$ being the Bohr radius. The ZBL potential describes well the interaction between neutral atoms. In the case of ions considered here, the Coulomb potential must also be added, accounting for the repulsion between the ions. The introduction of the ZBL potential restricts the interatomic distances to the realistic values. The four terms in the ZBL potential are important for different range of energies, but for $\mathrm{K}^{+}$ions, with energies up to $200 \mathrm{KeV}$, which are much larger that the ones considered here, the interaction potential can be represented by a single term, which together with the Coulomb ionic part takes the form:

$$
U(r)=\frac{1}{r}+\frac{\alpha}{r} \exp \left(-\frac{r}{\rho}\right),
$$

with $\alpha=184.1$ and $\rho=0.0569$ in dimensionless units, which in physical units correspond to $2650.6 \mathrm{eVÅand} 0.29529 \AA$, respectively. Figure 1.15 represents ZBL and Coulomb potentials, with their sum and other details to be commented later. Note that around $r \simeq 0.4$, the combined potential $U(r)$ differs considerably from the Coulomb potential.

The dynamical equations become

$$
\frac{\partial^{2} u_{n}}{\partial t^{2}}=-G_{n+1}+G_{n}-F_{n+1}+F_{n},
$$

with $F_{n}$ given by Eq. (1.11) and $G_{n}$ given by

$$
G_{n}=\frac{\alpha}{1+v_{n}} \exp \left(-\frac{1+v_{n}}{\rho}\right)\left(\frac{1}{1+v_{n}}+\frac{1}{\rho}\right) \text {. }
$$

When the joint effect of both the screened Coulomb (ZBL) and bare Coulomb potentials is considered, i.e., Eq. (1.35), numerical simulations show that the behavior of the kinks is not much different from that observed in the bare Coulomb 


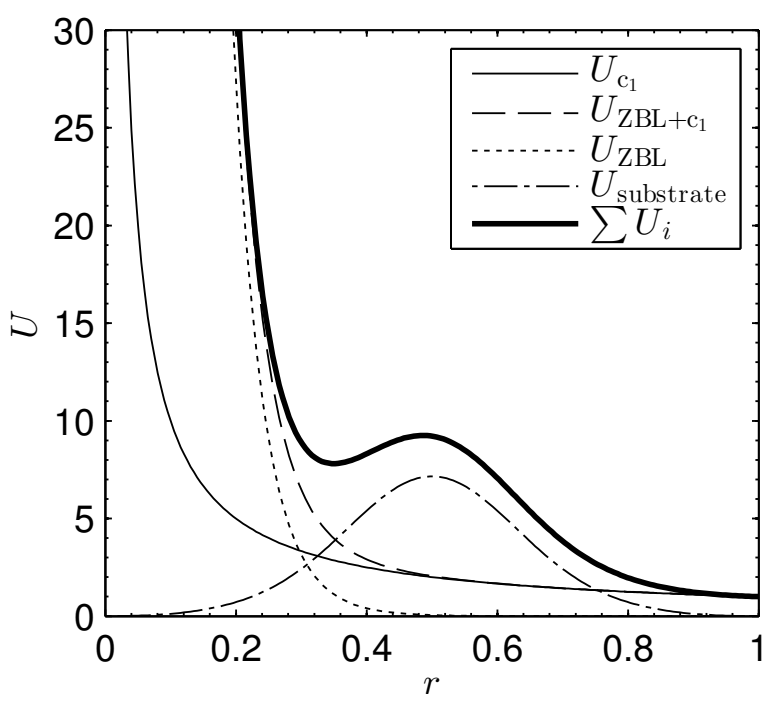

Figure 1.15: Interaction potentials $\mathrm{U}(\mathrm{r})$ in dimensionless units. Coulomb $\left(c_{1}\right) ; \mathrm{ZBL}\left(c_{\mathrm{ZBL}}\right)$; Coulomb+ZBL $\left(c_{1+\mathrm{ZBL}}\right)$; substrate potential $\left(c_{\mathrm{S}}\right)$ and the sum of the Coulomb, ZBL and substrate potentials $\left(c_{1+\mathrm{ZBL}+\mathrm{S}}\right)$. The scaled units are $2.77 \mathrm{eV}$ and the lattice unit a=5.19 Åfor $U$ and $r$, respectively.

case, discussed in the preceding sections. Supersonic kinks propagate equally well, changing from the magic wavenumber $q=2 \pi / 3$ to the proximity of $q=\pi$ and from the sinusoidal to nearly triangular waveform in increasing the amplitude $A$. Figures 1.13 and 1.14 also show the characteristic curves $V=V(A)$ and $q=q(A)$. The basic difference is that the high energy regime, i.e. the regime of high velocities at witch the waveform change from sinusoidal to triangular, is obtained for for lower (and also realistic) interatomic distances. On the other hand the sound velocity does not change since the ZBL potential is felt only for very large perturbations. Unfortunately, the RWA cannot be obtained analytically but the numerical RWA fits very well the results of the simulations.

\subsection{The effect of the substrate potential: lattice kinks or crowdions}

In the preceding sections, the interaction with the other atoms in the crystal was taken into account only implicitly, since the only effect of the surrounding atoms was to fix the equilibrium lattice period and to confine the particles within the crystal. To better model the properties of the kinks in a crystal like muscovite, we take into account explicitly the interaction with the surrounding atoms in a simplified mica geometry. 


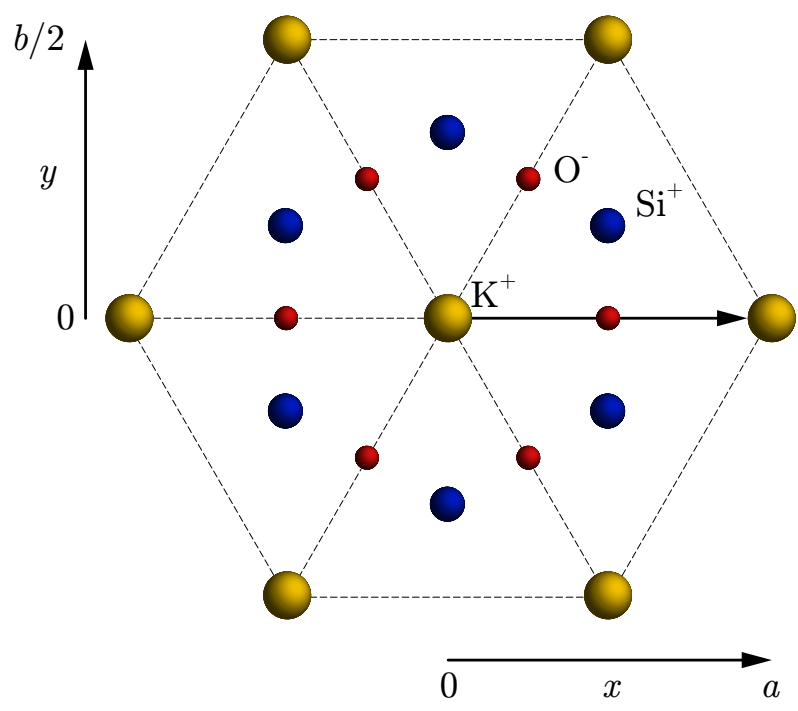

Figure 1.16: Projection on the [001] plane of the ions used in the calculation of the substrate potential. Four planes of ions are considered, two above and two symmetrical below the $\mathrm{K}^{+}$plane, the closest two with oxygen and the other two with silicon ions. The path for the central $\mathrm{K}^{+}$ion used the in calculation is shown, note that the $\mathrm{O}^{-2}$ ions in the middle of the path are actually at a distance of $1.68 \AA$ above and below. The interaction between the $\mathrm{K}^{+}$ions in the central $X$ axis is not taken into account in constructing the substrate potential as it is taken into account explicitly. The crystal is continued in the $\mathrm{K}^{+}$plane until the convergence is achieved. Distance between the longer marks is $1 \AA$.

The $\mathrm{K}^{+}$ions occupy the nodes of the hexagonal lattice with a lattice unit $a=5.19 \AA$. There are no other atoms in the $\mathrm{K}^{+}$plane, therefore we need to consider more atoms above and below. We consider two planes above and two symmetric below. The closest plane, situated at a height of $1.6795 \AA$, is occupied with oxygen ions with charge -2 , their projections on the $\mathrm{K}^{+}$plane lie in the middle of the two nearest-neighbor $\mathrm{K}^{+}$ions. The other layers, at at a height of $2.2227 \AA$ from the $\mathrm{K}^{+}$plane, are occupied by silicon ions. They are in the centers of tetrahedra whose three horizontal vertices are occupied by the oxygen ions. See the sketch on Fig. 1.16 for a projection of oxygen and silicon layers on the [001] plane, i.e. a top view of the $\mathrm{K}^{+}$layer. The $\mathrm{Si}$ sites are occupied by $\mathrm{Si}^{+4}$ and $\mathrm{Al}^{+3}$ ions in the proportion of $3: 1$, giving an average charge of +3.75 , but we assign them a smaller charge +2.75 to take into account other atoms in successive layers, particularly the oxygen ions at the top of the tetrahedra, and to achieve charge neutrality.

We suppose that all the atoms are in fixed positions except the moving $\mathrm{K}^{+}$ions in a row and only in the [100] direction. This is justified by the supersonic speed of the kinks, that we are interested in, and due to the weak interaction between the $\mathrm{Si}$ and $\mathrm{Al}$ ions compared to the ZBL interaction between potassium ions when they collide at high velocities. The interaction between the $\mathrm{K}^{+}$ions, that are in the central $X$-axis, are not considered in constructing the substrate potential as 
it is taken into account explicitly. The lattice is extended in the $[001] \mathrm{K}^{+}$plane until the convergence of the potential is achieved. Specifically the potentials used are the electrostatic interactions and Born-Mayer potentials of the form $V=$ $A \exp \left(-r / r_{g}\right)$, given in Ref. (Gedeon et al., 2002). The value $r_{g}=0.29 \AA$ is for all the interactions, and the pre-exponential constants in $\mathrm{eV}$ are: $A_{\mathrm{KO}}=3800.125$, $A_{\mathrm{KSi}}=2762.5, A_{\mathrm{OO}}=453.375, A_{0 \mathrm{Si}}=1851.25$, and $A_{\mathrm{SiSi}}=1173.125$. For the $\mathrm{K}^{+}-\mathrm{K}^{+}$interaction, we use the Coulomb-ZBL potential described above.

We obtain a substrate potential in scaled units $\left(u_{E} \simeq 2.77 \mathrm{eV}\right)$, which can be described with very good approximation by a truncated Fourier series up to fourth harmonic:

$$
U_{s}(x)=\sum_{h=0}^{4} U_{h} \cos (2 \pi h x)
$$

with coefficients $U_{h}$ equal to $[2.4473,-3.3490,1.0997,-0.2302,0.0321]$. The potential well of $20 \mathrm{eV}$ is consistent with molecular dynamics simulations (Collins et al., 1992). It is represented in Fig. 1.15 together with the other potentials, such that their relative magnitudes can be compared.

\subsubsection{Dispersion relations with substrate}

Our dynamical equations with a substrate potential $U_{s}(x)$ in the form given by Eq. (1.38) and for $M$ neighbour Coulomb interactions can be written as

$$
\frac{\partial^{2} u_{n}}{\partial t^{2}}=-\frac{\partial U_{s}\left(u_{n}\right)}{\partial u_{n}}+\sum_{m=1}^{M}\left(-\frac{1}{\left(m+u_{n+p}-u_{n}\right)^{2}}+\frac{1}{\left(m+u_{n}-u_{n-m}\right)^{2}}\right)
$$

where the restoring forces of the substrate potential are

$$
-\frac{\partial U_{s}\left(u_{n}\right)}{\partial u_{n}}=\sum_{h=1}^{4} 2 \pi h U_{h} \sin \left(2 \pi h u_{n}\right)
$$

The latter equations, for small amplitude oscillations of $u_{n}$ becomes

$$
-\frac{\partial U_{s}\left(u_{n}\right)}{\partial u_{n}} \simeq-\omega_{0}^{2} u_{n}
$$

with the linear frequency $\omega_{0}$ as

$$
\omega_{0}^{2}=\sum_{h=1}^{4}(2 \pi h)^{2} U_{h}
$$



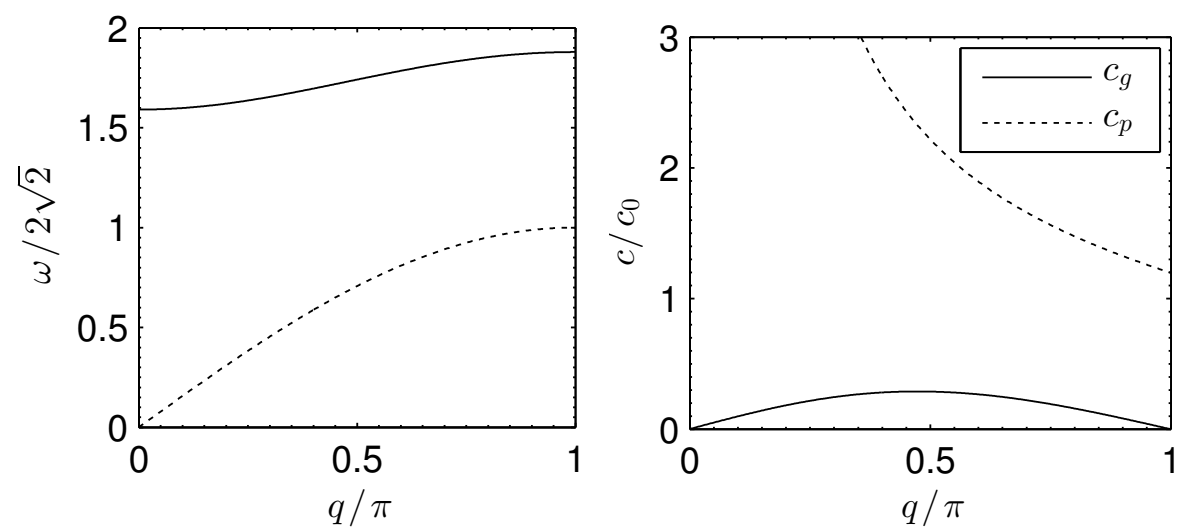

Figure 1.17: Left: Dispersion relation for the linearized Coulomb lattice with (continuous) and without (dotted) substrate potential. Right: Phase (dotted) and group (continuous line) velocity for linearized Coulomb lattice. All dispersion curves are for $M=1$ neighbour interaction.

The corresponding value for linear frequency is $\omega_{0}=4.48$ or $119 \mathrm{~cm}^{-1}$ in physical units, which is close to $110 \mathrm{~cm}^{-1}$ determined experimentally (Diaz et al., 2000). Therefore the dynamical equations for $u_{n}$ small become

$$
\frac{\partial^{2} u_{n}}{\partial t^{2}}=-\omega_{0}^{2} u_{n}+c_{0}^{2} \sum_{m=1}^{M} \frac{1}{m^{3}}\left(u_{n-m}-2 u_{n}+u_{n+m}\right) .
$$

Substitution of $u_{n}=\exp (\mathrm{i}(q n-\omega t))$ leads to

$$
-\omega^{2}=-\omega_{0}^{2}+c_{0}^{2} \sum_{m=1}^{M} \frac{1}{m^{3}}(\exp (-\mathrm{i} q m)-2+\exp (\mathrm{i} q m)) .
$$

Therefore the phonon dispersion relation become

$$
\omega^{2}=\omega_{0}^{2}+4 c_{0}^{2} \sum_{m=1}^{M} \frac{1}{m^{3}} \sin ^{2}\left(\frac{q m}{2}\right) .
$$

In the following we particularize for a single neighbour,i.e. $M=1$. The phonon spectrum becomes an optical one, and the frequency and group velocity are

$$
\begin{aligned}
\omega^{2} & =\omega_{0}^{2}+4 c_{0}^{2} \sin ^{2}(q / 2), \\
c_{g} & =\frac{\partial \omega}{\partial q}=\frac{c_{0}^{2} \sin q}{\sqrt{\omega_{0}^{2}+4 c_{0}^{2} \sin ^{2}(q / 2)}} .
\end{aligned}
$$

Note that $c_{0}$ is still the sound speed in the system without substrate. Thus, as Fig. (1.17) shows, the dimensionless phonon frequencies $\omega$ are in the interval 


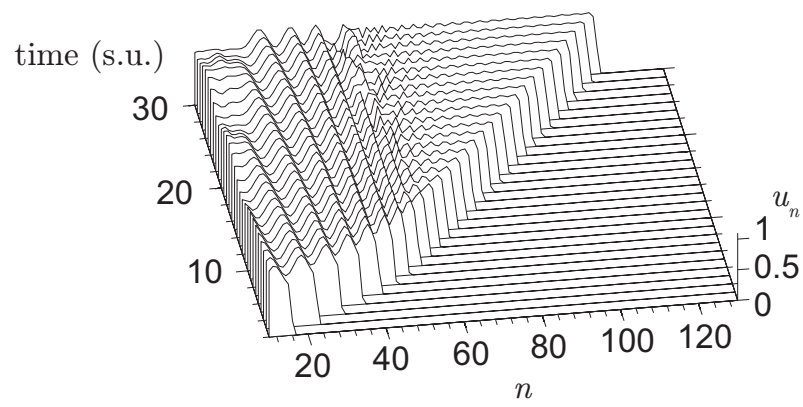

Figure 1.18: Representation of the process of dynamical slowing down of the kink. The nonlinear and linear radiation processes can be easily distinguished. Nonlinear waves with large amplitude are first emitted. Later, the phonons with the wavelength close to $\lambda \simeq 4$, which corresponds to maximal group velocity, and with exponentially decaying amplitudes are emitted while the kink approaches the limit velocity. Scaled units for time and distance are $0.2 \mathrm{ps}$ and lattice constant $5.19 \AA$.

between $\omega_{0}=4.48$ and $\omega_{\max }=5.31$. The group velocity is zero at $q=0$ and $q=\pi$ and reaches its maximum $c_{\mathrm{c}, \max } \simeq 0.4$, in the proximity of $q=\pi / 2$ with $\lambda \simeq 4$. These features are also observed in the simulations for low amplitude travelling waves.

\subsubsection{Qualitative description}

The introduction of the substrate potential does not prevent the existence of supersonic lattice kinks. The lattice kink, also called crowdion, consists of an interstitial atom propagating very fast in the lattice and leaving behind a vacancy. The specific feature of the kinks found in the present work is that its velocity and energy are fixed by the layer+substrate system, let us denote them as $V_{c}$ and $E_{c}$ ( $c$ for crowdion). If the initial energy of the excitation is smaller than the threshold $E_{c}$, the kinks are rapidly dispersed and disappear and cannot propagate more than few lattice units. However, if it is larger, a kink is formed and its speed depends on its amplitude following the relation $V(A)$ of the ZBL interaction without substrate. However this velocity is not constant with time: the kink always slows down to a fixed stable speed $V_{c}$, and the excess energy is backward radiated. The specific values for the stable kink velocity and energy in scaled units are $V_{c}=2.7387 \simeq 2 c_{0}$ and $E_{c}=9.4374$, corresponding to $7.16 \mathrm{~km} / \mathrm{s}$ and $26.2 \mathrm{eV}$, respectively.

The lattice kink is supersonic in the two meanings a) $V_{c}>c_{0}$, where $c_{0}$ is the sound velocity without substrate; b) $V_{c}$ is much larger than the maximum phonon group speed $V_{\mathrm{c}, \max } \simeq 0.4$ in the system with the substrate, but is not larger than the maximum phase velocity which is unbounded for $q \rightarrow 0$, see Fig. (1.17). 

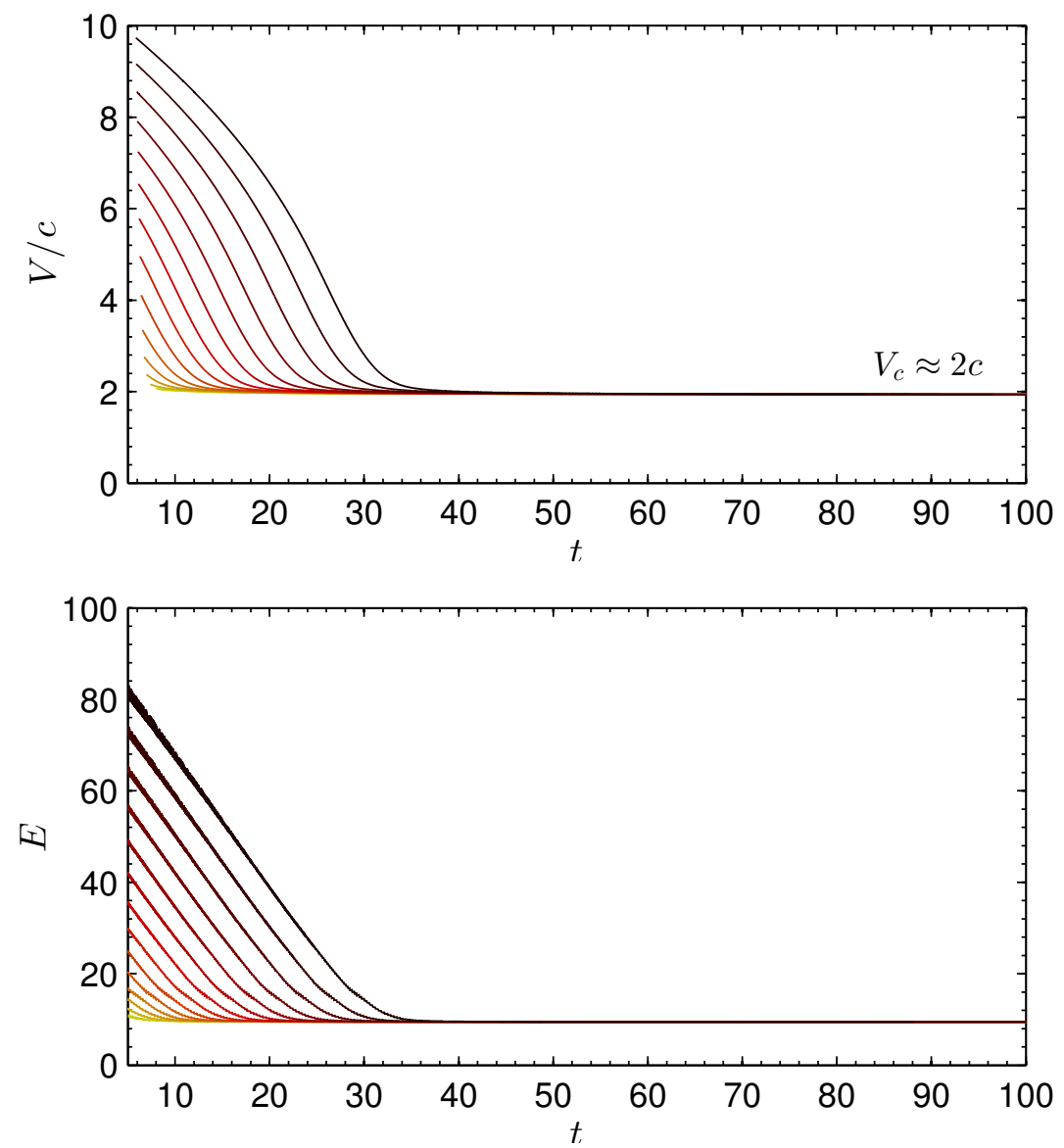

Figure 1.19: (top) Energies of several kinks with respect to time. When more energy than the crowdion's one is delivered a faster kink is produced and a radiation process takes place until the supersonic crowdion is formed. Thereafter, the crowdion is extremely stable. If the initial energy is smaller than the crowdion's one the kink dissipates into phonons. The scaled units are approximately $2.77 \mathrm{eV}$ for energy and $0.2 \mathrm{ps}$ for time. (bottom) Velocities of lattice kinks or crowdions versus time in scaled units for different initial conditions. The final velocity and energy are approached asymptotically, being $V_{c}=2.7(7.2 \mathrm{~km} / \mathrm{s})$ and $E_{k}=9.5(26.2 \mathrm{eV})$ in scaled and physical units. For $V<V_{c}$, the kinks are dissipated into low-amplitude phonons.

\subsubsection{Slowing down processes}

The process of slowing down to $V_{c}$ is shown in Figs. (1.18) and (1.19), where two well distinguished phases can be identified.

a) Nonlinear radiation: For an initial energy $E>E_{c}$, the kink progressively loses energy. The particles immediately after the kink perturbation are left with enough energy for nonlinear vibrations in the potential well bringing about 
nonlinear wave. Their frequencies obtained numerically are above the phonon band with a maximum value of about 6.3 . This strong radiative process is shown in Fig. 1.18 for an initial velocity $V(t=0)=7$. This process is very fast and the decay of kink energy is almost linear with time. As long in this regime the waveform is nearly triangular, kink kinetic energy can be approximated by $K=\frac{1}{2} A^{2} V^{2}$ as explained is Sec. (1.4.5). Thus, as the potential energy is negligible for this regime, the linear decay of energy is consistent with the decreasing of velocity at the initial part seen in the of Fig. 1.19.

b) Linear radiation: As the lattice kink energy approaches $E_{c}$, the amplitude of the tail oscillations and its frequency decrease, the kink frequency enters the phonon band, radiating low amplitude phonons (Braun et al., 1998; Braun et al., 2004). The energy decreases exponentially with time towards $E_{c}$. The wave number of the radiated phonons can be deduced from the kink speed as each particle left behind the kink is excited with a delay $\Delta t=1 / V_{c}$ and, therefore, with a phase difference $q=\omega(q) \Delta t=\omega(q) / V_{c}$. As the phonon wave vector is given by $q=\omega(q) / c_{\mathrm{ph}}$, where $c_{\mathrm{ph}}$ is the phase speed of the phonons, $V_{\mathrm{ph}}=V_{c}$. Using the phonon dispersion relation in Eq. (1.46) it is possible to obtain the phonon wavenumber and wavelength $\lambda_{\mathrm{ph}}=3.5$ which is the observed one in the simulations. Similar process has been described in Ref. (Braun et al., 1998) and in references therein for subsonic kinks. However, there is an important difference: the subsonic kinks, described in those works, radiate continuously and eventually stop. Here the amplitude of the radiation decreases exponentially, allowing the kink to achieve a constant energy value and propagate stable along the lattice.

From the comparison of Figs. (1.13) and (1.19) with Fig. (1.15) we conclude that supersonic kink's amplitude $A$ of relative particle displacements is determined by the interatomic distance $R$ at the local minimum of the combined interatomic plus substrate potential, $R=1-A \approx 0.33$ in lattice units. This value of the kink amplitude ensures the absence of kink oscillations and correspondingly the absence of phonon radiation into the chain by the supersonic kink. We also want to emphasize that we do not observe discrete spectrum (a set of possible values) of the velocity of the supersonic kink in our system, even when we start with high initial kink velocity, see Fig. (1.19). This is in contrast with the prediction of supersonic multi-solitons (lattice $N$-solitons) in Frenkel-Kontorova model with nonlinear intersite coupling in Refs. (Braun et al., 2004; Savin, 1995; Zolotaryuk et al., 1997). We relate this finding with the extreme discreteness of our kink of effective wavenumber $q \simeq 2 \pi / 3$, which as shown in Fig. (1.14) corresponds to only two particles moving at a given time, cf. Ref. (Kosevich et al., 2004), which does not allow for different matching with the substrate potential, and also with possible dynamical instability of lattice multi-solitons (bound states of supersonic kinks) in the considered system. 


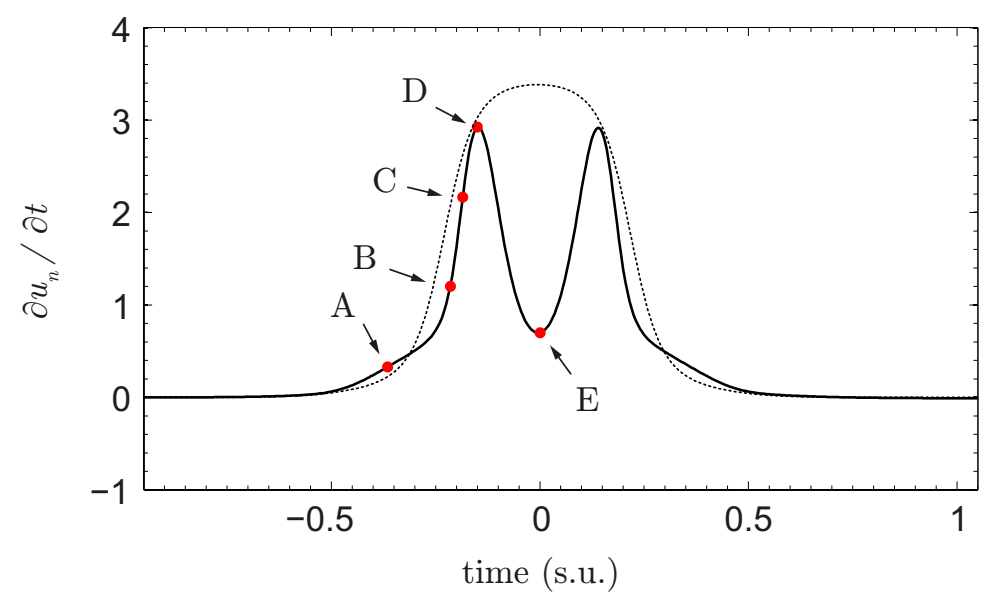

Figure 1.20: Particle velocity waveform measured at $n=470$ in supersonic kink with amplitude $A=0.67$ and minimal interatomic distance in lattice units $R=1-A=0.33$ for Coulomb $+\mathrm{ZBL}$ potential with (continuous line) and without (dotted line) substrate potential. The instants when the ZBL interaction acts (near A) and the minimum velocity at the top of the potential barrier (E) are easily identified. The configurations at those times can be seen in Fig. 1.21. Scaled units are $0.2 \mathrm{ps}$ and $2.6 \mathrm{~km} / \mathrm{s}$.

\subsubsection{Double kink}

The particle velocity $\dot{u}_{n}$ as a function of time for the stable kink in the substrate is represented in Fig. 1.20. Due to the extreme discreteness of the kink, it is not practical to represent $\dot{u}_{n}$ an a function of $n$. The profile of a double soliton for the particle velocity corresponds to the double kink shown in Fig. (1.21)-(a), is evident as a function of time.

Supersonic multi-solitons in Frenkel-Kontorova system with anharmonic coupling has been described in Refs. (Kosevich et al., 1973; Savin, 1995; Zolotaryuk et al., 1997). They correspond precisely to the discrete set of velocities for which the kinks do not radiate phonons. Although roughly similar in the shape, the kink in our system does not correspond to the mathematical forms obtained in those references. The kink in our system is more discrete, very close to the limit of discreteness, its wavenumber $q \simeq 2 \pi / 3$, shown in Fig. (1.14), corresponds to only two particles moving at a given time, cf. Ref. (Kosevich et al., 2004).

This discreteness makes possible the exact description of the double kink process as shown in Fig. 1.21 (c): a particle $n$, represented by a white circle, initially at rest at the bottom of a potential well, experiences two collisions, one when it was hit by the particle $n-1$ and is accelerated afterwards, and a second one when it hits the particle $n+1$ and is decelerated, attaining almost zero velocity at the 

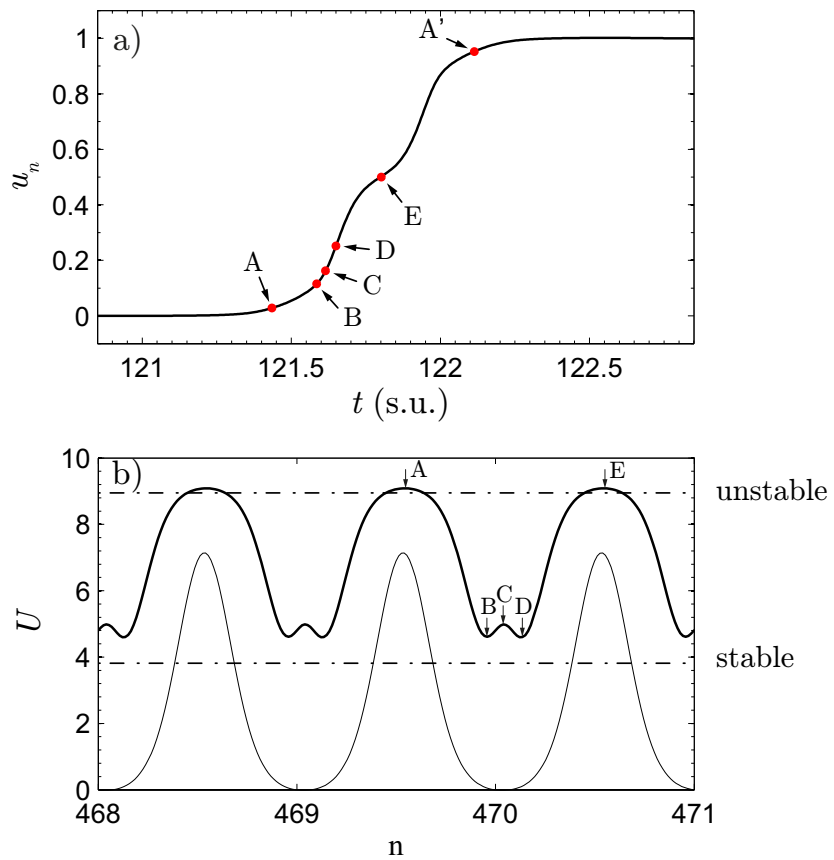

c)

A

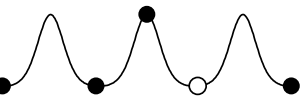

B

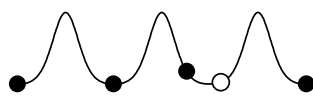

C

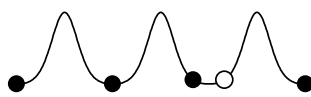

$\mathrm{D}$

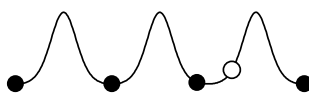

$\mathrm{E}$

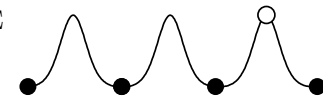

Figure 1.21: (a) Particle displacement waveform for the established lattice kink. (b) Potential energy seen by the kink during propagation (thick line) and periodic substrate potential (thin line), horizontal lines are the energies corresponding to the equilibrium stable (C) and unstable (A) interstitials. (c) Particle configurations corresponding to the points $A-E$, shown in (a) and (b). Scaled units are lattice unit $5.19 \AA$ for distances, $0.2 \mathrm{ps}$ for time and $2.77 \mathrm{eV}$ for energies.

bottom of the potential well (during the radiation process the particle velocity is different from zero). Between the two collisions, the particle finds the substrate potential barrier between sites and experiences a decrease in velocity while going uphill which is followed by an acceleration while going downhill.

Note that the states $\mathrm{A}$ and $\mathrm{E}$ in Fig. 1.21 have exactly the same energy, but the kink has only moved half a lattice site. This is the first kink of the double-kink process shown in Fig. 1.21 (a). The process of the particle $n$ going downhill and hitting the $n+1$ particle until it stops forms the second kink.

This process can be seen in terms of the kink energy in Fig. 1.21 (b). Two identical oscillations of particle's potential energy $U$ happen for the kink to move a lattice site. There is a local maximum at point $C$, corresponding to the minimum distance between particles and the short range ZBL interaction. The horizontal dashed-dotted lines indicate the energies for the equilibrium interstitial configurations, with two particles inside a potential well (stable), or one particle at the top of the potential barrier (unstable), with the energy difference corresponding to the Peierls-Nabarro (PN) barrier. The potential energy is always above the stable interstitial energy as the lattice has no time to relax, bringing about an $a d i$ - 
abatic PN barrier. The kink always has finite kinetic energy, with the minimum corresponding to configuration $A$.

\subsection{Some numerical simulations with ultradiscrete kinks or crowdions}

In this section we present the results of different simulations to show the capacity of the crowdions to survive a perturbed environment when larger energy is initially delivered and, second, the behaviour of the crowdions with temperature.

\subsubsection{Excess energy}

We present some examples of simulations when the lattice is given more energy than the $26.2 \mathrm{eV}$ needed to produce the supersonic crowdion. The energies range from $130 \mathrm{eV}$ to $520 \mathrm{eV}$. They are represented in Fig. 1.22. In (a) a single crowdion is formed after nonlinear waves are emitted. In (b) two crowdions are formed leaving behind an stationary linear wave. Note how the second crowdion survives to the tail of the first and the common velocity $V_{c}$ of both. In (c) the excessive energy destroys the second crowdion which transforms into a highly localized nonlinear stationary wave. In (d) the second crowdion survives again, while in (e) it is again destroyed. Extensive phonon radiation and wandering kinks can be seen in the latter figure. In (f) a second crowdion survives 150 sites in a highly perturbed media but it is finally pinned down.

\subsubsection{Thermalized medium}

An interesting question is whether the crowdion can travel trough a previously thermalized medium. This is not only a question of general interest but particulary important for mica muscovite. As it has been calculated in the chapter Tracks in mica: 50 years later in this book, the recording process of tracks happens a few kilometers underground under large pressure and temperatures of 700-1000 K. Although much more work is necessary, the answer is positive. For comparison Fig. 1.23 (a-b) shows two simulations at $300 \mathrm{~K}$ and $1000 \mathrm{~K}$ in the system without substrate potential where the kink survives over hundreds of lattice sites. It is not really surprising as if we compare the energy of the crowdion $26.2 \mathrm{eV}$ with the mean thermal energy of a particle $k_{B} T$, the crowdion energy is 1000 and 300 times larger at $300 \mathrm{~K}$ and $1000 \mathrm{~K}$ respectively.

In the case of including the substrate potential, as shown in Fig. 1.23 (c-d) for $300 \mathrm{~K}$ and $1000 \mathrm{~K}$ respectively, the crowdion can also travel hundreds of sites of the previously thermalized media. As it was studied in Ref. (Archilla et al., 

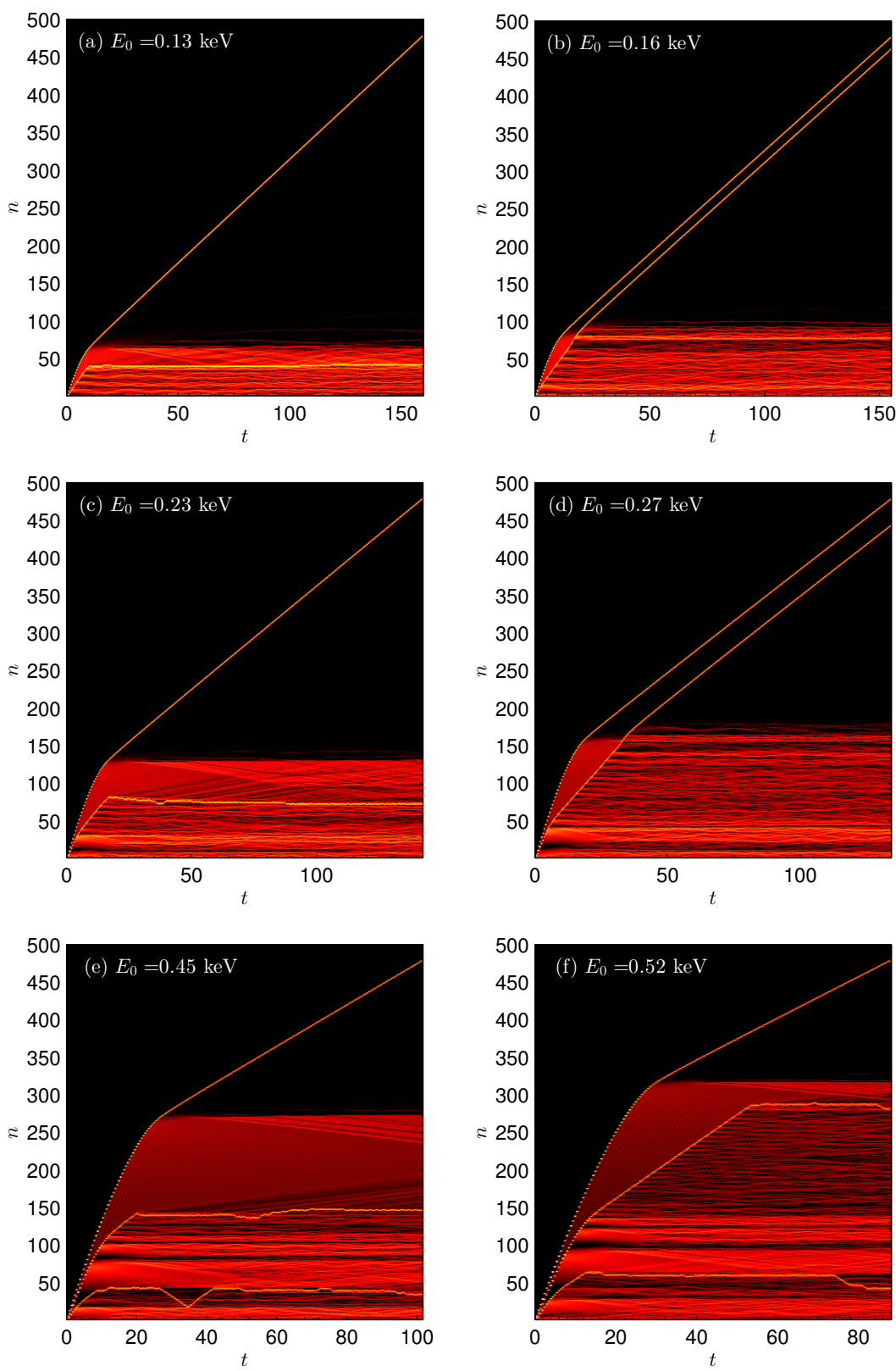

Figure 1.22: (Color) Particle energy plots of several examples of crowdion formation in arbitrary units of $\simeq 3 \mathrm{eV}$. Initial energy increases form (a) to (f). Many features can be observed, among them the specific velocity of the crowdion $V_{c}$, the formation of nonlinear waves and phonons, the formation of two crowdions and the survival of the crowdion to the severely perturbed media during hundreds of sites 

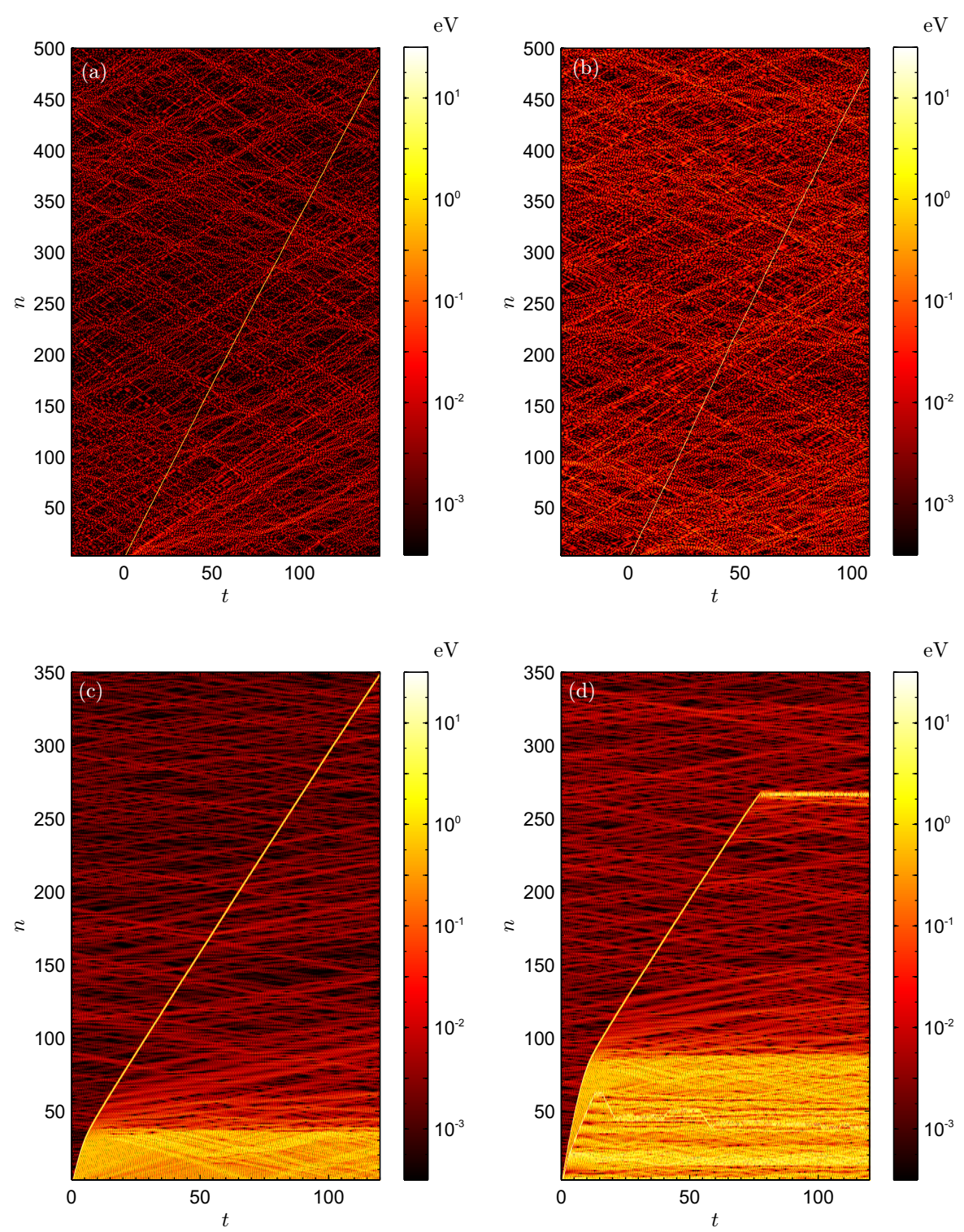

Figure 1.23: (Color) Particle energy plots of two crowdions travelling on a previously thermalized medium at (a,c) $300 \mathrm{~K}$ and (b,d) $1000 \mathrm{~K}$, top without and bottom with substrate potential. Color bars are in $10 \log _{10}(E)$ units

2015), the crowdion always has finite kinetic energy, but the final total energy of the kink, $E_{k}$, is always on the order of magnitude of the Peierls-Nabarro (PN) barrier. The equivalent kinetic energy equivalent for the thermalized media is 0.005 
$(0.013 \mathrm{eV})$ at $300 \mathrm{~K}$ and $0.016(0.043 \mathrm{eV})$ at $1000 \mathrm{~K}$ in normalized and physical units. These values are far below the energy difference between the PN barrier and the kink energy. However, in some simulations, for temperatures of $1000 \mathrm{~K}$ the thermalization is not completely achieved due to appearance of nonlinear waves instead of phonons. Therefore, localized peaks of the background vibrations can interfere with the crowdion where, in some cases, it can be trapped leading to a highly localized nonlinear stationary perturbation. Figure 1.23 (d) shows and example of this situation, where the crowdion is eventually trapped forming an interstitial defect.

Thermal effects discussed in this section lead to different survival path length of the crowdions. It the hypothesis of crowdions propagating in mica muscovite is correct, they might be related with some the tracks observed in the mineral. Other feature of the presented simulations worth remarking is that the high equivalent temperature of the nonlinear tail radiation of the crowdion is likely to favor a change of structure and the formation of tracks.

\subsection{Conclusions}

In this chapter we have modeled a low dimensional system using realistic potentials corresponding to a row of ions in a silicate layer, mica muscovite sheet. Our objective was to determine what kind of nonlinear localized modes can exist with realistic parameters and with what characteristics. The choice of the parameters of mica muscovite is motivated by the fact that many of the dark tracks that appear in sheets of this material are consistent with the in-layer propagation of vibrational excitations along close-packed lattice lines of ions, and an experiment has demonstrated that localized energy can travel along lattice directions, being able to eject an atom at the opposite surface.

The modeling of the system has followed a process of increasing complexity to better understand which effect is responsible for which characteristic of the model. In the starting model in preliminary publications (Archilla et al., 2013; Archilla et al., 2014), only $\mathrm{K}^{+}$ions with nearest-neighbor Coulomb repulsion were present, for which we have found that very fast supersonic kinks can propagate. They are extremely localized, only two particles or for higher energies only a single particle is moving at the same time.

We have added analytical calculations of the energies and compare them with numerical simulations. We have also shown that the introduction of interaction with several neighbours do not produce significant changes as long as the sound speed in each system is taken as a reference. But the extremely short minimal interatomic distances in the kink has motivated the introduction of more realistic short-range ZBL potential. In the improved dynamical model, kinks propagate equally well and with as much energy as desired, but now with realistic minimal 
interatomic distances.

The next step was the introduction of a periodic substrate potential using empirical potentials and the geometry of the crystal. Supersonic kinks continue to propagate without losing energy but with several important properties:

a) The main one is that the system selects only one velocity and one energy of the kink;

b) The lattice kink energy is larger than the one which is needed for atom ejection at the surface, and smaller than the one of the proposed sources of energy, the recoil of a $\mathrm{K}^{+}$ion due to beta emission;

c) The found kinks can be described as the double-kinks or bi-solitons depending of the variable chosen. The unique velocity of the supersonic kink on the periodic substrate potential we relate with the kink amplitude of relative particle displacements which is determined by the interatomic distance corresponding to the minimum of the combined intersite plus substrate potential.

The extreme discreteness of the system with basically two particles moving at the same time allows for the detailed interpretation of the observed doublekinks, which was not possible for multi-kinks without dispersion presented in other works. The double-kink structure is produced by the matching of the two collisions experienced by a particle and the process of going over the substrate potential barrier between neighboring sites. We have shown that realistic potentials allow for the propagation of ultra discrete lattice kinks with energies that are consistent with the properties observed in experiments. Although we cannot claim that those kinks are responsible for those phenomena, it seems likely that they play an important role.

\section{References}

Alba, M. D., A. I. Becerro, M. A. Castro, and A. C. Perdigón (2001). "Hydrothermal reactivity of Lu-saturated smectites: Part I. A long-range order study". In: Amer. Mineral. 86, p. 115.

Archilla, J. F. R., J. Cuevas, M. D. Alba, M. Naranjo, and J. M. Trillo (2006). "Discrete Breathers for Understanding Reconstructive Mineral Processes at Low Temperatures". In: J. Phys. Chem. B 110.47, pp. 24112-24120.

Archilla, J. F. R., Yu. Kosevich, N. Jiménez, V. Sánchez-Morcillo, and L. M. García-Raffi (2013). "Moving Excitations in Cation Lattices". In: Ucr. J. Phys. 58.7, pp. 646-656.

Archilla, J. F. R., Yuriy A. Kosevich, N. Jiménez, V. J. Sánchez-Morcillo, and L. M. GarcíaRaffi (2015). "Ultradiscrete kinks with supersonic speed in a layered crystal with realistic potentials". In: Phys. Rev. E 91 (2), p. 022912.

Archilla, J.F.R., Y. A. Kosevich, N. Jiménez, V.J. Sánchez-Morcillo, and L.M. García-Raffi (2014). "Supersonic Kinks in Coulomb Lattices". In: Localized Excitations in Nonlinear Complex Systems. Ed. by R. Carretero et al. Springer, pp. 317-331.

Biersack, J., J.P. Ziegler, and M.D. Ziegler (2008). SRIM - The Stopping and Range of Ions in Matter. Published by J.P. Ziegler. 
Braun, O M and Yu S Kivshar (1998). "Nonlinear dynamics of the Frenkel-Kontorova model". In: Phys. Rep. 306, pp. 1-108.

Braun, O.M. and Yu.S. Kivshar (2004). The Frenkel-Kontorova Model. Springer.

Brudeylins, G. and D. Schmicker (1995). "Elastic and inelastic helium atom scattering at a cleaved mica sheet." In: Surf. Sci. 333, pp. 237-242.

Chaikin, P. M. and T. C. Lubensky (1995). Principles of Condensed Matter Physics. Cambridge: Cambridge University Press, pp. 601-602.

Chaplot, SL and et al (2002). "Inelastic neutron scattering and lattice dynamics of minerals". In: Eur. J. Min. 14, p. 291.

Collins, D R and C R A Catlow (1992). "Computer simulation of structure and cohesive properties of micas". In: Am. Min. 77, pp. 1172-1181.

Collins, D.R., W.G. Stirling, C.R.A. Catlow, and G. Rowbotham (1993). "Determination of Acoustic Phonon Dispersion Curves in Layer Silicates by Inelastic Neutron Scattering and Computer Simulation Techniques." In: Phys. Chem. Miner. 19, pp. 520-527.

Diaz, M, V. C. Farmer, and R Prost (2000). "Characterization and assignment of far infrared absorption bands of $\mathrm{K}^{+}$in muscovite". In: Clays Clay Miner. 48, pp. 433-438.

Dou, Q., J. Cuevas, J. C. Eilbeck, and F. M. Russell (2011). "Breathers and kinks in a simulated crystal experiment". In: Discret. Contin. Dyn. S.-S 4, pp. 1107-1118.

Dubinko, V. I., P. A. Selyshchev, and J. F. R. Archilla (2011). "Reaction-rate theory with account of the crystal anharmonicity". In: Phys. Rev. E 83, p. 041124.

Durrani, S. A. (2001). "Nuclear tracks: A success story of the 20th century". In: Rad. Meas 34, pp. $5-13$.

Durrani, S. A. (2008). "Nuclear tracks today: Strengths, weaknesses, challenges". In: Rad. Meas 43, S26-S33.

Fleischer, R.L. (2011). Tracks to Innovation. Nuclear Tracks in Science and Technology. Springer.

Frenkel, Ya. and T. Kontorova (1938). “-”. In: Phys. Z. Sowjetunion 13 (1), p. 1.

Friesecke, G. and K. Matthies (2002). "Atomic-scale localization of high-energy solitary waves on lattices". In: Physica D 171.4, pp. $211-220$.

Gedeon, O., J. Machacek, and M. Liska (2002). "Static energy hypersurface mapping of potassium cations in potassium silicate glasses". In: Phys. Chem. Glass. 43.5, pp. 241-246.

Hong, Z. L., H. Yoshida, Y. Ikuhara, T. Sakuma, T. Nishimura, and M. Mitomo (2002). "The effect of additive on sintering behavior and strength retention in silicon nitride with REdisilicate". In: J. Eur. Ceram. Soc. 22, p. 527.

Kosevich, A.M. and A.S. Kovalev (1973). "The supersonic motion of a crowdion. The one dimensional model with nonlinear interaction between the nearest neighbors". In: Solid State Commun. 12, pp. 763-764.

Kosevich, Yu. A. (1993). "Nonlinear sinusoidal waves and their Superposition in anharmonic lattices." In: Phys. Rev. Lett. 71, pp. 2058-2061.

Kosevich, Yu. A., R. Khomeriki, and S. Ruffo (2004). "Supersonic discrete kink-solitons and sinusoidal patterns with magic wave number in anharmonic lattices". In: Europhys. Lett. 66, pp. $21-27$.

Kudriavtsev, Y., A. Villegas, A. Godines, and R. Asomoza (2005). "Calculation of the surface binding energy for ion sputtered particles". In: Appl. Surf. Sci. 239.3-4, pp. 273-278.

Milchev, Andrey (1990). "Breakup threshold of solitons in systems with nonconvex interactions". In: Phys. Rev. B 42 (10), pp. 6727-6729.

Molerón, M., A. Leonard, and C. Daraio (2014). "Solitary waves in a chain of repelling magnets". In: J. Appl. Phys. 115.18, p. 184901.

Ni, Y, Yu A. Kosevich, S. Xiong, Y. Chalopin, and S. Volz (2014). "Substrate-induced cross-plane thermal propagative modes in few-layer graphene". In: Phys. Rev. B 89 (20), p. 205413.

Price, P. B. and R. M. Walker (1962). "Observation of fossil particle tracks in natural micas". In: Nature 196, pp. 732-734.

Russell, F. (1988a). "Identification and selection criteria for charged lepton tracks in mica". In: Nucl. Tracks. Rad. Meas. 15, pp. 41-44.

Russell, F. M. (1967a). "Duration of sensitive period for track recording in mica". In: Nature 217, pp. 51-52.

Russell, F. M. (1967b). "Tracks in mica caused by electron showers". In: Nature 216, pp. 907-909. 
Russell, F. M. (1988b). "Positive charge transport in layered crystalline solids". In: Phys. Lett. A 130, pp. 489-491.

Russell, F. M. and J. C. Eilbeck (2007). "Evidence for moving breathers in a layered crystal insulator at 300K". In: Europhys. Lett. 78, p. 10004.

Savin, A. V. and Yu A. Kosevich (2014). "Thermal conductivity of molecular chains with asymmetric potentials of pair interactions". In: Phys. Rev. E 89 (3), p. 032102.

Savin, A.V. (1995). "Supersonic regimes of motion of a topological soliton". In: Sov. Phys. JETP 81, pp. 608-613.

Schlößer, D., K. Kroneberger, M. Schosnig, F. M. Russell, and K. O. Groeneveld (1994). "Search for solitons in solids". In: Rad. Meas 23, pp. 209-213.

Silk, E C H and R S Barnes (1959). "Examination of fission fragment tracks with an electron microscope". In: Phylos. Mag. 4, pp. 970-971.

Snowden-Ifft, D. P., E. S. Freeman, and P. B. Price (1995). "Limits on dark-matter using ancient mica". In: Phys. Rev. Lett. 74.21, pp. 4133-4136.

Wada, N and WA Kamitakahara (1991). "Inelastic neutron and Raman scattering studies of muscovite and vermiculite layered silicates." In: Phys. Rev. B 43, p. 2391.

Zolotaryuk, Y., J.C Eilbeck, and A.V. Savin (1997). "Bound states of lattice solitons and their bifurcations". In: Physica D 108, pp. 81-91.

\section{Publications}

The contents of this chapter has been presented in the following publications:

\section{Journal papers}

- Archilla, J. F., Kosevich, Y. A.; Jiménez, N., Sánchez-Morcillo, V. and GarcíaRaffi, L. M. "Ultradiscrete kinks with supersonic speed in a layered crystal with realistic potentials" Phys. Rev. E, 91, 022912 (2015)

- Archilla, J. F., Kosevich, Y. A., Jiménez, N., Sanchez-Morcillo, V. and GarcíaRaffi, L. M. "Moving excitations In cation lattices" Ukranian Journal of Physics, $58,646-656(2013)$

\section{Conference proceedings}

- Jiménez, N., Archilla, J. F., Kosevich, Y. A., Sanchez-Morcillo, V. and GarcíaRaffi, L. M. "A crowdion in mica. Between K40 recoil and transmission sputtering" Quodons in mica: nonlinear localized travelling excitations in crystals, Altea, Spain. September 18-21, (2013)

- Jiménez, N., Sánchez-Morcillo, V., García-Raffi, L. M. and Archilla, J. F. "Kinks supersónicos en redes repulsivas" XXXIV Reunión Bienal de la Real Sociedad Española de Física, Valencia, Spain. July 17, (2013)

- Archilla, J. F., Kosevich, Y. A., Jiménez, N., Sanchez-Morcillo, V. and GarcíaRaffi, L. M. "Effect of ZBL potential on kinks in repulsive lattices" IV International Symposium on Strong Nonlinear Vibronic and Electronic Interactions in Solids. Tartu, Estonia, May 1-3, (2013) 
- Archilla, J. F., Kosevich, Y. A., Jiménez, N., Sanchez-Morcillo, V. and GarcíaRaffi, L. M. "Moving excitations in cation lattices" Problems of theoretical physics (dedicated to the 100th anniversary of Alexander Davydov). Kiev, Ukraine, October 8-11, (2012)

- Archilla, J. F., Kosevich, Y. A., Jiménez, N.; Sanchez-Morcillo, V. and GarcíaRaffi, L. M. "Supersonic solitons and kinks in repulsive lattices" Lattice Solitons and Irradiation-Induced Nonlinear Phenomena in Solids. Tartu, Estonia, October 31-November 2, (2012)

\section{Book chapters}

- Carretero-González, R. \& others (Eds.) Localized Excitations in Nonlinear Complex Systems, Springer (2014). Archilla, Juan F. R. and Kosevich, Yuriy A. and Jiménez, Noé and Sánchez-Morcillo, Víctor J. and García-Raffi, Luís M. "Supersonic Kinks in Coulomb Lattices", pp. 317-331 (2014),

- L. M. Archilla, J. F. R.; Jiménez, N.; Sánchez-Morcillo, V. J. \& García-Raffi, L. M. (Eds.) Quodons in mica: nonlinear localized travelling excitations in crystals, Springer, (2015). Archilla, J. F., Kosevich, Y. A., Jiménez, N. Sanchez-Morcillo, V. and García-Raffi, L. M. " A supersonic crowdion in mica: Ultradiscrete kinks with energy between $\mathrm{K}^{4} 0$ recoil and transmission sputtering", pp 69-98 (2015). 



\subsection{Introduction}

In the previous chapter we have considered waves propagating in nonlinear lattices. Such lattices can be macroscopic, idealized as masses coupled by springs, or even microscopic, as atoms/ions coupled by electromagnetic forces. Lattices can be then considered of basic model of solid matter, with its elements distributed periodically in a regular array. Many properties of solids, such as thermal conductivity, can be interpreted in terms of fundamental vibrations of the lattice, or phonons.

The study of matter as a periodic medium (a crystal of atoms), was originally performed by Bloch and Brillouin (Brillouin, 2003) in the 30-40's, and gave rise to an entire new discipline in physics, the solid state (or condensed matter) physics. One of the most celebrated effects of wave propagation in periodic media are the appearance of forbidden propagation regions in the energy spectrum of electrons, or band-gaps. Most of the physics of semiconductors, and therefore many electronic devices, are somehow based on this concept. In the late 80's, these ideas where extended by Yablonovich and John to light waves (electromagnetic waves in general) propagating in materials where the optical properties like the index of refraction were distributed periodically. These materials were named, by analogy with metals, as photonic crystals. The typical scale is given by the wavelength. Actually, not only light but any wave propagating in a periodic medium may experience the same effects, and acoustic waves are not an exception. Sound wave propagation in periodic media has become very popular in the last 20 years in acoustics, after the introduction of the concept of sonic crystals. Exploiting the analogies with other type of waves many interesting effects, as the mentioned forbidden propagation bands (band-gaps), but also focalization, self-collimation, negative refraction, and many others have been proposed. We consider in this chapter the simplest case plane waves propagating in a 1D structure, formed by a periodic alternation of layers with different properties. Depending on the context, such a structure has been named a multilayer, a superlattice (particularly in the context of semiconductors) or a 1D phononic crystal (this include more exotic structures, as the granular crystal or lattice).

The huge majority of the studies considered so far have assumed a low-amplitude (linear) regime, neglecting the nonlinear response of the medium. Intense wave propagation in nonlinear periodic media, and in particular the case of sound waves, is almost unexplored. In this chapter we present different examples of new phenomena related to sound wave propagation in multilayers, where each of the layer has a nonlinear cuadratic elastic response. Nonlinear acoustical effects in such structure have been studied only in a few works. For example, in (Yun et al., 2005) the harmonic generation process is described in a fluid/fluid multilayered structure (water/glicerine), based in a nonlinear wave equation. Also, acoustic solitons in solid layered nonlinear media have been presented (Yong et al., 2003). More recently, nonlinearity plus periodicity has been considered in (Liang et al., 2009), where an asymmetric propagation device (acoustic diode) was proposed. 
There, the nonlinearity and the periodicity appear in different position and its effect is consider separately.

The effects discussed in this Chapter are the result of the interplay between nonlinearity and periodicity. We mostly concentrate in two phenomena: the control of harmonic distortion and the generation and propagation of solitons in the multilayer. The first case is analyzed in depth, and the conditions needed to selectively act on the nonlinearly generated spectrum, and therefore manipulate the waveform in the desired way, are obtained and discussed.

The theory in this Chapter has been developed for fluid-fluid (scalar) structures, however the main conclusions are extendable to fluid-solid or to solid-solid multilayers, if particular conditions are given. Also, the main conclusions in the Chapter are independent on the regime of the waves (audible, ultrasound,...), and therefore on the size or scale of the structure. However, there is a domain, when ultrasound waves reach the Teraherz regime, where these ideas may find a great potential. The progress in miniaturization and the technological development allows currently to create multilayers at scales even in the nanometer range (each layer contains then a small number of atoms). This structures are usually made of semiconductors and are often used in particular applications as phononic mirrors to form phonon nanocavities, (Huynh et al., 2006) or microcavities to obtain a strong optomechanical coupling (Fainstein et al., 2013) (for a revent survey, see Huynh et al. (2015)). Notably, acoustic amplification was realized in doped GaAs/AlAs superlattices and recently a saser, for sound amplification by the stimulated (acoustic phonons) radiation was demonstrated, in a device including a superlattice gain medium and GaAs/AlAs SLs acoustic mirrors (Maryam et al., 2013).

Some phenomena are common to lattices and superlattices (both are nonlinear, dispersive, periodic media), and the results of this Chapter and the previous are connected. Harmonic generation has been described in granular chains, modeled by FPU equation. Such quadratic FPU is actually a basic model for lattices with different interaction potentials (like Hertz, Coulomb,...) for small displacements. The continuous limit of this equation gives the nonlinear acoustic equation, which actually connects the propagation of sound waves with the microscopic theory of matter.

\subsection{Dispersion relations}

We consider the simple case of a 1D periodic medium made of an arrangement of homogeneous fluid media of thickness $a_{i}$ with varying material properties. For the shake of simplicity only longitudinal waves under normal incidence are considered. A scheme of the system is shown in Fig. 2.1.

The propagation of small amplitude waves in an infinite periodic system is completely described by its dispersion relation, often known as band structure, 


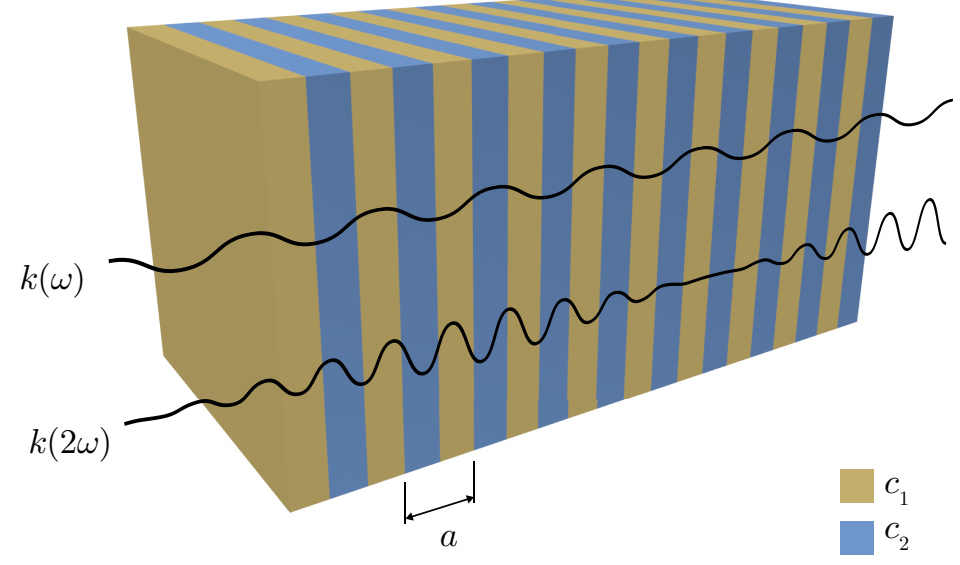

Figure 2.1: Layered acoustic system with two different layers and second harmonic generation scheme. Here the lattice period is $a=\sum a_{i}$.

that for $1 \mathrm{D}$ systems consisting in a periodic alternation of two different layers of length $a_{1}$ and $a_{2}$ can be expressed analytically as (Kosevich, 2005):

$$
\cos (k a)=\cos \left(k_{1} a_{1}\right) \cos \left(k_{2} a_{2}\right)-\frac{1}{2}\left(\frac{k_{1}}{k_{2}}+\frac{k_{2}}{k_{1}}\right) \sin \left(k_{1} a_{1}\right) \sin \left(k_{2} a_{2}\right),
$$

also known as the Rytov formula, where $k$ is the Bloch wave-number, the lattice period is defined by $a=a_{1}+a_{2}$ and $k_{i}=\omega / c_{i}$ with $c_{i}$ the sound speed in the $i$ layer. For a wave of frequency $\omega$ incident in a medium with known acoustical $c_{i}$ and geometrical $a_{i}$ parameters, the above equation results in a band structure of propagating and nonpropagating (bandgap) regions, as shown in Fig. 2.2. Thus, using Eq. (2.1), we can estimate the effect of periodicity on the different harmonics of the incident wave as they propagate trough the multilayer.

The ratio between layer thickness can be defined as

$$
\alpha=a_{1} / a
$$

leading to $a_{2}=(1-\alpha) a$.

An example of dispersion relation plot is shown in Fig. 2.2 for normalized parameters $a=0.5$ and for different sound speed ratios $c_{1} / c_{2}$. Increasing the impedance ratio between layers increases the reflected intensity in the trans-layer 

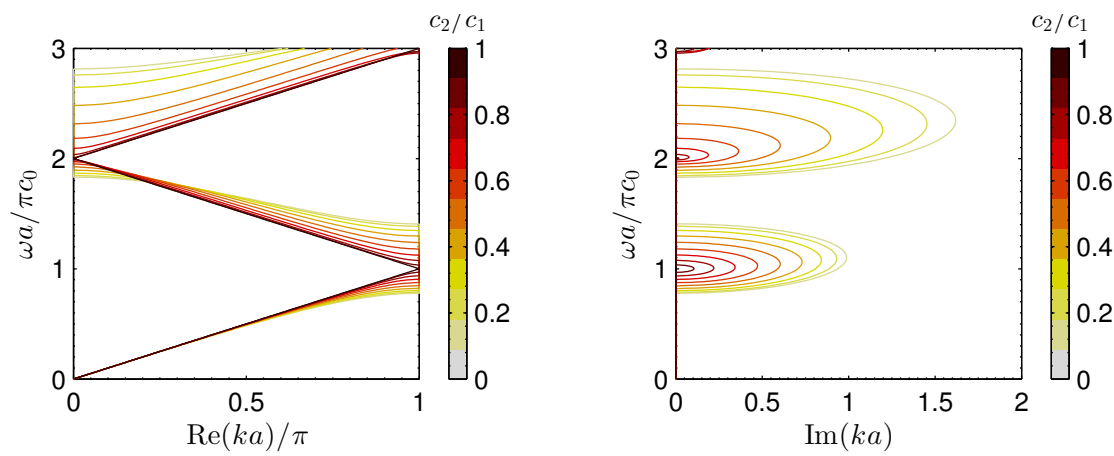

Figure 2.2: Dispersion relation of the two-layers system for layer proportion $\alpha=0.5$ and for different $c_{2} / c_{1}$ ratio. Left: real wavenumbers. Right: imaginary part of the complex wavenumber.

propagation, while the transmitted energy of the multiple internal reflections diminishes. As can be seen, due to these scattering processes band-gaps are progressively open for wavenumber $k=n \pi / a$ with $n=1,2, \ldots$. Thus, the bandwidth of these band-gaps also increases when the impedance ratio grows.

On the other hand, its imaginary part increases in amplitude with $c_{1} / c_{2}$, leading to shorter evanescent propagation inside the band-gap for high sound speed contrast layers, while remains zero (no attenuation) in the propagation band. It is worth noting here that the system is conservative: the physical interpretation of the complex wavenumber is not energy absorption, but back reflection of the incident wave. Thus, at band-gap frequencies waves penetrate only a short distance into the medium with a forward evanescent mode, and if the medium is perfectly periodic and lossless the energy is back-reflected (it behaves as a mirror).

Furthermore, the curvature of the real part of the dispersion relations in the propagation band indicates dispersive propagation. However, in the low frequency regime the curve $\omega(k)$ is nearly linear. Here, a relation between $c_{1}$ and $c_{2}$ can be obtained for estimating the ratio in the limit $\omega \rightarrow 0, k \rightarrow 0$, that is the effective sound speed of the layered system $\tilde{c_{0}}$.

In order to obtain $c_{0}$ we first expand in Taylor series Eq. (2.1) in powers of $\omega$ around $\omega=0$, and collecting terms up to second order we get

$$
\omega \approx \sqrt{(1-\cos (k a)) \frac{2 c_{1}^{2} c_{2}^{2}}{a\left(a_{2} c_{1}^{2}+a_{1} c_{2}^{2}\right)}} .
$$

Then, we expand again in Taylor series in powers of $k$ around $k=0$, and collecting terms up to first order we get

$$
\tilde{c_{0}}=\frac{\omega}{k} \approx \sqrt{\frac{a c_{1}^{2} c_{2}^{2}}{a_{2} c_{1}^{2}+a_{1} c_{2}^{2}}} .
$$


This value characterizes the layered media in the low frequency regime where the wavelength is much greater than the distance between layers. Furthermore, $\tilde{c_{0}}$ is useful also for frequency normalization, as long the first band-gap appears at $\omega=\pi \tilde{c_{0}} / a$ as can be appreciated in the common slope in the low frequency regime in Fig. 2.2. This quantity can be also obtained by assuming a mean density, $\tilde{\rho}$, and compressibility, $\tilde{\kappa}$ as (Yong et al., 2003):

$$
\tilde{c_{0}}=\sqrt{\frac{\tilde{\kappa}}{\tilde{\rho}}},
$$

where the mean values for density and compressibility can be obtained as

$$
\begin{aligned}
\tilde{\rho} & =\alpha \rho_{1}+(1-\alpha) \rho_{2}, \\
\frac{1}{\tilde{\kappa}} & =\frac{1}{\alpha \kappa_{1}}+\frac{1}{(1-\alpha) \kappa_{2}},
\end{aligned}
$$

being $\kappa_{i}=\rho_{i} c_{i}^{2}$ the compressibility of each layer.

\section{$2.3 \quad$ Full-wave model}

\subsubsection{Nonlinear constitutive mode}

The nonlinear propagation of sound in the acoustic inhomogeneous media, and in particular in multi-layered media can be described by several models, with different levels of accuracy. Here, we start from the equations of continuum mechanics for ideal fluids with space dependent parameters. Thus, applying mass conservation principle over a fluid control volume, the continuity equation reads (Naugolnykh et al., 1998):

$$
\frac{\partial \rho}{\partial t}+\nabla \cdot(\rho \mathbf{v})=0
$$

On the other hand, the conservation of momentum over the same fluid test volume leads to the equation of motion

$$
\rho \frac{D \mathbf{v}}{D t}+\nabla p=0
$$

where $\rho$ is the total density, $\mathbf{v}$ is the particle velocity vector over a Eulerian reference frame, $p$ is the acoustic pressure, $t$ is the time and $D$ is the material derivative operator 


$$
\frac{D}{D t}=\frac{\partial}{\partial t}+\mathbf{v} \cdot \nabla
$$

For non homogeneous media, the ambient properties of the fluid in the absence of sound can be space dependent, so the total density becomes $\rho(t, \mathbf{x})=\rho^{\prime}(t, \mathbf{x})+$ $\rho_{0}(\mathbf{x})$, where $\rho_{0}(\mathbf{x})$ is the spatially dependent ambient density and $\rho^{\prime}(t, \mathbf{x})$ is the perturbation of the density or acoustic density, that is space and time dependent. Then, using the material derivative, Eq. (2.9) becomes

$$
\rho_{0} \frac{\partial \mathbf{v}}{\partial t}+\nabla p=-\rho^{\prime} \frac{\partial \mathbf{v}}{\partial t}-\left(\rho^{\prime}+\rho_{0}\right)(\mathbf{v} \cdot \nabla) \mathbf{v} .
$$

In this equation, the first two terms in the left-hand-side accounts for linear acoustic propagation, where the terms in the right-hand-side introduces nonlinearity in the Eulerian reference frame through momentum advection processes.

On the other hand, we can expand Eq. (2.8) for non-homogeneous media as

$$
\frac{\partial \rho^{\prime}}{\partial t}+\rho_{0} \nabla \cdot \mathbf{v}+\mathbf{v} \cdot \nabla \rho_{0}=-\rho^{\prime} \nabla \cdot \mathbf{v}-\mathbf{v} \cdot \nabla \rho^{\prime}
$$

Here, the first two terms on the left-hand-side account for linear acoustic propagation, the third, also linear, accounts for the magnitude of the changes in the ambient layer properties. Note this term is space dependent but only changes at the interface between adjacent layers. For density matched layers, $\rho_{i}=\rho_{i-1}$, this terms vanishes. The terms on the right-hand-side are nonlinear and accounts for mass advection. This equation remains exact.

Finally, a fluid thermodynamic state equation $p=p(\rho, s)$ is needed to close the system, with $s$ the entropy. The local nonlinear media response relating density and pressure variations, retaining up to second order terms, can be written as

$$
p=c_{0}^{2} \rho^{\prime}+\frac{B}{2 A} \frac{c_{0}^{2}}{\rho_{0}} \rho^{\prime 2},
$$

where $B / A(\mathbf{x})$ is the quadratic nonlinear parameter and $c_{0}(\mathbf{x})$ is the sound speed, that can be also spatially dependent.

In this system of equations, quadratic nonlinearity appears in the equation of motion (2.11) and in the continuity equation (2.12), in the momentum and mass advection terms respectively, and also in the equation of state, Eq. (2.13), relating pressure and density acoustic perturbations. It is worth noting here that we only take into account nonlinear processes through the layer's bulk. The nonlinear effects at the boundary between adjacent sheets are neglected. These nonlinear boundary effects include cavitation processes, that in the case of fluids with very 
different compressibility can be very important. In the case of solid layers, other local nonlinear effects relative to boundaries, e.g. clapping phenomena between surfaces, can lead to nonlinearities that are orders of magnitude in importance compared to the bulk cumulative nonlinearities (Tournat et al., 2004).

\subsubsection{Second-order model}

For moderate amplitudes, the system of Eq. (2.11-2.13) can be simplified. In the following, we use same ordering scheme as in Ref. Hamilton et al. (1998a), where $\mathcal{O}(\varepsilon), \mathcal{O}\left(\varepsilon^{2}\right)$ and $\mathcal{O}\left(\varepsilon^{3}\right)$ represents the terms of generic smallness parameter $\varepsilon$. The derivation of a second-order nonlinear wave equation requires the substitution of the linearized acoustic approximations (first order) into second order terms of Eq. $(2.12,2.21)$. This substitution procedure will give third order errors, so the final nonlinear wave equation will be a second order approximation of the full constitutive relations.

Therefore, the first order equations of momentum and continuity can be obtained by linearization of Eq. (2.11-2.13) by assuming the acoustical magnitudes, $\rho^{\prime}, p^{\prime}$, and $\mathbf{v}$, are of small amplitude compared to the ambient values. Thus, for small amplitude perturbations the constitutive equations become

$$
\begin{aligned}
\frac{\partial \rho^{\prime}}{\partial t} & =-\rho_{0} \nabla \cdot \mathbf{v}+\mathcal{O}\left(\varepsilon^{2}\right) \\
\rho_{0} \frac{\partial \mathbf{v}}{\partial t} & =-\nabla p+\mathcal{O}\left(\varepsilon^{2}\right) \\
\rho^{\prime} & =\frac{p}{c_{0}^{2}}+\mathcal{O}\left(\varepsilon^{2}\right) .
\end{aligned}
$$

Then, we begin to manipulate the second order terms of the full constitutive relations with these first order approximations. Thus, using Eq. (2.14), the third right-hand-side term of the exact continuity Eq. (2.12) can be approximated up to second order as

$$
-\rho^{\prime} \nabla \cdot \mathbf{v}=\frac{\rho^{\prime}}{\rho_{0}}\left(\frac{\partial \rho^{\prime}}{\partial t}\right)+\mathcal{O}\left(\varepsilon^{3}\right)
$$

and using the linearized state Eq. (2.16) and rearranging terms leads to

$$
-\rho^{\prime} \nabla \cdot \mathbf{v}=\frac{1}{2 \rho_{i} c_{0}^{4}} \frac{\partial p^{2}}{\partial t}+\mathcal{O}\left(\varepsilon^{3}\right) .
$$

On the other hand, the fourth right-hand-side term of continuity Eq. (2.12) can be rewritten using the first order Eq. $(2.15,2.16)$ as 


$$
-\mathbf{v} \cdot \nabla \rho^{\prime}=\frac{\rho_{0}}{2 c_{0}^{2}} \frac{\partial u^{2}}{\partial t}+\frac{\mathbf{v} \rho^{\prime}}{c_{0}^{2}} \nabla c_{0}^{2}+\mathcal{O}\left(\varepsilon^{3}\right)
$$

Note that the last term of the above equation, that accounts for the local effect of the sound speed spatial variations, is $\mathcal{O}\left(\varepsilon^{3}\right)$, so it will be discarded, in contrast to the local effect of the ambient-density spatial variations (third term of Eq. (2.12)), that is $\mathcal{O}\left(\varepsilon^{2}\right)$ and it will remain in the derivation of the second order nonlinear wave equation for the layered media.

On the other hand, using the vector identity $(\mathbf{v} \cdot \nabla) \mathbf{v}=\frac{1}{2} \nabla v^{2}-\mathbf{v} \times \nabla \times \mathbf{v}$ with $v^{2}=\mathbf{v} \cdot \mathbf{v}$, and collecting terms up to second order, the equation of motion, Eq. (2.11), can be rewritten as

$$
\rho_{0} \frac{\partial \mathbf{v}}{\partial t}+\nabla p=\frac{1}{2} \rho_{0} \nabla v^{2}-\rho^{\prime} \frac{\partial \mathbf{v}}{\partial t}+\rho_{0} \mathbf{v} \times \nabla \times \mathbf{v}+\mathcal{O}\left(\varepsilon^{3}\right)
$$

In general, although it is second order, the vorticity term (last term in the righthand-side) decays exponentially away from rigid boundaries and can be discarded. However, it is essential to the analysis of other second order phenomena as the acoustic streaming (Hamilton et al., 1998a). In the following we will assume irrotational fluid. For a layered system, where the layer boundaries are orthogonal to the acoustic wave-vector, the vorticity modes can be neglected, while for strictly 1D propagation vanishes. Therefore, Eq. (2.20) becomes

$$
\rho_{0} \frac{\partial \mathbf{v}}{\partial t}+\nabla p=-\frac{1}{2} \rho_{0} \nabla v^{2}-\rho^{\prime} \frac{\partial \mathbf{v}}{\partial t}+\mathcal{O}\left(\varepsilon^{3}\right)
$$

On the other hand, the second term of the right-hand-side of the above equation can be approximated up to second order using the first order Eqs. $(2.14,2.16)$ as

$$
-\rho^{\prime} \frac{\partial \mathbf{v}}{\partial t}=\frac{1}{2 \rho_{0} c_{0}^{2}} \nabla p^{2}+\mathcal{O}\left(\varepsilon^{3}\right)
$$

Therefore, the second order approximation of the full constitutive relations in nonhomogeneous media can be expressed as:

$$
\frac{\partial \rho^{\prime}}{\partial t}+\rho_{0} \nabla \cdot \mathbf{v}+\mathbf{v} \cdot \nabla \rho_{0}=\frac{1}{2 \rho_{0} c_{0}^{4}} \frac{\partial p^{2}}{\partial t}+\frac{\rho_{0}}{2 c_{0}^{2}} \frac{\partial u^{2}}{\partial t}+\mathcal{O}\left(\varepsilon^{3}\right)
$$

and

$$
\rho_{0} \frac{\partial \mathbf{v}}{\partial t}+\nabla p=-\nabla\left(\frac{1}{2} \rho_{0} v^{2}-\frac{1}{2 \rho_{0} c_{0}^{2}} p^{2}\right)+\mathcal{O}\left(\varepsilon^{3}\right)
$$


Using the definition of the Lagrangian density

$$
\mathcal{L}=\frac{\rho_{0}}{2 c_{0}^{2}} u^{2}-\frac{p^{2}}{2 \rho_{0} c_{0}^{2}}
$$

the second order relations can be rewritten in a compact form

$$
\frac{\partial \rho^{\prime}}{\partial t}+\rho_{0} \nabla \cdot \mathbf{v}+\mathbf{v} \cdot \nabla \rho_{0}=\frac{1}{\rho_{0} c_{0}^{4}} \frac{\partial p^{2}}{\partial t}+\frac{1}{c_{0}^{2}} \frac{\partial \mathcal{L}}{\partial t}+\mathcal{O}\left(\varepsilon^{3}\right)
$$

and

$$
\rho_{0} \frac{\partial \mathbf{v}}{\partial t}+\nabla p=-\nabla \mathcal{L}+\mathcal{O}\left(\varepsilon^{3}\right)
$$

These equations can be combined to form a unique nonlinear wave equation by subtracting the time derivative of Eq. (2.26) to the divergence of Eq. (2.26) to obtain

$$
\nabla^{2} p-\frac{\partial^{2} \rho^{\prime}}{\partial t^{2}}-\frac{\partial \mathbf{v}}{\partial t} \nabla \rho_{0}=-\frac{1}{\rho_{0} c_{0}^{4}} \frac{\partial^{2} p^{2}}{\partial t^{2}}-\left(\nabla^{2}+\frac{1}{c_{0}^{2}} \frac{\partial^{2}}{\partial t^{2}}\right) \mathcal{L}+\mathcal{O}\left(\varepsilon^{3}\right)
$$

Here, the third term can be expressed in terms of the acoustic pressure using the local instantaneous media response described by the state equation

$$
\rho^{\prime}=\frac{p}{c_{0}^{2}}-\frac{1}{\rho_{0} c_{0}^{4}} \frac{B}{2 A} p^{2} .
$$

The fourth term of Eq. (2.28) can be also approximated using the first order Eq. (2.15), to finally obtain the second order nonlinear wave equation for nonhomogeneous media

$$
\nabla^{2} p-\frac{1}{c_{i}^{2}} \frac{\partial^{2} p}{\partial t^{2}}-\frac{1}{\rho_{0}} \nabla \rho_{0} \nabla p=-\frac{\beta}{\rho_{0} c_{0}^{4}} \frac{\partial^{2} p^{2}}{\partial t^{2}}-\left(\nabla^{2}+\frac{1}{c_{0}^{2}} \frac{\partial^{2}}{\partial t^{2}}\right) \mathcal{L}+\mathcal{O}\left(\varepsilon^{3}\right)
$$

where we introduced the coefficient of nonlinearity $\beta=1+\frac{B}{2 A}$ that accounts for material and mass advection quadratic nonlinearities. It is worth noting here (and discussed elsewhere (Hamilton et al., 1998a)) that the second-order Lagrangian density vanish for plane progressive waves due to the first order relation $p=u c_{0} \rho_{0}$ that leads to $\mathcal{L}=0$. In this case, Eq. (2.28) simplifies to the well-known Westervelt equation for inhomogeneous media 


$$
\nabla^{2} p-\frac{1}{c_{0}^{2}} \frac{\partial^{2} p}{\partial t^{2}}-\frac{1}{\rho_{0}} \nabla \rho_{0} \nabla p=-\frac{\beta}{\rho_{0} c_{0}^{4}} \frac{\partial^{2} p^{2}}{\partial t^{2}}+\mathcal{O}\left(\varepsilon^{3}\right)
$$

In general, the Lagrangian density term can be discarded based on the distinction of cumulative and local nonlinear effects. In this way, for progressive quasi-plane wave propagation in homogeneous media the nonlinear local effects become insignificant in comparison to the nonlinear cumulative effects, where in most practicals situations, beyond a distance of only one wavelength away from the source local nonlinear effects can be neglected. However, local nonlinear effects can become significant in other complex situations including standing-wave fields. Concerning the layered media, in this Chapter we solve numerically the full constitutive relations, and we use the second order approximation Eq. (2.31) to obtain analytical solutions where the effect of the Lagrangian density term is assumed to be negligible.

\subsubsection{Numerical solution}

For one-dimensional acoustic waves $\mathbf{v}=\left(v_{x}, 0,0\right)$, so the full constitutive relations Eqs. (2.8-2.11) can be expressed for nonhomogeneous media as

$$
\begin{gathered}
\frac{\partial \rho}{\partial t}=-\frac{\partial\left(\rho v_{x}\right)}{\partial x} \\
\frac{\partial v_{x}}{\partial t}=-\frac{1}{2} \frac{\partial v_{x}^{2}}{\partial x}-\frac{1}{\rho} \frac{\partial p}{\partial x}
\end{gathered}
$$

The system of equations $(2.13,2.32-2.33)$ can be solved by several numerical methods (Botteldooren, 1996; Ginter et al., 2002; Vanhille et al., 2004; Albin et al., 2011). In this chapter we use the Finite-Differences in Time-Domain (FDTD) method: the density and pressure domain are discretized staggered in time and space respect to the particle velocity field, spatial and temporal derivatives are solved by centered finite difference operators, while the temporal integration is an explicit leap-frog scheme that consecutively solves density, pressure and particle velocity from continuity, momentum and state equations respectively. Thus, due to the 2-point stencil of the centered finite differences, the algorithm presents second order accuracy. Stability is achieved by the well-known Courant- Fiedrich-Levy (CFL) time step restriction and numerical FDTD dispersion is controlled due to a high enough grid refinement. Due to the low-dimensional space, a refinement $>100$ elements per wavelength gives a negligible numerical dispersion compared to the physical one due to the layered system.

The dispersion relation (band structure) can be numerically obtained for the $1 \mathrm{D}$ system by a time-domain simulation of the linearized system. Linearization of 

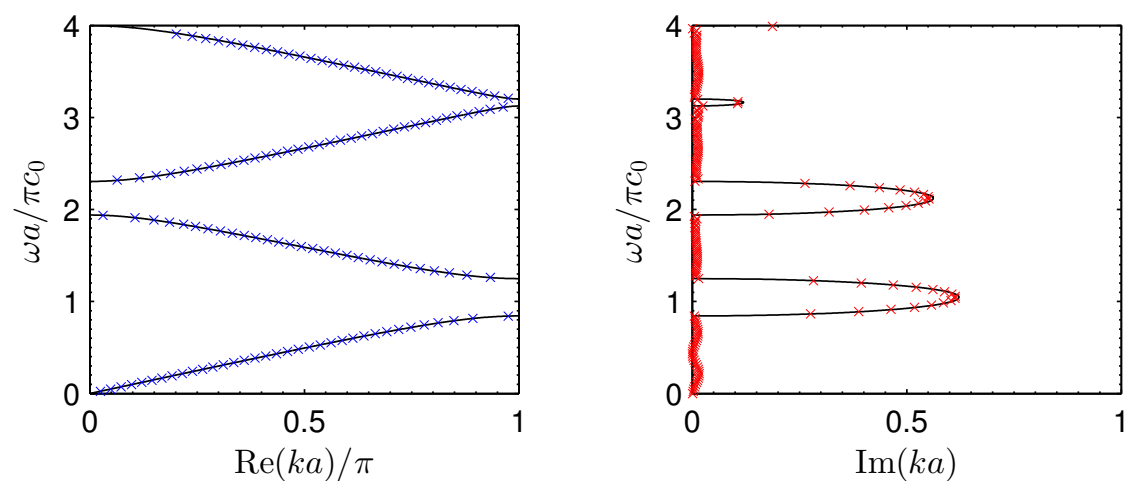

Figure 2.3: Dispersion relation of the two-layers system for normalized parameters $\alpha=0.5$ and $c_{2} / c_{1}=0.5$. Left: real wavenumbers. Right: imaginary part. Rytov analytical Eq. (2.1) (Continuous line) and numerical solution (markers).

equations $(2.13,2.32-2.33)$ leads to the small amplitude version of Eq. (2.30), that is the nonhomogeneous wave equation, so both models present the exact same solution. Simulated pressure of Eqs. $(2.13,2.32-2.33)$ using a small amplitude excitation $\left(p_{0}=10^{-6} \mathrm{~Pa}\right.$ or $\left.\varepsilon=u_{0} / c_{0} \approx 10^{-16}\right)$ was recorded at two locations $x_{0}$ and $x_{1}$, located in different layers. Attenuation and phase velocity were estimated from the spectral components over the bandwidth of the recorded signals, that in this case was a numerical Dirac delta function in order to excite the entire numerical bandwidth. The numerical attenuation was calculated as

$$
\alpha(\omega)=\frac{\ln \left(\left|P\left(\omega, z_{2}\right) / P\left(\omega, z_{1}\right)\right|\right)}{\left(z_{1}-z_{0}\right)},
$$

where $P(\omega)$ is the Fourier transform of the measured pressure waveforms at points $x_{1}$ and $x_{2}$. On the other hand, the phase velocity was computed as

$$
c_{p}(\omega)=\frac{\omega \cdot\left(z_{2}-z_{1}\right)}{\arg \left(P\left(\omega, z_{2}\right) / P\left(\omega, z_{1}\right)\right)},
$$

where a correct phase unwrapping is needed in the arg function. Figure 2.3 shows the numerical and theoretical estimations of the dispersion relations, where excellent agreement is observed between them. 


\subsection{Harmonic generation in layered media}

\subsubsection{Overview}

We start studying the response of the layered system for plane-harmonic wave excitation. Then, as sketched in Fig. (2.1), the source is placed in one boundary of the layered system, and the acoustic relevant magnitudes are calculated and measured along space and time. As the wave propagates, cumulative nonlinear effects generate harmonics of the fundamental frequency, $\omega_{0}$, and due to the multiple scattering processes into the layers, local nonlinear effects also distorts the wave. However, the high dispersion of the layered system have a strong impact on the nonlinear harmonic generation. Dispersion modify the resonance conditions between fundamental and second harmonic wave, and also for other nonlinearly generated higher frequencies. In this way, nonlinear energy transfer efficiency from one component to another is modified in a wide variety of configurations, leading to the possibility of engineering and controlling the nonlinear wave processes by tuning the dispersion relation.

Figures 2.4 Figure 2.5 show an overview of the nonlinear harmonic generation over the propagation distance in a sample of layered media for normalized parameters $\alpha=0.5$ and $c_{2}=0.7 c_{1}$. From now on, if it is not explicitly said, the rest of the medium parameters are kept constant for the sake of simplicity, namely $\rho_{1}=\rho_{2}$ and $B_{1} / A_{1}=B_{2} / A_{2}$. If the frequency of the fundamental wave is varied, the harmonic generation exhibit various phenomena depending on the dispersion relation (plotted on the left of Fig 2.4a and Fig. 2.4b). Thus, we difference different frequency areas that will be explained below. It is worth noting here that the specific limiting frequency values and bandwidth between areas are strongly depending in the layered parameters However, the ordering of zones for different phenomena are, in general, the following:

(A) Fubini-homogeneous: The first zone is the very-low frequency band where the dispersion relation is nearly flat not only for the fundamental wave, but also for higher spectral components. In this region, the layered system behaves as an homogeneous material with effective parameters and dispersion can be neglected.

(B) Weak-dispersion: As the frequency is increased dispersion start to appear and different harmonics propagate with different wave speed. In the low dispersive regime, second harmonic dispersion is weak and even can be neglected, but third harmonic experiment strong dispersion and higher harmonics can fall in a band-gap where its propagation will be evanescent. Due to this situation, the energy transfer from the fundamental to its second harmonic is almost constant, and the second harmonic grows linearly with propagation. However, higher order cascade processes can be modified due to dispersion and the energy transfer from second to higher harmonics is decreased. This 

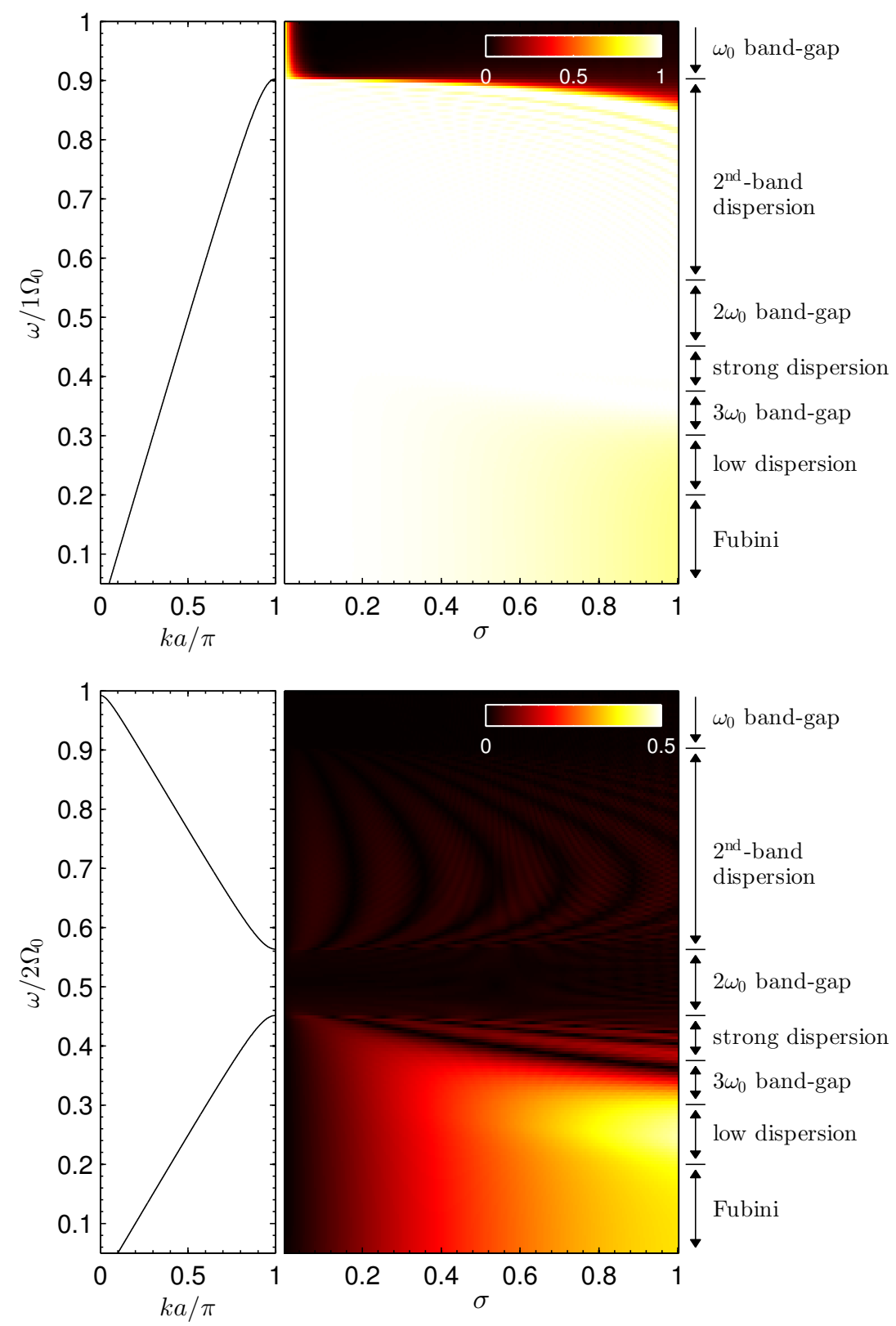

Figure 2.4: Overview of the harmonic generation processes in a nonlinear layered acoustic media. Top: fundamental component field, bottom: second harmonic field. Left: dispersion relation of the two-layers system for normalized parameters $\alpha=0.5$ and $c_{2} / c_{1}=0.7$. Right: pressure amplitude (color) normalized to the source amplitude versus fundamental wave $(\omega)$ frequency and distance normalized to shock distance $(\sigma)$ 


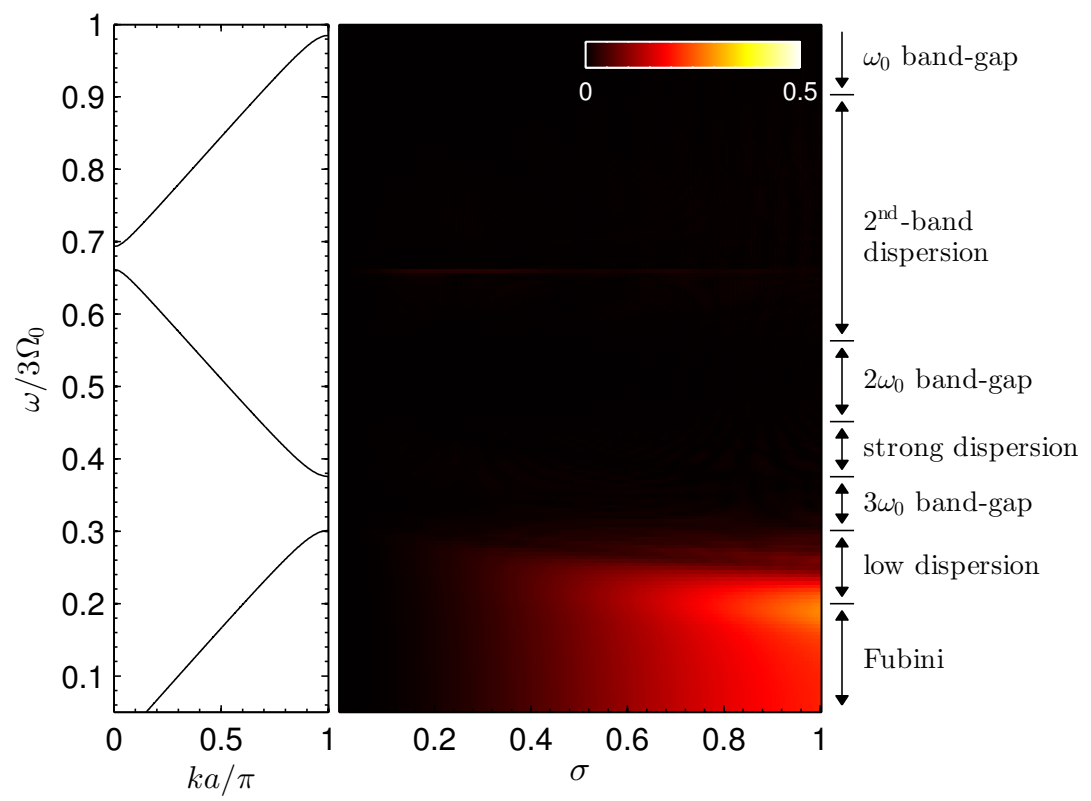

Figure 2.5: Overview of the harmonic generation processes in a nonlinear layered acoustic media for third harmonic. Left: dispersion relation of the two-layers system for normalized parameters $\alpha=0.5$ and $c_{2} / c_{1}=0.7$. Right: pressure amplitude (color) normalized to the source amplitude versus fundamental wave $(\omega)$ frequency and distance normalized to shock distance $(\sigma)$

situation avoids the emergence of shocks and underthat conditions the second harmonic amplitude can overcomes the second harmonic field amplitude observed in the absence of dispersion.

(C) $3^{\text {rd }}$ harmonic in band-gap: In this frequency area the propagation of third harmonic is evanescent and, in general, strong dispersion is observed on the second harmonic.

(D) Strong-dispersion: Where strong dispersion is observed for the second harmonic component, its field develops beatings in space, where its amplitude and spatial frequency depends on the matching conditions.

(E) $2^{\text {nd }}$ harmonic in band-gap: In this frequency area second harmonic propagation is evanescent and the field does not grow with distance, but achieves a nearly constant value, being locally forced by the first.

(F) $2^{\text {nd }}$ band dispersion: When second harmonic is in the second band, a high variability of phenomena can be observed depending on the material parameters. In the sample plot in Fig. 2.4 the second harmonic develops oscillations whose period varies by changing the frequency due to different phase matching conditions. By tuning material parameters and frequency different phase 
matching conditions can be obtained, leading to a wide variety of possibilities. A sample is observed in Fig. 2.5 for $\omega_{0}=0.6 \Omega_{0}$, where third harmonic falls in the limit of the second band-gap and also phase-matching is higher for third that for second harmonic, leading to third harmonic field higher than the second for this specific situation.

(G) $1^{\text {st }}$ harmonic in band-gap: When fundamental frequency falls in the bandgap evanescent propagation is achieved and only local nonlinearities can be exited. However, due to the imaginary part of the wavevector inside the band-gap, that depends on the frequency, (see Fig. 2.3), the length of the exponentially decaying propagation inside the layered media can be tuned and therefore second harmonic can be produced at the beginning of the layered media while fundamental cannot propagate at the exist of the system.

These scenarios will be studied in the following subsections.

\subsubsection{Homogeneous-Fubini}

We start studying the rpropagation in the layered system for harmonic excitation in the very low frequency regime, where we assume that $k a \ll 1$ holds. As the Rytov's Eq. (2.1) predicts, in the very low frequency regime the slope of the $\omega(k)$ curve is nearly flat, and after sound speed normalization using using Eq. (2.4), the dispersion of the layered system should be negligible. Thus, the medium behaves as a continuum-lossless homogeneous material with effective parameters. This is known as the homogenization limit. However, attention should be paid here if the fluid compressibility highly differs between layers because the validity of the effective parameters may fail. It is the case, for example, of a liquid with gas inclusions (bubbly liquids) (Gurbatov et al., 2011).

In limit of low-frequency, the dispersion of all the spectral components is negligible, and they all propagate at nearly the same velocity. Thus, in the absence of dispersion and attenuation process, the system of Eq. (2.13, 2.32-2.33) can be reduced for harmonic-plane wave to a Burger's evolution equation expressed in traveling coordinates with effective parameters, namely $\tilde{c_{0}}, \tilde{\rho_{0}}$ and $\tilde{\beta}$. Therefore, the approximate evolution equation in terms of the acoustic pressure can be written as:

$$
\frac{\partial p}{\partial t}+\tilde{c_{0}} \frac{\partial p}{\partial x}=\frac{\tilde{\beta}}{2 \tilde{\rho_{0}}{\tilde{c_{0}}}^{2}} \frac{\partial p^{2}}{\partial t}
$$

An analytic solution of this equation is also available through the Fubini's representation for the $n$ th-harmonics of the fundamental wave of frequency $\omega$ and initial amplitude $p_{0}$ as 

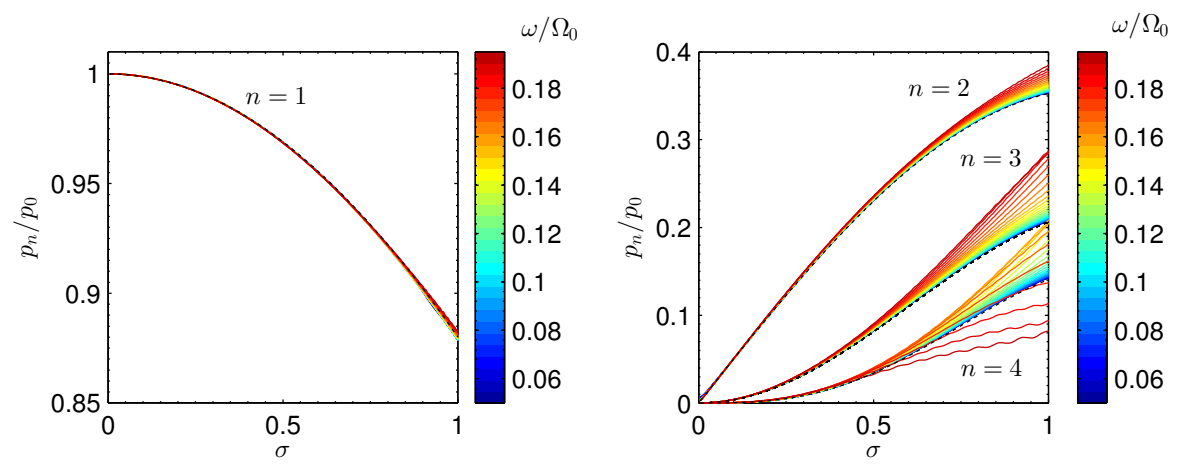

Figure 2.6: Harmonic generation in homogeneous-Fubini regime of the layered media

$$
p(\sigma, \tau)=p_{0} \sum_{n=1}^{\infty} \frac{2}{n \sigma} J_{n}(n \sigma) \sin (n \omega \tau),
$$

where $J_{n}$ is the Bessel function of order $n$, and $\sigma=x / x_{s}$ is the propagation coordinate, normalized to the shock formation distance:

$$
x_{s}=\frac{1}{\tilde{\beta} \tilde{\varepsilon} k},
$$

with the effective match number $\tilde{\varepsilon}=u / \tilde{c_{0}}$ and the effective wavenumber $k=\omega / \tilde{c_{0}}$, that can be also found from Eq. (2.1). This celebrated solution is valid for $\sigma<1$ (pre-shock region).

Simulations in this regime were carried out using a full-wave constitutive relations solver. Thus, we shall define the normalized frequency as $\Omega_{0}=\pi \tilde{c_{0}} / a$ (located in the first band-gap). Therefore, the source frequency was set to $\omega=0.1 \Omega_{0}$.

Figure 2.6 shows the analytical and numerical solutions for the low frequency limit of the layered system, where an excellent agreement is obtained between Fubini and numerical solutions in the pre-shock region, $\sigma<1$ and for low excitation frequencies. As commented above, when the fundamental frequency is increased the higher harmonics fall in dispersive region of the frequency bands, and thus its wave speed is reduced. In this situation, phase matching conditions are no longer fulfilled and therefore the energy transfer from fundamental to higher harmonics is modified. Thus, the Fubini solution can be only applied as an ideal solution for the low-frequency limit or as a good approximation for the first harmonics and for frequencies below $\omega \lesssim 0.1 \Omega_{0}$.

However, it should be noted that for observing a shock wave, i. e. a discontinuous solution, all the spectral components must be phase matched, as occurs in 

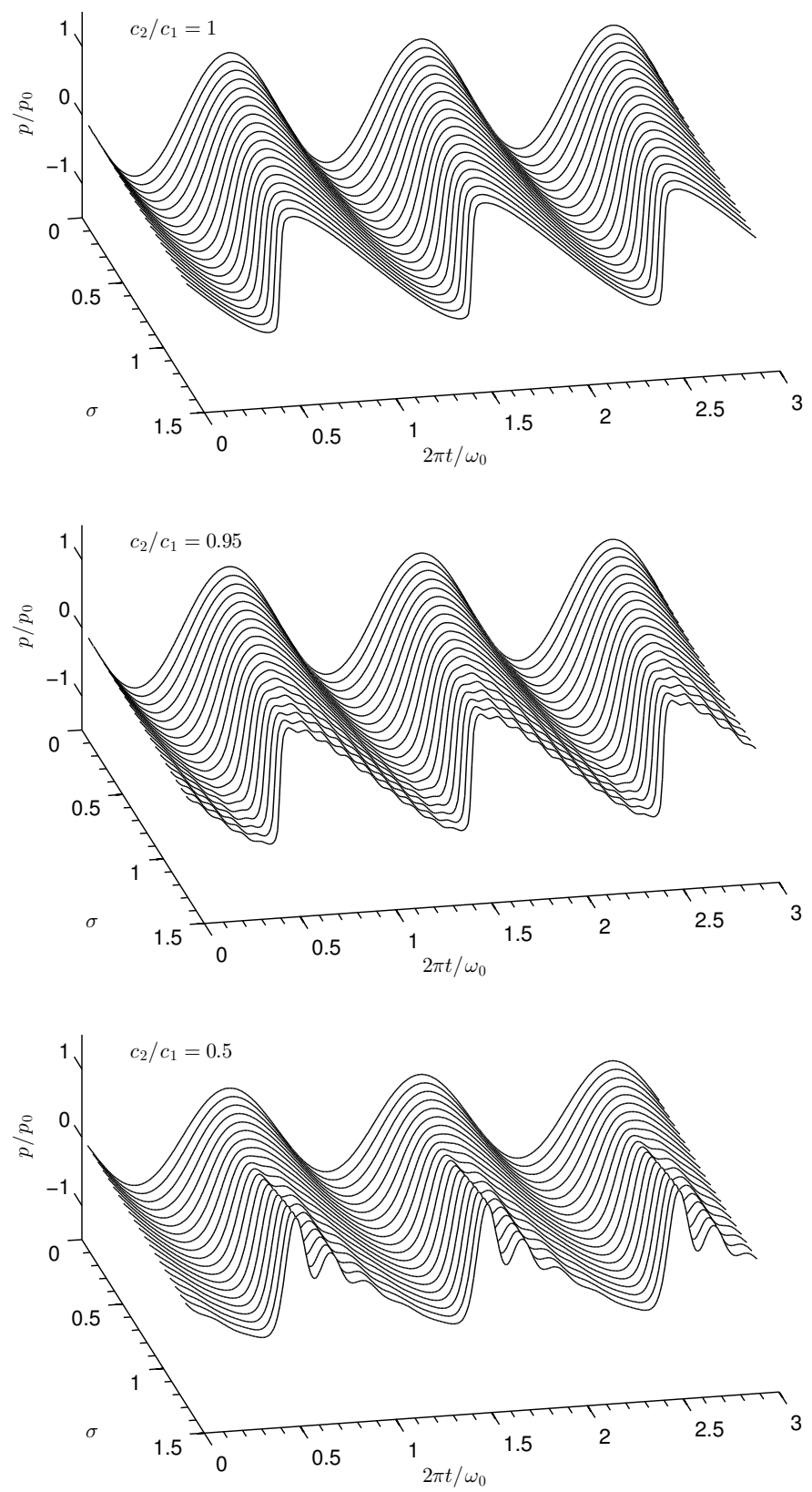

Figure 2.7: Waveform evolution beyond shock formation distance in layered media with parameters $\omega_{0} / \Omega_{0}=0.1$ and $c_{2} / c_{1}=1$ (top), $c_{2} / c_{1}=0.95$ (middle) and $c_{2} / c_{1}=0.5$ (bottom). Instead a discontinuity, pulsations around the sharp front are formed. 
the nondispersive (homogeneous) media. Then, even for a low finite fundamental frequency, a small (but non negligible) dispersion will appear for the higher frequency components, and a phase mismatch is introduced on these high spectral components. The differences on phase speed on the different spectral components over a discontinuity impedes the formation of a stable traveling shock wave, as long sound speed dispersion spreads the high-frequency wave vectors that describe the discontinuous solution. Instead of a shock wave, pulsations or ripples are formed over the sharp nonlinear wavefront for $\sigma>1$. This scenario reduces the validity of the homogeneous Eq. (2.36) to distances shorter than shock formation in lossless media, where the specific limit is dependent on the flatness of the dispersion relation near the band-gap, i.e. the layered media parameters: $a, c_{2} / c_{1}, \rho_{2} / \rho_{1}$ and $\alpha=a_{1} / a$.

Figure 2.7 shows the waveform evolution calculated at different distances in the shock-free region and also beyond shock formation distance for a homogeneous media $\left(c_{2} / c_{1}=1\right)$ for a nearly-flat dispersion relation $\left(c_{2} / c_{1}=0.95\right.$ and $\left.\alpha=0.5\right)$ and for a more dispersive media $\left(c_{2} / c_{1}=0.5, d_{2} / d_{1}=0.5\right)$. The above described effects are clearly visible: the waveform evolution follows the Fubini analytical solution for $\sigma<1$ in both layered systems. However, for longer distances, pulsations around the discontinuity are formed due to different phase speed of the higher spectral components, spreading the sharp solution into a solitonic pulsations. In the absence of dissipation, these pulsations evolves in a train of weakly interacting solitons. Solitons in the layered media will be studied later on Sec. 2.6.

\subsubsection{Dispersive regime}

For frequencies above the (idealized) homogeneous-Fubini regime, finite (weak and strong) dispersion effects are observed. The dispersive effects of the layered system deeply affects harmonic generation processes.

As intense waves propagate through a quadratic nonlinear medium, their frequency component interact with each other and new frequencies arise at combination frequencies, including higher harmonics. The cumulative energy transfer from the interacting waves to the harmonics is dependent on the resonance conditions:

$$
\omega_{1} \pm \omega_{2}=\omega_{3}, \mathbf{k}_{1} \pm \mathbf{k}_{2}=\mathbf{k}_{3}
$$

Note these conditions express the laws of conservation of energy $(\hbar \omega)$ and momentum $(\hbar \mathbf{k})$ in the quantum description for the disintegration and merging of quanta (Naugolnykh et al., 1998).

These conditions can be satisfied in a variety of situations. The most simple case is observed in nondispersive media and for collinear waves $k_{i}=\omega_{i} / c_{0}$. In this situation the resonance conditions are fulfilled all over the spectra and a large number of harmonics interacts synchronously: when there exist in the system a free 


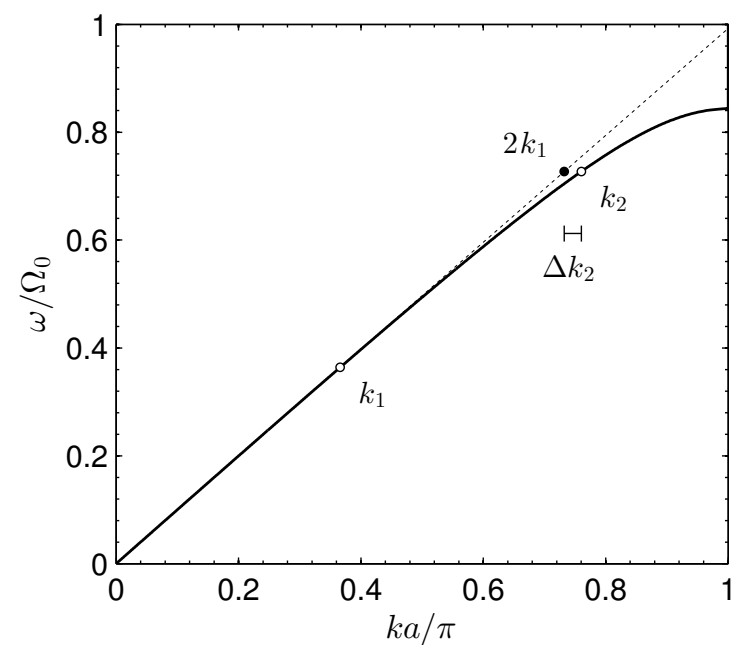

Figure 2.8: Scheme of the phase miss-matching situation. The fundamental wave vector $k_{1}$ at frequency $\omega$ generates a forced wave $2 k_{1}$ at frequency $2 \omega$. The free wave that the system allows to propagate is $k_{2}$, located in the dispersion relation curve. Due to dispersion, $k_{2} \neq 2 k_{1}$, thus there exist a phase mismatch, $\Delta k_{2}$ between both waves and the generation is therefore asynchronous.

wave with velocity $\omega_{3} /\left|\mathbf{k}_{3}\right|$ that matches the excited (forced) wave $\omega_{1} \pm \omega_{2} /\left|\mathbf{k}_{1} \pm \mathbf{k}_{2}\right|$, the free wave is excited in a resonant way. The resonant interaction leads therefore to synchronous, cumulative energy transfer from the initial wave to the secondary wave fields.

In the case of an initial monochromatic wave, the main wave generates its second harmonic. The resonant conditions Eq. (2.39) in this situation read

$$
2 \omega_{1}=\omega_{2}, 2 \mathbf{k}_{1}=\mathbf{k}_{2}
$$

that holds true for nondispersive collinear waves, leading to the simple relation $2 \mathbf{k}\left(\omega_{1}\right)=\mathbf{k}\left(2 \omega_{1}\right)$. However, in the case of dispersive media this condition is, in general, not fulfilled and the forced and free waves interact asynchronously. Figure 2.8 shows a scheme of this situation for a layered media with a fundamental wave in the first dispersion band.

In order to study asynchronous second harmonic generation processes, we recall here for the lossless second-order wave equation Eq. (2.31) for one-dimensional propagation

$$
\frac{\partial^{2} p}{\partial x^{2}}-\frac{1}{c_{0}^{2}} \frac{\partial^{2} p}{\partial t^{2}}-\frac{1}{\rho_{0}} \frac{\partial \rho_{0}}{\partial x} \frac{\partial p}{\partial x}=-\frac{\beta}{\rho_{0} c_{0}^{4}} \frac{\partial^{2} p^{2}}{\partial t^{2}}
$$


This equation does not include dispersion by itself, dispersion arises from the solution of the linearized wave equation with the layered media boundary conditions, where the eigenvalue problem leads to the Rytov's dispersion relation Eq. (2.1).

In the following, we apply a perturbation method to obtain an approximate solution for the second harmonic field. We expand the pressure field as sum of contributions of different orders, as

$$
p=p^{(1)}+\varepsilon p^{(2)}+\cdots,
$$

where $\varepsilon$ is the smallness perturbation parameter, which we identify with the acoustic Match number. Thus, $p^{(1)}$ is the first order (linear) solution of the problem and $p^{(2)}$ its the second order contribution. By substituting Eq. (2.42) in the second order wave Eq. (2.41), assuming constant density ${ }^{1}$ and neglecting $\mathcal{O}\left(\varepsilon^{3}\right)$ terms we get the coupled set of equations

$$
\begin{aligned}
\frac{\partial^{2} p^{(1)}}{\partial x^{2}}-\frac{1}{c_{0}^{2}} \frac{\partial^{2} p^{(1)}}{\partial t^{2}} & =0 \\
\frac{\partial^{2} p^{(2)}}{\partial x^{2}}-\frac{1}{c_{0}^{2}} \frac{\partial^{2} p^{(2)}}{\partial t^{2}} & =-\frac{\beta}{\rho_{0} c_{0}^{4}} \frac{\partial^{2} p^{(1) 2}}{\partial t^{2}} .
\end{aligned}
$$

The first order Eq. (2.43) for a monochromatic plane wave of frequency $\omega$ has the trivial solution

$$
p^{(1)}=p_{0} \sin \left(\omega t-k_{1} x\right)
$$

where $k_{1}=k(\omega)$ is the wave vector associated with the primary frequency $\omega$, and $p_{0}$ is the excitation pressure amplitude. Substitution of Eq. (2.45) into the right-hand side term of Eq. (2.44), using the trigonometric identity $\sin ^{2}(\theta)=(1-\cos (2 \theta)) / 2$, an inhomogeneous equation for the second harmonic field is obtained:

$$
\frac{\partial^{2} p^{(2)}}{\partial x^{2}}-\frac{1}{c_{0}^{2}} \frac{\partial^{2} p^{(2)}}{\partial t^{2}}=-\frac{4 \beta \omega^{2} p_{0}^{2}}{\rho_{0} c_{0}^{4}} \sin \left(2 \omega t-2 k_{1} x\right) .
$$

The general solution of the this equation will be the sum of the solution of the homogeneous equation $\left(p_{0}=0\right)$, and the particular solution of the inhomogeneous equation $\left(p_{0} \neq 0\right)$. Therefore the field for the second harmonic can be expressed as $p^{(2)}=p_{h}^{(2)}+p_{f}^{(2)}$, where the corresponding waves for this two solutions are the free, and forced waves respectively. Following Ref. Rudenko et al. (1977), we can obtain the homogeneous and particular solutions as:

\footnotetext{
${ }^{1}$ We neglect the ambient density variations for the sake of simplicity. Dispersion arise also for sound speed variations, that are assumed to be implicit in the boundary conditions.
} 


$$
\begin{aligned}
& p_{h}^{(2)}=p_{h}^{(2)}(x=0) \sin \left(2 \omega_{1} t-k_{2} x\right), \\
& p_{f}^{(2)}=\frac{A}{\left(k_{2}+2 k_{1}\right)\left(k_{2}-2 k_{1}\right)} \sin \left(2 \omega_{1} t-2 k_{1} x\right),
\end{aligned}
$$

where $k_{2}=k\left(2 \omega_{1}\right)$ is the wavenumber of the free wave at second harmonic frequency, and the constant $A=-4 \beta \omega_{1}^{2} p_{0}^{2} / \rho_{0} c_{0}^{4}$. It is worth noting here that as long $2 k_{1} \neq k_{2}$, the forced and free waves in dispersive media have different phase speed, i. e. the forced and free waves are phase mismatched as can be seen in the argument of the sin function in Eq. (2.47-2.48). On the other hand, as long as there is not second harmonic at $x=0$ (boundary condition), we must set

$$
p_{h}^{(2)}(x=0)=-\frac{A}{\left(k_{2}+2 k_{1}\right)\left(k_{2}-2 k_{1}\right)} .
$$

Therefore, combining Eqs. (2.47-2.49) the evolution of the second harmonic field can be expressed as

$$
p^{(2)}=\frac{A}{k_{2} \Delta k} \sin \left(\frac{\Delta k}{2} x\right) \cos \left(2 \omega_{1} t-k_{2}^{\prime} x\right),
$$

where the effective wave number is $k_{2}^{\prime}=\left(k_{2}+2 k_{1}\right) / 2 \approx k_{2}$ and the detuning parameter that describes the asynchronous second harmonic generation is defined as

$$
\Delta k=\left|k_{2}-2 k_{1}\right|=|k(2 \omega)-2 k(\omega)|
$$

Equation (2.50) describes the well-known effect in second harmonic generation, that is the beatings in space of the second harmonic field when the resonant conditions are not fulfilled. Thus, as $\Delta k$ increases, the beating spatial period and also its maximum amplitude decreases. The position of the maximum of the beating, also called the coherence length, can be related to the second-harmonic phase-mismatching frequency as

$$
x_{c}=\frac{\pi}{\Delta k}=\frac{\pi}{|k(2 \omega)-2 k(\omega)|} .
$$

This length corresponds to the half of the spatial period of the beating, where the maximum of the field is located. It can be expressed also for other higher harmonics simply as $x_{c}(n)=\pi / \Delta k_{n}=\pi /|k(n \omega)-n k(\omega)|$.

In the limiting case of $\Delta k \rightarrow 0$, the second harmonic field is generated synchronous and accumulates with distance, so linear growth is predicted. In this 

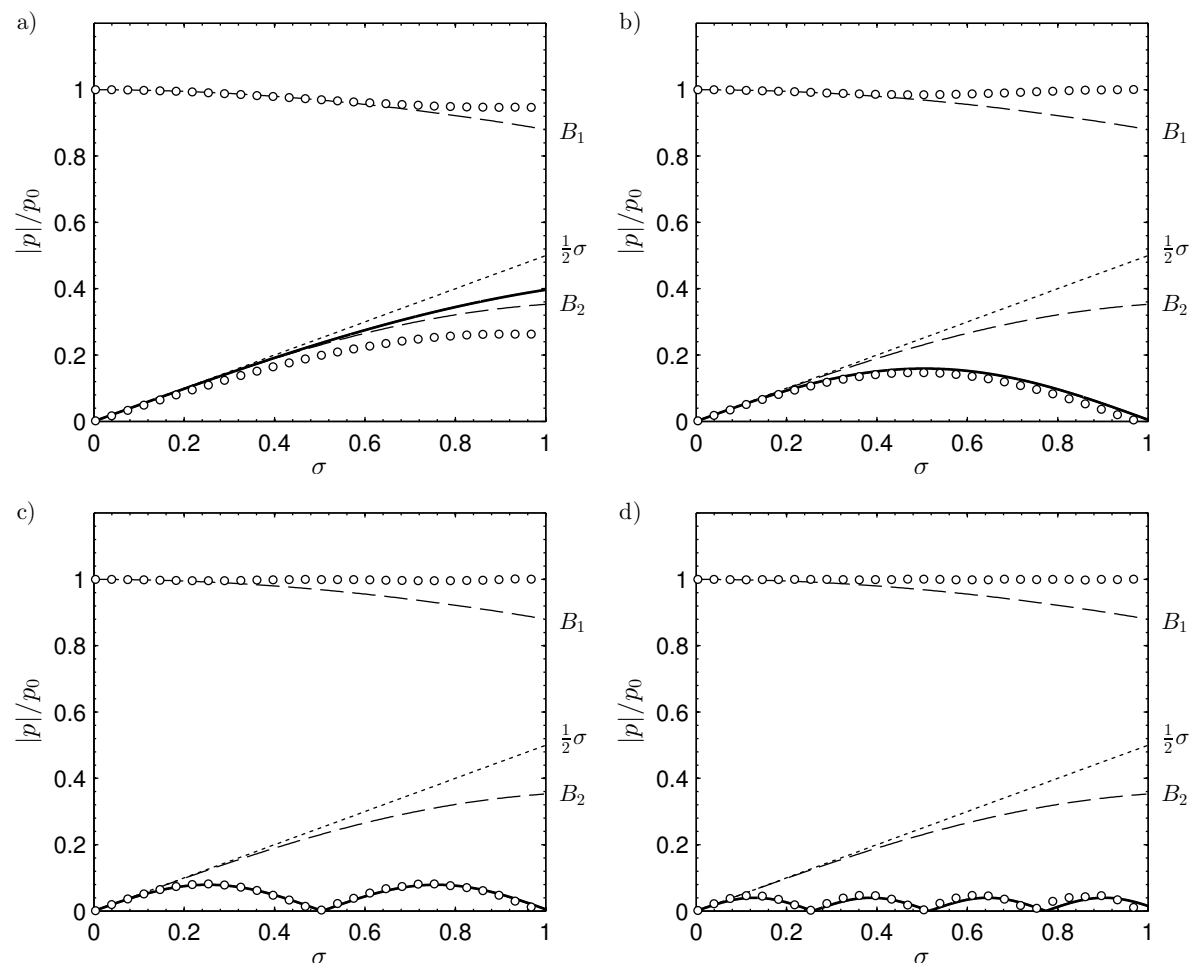

Figure 2.9: Second harmonic evolution for $x_{c} / x_{s}=[1,1 / 2,1 / 4,1 / 8]$ obtained using Eq. (2.50) (continuous line), numerically (white circles), nondispersive linear law of growing (dotted line) and Bessel-Fubini nondispersive solution (dashed).

case, phase matching conditions are fulfilled and the free wave is excited synchronous to the forced wave. Note here that in the derivation of Eq. (2.50) only second order processes are taken into account and therefore, only second harmonic is predicted. This leads to overestimate the second harmonic field: as long no energy is transferred to third harmonic, second harmonic predicted by Eq. (2.50) in the absence of dispersion grows indefinitely. The validity of this model can be explored expanding the Bessel functions of Fubini series near the source. A simple comparison between the full Fubini solution and linear second harmonic growth gives a reasonable approximation for distances $\sigma<0.5$ or for second harmonic field values of $p(2 \omega)<p_{0} / 4$.

Figure 2.9 shows four different simulations in the dispersive regime of the layered media where the wave amplitude and frequency has been selected to match $x_{c} / x_{s}=[1,1 / 2,1 / 4,1 / 8]$. The higher beating spatial period waves corresponds to lower frequencies. The analytical solution for the second harmonic matches the full-wave numerical solution. However, differences can be observed in the second harmonic amplitude estimation for $x_{c} / x_{s}=1$ (left plot in Fig. (2.9)). This 
overestimation by the analytical solution can be related to the absence of energy transfer to higher harmonics, that is not considered by the perturbation solution but is included in the simulation. Therefore, this model is specially suitable in situations where the third harmonic does not grow cumulative with distance. In the lossless layered media, this situations include frequencies that leads to very high-third harmonic detuning and also when the third harmonic falls in band gap.

\subsubsection{Second harmonic in band gap}

Waves with frequencies falling into the band-gap of the dispersion relation are evanescent due the non negligible imaginary part of its complex wave number. Thus, its amplitude decays exponentially with distance. When the second harmonic frequency falls into the band-gap, a characteristic effect, studied also in other nonlinear lattices (Sánchez-Morcillo et al., 2013) can be observed: its amplitude reaches a constant value. Figure 2.10 present an example of this case for four
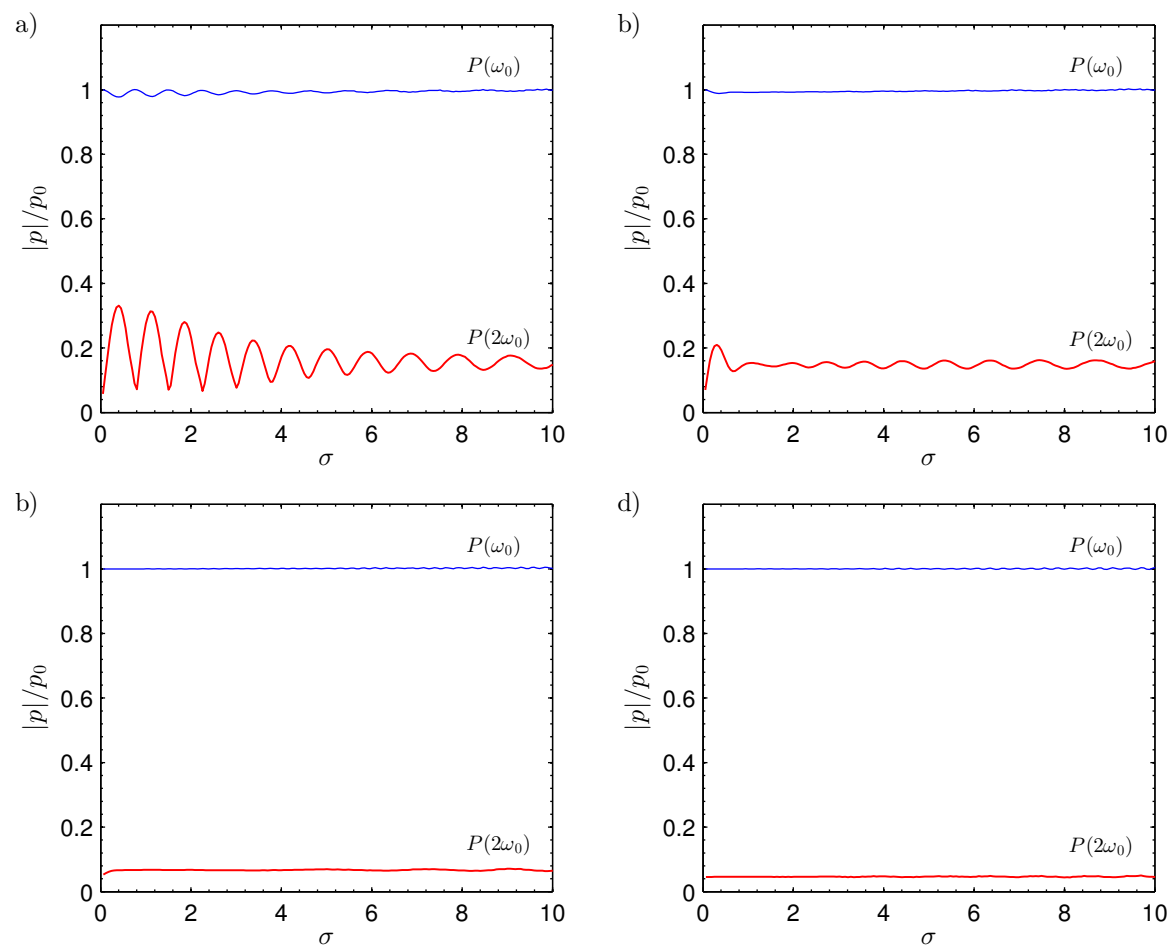

Figure 2.10: Evolution of the second harmonic field propagating in bang-gap for second harmonic frequencies (a) just above band-gap $2 \omega_{0}=0.84 \Omega_{0}$, (b) $2 \omega_{0}=0.85 \Omega_{0}$, (c) in the middle of the bandgap $2 \omega_{0}=\Omega_{0}$ and (d) $2 \omega_{0}=1.05 \Omega_{0}$ at the maximum of the imaginary part for the first band gap. All results for a layered system of $\alpha=1 / 2$ and $c_{1} / c_{2}=1 / 2$. 
different frequencies, where can be observed that the constant value of the second harmonic field depends on the imaginary part of the wave vector.

This effect can be understood in terms of the free and forced waves. If the second harmonic is evanescent (as follows from the dispersion relation), the wave will not accumulate with distance. But the fundamental wave is still "pumping" energy to the second harmonic field at every point in space. Thus, the second harmonic field is generated locally and remains trapped inside the layered media. It reaches a constant level that depends on three main factors. In first place, the "pumping" rate, characterized by the fundamental wave amplitude and medium nonlinearity, or more strictly the ratio between the layer thickness and the shock distance $d / x_{s}$. Secondly, it also strongly depends on the magnitude of the imaginary part of the complex wave number, i.e. the ratio between its characteristic exponential decay length and the shock distance in a layer. The characteristic decay length of the evanescent propagation is always shorter when the second harmonic is in the middle of the band-gap, leading to less second harmonic field in this frequency region, as can be seen in Fig. 2.10 (c-d). Finally, it depends also on the detuning of real part of the wave number, where for the first band-gap is minimum at the center. The first factor can be isolated and studied separately. However, the two last factors are linked through the specific dispersion relation of the medium.

Figure 2.11 shows the detuning of the second harmonic and the imaginary part as a function of the frequency for a medium with $\alpha=1 / 2$ and $c_{1} / c_{2}=1 / 2$, showing that at the middle of the band-gap these two factors have opposite effects: detuning is minimized when evanescence decay is maximized and vice versa. However, the magnitude of the effects can be very different. As the rate of the second harmonic generation (see the initial slope in Fig. 2.9) is independent on the detuning, and the evanescence implies that the wave decays after few layers, there not exist a practical

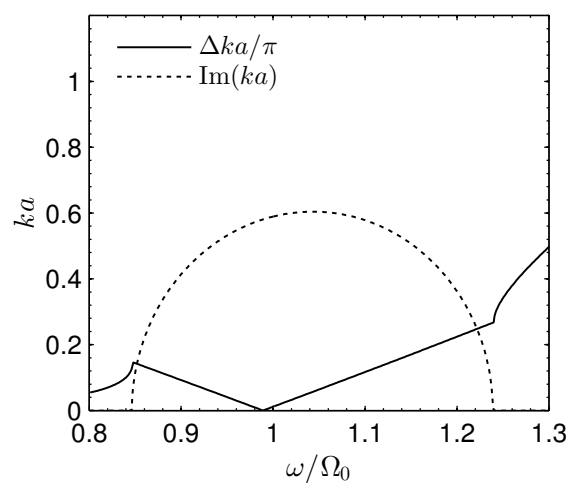

Figure 2.11: Detuning of the second harmonic (continuous line) and imaginary part (dotted line) in function of the normalized frequency. 

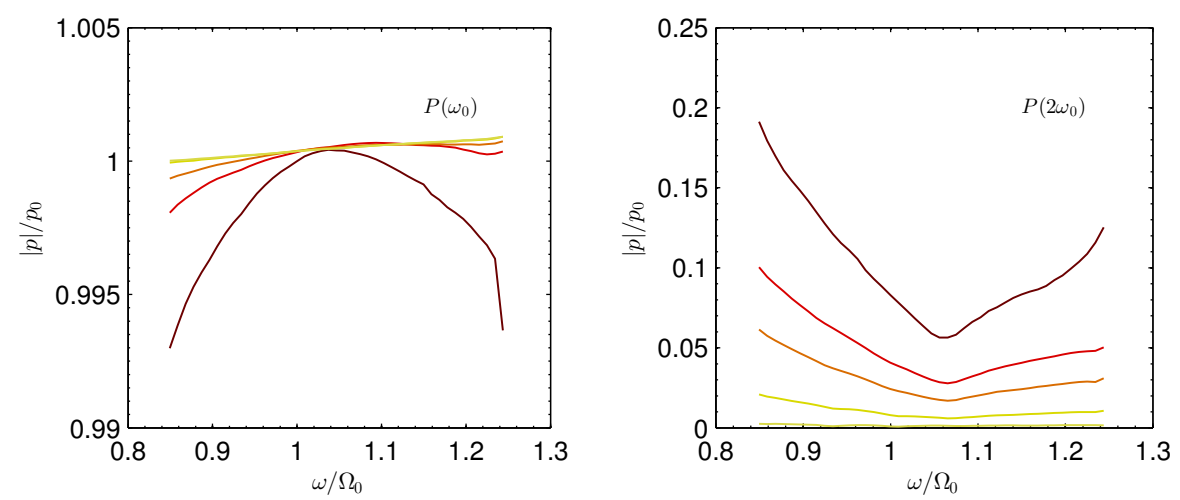

Figure 2.12: (left) Amplitude of the constant field value for fundamental (dashed) and (right) its second harmonic field (continuous line) for amplitudes matching $a / x_{s}=0.08,0.04,0.02,0.001$

compensation of the effects at the center of the band-gap. However, the situation is different for frequencies around the limits of the band-gap, where the coherence length is of the order of the exponential decay characteristic length. Thus, for frequencies just above bad-gap and for amplitudes with shock distance comparable to the evanescent characteristic decay length, the beatings can be also observed, as shown in Fig. 2.10 (a). Then, if frequency is increased the characteristic decay length becomes shorter than the shock wave distance and beatings cannot be observed, leading to to the characteristic constant second harmonic field shown in Fig. 2.10 (c-d).

Figure 2.12 (right) shows the second harmonic amplitude (of the constant field) in function of the frequency for different amplitudes matching $a / x_{s}=0.08,0.04$, $0.02,0.001$, where it is clearly visible that the minimum field value is obtained at the frequency of the maximum imaginary part of the complex wave number. On the other hand, as more energy is transferred to second harmonic the fundamental amplitude decays in an inverse way of its second harmonic field, as shows in Figure 2.12 (left).

\subsubsection{Fundamental harmonic in band gap}

When the fundamental frequency of the wave lies within the band-gap, small amplitude waves propagates evanescently. Essentially, the same applies to finite amplitude harmonic waves. In general, if the shock distance is large compared to the exponential characteristic length of the evanescent wave, the nonlinear effects have no time to accumulate and harmonic amplitude is negligible. Since the characteristic exponential decay is about few lattice sites, this means that the initial amplitudes necessary to achieve nonlinear effects in this configuration are much higher than those in the preceding sections. Figure 2.13 shows the evolution of the 
first and second harmonics for a fundamental frequency falling in the middle of the bad-gap, $2 \omega_{0}=1 \Omega_{0}$, and with a frequency just above but into the band-gap, $\omega_{0}=0.87 \Omega_{0}$ for a layered media of $\alpha=1 / 2$ and $c_{1} / c_{2}=1 / 2$. In the first case, the imaginary part of the wave vector is remarkable high and the waves decay fast after few lattice units. Due to this fast decay, the second harmonic interacts over a short distance with the first, and its amplitude is very limited. After few lattice units, fundamental wave can be treated as a small-amplitude evanescentwave. The second harmonic, that also falls in bandgap (but in the second band) also decays exponentially.

On the other hand, if the fundamental frequency is set just above the bandgap, where the imaginary part of the wave-vector is smaller, the amplitude of the fundamental wave decays more slowly, penetrating deeper into the material. The interaction region with the second harmonic is larger, and nonlinear effects resultv in a more efficient generation of the second harmonic. Furthermore, as long the different (higher order) bandgaps in the layered media can have different bandwidth, in this configuration at $\omega_{0}=0.87 \Omega_{0}$ second harmonic does not fall inside a bandgap. Therefore, the generated second harmonic wave at the beginning of the lattice propagates through the medium essentially without amplitude change. Due to the evanescence of the fundamental wave, there is only forced wave at the beginning of the medium. Therefore, although in this configuration waves are phase mismatched, beatings are not present: only the free wave propagates through the medium.
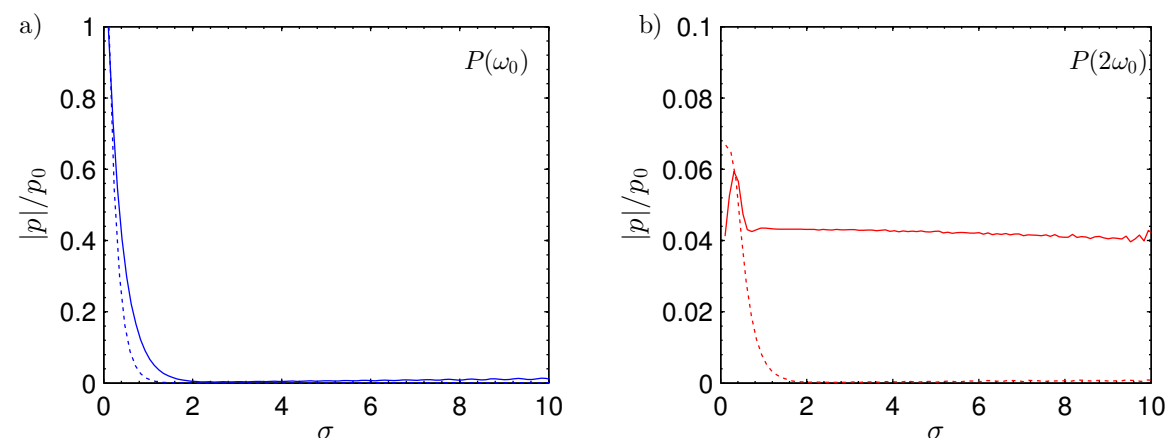

Figure 2.13: (a) Evolution of the fundamental harmonic wave field with its fundamental frequency falling just above into band-gap, $\omega_{0}=0.87 \Omega_{0}$, (continuous line), and in the middle of the bad-gap, $2 \omega_{0}=1 \Omega_{0}$ (dotted line). (b) Corresponding second harmonic field, where for $\omega_{0}=1 \Omega_{0}$ (dotted line) second harmonic frequency falls in the 2nd band band-gap while for $\omega_{0}=0.87 \Omega_{0}$, (continuous line) lies into a propagating band. 


\subsection{Nonlinear acoustic field management}

\subsubsection{Tuning nonlinearity with dispersion}

In the preceding sections we have explored the fundamental behavior of nonlinear waves generated inside layered media. However, the layered medium parameters can be tuned to provide specific conditions. These conditions are, among others, looking for detuning or coherence at specific frequencies and using the evanescent propagation in the bad-gap for another specific frequency.

The material parameters can be tuned to get coherence at one frequency of interest, e.g. at one of the harmonics of the fundamental wave, or to get detuning or evanescent propagation at other specific harmonics. Using these mechanisms the layered system provides a control of the nonlinear process inside the medium. Thus, the layered media can be tuned in order to obtain specific balance of the harmonic amplitude or specific nonlinear waveforms.
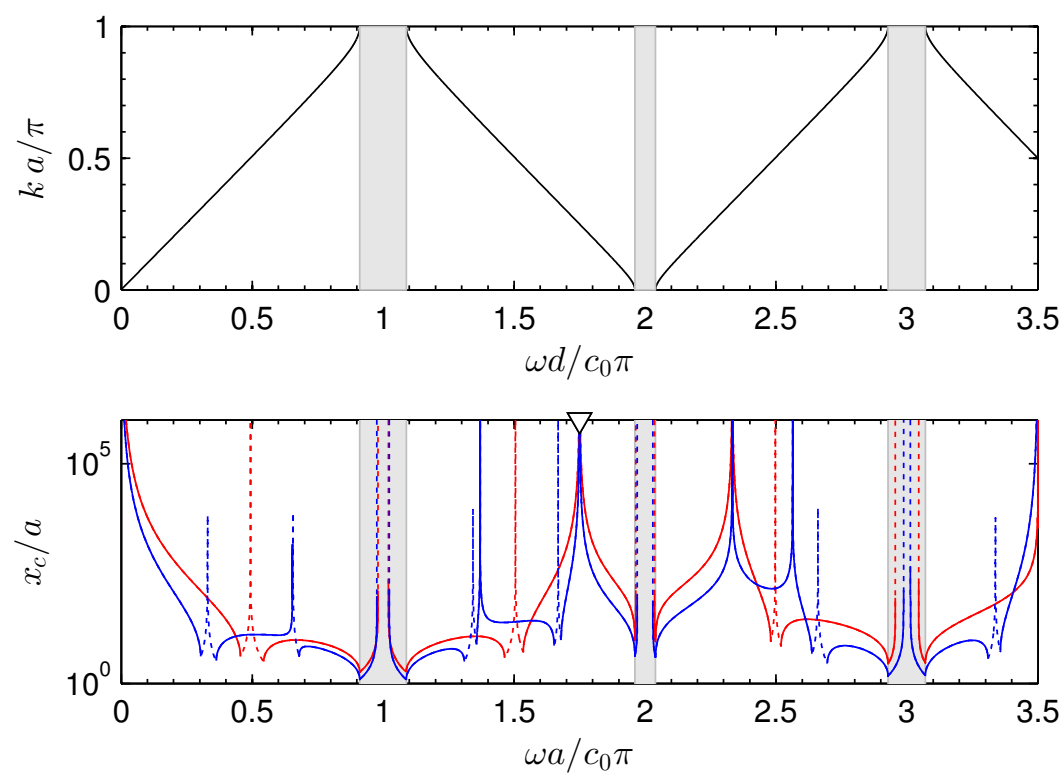

Figure 2.14: (Top) dispersion relation for a layered media of $c_{1} / c_{2}=1.33$ and $\alpha=1 / 2$. (Bottom) coherence length for second (red) and third (blue) harmonic in function of the fundamental frequency. Phase matched frequencies are those with $x_{c} \rightarrow \infty$, while asynchronous generation is predicted for $x_{c} \rightarrow 0$. Frequencies at which the fundamental frequency is in band-gap are is marked in gray regions, while band-gap regions for second and third harmonic are marked in dashed lines. 


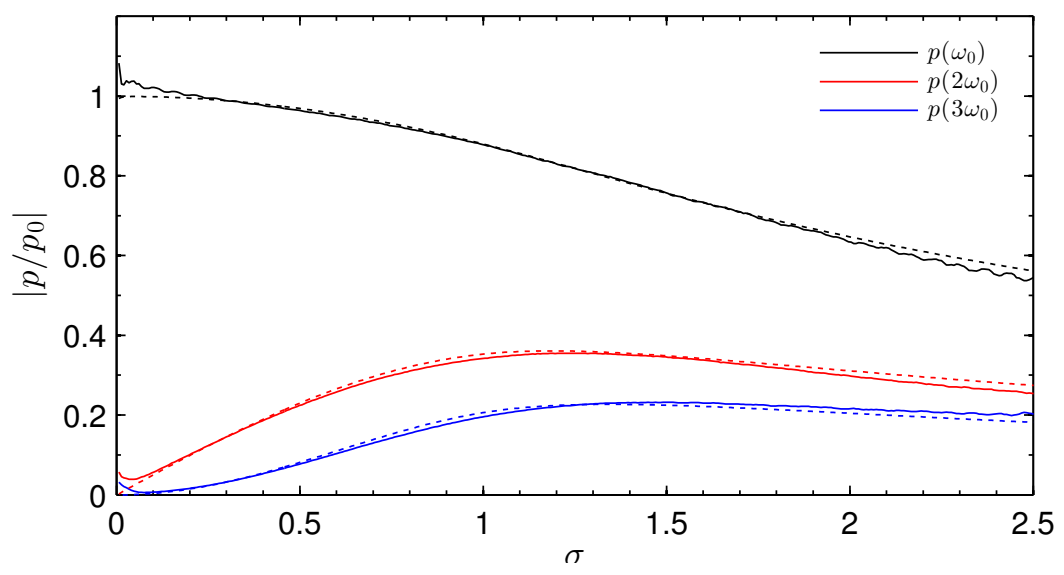

Figure 2.15: Harmonic distribution for the frequency $\omega_{0}=1.75 / \Omega$. Coherence is recovered for at least the lowest spectral components. Blackstock solution (dotted lines).

In the design of a system for this purpose, the coherence length is a useful control parameter. Thus, Fig. 2.14 (top) shows an example of the dispersion relation with parameters $c_{1} / c_{2}=1.333$ and $a=1 / 2$. Then, in Fig. 2.14 (bottom) the coherence length for the second and third harmonic is shown. The propagating bands are marked with continuous lines while the band-gap frequencies are marked in dotted lines. Thus, the abscissa axes marks the frequency of the fundamental wave. It can be observed that there exist singular frequencies, e.g. frequencies where all the harmonics are phase matched, $x_{c} \rightarrow \infty$. In Fig. 2.14 it happens for $\omega_{0}=(0,1.75,2.333, \ldots) / \Omega$. On the other hand, there also exist frequencies at which there exist coherence for the second but a non-negligible detuning is observed for the third. Opposite effect can be observed, where coherence is achieved for the third harmonic but second harmonic presents strong dispersion. Finally, other interest regions are those where second harmonic component is almost phase matched and for the same frequency third harmonic falls into a band-gap. ${ }^{2}$

Thus, the analytic Eq. (2.1) provides an excellent framework to tune-up the layered parameters to obtain the desired balance between detuning, evanescent propagation, synchronous generation and, at the same time, it allows to find those conditions for a specific phase/group speed.

We start studying the harmonic distribution for an initial monochromatic wave of $\omega_{0}=1.75 / \Omega$, corresponding to the triangle marked in Fig. 2.14 (bottom). Figure 2.15 shows the harmonic distribution, where, as expected, synchronous cumulative harmonic generation inside the dispersive media is recovered. The harmonic evolution in the second band follows again the Bessel-Fubini-Blackstock harmonic

\footnotetext{
${ }^{2}$ This singular situations can be also obtained with no difficulty for physical values of sound speed and other layer proportions. We use here normalized values for the sake of simplicity.
} 
distribution for the lower spectral components. However some remark must be underlined. First, there exist some detuning for the high frequency components that avoids the formation of shock waves for $\sigma>1$. Figure 2.16 shows the waveforms measured at $\sigma=(0.5,1, \pi / 2,2)$. It can be observed that, although second and third harmonics are phase matched a shock is not produced. Due to finite detuning of the higher harmonic components, oscillations instead of a shock wave profile appears for $\sigma>1$, as those presented in the low frequency limit in Sec. 2.4.2.

It is worth noting here that coherence is achieved in theoretically lossless media for all spectral components. However, losses can modify the dispersion relation specially for frequencies near the band-gap. Thus, in practical cases, where losses exist and typically increases quadratically with frequency, the dispersion relation will be modified specially at frequencies of the higher spectral components. In the presented cases, due to the introduction of some amount of artificial attenuation necessary for nonlinear numerical stability and convergence, it is observed how appears a finite detuning for high spectral components, causing the characteristic dispersive oscillations. On the other hand, the finite difference method used always introduces some amount of artificial dispersion that modify the dispersion relations. However, the oscillatory solutions, e.g. Fig. 2.16, are therefore not caused directly by numerical artifacts. They are not present in homogeneous simulations. Note the grid used is 300 elements per wavelength and the artificial attenuation was only $10^{-3} \mathrm{P} / \mathrm{s}$, ten times greater than the water viscosity. The effect of computational restrictions is to slightly modify the dispersion relations for higher harmonics causing finite detuning. We expect those effects to be present in real materials, and therefore, limit the ability of recovering perfect coherence for all spectral components and create shock waves ${ }^{3}$.

Other interest feature is that in these conditions phase speed at the fundamental and at higher harmonic frequencies always match $c_{p}(n \omega)=\bar{c}_{0}$. In contrast, in this situation the group velocity does not match necessary the low frequency sound speed. In this case was close, with a value of $c_{g}=0.97 \bar{c}_{0}$.

Other situations can be explored by changing the layered parameters. Figure 2.17 (top)shows the dispersion relation for $c_{1} / c_{2}=2$ and $\alpha=0.1$. It is shown in Fig. 2.17 (bottom) that coherence is achieved, at least for the lowest components, for $\omega / \Omega=1.22$. In this case, the phase speed matches $c_{p}\left(n \omega_{0}\right)=\bar{c}_{0}$, but the group velocity is reduced to $c_{g}\left(\omega_{0}\right)=0.830$. Figure 2.18 shows the evolution of the fundamental and its first two harmonics. A remarkable effect that can be observed here is the appearance of high-amplitude oscillatory fields due to the strong impedance contrast between layers, actually nonlinear Bloch waves. In these reduced group velocity conditions, the analytical solution for homogeneous media (dashed lines) does not match the numerical results. However, no beating are produced and the shape of harmonic growing is essentially the same. It can be seen that the rate of second and third harmonic growth is increased. The distance at

\footnotetext{
${ }^{3}$ Other interesting topic, not studied here, is to find conditions for recovering coherence in media with attenuation by tuning its lossy dispersion relations
} 

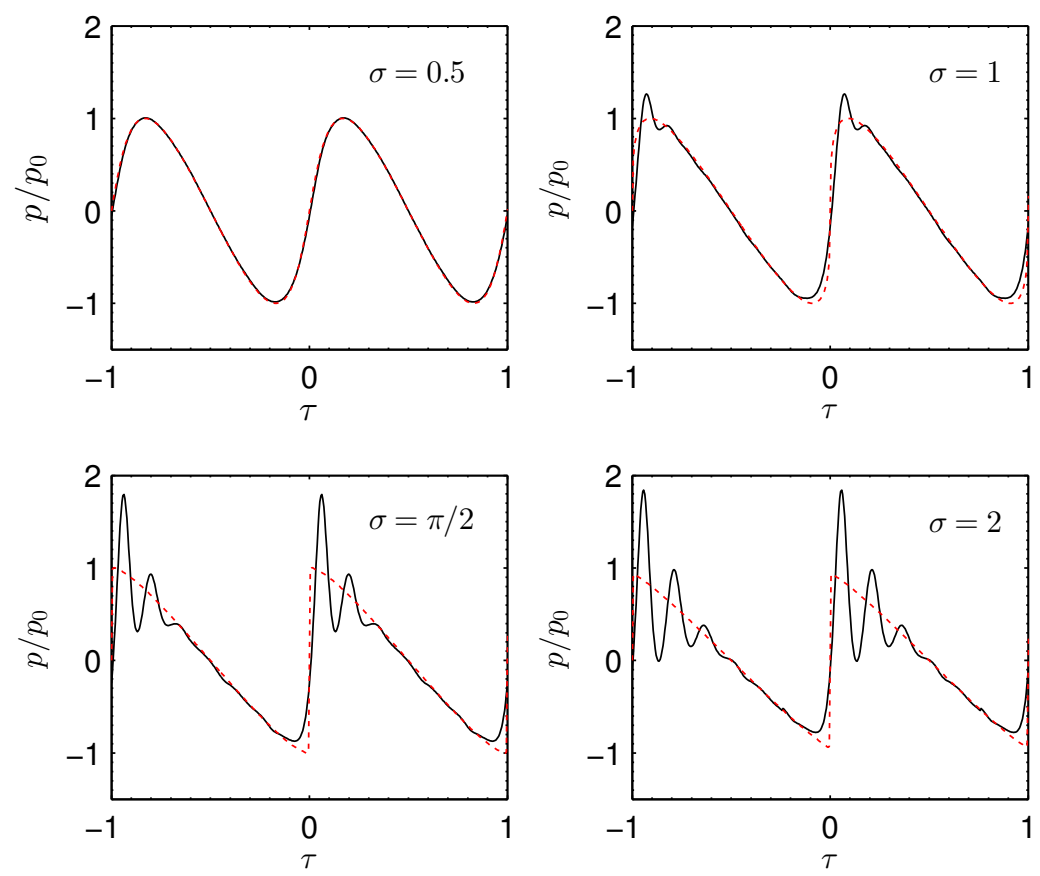

Figure 2.16: (continuous lines) Waveforms at different distances $(\sigma=0.5,1, \pi / 2,2)$ for $\omega_{0}=$ $1.75 / \Omega$ where a coherence is recovered for the lowest harmonics. Blackstock analytic solution (red dotted lines).

which the maximum second harmonic field is produced is located at $\sigma \approx 1.2$ in the homogeneous media while in the numerical simulations appears at $\sigma=0.7$. Same scaling is observed for the rate of harmonic generation, where in the homogeneous media (near the source) second harmonic grows at a rate $p_{0} \sigma / 2 \mathrm{~Pa} / \sigma$, and in the numerical simulations the rate of growing was $0.86 p_{0} / \sigma \mathrm{Pa} / \sigma$. The whole effect is that the effective nonlinearity of the system in these conditions is increased. For the present case, a factor of increased nonlinearity can be obtained by fitting the analytical solution to the numerical results, letting as the free variable an effective parameter o nonlinearity, namely $\bar{\beta}$. Thus, for the presented results, a factor of $\bar{\beta}=1.72$ is estimated. A new solution using this effective parameter is shown in Fig. 2.17 (bottom) in thick lines. It can be observed how the fitted solution agrees the slow-group speed for distances up to the effective shock distance $(0.58 \sigma$ in the figure). The observed effect of retaining coherence and reduce group velocity is to increased the effective nonlinearity of the media together with the apparition of high-amplitude nonlinear Bloch waves.

In the following subsections, we propose and analyze different interest layered 

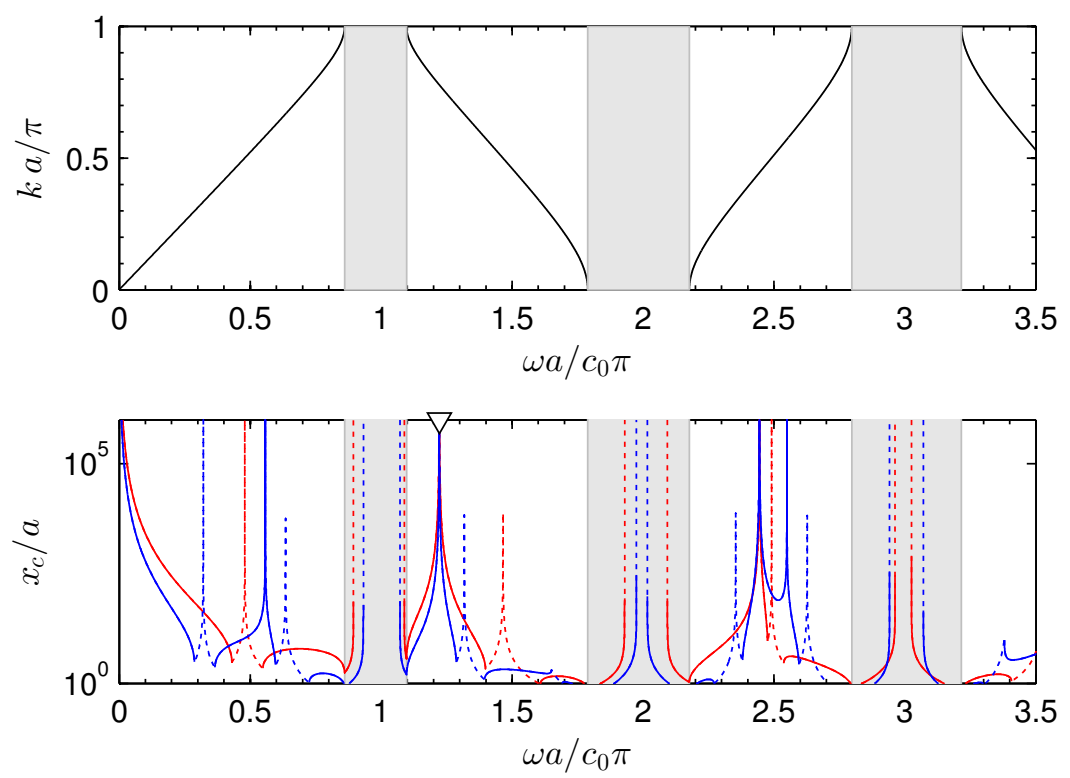

Figure 2.17: (Top) dispersion relation for a layered media of $c_{1} / c_{2}=2$ and $\alpha=0.1$. (Bottom) coherence length for second (red) and third (blue) harmonic in function of the fundamental frequency. Frequencies at which the fundamental frequency is in band-gap are is marked in gray regions, while band-gap regions for second and third harmonic are marked in dashed lines.

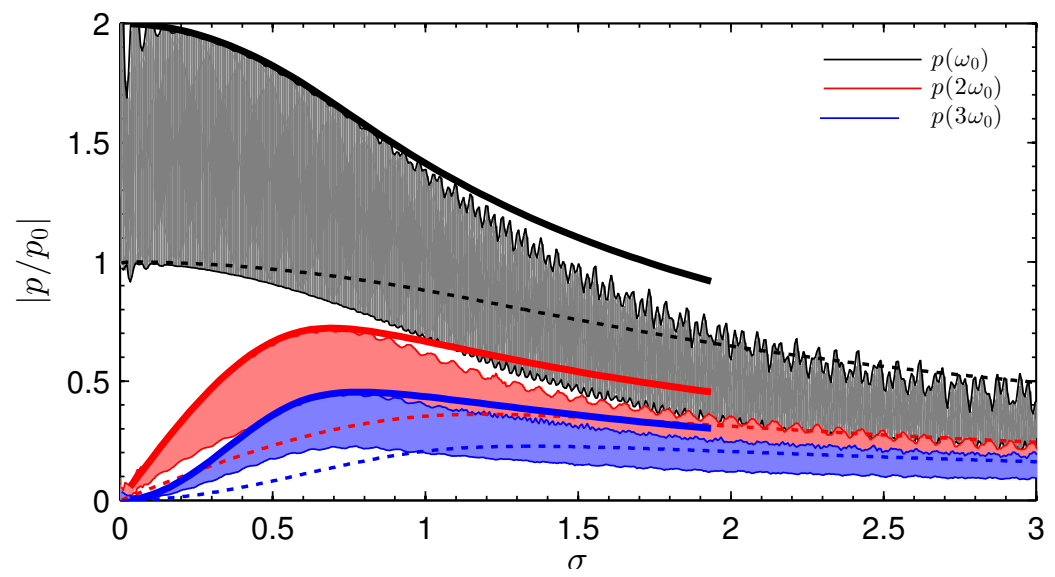

Figure 2.18: Harmonic distribution for the frequency $\omega_{0}=1.22 / \Omega$. Blackstock solution (dotted lines). 


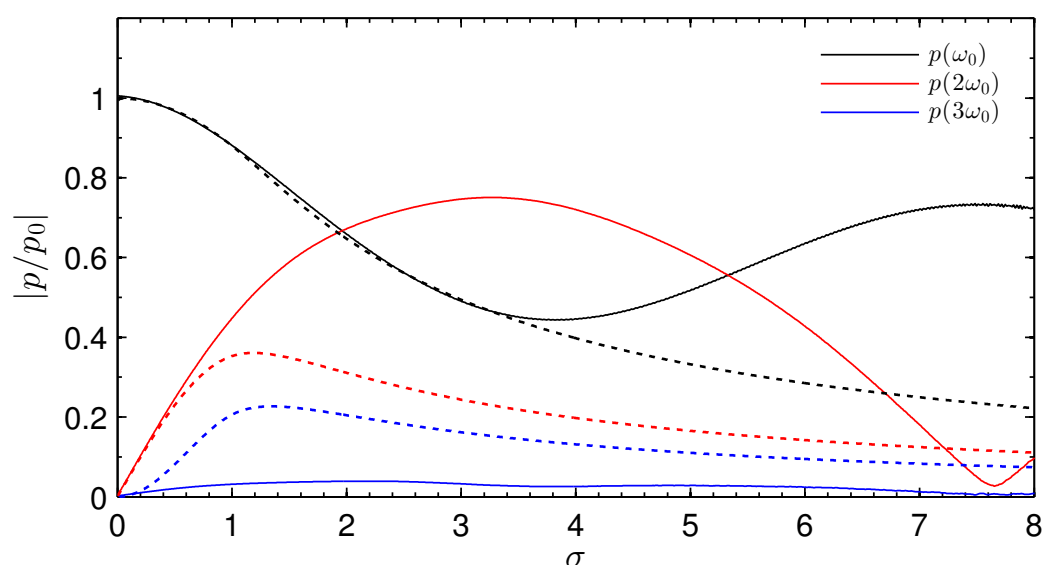

Figure 2.19: Harmonic distribution for $\omega_{0}=1.668 / \Omega$ where a coherence is achieved for second harmonic while the frequency of the third harmonic falls into the bad-gap.

media designs with specific balance between between detuning, evanescent propagation and synchronous generation.

\subsubsection{Increasing second harmonic generation}

One can expect that second harmonic generation is maximized in homogeneous nondispersive media. However, in nondispersive media coherence is achieved not only at second harmonic frequency, but also in the higher spectral components. As a result, energy is transferred from second harmonic field to higher spectral components and therefore second harmonic field does not grow indefinitely. Moreover, shock waves are formed and nonlinear absorption reduces wave intensity for $\sigma>\pi / 2$ even in lossless media (Hamilton et al., 1998a).

The controlled dispersion of the layered system can modify this situation by including dispersion that alters the higher harmonic cascade processes while maintaining coherence for second harmonic. Figure 2.14 shows an example of a dispersion relation where for $\omega_{0}=1.668 / \Omega$ it can be observed that there exist a reasonable coherence for the second harmonic $\left(x_{c} / a \approx 1000\right.$ lattice units), while the third harmonic falls in a band-gap. Figure 2.19 shows the harmonic distribution in this situation. Here, energy is transferred to second harmonic field that grows almost linearly for $\sigma<2$. On the other hand, the energy transferred from second to third harmonic is not cumulative and its amplitude does not grow with distance. Third harmonic experiment evanescent propagation due to the imaginary part of the complex wave-vector at this frequency. A constant field, as studied in Sec. 2.4.4, is obtained for the third harmonic. 

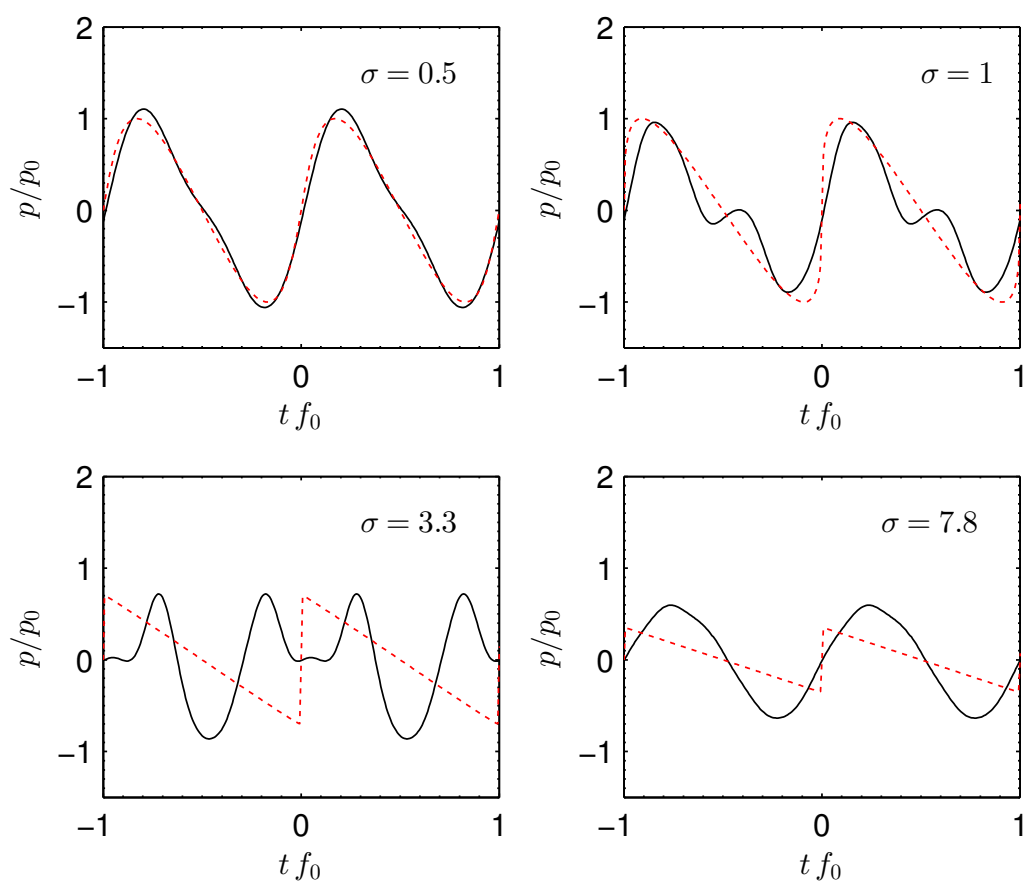

Figure 2.20: (continuous lines) Waveforms at different distances for $\omega_{0}=1.668 / \Omega$. At $\sigma=$ 3.3 second harmonic generation field is maximize and can be seen the period doubling in the waveform. Then, at $\sigma=7.8$ due to second harmonic detuning nearly sinusoidal wave is recovered. Blackstock analytic solution (red dotted lines).

The total amount of the second harmonic amplitude in nondispersive media is $\left.p_{2}\right|_{\max } \approx 0.36 p_{0}$, while in the example of Fig. 2.19 a maximum second harmonic amplitude of $\left.p_{2}\right|_{\max } \approx 0.75 p_{0}$ is predicted. As can be shown the decreasing of the first harmonic follows the analytic nondispersive Blackstock solution for $\sigma \lesssim 3$. Thus, in this regime all the energy of the first harmonic is being transferred to the second harmonic field. However, due to finite detuning of the second harmonic a long spatial beating is produced, with period $8 \sigma$, and energy is returned back to the first harmonic component.

It is worth noting here that at distance $\sigma \approx 3$ sawtooth profile is observed in nondispersive media. In contrast, only second and first harmonic have remarkable amplitude into the layered media. Waveforms are shown in Fig. 2.20. Near the source, where the amplitude of higher harmonics in not relevant the nondispersive waveform (in red dotted) is well approximated by the fundamental and its second harmonic of the layered medium. However, due to the evanescent propagation of the third harmonic for longer distances the nonlinear solution of the layered medium is mainly composed by the fundamental and its second harmonic. The 
maximum second harmonic in this configuration is observed at $\sigma=3.3$, where it can be appreciated in the waveforms of Fig. 2.20 the period doubling. Moreover, due to finite detuning of the second harmonic the process is not cumulative for all distances and at $\sigma=7.8$ the energy is restored in the first harmonic again and a sinusoidal wave is obtained ${ }^{4}$. An analogous effect has been also studied (Naugolnykh et al., 1998) where instead of dispersion, selective absorption at specific frequencies is used to modify and enhance harmonic generation.

\subsubsection{Increasing third harmonic generation}

In the first band $\left(\omega<\Omega_{0}\right)$, coherence is always lower for the third harmonic than for the second. However, in the superior bands the layered medium parameters can be tuned to obtain higher coherence for the third than for the second harmonic. Essentially we follow same ideas on the preceding section but for the third harmonic. In this case, the lattice is designed forcing the second harmonic to fall in bandgap. A the same time, perfect coherence can be found for the third harmonic at $\omega=0.529 \Omega_{0}$. This situation is illustrated on Fig. 2.21. In this case, the dispersion relation was obtained for a layered medium with parameters $\alpha=0.3$ and $c_{2} / c_{1}=1 / 3$.

In this situation, as Fig.2.22 shows, second harmonic field present a constant value of about $0.04 p_{0}$. As discussed in Sec.2.4.4, this constant field does not grow with distance and is related to the evanescent solution of the free wave and the local nonlinear "pumping". On the other hand, due to the coherence of the third harmonic, all the energy transferred form second to third is accumulated with distance. Therefore, near the source the rate of energy transfer from second to third harmonic is constant. Thus, third harmonic start to grow almost linearly with distance, opposite to quadratically in homogeneous media.

Also it can be seen in the simulations how fourth and fifth harmonics grow (red dotted and blue dotted lines in Fig. 2.22). In this case only fifth harmonic have remarkable amplitude, growing near the source almost quadratically with distance. Therefore, the entire system behaves as an artificially cubic-like nonlinear medium formed by quadratic nonlinear layers.

The corresponding waveforms measured at $\sigma=(0.5,2,8,12)$ are shown in Fig. 2.23. For $\sigma=0.5$ and 2, it can be observed how the wave steepens with the characteristic shape of cubic nonlinearity. No shock waves are formed as long strong dispersion is present for high frequency harmonics. However, it is worth noting here a remarkable fact: it steepens in the positive time axis direction (to

\footnotetext{
${ }^{4}$ Note all the energy is not restored to first harmonic in Fig. 2.19 at $\sigma=7.8$, leading to a sinusoidal wave of different amplitude as can be observed in Fig. 2.20. The energy loss is mainly due to the artificial (numerical) viscosity necessary to nonlinear convergence(Hamilton et al., 1998a). For these simulations the total distance is 1200 lattice sites and therefore the effects of attenuation are not negligible. However, the main nonlinear effects related to strong lattice dispersion still appreciated.
} 

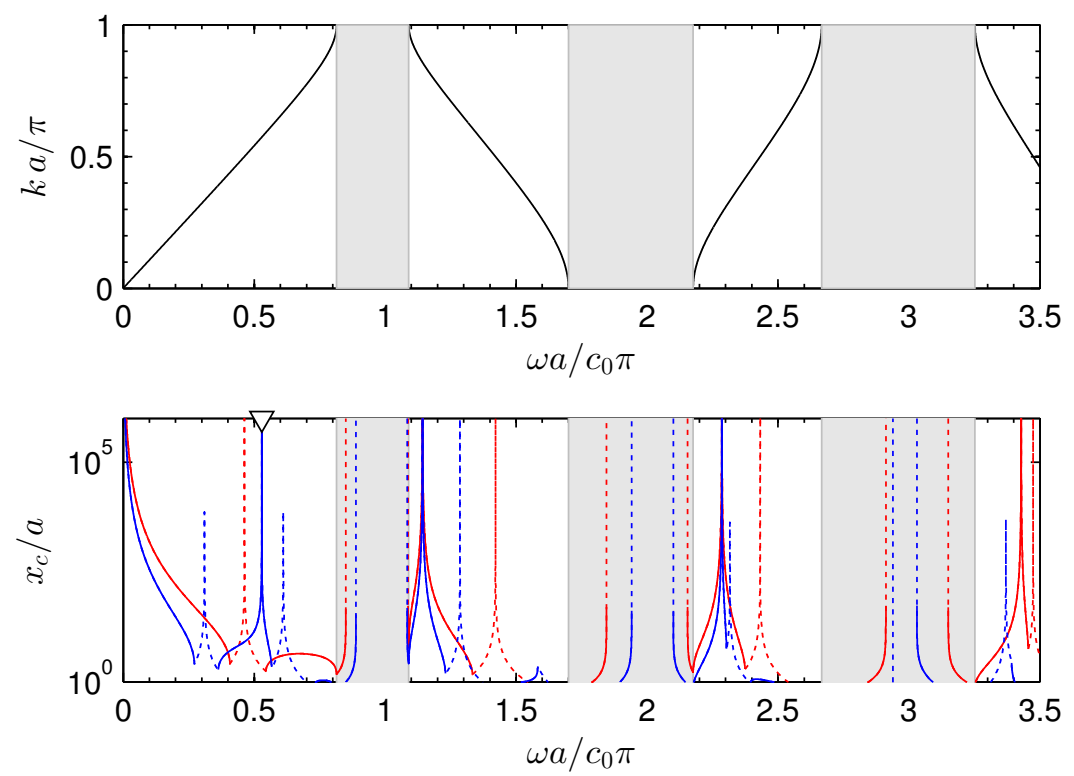

Figure 2.21: Harmonic distribution for $\omega_{0}=1.668 / \Omega$ where a coherence is achieved for second harmonic while the frequency of the third harmonic falls into the bad-gap.

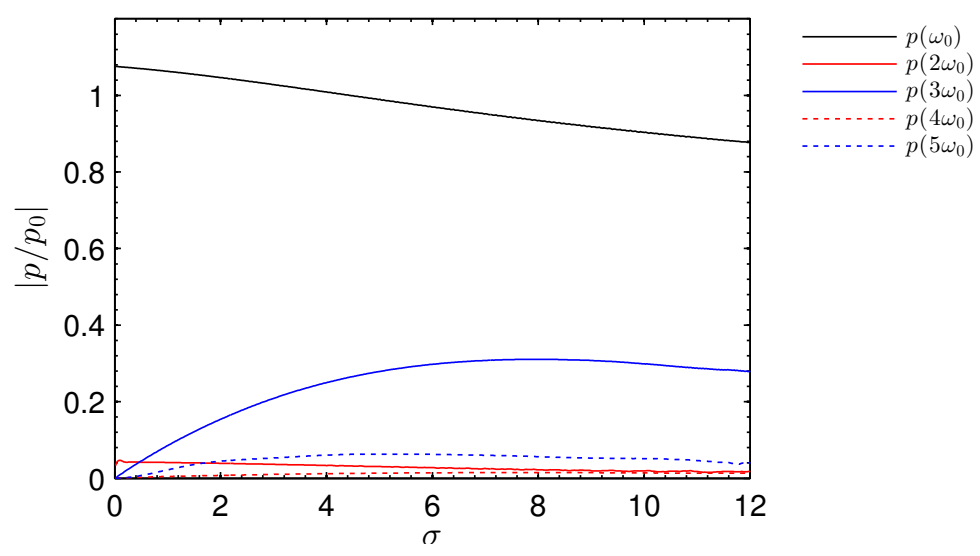

Figure 2.22: Harmonic distribution for $\omega_{0}=1.668 / \Omega$ where a coherence is achieved for second harmonic while the frequency of the third harmonic falls into the bad-gap. 

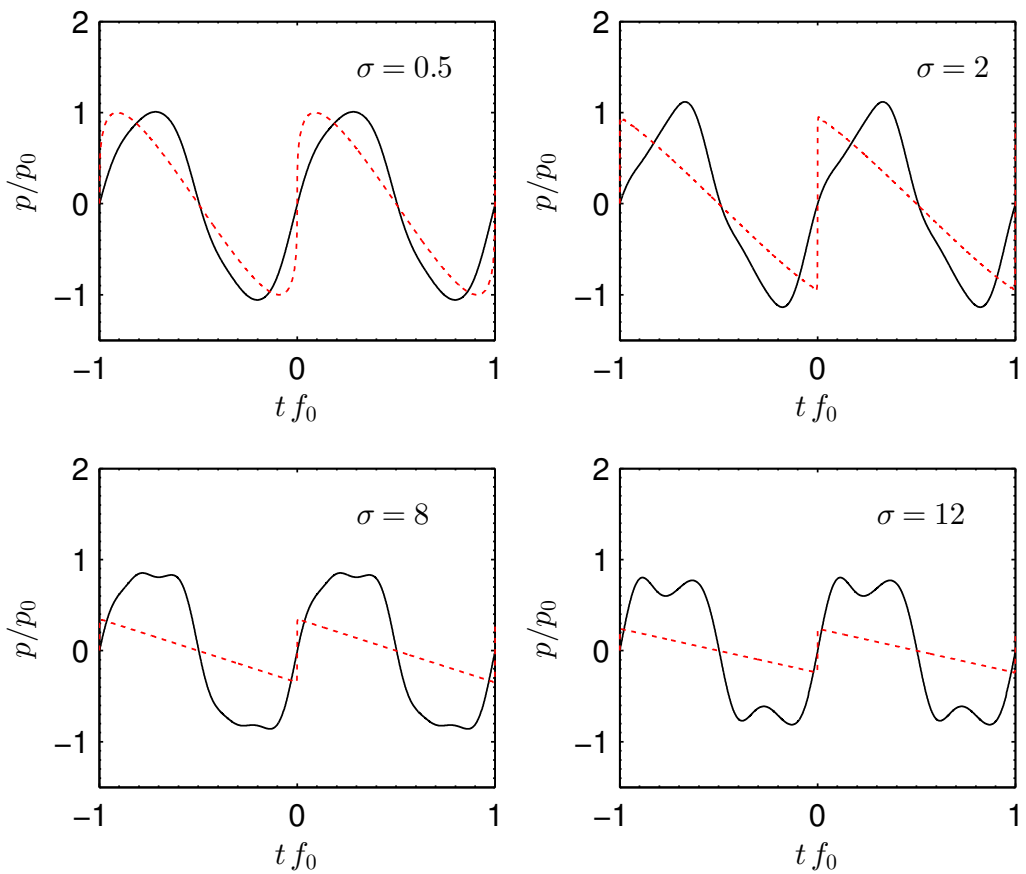

Figure 2.23: (continuous lines) Waveforms at different distances for $\omega_{0}=1.668 / \Omega$. At $\sigma=$ 3.3 second harmonic generation field is maximize and can be seen the period doubling in the waveform. Then, at $\sigma=7.8$ due to second harmonic detuning nearly sinusoidal wave is recovered. Blackstock analytic solution (red dotted lines).

the right in the figure), opposite than the quadratic nonlinearity plotted in red dotted as a reference. This effect, i.e. the steepening on the opposite side of the propagation direction, is characteristic of materials with negative parameter of nonlinearity. Therefore, the effective nonlinear behavior observed by the simulations in this conditions can be described as negative-cubic-like nonlinearity.

\subsubsection{Acoustic Fluorescence in chirped layered media}

Another interesting configurations can be achieved by adiabatically changing the lattice period, $a=a(\mathbf{x})$, of the layered medium, namely chirped layered media. Analogues to these configurations, chirped periodic media has been studied in linear regime in optics (Cheng et al., 2014) or in acoustics using arrays of distributed cylindrical scatterers (Romero-García et al., 2013). Using these chirped configurations band-gap can be placed at different locations for different frequencies. Here, we design a system with increasing lattice period, where the band-gap for 


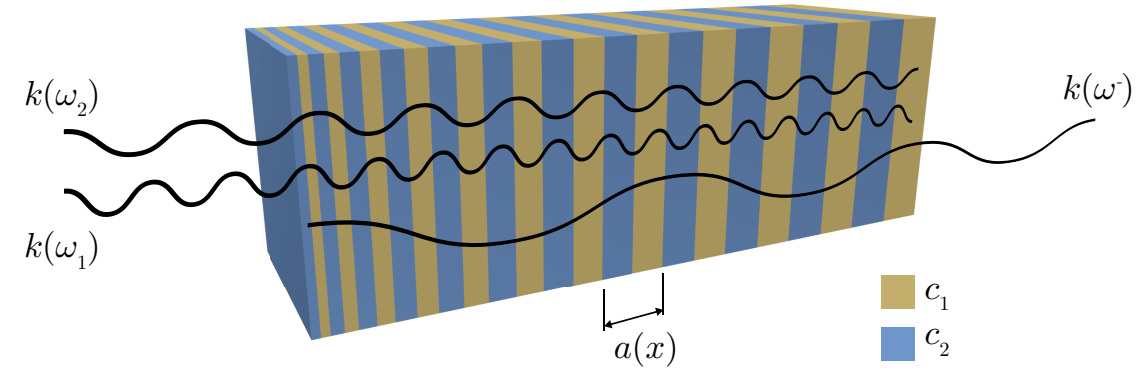

Figure 2.24: Scheme of the acoustic "fluorescence" effect. The chirped system is radiated with two waves of slightly different fundamental frequencies $\omega_{1}$ and $\omega_{2}$. Harmonics are generated at combination frequencies inside the medium and at the end of the system the period is tuned to achieve a band-gap at fundamental frequency. Thus, only the low-frequency difference-component is transmitted.

the fundamental component of two nearly similar frequency waves is located at the end of the chirped layered medium. Thus, as sketched in Fig. 2.24, two harmonic waves with close frequencies are injected at the beginning of the lattice. In this region, the lattice period is designed small enough to achieve good coherence so harmonics at combination frequencies can be generated progressively through the layered system. Then, at the end of the lattice the period is decreased in such a way that it correspond to a forbidden band for the fundamental component.

In this case, the frequency of the waves was selected to be $f_{1}=f_{0}$ and $f_{2}=f_{0}+$ $f_{0} / 3$. Figure 2.25 (top) shows the bandgap location in the lattice, that is located at $x=0$ until $x=200 a$. Then, the rest of the domain is homogeneous. Although it is calculated for infinitely period media, we assume that the band gap exist for the adiabatic chirped lattice. Figure 2.25 (bottom) shows the harmonic evolution for this configuration where it can be seen how harmonics at combination frequencies are generated at the beginning of the lattice. The bandgaps of layered medium act as spatial filtering for the fundamental components that are reflected back around $x / a=100$, leading to an enhancement of the field discussed in (Romero-García et al., 2013). This enhancement is clearly visible in Fig.2.25 (bottom). Furthermore, not only fundamental frequency is filtered. As long the bandgap central frequency change with distance, the superior bandgaps cover the entire frequency range, reflecting back higher harmonics. 

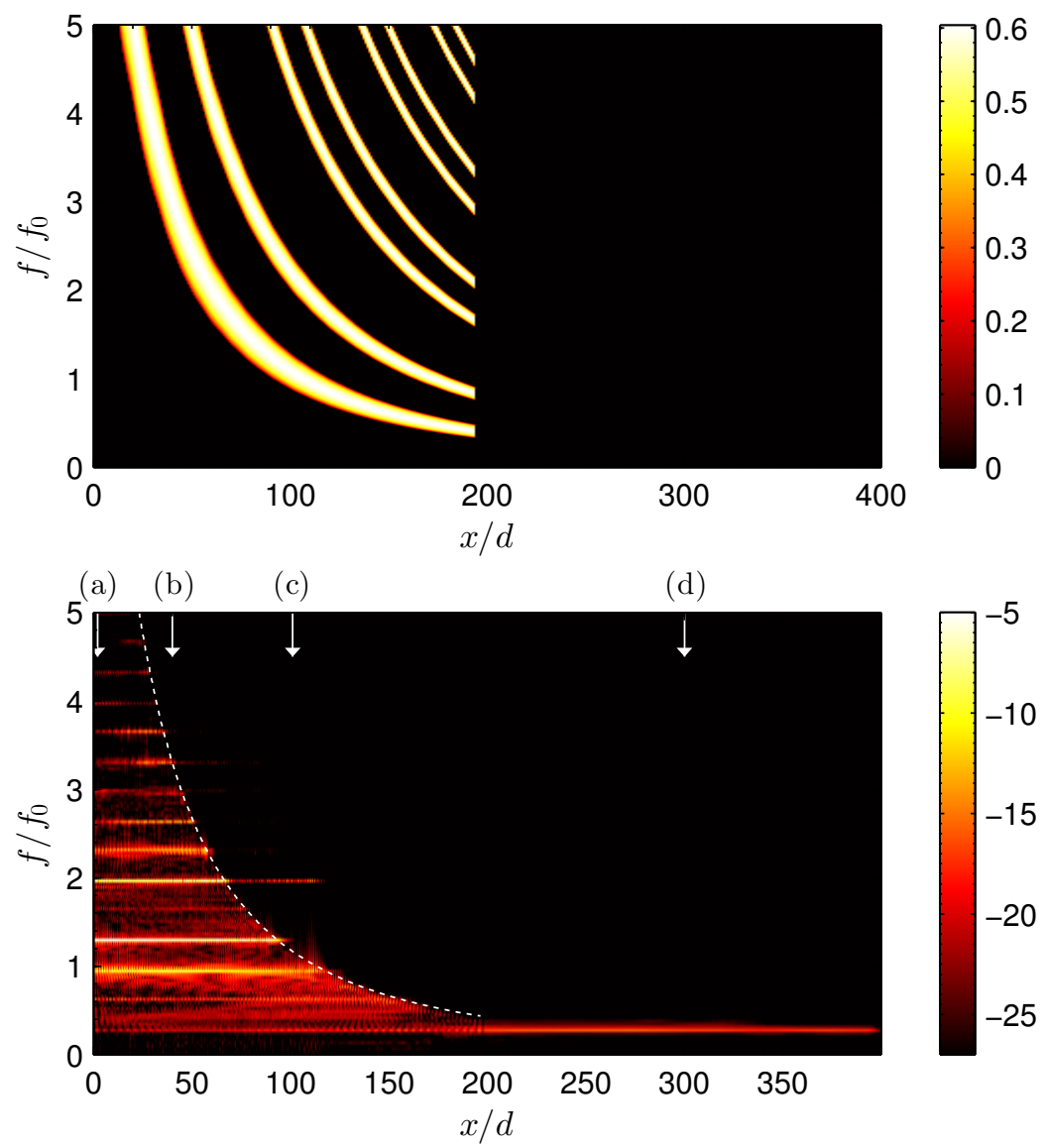

Figure 2.25: (top) Bandgap distribution in function of space. The chirped lattice ranges from $x=0$ to $x / a=200$, (middle) Spectrum-spatial distribution, (bottom) harmonic distribution for fundamental waves, its second harmonics and the difference component.

Therefore, only the difference frequency wave can be transmitted trough the medium. The final effect is that in transmission an effective demodulation is achieved: an amplitude modulated wave is injected, see Fig. 2.26 (a), the difference frequency is nonlinearly generated among other combination frequencies Fig. 2.26 (b-c), and the system output is only the low frequency component.

The whole effect presents some analogy with fluorescence, where electrons reemit a photon when relaxing to a lower quantum state after being exited with some electromagnetic wave. The emitted light has, in most cases, a longer wavelength and lower energy, than the absorbed radiation. Obviously, this analogy is not formal and only conceptual. 

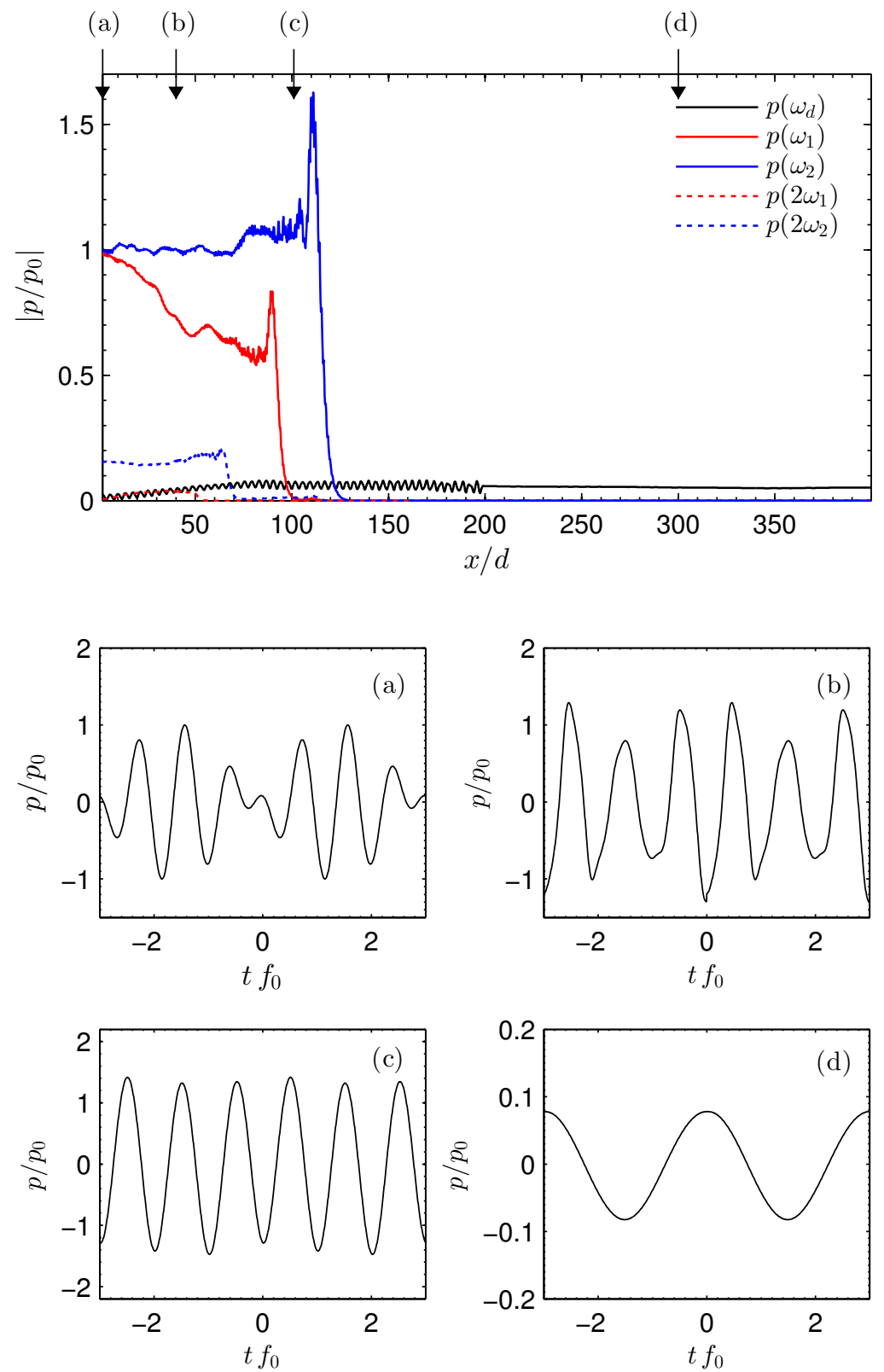

Figure 2.26: (Top) Harmonic distribution in the chirped layered media where the acoustic "fluorescence" is observed. The self-demodulation of the two collinear modes is achieved inside the nonlinear media, while the band-gap at the end of the chirped layered media limits the transmission of the high frequency components. Therefore, only the self-demodulated component is transmitted. (Bottom) Corresponding waveforms observed at locations (a-d). 


\subsubsection{Frequency Conversion Mirror in chirped layered me- dia}

We propose a setup of the chirped layered media in which the bandgap is located for the frequency of the second harmonic at the end of the structure. Actually, the structure is equivalent to the preceding subsection, but a monochromatic fundamental wave is selected here at frequency $\omega^{-}$. The fundamental wave is therefore a propagating mode over all the structure. On the other hand, its second harmonic only can propagate trough the beginning part of the structure. At the end of the layered media, its corresponding wavevector presents an imaginary part and the propagation is evanescent. Therefore, it is reflected back to the source. From the point of view of the source, the whole process can be treated as a nonlinear mirror: the incident wave is reflected with doubled frequency.

In order to visually illustrate the process, simulations were carried out using a $k$-space method over a $2 \mathrm{D}$ domain. The incident wave was generated by a Gaussian beam at frequency $\omega$, steered $10^{\circ}$ from the axis of the structure, see Fig. 2.27. It is worth noting here that the theoretical dispersion relation is one-dimensional and for non-normal incidence angles it will change. However, for illustrate the processes and for the selected incident angles is enough.

Figure 2.28 shows the first and second harmonic field, with the structure in the middle and the source below. It can be seen that when the intense wave impact on the layered, its first harmonic is slightly refracted due to different phase speed in the outside media and in the layered structure. Then, it shows very high transmission out of the structure. Only spurious reflection of the first harmonic is observed due to the impedance mismatching at the boundaries of the structure: in both limits of the crystal (top and bottom) reflection can be observed. On the other hand, the second harmonic is generated at the beginning of the propagation and inside the structure. The transmission of second harmonic is negligible. It can be seen how its amplitude becomes negligible in the bandgap area marked by

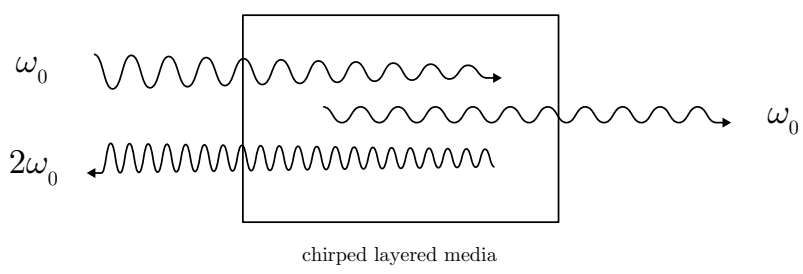

Figure 2.27: Scheme of the frequency conversion mirror effect. A wave with fundamental frequency $\omega_{0}$ enters in the chirped system. Second harmonic is generated inside the medium and at the end of the system the period is tuned to achieve a band-gap at second harmonic frequency. Thus, second harmonic is reflected while fundamental field is transmitted. 
(a)

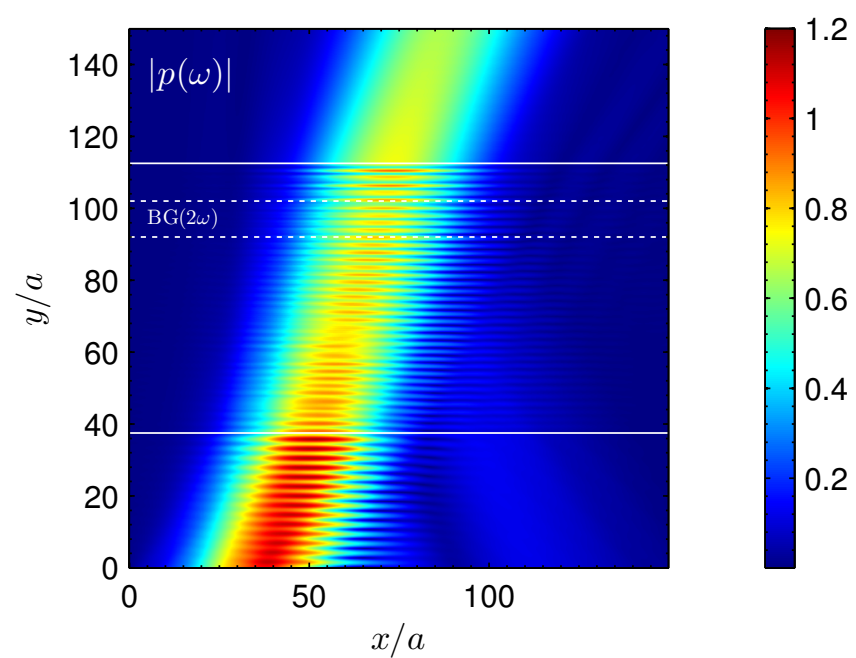

(b)

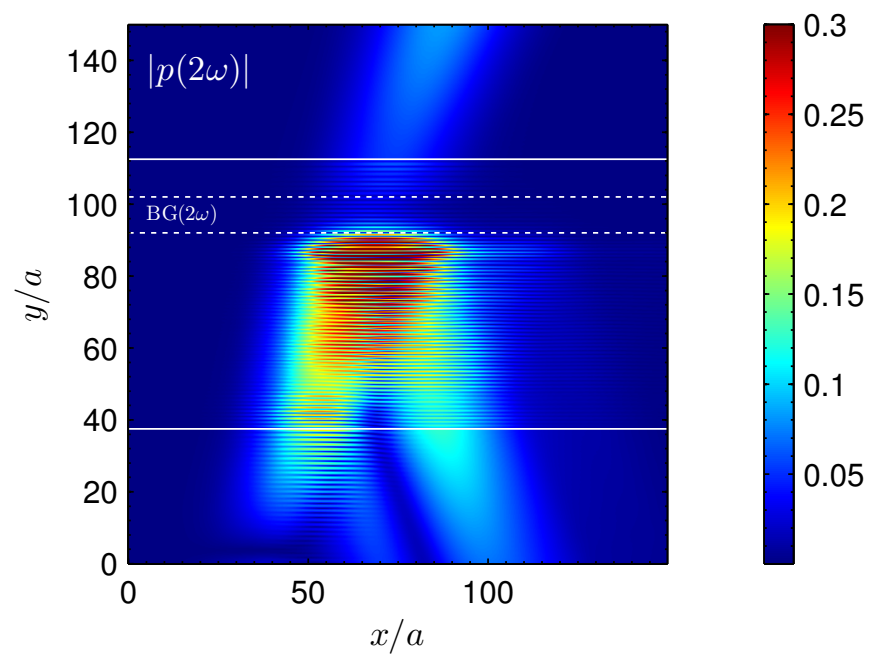

Figure 2.28: Field distribution for (a) first harmonic and (b) second harmonic field in the frequency conversion mirror configuration. The chirped layered media extends between white lines. White dotted lines marks the extension of the bandgap for the second harmonic.

the dotted lines. After the end of the gap, first harmonic start again generate harmonics by cascade processes, and second harmonic field increases, but the second harmonic generated at the beginning is not transmitted. The energy that is not transmitted is reflected back, as can be seen in Fig. 2.28 (b). In addition, reflection at the bottom boundary can be also observed due to impedance mismatching.

The effects of the present configuration can be enhanced by using very high 
nonlinear material in the construction of the layered media than in the surrounding fluid. Thus, for instance using layers of bubbly fluids and solid plates with micro cracks the nonlinear generation of second harmonic can be located only inside the layered structure and if the bandgap is properly designed at the end of the lattice the transmission of second harmonic will be negligible. On the other hand, controlling the length of the crystal and the steering of the beam the reflection of the second harmonic can be uncoupled totally from the reflections of the first harmonic field. Another improvements include focalization of the reflected harmonics as achieved in optics for linear waves (Cheng et al., 2014).

\subsection{6 $\beta$-Modulated media}

Finally, instead of modulate the sound speed and density of the layers, the nonlinear parameter of the media can be also modulated. Thus, it is interesting to take into account that there exist some materials, most of them solid, that exhibit negative nonlinearity. These materials are well-known and also common, e.g. some glasses as Pyrex or fused silica (Hamilton et al., 1998a). The nonlinear processes in negative nonlinear parameter media are not completely different from the positive ones. The nonlinear parameter shows the dependence of sound speed on pressure. Thus, the difference between positive and negative nonlinear media is that in negative media the rarefaction cycles of the wave travels faster than the compression cycles. Thus, wave profile distortion, as Fig. 2.29 sketches, is simply inverted.
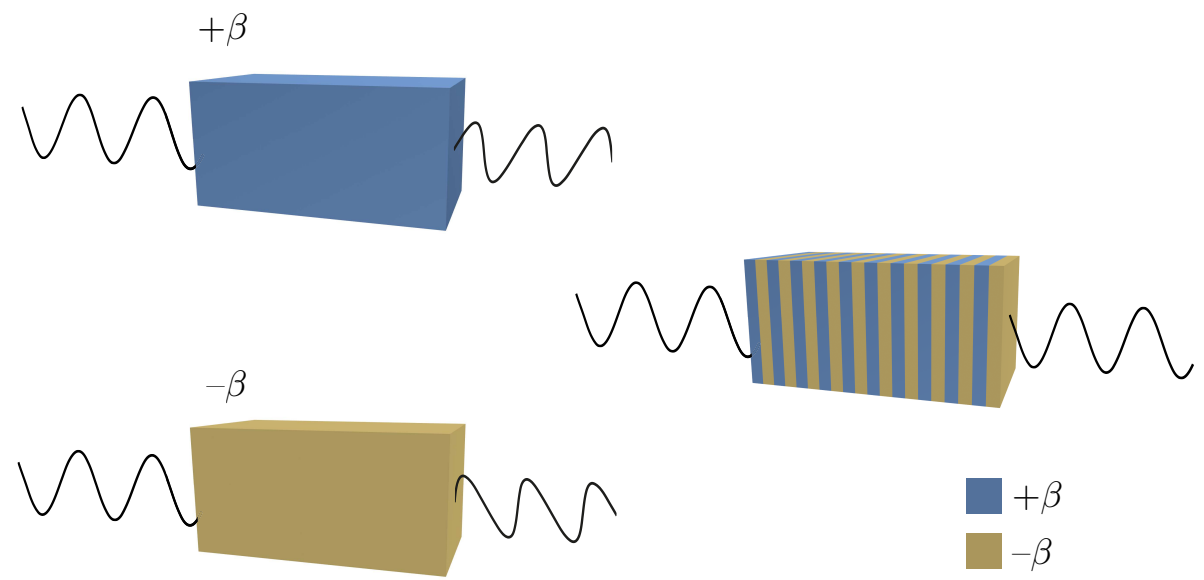

Figure 2.29: Scheme of the $\beta$-modulated acoustic layered media. (top-left) The nonlinear distortion in an homogeneous layer with $+\beta$ leads to steepening in the $+z$ direction, while for $-\beta$ the steepening is produced in the opposite direction. Therefore, alternating thin layers of positive and negative nonlinearity the steepening distortion can be mitigated. 

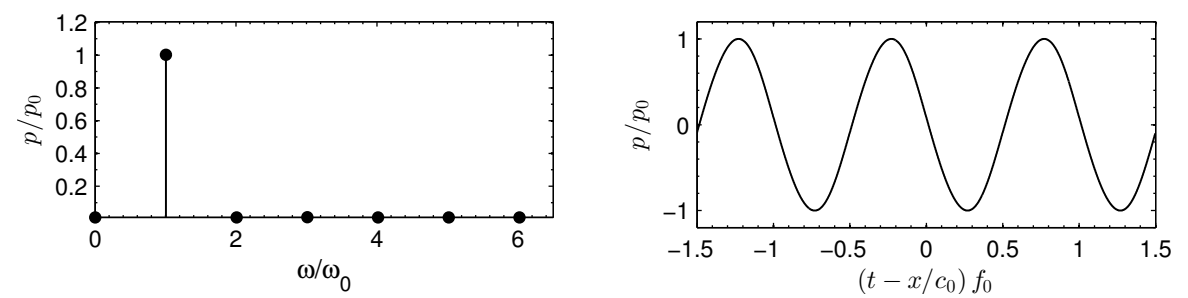

Figure 2.30: (Left) Spectrum obtained at $\sigma=1$ for a wave traveling a the $\beta$-modulated material. (Right) Corresponding waveform.

Then, we propose a configuration where thin layers of two materials with inverted nonlinear parameter are alternated. The idea is that the distortion during propagation in one layer is compensated by the distortion in the following and thus, a extremely linear material can be generated for supporting very intense waves at distance much longer than the shock formation distance. For the sake of simplify, the density and sound speed will be maintained equal in both media. The layer thickness was set to $a=0.1 \lambda$ and the nonlinear parameters as $\beta_{1}=-\beta_{2}=5$, of the order of the common materials.

\section{Extremely linear media}

Figure 2.30 shows the waveform and its spectrum recorded at the shock distance. It can be observed the absence of nonlinear distortion. due to the negligible energy transference to higher harmonics the fundamental component retains its initial amplitude. The evolution of the spectral components versus distance is shown in Fig. 2.31. It can be observed that the monochromatic wave can travel at the shock distance without experiment distortion.

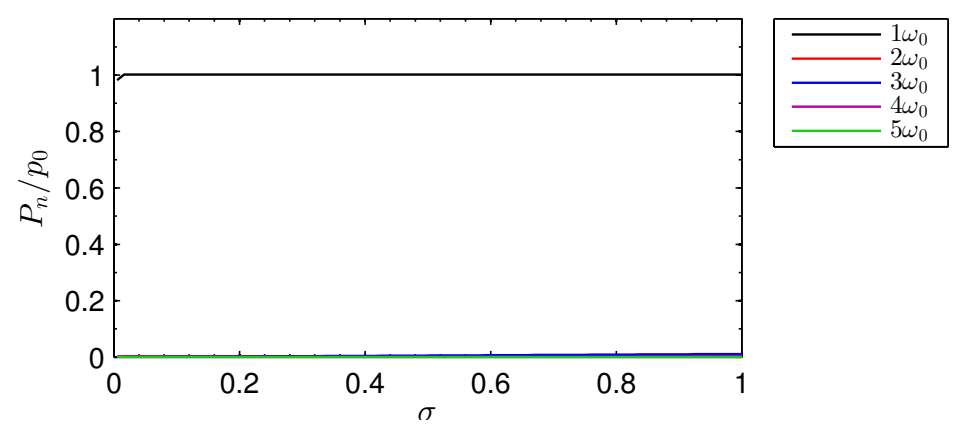

Figure 2.31: Harmonic evolution in the $\beta$-modulated material. Up to the shock wave distance almost all the energy remains in the fundamental harmonic and the harmonic amplitude for the higher spectral components is negligible. 

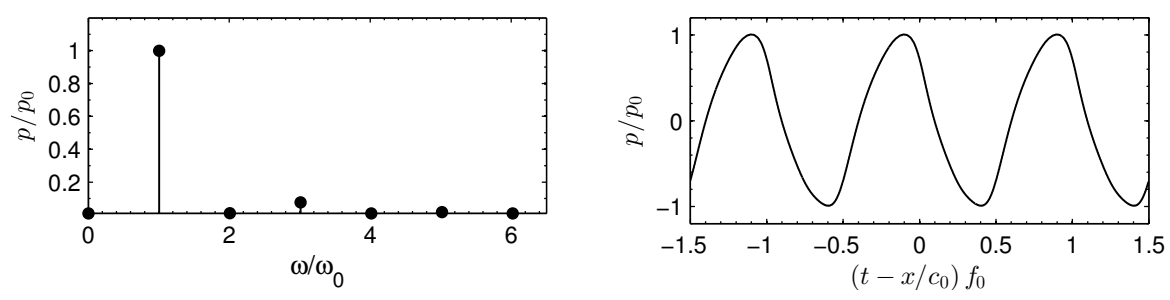

Figure 2.32: (Left) Spectrum obtained at $\sigma=10$ for a wave traveling a the $\beta$-modulated material. (Right) Corresponding waveform.

\section{Cubic nonlinear composite media}

However, an interesting effect is also observed. If the propagation is long enough, some harmonics of the fundamental frequency appears, but only the odd harmonics are generated. Thus, response of the media appears to be effectively equivalent to a cubic media. An explanation of this phenomena can be obtained for nondispersive propagation. The asymptotic expressions for the amplitude of the harmonics near the source can be obtained by expanding the Bessel-Fubini Eq (2.37). The expansion leads to the expressions for the second and third harmonics:

$$
\begin{aligned}
p_{2}(x, \tau) & =\frac{\beta p_{0}^{2} \omega}{2 \rho_{0} c_{0}^{3}} x \sin 2 \omega \tau, \\
p_{3}(x, \tau) & =\frac{3 \beta^{2} p_{0}^{3} \omega^{2}}{8 \rho_{0}^{2} c_{0}^{5}} x^{2} \sin 3 \omega \tau .
\end{aligned}
$$

It can be seen that the second harmonic grows linearly with the distance and it is proportional to the nonlinear parameter $\beta$. On the other hand, the third harmonic depends quadratically on both variables. Then, if a wave travels trough a layer of the proposed structure with $\beta_{1}=+\beta$, its second harmonic will increase

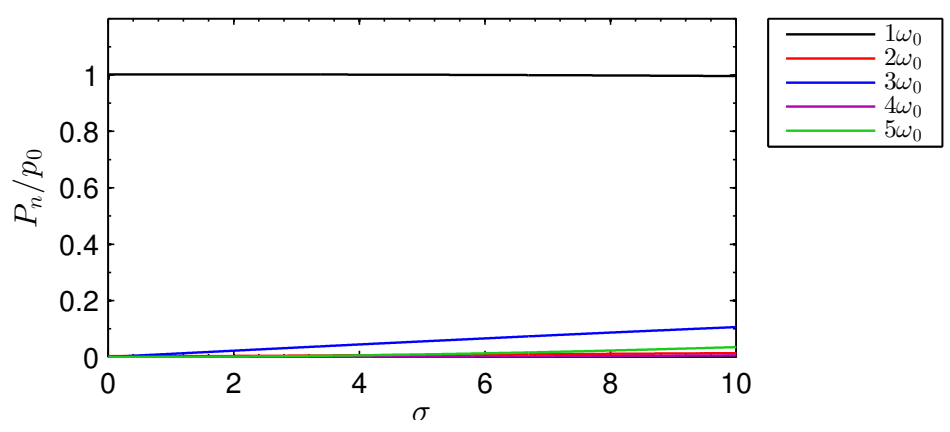

Figure 2.33: Harmonic evolution in the $\beta$-modulated material, where it can be seen the generation of odd harmonics. 
linearly and the third, almost absent, will do quadratically. When the wave crosses the next layer with inverted nonlinearity $\beta_{2}=-\beta$, Eq. 2.53 contributes creating the same amount of second harmonic than in the preceding layer but inverted due to the $-\beta$ coefficient, i.e. with opposite phase. Therefore, second harmonic at the end of the second layer vanishes. This process holds for thin layers were the asymptotic expressions can be applied. However, the generation of third harmonic depends on $\beta^{2}$, so there does not exist cancellation between layers and the generation is cumulative with distance. Third harmonic is generated by cascade processes and needs second harmonic field. Due to second harmonic is generated and compensated periodically, the evolution of the third does not grows quadratically with distance and its rate of the generation is very slow.

Figure 2.33 presents the evolution of the spectral components for distances up to $x=10 x_{s}$. It can be observed that the odd harmonics are generated and the even ones vanishes. On the other hand, the generated third harmonic shows an almost linear dependence with propagation distance, where the fifth harmonics grows at a quadratic rate. Figure 2.32 shows a waveform recorded at $\sigma=10$, where the characteristic shape of cubic nonlinearity can be observed, see e.g. (Jacob et al., 2007). On the right plot, its spectrum revels an almost negligible contribution of the even harmonics.

The configuration proposed, opens a way to obtain cubic nonlinearity for the longitudinal waves from a layered distribution of quadratic nonlinear materials. Therefore, such structure can be designed also using solid composites in the micro scale to obtain new ways of management of acoustic waves as low distortion waveguides for intense perturbations where the acoustical saturation distance can be extended. In the more general case including dispersion, the system offers the possibility of designing new tunable nonlinear materials where phase velocity and effective nonlinearity can be modified to achieve exotic configurations, while the structure is made from common material composites.

\subsection{Dispersion managed acoustic solitons}

One of the most widely studied phenomena in most nonlinear and strongly dispersive media is the existence of solitons. Solitons are nonlinear localized perturbations that propagate without changing its shape, emerging from the interaction of nonlinearity and dispersion. The first observation of the solitary waves was in 1834 by John Scott Russell at the Union Canal in Scotland, who called them Waves of Translation. In its experiments Russel described the main properties of these shallow-water waves (Russell, 1844). Starting from the theoretical works of Boussinesq and Rayleigh (Boussinesq, 1871; Boussinesq, 1877; Rayleigh, 1876), these waves could not been completely explained by the existent theory of water waves until 1895 when the Diederik Korteweg and Gustav de Vries derived a simple partial differential equation for shallow-water waves (Kordeweg et al., 1895). 
Their model, now known as the KdV equation, is completely integrable and provides exact solutions as solitons and cnoidal waves. However, it was not widely studied until 1965 with the advent of the computational physics when Zabusky and Kruskal found that the KdV model arises as the continuum limit of the FPU nonlinear lattice (Zabusky et al., 1965).

From these seminal works, solitary-wave solutions have been observed and deeply studied in most physical systems that can support nonlinear and dispersive waves: electromagnetism and nonlinear optics; hydrodynamics; acoustics, elastodynamics and granular media; phononics and nonlinear molecular dynamics, DNA dynamics, Bose-Einstein condensates, plasmas and so on. The solutions presented Chapter 1, in the limit of weak dispersion and weak nonlinearity, can also be described by the KdV equation. The acoustic layered media is not an exception.

Solitons have been studied by Yong et al. (2003) in layered acoustic media and in also in media with alternated solid and fluid layers (Fainstein et al., 2013; Huynh et al., 2015). Here, we extend the analysis using numerical techniques in the limits of strong nonlinearity and strong dispersion, i.e. from the long and slow solitons to sharp and intense acoustic solitary-waves.

\subsubsection{KdV solitons}

The Korteweg-de-Vries (KdV) equation arises as the continuum limit of the FPU quadratic nonlinear lattice (Zabusky et al., 1965). Thus, in the low frequency limit both models can be described by the same dispersion relation. Consider the dispersion relation of a mono-atomic lattice as

$$
\omega^{2}=\omega_{M}^{2} \sin \left(\frac{k a}{2}\right)^{2},
$$

where $c_{0}$ is the small amplitude sound speed and $a$ the oscillator separation, and the upper cut frequency is $\omega_{M}=2 c_{0} / a$. We can expand in Taylor series this lattice dispersion relation around $k=0$, retaining up to cubic terms leads to the $\mathrm{KdV}$ dispersion relation:

$$
\omega(k)=c_{0} k-b k^{3},
$$

with

$$
b=\frac{1}{24} c_{0} a^{2}
$$

as the dispersion parameter of the low frequency limit for longitudinal vibrations in any mono-atomic lattice. Figure 2.34 shows the normalized dispersion relation for the lattice and its KdV low frequency approximation, where it can be appreciated 


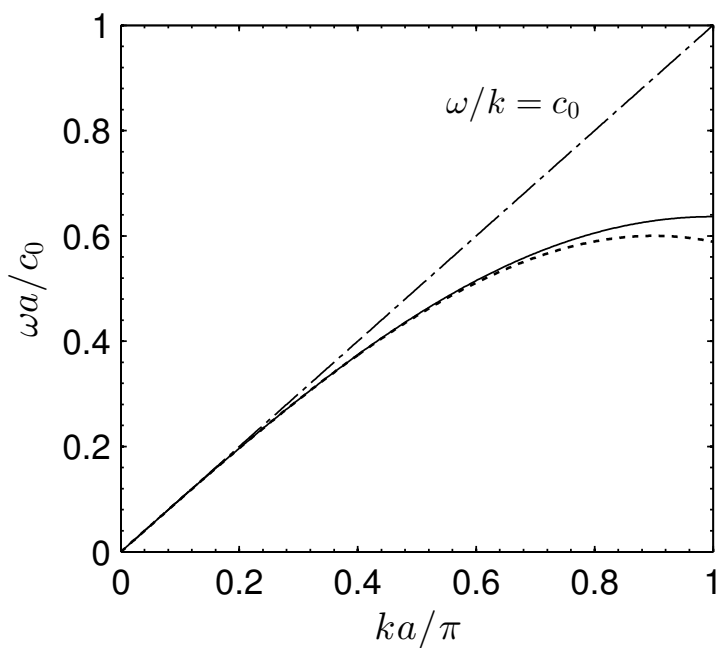

Figure 2.34: Dispersion relation for the mono-atomic lattice (continuous) and its KdV low frequency regime (dotted). Nondispersive slope is plotted in dashed-dotted line for comparison.

that both models fit very well for long wavelengths, but also for remarkable high wavenumbers. Relative difference is below $1 \%$ for wavenumbers below $k d / \pi<$ 0.64. However, the group velocity in this equivalent $\mathrm{KdV}$ is lower then in the full lattice, and it decreases faster with frequency.

Seen that similarity between band structures, and given the fact of the same quadratic nonlinearity is present in the KdV model and the FPU lattice, it is not surprising that similar solutions can be obtained in both systems, at least in the low frequency regime. The solutions explored in the Coulomb chain presented in Chapter 1 can be obtained also by the KdV equation provided that the kinks are far of the ultra-discrete regime, e.g. for energies below $0.5 \mathrm{eV}$.

Furthermore, homogenization techniques can applied to the layered media (Santosa et al., 1991), where, despite the complexity of the superior bands, the dispersion relation in the first band present the same shape under some assumptions than the FPU lattice. Therefore, a Taylor series expansion of Eq. (2.1) in powers of $\omega$ around $\omega=0$, collecting terms up to second order, leads to

$$
\omega^{2} \approx(1-\cos (k a)) \frac{2 c_{1}^{2} c_{2}^{2}}{a\left(a_{2} c_{1}^{2}+a_{1} c_{2}^{2}\right)} .
$$

Then, we expand again in Taylor series in powers of $k$ around $k=0$, and collecting terms up to third order we get 


$$
\omega \approx \sqrt{\frac{a c_{1}^{2} c_{2}^{2}}{a_{2} c_{1}^{2}+a_{1} c_{2}^{2}}} k-\frac{a^{2}}{24} \sqrt{\frac{a c_{1}^{2} c_{2}^{2}}{a_{2} c_{1}^{2}+a_{1} c_{2}^{2}}} k^{3},
$$

that can be expressed in the compact $\mathrm{KdV}$ form

$$
\omega(k)=c_{0} k-b k^{3},
$$

with the parameters

$$
\begin{aligned}
c_{0} & =\sqrt{\frac{a c_{1}^{2} c_{2}^{2}}{a_{2} c_{1}^{2}+a_{1} c_{2}^{2}}} \\
b & =\frac{a^{2}}{24} c_{0} .
\end{aligned}
$$

Note the dispersion parameter is exactly the same that obtained for the FPU mono-atomic lattice.

However, this result only states that both models are similar around $k=0$, but there can exist remarkable differences between the layered system and its equivalent $\mathrm{KdV}$ model for short waves when $k a \rightarrow 1$. Figure 2.35 shows the full dispersion relation for three different $\alpha$ ratios and its asymptotic solution for the low frequency regime by Eq. (2.60). It is clearly seen that good agreement between the mono-atomic lattice and its $\mathrm{KdV}$ expansion is only achieved in the case of $\alpha=0.9$ and $c_{2} / c_{1}=0.1$. For the other cases $(\alpha=0.1$ and $\alpha=0.5)$, the first propagation band becomes flatten, the frequency at which the band-gap start becomes higher and therefore the band-gap stretches. In the limit of Eq.(2.1) for $\alpha \rightarrow 1$ and $c_{2} / c_{1} \rightarrow 0$, the first band of the layered matches exactly the monoatomic chain dispersion relation Eq. (2.55), while the second band does not start in a finite frequency, i.e. there does not exist superior bands. This limit shows that the first band most dispersive shape for the layered medium is the dispersion relation Eq. (2.55). This situation corresponds to the case of a homogeneous media with infinitesimal-sized soft inclusions. Note that the inclusions impedance contrast must be large and also that the sound speed of the inclusions must be is low than the host media. A good analogy of this situation, for small amplitude waves $^{5}$, is a one dimensional version of a liquid with period inclusions of gas bubbles. Also note in one dimensional system a rigid inclusion $\left(c_{2} / c_{1}=0\right)$ implies zero transmission and therefore no wave inside the structure, increasing $c_{2} / c_{1}$ to low but physical values mean strong dispersion.

On the other hand, the effect of the layered medium dispersion is to flatten the slope of the first propagation band for high spatial frequencies, from the critical

\footnotetext{
${ }^{5}$ Soft inclusion typically shows large compressibility values and for finite amplitude waves the inclusions, in this case bubbles, are large compressed. Therefore, large strains are produced and the material response is described not only by its compressibility, but a large fraction of its local nonlinearity.
} 


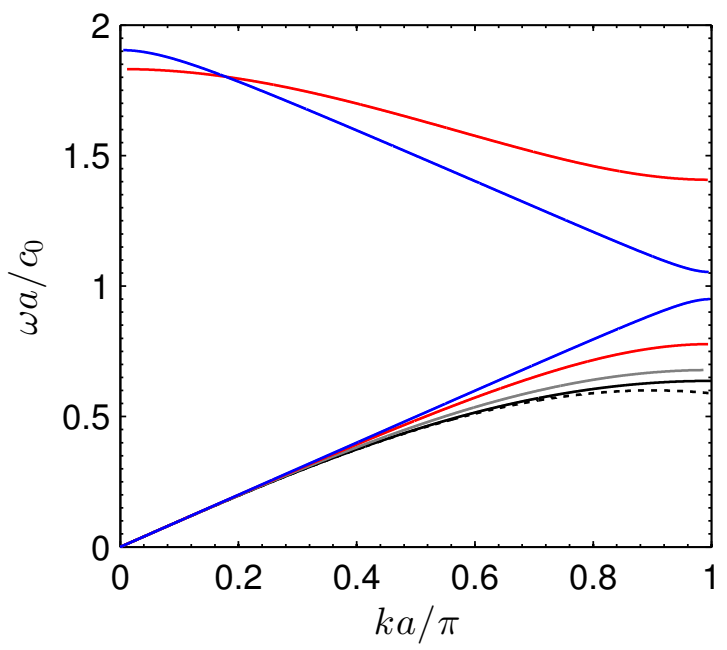

Figure 2.35: Dispersion relation for the layered media using $c_{2} / c_{1}=0.1$ and $\alpha=0.1$ (blue), $\alpha=0.5$ (red) and $\alpha=0.9$ (gray), mono-atomic lattice (black) and the KdV homogeneous limit (dotted line). It can be observed that the layered linear dispersion relation converges to the lattice dispersion for small inclusion of low sound speed.

case of the mono-atomic chain dispersion (gray line in Fig. 2.35), to situations in which infinitesimal bandgap are created and the group speed is $c_{0}$ for all the frequencies (blue line in Fig. 2.35).

As can be seen, the layered media dispersion can modify its shape in a large amount of different configurations. Soliton shape, width and amplitude will depend in those parameters.

Let us recall here the second order lossless-Westervelt Eq. (2.31), assuming $\rho_{0}$ does not change with space and for plane waves travelling in the $x$ direction

$$
\frac{\partial^{2} p}{\partial t^{2}}-c_{0}^{2} \frac{\partial^{2} p}{\partial x^{2}}-\frac{\beta}{\rho_{0} c_{0}^{2}} \frac{\partial^{2} p^{2}}{\partial t^{2}}=0
$$

Here, as usual in the literature ${ }^{6}$, we introduce the "travelling" coordinate system $z=x-c_{0} t$ in which the reference frame $z$ moves "with the wave" at speed $c_{0}$. Also, we define the new slow changing $\tau=t$ that corresponding to the travelling coordinates. The changes in the new coordinates can be related to the standard ones as

$$
\frac{\partial}{\partial x}=\frac{\partial}{\partial z} \quad, \quad \frac{\partial}{\partial t}=\frac{\partial}{\partial \tau}-c_{0} \frac{\partial}{\partial z}
$$

\footnotetext{
${ }^{6}$ Although for boundary problems is more convenient to transform the reference frame to a "retarded time" coordinate, we introduce the spatial "travelling" coordinates for convenience. The transformation here would be more appropriate for initial value problems.
} 
After some algebra, substitution of Eqs. (2.64) in Eq. (2.63) yields the lossless version of the Burgers equation

$$
\frac{\partial p}{\partial \tau}+\frac{\beta}{\rho_{0} c_{0}} p \frac{\partial p}{\partial z}=0
$$

where the term $\partial^{2} p / \partial \tau^{2}$ has been neglected due to the slowness change in the time $\tau$. This equation can be rewritten in terms of particle velocity using the first order relation $v \approx p / \rho_{0} c_{0}$ as

$$
\frac{\partial v}{\partial \tau}+\beta v \frac{\partial v}{\partial z}=0
$$

That is actually more close to its original form derived by Burgers. For convenience, let us return again to the static reference coordinate system, where Eq. (2.66), can be expressed as

$$
\frac{\partial v}{\partial t}-\left(c_{0}+\beta v\right) \frac{\partial v}{\partial x}=0 .
$$

In this evolution equation is clearly evident amplitude dependence of the wave speed $c=c_{0}+\beta v$, causing the well known steepening of the waveforms. As in previous sections, we know that the layered dispersion arises from the solution of the linearized wave equation with the layered media boundary conditions, where the eigenvalue problem leads to the Rytov's dispersion relation Eq. (2.1). In order to model KdV type dispersion, $\omega=c_{0} k-b k^{3}$, an additional term can be introduced into the Eq. (2.67):

$$
\frac{\partial v}{\partial t}-\left(c_{0}+\beta v\right) \frac{\partial v}{\partial x}+b \frac{\partial^{3} v}{\partial x^{3}}=0
$$

With this term, the leading equation (in traveling coordinates) is the $\mathrm{KdV}$ equation, the most widely model for nonlinear waves in dispersive media. One of the exacts solution of the $\mathrm{KdV}$ equation is the solitary wave

$$
v(x, t)=A \operatorname{sech}^{2}(\gamma(x-V t)),
$$

where the soliton width, $\gamma$, the amplitude, $A$, ant its speed, $V$, are related as

$$
\begin{gathered}
\gamma=\sqrt{\frac{\beta A}{12 b}}, \\
V=c_{0}+\frac{\beta A}{3}
\end{gathered}
$$

Here and beyond, we will demonstrate numerically the existence of this solution in the layered media, showing its limits of validity. Furthermore, we will investigate the behavior of solitary waves in the regime in which the layered media dispersion cannot be described by a KdV model with accuracy. 


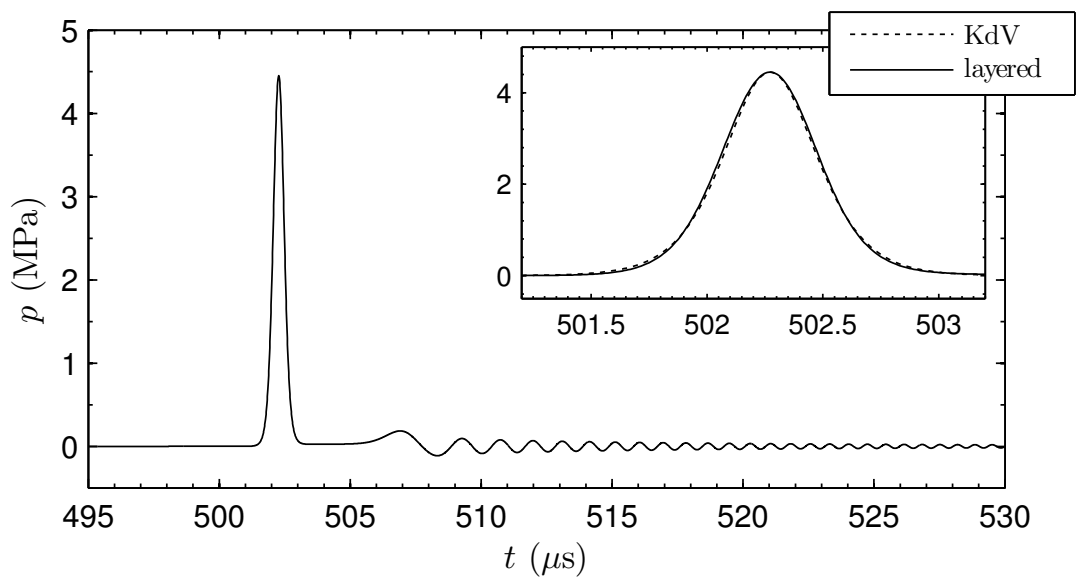

Figure 2.36: Solitary wave for the layered parameters $c_{2} / c_{1}=0.1$ and $\alpha=0.1$, (continuous line) numerical solution of the layered media (dotted line) KdV solution.

Figure 2.36 shows the evolution of solitary waves for the layered parameters $c_{2} / c_{1}=0.1$ and $\alpha=0.1$. As shown previously in Fig. 2.35, in this regime of parameters the layered dispersion can be described precisely by the KdV dispersive model. Due to both models are not exactly the same (even for the mono-atomic chain this is not fulfilled), we cannot claim the solitary wave solution observed in the layered system is strictly described by the solitary solution Eq. (2.69). However, as can be seen from the numerical tests, both solution closely agrees.

\subsubsection{Non-cubic dispersion solitons}

The presented KdV-like soliton exist only for a particular set of condition. In general, the dispersion near the band gap cannot be accurately described by the KdV dispersion. Furthermore, in case of high-amplitude/low-width solitary waves, the soliton spectrum can reach superior bands, that are in-existent in the KdV model. Here, we briefly present the existence of solitons with its width comparable to the lattice separation. Figure 2.37 shows four examples of propagating localized waves with different amplitudes. As can observed, as the soliton amplitude increases its speed increases and its width reduces, as commonly observed is any solitary wave. However, it can be seen that for high amplitude solitons, in which the width of the localized wave is of the order of the lattice, there exist remarkable differences with the continuum KdV approximation. In this simulations, the initial excitation is the KdV solution. Thus, for the low amplitude regime (Fig. 2.37 (a)) the soliton solution matches the excitation and propagates without changing its shape, amplitude and velocity. However, for higher amplitudes (Fig. 2.37 (b, c)) , it can be observed that the $\mathrm{KdV}$ is not the exact solution: the solitary wave breaks into 

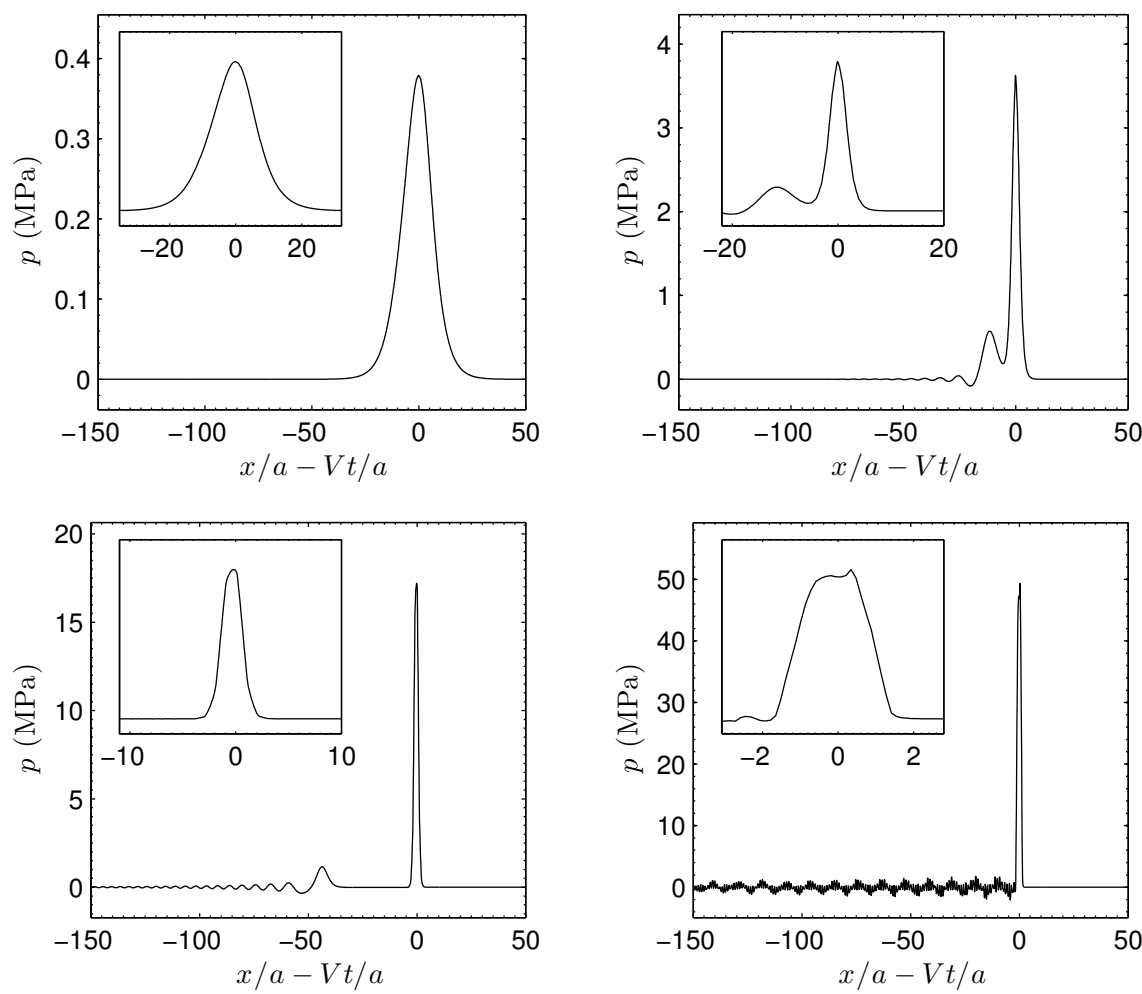

Figure 2.37: Examples of 4 solitons with increasing amplitudes.

other wave-packets plus an oscillatory tail that its left behind. Finally, in the regime width the soliton spectrum extend over the band-gap and up to second band, i.e. for very localized waves waves (Fig. 2.37 (d)) , the solitary wave suffers from a process of radiation. Note this radiation is totally different from the oscillatory tail shown in Figs. 2.37 (b, c): this radiation is not left behind and the localized wave continuously radiate low linear waves or phonons on its tail.

On the other hand, Fig. 2.38 shows the space time diagram of these waves. It can be seen clearly how the speed increases from $c_{0}$ to $V$, being $V$ the speed of the localized wave. Here, the radiation is not visible due to the scaling of the color map but another particular feature of this system can be observed. For high amplitude solitons, the amplitude oscillates periodically in time and space, showing variations that in space matches the lattice period $a$ and in time $V t / a$. Therefore, the main features of the solitons in the discrete limit, where the width of the wave is comparable to the layer thickness is the existence of tail radiation and periodic oscillatory amplitude of the strongly localized waves.

A more detailed study is presented in Fig. 2.39, where we show the amplitude 

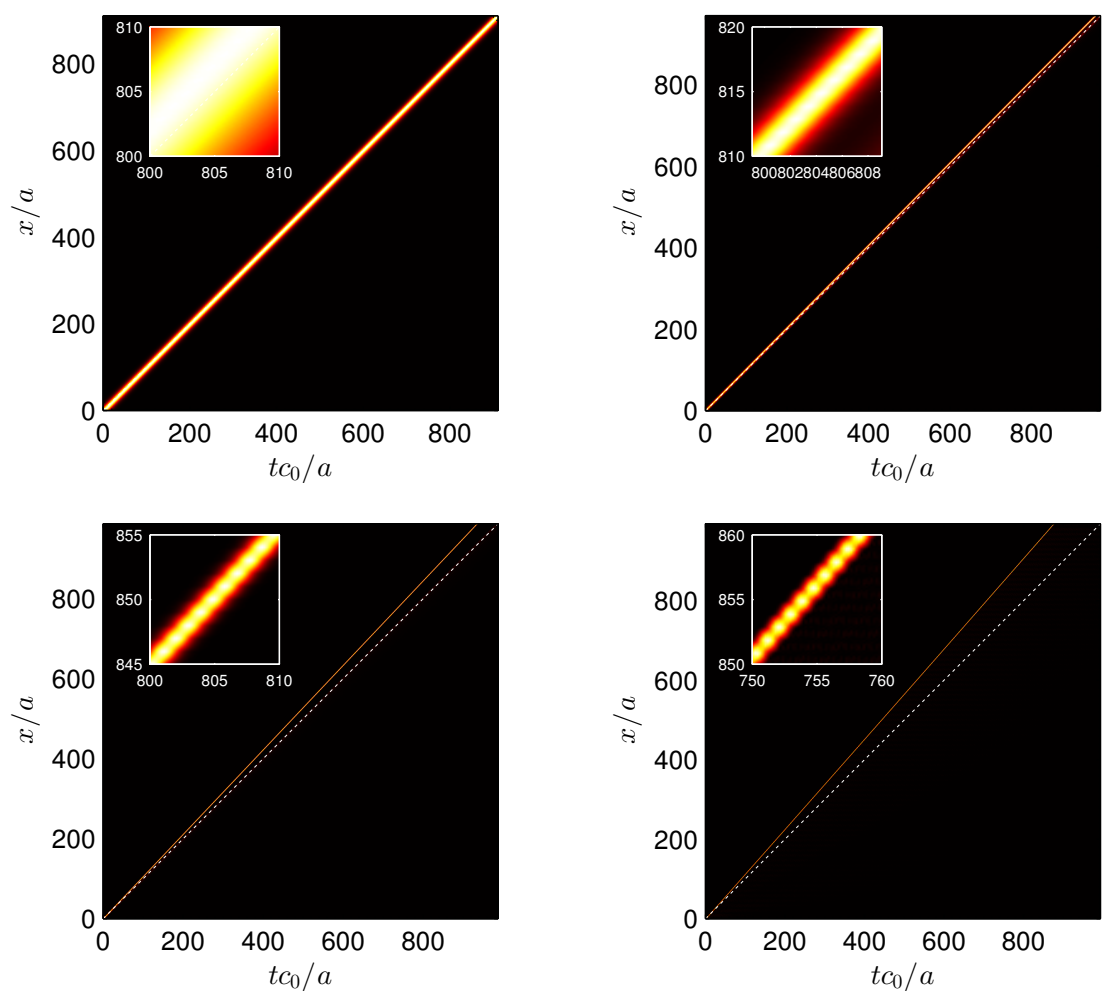

Figure 2.38: Space-time diagram representation of the solitons in Fig. 2.37, with increasing amplitude and velocity.

dependence of the soliton amplitude in function of its velocity. It can be seen that the $\mathrm{KdV}$ regime is only valid for weakly nonlinear excitations, of in other words, for wide solitons. For amplitudes of several $\mathrm{MPa}^{7}$ the soliton velocity observed in simulations is considerably higher than in the $\mathrm{KdV}$ model.

\subsubsection{D Layered chirped: progressive amplification of soli- tons}

The layered media offers the possibility of artificially modify the dispersion relation. As seen in previous sections, the modification of the lattice parameters can be made adiabatically, increasing or decreasing the lattice period progressively with distance forming chirped layered structure. Along the solitary wave amplitude, speed an thickness depends on the dispersion, the chirped la provides an usefully way to progressively amplify solitons. Thus, we present a test of this idea where

${ }^{7}$ The lattice parameters were $a=0.85, c_{2}=1500 \mathrm{~m} / \mathrm{s}, c_{1}=450 \mathrm{~m} / \mathrm{s}$ and $d=0.225 \mathrm{~mm}$ 


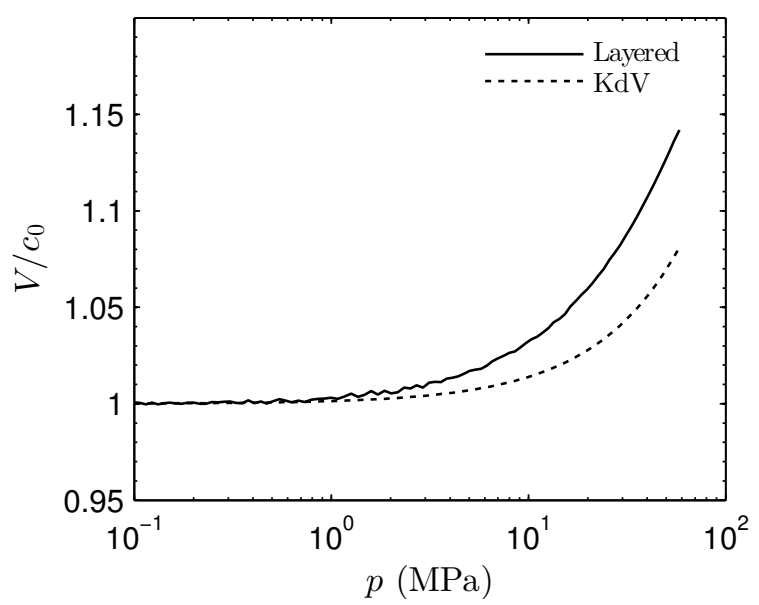

Figure 2.39: Soliton speed as a function of its amplitude for (continuous line) numerical results and (dotted line) KdV solution.

a chirped lattice period decreases with distance.

The initial excitation at the beginning of the lattice is a wide, KdV-type excitation. Thus, as Fig. 2.40 shows, soliton start to propagate at a supersonic, but low speed. As long the period is decreased, the dispersion seen by the wave adiabatically changes. Thus, the solitary wave solution slowly adapts it amplitude and thick according to the local dispersion. The result is that the wave can be stretched, amplified and accelerated to higher supersonic speeds. This proof of concept provides a method to manage the parameters of localized waves that can be applied to any other lattice. Configurations of special interest are probably the dispersion management of strain solitons in micro-structured composites, high intensity ultrasonic cutting devices for medical applications triggered by trains of amplified and pulsed excitations (instead of continuous mode) to reduce the heating and material fatigue (Harvey et al., 2014), or even period-varying granular beads composed of metallic spheres for drilling devices that can be usefully planetary exploration (Harkness et al., 2012).

\subsection{Conclusions}

In this Chapter we have analyzed the nonlinear propagation of acoustic waves in a layered media. The harmonic generation processes are presented and discussed mainly with numerical solutions considering a fluid-fluid structure. The strong characteristic dispersion of a layered media, in addition to the fluid nonlinearity, provides a wide range of possibilities for harmonic generation and control. In this 


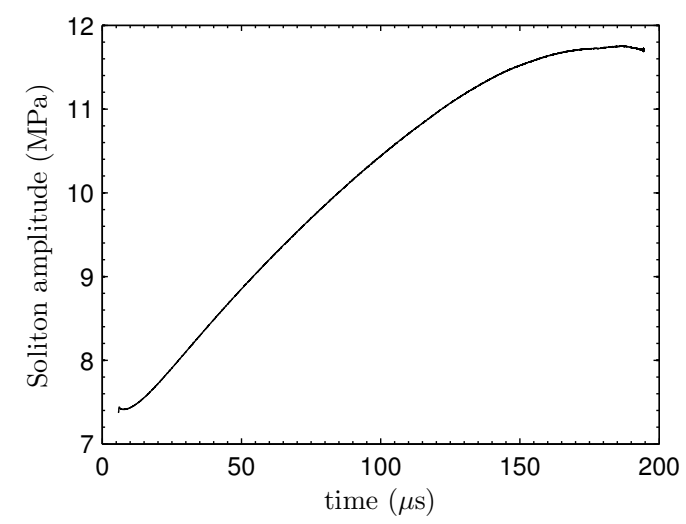

Figure 2.40: Progressive amplification of a soliton traveling in a chirped lattice.

sense, the effective nonlinearity of the composite media can be tuned for obtaining the desired harmonic balance. We provide the methods for selective harmonic generation, where the material layers can be adjusted for generate only second harmonic, only third harmonic, discard the even harmonics creating a cubic-nonlinear medium composed of quadratically-nonlinear layer. We provided also conditions for fabrication of waveguides for intense acoustic waves with extraordinary nonlinear distortion. Finally, the layered media provides conditions for dispersion management solitons, that are briefly presented, extending the work existent in the literature to the discrete limit of the soliton in which the width is of the order of the media periodicity.

On the other hand, much work on modeling the presented results remains. In this sense, we are currently working on Coupled Mode Theory. This analytical method widely used in optics (Malomed, 2000) can provide simple explanation of the dynamics of the system in terms of forward and backward coupled fields for fundamental and higher harmonics.

\section{References}

Albin, Nathan and Oscar P. Bruno (2011). "A spectral FC solver for the compressible Navier Stokes equations in general domains I: Explicit time-stepping". In: J. Comput. Phys. 230.16, pp. $6248-6270$.

Botteldooren, D. (1996). "Numerical model for moderately nonlinear sound propagation in threedimensional structures". In: J. Acoust. Soc. Am. 100.3, pp. 1357-1367.

Boussinesq, Joseph (1877). Essai sur la théorie des eaux courantes. Vol. 2. Imprimerie nationale.

Boussinesq, Joseph Valentin (1871). "Théorie générale des mouvements qui sont propagés dans un canal rectangulaire horizontal". In: CR Acad. Sci. Paris 73, pp. 256-260.

Brillouin, L. (2003). Wave Propagation in Periodic Structures: Electric Filters and Crystal Lattices. Dover phoenix editions. Dover Publications. 
Cheng, YC, S Kicas, J Trull, M Peckus, C Cojocaru, R Vilaseca, R Drazdys, and K Staliunas (2014). "Flat Focusing Mirror". In: Scientific reports 4, pp. -.

Fainstein, Alejandro, N Daniel Lanzillotti-Kimura, Bernard Jusserand, and B Perrin (2013). "Strong optical-mechanical coupling in a vertical GaAs/AlAs microcavity for subterahertz phonons and near-infrared light". In: Physical review letters 110.3, p. 037403.

Ginter, Siegfried, Marko Liebler, Eckard Steiger, Thomas Dreyer, and Rainer E. Riedlinger (2002). "Full-wave modeling of therapeutic ultrasound: nonlinear ultrasound propagation in ideal fluids." In: J. Acoust. Soc. Am. 111.5, pp. 2049-2059.

Gurbatov, S.N., O.V. Rudenko, and A.I. Saichev (2011). "Waves and Structures in Nonlinear Nondispersive Media. General Theory and Applications to Nonlinear Acoustics". In: ed. by S.N. Gurbatov, O.V. Rudenko, and A.I. Saichev. Springer. Chap. Chapter 8. Types of Acoustic Nonlinearities and Methods of Nonlinear Acoustic Diagnostics, pp. 271-304.

Hamilton, Mark F, David T Blackstock, et al. (1998a). Nonlinear acoustics. Vol. 237. Academic press San Diego.

Harkness, Patrick and Margaret Lucas (2012). "A brief overview of space applications for ultrasonics". In: Ultrasonics 52.8, pp. 975-979.

Harvey, Gerald, Anthony Gachagan, and Tapiwa Mutasa (2014). "Review of high-power ultrasoundindustrial applications and measurement methods". In: Ultrasonics, Ferroelectrics and Frequency Control, IEEE Transactions on 61.3, pp. 481-495.

Huynh, A, ND Lanzillotti-Kimura, B Jusserand, B Perrin, A Fainstein, MF Pascual-Winter, E Peronne, and A Lemaître (2006). "Subterahertz phonon dynamics in acoustic nanocavities". In: Physical review letters 97.11, p. 115502.

Huynh, Agnes, B Perrin, and A Lemaître (2015). "Semiconductor superlattices: A tool for terahertz acoustics". In: Ultrasonics 56, pp. 66-79.

Jacob, Xavier, Stefan Catheline, Jean-Luc Gennisson, Christophe Barrière, Daniel Royer, and Mathias Fink (2007). "Nonlinear shear wave interaction in soft solids". In: The Journal of the Acoustical Society of America 122.4, pp. 1917-1926.

Kordeweg, DJ and G de Vries (1895). "On the change of form of long waves advancing in a rectangular channel, and a new type of long stationary wave". In: Phil. Mag 39, pp. 422443.

Kosevich, Arnold M. (2005). "The Crystal Lattice: Phonons, Solitons, Dislocations, Superlattices, Second Edition". In: The Crystal Lattice. Ed. by Arnold M. Kosevich. Wiley-VCH Verlag GmbH \& Co. KGaA. Chap. Chapter 5. Acoustics of Elastic Superlattices: Phonon Crystals, pp. 153-162.

Liang, Bin, Bo Yuan, and Jian-chun Cheng (2009). "Acoustic diode: Rectification of acoustic energy flux in one-dimensional systems". In: Physical review letters 103.10, p. 104301.

Malomed, Boris A (2000). "Solitons in optical media with quadratic nonlinearity". In: Nonlinear Science at the Dawn of the 21st Century. Springer, pp. 247-262.

Maryam, W, AV Akimov, RP Campion, and AJ Kent (2013). "Dynamics of a vertical cavity quantum cascade phonon laser structure". In: Nature communications 4.

Naugolnykh, K. and L. Ostrovsky (1998). Nonlinear Wave Processes in Acoustics. Cambridge Texts in Applied Mathematics. Cambridge University Press.

Rayleigh, Lord (1876). "On waves". In: Phil. Mag 1.5, pp. 257-279.

Romero-García, Vicente, R Picó, A Cebrecos, Víctor José Sánchez-Morcillo, and K Staliunas (2013). "Enhancement of sound in chirped sonic crystals". In: Applied Physics Letters 102.9, p. 091906.

Rudenko, O.V. and S.I. Soluian (1977). Theoretical foundations of nonlinear acoustics. Studies in Soviet science. New York, NY 10011, USA: Consultants Bureau, p. 274.

Russell, J Scott (1844). "Report on waves". In: 14th meeting of the British Association for the Advancement of Science. Vol. 311, p. 390.

Sánchez-Morcillo, V., I. Pérez-Arjona, V. Romero-García, V. Tournat, and V. Gusev (2013). "Second-harmonic generation for dispersive elastic waves in a discrete granular chain". In: Physical Review E 88.4, p. 043203.

Santosa, Fadil and William W Symes (1991). "A dispersive effective medium for wave propagation in periodic composites". In: SIAM Journal on Applied Mathematics 51.4, pp. 984-1005. 
Tournat, V, VE Gusev, V Yu Zaitsev, and B Castagnede (2004). "Acoustic second-harmonic generation with shear to longitudinal mode conversion in granular media". In: Europhysics Letters 66.6, p. 798.

Vanhille, Christian and CleofCampos-Pozuelo (2004). "Numerical simulation of two-dimensional nonlinear standing acoustic waves". In: J. Acoust. Soc. Am. 116.1, p. 194.

Yong, Darryl H and Randall J LeVeque (2003). "Solitary waves in layered nonlinear media". In: SIAM Journal on Applied Mathematics 63.5, pp. 1539-1560.

Yun, Y, GQ Miao, P Zhang, K Huang, and RJ Wei (2005). "Nonlinear acoustic wave propagating in one-dimensional layered system". In: Physics Letters A 343.5, pp. 351-358.

Zabusky, Norman J and Martin D Kruskal (1965). "Interaction of solitons in a collisionless plasma and the recurrence of initial states". In: Phys. Rev. Lett 15.6, pp. 240-243.

\section{Publications}

The contents of this chapter has been presented in the following publications:

\section{Journal papers}

- Jiménez, N., Mehrem, A., Picó, R., García-Raffi, L. M., Sánchez-Morcillo, V. J. "Nonlinear propagation and control of acoustic waves in phononic superlattices" Comtes Rendus Physique: Special issue on phononic crystals, Elsevier. Submitted 19 May (2015).

\section{Conference proceedings}

- V.J. Sánchez-Morcillo, N. Jiménez, A. Mehrem, R. Picó, Ll. García-Raffi, V. Romero-García, V. Tournat. "Nonlinear waves in periodic media: from lattices to superlattices and phononic crystals". PHONONICS 2015: 3nd International Conference on Phononic Crystals/Metamaterials, Phonon Transport and Phonon Coupling. Paris, France. May 31-June 5 (2015)

- V. J. Sánchez-Morcillo, N. Jimenez, A. Mehrem, E.M. HamHam, L. García-Raffi and R. Picó. "Propagation of Intense Acoustic Waves in Sonic Crystals", ICU 2015: International Congress on Ultrasonics. Metz, France. May 11-14 (2015)

- Mehrem, A., Hamham, E. M., Jiménez, N., Cebrecos, A., Pico, R.; SanchezMorcillo, V. and García-Raffi, L. M. "Nonlinear Acoustic Waves Periodic Media" 7th Forum Acusticum 2014. Krakow, Poland. September 7 (2014) 
Part II

Nonlinear Singular Beams 



\title{
Chapter 3
}

\section{Nonlinear Self-Collimated Beams in Periodic Media}

\begin{abstract}
We report the propagation of high-intensity sound beams in a sonic crystal, under self-collimation or reduced-divergence conditions. The medium is a fluid with elastic quadratic nonlinearity, where the dominating nonlinear effect is harmonic generation. The conditions for the efficient generation of narrow, non-diverging beam of second harmonic are discussed. Numerical simulations are in agreement with the analytical predictions made, based on the linear dispersion characteristics in modulated media and the nonlinear interaction in a quadratic medium under phase matching conditions.
\end{abstract}




\subsection{Introduction}

The beams of different kind of waves, electromagnetic, sonic, etc..., experience diffraction and broaden as they propagate in a homogeneous medium. This spreading of energy in space diminishes the wave amplitude of the beam in the axis along propagation, unless the spreading is balanced by some focusing mechanism. Still, it is possible to prevent this fundamental wave propagation property and create non-diverging beams. Among the beam patterns without divergence the most popular are the linear Bessel beams (J. Durnin et al., 1987), and the nonlinear solitonic or self-trapped beams (Stegeman et al., 1999). Recently, another method for creating linear non-diverging beam was proposed, for waves propagating in a periodic medium. Such beams have been named self-collimated beams, and were first proposed for light beams in photonic crystals (Kosaka et al., 1999), and later extended to other type of waves. The phenomenon of self-collimation has attracted much attention, as a technique to propagate optical, acoustical or even matter wave beams at long distances without a sensible loss of amplitude. Selfcollimation is highly sensitive to the frequency: the divergence of beams can be reduced or even suppressed only at particular frequencies, those presenting particular dispersion characteristics, namely flat regions in the isofrequency contours (Kosaka et al., 1999). The size of the self-collimated beam is also limited by the extension of such flat region in angular space.

Self-collimation of low amplitude (linear), monochromatic acoustic waves has been demonstrated in 2D (Espinosa et al., 2007) and 3D (Soliveres et al., 2009) sonic crystals. More recently, the simultaneous self-collimation of two beams of different frequencies was also demonstrated experimentally (Soliveres et al., 2011). These results show that the conditions for self-collimation can be achieved also for non-monochromatic beams; in particular the case of the superposition of beams of one frequency and its second harmonic was considered in (Soliveres et al., 2011). The latter results are valid in linear regime; actually in (Soliveres et al., 2011) both frequency components were present in the input beam, and the corresponding beams propagated in the crystal without nonlinear interaction between them.

In the linear case, the propagation of light and sound beams obey similar equations, and similar propagation characteristics are expected. The similarities between photonic and sonic crystals are well established (Miyashita, 2005), and have motivated many studies, where analogous effects in both systems have been investigated. The analogy, however, breaks for high amplitude waves, where nonlinear effects appear. For example, second and higher harmonic generation processes may be essentially different in optics and acoustics. One reason is the absence of intrinsic dispersion for acoustic waves propagating in homogeneous media. Nonlinear acoustical waves in nondispersive media as homogeneous fluids, eventually generate shock waves, which are not observed in optics. Also, the type and strength of nonlinearity may be different. While most common optical nonlinearities are cubic (Kerr-type), in fluids and homogeneous solids, quadratic 


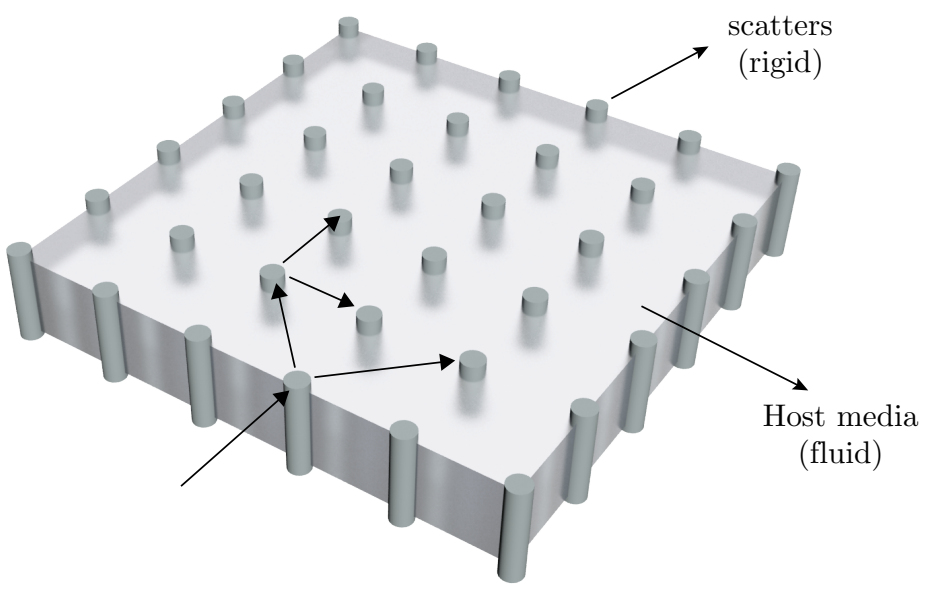

Figure 3.1: Basic scheme of a sonic crystal. The strong dispersion of the system is caused by the multiple scattering of waves over periodically distributed rigid inclusions. The resulting waves that propagates in the periodic media are Bloch waves, where the specific band structure of the media depends on the scatters and host media parameters. In this work we consider a fluid host media (nonlinear) and perfectly rigid scatters.

nonlinearity is dominant in acoustics. Even, nontraditional acoustic nonlinearities (power-law, hysteretic,...) are typical of some complex or microstructured acoustic media. In this sense, nonlinear effects of acoustic waves in periodic media, and in particular the self-collimation problem considered here are not a direct extension of the same effects in the optical case. Furthermore, the propagation of nonlinear acoustic beams in sonic crystals has never been addressed before.

The basic effect in nonlinear acoustics is harmonic generation (Hamilton et al., 1998b). It is known that efficient harmonic generation is only possible under fulfillment of phase matching conditions. For acoustic waves in fluids, this condition is rather natural, being always fulfilled for all harmonics due to the absence of dispersion, however in optics this requires special materials and special phase matching techniques (Boyd, 2003).

Acoustic harmonic generation has been studied in a variety of highly-dispersive nonlinear media, as bubbly liquids, or acoustic waveguides (Hamilton et al., 1998b), and weakly dispersive media as elastic plates (De Lima et al., 2003; Müller et al., 2010), nonlinear porous-elastic media (Donskoy et al., 1997) and in granular media (Legland et al., 2012). It has been proven as a useful effect in different applications, as material characterization (Hirsekorn et al., 1994; Zheng et al., 2000), ultrasound imaging and echography, (Humphrey, 2000), biological tissue characterization (Law et al., 1981) and other medical ultrasound applications.

The purpose of this Chapter is to study nonlinear propagation of high intensity 
sound beams in periodic media, and in particular to demonstrate the formation of nonlinear self-collimated acoustic beams in sonic crystals, and discuss the conditions under which this process occurs with maximal efficiency. A sonic crystal, as sketched in Fig 3.1 is designed, by using an iterative method, to fulfill the three conditions for optimal energy transfer between harmonics: flatness of isofrequency contour for each harmonic, phase matching and large overlap between distributions of the interacting Bloch modes. The predictions are checked by FDTD simulations of the nonlinear problem, that demonstrate the efficient generation of fundamental and second harmonic acoustic narrow beams.

\subsection{Nonlinear sound beam propagation model}

Several models can be used to describe nonlinear sound wave propagation though a fluid medium, with different levels of accuracy. An accurate description, when thermal and viscous effects are negligible, follows from the conservation laws of mass and momentum, can be written, respectively, in a Eulerian form (Hamilton et al., 1998b):

$$
\begin{array}{r}
\frac{\partial \rho}{\partial t}=-\nabla \cdot(\rho \mathbf{v}) \\
\rho\left(\frac{\partial \mathbf{v}}{\partial t}+\mathbf{v} \cdot \nabla \mathbf{v}\right)=-\nabla p
\end{array}
$$

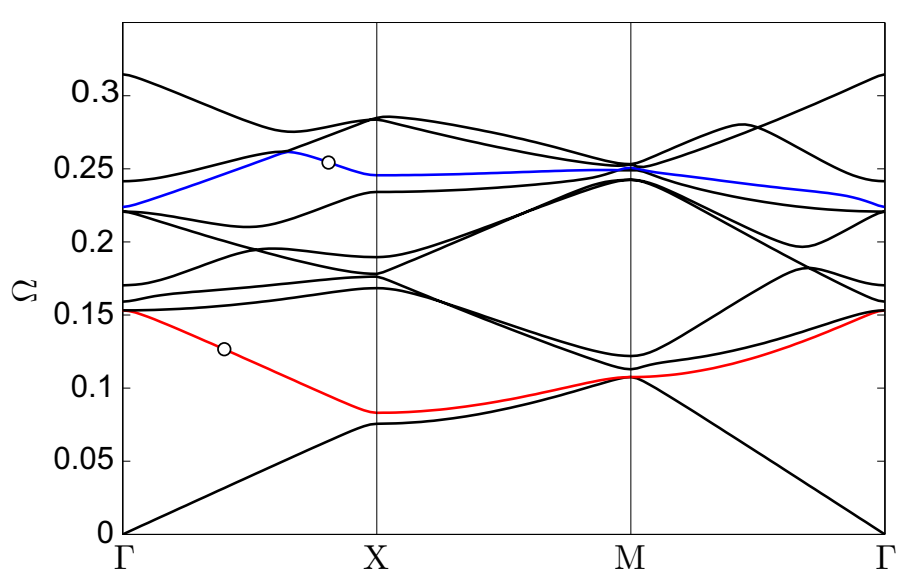

Figure 3.2: Band structure obtained by the Plane Wave Expansion (PWE) method for a SC made by a square lattice of rigid cylinders with $r=0.11 a$, where $a$ is the lattice constant, immersed in water. Red and blue lines mark the bands (2nd and 8th) for which simultaneous nondiffractive propagation in $\Gamma-\mathrm{X}$ direction is explored for both $\mathrm{FW}$ and $\mathrm{SH}$ respectively. 

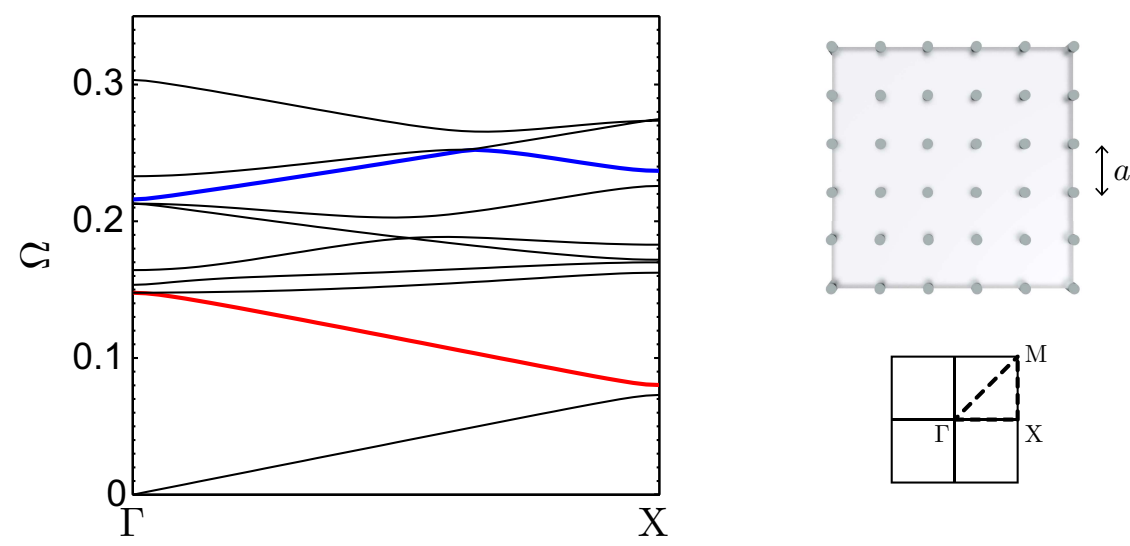

Figure 3.3: Top: Band structure (left) of a square lattice of rigid cylinders with $r=0.11 a$, where $a$ is the lattice constant, immersed in water (right). Red and blue lines mark the bands (2nd and 8th) for which simultaneous self-collimation for both fundamental and second harmonic searched, along $\Gamma-\mathrm{X}$ direction.

where $\mathbf{v}$ is the particle velocity vector, $p$ is the acoustic pressure, $\rho$ is the total density field that can be expressed as $\rho=\rho_{0}+\rho^{\prime}$, where $\rho_{0}$ the ambient fluid density and $\rho^{\prime}$ is the acoustic density. The system is closed by the equation of state of the fluid, that under our assumptions is a pressure-density relation, $p=p(\rho)$. A commonly used expression is obtained after Taylor expansion, keeping nonlinear terms up to second order. Then

$$
p=c_{0}^{2} \rho+\frac{c_{0}^{2}}{\rho_{0}} \frac{B}{2 A} \rho^{2},
$$

where $B / A$ is the nonlinearity parameter of the medium (which is known for most of materials, see e.g. (Naugolnykh et al., 1998)) and $c_{0}$ the sound speed in the medium. Note that Eqs. (3.1) and (3.2) also contain nonlinearities related to (1) mass and momentum advection, or (2) geometrical nonlinearities. However, for the second harmonic generation they are of minor importance compared with the nonlinear terms in the equation of state, Eq. (3.3).

The above formulation of nonlinear propagation problem remains valid when the propagating medium is inhomogeneous, including the case of sonic crystals where inhomogeneity is periodically distributed in space. In such case, the medium parameters $c_{0}$ and $\rho_{0}$ are space-dependent, represented by periodic functions. To our knowledge, the propagation of acoustic beams in periodic media has been only studied in the linear regime, and the corresponding nonlinear problem is addressed here for the first time. 

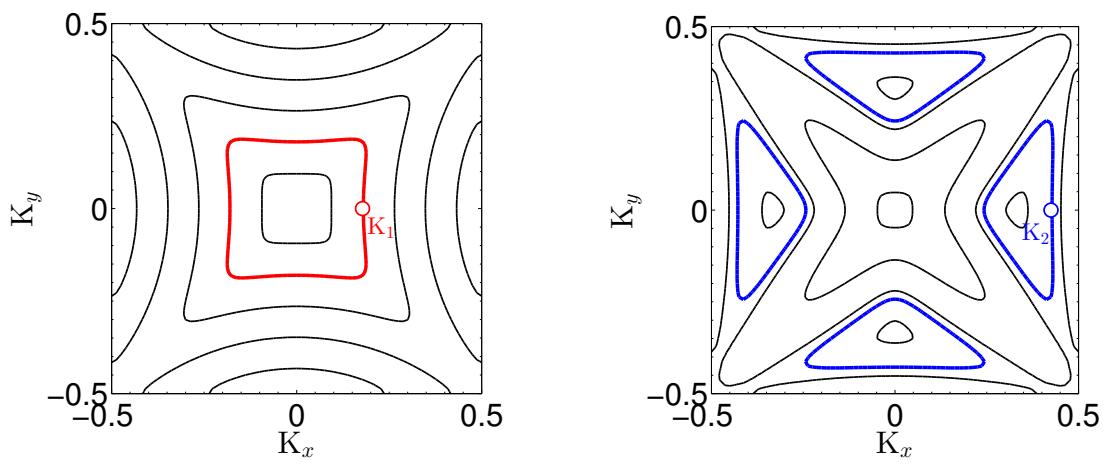

Figure 3.4: Isofrequency contours for the 2 nd (left) and 8 th bands (right). Points denote the wavevectors for both waves, lying on flat segments respectively.

\subsection{Self-collimation of intense acoustic beams}

We consider a narrow, intense acoustic beam incident on a 2D sonic crystal made of cylindrical scatterers of radius $r$ embedded in a fluid, arranged in a square-lattice with lattice constant $a$. The corresponding filling fraction is $f f=\pi(r / a)^{2}$. The beam width is roughly 6 lattice periods. For the sake of simplicity the scatterers are considered perfectly rigid (the sound field is totally reflected from the wall of scatterer). Assuming water as a host fluid, the material parameters are $\rho_{0}=1000$ $\mathrm{kg} / \mathrm{m}^{3}, c_{0}=1490 \mathrm{~m} / \mathrm{s}$.

The special conditions required for a sound beam to propagate without diffraction are presented in this section. The problem of self-collimation has been already discussed for linear, monofrequency (Pérez-Arjona et al., 2007; Espinosa et al., 2007) and bi-frequency (Soliveres et al., 2011) beams. Since a nonlinear beam is composed by a fundamental frequency component and its high frequency harmonics, self-collimation of the nonlinear beam requires self-collimation of its constituent frequency components. We remind that in self-collimation regime the sonic beam does not spread diffractively because Bloch wave vectors lying on the flat segment of the spatial dispersion curve have equal longitudinal components and thus do not dephase mutually in propagation. In general, this flatness of the dispersion curve appears at a particular frequency, but as shown in (Soliveres et al., 2011) it can be also obtained for a wave and its second harmonic regarded they propagate in different propagation bands.

To illustrate this case, we show in Fig. 3.3 the dispersion diagram of the sonic crystal for small-amplitude excitations, obtained using the Plane Wave Expansion (PWE) method on a linearized version of Eqs. (3.1)-(3.3). The conventional form of the band diagram is represented on the trajectory along the principal directions of the crystal, $\Gamma-\mathrm{X}-\mathrm{M}-\Gamma$, which are the boundary of the irreducible Brillouin 
zone (BZ). Figure 3.3 (top) shows the dispersion diagram for $\Gamma-\mathrm{X}$ direction. The red line in Fig. 3.3 denotes the 2nd propagation band. The fundamental (driving) field lies on this band. Its frequency $\Omega$ is chosen such that the corresponding isofrequency contour contains flat regions. The blue line in Fig. 3.3 denotes the 8th propagation band. The second harmonic frequency, $2 \Omega$, lies in this band for the particular crystal parameters considered.

As shown in (Soliveres et al., 2011), for a given crystal, it is possible to choose the fundamental frequency such that the isofrequency contours for both frequencies present flat regions. In our particular crystal, this happens when $\Omega=0.125$. These are dimensionless frequencies, related to physical frequencies $\omega$ as $\omega=\Omega\left(2 \pi c_{0} / a\right)$. Similarly, a normalized Bloch wavevector is defined as $\mathrm{K}=\mathrm{k}_{x}(a / \pi)$

Such condition is necessary to achieve self-collimated propagation of the nonlinear beam. In order to obtain an efficient generation of the second harmonic, together with simultaneous self-collimation for both waves two additional geometric conditions must be fulfilled, related to the wavenumber and spatial shape of the interacting beams. These conditions have been discussed for photonic crystals (Nistor et al., 2008; Nistor et al., 2010). The analysis can be extended to higher harmonics $($ third,...), however numerical simulations show that the amplitude of harmonics higher than second is small and does not contribute significantly to the beam profile, and therefore they will not be considered for the design of the structure.

\subsubsection{Phase matching conditions}

It is well known from nonlinear optics (Boyd, 2003) that a proper phase relationship between the fundamental and second harmonic waves must be satisfied for an efficient nonlinear frequency conversion along the propagation direction. In a dispersive medium, the wavenumbers of first harmonics do not combine to result precisely in wavevector of second harmonics, and a phase mismatch $\Delta \mathrm{K}=2 \mathrm{~K}(\Omega)-\mathrm{K}(2 \Omega)$ occurs. As a consequence, the second harmonic field is limited in amplitude: it does not grow linearly but oscillates in propagation, with a characteristic period given by the coherence length (Boyd, 2003)

$$
l_{c}=\frac{\pi}{\Delta \mathrm{k}}=\frac{a}{\Delta \mathrm{K}} .
$$

The conversion efficiency into second harmonics generally is smaller in optics than in acoustics, because of the inherent material dispersion for light waves (absent for sound waves in fluids), that causes the fundamental and second harmonic waves to travel along the crystal with different phase velocities. Thus, the presence of the scatterers is the only important source of dispersion in the acoustic case.

Phase matching corresponds to $\Delta \mathrm{K}=0$. Figure 3.5 shows that it can be actually achieved for the pair of frequencies where self-collimation occurs, as follows 
from the previous analysis. There, we represent the dispersion branches involved in self-collimation, along $\Gamma-\mathrm{X}$ direction, as in Fig. 3.3. Fundamental and second harmonic modes correspond to the crossings of the dotted horizontal lines with the corresponding dispersion branches. For a given fundamental frequency $\Omega$, in order to check the fulfillment of the phase matching condition, the curve corresponding to the "double" of the 2 nd band dispersion curve, $2 \Omega(2 \mathrm{~K})$, has been represented in Fig. 3.5 as a red dashed line. Phase matching is satisfied at the intersection between this curve with the corresponding curve at the 8th band. This corresponds to the mode labeled $\mathrm{A}$ in Fig. 3.5, which is phase-matched with the fundamental mode.

Note that due to the concavity of the 8 th band, at frequency $2 \Omega$ a second mode labelled with B in Figure. 3.5 can be also excited. This solution presents a large phase mismatch with the fundamental mode, and its contribution to the second harmonic field is negligible.

The simultaneous fulfillment of both conditions is obtained by an iterative procedure, which implies a re-design of the crystal parameters. The procedure is as follows: we start from a pair of frequencies $(\Omega, 2 \Omega)$ showing self-collimation for a given crystal parameters. Around this doublet, we seek the closest pair of frequencies $\Omega^{\prime}=\Omega+\delta \Omega$ and $2 \Omega^{\prime}$ showing phase-matching. Then the isofrequency curves (Fig. 3.3) are again calculated in order to evaluate the deviation of flatness

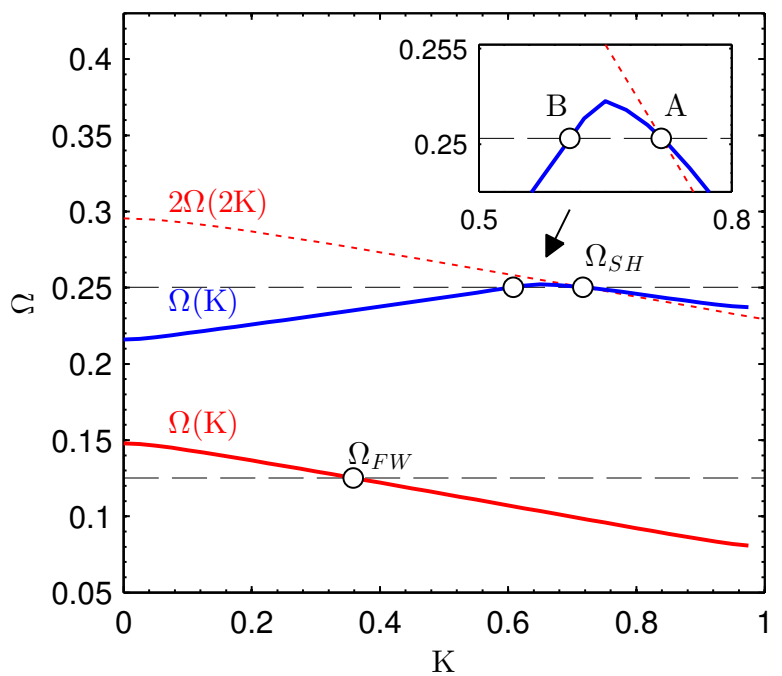

Figure 3.5: Dispersion curves involved in simultaneous self-collimation for the fundamental (red line) at the 2nd band and second harmonic (blue line) at the 8th band. The "doubled" dispersion curve is represented (dashed red line) to identify phase matching of harmonics. The intersection denotes the frequency presenting phase matching. A closest view (inset) shows that for the self-collimated second harmonic, two solutions (modes A and B, with distinct K) are found, phase and non-phase matched, respectively 

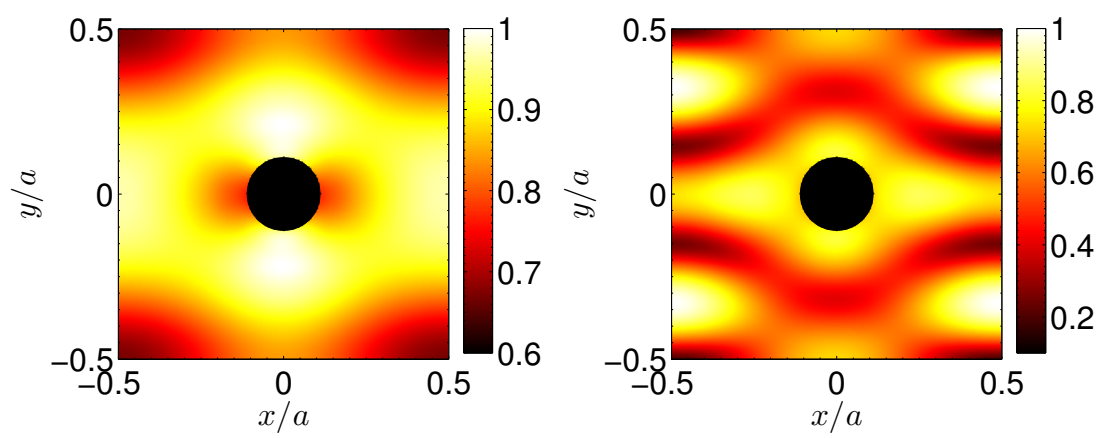

Figure 3.6: Spatial distribution of the pressure field for the Bloch modes of the fundamental (left) and the second harmonic (right) waves. The coupling coefficient is estimated to be $\kappa=0.85$.

in the isofrequency contours. The sonic crystal parameters are then modified, e.g. by a slight variation of the filling fraction, in order to tune the dispersion relations to get again self-collimation conditions. The process is repeated again until both conditions (flatness and phase matching) are simultaneously satisfied. We note that, despite this is out of the scope of this paper, optimization techniques as genetic algorithms can be applied here in order to find an optimal structure.

\subsubsection{Nonlinear coupling of Bloch modes}

Efficient energy transfer between harmonics requires also a strong mode coupling, which depends on the spatial overlapping between the two interacting waves. For plane waves in a homogeneous medium a perfect spatial nonlinear coupling between first and second harmonic is assured, since mode overlapping is maximal. The propagation eigenmodes in a periodic medium are Bloch waves, whose amplitudes are spatially modulated and does not necessarily overlap. If two modes do not overlap in space, the energy transfer is less efficient even if they are phase matched. The amount of energy transfer can be estimated evaluating the spatial overlap between the envelopes of the corresponding Bloch modes.

Let $B_{1}$ and $B_{2}$ be the spatial envelopes of the Bloch modes of the fundamental and and second harmonic waves, respectively. The nonlinear coupling coefficient is calculated as the cross-correlation between the functions $B_{1}^{2}$ and $B_{2}$ normalized in such a way that unity would correspond to the perfect matching of the modes (Nistor et al., 2008). We define the coupling coefficient as

$$
\kappa=\frac{\left|\int_{M} B_{1}^{2} B_{2}^{*} \mathrm{~d} r\right|}{\sqrt{\int_{C}\left|B_{1}^{4}\right| \mathrm{d} r \int_{C}\left|B_{2}\right|^{2} \mathrm{~d} r}},
$$

where the upper integral is calculated in the nonlinear medium from one unit cell, while the lower integrals are taken over the entire unit cell. 
To calculate $B_{1}$ and $B_{2}$ we solve the eigenvalue problem for the pressure field by means of the PWE method, which converts the differential equation to an infinite matrix eigenvalue problem that can be truncated and solved numerically. For that, we follow the same procedure as in (Pérez-Arjona et al., 2007), however, the problem is solved inversely, i.e. for a given frequency, the corresponding wave vectors satisfying the phase matching condition are obtained. Then $B_{1}$ and $B_{2}$ are obtained as the eigenvectors corresponding to fundamental and second harmonic frequencies, respectively. In Fig. 3.6 we plot the spatial distributions of $B_{1}$ and $B_{2}$, respectively, for the selected final design where a coupling coefficient of $\kappa=0.85$ is obtained. This value is of the same order as the coupling in homogeneous media, $\kappa=1$, and therefore sufficiently large for an efficient harmonic generation.

\subsection{Numerical simulation}

A full-wave nonlinear simulation was performed, using the FDTD method, to validate the efficiency of the second harmonic generation in the proposed structure. The crystal parameters, obtained after the iterative procedure describe above, are as in Fig. 3.3 and Fig. 3.5. The source is a plane piston with a width of $6 a$, located near the crystal, and radiating a harmonic wave with normalized frequency $\Omega=0.125$. In order to minimize numerical dispersion a computational grid with $N_{\lambda}=45$ elements per wavelength and a Courant-Friedrich-Levy number of $S=0.95$ was used. In Fig. 3.7 we present the numerically obtained spatial distributions of the fundamental and its nonlinearly generated second harmonic. As predicted, both beams are nearly collimated. For comparison, the beam spatial distribution calculated for an homogeneous material (removing the crystal) are represented in Fig. 3.7 (e-f), where the diffractive broadening of the beams is visible. Transversal intensity distributions are shown in Fig. 3.8 (a-b) for a distance $80 a$, where beam widths are compared with the reference beams in the homogeneous medium, broadened by diffraction.

The pressure amplitudes along the beam axis are shown in Fig. 3.9 for each harmonic. Here, the analytic solution for a nonlinear plane wave propagating in a homogeneous (nondispersive) medium is plotted for reference (dashed lines) (Naugolnykh et al., 1998), given by $p_{n} / p_{0}=2 J_{n}(n \sigma) / n \sigma$, where $J_{n}$ is the Bessel function of order $n, \sigma=\left(\beta \omega p_{0} / \rho_{0} c_{0}^{3}\right) x$ is coordinate normalized to the shock formation distance, $p_{0}$ is the pressure at the source and $\beta=1+B / 2 A$ the nonlinearity parameter. Such analytical solution is valid in the preshock region $\sigma<1$. The growth rate of the self-collimated second harmonic beam propagating in the crystal matches well the growth rate of a plane wave in a homogeneous medium in such preshock region, which is a consequence of the weak divergence of the beam and the high degree of phase matching. Also, the second harmonic field can reach even higher amplitudes than those corresponding to nondispersive media (where harmonics decay beyond the shock formation distance). The latter effect can be 

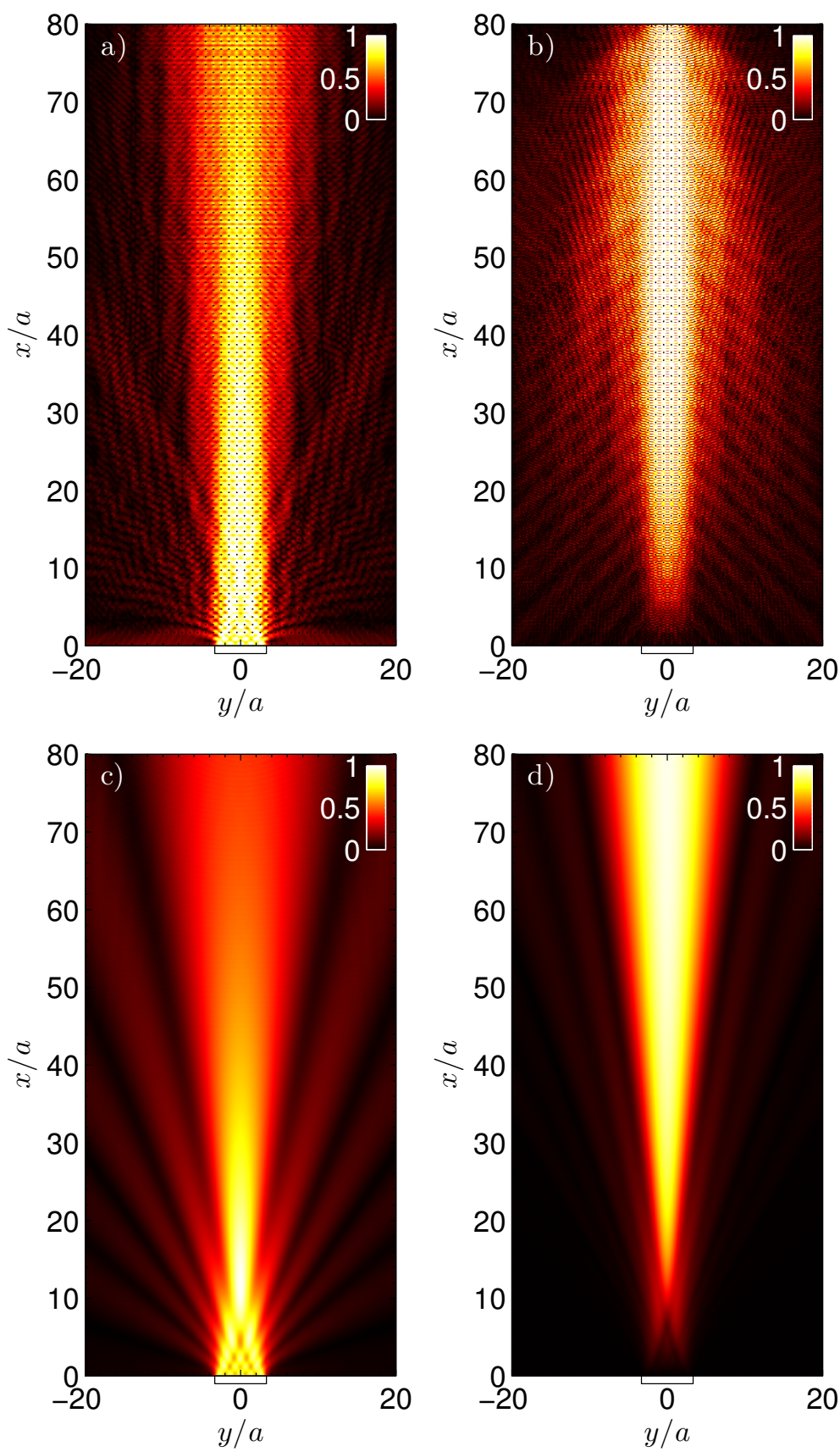

Figure 3.7: Beam spatial distribution for simultaneously self-collimated harmonics in the sonic crystal (c-d) and in homogeneous fluid (e-f). Pressures are normalized to the maximum pressure. 

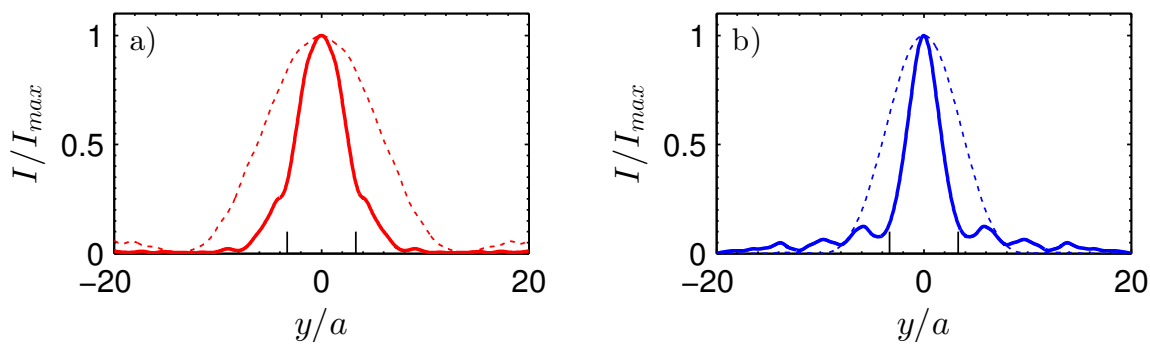

Figure 3.8: Pressure distributions obtained by FDTD simulations. Normalized intensity cross section of fundamental (a) and second harmonic (b) at $x=80 a$ propagating in the crystal (continuous line) and in a homogeneous (water) medium (dashed lines).

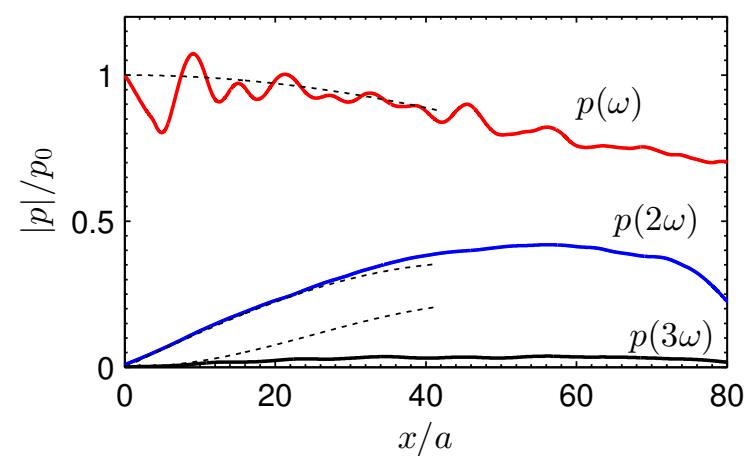

Figure 3.9: Normalized field amplitude along the acoustical axis $y=0$, for the fundamental, second and third harmonic beams. The dashed lines represent the analytical solutions for harmonic evolution on a plane wave propagating in a nondispersive medium.

understood in terms of phase mismatch of higher harmonics: in homogeneous media all harmonics are phase matched while in the crystal only the second harmonic is phase matched. In Fig. 3.9 the third harmonic is also plotted, where its small contribution to the beam is evident. The phase mismatch in third and higher harmonics decrease the energy flow into these components. Finally, the amplitude in Fig. 3.9 decays because non-perfect conditions for self-collimation, that makes the beam to start diverging after a long distance, or non-perfect phase matching, which results in a beating period with long coherence length.

\subsection{Conclusions and Remarks}

We have demonstrated the possibility of efficient second harmonic generation of sound in a sonic crystal, by means of the formation of narrow, weakly diverging nonlinear acoustic beams. Three conditions must be simultaneously present for a 
efficient second harmonic generation, which are: 1) simultaneous self-collimation, 2) phase mathing and 3) high spatial coupling of interacting harmonics. The use of simultaneous self-collimation regime limits the diffraction of both harmonic beams, maintaining the amplitude at the axis and therefore the nonlinear interaction. Under ideal conditions (no divergence and losses), the decrease of the first harmonic beam is mainly attributed to the energy transfer to second and higher harmonics. The sonic crystal parameters can be chosen to fulfill phase matching with the second harmonic, maximizing second harmonic generation due to synchronous cumulative interaction. Finally, the spatial coupling (overlapping) between interacting modes is also analyzed by calculating a nonlinear coupling coefficient. It is shown that its value $(\kappa=0.85$ for the case studied) is not far from the ideal case, revealing a strong spatial overlap between both Bloch modes that leads to high energy transfer.

The study show that linear dispersion characteristics (band structures, isofrequency contours) can be used to predict the behaviour of nonlinear beams propagating in periodic media. This opens the possibility of extending the study of nonlinear sound beam propagation in sonic crystals to other cases of interest. For example, crystals with higher filling factors present full frequency bandgaps, that may be used to filter out the propagation of selected higher harmonics. In this sense, sonic crystals can be a way to control the spectrum of intense acoustic waves, using the strong dispersion properties introduced by the periodicity.

\section{References}

Boyd, Robert W (2003). Nonlinear optics, 2nd edition. Academic press.

De Lima, WJN and MF Hamilton (2003). "Finite-amplitude waves in isotropic elastic plates". In: Journal of sound and vibration 265.4, pp. 819-839.

Donskoy, D. M., K. Khashanah, and T. G. McKee (1997). "Nonlinear acoustic waves in porous media in the context of Biot???s theory". In: The Journal of the Acoustical Society of America 102.5, pp. 2521-2528.

Espinosa, Víctor, Víctor J Sánchez-Morcillo, Kestutis Staliunas, Isabel Pérez-Arjona, and Javier Redondo (2007). "Subdiffractive propagation of ultrasound in sonic crystals". In: Physical Review B 76.14, p. 140302.

Hamilton, M.F. and D.T. Blackstock (1998b). Nonlinear Acoustics. Academic Press.

Hirsekorn, S and S Pangraz (1994). "Materials characterization with the acoustic microscope". In: Applied physics letters 64.13, pp. 1632-1634.

Humphrey, Victor F (2000). "Nonlinear propagation in ultrasonic fields: measurements, modelling and harmonic imaging". In: Ultrasonics 38.1, pp. 267-272.

J. Durnin, J.J. Miceli and J.H. Eberly (1987). "Diffraction-free beams". In: Phys. Rev. Lett. 58.4, pp. 1499-1501.

Kosaka, Hideo, Takayuki Kawashima, Akihisa Tomita, Masaya Notomi, Toshiaki Tamamura, Takashi Sato, and Shojiro Kawakami (1999). "Self-collimating phenomena in photonic crystals". In: Applied Physics Letters 74.9, pp. 1212-1214.

Law, WK, LA Frizzell, and F Dunn (1981). "Ultrasonic determination of the nonlinearity parameter B/A for biological media". In: The Journal of the Acoustical Society of America 69.4, pp. 1210-1212. 
Legland, JB, V Tournat, O Dazel, A Novak, and V Gusev (2012). "Linear and nonlinear Biot waves in a noncohesive granular medium slab: Transfer function, self-action, second harmonic generation". In: The Journal of the Acoustical Society of America 131.6, pp. 4292-4303.

Miyashita (2005). "Sonic crystals and sonic waveguides". In: Meas. Sci. Technol. 16, R47-R63.

Müller, Martin F, Jin-Yeon Kim, Jianmin Qu, and Laurence J Jacobs (2010). "Characteristics of second harmonic generation of Lamb waves in nonlinear elastic plates". In: The Journal of the Acoustical Society of America 127.4, pp. 2141-2152.

Naugolnykh, K. and L. Ostrovsky (1998). Nonlinear Wave Processes in Acoustics. Cambridge Texts in Applied Mathematics. Cambridge University Press.

Nistor, C, C Cojocaru, Yu Loiko, J Trull, R Herrero, and K Staliunas (2008). "Second-harmonic generation of narrow beams in subdiffractive photonic crystals". In: Physical Review A 78.5, p. 053818.

Nistor, Cristian, Crina Cojocaru, Jose Trull, and Kestutis Staliunas (2010). "Broad angle phase matching in subdiffractive photonic crystals". In: Optics Communications 283.18, pp. 35333535 .

Pérez-Arjona, Isabel, Víctor J Sánchez-Morcillo, Javier Redondo, Víctor Espinosa, and Kestutis Staliunas (2007). "Theoretical prediction of the nondiffractive propagation of sonic waves through periodic acoustic media". In: Physical Review B 75.1, p. 014304.

Soliveres, Ester, Víctor Espinosa, Isabel Pérez-Arjona, Víctor J Sánchez-Morcillo, and Kestutis Staliunas (2009). "Self collimation of ultrasound in a three-dimensional sonic crystal". In: Applied Physics Letters 94.16, p. 164101.

Soliveres, Ester, Isabel Pérez-Arjona, R Picó, V Espinosa, Víctor José Sánchez-Morcillo, and K Staliunas (2011). "Simultaneous self-collimation of fundamental and second-harmonic in sonic crystals". In: Applied Physics Letters 99.15, p. 151905.

Stegeman, George I and Mordechai Segev (1999). "Optical spatial solitons and their interactions: universality and diversity". In: Science 286.5444, pp. 1518-1523.

Zheng, Yongping, R Gr Maev, and I Yu Solodov (2000). "Review/Sythèse Nonlinear acoustic applications for material characterization: A review". In: Canadian Journal of Physics 77.12, pp. 927-967.

\section{Publications}

The contents of this chapter have been presented in the following publications:

\section{Journal papers}

- Hamham, E.M., Jiménez, N., Picó, R., Sánchez-Morcillo, V., García-Raffi, L., Staliunas, K. "Nonlinear self-collimated sound beams in sonic crystals". Phys. Rev. B. Accepted for publication May (2015)

\section{Conference Proceedings}

- El-M. Hamham, N. Jiménez, R. Picó, L. M. García-Raffi, V. J. Sánchez-Morcillo, K. Staliunas. "Efficient Second Harmonic Generation of Narrow Beams in 2D Sonic Crystals". PHONONICS 2015: 3nd International Conference on Phononic Crystals/Metamaterials, Phonon Transport and Phonon Coupling. Paris, France. May 31-June 5 (2015) 
- Mehrem, A., Hamham, El-M., Jiménez, N., Cebrecos, A., Pico, R.; SanchezMorcillo, V. and García-Raffi, L. M. "Nonlinear Acoustic Waves Periodic Media" 7th Forum Acusticum 2014 Krakow, Poland. September 7 (2014) 
Chapter 3. Nonlinear self-collimated sound beams in periodic media. 


\title{
Chapter 4
}

\section{Acoustic Bessel Beams generated by Axisymmetric Gratings}

\begin{abstract}
We report Bessel-like beam formation of acoustic waves by means of an axisymmetric grating of rigid tori. The results show that the generated beam pattern is similar to that of Bessel beams, characterized by elongated non-diffracting focal spots. A multiple foci structure is observed, due to the finite size of the lens. The dependence of the focal distance on the frequency is also discussed, on the basis of an extended grating theory. Experimental validation of acoustic Bessellike beam formation is also reported for sound waves. The results can be generalized to wave beams of different nature, as optical or matter waves.
\end{abstract}




\subsection{Introduction}

Bessel beams, originally proposed in optics (Durnin, 1987; Maddox, 1987), are now at the basis of many applications due to their unusual propagation properties (Duocastella et al., 2012; Fahrbach et al., 2011; Chu, 2012; Matsupka et al., 2006; Hsu et al., 1989a; Katchadjian et al., 2010). The most celebrated property of a Bessel beam is that, in the ideal case, the field propagates invariantly, i.e. without any diffracting broadening, in contrast to the other canonical case, the Gaussian beam, where the beam experiences diffractive broadening in free space propagation. As a consequence, the field pattern in a Bessel beam possesses an infinitely extended focal line.

Strictly speaking, Bessel beam is a solution of the wave equation in the form of a monochromatic wave with a transverse profile given by a Bessel function of the first kind, which by definition presents an infinite spatial extension. This ideal case cannot be realized in practice (in the same way as ideal, infinitely extended plane waves cannot exist). However, approximate or imperfect Bessel beams of finite transverse extent can be excited by different means, displaying not an infinite but an extremely elongated focal line.

In optics, Bessel-like beams are usually formed by focusing a Gaussian beam by an axicon (McLeod, 1954), a transparent refractive element of conical shape, as shown in Fig. 4.1 (a). The beam in propagation through the axicon acquires linearly tilted (conical) wave-fronts, which results in an elongated focus behind the axicon. As the axicon is not infinitely extended in transverse space, the resulting Bessel beam is not perfect, and displays a focal line of finite extent. Optical Bessel beams have been also obtained by acoustic gradient index lenses (McLeod et al., 2006). In electromagnetism, Bessel-like beams have been generated from a subwavelength aperture by adding a metallic circular grating structure in front of the aperture (Li et al., 2009). Such imperfect Bessel beams find multiple applications, e.g. in optics for laser inscription of patterns deep into transparent materials, or for etching of deep narrow holes in laser manufacturing of opaque materials, among others (Duocastella et al., 2012; Matsupka et al., 2006; Fahrbach et al., 2011).

In acoustics, Bessel beams of sound waves were also reported (Hsu et al., 1989a; Lu et al., 1990a), however are still not so broadly applied as in optics, which is perhaps related with the lack of convenient techniques of formation of such kind of acoustic waves. Acoustic Bessel beams have been excited using acoustical axicons (Katchadjian et al., 2010), in analogy to the optical case. However the most convenient way to form acoustic Bessel beams is by using annular transducer arrays (Masuyama et al., 1999). Related theoretical studies include the scattering of Bessel beams by spheres (Marston, 2007b), nondiffracting bulk-acoustic $X$ waves (Salo et al., 1999) or non linearly generated Bessel beams of higher harmonics (Ding et al., 2000a; Cunningham et al., 2000a). More recently, ultrathin planar acoustic metasurfaces by coiling up space have been proposed to generate acoustic Bessel beams (Li et al., 2012). 


\subsection{Generation of Zero th-order Bessel beams by axisymmetric gratings}

The present work proposes and demonstrates a technique for acoustic Bessel-like beam formation using a structure made of concentric tori of circular section, called here rings for simplicity. We show that, under specific conditions, part of a diffracted wave collimates, producing an elongated focus. Moreover, different diffraction orders can result in different elongated foci, as illustrated in Fig. 4.1(b). In the present work we demonstrate the feasibility of this idea by analytical estimations, numerical simulations and experiments. A simple analytical model based on an approach of axisymmetric diffraction gratings is used to estimate the focal positions and the extent of the focal line. Numerical calculations (using finite difference time domain (FDTD) techniques (Taflove et al., 2000)) of acoustic waves propagating through such axisymmetric gratings were used to observe the complete acoustic field. Finally, the experimental verification of Bessel-like beam formation by an axisymmetric grating is reported.

Each element of the concentric ring structure is characterized by two parameters, $(i)$ the toroidal radius, $r_{m}$ and $(i i)$ the radius of the tube (circular section), $R_{m}$. The rings in the axisymmetric grating have increasing toroidal radii as $r_{m}=m a$, where $a$ is the separation between rings and $m$ is an element index, as shown in Fig. 4.1 (b). The continuity of the transversal component of the wave vector at the interface between the free propagation medium and a linear diffraction grating with periodicity $a$, results in diffraction of normal incident plane waves at diffraction angles given by

$$
\sin \beta_{n}=n \lambda / a
$$

where $\lambda$ is the wavelength and $n$ is the diffraction order. It is worth noting that approximately half part of the diffracted radiation converges towards the symmetry axis and the other half diverge. Resulting from the converging radiation, as it follows from simple trigonometry considerations, each ring with major radius $r_{m}$ is mapped to a particular distance along the symmetry axis, given by

$$
f_{n}\left(r_{m}\right)=f_{n m}=\frac{r_{m} a}{n \lambda} \sqrt{1-\left(\frac{n \lambda}{a}\right)^{2}} .
$$

If the system of concentric rings extends from $r_{1}$ (toroidal radius of inner ring) to $r_{M}$ (radius of outer ring) in the transverse plane, the focal line for the $n$-th diffraction order will extend approximately from $f_{n 1}$ to $f_{n M}$. In a limiting case of infinitely extended ring structure $\left(r_{1}=0, r_{M}=\infty\right)$ Eq. (4.2) predicts an infinitely extended focus, similarly to that of an ideal Bessel beam.

First, we perform numerical simulations in order to explore the character of the elongated focal line (also different foci) due to the axisymmetric diffraction. For 
a)

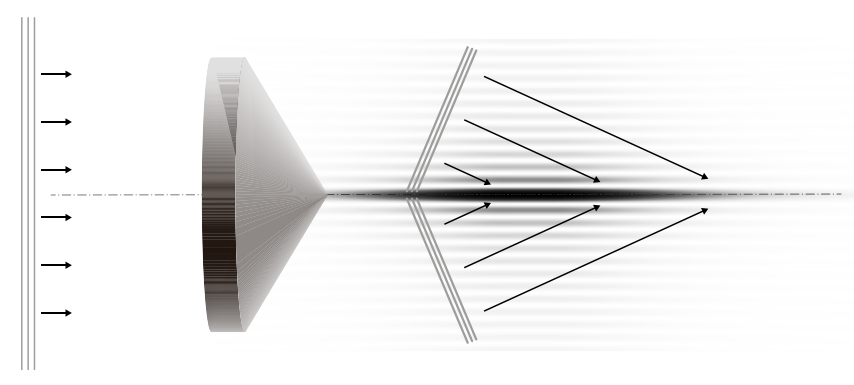

b)

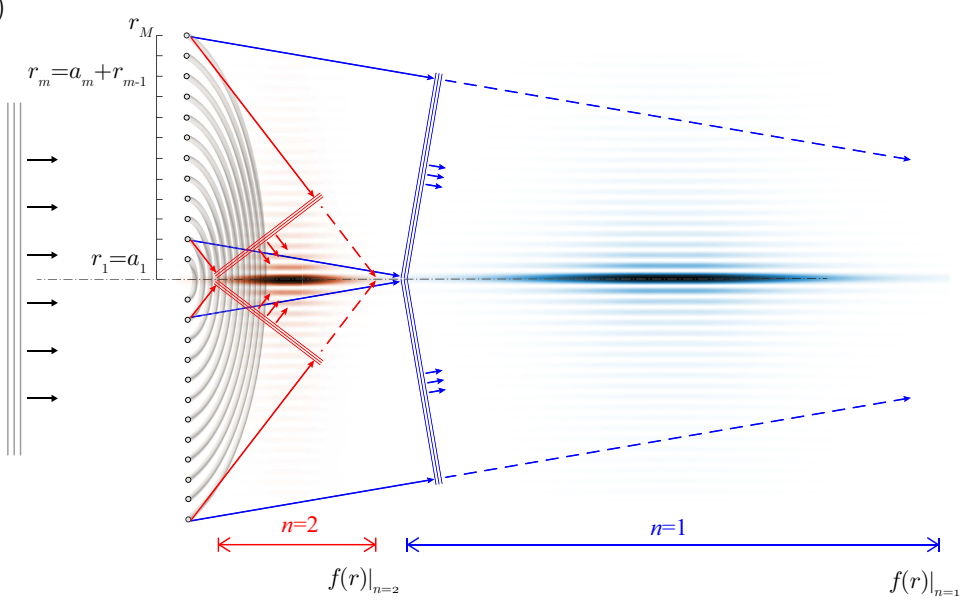

Figure 4.1: Illustration of the formation of Bessel beam by an axicon resulting in imperfect Bessel beam showing a focus-line of finite extent; (b) Illustration of the formation of Bessel-like beams by a plane of concentric rings, where converging diffracted waves result in two elongated foci.

that, we use a structure composed by a set of 50 concentric rings with constant minor radius $R=a / 3$, irradiated by a plane wave. We notice that this structure is much larger than that used in experiments (detailed below), therefore, the Bessel-like features of focal line are more pronounced. For the numerical simulations we calculate the wave propagation using the FDTD technique considering an incident plane wave of unity amplitude, $\left|p_{0}\right|=1 \mathrm{~Pa}$. Figures 4.2 (a) and 4.2 (b) represent the frequency dependence of the on-axis amplitude and radial far field amplitude respectively. Color map represents the amplitude of the acoustic field, $|p|$, and continuous lines in Fig. 4.2 (a) show the predictions from Eq. (4.2) for the cases $m=1$ and $m=50$ for the first three diffraction orders, $n=1,2,3$. The focal spots appear at normalized frequencies $a / \lambda=n$, so the diffraction an- 

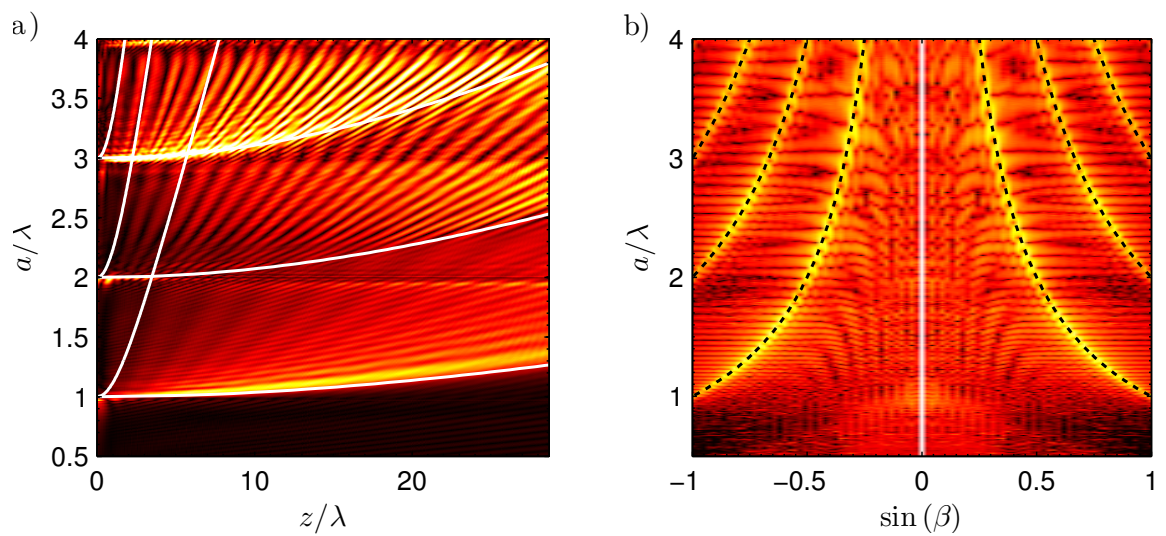

Figure 4.2: Map of acoustic pressure along the symmetry axis (horizontal axis) for varying frequency (vertical axis). Analytical estimations from Eq. (4.2) of focal line extent from $f_{n 1}$ to $f_{n M}$ are shown by solid white lines.

gles at these frequencies corresponds to $\beta_{n}=\pi / 2\left(\sin \beta_{n}=1\right)$. With increasing frequency, as shown in Fig. 4.2 (a), foci elongate corresponding to analytical estimations by Eq. (4.2) (white lines in Fig. 4.2 (a)). The diffraction angle decreases with the increasing frequency. Concerning the angular far field distribution shown in Fig. 4.2 (b), excellent agreement between theory (Eq. 4.1) and numerical results are observed, where the characteristic rings appear in the far field pressure distribution. Different maxima appear due to different diffraction order, as a consequence of the focusing effect at near field due to the finite size of the structure, in agreement with Fig. 4.2 (a).

Next, we analyze the acoustic field behind the axisymmetric grating for a particular frequency corresponding to $a=1.033 \lambda$. We study here the case when only one focal line appears. Fig. 4.3 (a) shows the field distribution on axial cross-section. The focus is substantially long, which is a signature of a Bessel-like beam. The latter is proven and illustrated in Fig. 4.3 (b), where the transverse field profiles at the indicated distance behind the ring structure is plotted and compared with Bessel function.

Finally, Fig. 4.4 shows the intensity distribution along the symmetry axis for two different frequencies: important to note that the intensity increases with distance until a maximum focusing distance, where it drops. The scattered energy is proportional to the area between neighbouring rings. Henceforth, the acoustic intensity along the focus increases with a linear trend overlaid of stray oscillations from $f_{n 1}$ to $f_{n M}$, as shown in Fig. 4.4. These oscillations are mainly due to the finite size of the structure: the edges of the diffraction grating result in fringes. The linear dependence of acoustic intensity along the focus is however modified if the thickness of the rings depends on their radius (see the experiments below). Moreover, the transmission efficiency of the structures is high as shown 

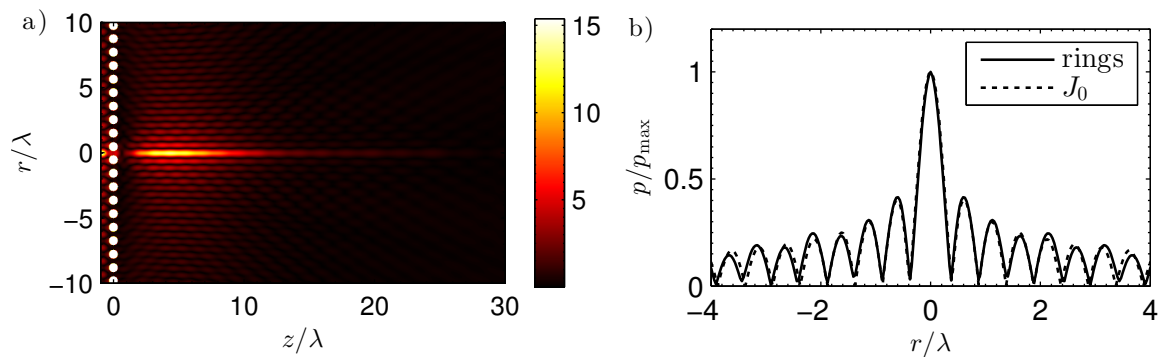

Figure 4.3: 2D pressure distribution for case of single focus $(a=1.03 \lambda)$ on a cross section along the symmetry axis. (i) Inset shows axial cross-section of the pressure field at a distance $z=5 \lambda$ (solid black) compared with Bessel beam (solid gray).

in Fig. 4.4, with a gain of amplitude $g=|p| /\left|p_{0}\right|=12.5$ for $a / \lambda=1.17$ (red line) and of $g=9.76$ for $a / \lambda=1.63$ (blue line). We also note, that although the focal lines associated with different diffraction orders partially overlap, the radiation due to higher order diffraction clearly dominates in the interference picture being the higher diffraction order presenting the biggest gain (see maximum values in Fig. $4.2(\mathrm{a}))$.

\subsection{Experimental validation}

For the experimental validation we designed a system composed by a set of 7 concentric rigid rings embedded in air. The rings are made of methyl methacry-

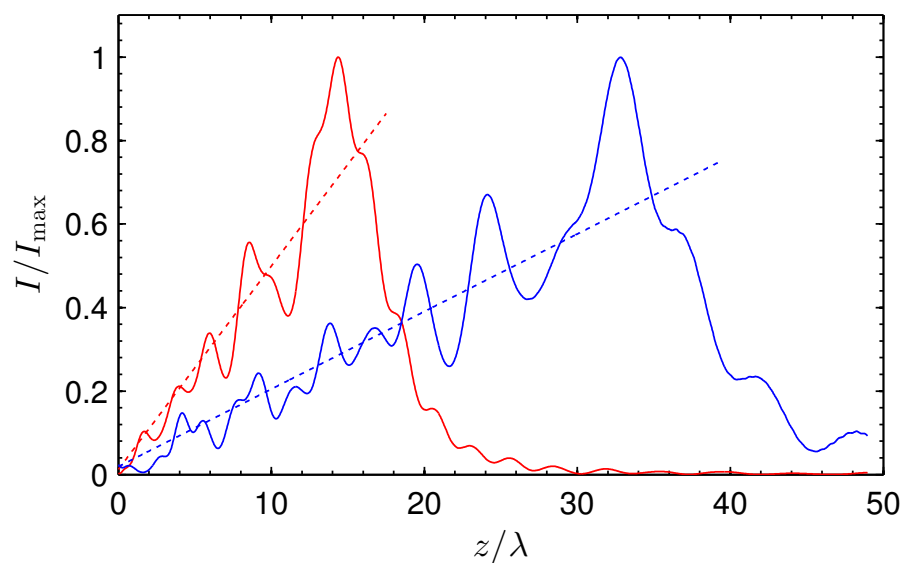

Figure 4.4: On-axis intensity for the cases $a / \lambda=1.17$ (red line) and $a / \lambda=1.63$ (blue line). Dashed line is an eye-guide to show the linear dependence. 
late (plexiglass), which acoustic impedance is much larger than that of the air $\left(Z_{\text {plexiglass }} / Z_{\text {air }} \sim 6000\right)$; therefore, they can be considered acoustically rigid. The variation of the toroidal radius in the structure is $r_{m}=m a$, where $a=4$ $\mathrm{cm}$. The minor radius now is a function of the major radius, $R\left(r_{m}\right)$, following a hyperbolic secant profile like in the axisymmetric gradient index lens analyzed in Ref. (Romero-García et al., 2014). This profile slightly modifies the linear dependence of the intensity along the focus because of the changing of the area between the different tori. The hyperbolic secant used here increases the intensity of the components at the end of the foci because the area between the external tori is bigger than the area between the inner tori. In the same way, if the profile is inverted, i.e. the area is bigger between the inner tori, then the near components are increased. Therefore, as we want to show the elongated focus produced by these structures, we use the hyperbolic secant profile with decreasing $R$ with $r_{m}$, in order to increase the far components. This profile produces clearer and better reproducible diffraction pattern with smaller number of concentric rings. All measurements were performed in anechoic chamber to avoid unwanted reflections. The source was a loudspeaker radiating a sound wave with a white noise spectrum placed at $1.5 \mathrm{~m}$ in front of the plane of concentric rings, a sufficient distance in order to ensure that a nearly plane wave radiates the structure at the frequencies of interest. A movable microphone located behind the structure recorded the transmission spectrum. The experimental set-up is shown in Fig. 4.5 (a).

The quantitative study of Bessel-like beam formation is summarized in Figs. 4.5(b) and 4.5(c). Pressure color maps of Figs. 4.5(b) and 4.5(c) show the numerical and experimental frequency dependence of the on-axis amplitude produced by the used structure. In the experiment, we were able to measure frequencies until $20 \mathrm{kHz}\left(\frac{a}{\lambda}<2.3\right)$, i.e. we could achieve Bessel beam formation by the first diffraction order only. The expected dependence of the focal distance on frequency (compare with Fig. 4.4) is also evident in both numerical and experimental plots.

We have also measured field cross-sections: an horizontal plane containing the symmetry axis (see Figs. 4.6 (a-d)) as well as several axial cross-sections (see Figs. $4.6(\mathrm{e}-\mathrm{j}))$. We focused on two particular frequencies, $10 \mathrm{kHz}(a / \lambda=1.17)$ and $16 \mathrm{kHz}(a / \lambda=1.88)$ (indicated by white dashed-dotted horizontal lines in Figs. 4.5 (b) and 4.5(c)). For the analyzed structure the frequency of $10 \mathrm{kHz}$ is close to the condition $a / \lambda=1$, consequently, the first diffraction spot appears just behind the structure (see white dashed line in Fig. 4.5(c) as a reference). However, the frequency of $16 \mathrm{kHz}$ produces the elongated focus or Bessel-like beam. These two phenomena are both numerically and experimentally shown in Figs. 4.6(a)-(c) and Figs. 4.6(b)-(d) with good agreement.

In order to see the symmetry quality of the beams produced experimentally, we have also measured the axial cross-sections of the pressure field at different $z$-positions for above discussed cases of $10 \mathrm{kHz}$ and $16 \mathrm{kHz}$ (Fig. 4.6(e)-(j)). In both cases $(i)$ the diffracted pattern is highly axisymmetric and ( $i i)$ the diffracting broadening of the central beam, along the extended focus, is almost negligible. 
a)

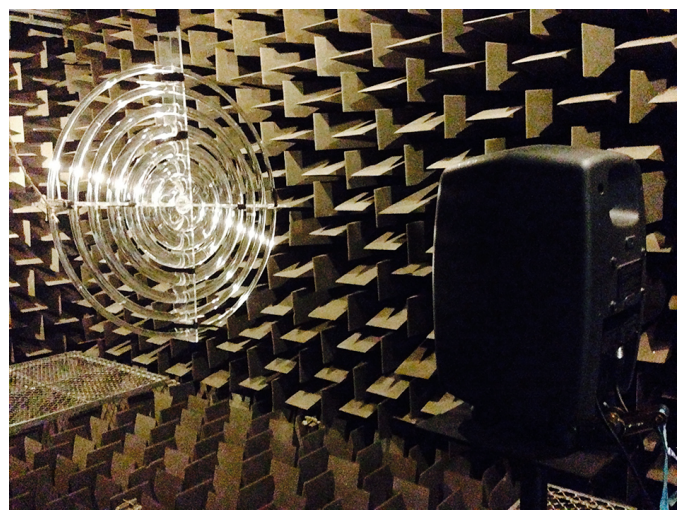

b)

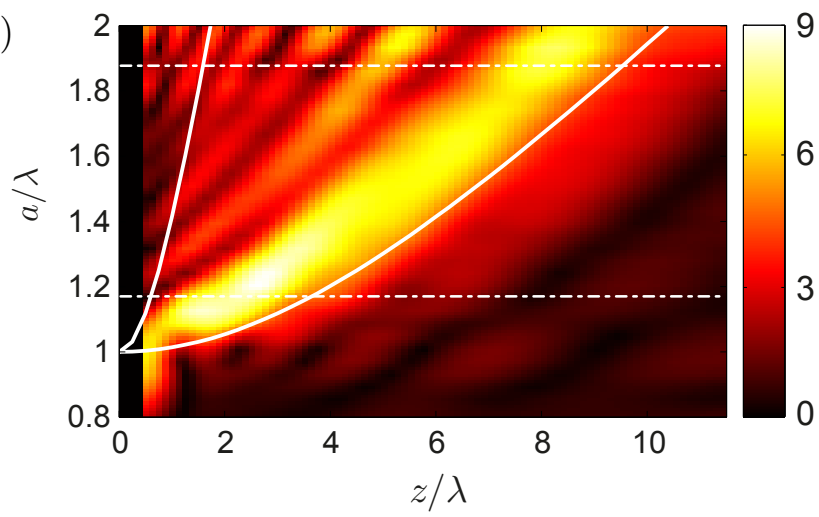

c)

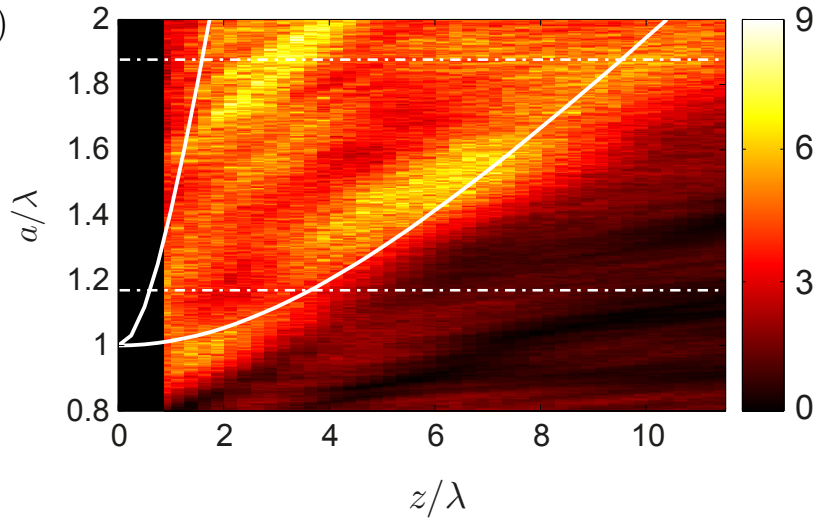

Figure 4.5: (Color online) (a) Experimental set-up. (b) Numerically calculated and (c) experimentally measured maps of on-axis amplitude dependence for varying frequency (vertical). Solid white lines indicate the extend of the focal line from analytical estimations using Eq. (4.2) for $f_{1}\left(r_{1}\right)$ and $f_{1}\left(r_{7}\right)$. Dashed white lines represent the frequencies shown in Fig. 4.6 

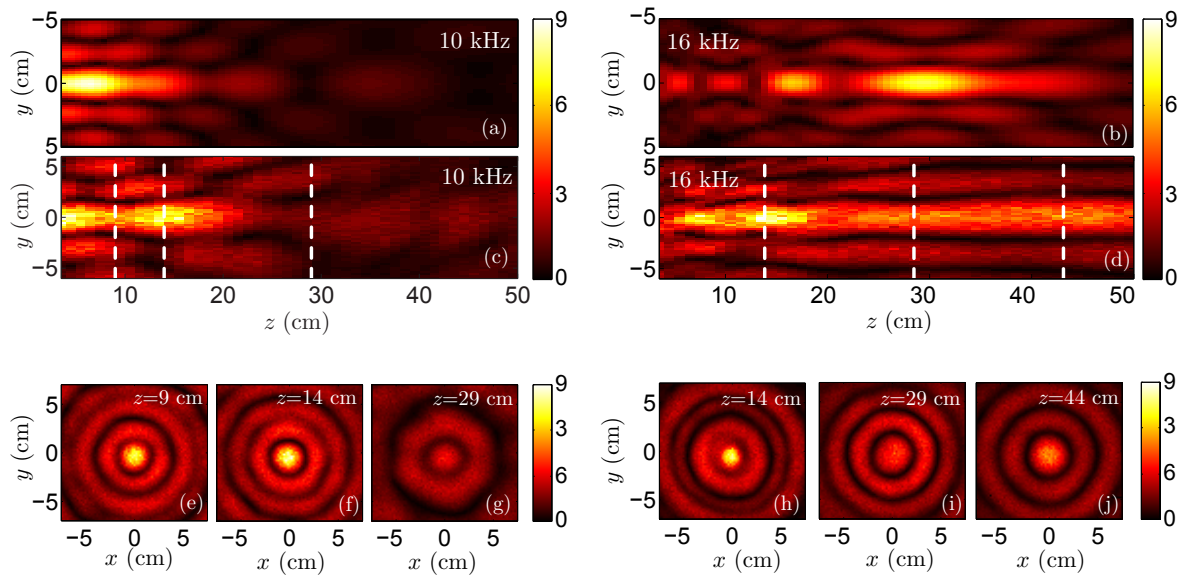

Figure 4.6: (Color online) (a-d) Amplitude distributions in planes along the symmetry axis $r(z, x)$ obtained by numerical simulations $(\mathrm{a}, \mathrm{b})$ and experimental $(\mathrm{c}, \mathrm{d})$ measurements for two different frequencies, showing the formation of elongated foci. Experimental transversal amplitude cross-sections $(x, y)$ at different distances behind the ring structure, showing the formation of the Bessel-like beam. (e-g) correspond to the experimental transversal planes at $10 \mathrm{kHz}$ marked with dashed lines in (c). (h-j) correspond to the experimental transversal planes at 16 $\mathrm{kHz}$ marked with dashed lines in $(\mathrm{d})$.

The transversal profiles in Figs. 4.6(e)-(j) also illustrate the typical shape of the truncated Bessel function. It is simple to predict the tendencies of the amplitude distribution along the focus for such a small number of rings. The longitudinal shape along the elongated focus seems to be not linearly increasing/sharply dropping, but correspondingly smoother. This is due to the small number of rings in the structure; also due to the difference in thickness of the rings.

\subsection{Conclusions}

Concluding, we have demonstrated the principle of Bessel-like focusing in a system of concentric rigid rings. Although the size of experimental system was reduced (to 7 rings), the main properties of the Bessel beam formation were demonstrated: the elongated foci along the symmetry axis, the Bessel-like distributions of the field in axial cross-sections, and the expected dependence of the focal distance with the frequency. The Bessel beam formation can be substantially improved, as follows from numerical calculations with larger number of rings.

For technical applications, the Bessel beam formation can be modified and improved, according to specific needs, by some means as for example: $(i)$ the use of not toroidal rings, but rather the ones with more sophisticated shapes, which would favor the converging part of the diffracted wave (i.e. to convert into Bessel 
beams nearly all radiation (note that the rings can converge as maximum the half of initial radiation); (ii) the use of a ring structure of adequate thickness which would allow tailoring the longitudinal profile of the focus, according to the requirements; (iii) modifying the radii of the rings (making the radii incrementing not linearly) which is another parameter allowing the engineering of the focusing, and allowing to optimize the focal structure; finally $(i v)$ by using multiple layers of rings at equidistant separations along the symmetry axis one could not only enhance the effect, but also introduce another possibility of tailoring the focal spot, as the interference from different planes will come into play.

\section{References}

Chu, X. (2012). "Analytical study on the self-healing property of Bessel beam". In: Eur. Phys. J. D 66, p. 259.

Cunningham, Kevin B. and Mark F. Hamilton (2000a). "Bessel beams of finite amplitude in absorbing fluids". In: J. Acous. Soc. Am. 108, p. 519.

Ding, D. and J. y. Lu (2000a). "Higher-order harmonics of limited diffraction Bessel beams". In: J. Acous. Soc. Am. 107, p. 1212.

Duocastella, M. and C.B. Arnold (2012). "Bessel and annular beams for material processing". In: Laser Photonics Rev. 6, p. 607.

Durnin, J. (1987). "Exact solutions for nondiffracting beams. I. The scalar theory". In: J. Opt. Soc. Am. A 4, p. 651.

Fahrbach, F.O. and A. Rohrbach (2011). "Propagation stability of self-reconstructing Bessel beams enables contrast-enhanced imaging in thick media". In: Nat. Commun. 3, p. 632.

Hsu, D.K., F.J. Margetan, and D. O. Thompson (1989a). "Bessel beam ultrasonic transducer: fabrication method and experimental results". In: Appl. Phys. Lett. 55, p. 2066.

Katchadjian, P., C. Desimone, and A.D. Garcia (2010). "Application of Axicon Lenses in Ultrasonic Techniques". In: AIP Conf. Proc. 1211, p. 1043.

Li, Yong, Bin Liang, Zhong ming Gu, Xin ye Zou, and Jian chun Cheng (2012). "-”. In: Sci. Rep. 3, p. 2546.

Li, Zhaofeng, Kamil Boratay Alici, Humeyra Caglayan, and Ekmel Ozbay (2009). "Generation of an Axially Asymmetric Bessel-Like Beam from a Metallic Subwavelength Aperture". In: Phys. Rev. Lett. 102, p. 143901.

Lu, J. and F.J. Greenleaf (1990a). "Ultrasonic nondiffracting transducer for medical imaging". In: IEEE Trans. Ultrason. Ferroelec. Freq. Contr. 37, p. 438.

Maddox, J. (1987). "Making Light Spots travel further". In: Nature 327, p. 183.

Marston, Philip L. (2007b). "Scattering of a Bessel beam by a sphere". In: J. Acous. Soc. Am. 121, p. 753.

Masuyama, Hiroyuki, Tomoki Yokoyama, Keinosuke Nagai, and Koichi Mizutani (1999). "Generation of Bessel Beam from Equiamplitude-Driven Annular Transducer Array Consisting of a Few Elements". In: Jpn. J. Appl. Phys. 38, p. 3080.

Matsupka, Y., Y. Kizuka, and T. Inoue (2006). "The characteristics of laser microdrilling using a Bessel beam". In: Appl. Phys. A-Mater. 84, p. 423.

McLeod, Euan, Adam B. Hopkins, and Craig B. Arnold (2006). "Multiscale Bessel beams generated by a tunable acoustic gradient index of refraction lens". In: Opt. Lett. 31, p. 3155.

McLeod, J. H. (1954). "The axicon: A new type of optical element". In: J. Opt. Soc. Am. 44, p. 592 .

Romero-García, V., A. Cebrecos, R. Picó, V. Sánchez-Morcillo, L.M. Garcia-Raffi, and J.V. Sánchez-Pérez. (2014). "Wave focusing using symmetry matching in axisymmetric acoustic gradient index lenses". In: App. Phys. Lett. 103, p. 264106. 
Salo, J., J. Fagerholm, A. T. Friberg, and M. M. Salomaa (1999). "Nondiffracting Bulk-Acoustic X waves in Crystals". In: Phys. Rev. Lett. 83, p. 1171.

Taflove, A. and S.C. Hagness (2000). Computational Electrodynamics: The Finite-Difference Time-Domain Method. Artech House, Boston.

\section{Publications}

The contents of this chapter have been presented in the following publications:

\section{Journal papers}

- Jimenez, N.; Romero-García, V.; Picó, R.; Cebrecos, A.; Sanchez-Morcillo, V.; García-Raffi, L.; Sánchez-Pérez, J. V. and Staliunas, K. "Acoustic Bessel-like beam formation by an axisymmetric grating" Europhysics Letters, 106, 24005 (2014) 


\subsection{Introduction}

Acoustic focusing is an interdisciplinary topic covering a rich set of applied sciences that include nondestructive testing and acoustic microscopy techniques, detection of fish and undersea exploration, ultrasound and elasticity diagnostic imaging or ultrasound therapeutic techniques among others. Particularly interesting is the fact that focusing results in high intensities at a specific location and over only a small volume, triggering nonlinear acoustics effects and enhancing specific mechanical and thermal effects. Mechanical phenomena include cavitation, microstreaming, and acoustic radiation forces while the thermal effects include heat generation due to acoustic energy absorption.

These nonlinear effects are exploited by a broad range of applications. In ultrasound medical applications second harmonic generation is widely used in harmonic imaging techniques as in Tissue Harmonic Imaging (THI) (Averkiou et al., 1997), microbubble cavitation processes are commonly used for increase contrast in ultrasound imaging (Duck, 2002), to induce mechanical interaction with the surrounding tissues in ultrasound-enhanced drug delivery techniques (Bailey et al., 2003) or for noninvasive blood-brain barrier opening using focused ultrasound (Choi et al., 2007). Acoustic radiation forces are exploited to map media elasticity in ultrasound elastography techniques such as in Shear Wave Elasticity Imaging (SWEI) (Sarvazyan et al., 1998), Acoustic Radiation Force Impulse imaging (ARFI) (Nightingale et al., 2002), Harmonic Motion Imaging (HMI) (Konofagou et al., 2003) or in Supersonic Shear Imaging (SSI) (Bercoff et al., 2004). Moreover, at high intensities nonlinearity increase energy deposition rate, that is exploited in High Intensity Focused Ultrasound (HIFU) for enhancing thermal ablation (Bailey et al., 2003). Finally, in the case of Shock Wave Lithotripsy (SWL) acoustic pulsed-shock waves are focused to produce high-amplitude localized shear stresses and induce calculus fragmentation, as widely used for kidney stone treatments (Bailey et al., 2003). In all of these techniques, the design of devices to easily manage the focusing properties and the enhancement of the focal gain is of pivotal importance.

Acoustic focusing can be achieved by arrays of transducers where the beam focusing and steering can be controlled actively, where its focusing performance is limited by the number of active channels. It is attractive the design of passive acoustic focusing devices in order to reduce costs.

Generally, there are four main different procedures to generate strongly focused acoustic field by passive devices: by optimizing the geometry of the transducer, by using refraction lenses, by using reflective systems or by using diffraction gratings. The most common approach is to use curved shaped radiation surfaces. These can be made by curved bowl single element piezoelectric transducers, but also from different arrangements of small plane transducers distributed over a curved surface and excited in-phase. Curved acoustic lenses made of different refraction 
index material are also common, as long the lens is also used as an impedance matching layer between the active element, and the surrounding media. However, the transmission efficiency of these lenses is strongly reduced because the contrast of refraction index between the lens and the surrounding media is remarkable. On the other hand, focusing can be achieved by curved reflectors, e.g. elliptic reflectors in SWL. Due to the low dispersion of the acoustic media, all these focusing devices based on refraction, and also based on reflection of curved surfaces, present the feature that focusing does not strongly depends on frequency and therefore are suitable for impulsive excitations. However, in these devices, in which the focal sport is limited by the diffraction limit (Rayleigh criterion), the focal size is larger than one wavelength, i.e. the focal resolution is bigger than the sub-wavelength regime.

The last group of passive devices to generate strongly ultrasound focusing is based on the diffraction grating. The diffraction of waves on flat grating plates have been proposed as acoustic analogues of the Fresnel Zone Plates (FZP) (Farnow et al., 1974; Sleva et al., 1994; Schindel et al., 1997; Wang et al., 2008). As in optics, acoustic Fresnel Zone Plates consist of alternating rigid (opaque) and open (transparent) annuli. The radius and width of the open annuli are specially designed to only transmit the waves that constructively interferes at focus, while the radiation that destructively interferes is blocked by the rigid annuli. There exist other approaches that exploit the diffraction over concentric ringed-shaped structures to achieve focalization. These include axisymmetric gratings based on Fresnel Zone Plates in which the area of the annuli is not equal for opaque and transparent zones (Clement et al., 2015) or acoustic Fresnel lenses that exploit resonances to enhance the transmission (Molerón et al., 2014).

It is worth noting here that the diffraction grating over equally spaced toroidal scatters, as those presented in Chapter 4, can generate Bessel beams at the axis (Jimenez et al., 2014). This approach is roughly equivalent to the Bessel beams created by annular piezoelectric annuli (Hsu et al., 1989b). A characteristic feature of Bessel beams is that the transverse beam-width can be reduced below the subwavelength limit. However, these beams present also high amplitude side-lobes. Thus, when used for imaging, these devices provides high spatial resolution but low signal-to-noise (S/N) ratios (Lu et al., 1990b).

As soon as high amplitudes are applied to these focusing devices, it is wellknown that the second harmonic field is generated mainly around the focal spot due to the local activation of the nonlinear response of the media. The focus of the second harmonic has reduced side-lobes as long the nonlinear processes are mainly activated at the focal spot (Duck, 2002). In the case of ideal Bessel beams, it has been observed a reduction of half intensity of the side-lobe amplitude for the second harmonic component (Ding et al., 1996), while its transverse profile is reduced to the half, i.e. exactly maintain its narrowness if normalized to the wavelength (Ding et al., 2000b). These effects have been also observed in more realistic configurations including absorption (Cunningham et al., 2000b) and limited 
a)

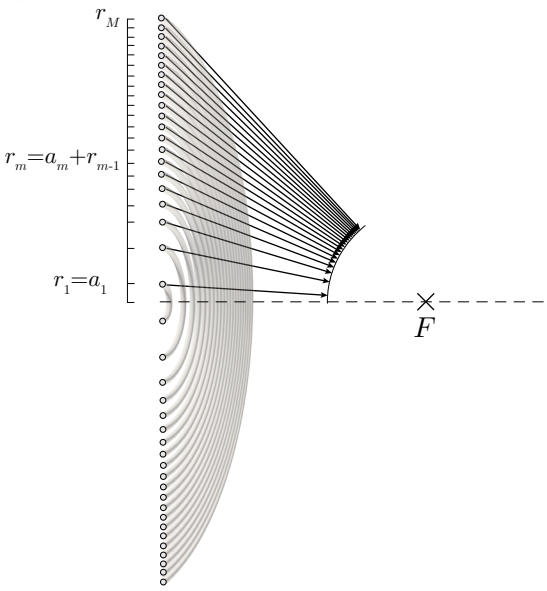

b)

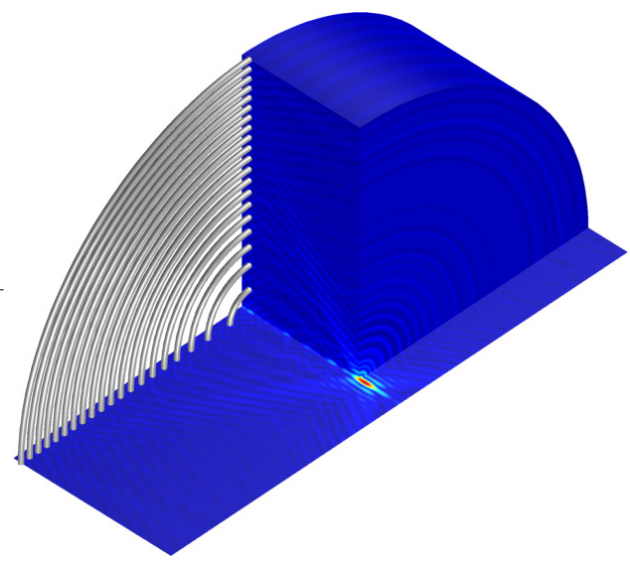

Figure 5.1: (a) Scheme of the passive focusing device composed of concentric rings. (b) Simulation example of focusing by this device.

aperture Bessel beams (Holm et al., 2010). Recently, the reduction of side-lobes of second harmonic has been observed for a focused Fresnel field, where the focalization, instead of passive lenses, has been achieved by active Fresnel beamforming in the context of second harmonic imaging (Nguyen et al., 2014).

In this Chapter, we present the acoustical focusing properties of an axisymmetric diffraction grating in both the linear and the nonlinear regime. We show that in the linear regime the system presents a focal spot in the sub-wavelength regime with high gain, around $30 \mathrm{~dB}$, with relative high amplitude side-lobes. Interestingly, nonlinear effects can be excited locally mainly at the sharp focal spot. Thus, in the nonlinear regime the high intensity at the focal spot activates the harmonic generation, producing a focus of the second harmonic with particular spatial features. Mainly, second harmonic beam-width is between sub-diffraction and sub-wavelength limits, but having strongly reduced lateral lobes.

We demonstrate these results by analytical estimations, numerical simulations and experiments. A simple analytical model based on an approach of axisymmetric diffraction gratings is used to estimate the focal positions and the extent of the focal spot. Numerical calculations (using finite-difference time domain (FDTD) techniques (Botteldooren, 1995)) of acoustic waves propagating through such axisymmetric gratings were used to observe the complete acoustic field in both the linear and the nonlinear regime. The experimental verification of the theoretical prediction is also reported showing very good agreement with the theory. 


\subsection{Axisymmetric diffraction grating design}

We consider $M$ concentric toroidal scatterers of minor radius $R$ and major radius $r_{m}$ with $m=1, \ldots, M$. A detailed scheme is presented in Fig. 5.1 (a). A plane wave radiates the axisymmetric grating and as a consequence it is scattered. We consider that $R$ is small in comparison to the wavelength of the incident wave, in such a way that each scatterer can be considered as a point source. Then, in order to have an optimal superposition at a given focal point, $F$, behind the axisymmetric grating, all the incoming waves should arrive precisely in phase to $F$. This suggests an ideal axisymmetric grating would consist of a series of infinitesimal toroidal scatterers located in a plane at radial distances given by the geometric focal law

$$
r_{m+1}^{2}=r_{m}^{2}+n \lambda\left(n \lambda+2 \sqrt{r_{m}^{2}+F^{2}}\right),
$$

where $n$ indicates the diffraction order of the axisymmetric diffraction grating. Equivalently, given the distribution of toroidal scatterers, $r_{m}$, the focal position can be determined by the following expression,

$$
F=\sqrt{\left(\frac{r_{m+1}^{2}-r_{m}^{2}}{2 n \lambda}-\frac{n \lambda}{2}\right)^{2}-r_{m}^{2}}
$$

Here, it is important to note the relation of Eq. (5.1) to the standard Fresnel Zone Plate. As explained above, FZP consist in alternating opaque and open annuli. The location of the edges for the $m$ zone is given by

$$
r_{m}^{\prime}=\sqrt{\left(\sqrt{r_{0}+F^{2}}+m \frac{\lambda}{2}\right)^{2}-F^{2}} .
$$

The location of the center of each annuli in the standard FZP is given by (Clement et al., 2015)

$$
r_{m}=\sqrt{\left(\sqrt{r_{0}+F^{2}}+m \lambda\right)^{2}-F^{2}}
$$

which gives same radial distribution of the toroidal rings as the succession obtained by diffraction grating in Eq. (5.1) if the proper first toroidal radius is matched for both lenses. On the other hand, in the work of Clement et al. (2015) they study a lens formed by slits of different thickness located at $r_{m}$, while in the present work we used rigid scattered located at $r_{m}$. Following Babinet's principle considerations, both approach can be considered equivalent.

Figure 5.1 (b) presents a simulation example of the focalization. As long the separation between the external rings is about one wavelength, it can be seen qualitatively the focusing of the system, the focal spot and the good spatial resolution 
(a)

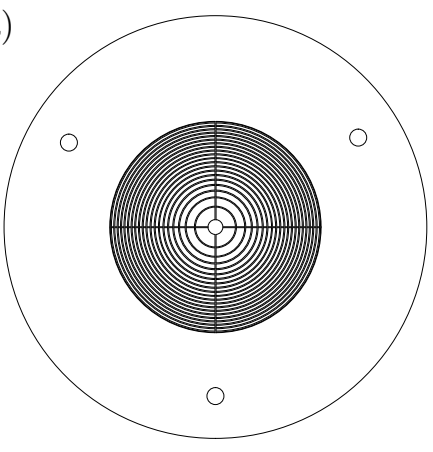

(c)

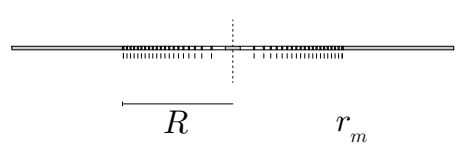

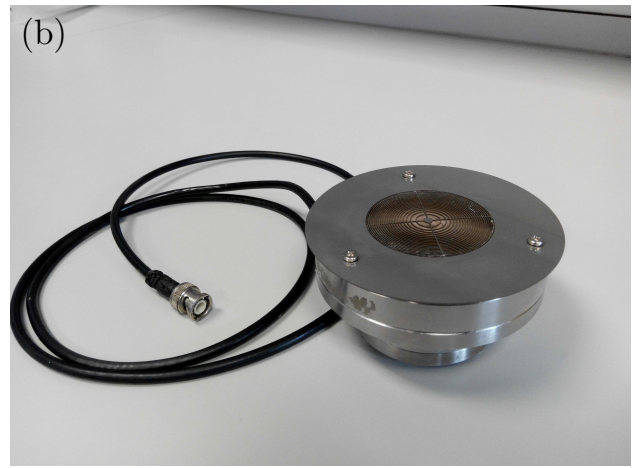

(d)

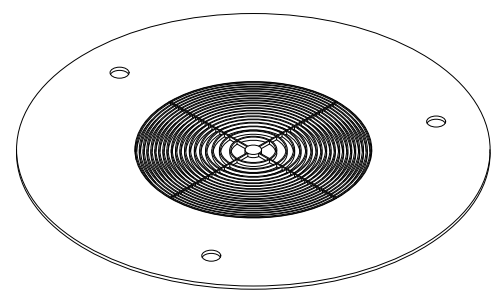

Figure 5.2: (a,c,d) Design of the axisymmetric grating plate. (b) Image of the constructed grating mounted over a plane piezoelectric transducer of $50 \mathrm{~mm}$ aperture.

compared to the wavelength. In the following sections a further analysis of the focusing quality of this design is presented.

\subsection{Small amplitude focusing}

An experiential test was performed in order to validate model results. Following the design Eq. (5.1), an axisymmetric grating was designed by using a focal distance of $F=15 \mathrm{~mm}$ and focal frequency $f_{0}=2.29 \mathrm{MHz}$. The passive system was fabricated by laser cutting over a stainless steel plate of $0.8 \mathrm{~mm}$ thickness. Figures 5.2 (a,c,d) show a scheme of the axisymmetric grating. The minor radius of the toroidal scatterers was $R=0.75 \mathrm{~mm}$ (solid parts). The number of rings was $M=21$ and the grating total radius was $r_{M}=25 \mathrm{~mm}$.

The axisymmetric grating lens was placed in front of an ultrasound plane transducer as shown in Fig. 5.2 (b). This source was formed by a plane single element piezoceramic crystal (PZ 26, Ferroperm Piezoceramics, Denmark) mounted in a custom designed steel housing with aperture $R=25 \mathrm{~mm}$, matching the area of the grating. The separation distance between the grating and the source plane was adjusted to $0.5 \mathrm{~mm}$. 
The assembled grating-transducer system was immersed in a degassed and distilled water tank of $350 \times 350 \times 350 \mathrm{~mm}^{3}$, the temperature of which during the measurements was constant at $26{ }^{\circ} \mathrm{C}$. The source was driven with a sinusoidal sweep burst ranging from frequency $f_{\min }=1 \mathrm{MHz}$ to $f_{\max }=3 \mathrm{MHz}$ and 50 cycles using a function generator (14 bits, $100 \mathrm{MS} / \mathrm{s}$, model PXI5412, National Instruments) and a linear RF amplifier (ENI 1040L, 400W, 55dB, ENI, Rochester, NY). The pressure waveforms were acquired with a HNR $500 \mu \mathrm{m}$ needle PVDF hydrophone (Onda Corp, CA), and a digitizer (64 MS/s, model PXI5620, National Instruments) were used. A three-axis micropositioning system (OWIS GmbH, Germany) was used to move the hydrophone in three orthogonal directions with an accuracy of $10 \mu \mathrm{m}$ and a National Instruments PXI-Technology controller NI8176 was used to control all the devices. The amplitude frequency response of the hydrophone was compensated in post-processing.

In addition to measurements, we perform numerical simulations in order to explore the linear character of the designed axisymmetric diffraction grating. For the numerical simulations we calculate the wave propagation using the FDTD technique considering an incident plane wave of unity amplitude, $p_{0}=1 \mathrm{~Pa}$. From
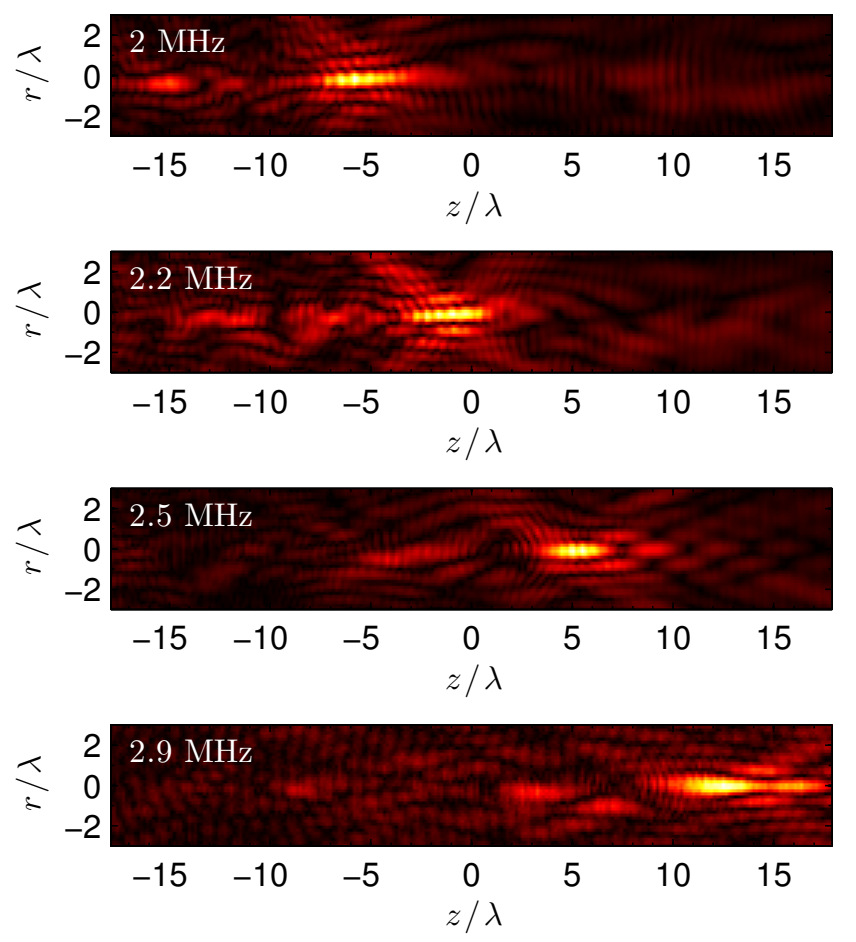

Figure 5.3: Field distribution for four different frequencies obtained by the experiment. It can be seen how the focal spot displaces in the axial location. 

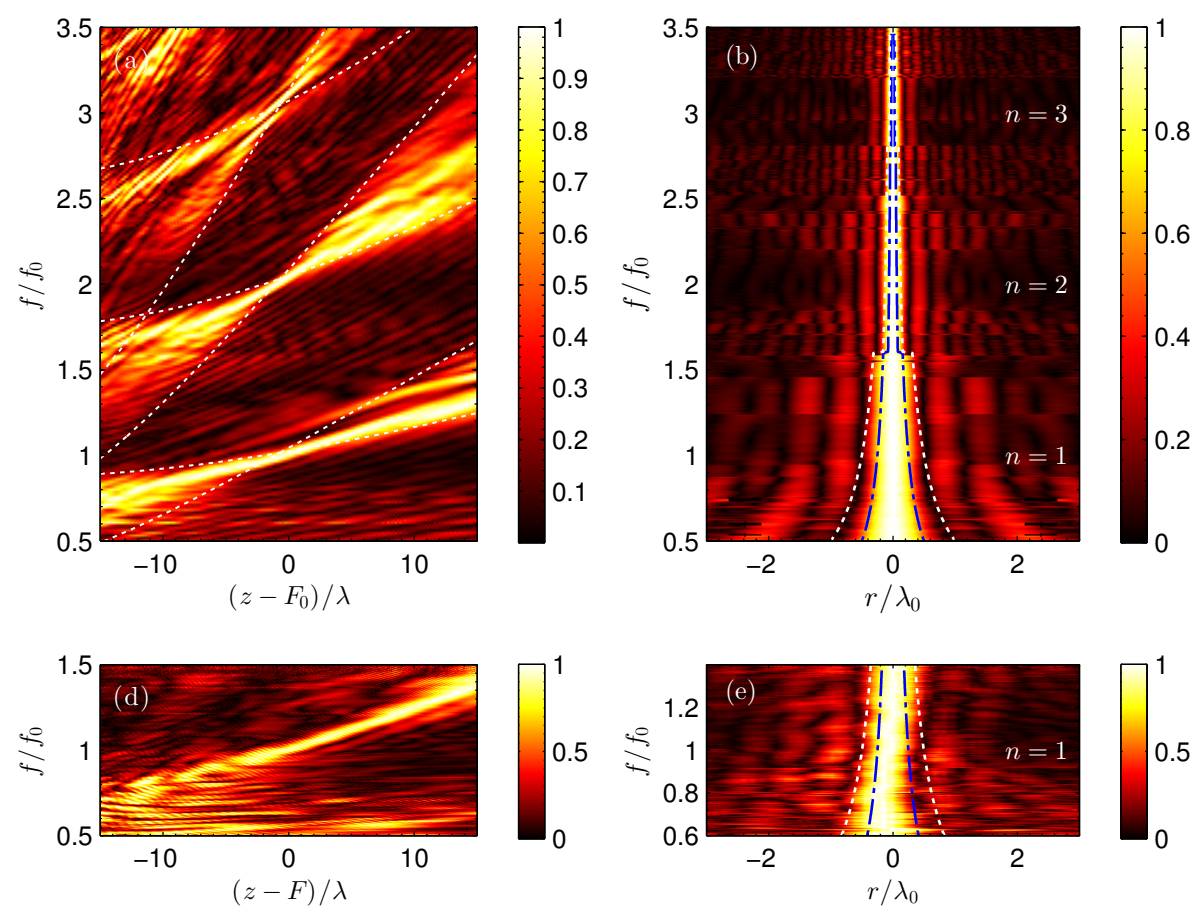

Figure 5.4: On axis field distribution as a function of frequency obtained by (a) simulation and (c) experiment. White lines indicates theoretical focus by using Eq. (5.2). Transverse field distribution measured at the focal spot axial distance as a function of frequency obtained by (b) simulations and (d) experiment. White lines mark the sub-wavelength and blue lines the sub-diffractive limit.

now we will show the results in normalized units. Distances will be normalized with respect to the wavelength, $\lambda$, and in the case of the distances in the symmetry axis will be referred to the focal distance $F$; frequencies will be normalized to the design frequency, $f_{0}$.

Figure 5.3 shows the experimental pressure distribution for different frequencies around the design frequency $f_{0}=2.229 \mathrm{MHz}$. It can be seen that a sharp focusing is observed for the design frequency. Furthermore, if frequency is modified the focal position displaces in the axial direction according to Eq. (5.2). In these cases, some focus aberration appears as long the design model holds exact only for the design frequency (Fig. 5.3 (b)). However, the focal spot maintains its shape and focusing properties for a reasonably bandwidth that we will study in the following sections.

The axial pressure distribution as a function of frequency is presented in Fig. 5.4 (a) for the simulations and Fig. 5.4 (c) for the experiment. Note that, due to the finite bandwidth of the ultrasonic source, in the experimental measurements 

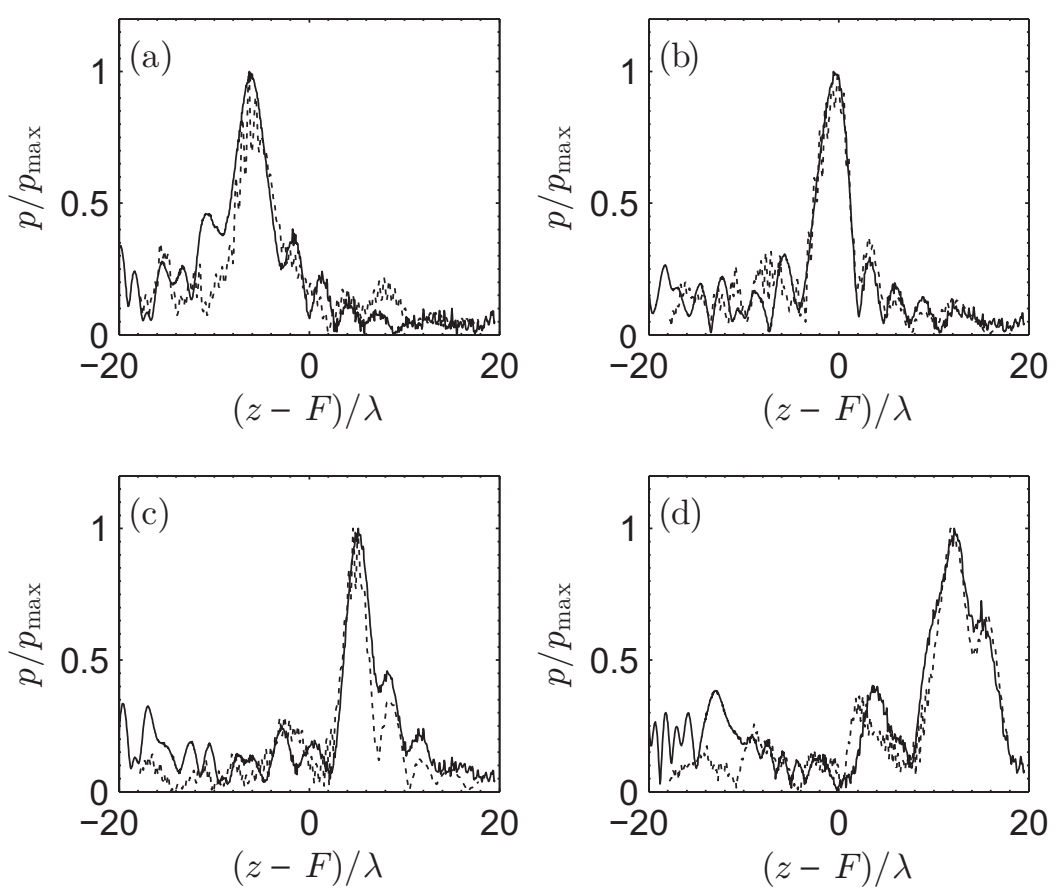

Figure 5.5: (a-d) On-axis pressure measured for the frequencies $f=2,2.2,2.5,2.9 \mathrm{MHz}$ respectively obtained by (doted lines) experiments and (continuous lines) simulations.

the characterization is restricted to a frequency range covering the first diffraction grating mode, i.e., $n=1$, while in the simulations the characterization is extended for frequencies covering up to $n=3$ diffraction orders. White dotted line represents the curves obtained from Eq. (5.2) for two different pairs of $m,(m=1$ and $m=21$ ), the crossing of this line represents the focal point. The agreement with the numerical simulation is very good. Moreover, one can see that although the lens is designed to focus waves in the point $F$ at frequency $f_{0}$, the lens produces a focal shift with frequency giving the possibility to manage the position of the focus with frequency. However, as we will see later, the gain at focal spot changes considerably with frequency. Finally, we notice that as we increase frequency in the simulations new focal spots are generated for the higher diffraction grating orders. according to Eq. (5.2).

Figures 5.4 (b, d) represents the radial distribution of the normalized acoustic field at the position of the focal spot: Fig. 5.4 (b) shows the simulation while Fig. 5.4 (d) shows experimental results. Figures 5.4 (a, c) represents the equivalent radial distribution to the results of Figs. 5.4 (b, d) respectively. We can observe a main lobe aligned with the center of the axisymmetric lens. Secondary smaller lobes also appears. We also represent the the theoretical Full Width at Half Maximum (FWHM) corresponding to (dotted white lines) sub-wavelength $(<\lambda)$ and 

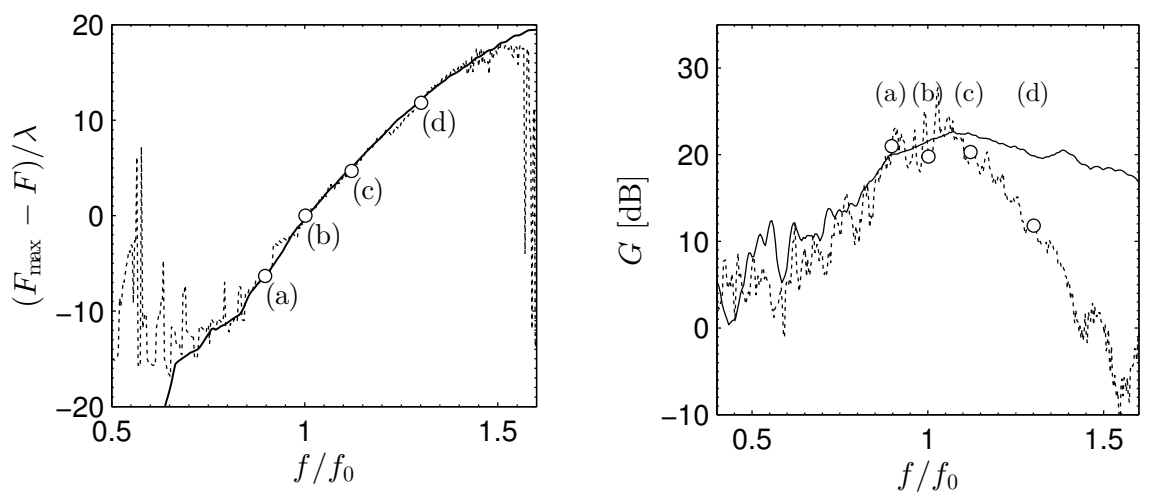

Figure 5.6: (Left) Location of the focal obtained by (dotted lines) experiments and (continuous line) simulations. (Right) Gain value obtained by (dotted lines) experiments and (continuous line) simulations. Markers (a-d) corresponds to the frequencies $f=2,2.22 .5,2.9 \mathrm{MHz}$ respectively.

(dashed blue lines) subdiffractive $(<\lambda / 2)$ limits respectively. At first glance, we can observe that in both, simulations and experiments, the width of the focal spot between the sub-diffractive and sub-wavelength regime, overcoming the diffraction limit. A deeper analysis is presenter later.

In order to see the agreement between experiments and theory, we have plotted in Fig. 5.5 the longitudinal cuts of the normalized acoustic pressure for the cases $f=2,2.2,2.5,2.9 \mathrm{MHz}$, in the subplots (a-c) respectively. Continuous line represents the numerical predictions while dashed lines show the experimental results. Good agreement is seen in all cases, where again it can be seen how the focal spot is shifted in axial direction for increasing frequencies. The shifting of the focal position can be seen in detail in Fig. 5.6 (left), where it is represented the location of the peak pressure for the (continuous line) simulations and (dashed lines) experiment. Excellent agreement is observed between them. The frequencies corresponding to $f=2,2.2,2.5,2.9 \mathrm{MHz}$, are marked as the points (a-c).

In order to evaluate the focusing capability of the axisymmetric grating we are discussing in this work, we have analyzed the gain of the lens evaluating the expression $G=20 \log \left(\left|p_{\max }\right| /\left|p_{0}\right|\right)[\mathrm{dB}]$, where $p_{\max }$ is the pressure at the focal point and $\left|p_{0}\right|$ is the incident wave pressure amplitude. Figure 5.6 (right) shows the gain numerically evaluated (continuous line) and experimentally measured (dotted line). For the design of this lens with $M=21$ scatterers, the gain is $20 \mathrm{~dB}$. We notice that this gain is constant around $f / f_{0}=1$, so the focusing properties of the axisymmetric lens is robust in terms of frequency, i.e., it is insensitive to possible errors in frequency around $f / f_{0}=1$.

Figure 5.7 (left) goes further and shows a general overview of the gain of a lens designed with the Eq. (5.1) as the number of toroidal scatterers, $M$ is increased. 

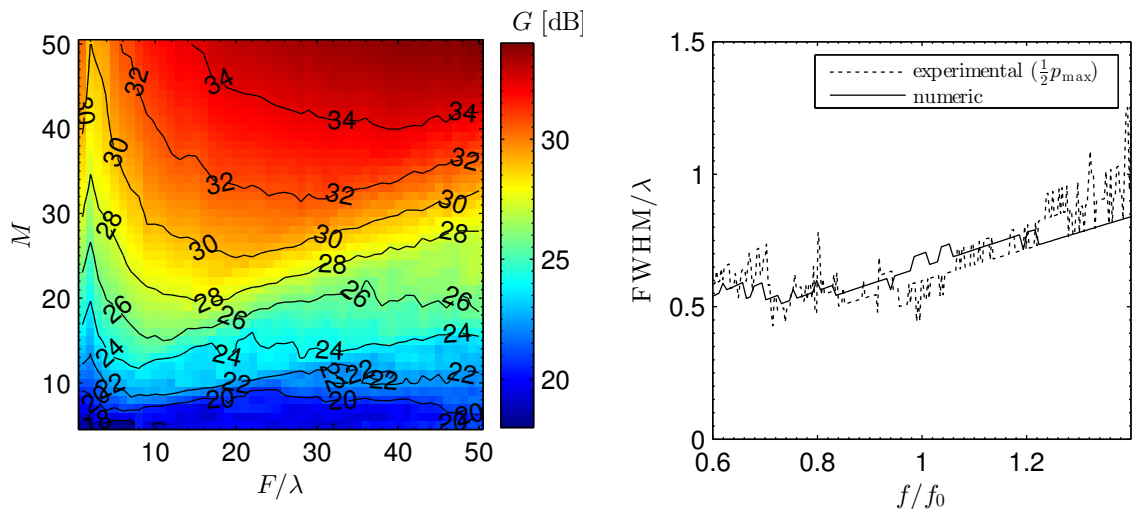

Figure 5.7: (Left) Simulated gain value measured at the design frequency for different gratings, as a function of the number of rings, $M$, and the design focal distance, $F$. (Right) Full Width at Half Maximum (FWHM) obtained by (dotted lines) experiments and (continuous line) simulations, measured at the half pressure.

By using double number of toroidal scatterers, $M=42$, the gain of the system can be increased to $34 \mathrm{~dB}$. We notice that this amount of gains are competitive with the usual systems used in high intensity focused ultrasound (Canney et al., 2008), but note we can achieve these values for very short distances. It is worth noting here that we can achieve these high gains at very short focal distances, of the order of wavelength. This property is almost impossible with with other common passive devices due to geometrical limitations. A further discussion is reported in the Conclusions section.

Finally, we have analyzed the Full Width at Half Maximum (FWHM) of the radial dimensions of the focal spot in the whole range of frequencies. Figure 5.7 (right) shows the (continuous line) numerical predictions and the (dotted line) experimental results for the FWHM. The axisymmetric lens presented in this work, shows a sub-wavelength full width at half maximum, i.e., FWHM $<\lambda$, for the whole range of analyzed frequencies. We also calculated the FWHM evaluated from the intensity data, i.e. the with of the spot at $p=p_{\max } / \sqrt{2}$. Obviously the width of the focal evaluated by intensity is thinner but the same concussions applies here: the central radial lobe corresponding to the focal spot is always between the sub-wavelength regime and the diffraction limit. This feature can be also observed by the white dashed lines in the maps shown in Figs. 5.4 (b, d). 


\subsection{Transition from linear-to-nonlinear focusing}

Now we start the study of the transition from the linear- to the nonlinear focusing of the axisymmetric grating by increasing the amplitude of the incident plane wave. The piezoelectric transducer is excited with an amplitude in which its behavior can be considered linear in the range of frequencies from $0.5 f / f_{0}$ to $1.5 f / f_{0}$. In this range of frequencies we reproduce the same acoustic pattern as in the linear (small amplitude excitation) regime showing the focal spot at $f / f_{0}=1$ and at $z=F$, as shown in Fig. 5.8 (a). However, now we can clearly see that the focal spot has contributions fro the double frequency $f / f_{0}=2$, i.e., at the second harmonic of the fundamental wave. The high amplitude around the spot generated at frequency $f / f_{0}=1$ activates the harmonic generation due to the nonlinearity of water, activating the contribution from $f / f_{0}=2$ due to the high amplitudes around the focal spot.

The focal spot generated by this nonlinear effect presents a spatial acoustic profile with different properties than the focal spot of the fundamental mode. Because of the local nonlinear generation of this spot, the longitudinal profile presents smaller lobes than in the case of the spot of the fundamental frequency. Figure 5.8 (b) shows the comparison between the experimental longitudinal profile for (continuous line) first harmonic and (dashed line) its corresponding second harmonic.

(a)

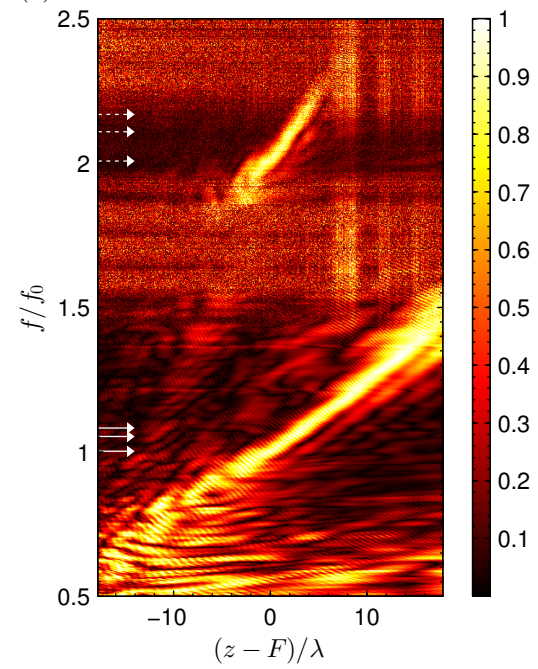

(b)
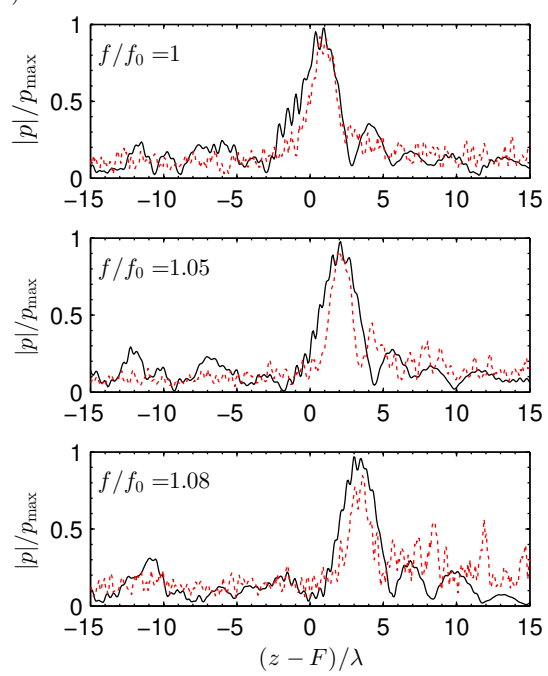

Figure 5.8: Weakly nonlinear experimental measurements of the grating. (a) On-axis field distribution as a function of the frequency. (b) On-axis field distribution for the second harmonic for three frequencies, marked by the arrows in (a). Experiments (Red dotted line) and simulation (continuous line). 


\subsection{High intensity regime}

We explore the high amplitude regime by using nonlinear simulation methods based also in FDTD technique, as used previously in Chapter 3. The simulations assumed perfectly rigid scatters over a cylindrical axisymmetric coordinate system, where the nonlinearity is only present on the bulk of the fluid, in this case we assumed water, with parameters: $\beta=1+B / 2 A=3.5$, density $\rho_{0}=1000$, sound speed $c_{0}=1500$.

An overview of the device focusing for increasing source amplitude is shown in Fig. 5.9. The source amplitude is expressed as the normalized quantity $F / x_{s}$, being $x_{s}=1 / k \varepsilon \beta$ the shock formation distance with $k$ the wavenumber, $\varepsilon$ the acoustic match number and $\beta$ the parameter of nonlinearity. In Fig. 5.9, we show the axial peak pressure distribution, i.e. the maximum of the nonlinear waveforms, $|p|^{+}$, and the minimum rarefaction distribution, $|p|^{-}$. On the other hand, in Fig. 5.10 it is shown the lateral pressure distribution corresponding also to $|p|^{+}$and $|p|^{-}$for the same range of excitation amplitudes.

The main nonlinear effects in the field distribution in high intensity focused ultrasound can be observed here. The effective gain of the system, the peak value corresponding to $p_{\max } / p_{0}$, is extraordinary increased from the linear value of $p / p_{0}=39(32 \mathrm{~dB})$, to $|p|^{+} / p_{0}=92(39 \mathrm{~dB})$ in the case of very strong nonlinearity. Thus, as peak pressure grows locally at the focus, the focal width stretches in both axial and lateral dimensions. In addition, the relative amplitude between the peak pressure and its lateral lobes is strongly increased.

In Figure 5.11 we illustrate the pressure distribution for 4 different source

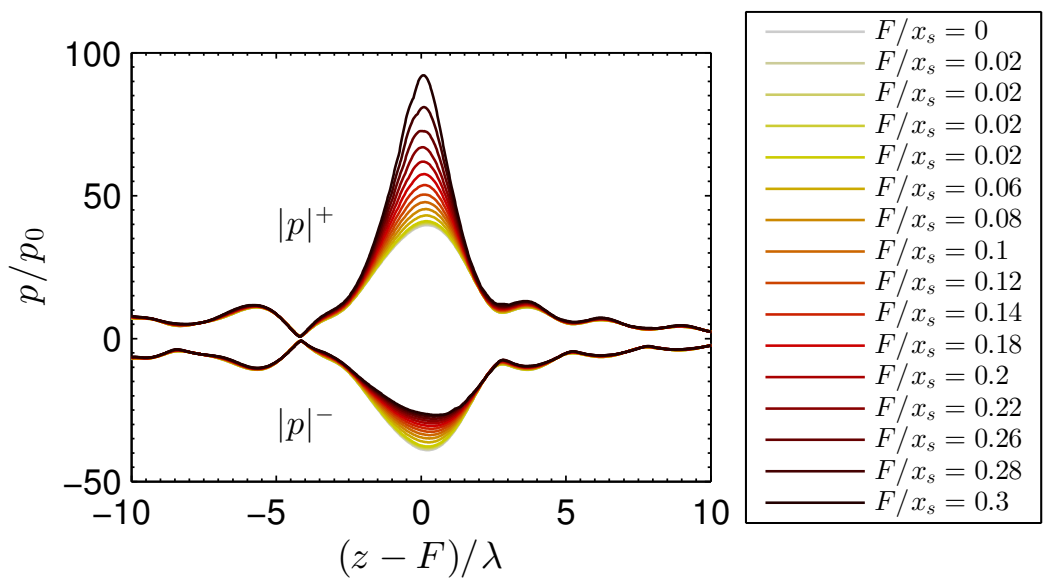

Figure 5.9: Axial simulated pressure profile for the positive envelope $\left(|p|^{+}\right)$and for the rarefaction minimum distribution $\left(|p|^{-}\right)$. Different excitation amplitudes are marked as different color lines. 


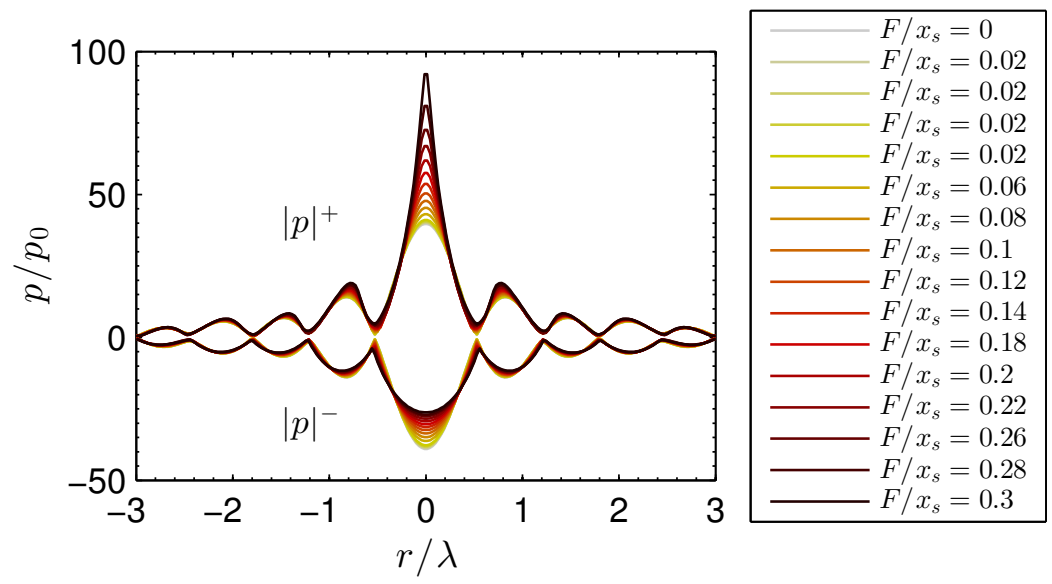

Figure 5.10: Transversal simulated pressure profile for the positive envelope $\left(|p|^{+}\right)$and for the rarefaction minimum distribution $\left(|p|^{-}\right)$. Different excitation amplitudes are marked as different color lines.

amplitudes, being $F / x_{s}=0.02,0.16,0.260 .3$ from low to high amplitude. The above described effects can be observed. The axial field distribution corresponding to these amplitudes can be observed in Fig. 5.12, where the amplitude of the first and second harmonic is extracted. It can be seen that the amplitude of the first harmonic is slightly reduced for increasing amplitudes and the second harmonic field grows locally at the focal spot.

The locally nonlinear generation of the higher harmonics contributes to modify the waveform, shown in the corresponding subplots of Fig. 5.12. Here, the characteristic shape of nonlinear focused waveforms can be observed, reaching a peak pressure of $|p|^{+}=126 \mathrm{MPa}$ and a rarefaction pressures of $|p|^{+}=-36 \mathrm{MPa}$. These peak pressures are of the order of those used in medical therapy applications for thermal ablation, with the rarefaction pressure close to the cavitation threshold of the water at room hydrodynamic pressure and temperature ${ }^{1}$ (Caupin et al., 2006).

\footnotetext{
${ }^{1}$ Note the cavitation threshold strongly depends of the dissolved oxygen content and bubbles in general of the water.
} 

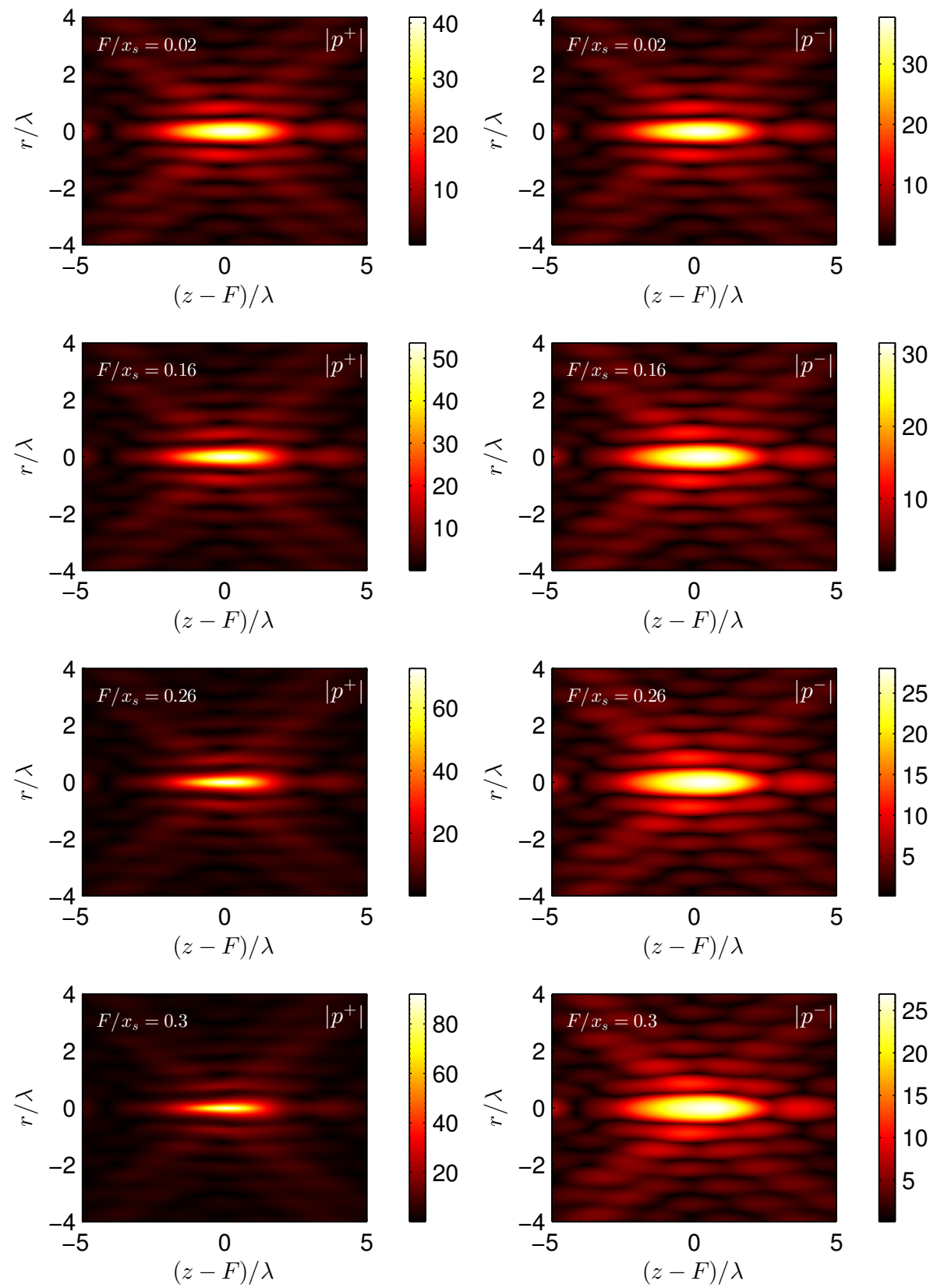

Figure 5.11: Field distribution for increasing amplitude excitation. (Left column) pressure profile for the positive envelope $\left(|p|^{+}\right)$and (right column) for the rarefaction minimum distribution $\left(|p|^{-}\right)$. 

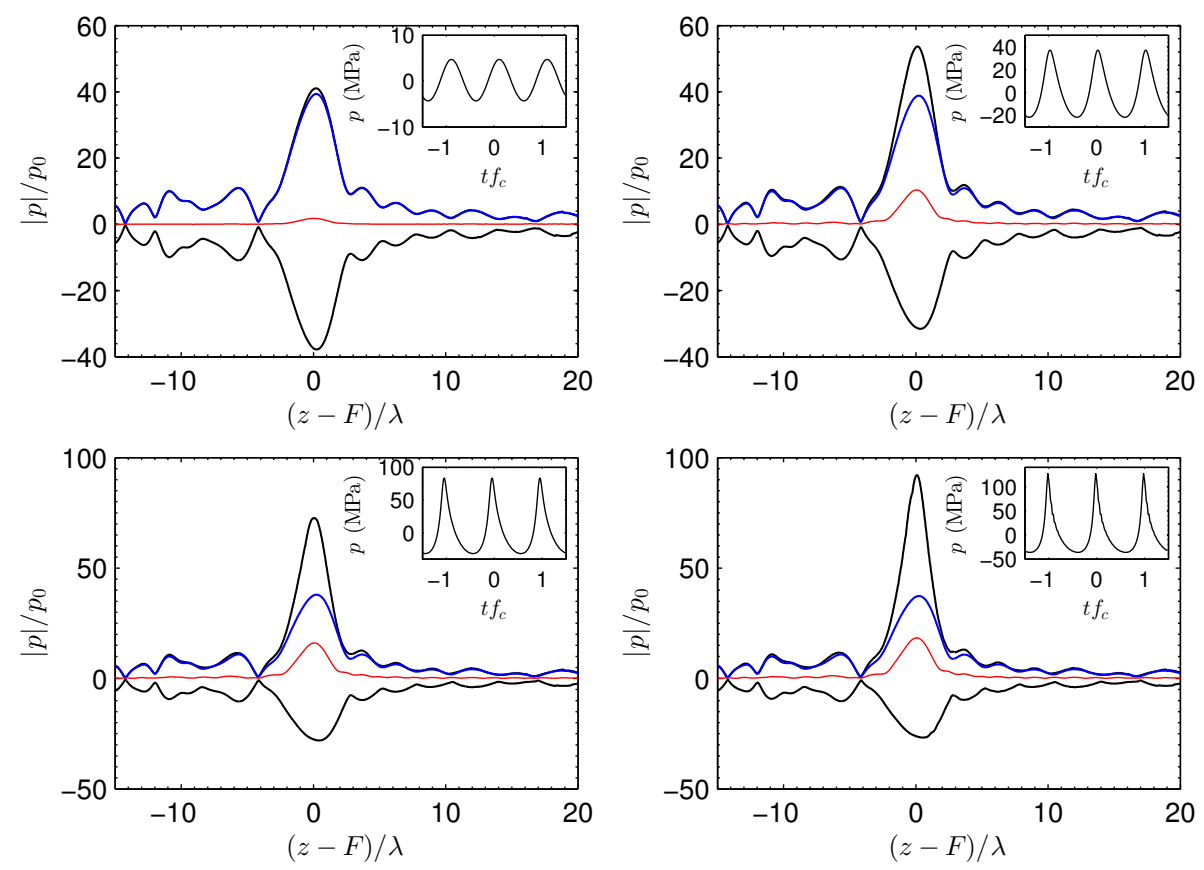

Figure 5.12: Axial field distribution for four increasing amplitudes, being $F / x_{s}=$ 0.02, 0.16, 0.260.3 from low to high amplitude. (Black lines) full envelopes $|p|^{+}$and $|p|^{-}$, (blue) fundamental harmonic component and (red) second harmonic field distribution. The inset shows the corresponding waveforms measured at the focal in physical units.

Finally, from all these the simulation data, the nonlinear dependence of the focusing parameters of the grating is calculated and presented in Fig. 5.13. In first place, the nonlinear gain of the system is shown in Fig. 5.13 (a), where it is plotted the gain for the full peak amplitudes $\left(|p|^{+} / p_{0}\right.$ and $\left.|p|^{-} / p_{0}\right)$, and also the corresponding gain for the first, second and third harmonics. It can be seen that the nonlinear effects dramatically increases the peak pressure gain due to the strong asymmetry of the waveforms. In the same way, the gain for the rarefaction pressure decreases with excitation amplitude also due to the asymmetry of the nonlinear distortion at focus (see insets in Fig. 5.12). The gain for the first harmonic is almost constant, around $40 \mathrm{~dB}$, but it slightly reduces for increasing amplitudes as long energy is transferred to higher harmonics. Finally, the gain for second and higher harmonics increases. In the case of second harmonic it varies from 0 to about the half of the linear gain of the system.

There exist also a nonlinear focal shift in the location of the peak pressure, shown in Fig. 5.13 (b). Thus, as the source amplitude is increased the location of the peak pressure is displaced in the axial direction beyond the linear focal position, a self-defocusing effect is observed. On the other hand, for the case of the rarefaction pressure it appears a nonlinear self-focusing effect, i.e. the focal 

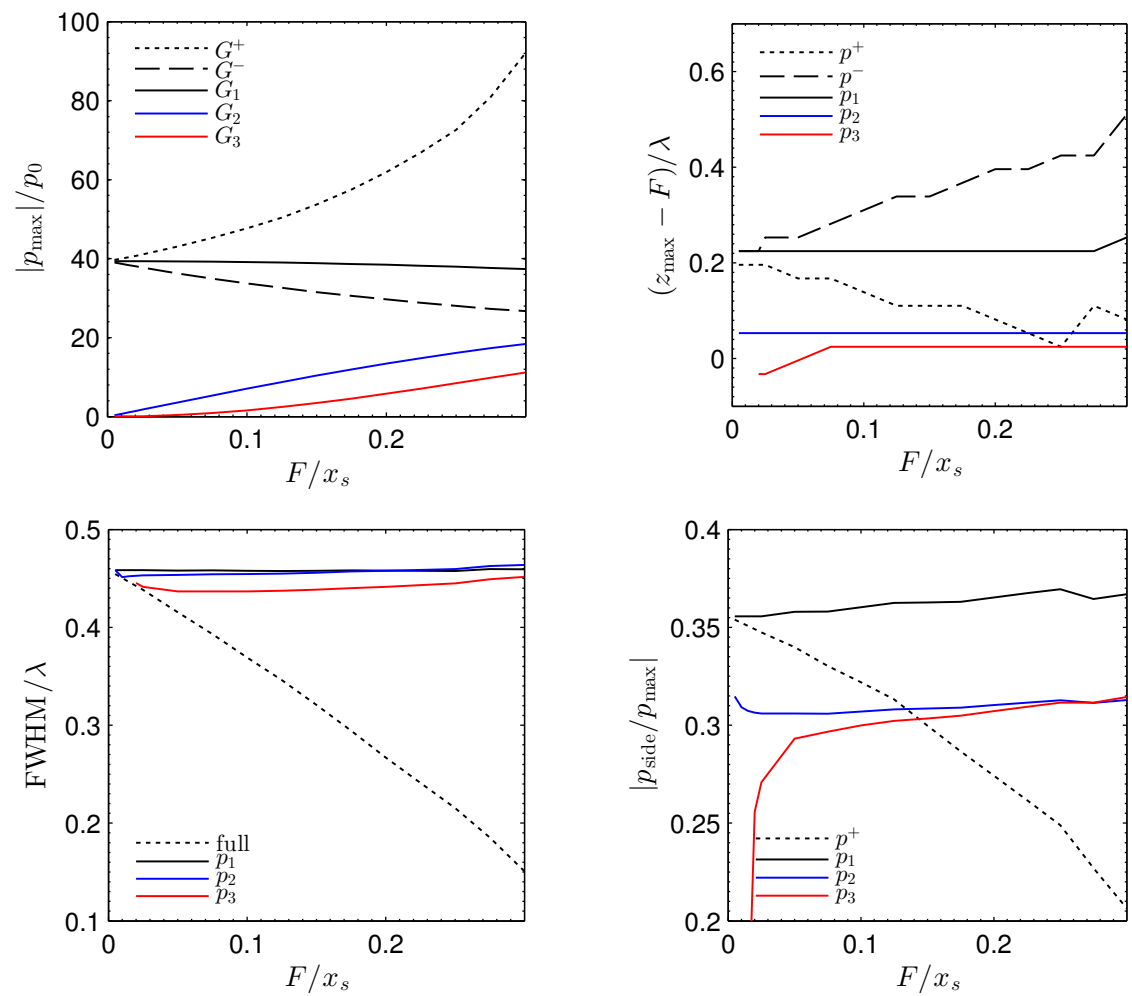

Figure 5.13: Focal characterization in the nonlinear regime. (Up left) Effective gain obtained as $\left|p_{\max }\right| / p_{0}$ as a function of the normalized excitation amplitude. (Up right) Location of the peak pressure. (Bottom left) Intensity Full Width at Half Maximum (FWHM), also normalized by the harmonic index. (Bottom right) Side lobe amplitude as a function of the excitation amplitude.

displaces towards the source. Both effects can be related to nonlinear self-refraction of the beam in the focal spot (Camarena et al., 2013a). However, due to the low focal size of this strongly focused device (Camarena et al., 2010) these effects are minor and the total displacements are not longer that $\lambda / 4$.

We have analyzed the FWHM of the several harmonics as the amplitude of the incident wave in increased. Figure 5.13 (c) shows the dependence of FWHM on the amplitude of the incident wave, measured again in terms of the shock distance normalized to the focal position in the linear regime, $F$. FWHM is constant for both the fundamental and the second harmonic focal spots, note here that the second harmonic focal size is effectively half of the size of the fundamental wave, but we show the value normalized to its wavelength. It is worth noting here, that as the nonlinearity of the medium is activated, the focal spot considering all the components, decreases dramatically its FWHM because of the strong asymmetry of the waveforms only at the focal spot. 
Finally, we can compare the different transversal profiles and observe the reduction of the side lobes of the second-and-higher harmonic profiles. The numerical results show the highly reduced lobes around the focal spot both in the radial and in the longitudinal directions. This effect is caused by the locally generation of the field only at the focal spot, where in the other hand, the nonlinear (local) harmonic generation at the lateral lobes is reduced due to its low amplitude. It is worth noting here that the reduction does not strongly depends on the excitation amplitude: very weak nonlinear effects are enough to provide second harmonic side-lobe reduction.

\subsection{Conclusions}

We have presented a characterization of the focusing properties of an axisymmetric grating constructed by toroidal scatters. The design is equivalent to a binary Fresnel lens of infinitesimally thicker slits. We demonstrated experimentally and numerically that the device presents a very narrow focal spot, that is between the sub-diffraction and the sub-wavelength limits. As common from other Fresnellike lenses and also in Bessel beams, the narrow focal is accompanied by high amplitude side lobes. On the other hand, the confined focal provides strong energy concentration over a small volume. Thus, nonlinear effects can be mainly activated locally at the focal spot, and second harmonic is generated mainly there. In this way, the field of the second harmonic maintain the excellent beam-width of the Fresnel lens, while its side-lobes are strongly reduced. The proposed lens in the weakly nonlinear regime is suitable for second harmonic imaging techniques: the excellent beam spatial resolution of the second harmonic (normalized to the wavelength) is maintained, while the side-lobe amplitude, responsible of signal-tonoise ratio, is reduced.

Another important feature is related to the energy concentration of the system. In the proposed system, an amplification of the incident field up to $30 \mathrm{~dB}$ has been observed numerically and experimentally. A parametric study shows that amplification values up to $35 \mathrm{~dB}$ can be also obtained if the number of rings, i.e. the aperture, are increased to $M=50$.

Finally, it is worth noting here that the device is designed for single frequency operation. The chromatic aberration for frequencies around the design frequency is not strong: for a relatively broad bandwidth, around one octave, the main observed effect in simulations and experiments is a focal shift. The focal spot shifts in the axial direction and the displacement can be estimated analytically. For the presented device a focal displacement of about 30 wavelengths has been observed in the frequency range studied. The gain also is modified by the chromatic aberration, but notice that these variations can be compensated by signal processing. In this way, the focal aberration provides a way to actively change the axial location of the sharp focus if the passive device is excited by a frequency modulated signal. 
Thus, it can provide a cheap alternative to ultrasound imaging techniques that requires a fast intense moving focal, as in Supersonic Shear Imaging.

\section{References}

Averkiou, Michalakis A, David N Roundhill, and JE Powers (1997). "A new imaging technique based on the nonlinear properties of tissues". In: Ultrasonics Symposium, 1997. Proceedings., 1997 IEEE. Vol. 2. IEEE, pp. 1561-1566.

Bailey, MR, VA Khokhlova, OA Sapozhnikov, SG Kargl, and LA Crum (2003). "Physical mechanisms of the therapeutic effect of ultrasound (a review)". In: Acoustical Physics 49.4, pp. 369388.

Bercoff, Jérémy, Mickaël Tanter, and Mathias Fink (2004). "Supersonic shear imaging: a new technique for soft tissue elasticity mapping". In: Ultrasonics, Ferroelectrics, and Frequency Control, IEEE Transactions on 51.4, pp. 396-409.

Botteldooren, Dick (1995). "Finite-difference time-domain simulation of low-frequency room acoustic problems". In: The Journal of the Acoustical Society of America 98.6, pp. 33023308.

Camarena, Francisco, Yuri Makov, Víctor Sánchez-Morcillo, Silvia Adrián, and Noé Jiménez (2010). "Nonlinear focal shift in medium Fresnel-number focused acoustic beams". In: Ultrasonics Symposium (IUS), 2010 IEEE. IEEE, pp. 1845-1848.

Camarena, Francisco, Silvia Adrian-Martinez, Noe Jimenez, and Victor Sanchez-Morcillo (2013a). "Nonlinear focal shift beyond the geometrical focus in moderately focused acoustic beams". In: The Journal of the Acoustical Society of America 134, pp. 1463-1472.

Canney, Michael S, Michael R Bailey, Lawrence A Crum, Vera A Khokhlova, and Oleg A Sapozhnikov (2008). "Acoustic characterization of high intensity focused ultrasound fields: A combined measurement and modeling approach". In: The Journal of the Acoustical Society of America 124.4, pp. 2406-2420.

Caupin, Frédéric and Eric Herbert (2006). "Cavitation in water: a review". In: Comptes Rendus Physique 7.9, pp. 1000-1017.

Choi, James J, Mathieu Pernot, Scott A Small, and Elisa E Konofagou (2007). "Noninvasive, transcranial and localized opening of the blood-brain barrier using focused ultrasound in mice". In: Ultrasound in medicine 85 biology 33.1, pp. 95-104.

Clement, G., H. Nomura, and T. Kamakura (2015). "Ultrasound field measurement using a binary lens". In: Ultrasonics, Ferroelectrics, and Frequency Control, IEEE Transactions on 62.2 , pp. $350-359$.

Cunningham, Kevin B and Mark F Hamilton (2000b). "Bessel beams of finite amplitude in absorbing fluids". In: The Journal of the Acoustical Society of America 108.2, pp. 519-525.

Ding, Desheng and Zuhong Lu (1996). "The second harmonic component in the Bessel beam". In: Applied physics letters 68.5, pp. 608-610.

Ding, Desheng and L Jian-yu (2000b). "Higher-order harmonics of limited diffraction Bessel beams". In: The Journal of the Acoustical Society of America 107, pp. 1212-1214.

Duck, Francis A (2002). "Nonlinear acoustics in diagnostic ultrasound". In: Ultrasound in medicine Es biology 28.1, pp. 1-18.

Farnow, SA and BA Auld (1974). "Acoustic Fresnel zone plate transducers". In: Applied Physics Letters 25.12, pp. 681-682.

Holm, Sverre and Fabrice Prieur (2010). "Low sidelobe limited diffraction beams in the nonlinear regime". In: The Journal of the Acoustical Society of America 128.3, pp. 1015-1020.

Hsu, DK, FJ Margetan, and DO Thompson (1989b). "Bessel beam ultrasonic transducer: Fabrication method and experimental results". In: Applied physics letters 55.20, pp. 2066-2068.

Jimenez, Noe, Vicente Romero-García, R Picó, A Cebrecos, Víctor José Sánchez-Morcillo, LM Garcia-Raffi, Juan Vicente Sánchez-Pérez, and K Staliunas (2014). "Acoustic Bessel-like beam formation by an axisymmetric grating". In: EPL (Europhysics Letters) 106.2, p. 24005. 
Konofagou, Elisa E and Kullervo Hynynen (2003). "Localized harmonic motion imaging: theory, simulations and experiments". In: Ultrasound in medicine $\mathcal{E}$ biology 29.10, pp. 1405-1413.

Lu, J.-y. and J.F. Greenleaf (1990b). "Ultrasonic nondiffracting transducer for medical imaging". In: Ultrasonics, Ferroelectrics, and Frequency Control, IEEE Transactions on 37.5, pp. 438447.

Molerón, M., A. Leonard, and C. Daraio (2014). "Solitary waves in a chain of repelling magnets". In: J. Appl. Phys. 115.18, p. 184901.

Nguyen, Man Minh, Junseob Shin, and Jesse Yen (2014). "Harmonic Imaging with Fresnel Beamforming in the Presence of Phase Aberration". In: Ultrasound in medicine \& biology 40.10, pp. 2488-2498.

Nightingale, Kathryn, Mary Scott Soo, Roger Nightingale, and Gregg Trahey (2002). "Acoustic radiation force impulse imaging: in vivo demonstration of clinical feasibility". In: Ultrasound in medicine 63 biology 28.2, pp. 227-235.

Sarvazyan, Armen P, Oleg V Rudenko, Scott D Swanson, J Brian Fowlkes, and Stanislav Y Emelianov (1998). "Shear wave elasticity imaging: a new ultrasonic technology of medical diagnostics". In: Ultrasound in medicine 83 biology 24.9, pp. 1419-1435.

Schindel, DW, AG Bashford, and DA Hutchins (1997). "Focussing of ultrasonic waves in air using a micromachined Fresnel zone-plate". In: Ultrasonics 35.4, pp. 275-285.

Sleva, Michael Z, William D Hunt, and Ronald D Briggs (1994). "Focusing performance of epoxyand air-backed polyvinylidene fluoride Fresnel zone plates". In: The Journal of the Acoustical Society of America 96.3, pp. 1627-1633.

Wang, Hui, Da Xing, and Liangzhong Xiang (2008). "Photoacoustic imaging using an ultrasonic Fresnel zone plate transducer". In: Journal of Physics D: Applied Physics 41.9, p. 095111.

\section{Publications}

The contents of this Chapter have been presented in the following publications:

\section{Journal papers}

- Jiménez, N.; Romero-García, V.; Picó, R.; Cebrecos, A.; Sánchez-Morcillo, V.; García-Raffi, L.; and Staliunas, K. "Transition from linear- to nonlinear-focusing regime of ultrasonic waves produced by an axisymmetric diffraction grating" In preparation (2015) 


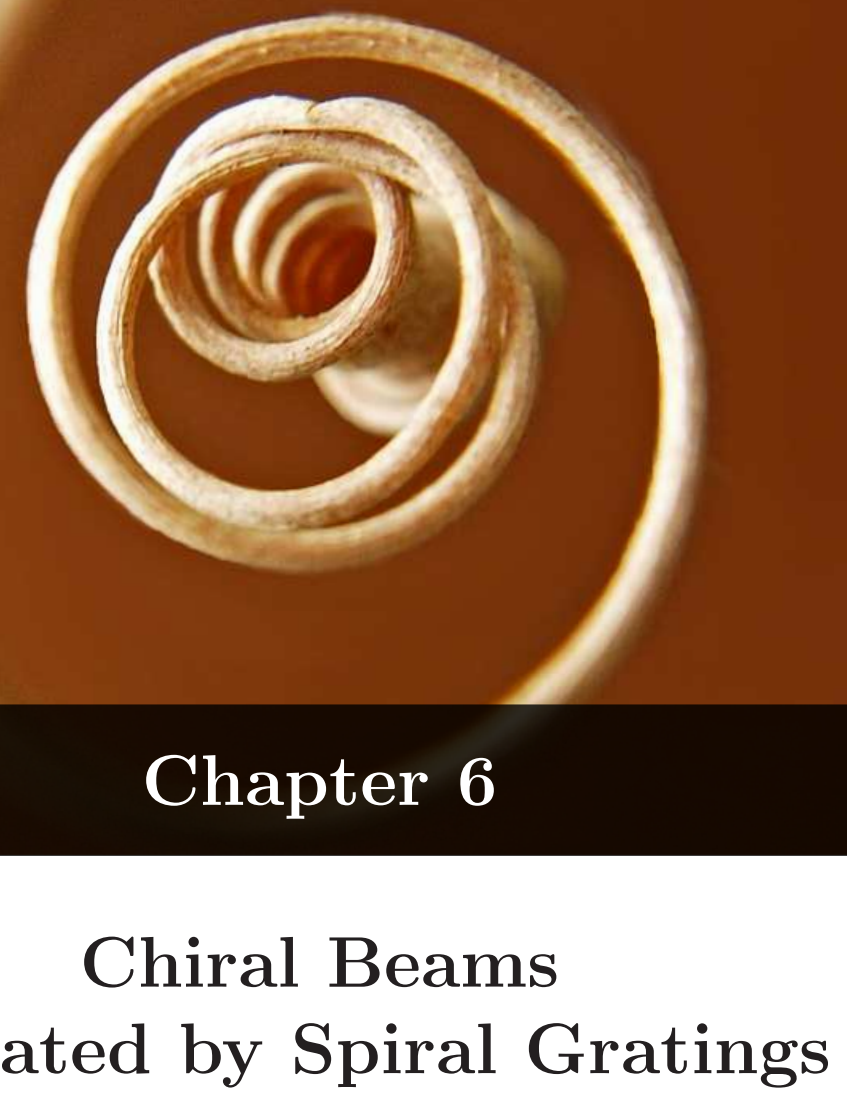

\title{
generated by Spiral Gratings
}

\begin{abstract}
Previously, zeroth order Bessel-like beams have been generated by axisymmetric diffraction gratings. Here, we report the generation of high order Bessel beams by the diffraction of plane waves over spiral shaped scatters. The acoustical beam vortex structure is analyzed and a method for generate arbitrary order Bessel beams based in multiple arms spirals is presented. A passive device is experimentally tested in the ultrasound regime and a first order Bessel vortex beam is observed. Simulations in the weak nonlinear regime are also included where the conservation law for the ratio between the topological charge of the beam and the generated harmonic order is proved. This novel technique can be used to generate cheap lenses for acoustic particle manipulation and acoustic radiation force applications.
\end{abstract}




\subsection{Introduction}

In the previous Chapters $(4,5)$ the diffraction generated by axisymmetric structures is analyzed for generate Bessel beams and strongly focused beams. Many of these axisymmetric structures, matching its symmetry with the usual sources, have been proposed for the control of the acoustic field. thus, zeroth-order Bessellike beams can be generated by these structures in Bragg regime (Jimenez et al., 2014). In the long-wavelength limit, GRaded INdex (GRIN) lenses amplify the field in the axis (Romero-García et al., 2013; Molerón et al., 2014). In all of these configurations the scattered field maintain the axisymmetry of the lenses.

Zeroth-order Bessel beams, as those reported in Chapter 4, present a maximum of the field trough the axis. On the other hand, higher-order Bessel beams exhibit a minimum of the field at the axis. Both, zeroth and higher Bessel beams propagates in free space without any diffraction spreading, as long they are propagationinvariant solutions of the Helmholtz equation (Durnin et al., 1987). Both types of beams are characterized by the appearance of high amplitude concentric rings surrounding the axis that its transverse profile follows a lth-order Bessel function. Is important to note also that the magnitude of the spatial spectrum of these beams does not depends of the order of the beam, i.e. in the far field all Bessel beam is characterized by a annular radiation. In other words, all Bessel beams can be generated by a conical wavefront of infinite extension. The difference between zeroth and higher order Bessel beams is that for higher order Bessel beams the azimuthal phase shows a linear variation across its annular spectrum. Thus, the central zero in the axis of the higher-order Bessel beams that appears in the near field is caused due to the phase singularity of charge $l$ associated with the azimuthal phase variations.

In this way, high order Bessel beams generates acoustical vortices with a spatial phase dependence that cannot be generated by axisymmetric radiating sources. In optics, different solutions have been proposed to break the axial symmetry in an appropriate way to generate vortices. These methods include direct Gaussian beam illumination by use of axicon-type computer generated holograms (Vasara et al., 1989; Heckenberg et al., 1992) or illuminating an axicon with a Laguerre-Gaussian mode (Arlt et al., 2000). Another solution is to impose an azimuth-dependent retardation on the optical field with Spiral Phase Plates (SPP) (Oemrawsingh et al., 2004). A diffraction grating with groove bifurcation (called "fork") is also able to generate a vortex from an optical beam falling onto the grating center with an arbitrary topological charge (Bekshaev et al., 2008).

Although phase dislocations were first studied in acoustics (Nye et al., 1974), the related experimental works have been developed lately. The acoustic analog of optical Spiral Phase Plates was proposed by Hefner et al. (1999). Here, the transducer surface is physically offset by twisting like the coil of a spring. This design has an important limitation: the transducer has a unique operating frequency of 
interest. Other approaches include the use of photoacoustic effeect to generate the Helical beam (Gspan et al., 2004). A common approach to overcome this problem is by using electronic means: an appropriate phase profile for an array of transducers generates the helical beam. Thus, signals emitted by each transducer of the array are delayed in order to control the angular phase shift. The interaction of high order Bessel beams with particles have been widely studied in the exhaustive collection of works of F. G. Mitri and G. T. Silva among others. Two main remarkable effects have been reported: (1) the transference of orbital momentum from the acoustical vortex to the particle (Mitri et al., 2012), and on the other hand, the appearance of negative axial acoustic radiation forces (Marston, 2006; Marston, 2007a; Mitri, 2008; Mitri, 2009). Thus, practical applications of vortex acoustic beams are multiple: particle manipulation (Demore et al., 2011) and acoustical tweezers (Wu, 1991), an acoustic spanner (Skeldon et al., 2008), rotation of fibers (Schwarz, 2013) or multiple-particle trapping (Yoon et al., 2014). However, although the array provides active steering and control of the vortex beam, in these active methods the discretization of the source is restricted by the number of transducers in the array. On the other hand, the screw dislocations generated by finite amplitude acoustical vortex has been also studied. In the case of weak nonlinear regime, it has been proved the conservation law for the ratio between the topological charge of the beam and the generated harmonic order (Thomas et al., 2003; Marchiano et al., 2008). ON the other hand, for intense acoustic beams, azimuthal shock wave have been reported numerically and experimentally (Marchiano et al., 2008; Brunet et al., 2009; Brunet et al., 2010).

In this Chapter we propose a passive system for the formation of acoustic vortex. The design consist of an Archimedes' spiral grating. An experimental setup is proposed in the ultrasound regime, where the results show that the diffracted field around the axis generates a first order Bessel beam with its characteristic screw dislocation. A method for synthesize high order Bessel beams is also provided and tested numerically, where it was proven that a $n$-arm spiral generates a $n$ th-order Bessel beam. Furthermore, weakly nonlinear propagation is considered, where the conservation law for the ratio between the topological charge of the beam and the generated harmonic order is also observed.

\subsection{From axisymmetric to chiral beams}

Let us start considering a pair of uniform axisymmetric gratings, $\mathrm{A}$ and $\mathrm{B}$, as those studied before in Chapter 4. Let the grating A only differ in one aspect from the grating B: a shift in the radial position of the toroidal rings. Figure 6.1 shows a scheme of the situation. Therefore, the first structure presents a radial distribution for the $M$ toroidal rings as $r_{m}=m a$, where $a$ is the separation distance between rings and $m=(1,2, \ldots, M)$ is the ring index. On the other hand, the second structure is designed as $r_{m}=m a+a / 2$. The incident plane wave is scattered by 

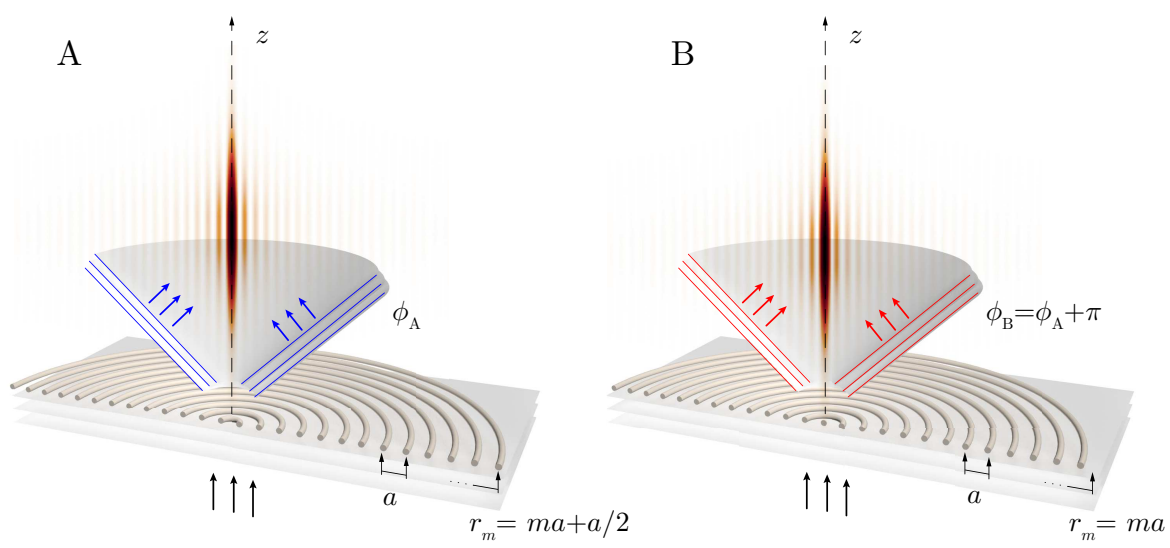

Figure 6.1: Scheme of the axisymmetric diffraction grating. The structure A differs from B by a shift of $\Delta r=a / 2$ in the radial distribution of the rings. The incident plane wave is scattered by the structures in a converging conical wavefront. When it converges to the axis a Bessel beam is formed. The beams generated have equal magnitude but are shifted $\Delta \phi=2 \pi \Delta r / a$, that in this example, for $\Delta r=a / 2$ leads to a phase difference of $\Delta \phi=p i$.

the structure creating a conical wavefront that converges to the axis of symmetry creating a limited-aperture Bessel beam. Same situation is also produced in the configuration $\mathrm{B}$, with the only main difference of the scattered field is phase shifted.

Numerical solutions for the scattering of the two structures are shown in Fig. 6.2. It is clearly visible how the magnitude of the field remains basically the same, where the transversal field is described by a Bessel function of zeroth order, namely $J_{0}$. In the other hand, the phase difference between the structure
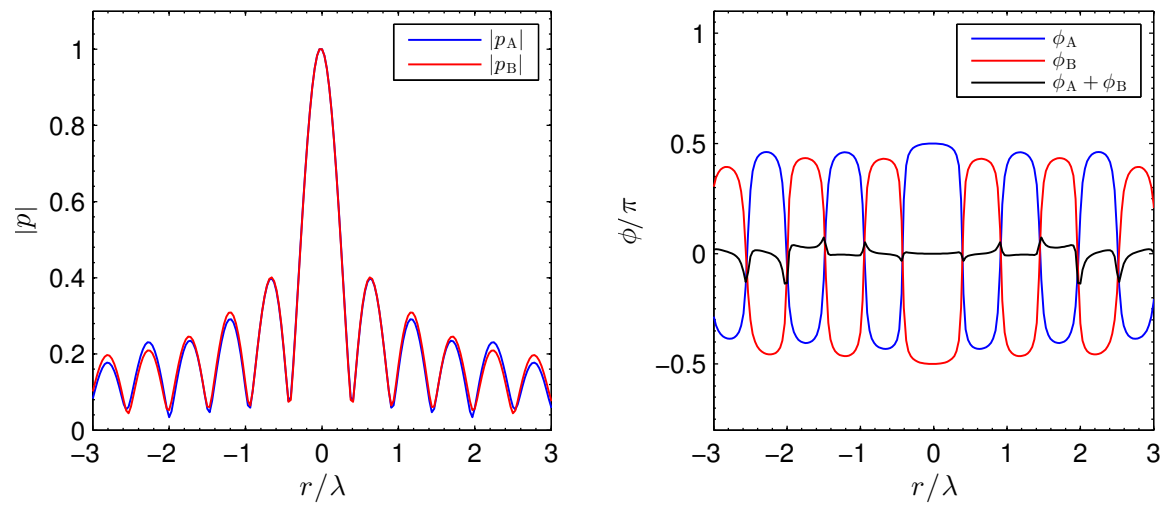

Figure 6.2: Radial distribution of the field generated by the axisymmetric structures A and B. (left) Magnitude, (right) phase. Note if the $\phi_{\mathrm{A}}+\phi_{\mathrm{B}}=0$, then $\Delta \phi=\pi$. 
A and the shifted grating B is $\pi$ radians. The field remains in anti-phase not only at the center, but over all the the lateral lobes of the Bessel function. Only at the zeros of the Bessel function there exist in the simulations some not perfect antiphase field distribution. In this example, the structure A is shifted in the radial coordinate by half of the radial period, this spatial shift between both structures is $\Delta r=a / 2$. In a more general case, the phase difference can be related to the radial shift simply by

$$
\Delta \phi=2 \pi \Delta r / a
$$

where $\Delta \phi=\phi_{\mathrm{A}}-\phi_{\mathrm{B}}$ is the phase difference between fields generated by a gratings shifted a distance $\Delta r$.

Thus, we propose a structure composed of curved tubular scatters, but not necessary of toroidal shape, that mixes the properties of the gratings $\mathrm{A}$ and $\mathrm{B}$ in order to have a destructive interference in the axis. First, is obvious that this structure is no longer axisymmetric, as long the scatters at azimuth angle $\theta=0$ must be shifted radially by $\Delta r=n a / 2$ respect to the plane $\theta=\pi$, being $n$ an integer. On the other hand, the tubular scatters must be continuous in azimuth, and the following design condition

$$
\phi(\theta)=\phi(\theta+\pi) \pm \pi
$$

must hold for any $\theta$. This condition is necessary in order to achieve a minimum at the axis. It essentially states that there exist a scattered field at some $\theta$ that have inverted phase respect to the scattered field at opposite azimuth $\theta+\pi$.

Let us here assume that the phase of the field changes linearly with the azimuth angle as $\phi=\alpha \theta$. Substituting this equation in Eq. (6.2) we get that, in order to fulfill the design conditions, that the rate of change of phase respect to the azimuth angle must be $\alpha= \pm 1$, leading to the simple relation $\phi= \pm \theta$.

Then, we can write Eq. (6.1) as

$$
\frac{\Delta r}{\Delta \phi}=\frac{a}{2 \pi}
$$

This relation states that the rate of change in the radial position of the tubes respect to the changes produced in the phase of the field is constant. Thus, using $\Delta \phi= \pm \Delta \theta$, we can relate the change of the radial position of the tubes respect to the azimuth coordinate as

$$
\frac{\Delta r}{\Delta \theta}= \pm \frac{a}{2 \pi}
$$

In the continuous limit, $\frac{\Delta r}{\Delta \theta} \rightarrow \frac{\partial r}{\partial \theta}$ and therefore Eq. (6.4) can be expressed as the parametric curve

$$
r= \pm b \theta
$$


with $b=a / 2 \pi$. Thus, if the tubular scatterers are bend following this parametric curve, the scattering of the structure in the axis must be zero. This expression is the Archimedes' spiral, a particular case of the general class of Archimedean spirals that can be described by

$$
r= \pm b \theta^{1 / \gamma}
$$

Here, the parameter $\gamma$ controls how fast the spiral is wrapped. For $\gamma=1$, we get the normal Archimedes' spiral, with uniform separation between turns, for $\gamma>1$ the spiral radius grows with each turn, while for $\gamma<1$ the separation distance between turns becomes thigh. Special cases of this family are the lituus $(\gamma=-2)$, the hyperbolic spiral $(\gamma=-1)$ or the Fermat's spiral $(\gamma=2)$.

\subsection{Chiral beams generated by spiral gratings}

In the previous section we have found that the spirals gratings are good candidates to provide chiral beams. These spirals have the propriety of having uniform separation between turns as $r(\theta+2 \pi)=r(\theta)+a$. This uniformly spaced grating, as occurs in the previously studied Bessel beams, diffracts plane waves in a converging conical wavefront ${ }^{1}$. In analogy to the well-known diffraction grating, the angle $\beta$ defined by the conical wavefront is

$$
\sin \beta=N \lambda / a
$$

where $\lambda$ is the wavelength and $N$ is the diffraction order. A scheme of this situation is shown in Fig. 6.3. It is well known that a converging conical wavefront generates a Bessel beam. The field of an ideal Bessel beam can be expressed for the velocity potential $^{2}, \psi$, as

$$
\psi(r, \theta, z)=\psi_{0} J_{n}\left(k_{r} r\right) \exp \left(i k_{z} z \pm i n \theta\right)
$$

where $J_{n}$ is the $n$ th-order Bessel function $\psi_{0}$ is the amplitude, and $k_{z}$ and $k_{r}$ are axial and transverse components of the wavevector $k=\sqrt{k_{z}^{2}+k_{r}^{2}}$. It is important the relation of this quantities to the cone angle of the Bessel beam

$$
\cos \beta=k_{z} / k, \sin \beta=k_{r} / k .
$$

\footnotetext{
${ }^{1}$ Note that the field is also scattered in a diverging cone with angle $\pi / 2-\beta$, but this waves does not contributes to the field distribution near the axis in free propagation. If the structure is embedded inside a tube, this field can be reflected by the boundaries and it will converge to the axis creating another Bessel beam.

${ }^{2}$ the velocity potential can be related to the acoustic pressure as $p=i \omega \rho_{0} \psi$ and to particle velocity as $\mathbf{v}=\nabla \psi$
} 


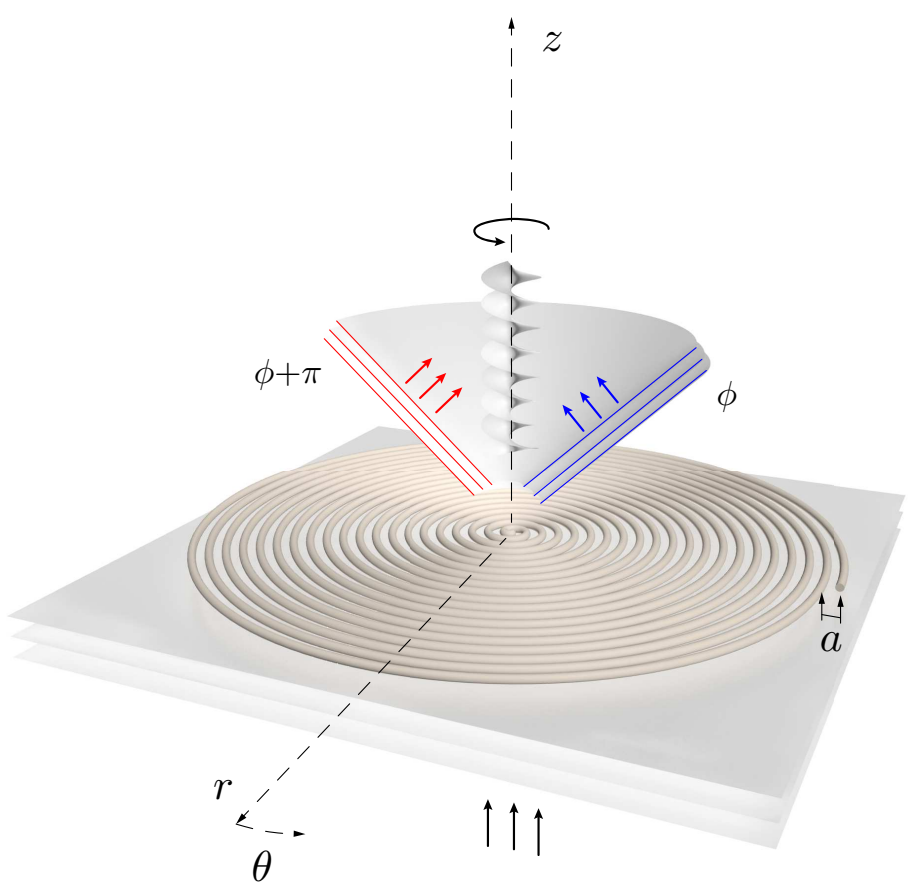

Figure 6.3: Scheme of the Archimedes' spiral scattering process. The incident plane wave is scattered into a converging conical wavefront which its phase changes linearly in azimuthal angle $\theta$. The field can be described as a first order Bessel beam, where in the axis a corkscrew dislocation is produced, leading to an acoustic vortex and a node at $r=0$.

Note the trivial solution for $\beta=0$, where the Bessel beam reduces to a plane wave traveling in the $z$ direction. Therefor, thee cone angle $\beta$ of the Bessel beam depends on the separation of scatters. Thus, combining Eq. (6.7) and (6.9) we get the relations

$$
k_{z}=\frac{2 \pi N}{a \tan \beta}, k_{r}=\frac{2 \pi N}{a} .
$$

This relations allow us to obtain the Bessel beam field, Eq. (6.8) in terms of the grating parameters as

$$
\psi(r, \theta, z)=\psi_{0} J_{n}\left(\frac{2 \pi N}{a} r\right) \exp \left(i \frac{2 \pi N}{a \tan \beta} z \pm i n \theta\right) .
$$

This idealized field is valid for a grating of infinite aperture. In the case of a real 
structure with finite aperture, the Bessel beam is bounded in the axial direction. Is common to refer to this limitation as limited diffraction Bessel beams. Therefore, if the grating have a total aperture of $R=M a$, where $M$ is the number of turns of the spiral, from simple trigonometrical considerations the cone angle will cover a region of the axis from $z=0$ to $z=F$, being

$$
F=\frac{R}{\tan \beta}=\frac{M a^{2}}{N \lambda} \sqrt{1-\left(\frac{N \lambda}{a}\right)^{2}}
$$

the maximum distance at which the Bessel beam is formed, showing the limit of the validity of the nondiffractive propagation.

Let us consider the radiation converging from each ring at angle $\beta$, in order to find the locus of the points around the axis where exact constructive interference is produced. The spiral grating is not axisymmetric, but chiral, that is, it is distinguishable from its mirror image and it cannot be superposed onto it. Thus, the radiation converging from each ring do not converge along the symmetry axis. Figure 6.4 shows a scheme, where if we suppose the grating diffracts the wave at angle $\beta$, and following simple trigonometry considerations the coordinates of the focal points can be obtained in function of the spiral geometry. Thus, for the axial component of the focal can be expressed as

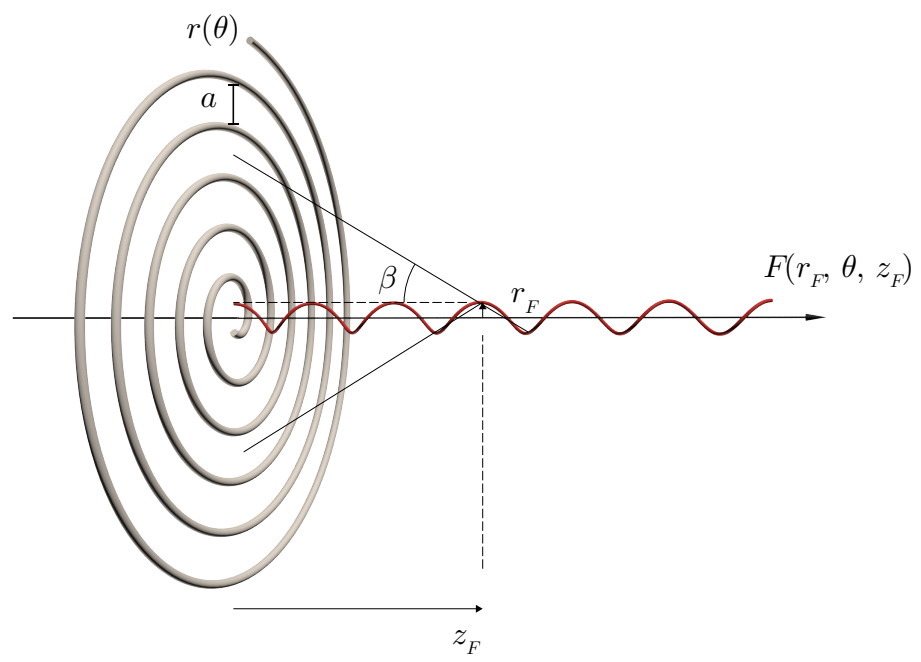

Figure 6.4: Scheme of the geometry of the spiral. Due to the scattering of the spiral at angle $\beta$, the locus of points in which perfect constructive interference is produced around the axis is an helix. 


$$
z_{F}(r, \theta)=\frac{r(\theta)+\frac{a}{4}}{\sin \beta} \sqrt{1-\sin ^{2} \beta} .
$$

Then, using the cone angle definition and the spiral curve $r(\theta)$ we get

$$
z_{F}(\theta)=\left(\frac{\theta}{2 \pi}+\frac{1}{4}\right) \frac{a^{2}}{N \lambda} \sqrt{1-\left(\frac{N \lambda}{a}\right)^{2}} .
$$

On the other hand, this interference is not produced at the axis, but shifted a constant radial distance. From the geometry, see Fig 6.4, we can see that the radial component of the focal is

$$
r_{F}=a / 4
$$

Equations (6.14-6.15) composes the curve $F\left(r_{F}, \theta, z_{F}\right)$, that is a liner helix of constant radius. Thus, in addition the beam generation, the scattering located in the points of the spiral achieves perfect constructive interference over the helix curve that rotates around the axis. Moreover, the distance between turn of this helix can be calculated as $z_{F}(\theta+2 \pi)-z_{F}(\theta)=\lambda \cos \beta / N$. Thus, for small diffraction angles, the focal rotates in $z$ with a period of the order of the wavelength, when for shorter Bessel cone is generated, the distance between rotation in the helix stretches ${ }^{3}$.

\subsubsection{First order Bessel beams}

Let us now analyze the Archimedes' spiral with $\gamma=1$ and $b=a / 2 \pi$. With this parameters the ideal Bessel beam can be expressed as

$$
\psi(r, \theta, z)=\psi_{0} J_{n}\left(\frac{N}{b} r\right) \exp \left(i \frac{N}{b \tan \beta} z \pm i n \theta\right) .
$$

In the other hand, we model the scattering of the grating by two methods. First, if the scatters are thing compared to the wavelength, we can assume the scattering of each fragment of the spiral can be modeled by a point source. This modeling is also valid in the opposite limit, where the scatterers are thick enough to provide narrow aperture between them. In this situation the structure forms a grating. From Babinet's principle considerations, both situations can be equivalent. Therefore, we calculate the Rayleigh-Sommerfeld diffraction integral along the path of the spiral, $r=b \theta$, as

$$
p(x, y, z)=\frac{-i \omega \rho_{0} v_{0}}{2 \pi} \int_{r} \frac{\exp (i k|\mathbf{r}|)}{|\mathbf{r}|} d r
$$

\footnotetext{
${ }^{3}$ In the limit $\beta \rightarrow 0$, where the helix period is the wavelength, the divergent and convergent conical wavefront sums again into a plane wave. Thus, the helicity disappears due to the topological charge of two chiral waves is inverted.
} 

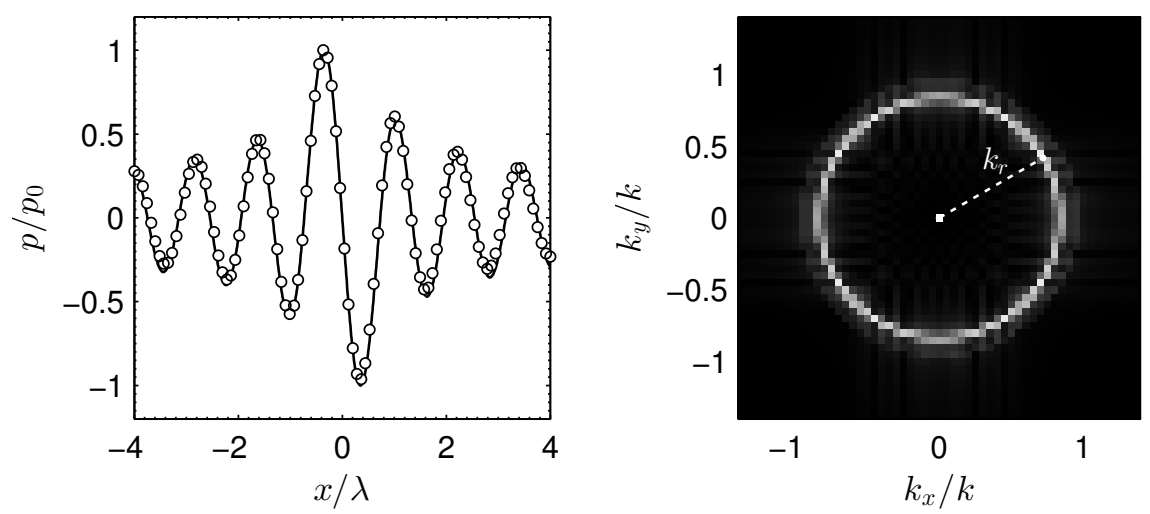

Figure 6.5: (Left) Transversal cut of beam field obtained by (continuous line) the analytic expression for ideal Bessel beam Eq. (6.16) and (circles) obtained by numerical evaluation of the Rayleigh-Sommerfeld diffraction integral Eq. (6.17) for a finite structure. (Right) Far-field of the spiral scattering showing the typical ring of the Bessel beam.

where $|\mathbf{r}|$ is the distance between each point in the path, $r=b \theta$, and the observation point $(x, y, z)$.

Second, we also approximate the diffraction with full-wave numerical integration of the acoustic wave equations. This approach provides an accurate estimation of the diffraction in situations where the wavelength is of the order of the scatter size, but due to the problem is $3 \mathrm{D}$, the full cartesian domain is integrated and is computationally intensive. In order to reduce the grid refinement requirements, we use the $k$-space method for solving the 3D computational problem. This method will be necessary also for the last section where we include the nonlinear propagation effects.

Figure 6.5 shows an example of first order Bessel-like beam generated by an uniform spiral with parameters $\beta=56.4^{\circ}, a / \lambda=1.2$ and aperture $R=60 \lambda$. We can see that the analytic expression for ideal Bessel beam Eq. (6.16) agrees with the solution obtained by the numerical evaluation of the Rayleigh-Sommerfeld diffraction integral Eq. (6.17) for this finite structure. On the right subplot, it can be appreciated how the typical ring of the Bessel beam appears in the far-field. This result shows that a conical wavefront is being generated by the structure, and it can be seen that the transversal wavenumber agrees with the estimated by the spiral parameters in Eq. (6.10), marked as a dotted line as $k_{r}$ in the figure. Therefore, we prove the field generated by the spiral structure is a first order Bessel-like beam. 

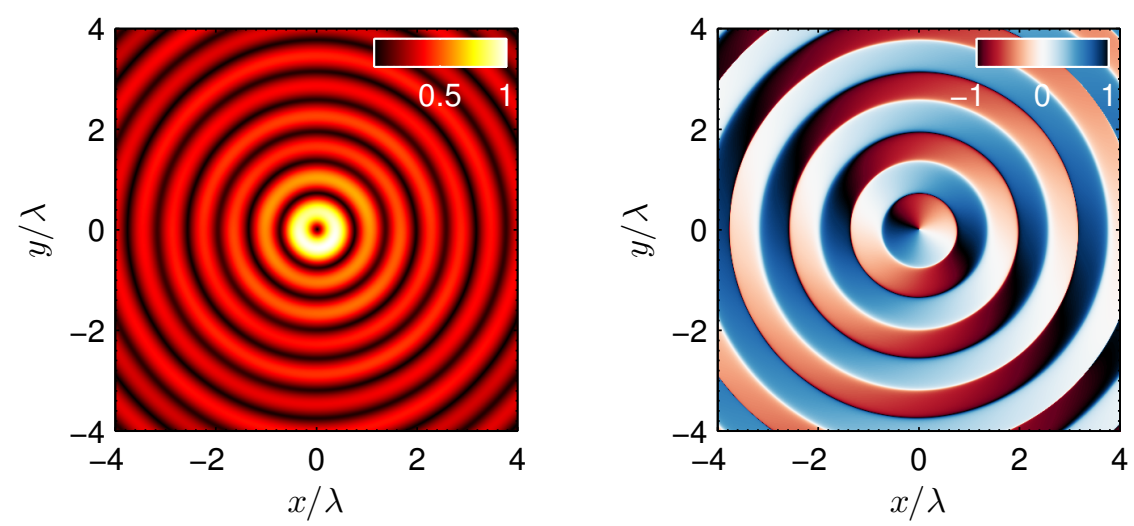

Figure 6.6: Transversal distribution of the field obtained by numerical evaluation of the Rayleigh-Sommerfeld diffraction integral. (Left) Magnitude, (right) phase. Colorbars in normalized units $\left(p / p_{0}\right.$ and $\left.\phi / \pi\right)$.

\section{Radial distribution: vortex}

One of the main features of the high order Bessel beams (HOBBs) is that all over the axis the field is zero, in contrast with the zeroth-order Bessel beam where a maximum appears in the symmetry axis. In the high order Bessel beams this zero is generated by destructive interference of a phase-rotating conical wavefront. Therefore, as a necessary condition, a vortex is generated around the axis. Figure 6.6 shows the transversal distribution of the field of a spiral at $F / 2$. It can be observed the node at the center in the magnitude, as long the high amplitude lateral lobes characteristic of the HOBBs.

On the right subplot, it can be appreciated that a vortex is generated. The number of complete phase shifts per turn is one, the vortex presents a topological charge of 1 . The nodes of the field connects 2 areas with opposite phase, while at the central minimum a screw-cork like dislocation is produced in the field. The ringed areas of the phase distribution corresponding to odd lateral lobes are phase inverted respect than the even ones, but all have the same rotation direction.

\section{Axial distribution}

Although the field distribution in the sagittal plane correctly corresponds to a truncated Bessel beam, there exist some features that must be underlined. Figure 6.7 shows the field in the $(x=0)$ plane of two first order Bessel beams. In the top subplot the spiral parameters are $a / \lambda=1.85$, that leads to a cone angle of $\beta=32.7^{\circ}$ and a beam extension distance of $F=115 \lambda$. As can be seen in the figure, the generated beam agrees with the analytical parameters. However, the farther part of the beam that is close to $F$ presents more amplitude compared to 

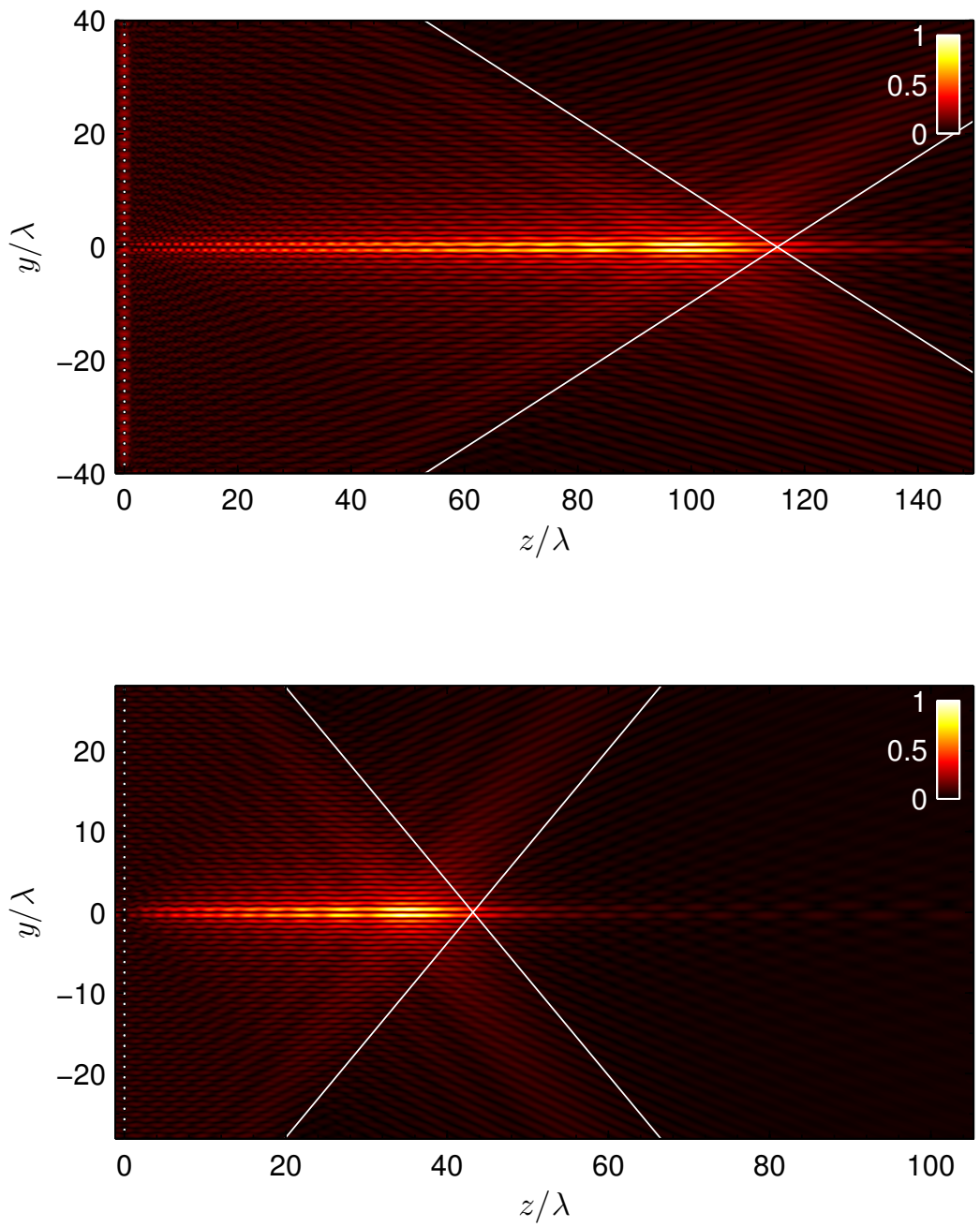

Figure 6.7: (Top) Distribution of the field for a spiral of $a / \lambda=1.85$ that leads to a (white line) cone angle of $\beta=32.7^{\circ}$ and beam extension distance of $F=115 \lambda$ (white line at $y=0$ ). (Bottom) Distribution of the field for a spiral of $a / \lambda=1.3$ that leads to a (white line) cone angle of $\beta=50^{\circ}$ and beam extension distance of $F=43.2 \lambda$ (white line at $y=0$ ). Colorbars in normalized units $\left(p / p_{\max }\right)$. 

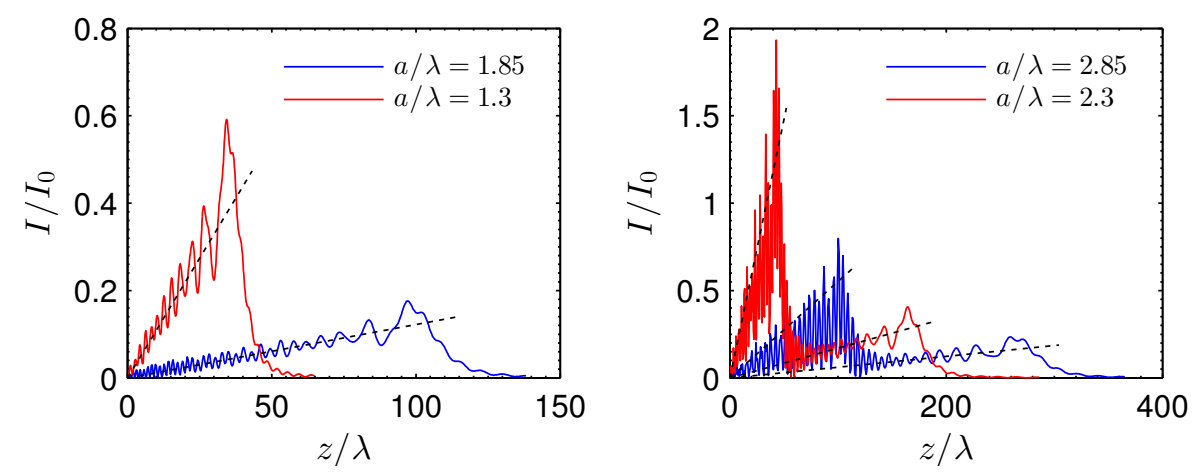

Figure 6.8: (Left) Axial Intensity distribution for a spiral of (blue) $a / \lambda=1.85$ and (red) $a / \lambda=1.3$. Black dashed lines shows the analytical extension of the truncated beam with a linear intensity profile. (Right) Axial Intensity distribution including higher diffraction orders for a spiral of (blue) $a / \lambda=2.85$ and (red) $a / \lambda=2.3$.

the part of the beam that is close to the grating. On the other hand, the field also present some oscillations in the $z$ direction.

Figure 6.7 (bottom) shows other example at lower frequency. Here, the spiral parameters are $a / \lambda=1.3$, that leads to a sharper cone angle of $\beta=50^{\circ}$ and a closest extension distance of $F=43.2$. Same properties can be observed in this situation.

In order to analyze the field amplitude, a axial cut is represented in Fig. 6.8. As long the field is zero in the axis, the intensity distribution shown in Fig. 6.8 is evaluated trough the maximum of the first lateral lobe. It can be clearly seen that the intensity increases linearly with the axial distance. This effect, is analogous to the studied in Sec. 4.2. As long the outermost rings scatters more energy, the external portions of the conical wavefront present more intensity. This energy converges to axial points around $F$, creating the same Bessel beam but with linearly increasing intensity.

Same effects are observed for the higher diffraction orders. As can be seen in Fig. 6.8 (right), when $a / \lambda>2$ the higher diffraction orders generates a secondary beam. Its corresponding parameters correctly follows the analytical estimations, as can be seen comparing the beam distribution to the dashed black lines. Due tho the appearance of this secondary beam, the amplitude of the fundamental diffraction order is reduced.

A parametric study of the longitudinal field is presented in Fig. 6.9 (left). It is analogous to the Fig. 4.2 for the zeroth-order Bessel beams. The field distribution extends with frequency following the analytical estimations (white lines), and for $a / \lambda>2$ higher diffraction orders appears forming secondary Beams close to the grating. On the other hand, similar study can be addressed for the transversal 

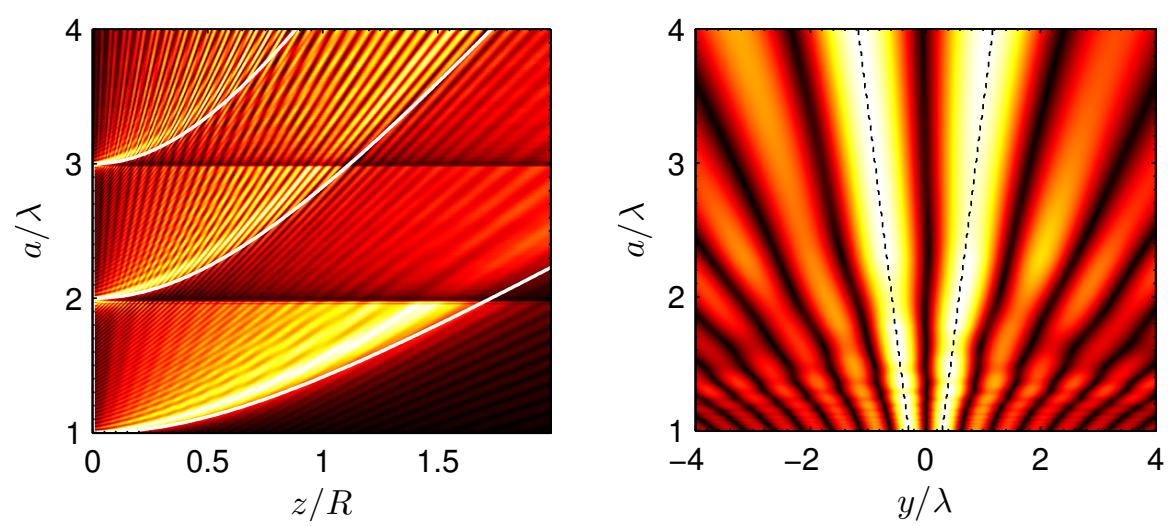

Figure 6.9: (Left) Axial distribution of the field in function of the quantity $a / \lambda$ obtained by numerical evaluation of the Rayleigh-Sommerfeld diffraction integral. White lines indicates the estimation of the focal by Eq. (6.12). (Right) Transversal distribution of the field in function of the quantity $a / \lambda$. Black dotted lines indicates the lateral location of the lobes estimated by Eq. (6.16). Colorbars in normalized units $\left(p / p_{0}\right.$ and $\left.\phi / \pi\right)$.

distribution. Figure 6.9 (right) shows the lateral distribution normalized to the wavelength in both axes. The separation distance is varied. As long the lateral distribution of the HOBBs depends on the transversal component of the wavevector, $k_{r}=2 \pi N / a$, there exist a lateral dependence of the lateral beam-width respect to spiral parameters. It essentially states that extending the Bessel beam at longer distances by spacing the spiral also spreads the beam-width. The numerical integration agrees with the analytical estimations (dashed black lines).

\subsubsection{Experimental validation}

An experimental test was realized in order to validate model results. A spiral grating was fabricated by laser cutting over a stainless steel plate of $0.8 \mathrm{~mm}$ thickness. Fig. 6.10 (a) shows a scheme of the grating designed. The grating aperture was $a=1 \mathrm{~mm}$. The thickness of the scatters was $0.75 \mathrm{~mm}$ (solid parts), leading to a grating aperture of $0.25 \mathrm{~mm}$ (open parts) ${ }^{4}$. The number of turns was $M=20$ and the grating radius was $R=25 \mathrm{~mm}$. The circular spiral plate was placed in front of a ultrasound plane transducer. This source was formed by a plane single element piezoceramic crystal (PZ 26, Ferroperm Piezoceramics, Denmark) mounted in a custom designed steel housing with aperture $R=25 \mathrm{~mm}$, matching the area of the spiral grating. The separation distance between the grating and the source plane was adjusted to $0.5 \mathrm{~mm}$.

\footnotetext{
${ }^{4}$ As pointed before, following Babinet's principle considerations, the scattered field by an structure of thin scatters or thin slits, in terms of the generated beam profile over the axis, is equivalent.
} 
(a)

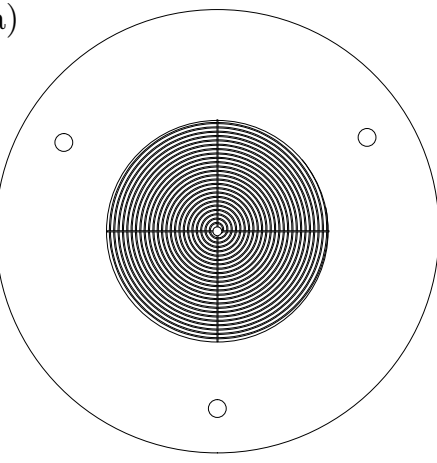

(c)

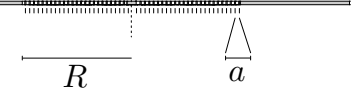

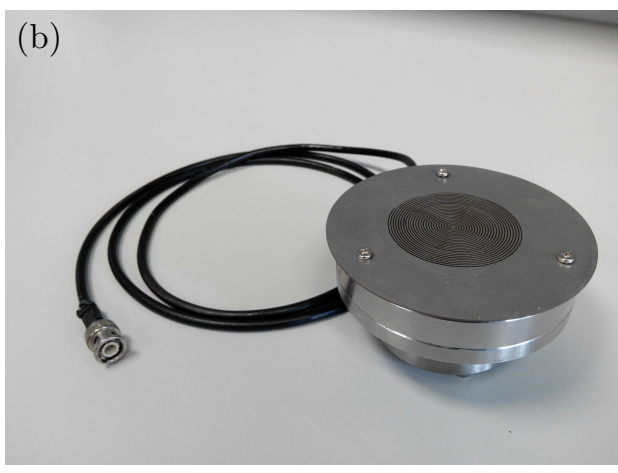

(d)

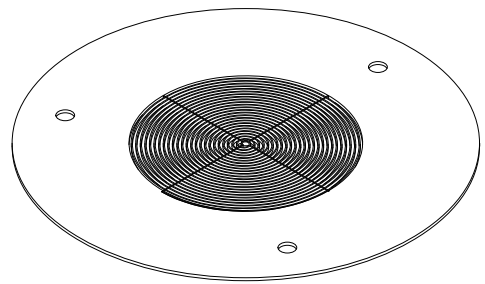

Figure 6.10: (a, c, d) Scheme of the grating used in the experiment. (b) The spiral grating mounted in front of a plane ultrasound transducer.

The transducer was immersed in a degassed and distilled water tank of $(350 \times$ $350 \times 350 \mathrm{~mm}$ ) were during the measurements the temperature was constant at $26^{\circ} \mathrm{C}$. Therefore, the theoretical beam extension distance of the proposed grating lens at the operating frequency was $F=27 \mathrm{~mm}$ and only one diffraction order appear.

The source was driven with a sinusoidal pulse burst of frequency $f_{0}=2.226$ $\mathrm{MHz}$ and 50 cycles using a function generator (14 bits, $100 \mathrm{MS} / \mathrm{s}$, model PXI5412, National Instruments) and a linear RF amplifier (ENI 1040L, 400W, 55dB, ENI, Rochester, NY). The pressure waveforms were acquired with a HNR $500 \mu$ m needle PVDF hydrophone (Onda Corp, CA), and a digitizer (64 MS/s, model PXI5620, National Instruments) were used. A three-axis micropositioning system (OWIS GmbH, Germany) was used to move the hydrophone in three orthogonal directions with an accuracy of $10 \mu \mathrm{m}$ and a National Instruments PXI-Technology controller NI8176 was used to control all the devices. The amplitude frequency response of the hydrophone was compensated in post-processing.

Figure 6.11 summarizes the experimental and numerical results, and the RayleighSommerfeld integration. It can be observed in Fig. 6.11 (a) that the steel grating conform a beam with a zero in its axis, and with a ring shaped maximum surrounding this zero. The experimental beam agrees the analytical Bessel beam presented in Fig. 6.11 (c). On the left column, the phase is shown and, again, 

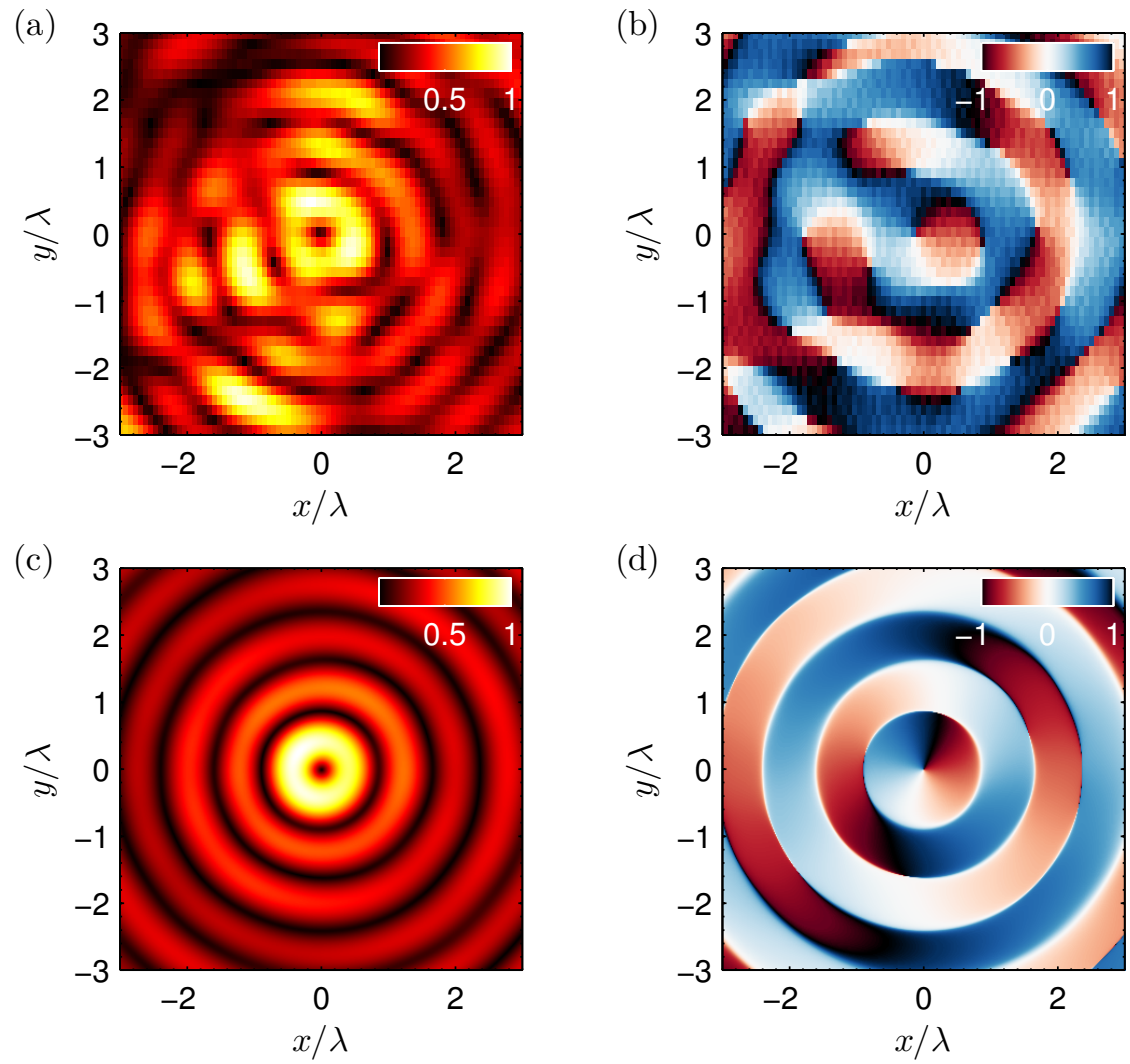

Figure 6.11: (a) Normalized field magnitude and (b) phase observed in the experiment and (c, d) its corresponding analytical estimation using Rayleigh-Sommerfeld integration. Colorbars in $p / p_{\max }$ units for the magnitude and $\phi / \pi$ units for the phase.

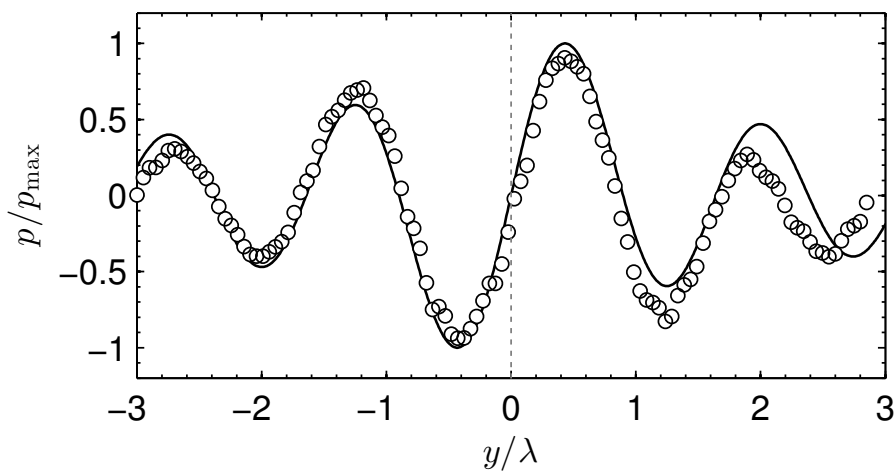

Figure 6.12: Transversal cut of the field observed by (circles) grating experiment and (line) predicted by the HOBB. Vertical line marks the axis were the zero field is expected. 
the generated beam in the experiment shows the correct topological charge of the analytic solution plotted in Fig. 6.11 (d). The characteristic screw-cork dislocation of the field at the center is also observed. Moreover, the phase distribution all over the secondary lateral lobes of the beam also shows, in some degree, the correct phase distribution of the HOBB.

However, some discrepancies can be observed in the experimental measurements. These aberrations are minor and can be produced by the misalignment between the grating and the transducer plane. A detailed transversal field profile is presented in Fig. 6.12. The transversal cut (markers) was selected for an azimuthal angle in the $(y, x)$ plane at -3 degrees, in order to present a good fit with the analytical solution (black line). The traversal profile correctly agrees its corresponding $J_{1}\left(k_{r} r\right)$ first order Bessel function. Minor aberration slightly modifies the lateral lobe amplitude, but the main features of the high order Bessel beam, i.e. its central zero and rotational vortex, are correctly observed by the proposed (and simple) experimental setup.

\subsection{High order Bessel beams generated by $n$-arm spirals}

In the preceding section a synthesis of first order Bessel beams is proposed. The basic principle of formation is the destructive interference trough the axis of a rotating-phase conical-wavefront. The phase rotation over a turn in the cone was $2 \pi$, and the topological charge of the generated vortex was 1 . Here, we extend this idea in order to generate vortex with higher topological charge. Therefore, the phase rotation of the cone must increase for each turn as $n 2 \pi$. Applying same ideas developed previously, Eq. (6.1), we must increase the spiral radius $r$ as $r(\theta)=n b \theta$ in order to increase the rotation of the phase by $n 2 \pi$.

However, if the spiral is constructed in that way, the spacing between turns is increased from $a$ to $n a$, and therefore, higher diffraction grating will appear and energy will spread into a multi focal beam as shown in Fig.6.8. Actually, the higher diffraction grating orders of a spiral generates high order Bessel beam. The beams presented in Fig.6.8 (right) corresponding to the diffraction order $N=2$ were second order Bessel beams. Figure 6.13 shows a more clear example. There, in the left subplot a first order Bessel beam is generated at $a / \lambda=1.2$. On the right, the frequency is increased to match $a / \lambda=7.2$, the beam of the first order is generated far a way from the grating plane. Near the source appear the higher diffraction gratings. The profile of the 7 th diffraction grating order generates a 7th order Bessel beam, where it can be appreciated that the numerical integration correctly agree the analytical estimation using $N=7$. Therefore, HOBBs can be generated from the higher diffraction grating of a simple Archimedes' spiral.

For practical applications it must be desirable to generate a single HOBB 

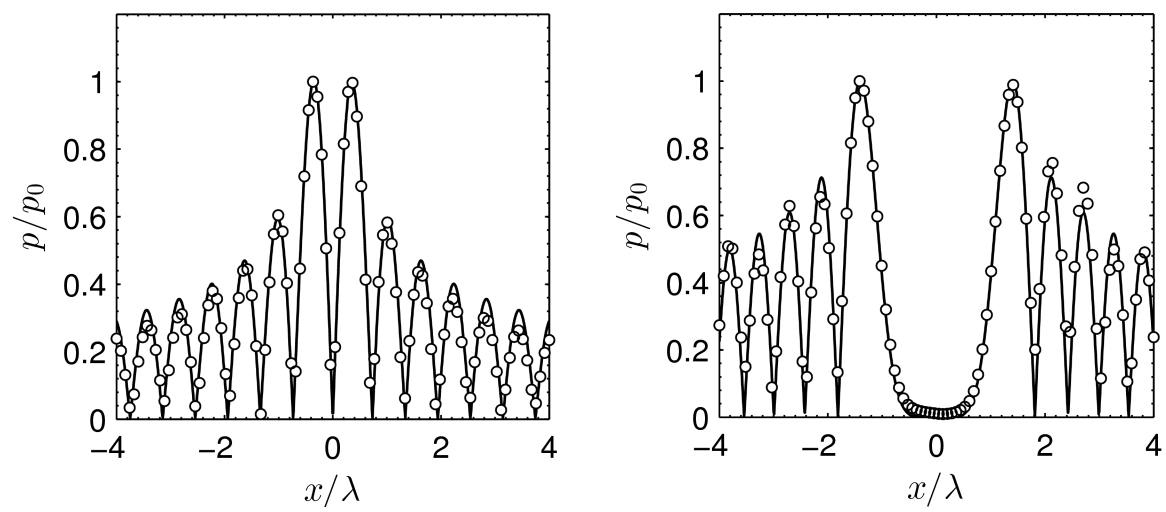

Figure 6.13: Generation of a high order Bessel beam from higher diffraction orders of a simple Archimedes' spiral grating. (continuous line) Analytical solution with (left) $N=1$ and (right) $N=7$, (circles) numerical integration at a distance $z=F(N) / 2$

without any diffraction orders in order to do not spread energy from the main beam to other secondary spots, e.g. in addition to the previously 7 th order Bessel beam of Fig. 6.13, all the inferior order Bessel beams are generated together.

The only way to increase the radius of the spiral as $r(\theta)=n b \theta$, while maintaining the grating spacing between turns, $a$, is to add additional arms to the spiral that fills the space between the stretched new curve. The general expression of $n$-arms spiral is

$$
r_{j}=n b\left(\theta+j \frac{2 \pi}{n}\right) .
$$

Therefore, there exists $n$ curves, each $j$ curve is separated a distance $a$ from its adjacent curve, and each curve start from the origin at different angles, equispaced $2 \pi / n$. Figure 6.14 shows various spirals with increasing number of branches. In the case of $n=2$, it can be observed that the curve for the $j=1$ branch (black spiral) have a growth slope of $\Delta r / \Delta \theta=2 b$. Therefore, the phase rotates at $\Delta \phi=\Delta \theta=2$. Same applies to the second arm, but this arms maintain the separation between turns constant, allowing the generation of the Bessel conical wavefront for the first diffraction order. As Fig. 6.14 shows, other possibilities are also feasible in order to obtain higher spiral arms. The main issue is that when the number of arms increases the aperture between them does not remain constant near the center, when obviously in the limit $n \rightarrow \infty$, the structure becomes completely opaque if it is formed by finite sized scatterers, or in-existent if the grating is formed by finite sized slits.

As long the spacing between spiral arms is kept constant at a distant $a$, the diffraction produced by the grating will create a cone with same angle that for 

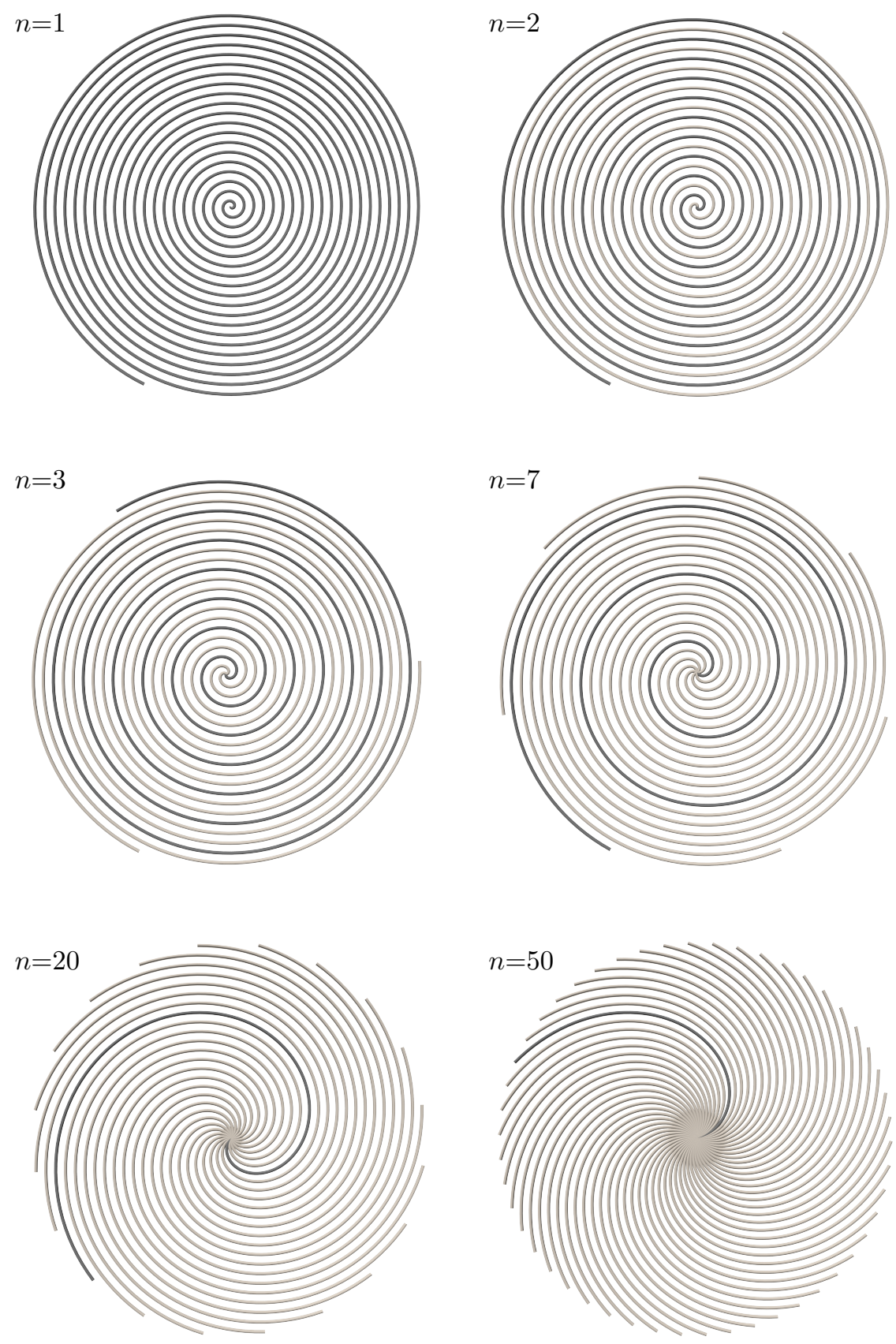

Figure 6.14: Scheme of a variety of $n$-arm spirals. 
the first order Bessel Beams. When this conical wavefront converges to the axis, a Bessel beam is also generated. However, due to the rotation of the phase is increased by a factor of $n$, the corresponding beam is a $n$ th-order Bessel beam. It can be appreciated in the last term of Eq. (6.11), that the topological charge will also be increased by a factor $n$. The number of arms of a spiral directly corresponds to the order of the Bessel beam generated and therefore to the topological charge of the acoustical vortex.

Beam profiles of the generated HOBBs are presented in Figs. 6.15-6.17. The magnitude of the field transversal sections are shown in Fig. 6.15 for $n$-arm spiral gratings of $n=1,2,3,7,20,50$. Here, the grating parameters were selected as $a / \lambda=1.2$ and $\beta=56.4^{\circ}$. First, in Fig. 6.15 we represent a transversal cut of the field measured at $z=F / 2$. As can be seen, the field is zero at the axis of the beam, and the central null spot increases in size according to the number of branches of the spiral. The generated field is nearly zero in a cylindrical volume around the axis: the higher the number of arms of the spiral, the bigger the volume around the axis where the field is nearly zero. Even in the case of a 50 branches spiral, the generated beam profile agrees with a 50th order Bessel beam. In this case, the corresponding central null is generated over a roughly cylindrical volume of $18 \lambda$ diameter (in agreement to Eq. (6.16) and a height of $F$ (in agreement to Eq. (6.12).

The location of the ring shaped lateral lobes, where the field increases to its maximum, also matches the beam profile of the function $J_{n}(2 \pi / a r)$. A close comparison will be presented later. On the other hand, it can be appreciated that the ringed lateral lobes present some oscillations or bumps. They are more evident in the higher order Bessel beams. If the transverse plane where the measurement is done moves from $z=F / 2$ to $z=F / 2+\Delta F$, these oscillation rotate around the axis. Furthermore, the number of oscillations is proportional to the order of the Bessel beam. They can be associated to the geometrical laws of perfect constructive interference around the axis for each arm of the spiral, in analogy to Eq. (6.14). Therefore, this results shows evidence of that the focal $F\left(r_{F}, \theta, z_{F}\right)$ for $n$-arms develop equispaced $n$ helix curves that rotates around the axis.

On the other hand, the phase of the $n$ th-order Bessel beam rotates around the axis forming an acoustic vortex. As can be seen in Fig. 6.16, the topological charge of the beam, i.e. the number of $n 2 \pi$ phase increments in a turn, is proportional to the number of branches. Furthermore, it is in agreement with the $n$-th order phase rotation characteristic HOBBs. These features are specially visible around the ringed areas of the field in which the field is maximum, according to Fig. 6.15.

Figure 6.17 shows the magnitude over the $(x=0)$ plane. The generated beams correctly agrees with the truncated HOBBs. The extension of the minimum at the axis is in all cases $F=X X X \lambda$. Another feature is that in the case of higher $n$, i.e. $n=50$, the extension of the maximum lateral lobe ring is shorter. This is due to the finite size structure, the beam only can be formed in the volume sonificated 
(a)

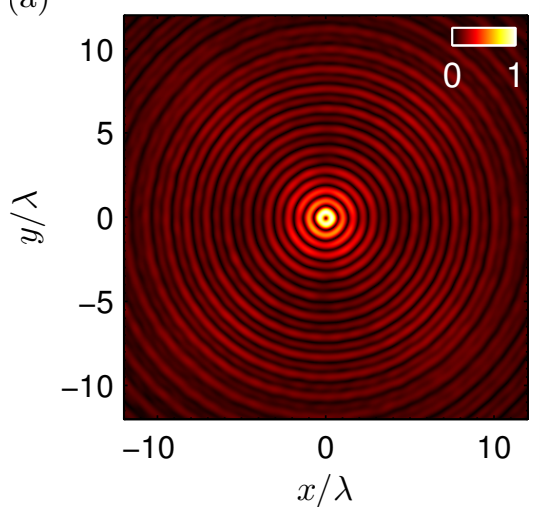

(c)

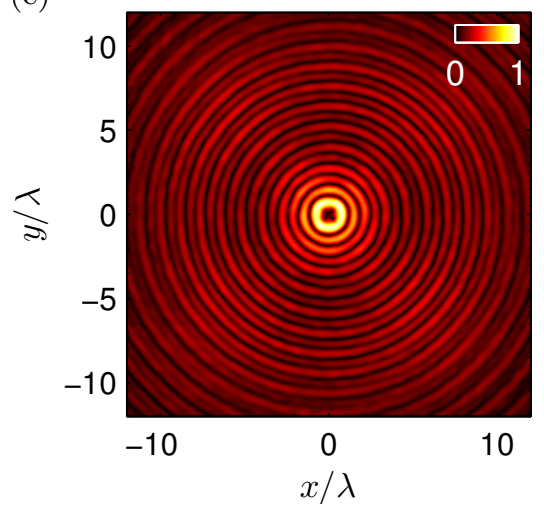

(e)

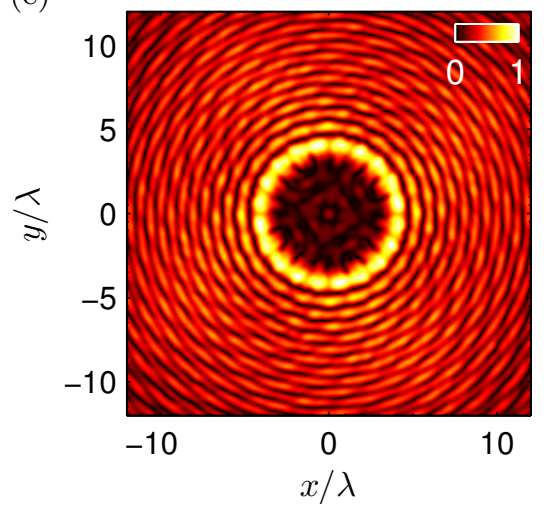

(b)

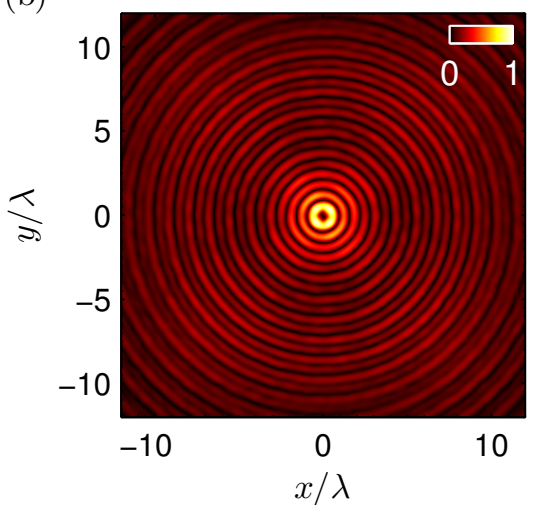

(d)

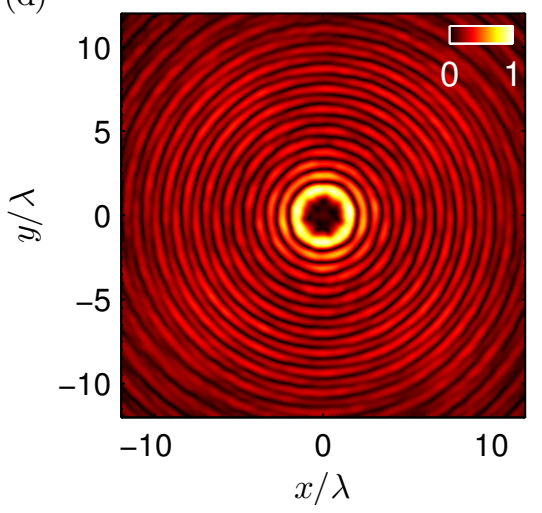

(f)

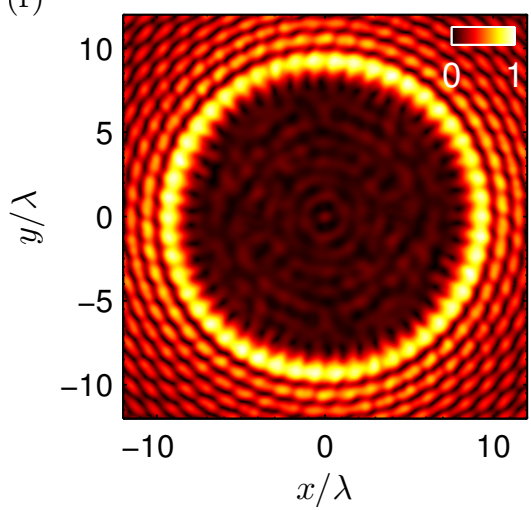

Figure 6.15: Magnitude of the transversal section of the field generated by a $n$-arm spiral grating, from (a-f), $n=1,2,3,7,20,50$. Colorbars in $p / p_{\max }$ units. 
(a)

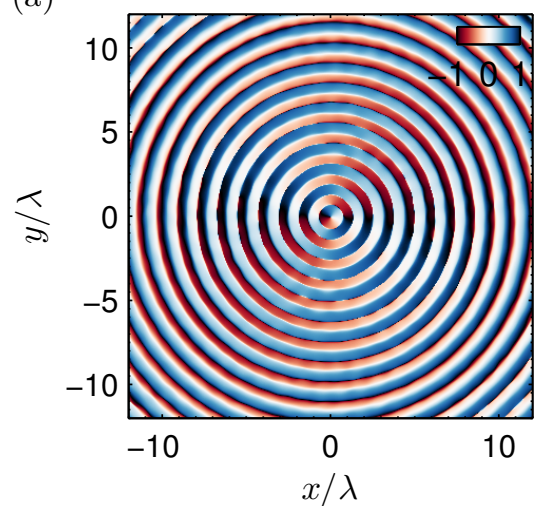

(c)

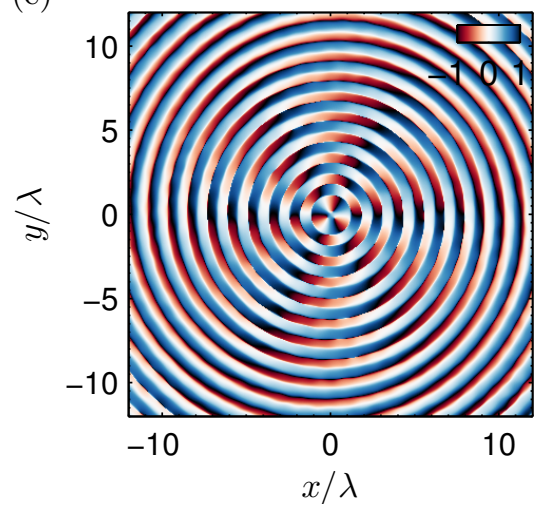

(e)

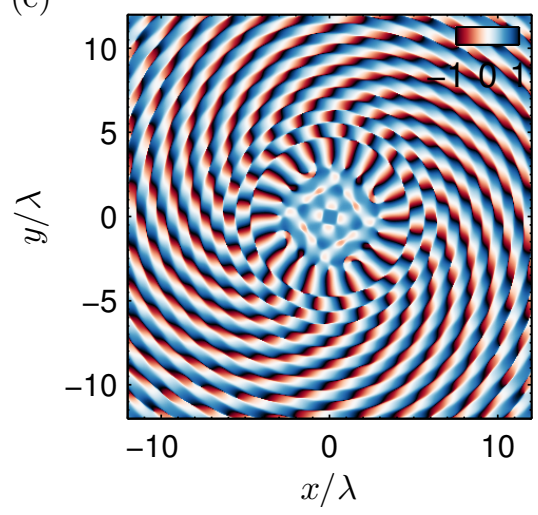

(b)

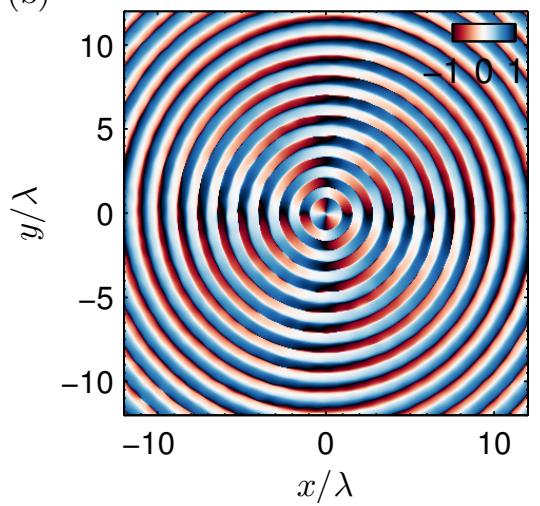

(d)

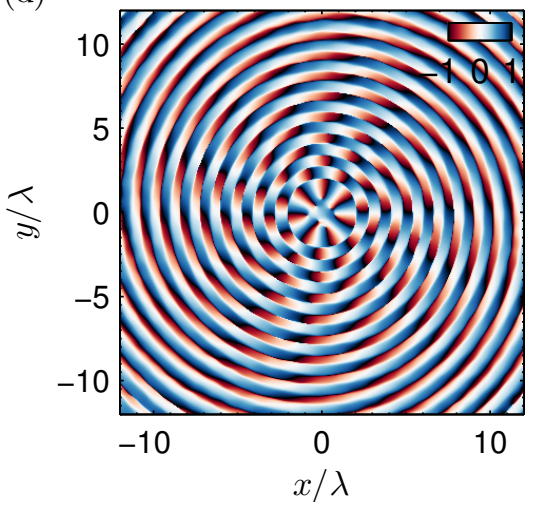

(f)

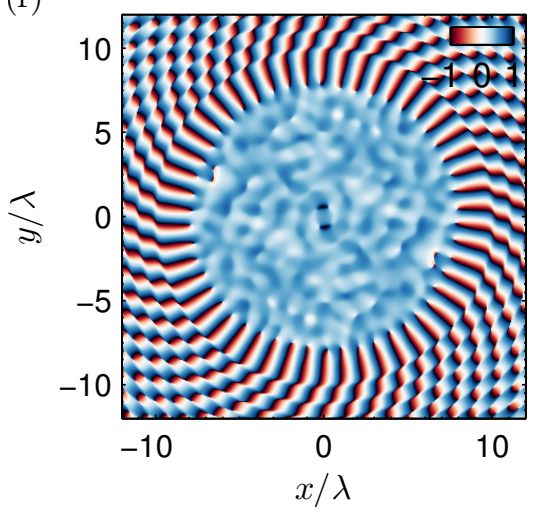

Figure 6.16: Phase of the transversal section of the field generated by a $n$-arm spiral grating, from (a-f), $n=1,2,3,7,20,50$. Colorbars in $\phi / \pi$ units. 

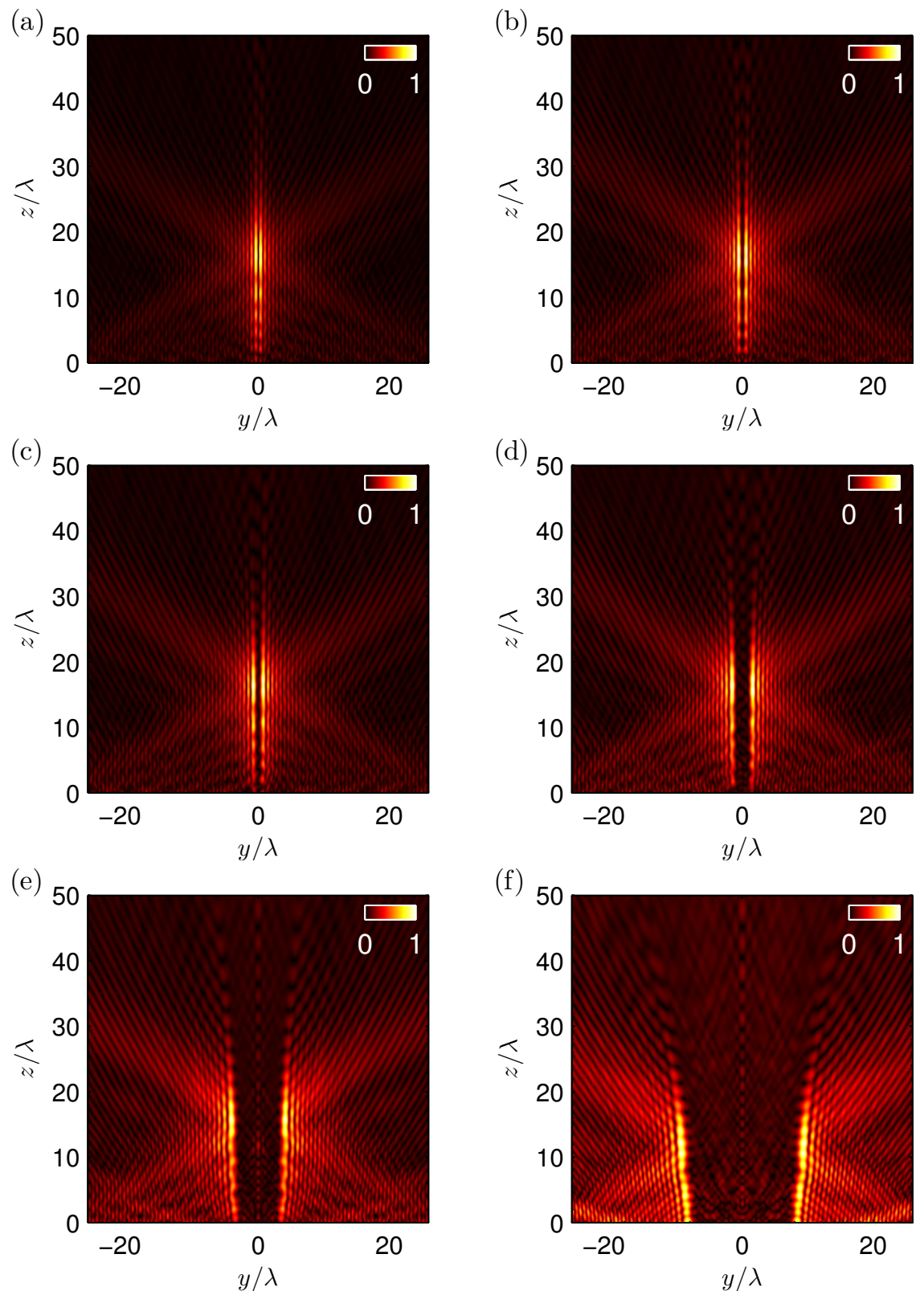

Figure 6.17: Magnitude of the axial distribution of the field for the $n$-arm spiral grating, from (a-f), $n=1,2,3,7,20,50$. Colorbars in $p / p_{\max }$ units. 

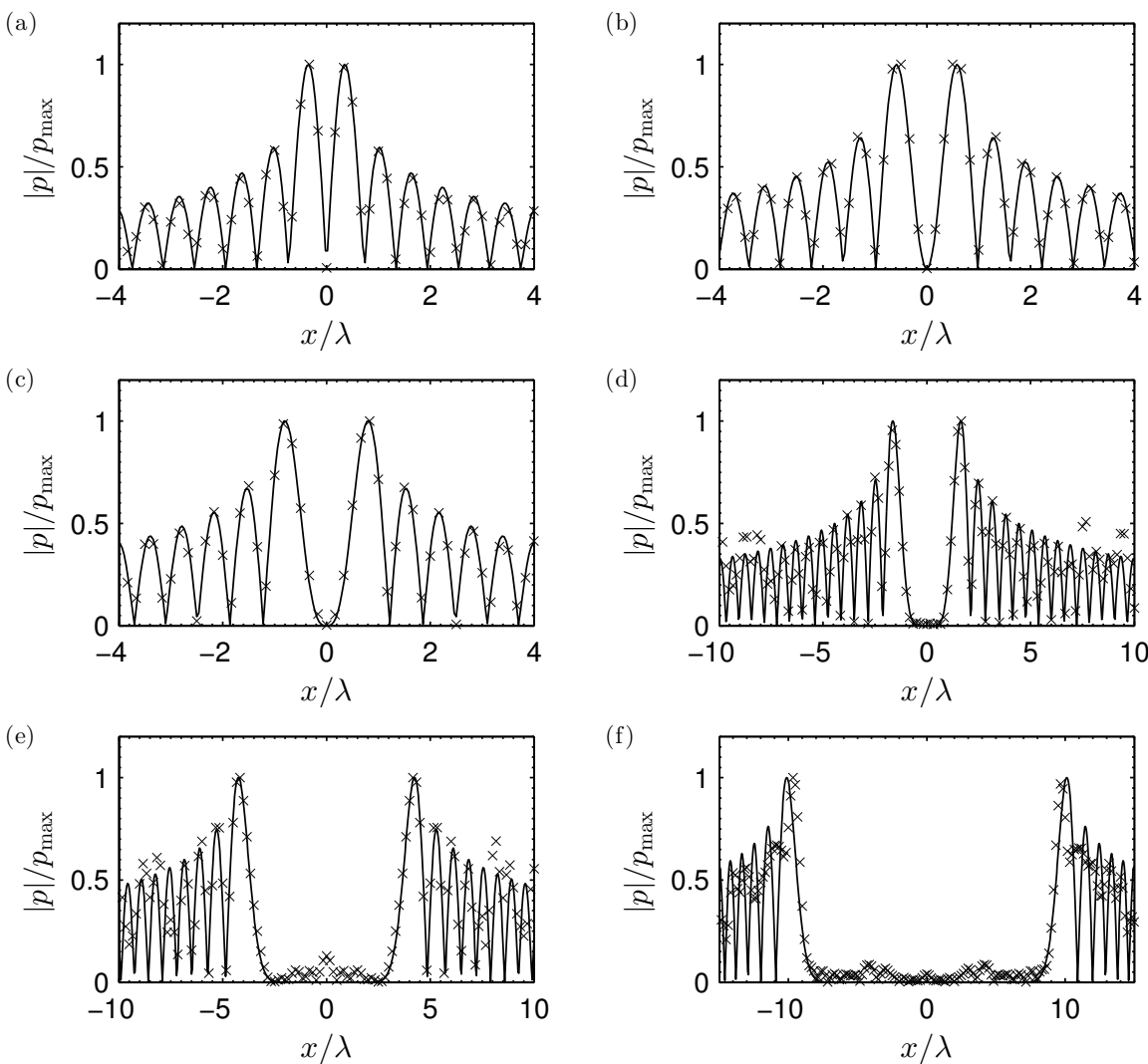

Figure 6.18: Magnitude of a transversal cut of the field for the $n$-arm spiral grating, from (a-f), $n=1,2,3,7,20,50$. (Markers) $k$-space numerical simulation, (continuous line) analytical HOBBs solutions.

by the conical wavefront. This volume extends from $z=0$ to $z=F$ at the axis, but for radially distant points, this distance is reduced. Any case, this limitation does not impedes the correct formation of the truncated Bessel beam, even in the case of $n=50$.

In order to demonstrate the multiple helix that conforms the acoustic vortex, we can represent the isosurface of the equal phase points. The modulations in the field magnitude over the rings of the high order Bessel beam correctly matches the helical shape shown of this reconstructed Fig. 6.21, in analogy with the analytical locus by Eqs. (6.14-6.15). The number of helix arms is equal to the number of spiral arms of the gratings, and its radius grows also withe the order of the Bessel beam generated.

Finally, in order to study these beams in a more realistic configuration, simulations of the full $3 \mathrm{D}$ problem of the same system were performed. In these 

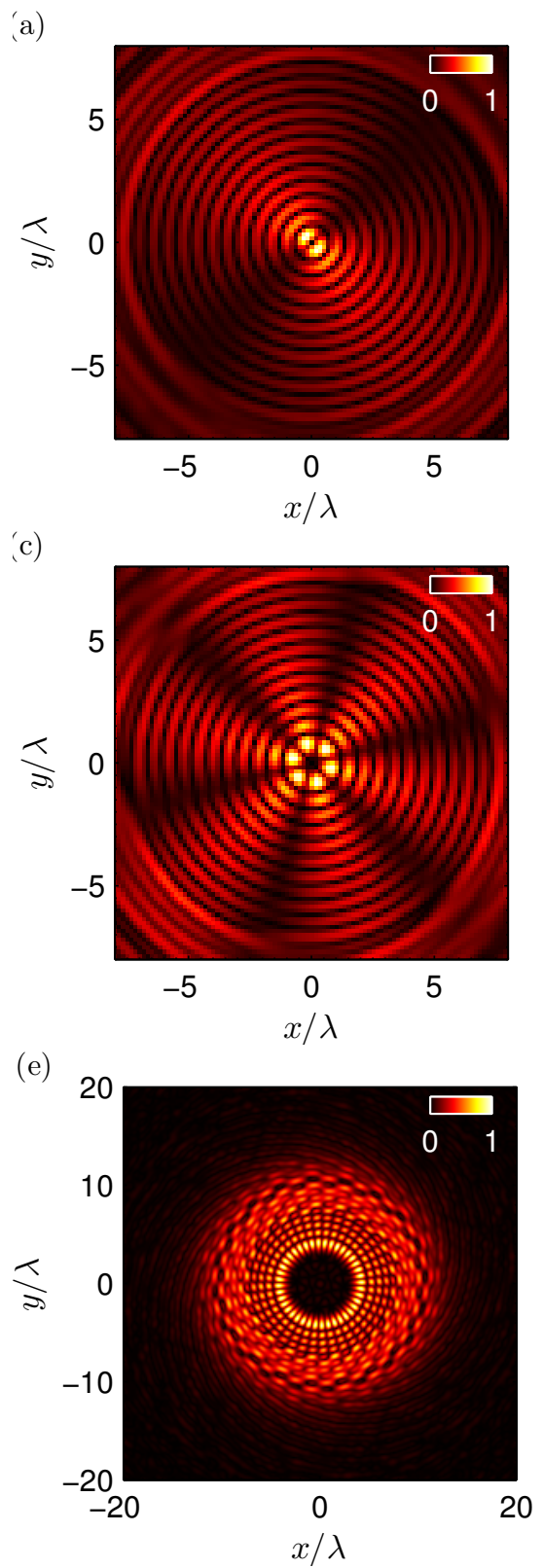
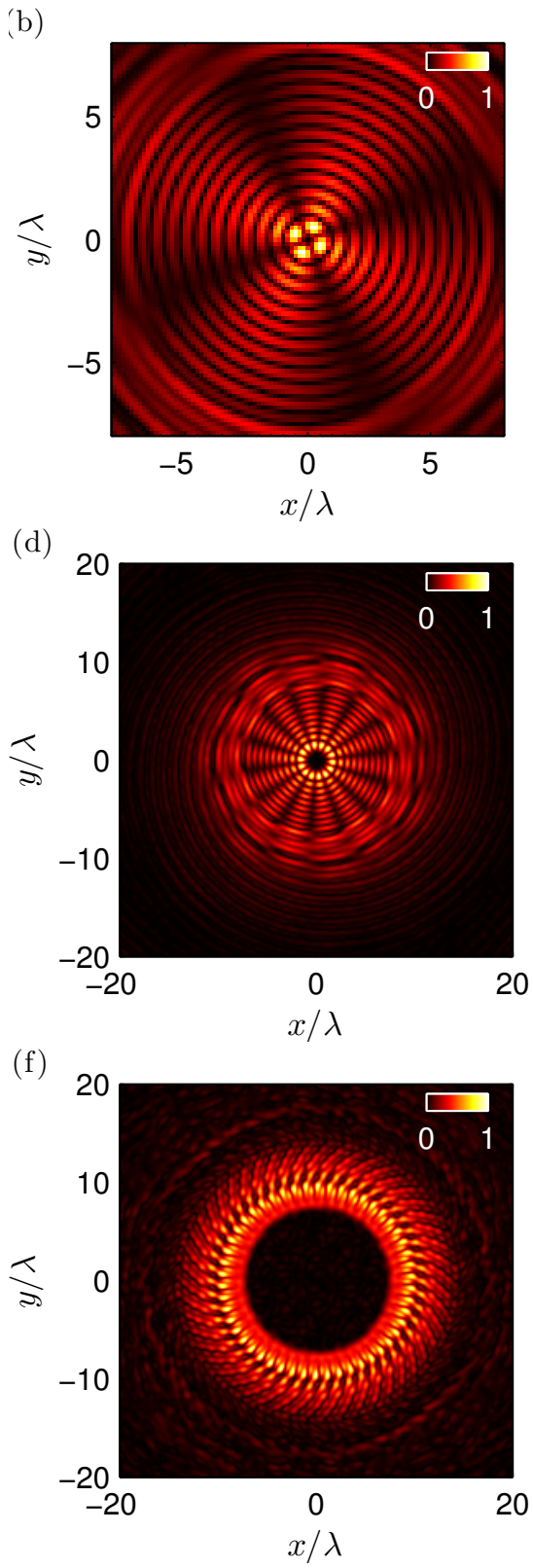

Figure 6.19: Magnitude of the field for the $n$-arm spiral grating, from (a-f), $n=1,2,3,7,20,50$ obtained by $k$-space numerical simulation. Colorbars in $p / p_{\max }$ units. 
(a)

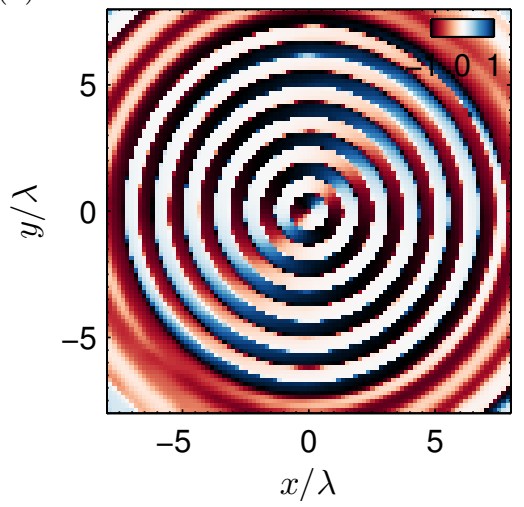

(c)
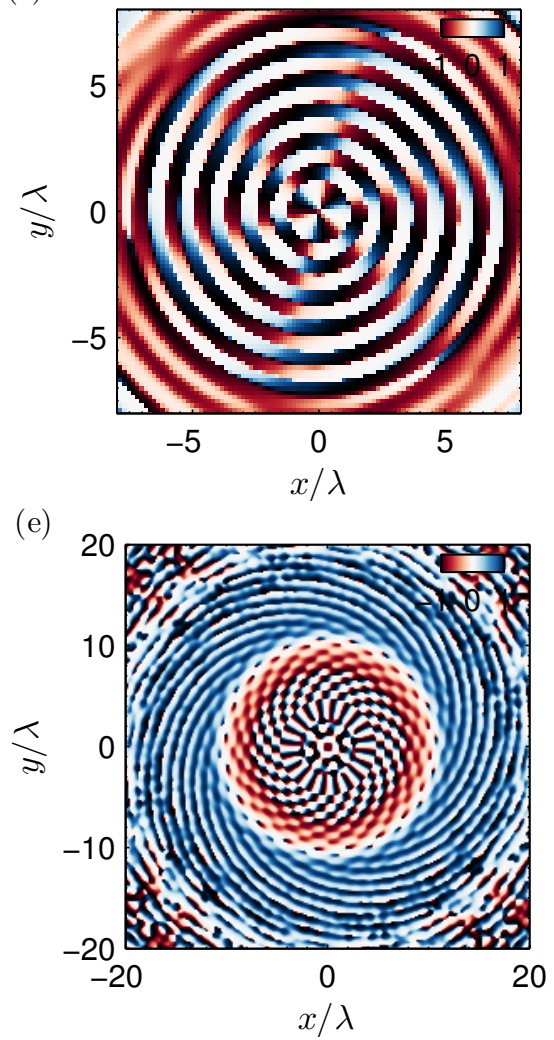

(b)

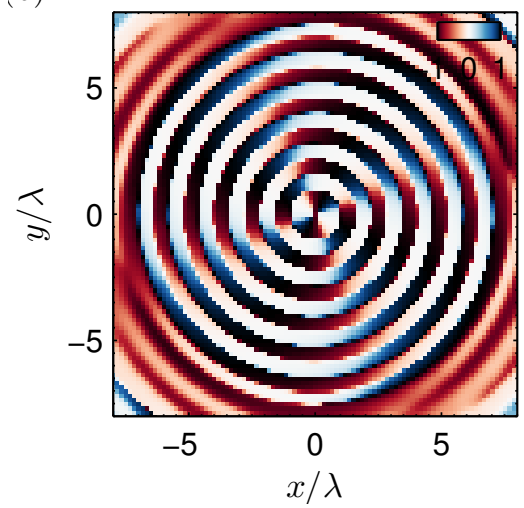

(d)

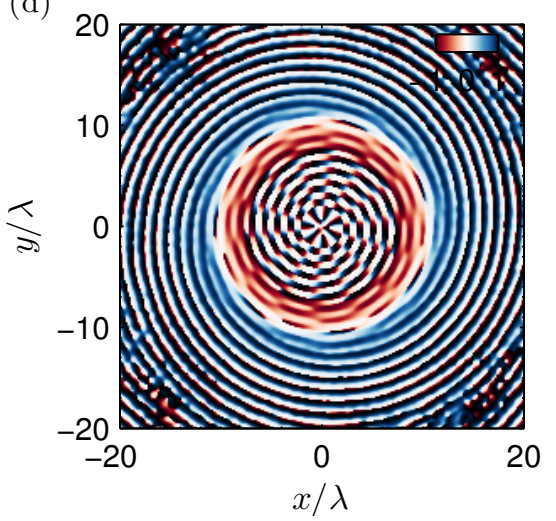

(f)

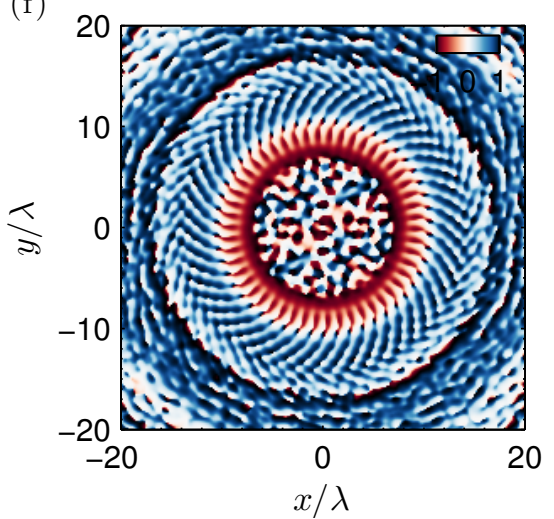

Figure 6.20: Phase of the field for the $n$-arm spiral grating, from (a-f), $n=1,2,3,7,20,50$ obtained by $k$-space numerical simulation. Colorbars in $\phi / \pi$ units. 


\section{$n=1$

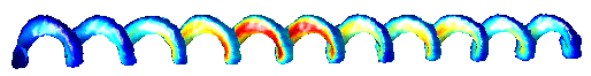

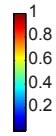 \\ $n=2$
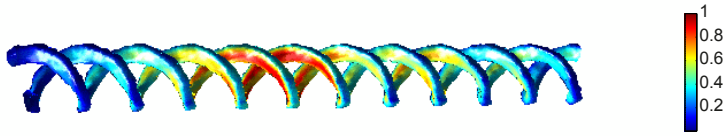 \\ $n=3$

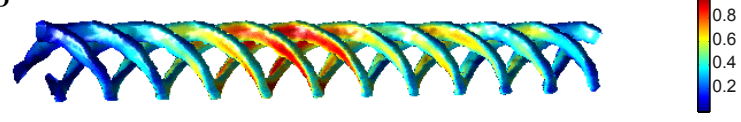

$n=20$

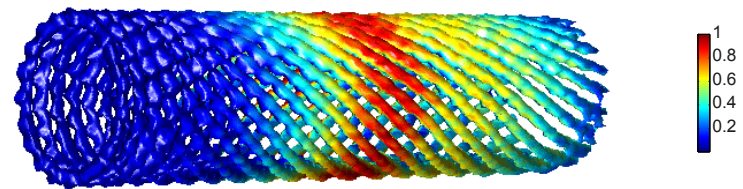

Figure 6.21: Reconstruction of the helical vortex by an isosurface of field points with equal phase. The number of helix arms is equal to the number of spiral arms of the gratings. Colorbars in $p / p_{\max }$ units.

simulations, instead of considering the scatterers perfectly rigid, the grating was included as a stainless steel plate, with same parameters than in the previous experimental setup. Thus, longitudinal wave propagation over the bulk of the steel plane were included. However, shear waves were not included in the simulations for the sake of simplicity. The simulation parameters used for the water media were $c_{0}=1490 \mathrm{~m} / \mathrm{s}$ and $\rho_{0}=1000 \mathrm{~kg} / \mathrm{m}^{3}$ and for the grating plate $c_{0}=6100 \mathrm{~m} / \mathrm{s}$ and $\rho_{0}=7850 \mathrm{~kg} / \mathrm{m}^{3}$. Losses were neglected in both media. Figure 6.18 shows the analytical HOBBs and the simulations of the proposed steel spiral gratings. Excellent agreement is obtained between the theory and the realistic simulations. Due the finite size of the structure, in this cases reduced to $R_{\max }=25 a$, the truncation effect of the Bessel function is more evident, as can be observed in the mismatching of the farther lateral lobes of the simulated beam. Furthermore, the characteristic oscillations of the maxima due to the helix focusing are more evident, as can be observed clearly in Fig. 6.19. It is worth noting here that those maxima are static, at this transversal cut $(z=F / 2)$, but rotates when the measurement is realized in a different plane according a multiple branch helix.

Concerning the vortex phase, the field also rotates with a topological charge equal to the spiral number of arms. Only in the case of $n=50$ the beam is roughly generate. This effect is related to the finite sized aperture grating, where the cone 
barely cover the volume necessary to generates the $18 \lambda$ diameter of the ring shaped beam. However, even in this situation the central dark spot is generated, due to at the axis the conical wavefront always covers the volume for $z<F$. Those results evidence the feasibility of high order Bessel beam generation by spiral grating in realistic configurations.

\subsection{Weakly nonlinear chiral beams}

The nonlinear propagation of acoustic vortex have been studied previously. Most of these studies employs arrays of transducers to electronically modify the phase profile at the source plane. Acoustic vortex beams including shocks has been tested numerically and experimentally, where rotational shock waves have been generated. If nonlinear effects are produced in the propagation path and not near or inside the spiral grating, the propagation of electronically generated beams and the diffraction grating HOBBs proposed here will present high similarities.

We present simulations of the full-wave Eqs $(3.1,3.2,3.3)$. The grating is designed as in previous simulations, where the nonlinearity of the water is introduced by the nonlinear parameter $B / A=5$. Nonlinearity in the bulk of the steel plate is neglected. As Figs (6.22-6.22) shows, nonlinear acoustic vortex are produced in a similar fashion than in the preceding sections. However, in order to correctly account for higher spectral components, the numerical grid have been refined compared to the numerical results presented below. Due to the computation requirements of the resulting $3 \mathrm{D}$ problem, the total size of the domain of integration has to be reduced, and therefore, the total aperture, i.e. the number of turns of the spiral, has been reduced dramatically. In this section, the grating spiral is composed by only by 5 turns. These computational limitation impedes the calculation of higher Bessel beams due to the ring lateral lobes falls out of the domain. Other restriction of the full 3D nonlinear numerical problem is that the number of harmonics is very limited, and therefore, the simulations only can take into account weakly nonlinear propagation.

However, the basic features of the nonlinear vortex up to $n=5$ can be presented. Figure 6.22 shows the magnitude of the first and its second harmonic transversal field distribution. It can be observed that due to the limited aperture grating, the Bessel beam is not correctly formed, but it can be appreciated how the characteristic lateral lobes appears. The zero at the axis roughly appears for the first and third order Bessel-like beams. In the case of $n=5$ cannot be formed correctly. Here, the focalization of each spiral arm that creates $n$ modulations over the lateral maxima is more evident. Again, if the measurement plane is shifted a small increment in $z$ axial direction, those maxima rotates. Thus, the maxima conforms an $n$-helix curve. The second harmonic field generated in propagation also focuses on these maxima. In addition, second harmonic field also present additional modulations in the positions between the first harmonic modulations, 

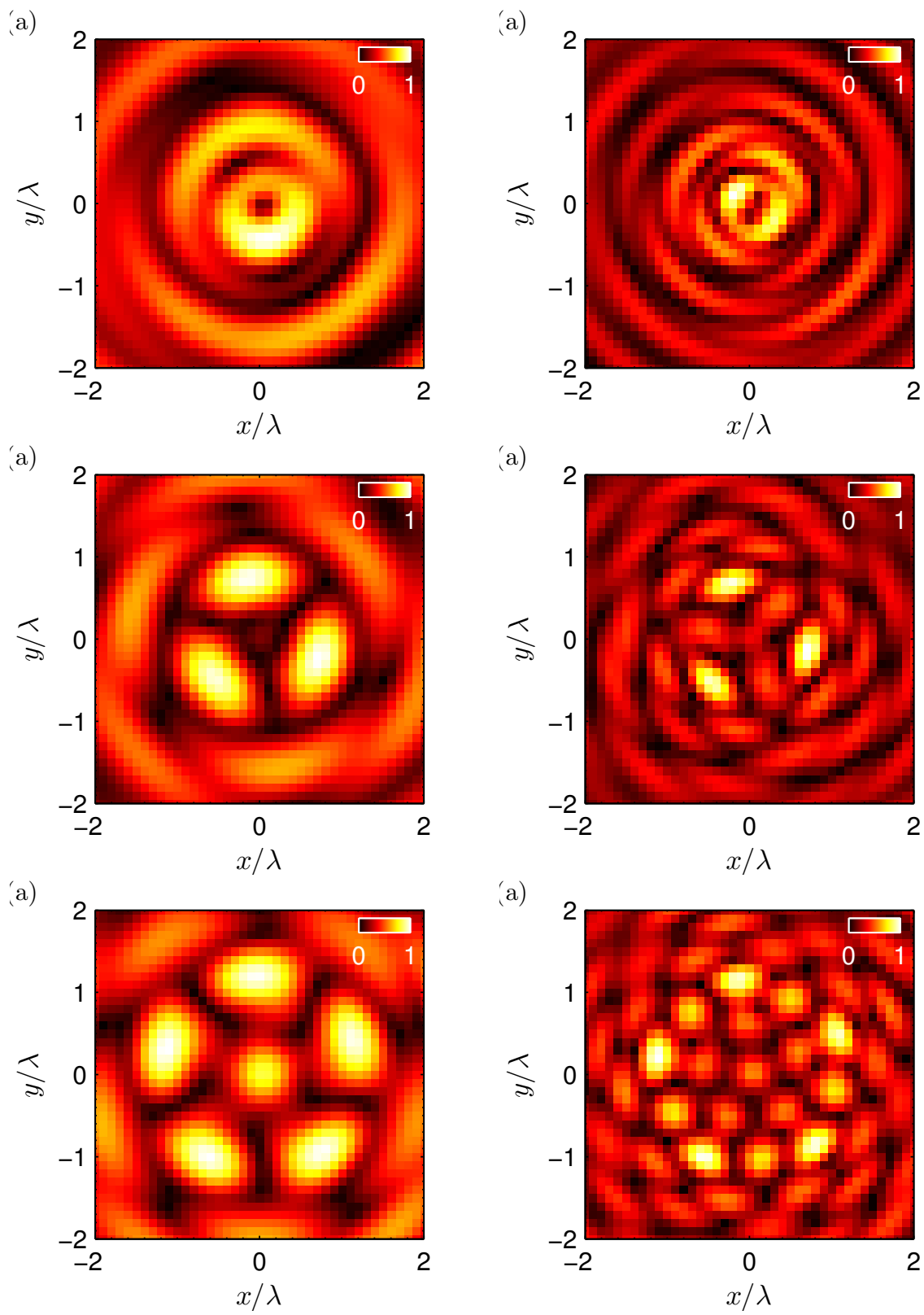

Figure 6.22: Magnitude of the field for the $n$-arm spiral grating, from top to bottom $n=1,3,5$, (left column) first harmonic, (right column) second harmonic. Colorbars in $p / p_{\max }$ units. 

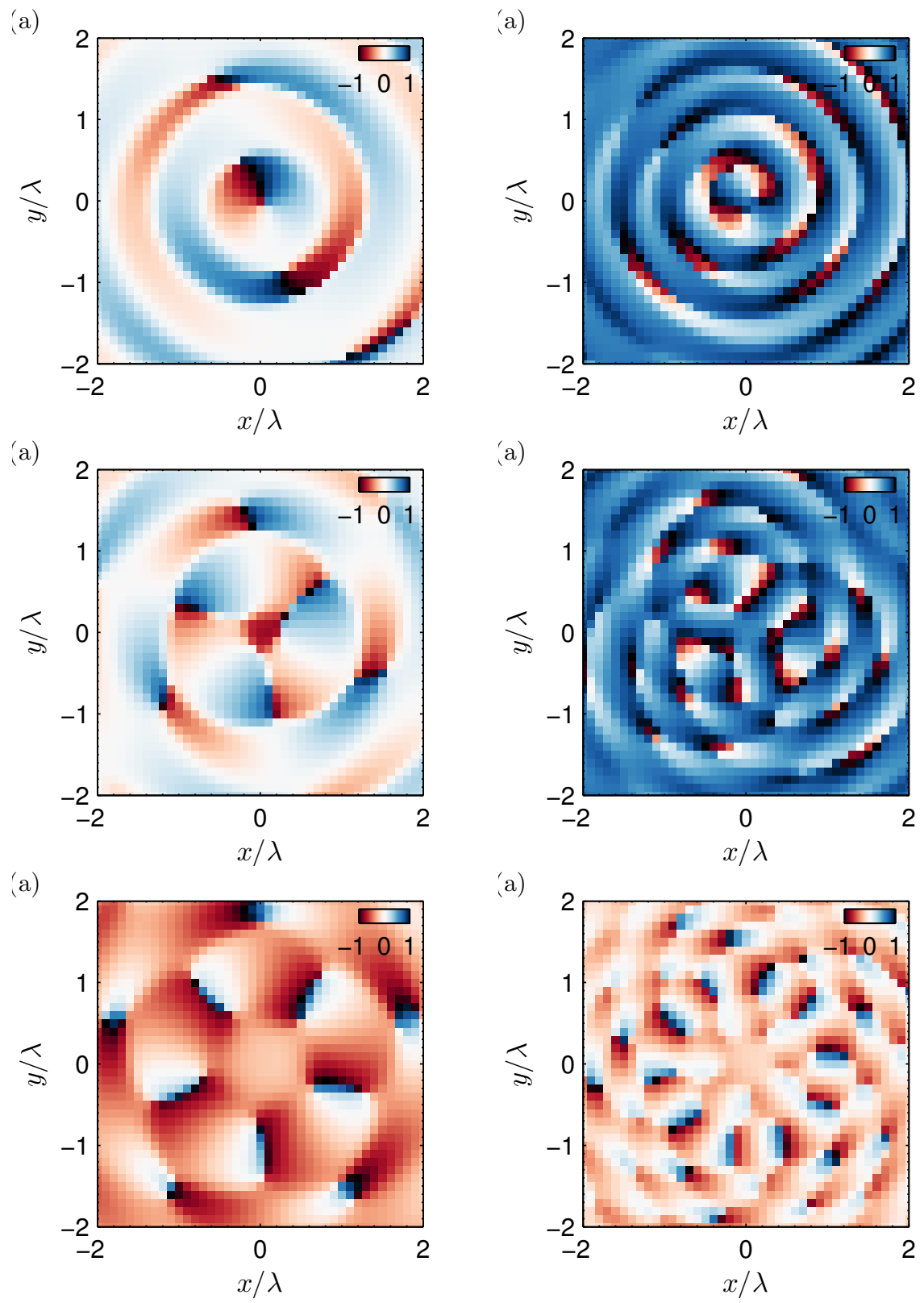

Figure 6.23: Phase of the field for the $n$-arm spiral grating, from top to bottom $n=1,3,5$, (left column) first harmonic, (right column) second harmonic. Colorbars in $\phi / \pi$ units. 
being these last of lower amplitude. Therefore, focalization around the axis of the higher harmonics are also helix shaped: the number of helix curves is the product of the harmonic index and the Bessel order.

Although the magnitude distribution of the field by this truncated grating hardly agrees to an ideal Bessel beam, the rotation of phase distribution is in agreement with the analytical vorticity of HOBBs. Thus, is clearly visible that the first harmonic in the simulations present a topological charge of $n$ for the presented cases. In the case of second harmonic it rotates with topological charge $2 n$. The topological charge is therefore the product of the the harmonic number and the order of the Bessel, i.e. the product of the harmonic and the number of arms. Thus, the conservation law for the ratio between the topological charge of the beam and the generated harmonic order is also observed (Marchiano et al., 2008). In this sense, nonlinearity present a opportunity to increase the topological charge of a generated beams.

\section{Intensity vortex}

One remarkable feature of the generated vortex is the acoustic intensity field. Due to the vortex rotation, the acoustic intensity vector presents, in addition to the axial, a rotational azimuthal component. The existence of this component evidences the possibility of using the HOBBs to transfer angular momentum to the media. Figure 6.24 presents an example of the transversal intensity distribution. The magnitude of the intensity maintains the characteristic ringed shape of the Bessel beam profile, wile the pointing vector of the intensity rotates around the axis.

The transfer of momentum from a wave to the media is closely related to the acoustic radiation force field. In the case of acoustic radiation force generated by scattering processes, i.e. when the gradient of the acoustic energy density is not null due to reflection on surfaces or objects, Bessel beams have been demonstrated high potential to generate interesting configurations for particle manipulation where negative axial radiation forces have been observed. On the other hand, if the gradient of the acoustic energy density is not null due to attenuation processes, there appears also a radiation force field. For plane waves, the acoustic radiation force exerted in the bulk of the lossy media is directly proportional to the intensity vector. The generated beams with acoustic intensity vortex can offer great potential in practical applications that uses acoustic radiation force generated by both, scattering or attenuation processes. The nonlinear acoustic radiation force will be studied in detail in Chapter 9 in the case of lossy media with frequency power law attenuation. 


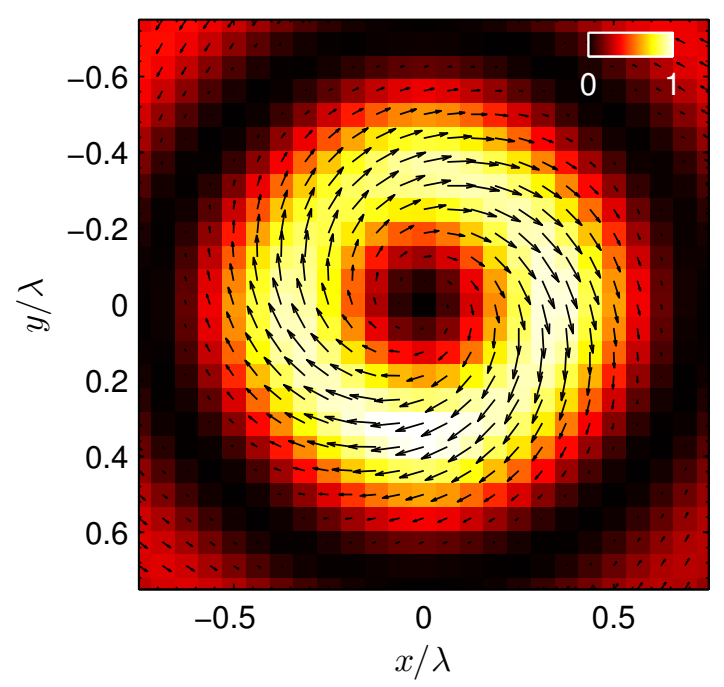

Figure 6.24: Intensity of a first order Bessel-like beam generated by a grating spiral. Colormap in $I / I_{\max }$ units, (arrows) intensity pointing vectors.

\subsection{Conclusions}

We have studied the scattering of Archimedes' spiral gratings, and demonstrated theoretically, experimentally and by numerical simulations that these diffraction gratings generates chiral beams. These beams are basically truncated high order Bessel beams, and are characterized by a null in the field all over it axis. It is demonstrated how the geometrical focal surrounds the axis in an helix shaped curve. It is of special interest the method presented for generation of arbitrary $n$ thorder Bessel beams using regular spiral patters with $n$-arms or arms. It was shown that the order of the Bessel beam is directly the number of arms of the spiral. The radial position of the ringed lateral lobes grows with the order of the Bessel beam, and over it an acoustic vortex is generated. The topological charge of these vortex was also proportional to the number of arms of the spiral. Furthermore, we have demonstrated that another way to generate HOBBs is using higher diffraction orders, but this approach generates also secondary secondary beams along the axis and the energy spreads into all. Finally, The topological charge can be also increased by cumulative nonlinear effects, where the number of the harmonic is proportional to the product between the number of arms and the harmonic index.

The spiral diffraction gratings offers the possibility of dramatically increase the order of the beam by using together the mentioned effects. Thus, a grating made of high number of gratings can radiated with high frequency wave in order to excite superior diffraction orders. Finally, the $i$ th-harmonic will present a topological 
charge of $i n N$, increasing its rotation and the possibility of transferring angular momentum to the media. On the other hand, currently work is being developed applying the technique of period varying grating exposed in Chapter 5 to an spiral configuration in order to achieve focusing of the vortex.

The presented method for synthesize HOBBs presents a high potential in ultrasound particle manipulation techniques and, in general, in acoustic radiation force applications in which the HOBBs have attracted great interest. This method provides the possibility of generation arbitrary order Bessel beams by a passive and extremely cheap device compared to active multiarrays. The generation of acoustic vortex by active means is limited by the amount of elements and its size. In contrast, the beam resolution by the proposed gratings is limited by the size of the scatterers and the space between them. Nowadays, with the increase in the performance of 3D printing and laser cutting techniques, the generation if HOBBs offer far more possibilities than its active counterpart to generate acoustical vortex.

\section{References}

Arlt, J and K Dholakia (2000). "Generation of high-order Bessel beams by use of an axicon". In: Optics Communications 177.1, pp. 297-301.

Bekshaev, A Ya and AI Karamoch (2008). "Displacements and deformations of a vortex light beam produced by the diffraction grating with embedded phase singularity". In: Optics Communications 281.14, pp. 3597-3610.

Brunet, Thomas, Jean-Louis Thomas, Régis Marchiano, and François Coulouvrat (2009). "Experimental observation of azimuthal shock waves on nonlinear acoustical vortices". In: New Journal of Physics 11.1, p. 013002.

Brunet, Thomas, Jean-Louis Thomas, Régis Marchiano, and François Coulouvrat (2010). "Experimental investigation of 3D shock waves on nonlinear acoustical vortices". In: Physics Procedia 3.1, pp. 905-911.

Demore, Christine, Zhengyi Yang, Alex Volovick, Han Wang, Sandy Cochran, Mike MacDonald, and Gabriel Spalding (2011). "A sonic screwdriver: Acoustic angular momentum transfer for ultrasonic manipulation". In: Ultrasonics Symposium (IUS), 2011 IEEE International. IEEE, pp. 180-183.

Durnin, Já, JJ Miceli Jr, and JH Eberly (1987). "Diffraction-free beams". In: Physical Review Letters 58.15, p. 1499.

Gspan, Stefan, Alex Meyer, Stefan Bernet, and Monika Ritsch-Marte (2004). "Optoacoustic generation of a helicoidal ultrasonic beam". In: The Journal of the Acoustical Society of America 115.3, pp. 1142-1146.

Heckenberg, NR, R McDuff, CP Smith, and AG White (1992). "Generation of optical phase singularities by computer-generated holograms". In: Opt. Lett 17.3, pp. 221-223.

Hefner, Brian T and Philip L Marston (1999). "An acoustical helicoidal wave transducer with applications for the alignment of ultrasonic and underwater systems". In: The Journal of the Acoustical Society of America 106.6, pp. 3313-3316.

Jimenez, Noe, Vicente Romero-García, R Picó, A Cebrecos, Víctor José Sánchez-Morcillo, LM Garcia-Raffi, Juan Vicente Sánchez-Pérez, and K Staliunas (2014). "Acoustic Bessel-like beam formation by an axisymmetric grating". In: EPL (Europhysics Letters) 106.2, p. 24005.

Marchiano, Régis, François Coulouvrat, Lili Ganjehi, and Jean-Louis Thomas (2008). "Numerical investigation of the properties of nonlinear acoustical vortices through weakly heterogeneous media". In: Physical Review E 77.1, p. 016605. 
Marston, Philip L (2006). "Axial radiation force of a Bessel beam on a sphere and direction reversal of the force". In: The Journal of the Acoustical Society of America 120.6, pp. 35183524 .

Marston, Philip L (2007a). "Negative axial radiation forces on solid spheres and shells in a Bessel beam". In: The Journal of the Acoustical Society of America 122.6, pp. 3162-3165.

Mitri, FG (2008). "Acoustic scattering of a high-order Bessel beam by an elastic sphere". In: Annals of Physics 323.11, pp. 2840-2850.

Mitri, FG (2009). "Negative axial radiation force on a fluid and elastic spheres illuminated by a high-order Bessel beam of progressive waves". In: Journal of Physics A: Mathematical and Theoretical 42.24, p. 245202.

Mitri, FG, TP Lobo, and GT Silva (2012). "Axial acoustic radiation torque of a Bessel vortex beam on spherical shells". In: Physical Review E 85.2, p. 026602.

Molerón, M., A. Leonard, and C. Daraio (2014). "Solitary waves in a chain of repelling magnets". In: J. Appl. Phys. 115.18, p. 184901.

Nye, JF and MV Berry (1974). "Dislocations in wave trains". In: Proceedings of the Royal Society of London A: Mathematical, Physical and Engineering Sciences. Vol. 336. 1605. The Royal Society, pp. 165-190.

Oemrawsingh, S. S. R., J. A. W. van Houwelingen, E. R. Eliel, J. P. Woerdman, E. J. K. Verstegen, J. G. Kloosterboer, and G. W. 't Hooft (2004). "Production and characterization of spiral phase plates for optical wavelengths". In: Appl. Opt. 43.3, pp. 688-694.

Romero-García, Vicente, R Picó, A Cebrecos, Víctor José Sánchez-Morcillo, and K Staliunas (2013). "Enhancement of sound in chirped sonic crystals". In: Applied Physics Letters 102.9, p. 091906.

Schwarz, Thomas (2013). "Rotation of particles by ultrasonic manipulation". PhD thesis. Diss., Eidgenössische Technische Hochschule ETH Zürich, Nr. 21572, 2013.

Skeldon, KD, C Wilson, M Edgar, and MJ Padgett (2008). "An acoustic spanner and its associated rotational Doppler shift". In: New Journal of Physics 10.1, p. 013018.

Thomas, Jean-Louis and Régis Marchiano (2003). "Pseudo angular momentum and topological charge conservation for nonlinear acoustical vortices". In: Physical review letters 91.24, p. 244302 .

Vasara, Antti, Jari Turunen, and Ari T Friberg (1989). "Realization of general nondiffracting beams with computer-generated holograms". In: JOSA A 6.11, pp. 1748-1754.

Wu, Junru (1991). "Acoustical tweezers". In: The Journal of the Acoustical Society of America 89.5, pp. 2140-2143.

Yoon, Changhan, Bong Jin Kang, Changyang Lee, Hyung Ham Kim, and K Kirk Shung (2014). "Multi-particle trapping and manipulation by a high-frequency array transducer". In: Applied physics letters 105.21, p. 214103.

\section{Publications}

The contents of this chapter have been presented in the following publications:

\section{Journal papers}

- N. Jimenez, V. J. Sanchez-Morcillo, R. Pico, L. Garcia-Raffi, V. Romero-Garcia and K. Staliunas "High-order acoustic Bessel beam generation by spiral gratings" Ultrasonics, In preparation 


\section{Conference proceedings}

- N. Jimenez, V. J. Sanchez-Morcillo, R. Pico, L. Garcia-Raffi, V. Romero-Garcia and K. Staliunas "High-order acoustic Bessel beam generation by spiral gratings" 2015 International Congress on Ultrasonics, Metz, France. May 10-15, (2015) 



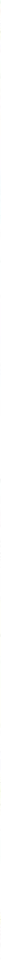

Part II

Nonlinear Acoustic Waves

in Biological Media

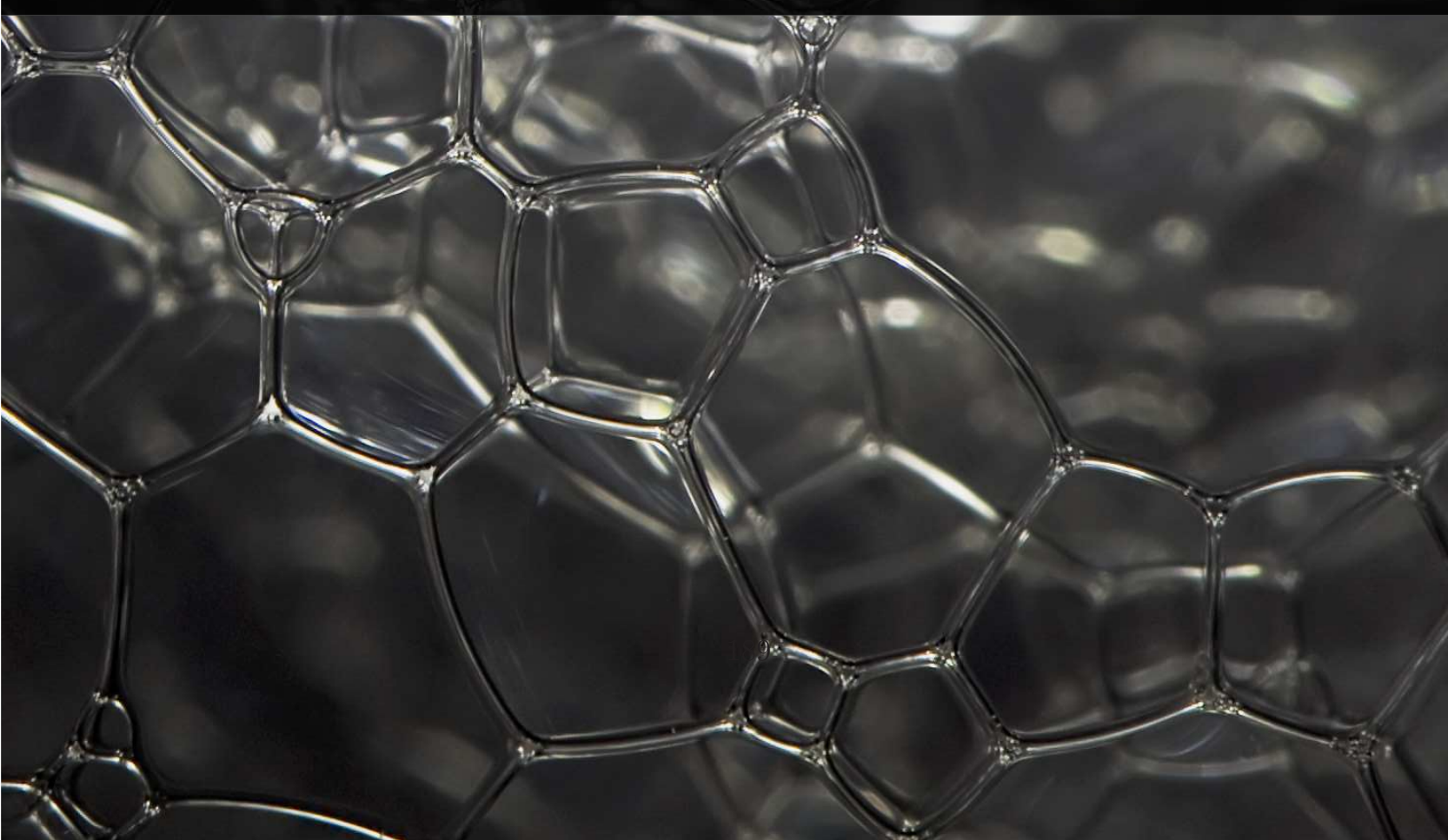





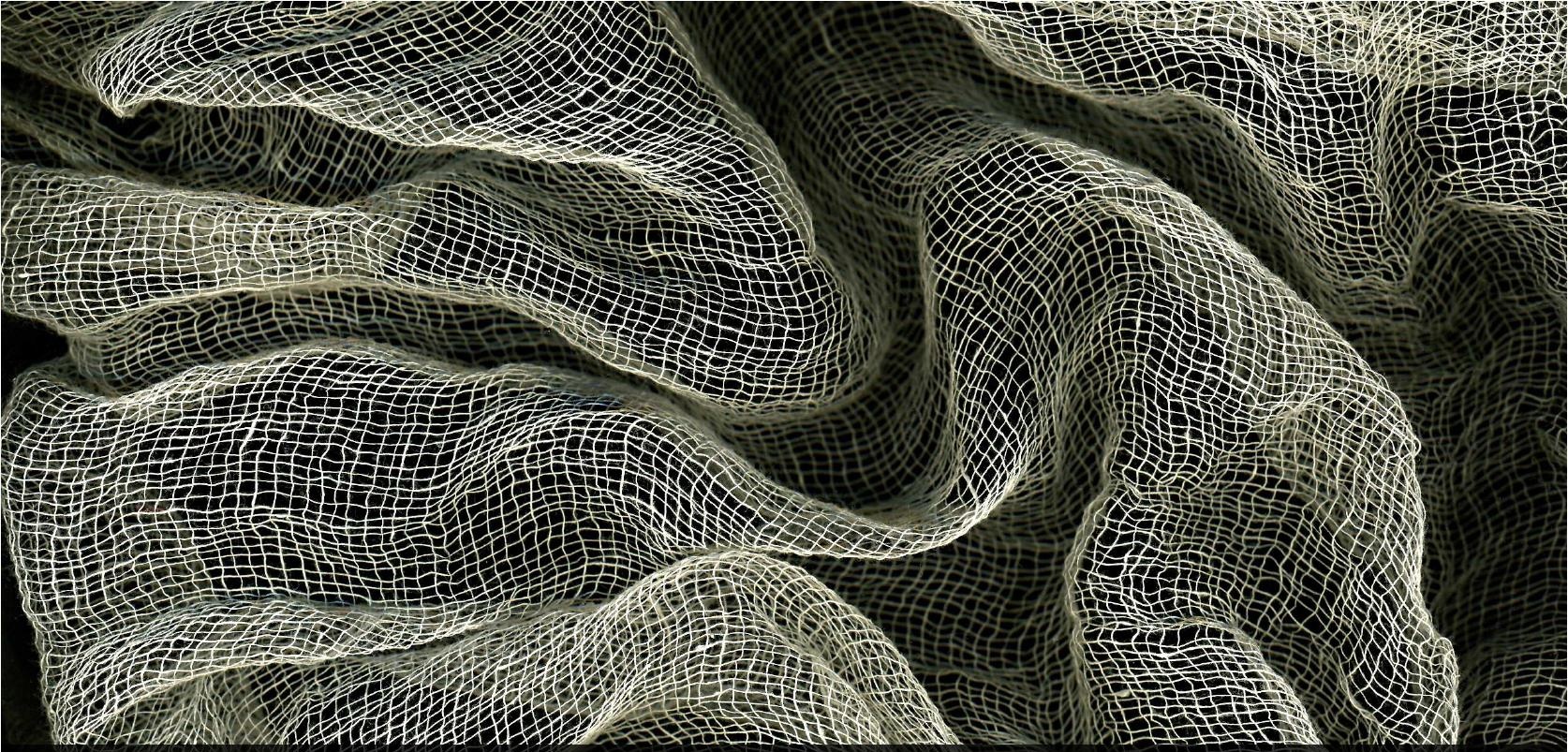

\title{
Chapter 7
}

\section{Time-Domain Nonlinear Acoustic Wave Modelling of Soft-Tissue Media}

\begin{abstract}
A time-domain numerical code based on the constitutive relations of nonlinear acoustics is presented. To model frequency power law attenuation, such as observed in biological media, multiple relaxation processes are included and relaxation parameters are fitted to both exact frequency power law attenuation and empirically measured attenuation data of a variety of tissues. A computational technique based on artificial relaxation is included to correct the non-negligible numerical dispersion of the numerical method and to improve stability when shock waves are present. This technique avoids the use of high order finite difference schemes, leading to fast calculations. The accuracy of the method is discussed by comparison with analytical and $k$-space numerical solutions, and also to focused beam experimental data.
\end{abstract}




\subsection{Introduction}

The propagation of acoustics waves trough tissue for biomedical applications is usually linked to nonlinear propagation (Carstensen et al., 1980). Nonlinear regime is commonly achieved in therapy techniques in which the waves interact with the tissue to achieve some ultrasound bio-effect. On the other hand, most tissues shows very large acoustic attenuation coefficients at interest frequencies (Duck, 1990). Therefore, for most ultrasound imaging techniques the acoustic amplitude necessary to achieve a good signal/noise ratio is high enough to generate weakly nonlinear effects, while, in some applications, nonlinear effects are used as the basis of imaging and therapy techniques.

Thus, accurate prediction of finite amplitude acoustic waves traveling through biological media are essential in developing new therapy and imaging techniques for medical ultrasound applications (Carstensen et al., 1980; Muir et al., 1980). Numerous experimental studies show that attenuation $\alpha(f)$ of biological media exhibits a power law dependence on frequency $\alpha(f)=\alpha_{0} f^{\gamma}$ over the frequency range used in medical applications (Hill et al., 2004; Duck, 1990). Considering an initial monochromatic plane wave traveling through nonlinear media, two opposite effects govern the final wave amplitude: on one hand, higher harmonics appear and their amplitude grows as a consequence of nonlinear progressive wave steepening; and on the other hand, the damping for each harmonic is different, following the above power law. Therefore, differences in the frequency power law model can lead to huge differences in attenuation of higher harmonics, and hence the inclusion of frequency dependence attenuation is critical for correctly predict nonlinear propagation.

\subsubsection{Models of frequency power law media attenuation}

Classical thermo-viscous sound attenuation exhibit a squared frequency dependence, $\gamma=2$ (Pierce, 1989), however the empirically fitted power law for soft tissues typically ranges between $\gamma=(0.6,2)$ (Goss et al., 1979; Duck, 1990; Hill et al., 2004). For most tissues the attenuation shows a dependence that can be modeled by $\gamma$ close to the unity, where the specific value varies from different tissues. Moreover, for some individual examples the local value of $\gamma$ exhibit lower values at low ultrasound frequencies and tends to 2 in the high frequency limit (Hill et al., 2004). Despite the study of the physical mechanism besides this complex frequency dependence is out of the scope of this work, there exist numerous phenomenological approaches for including the observed losses in the acoustic equations (Wismer et al., 1995; Kellya et al., 2009). On the other hand, is common in literature to describe the losses in soft tissues as multiple-relaxation processes (Nachman et al., 1990; Hill et al., 2004), from continuous distribution of relaxation processes (Jongen et al., 1986) to a discrete representation. Thus, relaxation parameters can be 
optimized to fit the observed tissue attenuation, where has been shown that two processes are enough to model tissue attenuation with acceptable accuracy over frequency range covering about 4 octaves (Yang et al., 2005). Moreover, fractional partial differential operators can describe frequency power law attenuation (Caputo, 1967) when inserted into a wave equation. These operators can be included into wave modeling equations by means of time-causal convolutions (Szabo, 1994; Chen et al., 2003), fractional spatial derivatives (Chen et al., 2004) and its causal correction (Treeby et al., 2010), and combined time-space fractional derivatives (Caputo, 1967; Wismer, 2006). The latter operators can be biological motivated and derived from tissue micro-structure using fractal ladders based on networks of springs and dashpots (Kellya et al., 2009). However, these fractional loss operators can be also derived from a continuum of relaxation process (Nasholm et al., 2011), that suggest that the two approaches can be equivalent under certain conditions (Treeby et al., 2012).

\subsubsection{Burgers equation}

The above lossy operators can be included in a variety of nonlinear acoustic wave propagation models and, while analytical solutions are not available for finite amplitude waves in frequency power law media even in simplest configurations, a wide range of numerical methods has been developed. The numerical resolution of the nonlinear constitutive equations in tissue-like medium supposes a difficult problem due to the large size of the region of interest in relation to the size of the acoustic wavelength and the complexity of the model. Simplifying assumptions have been needed in the past for modeling nonlinear propagation in the past. Forward or one-way methods are the simplest way to model progressive nonlinear acoustic waves. The general representation of a causal media response was introduced in the Burgers equation (Blackstock, 1985) leading to the generalized Burgers equation. Thus, by choosing an adequate time function in the convolution kernel the attenuation and dispersion of an specific medium can be accurately modeled. The memory function can be modeled by a discrete sum of relaxation processes (Cleveland et al., 1996), while fractional time derivatives can also be included into the augmented Burgers equation (Ochmann et al., 1993).

\subsubsection{KZK and one-way models}

In order to describe finite amplitude sound beam diffraction effects in a paraxial approximation the well-known Khokhlov-Zabolotskaya-Kuznetsov equation (KZK) (Aanonsen et al., 1984; Lee et al., 1995) can be used. The KZK model can be extended for accounting for tissue attenuation by including relaxation (Cleveland et al., 1996; Yang et al., 2005) or fractional derivatives (Prieur et al., 2011). The KZK equation is commonly solved by a split operator techniques (Lee et al., 1995) that allows the frequency domain representation of the progressive wave. In this 
way, the inclusion of tissue attenuation can be achieved efficiently in frequency domain (Khokhlova et al., 2006). Efficient and fast numerical solutions of the KZK model in power law media are also feasible under the assumption of Gaussian representation of the transverse field for each harmonic (Soneson et al., 2007). To overcome the validity of the parabolic approximation (i.e. when dealing with large aperture focused sound sources or modeling sound field near the acoustic source) many approaches has been proposed (Varslot et al., 2005). Also other phenomenological one-way approximations of finite amplitude sound beams has been developed (Christopher et al., 1991; Tavakkoli et al., 1998) and extended including tissue attenuation (Zemp et al., 2003). Furthermore, a numerical solution of the one-way version of the Westervelt equation (Yuldashev et al., 2011) has been also proposed.

\subsubsection{Full-wave Westervelt type equations}

Tissue inhomogeneity can be modeled in these one-way models (Jing et al., 2007), like transmission though tissue layers with refraction, but they do not take into account backscattering and multiple reflections. More realistic models, e.g. those accounting for scattering from internal tissue structures, are based on the Westervelttype full-wave equations. Finite-Differences in Time-Domain methods (FDTD) can be applied to obtain explicit numerical schemes that account for nonlinear propagation and temperature rising in thermo-viscous media (Hallaj et al., 1999), where the inclusion of temperature-dependent sound speed changes can be also modeled (Hallaj et al., 2001). Tissue attenuation can be achieved in the Westervelt nonlinear wave equation by including multiple relaxation processes (Pinton et al., 2009) with optimized relaxation parameters. The Westervelt equation can be solved also by the Iterative Nonlinear Contrast Source (INCS) (Huijssen et al., 2010) where power law attenuation is modeled as a compliance relaxation function (Demi et al., 2011; Verweij et al., 2013). Other approach to include tissue losses in the Westervelt equation is to solve the model in $k$-space and directly include tissue looses in spatial frequency domain (Jing et al., 2012). This full-wave equation has been validated for strongly focused sources (Jing et al., 2011). However, due to the assumptions taken in the derivation of the Westervelt equation, the accuracy of this model is limited in practical situations as $(i)$ the modeling of rigid boundaries where the thermo-viscous boundary layer effects are not-negligible, i.e. in general case where the particle velocity becomes rotational, (ii) situations where the second order Lagrangian density of acoustical energy not vanish, i.e. where plane progressive waves does not exist and the acoustic field becomes complex due to multiple scattering, reverberation or resonances, (iii) situations where the equilibrium-state particle velocity is not null, including the self generation of acoustic streaming. See Ref. Hamilton et al. (1998b) Chap. 3 for further discussion.

In this way, a physical model based on the constitutive relations of nonlinear 
acoustics include a more complete description of the nonlinear acoustic phenomena over arbitrary boundary conditions, i.e. the time-space relations between the involved acoustic magnitudes remains exact.

\subsubsection{Full-wave constitutive relations}

The recent development of computational capacity has made possible to consider the full constitutive relations (i.e. without the assumptions discussed above). Thus, for small-amplitude acoustic waves, the linearized pressure-velocity formulation of constitutive relations in inhomogeneous media was solved by means of Finite-Differences in Time-Domain (FDTD) methods with frequency independent losses by Manry et al. (Manry et al., 1996), or using two-step MacCormack finitedifferences scheme by Mast et al. (Mast et al., 1997). Also, relaxation processes can be included in finite difference methods in an efficient way in order to model tissue attenuation and dispersion (Yuan et al., 1999). Furthermore, $k$-space numerical methods have also been applied to solve the linearized first order equations in lossless inhomogeneous media (Mast et al., 2001). In order to account for soft tissue losses, the computational solution of the fractional Laplacian by $k$-space spectral methods have demonstrated to be extremely efficient due to the spatial frequency domain representation of the acoustic field (Treeby et al., 2010).

In the case of nonlinear constitutive relations models, the evolution of the acoustic magnitudes have been simulated in time-domain by means of finite differences schemes such as Dispersion Relation Preserving method (DRP) in ideal fluids and axisymmetric domains (Ginter et al., 2002). Thermo-viscous losses in finite-differences methods have been widely used, see Sparrow et al. (Sparrow et al., 1991). In order to introduce tissue attenuation in the governing equations time-dependent fractional derivatives can be included by convolutional operators. Thus, in Ref. Liebler et al. (2004) an efficient method has been presented, but although the memory requirements can be strongly reduced compared to direct convolutions the algorithm employs up to ten auxiliary fields and a memory buffer of three time steps. Furthermore, construction of specific causal memory functions that models soft tissue attenuation and dispersion in Navier-Stokes equations is also possible (Lobanova et al., 2014), but certain time history must be stored in memory and in this case the computational domain was restricted to one dimensional propagation.

In order to overcome those numerical limitations, recently $k$-space and pseudospectral numerical methods have been applied to constitutive relations in nonlinear regime to solve fractional Laplacian operators efficiently (Treeby et al., 2012). Furthermore, in the case of domains of hundreds of wavelengths, when the cumulative phase error due to numerical dispersion of standard finite-difference schemes can not be neglected, those spectral numerical methods have reported an improvement in accuracy of the numerical solution. This two factors, i.e. the negligible numerical dispersion and the efficient resolution of fractional Laplacian operators, have 
led the spectral methods to be widely used in practical applications. However, their main limitation is that the implementation of natural space discontinuities due to tissue layers or rigid boundary conditions leads to errors in the reconstruction of the spectral information due to the poor convergence of Fourier series at jumps, i.e. the well-known Gibbs oscillations. Preventing this kind of errors is typically achieved by filtering the spatial spectrum (Jing et al., 2012), so the theoretical spatial minimum sampling of two point per wavelength becomes larger. In addition, these errors propagate globally and affect to the accuracy all over the domain, in contrast with locally propagating errors in finite differences methods. On the other hand, taking into account the spatial discontinuity due to symmetry boundary condition, axisymmetric domains becomes not feasible by standard $k$-space methods, and full 3D domains must be employed even for axisymmetric configurations. Those errors can be prevented by means of the recently developed Fourier Continuation (FC) method (Albin et al., 2012). However, the discontinuities formed due to shock propagation are still not solved by FC methods and other additional numerical treatment must be applied for correctly describe shock formation, e.g. intensive computations by high order accurate weighted essentially non-oscillatory schemes (FC/WENO) (Shahbazi et al., 2011). Unlikely, the computational times increases by using those intensive computational techniques and the multiresolution analysis to detect discontinuities in the domain.

The aim of the present chapter is to present a generalization of the constitutive relations of nonlinear acoustics including multiple relaxation processes in a nonconvolutional formulation that allows the time-domain numerical solution by an explicit finite differences numerical scheme. Frequency power law attenuation based in relaxation have been applied in the same way than it has been applied to generalized Burgers equation (Cleveland et al., 1996), Khokhlov-ZabolotskayaKuznetsov (KZK) model (Cleveland et al., 1996; Yang et al., 2005) and Westervelt equation (Pinton et al., 2009). The relaxation parameters have been fitted to both exact frequency power law attenuation and empirically measured attenuation of a variety of tissues that does not fit an exact power law. Two processes have been enough to model tissue attenuation with acceptable accuracy over a frequency range covering about 4 octaves, as it was demonstrated by Yang et al. (Yang et al., 2005). A numerical technique based on artificial relaxation is included to control the non-negligible numerical dispersion of the FDTD method and improve stability when shock waves are present in the solution. The method includes backscattering and arbitrary propagation direction of finite amplitude beams, and can be specially suitable in axisymmetric configurations where the computational resources for full 3D $k$-space methods are prohibitive. 


\subsection{Generalized nonlinear acoustics model for mul- tiple relaxation media}

\subsubsection{Full-wave modeling}

The principles of mass and momentum conservation lead to the main constitutive relations for nonlinear acoustic waves, which for a fluid can be expressed as (Naugolnykh et al., 1998)

$$
\frac{\partial \rho}{\partial t}=-\nabla \cdot(\rho \mathbf{v})
$$

and

$$
\rho\left(\frac{\partial \mathbf{v}}{\partial t}+\mathbf{v} \cdot \nabla \mathbf{v}\right)=-\nabla p+\eta \nabla^{2} \mathbf{v}+\left(\zeta+\frac{\eta}{3}\right) \nabla(\nabla \cdot \mathbf{v})
$$

where $\rho$ is the total density field, $\mathbf{v}$ is the particle velocity vector, $p$ is the pressure, and $\eta$ and $\zeta$ are the coefficients of shear and the bulk viscosity respectively. The acoustic waves described by this model exhibit viscous losses with quadratic power law dependence on frequency. In order to include a power law frequency dependence on the attenuation, a multiple relaxation model will be added into the time domain equations.

The basic mechanism for energy loss in relaxing media is the appearance of a phase shift between the pressure and density fields. This behavior is commonly modeled as a time dependent relation at the fluid state equation, that for a fluid retaining the material nonlinear effects up to second order an be expressed as (Naugolnykh et al., 1998; Rudenko et al., 1977):

$$
p=c_{0}^{2} \rho^{\prime}+\frac{c_{0}^{2}}{\rho_{0}} \frac{B}{2 A} \rho^{\prime 2}+\int_{-\infty}^{t} G\left(t-t^{\prime}\right) \frac{\partial \rho^{\prime}}{\partial t} d t,
$$

where $\rho^{\prime}=\rho-\rho_{0}$ is the density perturbation over the stationary density $\rho_{0}, B / A$ is the nonlinear parameter, $c_{0}$ is the small amplitude sound speed, and $G(t)$ is the kernel associated with the relaxation mechanism. The first two terms of the right hand side of Eq. (7.3) describe the instantaneous response of the medium, where the convolutional third term accounts for the "memory time" of the relaxing media. Thus, by choosing an adequate time function for the kernel $G(t)$ the model can present an attenuation and dispersion response that fits the experimental data of the heterogeneous media. However, the direct resolution of the constitutive relations (7.1-7.3) in this integral form is a complex numerical task due to the convolutional operator. Thus, instead of describe $G(t)$ with a specific time domain waveform, the response of the heterogeneous medium can be alternatively described by a sum of $N$ relaxation processes with exponential time dependence 
as:

$$
\int_{-\infty}^{t} G\left(t-t^{\prime}\right) \frac{\partial \rho^{\prime}}{\partial t} d t=\sum_{n=1}^{N} G_{n} * \frac{\partial \rho^{\prime}}{\partial t},
$$

with the $n$-th order relaxation kernel expressed as

$$
G_{n}(t)=\eta_{n} c_{0}^{2} \mathrm{e}^{\frac{-t}{\tau_{n}}} H(t)
$$

where $H(t)$ is the Heaviside piecewise function $H(t<0)=0, H(t>0)=1$, $\tau_{n}$ is the characteristic relaxation time and $\eta_{n}$ the relaxation parameter for the $n$-th order process. This last dimensionless parameter controls the amount of attenuation and dispersion for each process as $\eta_{n}=\left(c_{n}^{2}-c_{0}^{2}\right) / c_{0}^{2}$, where $c_{n}$ is the sound speed in the high frequency limit associated to $n$-th order relaxation process, also known as the speed of sound in the "frozen" state (Pierce, 1989). In order to describe relaxation without the need of including a convolutional operator, we shall define a state variable $S_{n}$ for each process as

$$
S_{n}=\frac{1}{\tau_{n}} G_{n} * \rho^{\prime}
$$

Thus, using the convolutional property $\frac{\partial}{\partial t}\left(G(t) * \rho^{\prime}(t)\right)=\frac{\partial G(t)}{\partial t} * \rho^{\prime}(t)=$ $G(t) * \frac{\partial \rho^{\prime}(t)}{\partial t}$, the time derivative of the relaxation state variable obeys the following relation for the $\mathrm{n}$-th order process:

$$
\frac{\partial S_{n}}{\partial t}=\left(-\frac{1}{\tau_{n}} \frac{\eta_{n} c_{0}^{2}}{\tau_{n}} \mathrm{e}^{-\frac{t}{\tau_{n}}} H(t)+\frac{\eta_{n} c_{0}^{2}}{\tau_{n}} \mathrm{e}^{-\frac{t}{\tau_{n}}} \delta(t)\right) * \rho^{\prime}
$$

where $\delta(t)$ is the Dirac delta function. Using the Eq. (7.6) this relation becomes a simple ordinary differential equation for each process as

$$
\frac{\partial S_{n}}{\partial t}=-\frac{1}{\tau_{n}} S_{n}+\frac{\eta_{n} c_{0}^{2}}{\tau_{n}} \rho^{\prime}
$$

Using again convolutional properties, we can substitute Eq. (7.8) into (7.4), and the relaxing nonlinear state Eq. (7.3) becomes

$$
p=c_{0}^{2} \rho^{\prime}+\frac{c_{0}^{2}}{\rho_{0}} \frac{B}{2 A} \rho^{\prime 2}-\sum_{n=1}^{N} S_{n}+\sum_{n=1}^{N} \eta_{n} c_{0}^{2} \rho^{\prime} .
$$

Moreover, if "frozen" sound speed for $N$ mechanisms is defined as $c_{\infty}^{2}=$ $c_{0}^{2}\left(1+\sum_{n=1}^{N} \eta_{n}\right)$, Eq. (7.9) leads to

$$
p=c_{\infty}^{2} \rho^{\prime}+\frac{c_{0}^{2}}{\rho_{0}} \frac{B}{2 A} \rho^{\prime 2}-\sum_{n=1}^{N} S_{n}
$$


Due to the smallness of the relaxation parameter, $\eta_{n}$, i.e. when weak dispersion is modeled, the sound speed in the high frequency limit reduces to (Naugolnykh et al., 1998):

$$
c_{\infty}=c_{0}\left(1+\sum_{n=1}^{N} \frac{\eta_{n}}{2}\right) .
$$

Note Eq. (7.10) for a mono-relaxing media is equivalent to that can be found in literature (Rudenko et al., 1977)

$$
p=c_{\infty}^{2} \rho^{\prime}+\frac{c_{0}^{2}}{\rho_{0}} \frac{B}{2 A} \rho^{\prime 2}-\int_{-\infty}^{t} \frac{\eta c_{0}^{2}}{\tau} \mathrm{e}^{-\left(\frac{t-t^{\prime}}{\tau}\right)} \rho^{\prime}\left(t^{\prime}\right) .
$$

Thus, the constitutive relations to solve by means of the numerical method in the nonlinear regime are the continuity Eq. (7.1), the motion Eq. (7.2) and the second order fluid state relaxing Eq. (7.10), where the state variable $S_{n}$ obeys the relation (7.8) for the $n$-th order relaxation process. Although the aim of this work is to model biological media, the generalized formulation presented here can be used to describe the attenuation and hence the dispersion observed in other relaxing media, as the relaxation processes of oxygen and nitrogen molecules in air or the relaxation associated with boric acid and magnesium sulfate in seawater (Pierce, 1989).

\subsubsection{Small amplitude modeling}

If small amplitude perturbations are considered, an equivalent derivation of this model can be expressed for multiple relaxation media (Yuan et al., 1999). Thus, for an homogeneous inviscid relaxing fluid the linearized continuity and motion Eq. (7.1-7.2) reduces to

$$
\frac{\partial \rho}{\partial t}=-\rho_{0} \nabla \cdot \mathbf{v}
$$

and

$$
\rho_{0} \frac{\partial \mathbf{v}}{\partial t}=-\nabla p
$$

and linearizing the fluid state Eq. (7.10) we obtain:

$$
\rho^{\prime}=\frac{1}{c_{\infty}^{2}}\left(p+\sum_{n=1}^{N} S_{n}\right)
$$

These equations can be solved directly in this form, however, if expressed in pressure-velocity formulation the density field is no longer necessary and computational effort can be reduced. Thereby, assuming a linear "instantaneous" 
compressibility $\kappa_{\infty}=\rho_{0} c_{\infty}^{2}$, and substituting Eq. (7.15) into Eq. (7.13) yields

$$
\frac{\partial p}{\partial t}+\sum_{n=1}^{N} \frac{\partial S_{n}}{\partial t}=-\kappa_{\infty} \nabla \cdot \mathbf{v}
$$

Then, taking the time derivative of the state variable Eq. (7.8) we get

$$
\frac{\partial p}{\partial t}-\sum_{n=1}^{N} \frac{1}{\tau_{n}} S_{n}+\rho^{\prime} \sum_{n=1}^{N} \frac{\eta_{n} c_{0}^{2}}{\tau_{n}}=-\kappa_{\infty} \nabla \cdot \mathbf{v}
$$

Finally, substituting again the linearized state Eq. (7.15) and arranging terms the linearized continuity equation leads to

$$
\frac{\partial p}{\partial t}+p \sum_{n=1}^{N} \frac{\eta_{n} c_{0}^{2}}{\tau_{n} c_{\infty}^{2}}+\sum_{n=1}^{N}\left(\frac{\eta_{n} c_{0}^{2}}{\tau_{n} c_{\infty}^{2}}-\frac{1}{\tau_{n}}\right) S_{n}=-\kappa_{\infty} \nabla \cdot \mathbf{v}
$$

On the other hand, the state evolution equation can be expressed as a function of the acoustic pressure as

$$
\frac{\partial S_{n}}{\partial t}=-\frac{1}{\tau_{n}} S_{n}+\frac{\eta_{n} c_{0}^{2}}{\tau_{n} c_{\infty}^{2}}\left(p-\sum_{n=1}^{N} S_{n}\right)
$$

Thus, the linearized governing Eq. $(7.14,7.18)$ for a relaxing media are expressed in a pressure-velocity formulation and can be solved together with the coupled state evolution equation (7.19) by means of standard finite differences numerical techniques (Yuan et al., 1999). In this way, lossless linear acoustics equations can be obtained by setting $\eta_{n}=0$ or in the limit when the relaxation times $\tau_{n} \rightarrow \infty$. The relaxation behavior described by this linearized model is achieved too by the formulation described by (Yuan et al., 1999), where the relaxation coefficients $\eta_{n}$ and the relaxation variable $S_{n}$ are defined in a different, but analogous way.

\subsection{Numerical solution by finite differences in time domain}

In this section the numerical techniques for solving the complete set of equations (continuity Eq. (7.1), momentum Eq. (7.2), state Eq. (7.10) and the relaxation Eq. (7.8)) are presented. The numerical method is based on a second order FDTD method where multiple relaxation processes are included in order to: first, modeling physical attenuation and dispersion in the interest frequencies and second, 


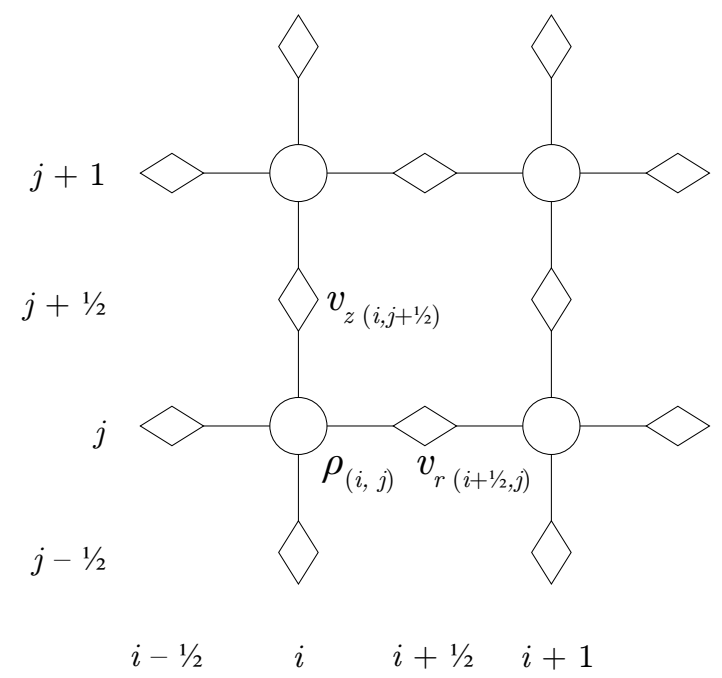

Figure 7.1: Spatial discretization as in standard acoustics FDTD method. The pressure $\left(p_{i, j}^{m}\right)$ and the $n$-th order relaxation process state fields $\left(S_{n, i, j}^{m}\right)$ are evaluated at same discrete location as the density $\left(\rho_{i, j}^{m}\right)$. Particle velocity fields are discretized staggered in both space and time respect to the density, pressure and the $n$-th order state fields.

correct the numerical dispersion and include artificial attenuation to guarantee convergence in nonlinear regime. Moreover the inclusion of relaxation processes in the presented formulation require only one extra field per relaxation process and no memory buffer is needed.

\subsubsection{Discretization}

Cylindrical axisymmetric $\mathbf{x}=(r, z)$ coordinate system is considered in this work, however, the method can be derived in other coordinate system. As in the standard acoustic FDTD method (Botteldooren, 1996), the particle velocity fields are discretized staggered in time and space respect to the density and pressure fields. As shown in Fig. 7.1 uniform grid is considered, where $r=i \Delta r, z=j \Delta z, t=m \Delta t$, with $\Delta r$ and $\Delta z$ as the radial and axial spatial steps, and $\Delta t$ is the temporal step.

Centered finite differences operators are applied over the partial derivatives of the governing equations. Thus, spatial interpolation is needed over the off-center grid variables in order to fulfill the conservation principles over each discrete cell of the domain (LeVeque, 1992). The $r$ component of Eq. (7.2) is expressed in a 
cylindrical axisymmetric system as

$$
\begin{aligned}
\frac{\partial v_{r}}{\partial t}= & -\frac{1}{\rho} \frac{\partial p}{\partial r}-v_{r} \frac{\partial v_{r}}{\partial r}-v_{z} \frac{\partial v_{r}}{\partial z} \\
& +\frac{\eta}{\rho}\left(\frac{\partial^{2} v_{r}}{\partial r^{2}}+\frac{1}{r} \frac{\partial v_{r}}{\partial r}+\frac{\partial^{2} v_{r}}{\partial z^{2}}-\frac{v_{r}}{r^{2}}\right) \\
& +\frac{1}{\rho}\left(\zeta+\frac{1}{3} \eta\right)\left(\frac{\partial^{2} v_{r}}{\partial r^{2}}+\frac{1}{r} \frac{\partial v_{r}}{\partial r}+\frac{\partial^{2} v_{z}}{\partial r \partial z}-\frac{v_{r}}{r^{2}}\right) .
\end{aligned}
$$

Each term of the above expression is approximated by centered finite differences evaluated at $r=\left(i+\frac{1}{2}\right) \cdot \Delta r, z=\left(j+\frac{1}{2}\right) \cdot \Delta z, t=\left(m+\frac{1}{2}\right) \cdot \Delta t$. This equation can be solved obtaining an update equation for $v_{r_{i+\frac{1}{2}, j}}^{m+\frac{1}{2}}$. In the same way, the $z$ component of the motion equation (7.2) is expressed as

$$
\begin{aligned}
\frac{\partial v_{z}}{\partial t}= & -\frac{1}{\rho} \frac{\partial p}{\partial z}-v_{r} \frac{\partial v_{z}}{\partial r}-v_{z} \frac{\partial v_{z}}{\partial z} \\
& +\frac{\eta}{\rho}\left(\frac{\partial^{2} v_{z}}{\partial r^{2}}+\frac{1}{r} \frac{\partial v_{z}}{\partial r}+\frac{\partial^{2} v_{z}}{\partial z^{2}}\right) \\
& +\frac{1}{\rho}\left(\zeta+\frac{1}{3} \eta\right)\left(\frac{\partial^{2} v_{r}}{\partial z \partial r}+\frac{1}{r} \frac{\partial v_{r}}{\partial z}+\frac{\partial^{2} v_{z}}{\partial z^{2}}\right) .
\end{aligned}
$$

This equation is approximated by centered finite differences and evaluated at $r=i \cdot \Delta r, z=\left(j+\frac{1}{2}\right) \cdot \Delta z, t=m \cdot \Delta t$. An update equation is obtained solving this equation for $v_{z_{i, j+\frac{1}{2}}}^{m+\frac{1}{2}}$. The equation (7.1) in cylindrical axisymmetric coordinate system is expressed as

$$
\frac{\partial \rho}{\partial t}=-\rho\left(\frac{\partial v_{r}}{\partial r}+\frac{v_{r}}{r}+\frac{\partial v_{z}}{\partial z}\right)-v_{r} \frac{\partial \rho}{\partial r}-v_{z} \frac{\partial \rho}{\partial z} .
$$

Following the same procedure, each term of the above expression is approximated by centered finite differences and evaluated at $r=i \cdot \Delta r, z=j \cdot \Delta z$, $t=\left(m+\frac{1}{2}\right) \cdot \Delta t$, and the update equation is obtained solving this expression for $\rho_{i, j}^{m+1}$.

A leap-frog time marching is applied to solve Eq. (7.20-7.22) for each time step until the desired simulation time is reached. Finally, Eq. (7.8) is locally solved for $m+1$ by and explicit fourth-order Runge-Kutta method and then Eq. (7.10) is evaluated.

\subsubsection{Boundary conditions}

The staggered grid is terminated on velocity nodes, so the boundary conditions are applied on these external nodes, allowing to prevent the singularity of the cylindrical coordinate system: due to the staggered grid, the only variable discretized at 


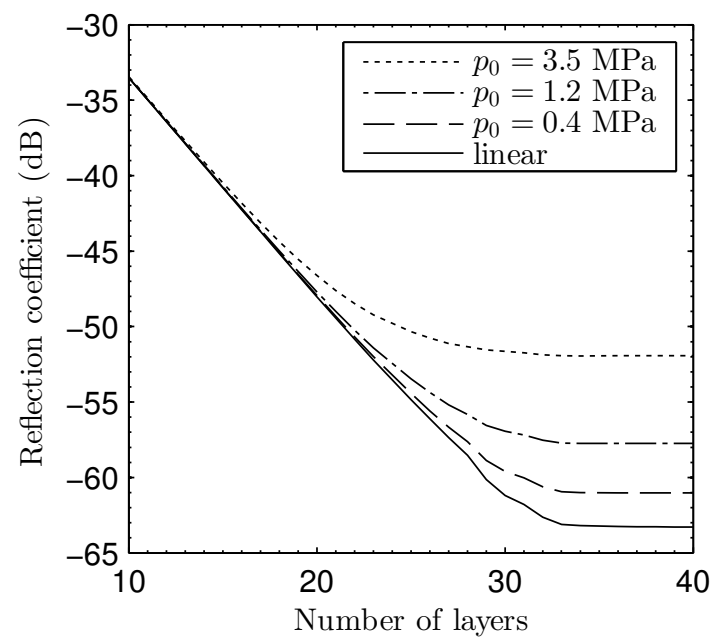

Figure 7.2: Reflection coefficient of the perfectly matched layer (PML) versus number of layers for different wave amplitudes.

$r=0$ is $v_{r}$, and axisymmetric condition $\left.v_{r}\right|_{r=0}=0$ is applied there. Furthermore, to solve spatial differential operators at boundaries some "ghost" nodes must be created with the conditions: $v_{r}(-r)=-v_{r}(r), v_{z}(-r)=v_{z}(r), \rho(-r)=\rho(r)$ and $p(-r)=p(r)$.

Perfectly matched layers (PML) (Liu et al., 1997) were placed in the limits of the domain $( \pm z$ and $+r)$ to avoid spurious reflections from the limits of the integration domain. Inside the PML domains linearized acoustic equations were solved using the complex coordinate screeching formulation (Liu, 1999). For a layer of 30 elements and a broadband incident wave with $1 \mathrm{MHz}$ central frequency and non-normal incidence angle, these absorbent boundary conditions have reported a reflection coefficient of $R=-55.2 \mathrm{~dB}$.

However, the performance of the PML is amplitude dependent as long the nonlinear terms are uncoupled to the PML domains. The amplitude dependence of the reflection coefficient is shown in Fig. 7.2, where a PML of 25 elements thickness have reported reflection coefficients $R<-50 \mathrm{~dB}$ for waves in linear regime and highly nonlinear waves including shocks.

\subsubsection{Minimizing numerical dispersion}

The stability for the lossless linear FDTD algorithm follows the Courant-FriedrichLevy (CFL) condition, that for uniform grid $(\Delta r=\Delta z=\Delta h)$ the maximum duration of the time step is limited by $\Delta t \leq \Delta h / c_{0} \sqrt{D}$ where $D$ is the number of dimensions (i.e. $D=2$ in cylindrical axisymmetric coordinate system). That 
condition essentially states that for a single time step information can not propagate in the numerical grid a distance longer that one cell. However, if relaxation is included numerical instabilities have been observed when $\tau_{f} / 2 \pi<\Delta t$. Due to this empirical relation, the maximum values for relaxation frequencies are limited too by the chosen spatial discretization by the simple relation $f_{n}<\sqrt{2} N_{\lambda} f_{0}$, where $f_{n}=2 \pi / \tau_{n}$ is the maximum relaxation frequency for all processes, $N_{\lambda}$ is the number of spatial samples per wavelength and $f_{0}$ the frequency of the propagating wave.

On the other hand, nonlinear effects induce the progressively growing of harmonics of the fundamental frequency of the initial wave. The diffusive viscous terms in Eq. (7.2), attenuates the small-amplitude high-spatial frequencies, damping the "node to node" numerical oscillations and ensuring numerical stability in weakly nonlinear regime. Thus, for a smooth solution the numerical algorithm shows consistency when $\Delta h \rightarrow 0$, so if stability is achieved by the CFL condition, the convergence is guaranteed. However, in strongly nonlinear regime, i.e. when sharp waveforms or even shocks are present in the solution, extra numerical techniques must be employed to guarantee convergence. Artificial viscosity can be added when shock waves are present in the solution where a common implementation follows a fourth order spatial filtering (Sparrow et al., 1991; Ginter et al., 2002). Thus, the artificial attenuation retrieved by this spatial operator is fourth power of frequency: the low frequency components of the solution remains quasi-undamped, while the higher spatial frequencies are strongly attenuated. In this way, the solution is smoothed and shock thickness depends on the artificial viscosity coefficient.

However, the main drawback for finite difference methods is numerical dispersion, where the analytic dispersion relation can be expressed in $1 \mathrm{D}$ as

$$
\sin ^{2} \frac{k \Delta h}{2}=\frac{1}{S^{2}} \sin ^{2} \frac{\omega \Delta t}{2}
$$

with the Courant number $S=c_{0} \Delta t / \Delta h$. In this way, numerical dispersion reduces phase speed for high frequency components so traveling sharp solutions develop tail oscillations: the low wavenumbers travels fast and left behind high spatial frequencies. In nonlinear regime, is well-known that the combined effects of nonlinearity and strong dispersion can lead rich phenomena, e.g. beatings in the generated harmonics, pulsations on the vertex of a sawtooth wave or soliton formation in strong dispersive media (Rudenko et al., 1977). In this way, the numerical dispersion by discreteness of the FDTD methods couple to the physical nonlinearity can lead to a great variety of non-physical or even unstable solutions.

In order to overcome those two limitations, i.e. the generation of harmonics over the discrete limit and the numerical dispersion, we propose the use of artificial relaxation. Physical relaxation processes introduces anomalous dispersion, i.e. the phase speed increases in the high frequency regime, opposite to the numerical (lattice) dispersion described above. Thus, by introducing a collection of 


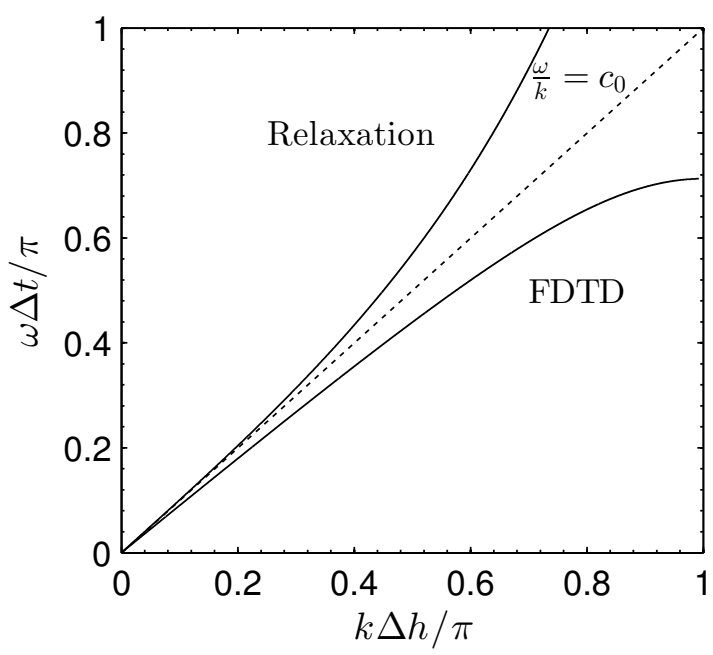

Figure 7.3: Normalized dispersion relation for of a FDTD lattice of Courant number $S=0.9$ and anomalous dispersion relation for a mono relaxing acoustic media. The straight line $c_{0}=\omega / k$ represents the reference nondispersive case.

relaxation processes and choosing its adequate relaxation parameters the high frequency numerical dispersion can be compensated. As a consequence, introduction of those relaxation processes in the high frequency lead to the inevitable inclusion of artificial attenuation. However, this numerical attenuation is then exploited to limit the growing of higher harmonics in a similar way than artificial viscosity (Sparrow et al., 1991). It is worth noting here that, due to the attenuation using artificial relaxation is, at maximum, only second power of frequency, the low frequency range of the solution is therefore also attenuated. Thus, the proposed method is restricted to lossy media.

The adequate relaxation parameters that corrects the numerical dispersion have been found by multi-objective optimization techniques, where two cost function are proposed: one for dispersion and other for attenuation. In the first case, the error between the goal (ideal) dispersion relation and the retrieved numerical dispersion corrected by multiple artificial relaxation and is evaluated in the high frequency regime. Finally, the second cost function is the error between the desired (ideal) attenuation and the artificial attenuation evaluated in the low frequency regime. 


\subsection{Validation}

\subsubsection{Single relaxation process}

A canonical case of a physical single relaxation process is presented. In order to correct numerical dispersion the parameters of two extra artificial relaxation processes have been found using the multi-objective genetic algorithms provided by the optimization toolbox in MATLAB R2014a v8.03. Linear propagation was considered and simulation parameters were set to typical values for water: $c_{0}=$ $1500 \mathrm{~m} / \mathrm{s}, \rho_{0}=1000 \mathrm{~kg} / \mathrm{m}^{3}, B / A=5, \eta=8.90 \cdot 10^{-4} \mathrm{~Pa} \cdot \mathrm{s}$. A single physical relaxation process was included, with a characteristic relaxation time of $\tau_{1}=$ $1 / 2 \pi f_{0}$ and $f_{0}=2 \mathrm{MHz}$, and relaxation modulus of $\eta_{1}=0.0134$ that leads to a frozen sound speed of $c_{\infty}=1510 \mathrm{~m} / \mathrm{s}$. In this case, the numerical parameters were set to $\Delta r=\Delta z=1.87 \cdot 10^{-7} \mathrm{~m}$ and $\Delta t=8.65 \cdot 10^{-11} \mathrm{~s}$. A plane wave traveling in $+z$ direction was considered.

Thus, the theoretical attenuation for the relaxation processes and including viscosity can be expressed as

$$
\alpha(\omega)=\frac{\omega^{2}}{2 \rho_{0} c_{0}^{3}}\left(\zeta+\frac{4}{3} \eta\right)+\sum_{n=1}^{N} \frac{\eta_{n}}{2 c_{0} \tau_{n}} \frac{\omega^{2} \tau_{n}^{2}}{1+\omega^{2} \tau_{n}^{2}},
$$

and the theoretical phase velocity can be predicted as (Pierce, 1989)

$$
c_{p}(\omega)=c_{0}\left(1+\sum_{n=1}^{N} \frac{\eta_{n}}{2} \frac{\omega^{2} \tau_{n}^{2}}{1+\omega^{2} \tau_{n}^{2}}\right) .
$$

In order to compute the attenuation and dispersion of the numerical method, simulated pressure was recorded at two locations $z_{0}$ and $z_{1}$, and attenuation and phase velocity were estimated from the spectral components over the bandwidth of the input signal. The numerical attenuation was calculated as

$$
\alpha(\omega)_{f d}=\frac{\ln \left(\left|P\left(\omega, z_{1}\right) / P\left(\omega, z_{0}\right)\right|\right)}{\left(z_{1}-z_{0}\right)},
$$

where $P(\omega)$ is the Fourier transform of the measured pressure waveforms at points $z_{0}$ and $z_{1}$. On the other hand, the phase velocity was computed as

$$
c_{p}(\omega)_{f d}=\frac{\omega \cdot\left(z_{1}-z_{0}\right)}{\arg \left(P\left(\omega, z_{1}\right) / P\left(\omega, z_{0}\right)\right)},
$$

where correct phase unwrapping is needed. 
In this way, Fig. 7.4 shows the retrieved Pareto front of the optimization, where 3 different areas can be distinguished. The first area, marked as (A) in Fig. 7.4, represents individuals those numerical dispersion is minimal but attenuation is not optimal. On the other hand, the individuals around area (B) represent a set of relaxation parameters that provides the best agreement between numerical and physical attenuation. A good compromise between both situations can be obtained in the central area of the Pareto front $(\mathrm{C})$, where retrieved attenuation and dispersion in the low frequency band shows good agreement with the physical, and the numerical dispersion has been corrected over a wide frequency range. However, as can be seen in the inset of Fig. 7.4, the dispersion relation retrieved by all the cases corrects the FDTD lossless numerical dispersion relation. The phase speed of those tree individuals is shown in Fig. $7.5 \mathrm{~b}$ ), where it can be seen that in the frequency range selected for the optimization the numerical phase speed is corrected for all the individuals, where the best fit is obtained for individuals in the Pareto front area A. On the other hand, the inclusion of artificial relaxation leads to an increasing of the attenuation in the high frequency range, as is shown in Fig. 7.5 a). In this way, as the phase speed error is reduced the effect of artificial attenuation increases. Although this increasing can be seen as a nondesired counterpart, the appearance of this attenuation is useful in order to control the harmonic growing in nonlinear regime in the same way as artificial viscosity

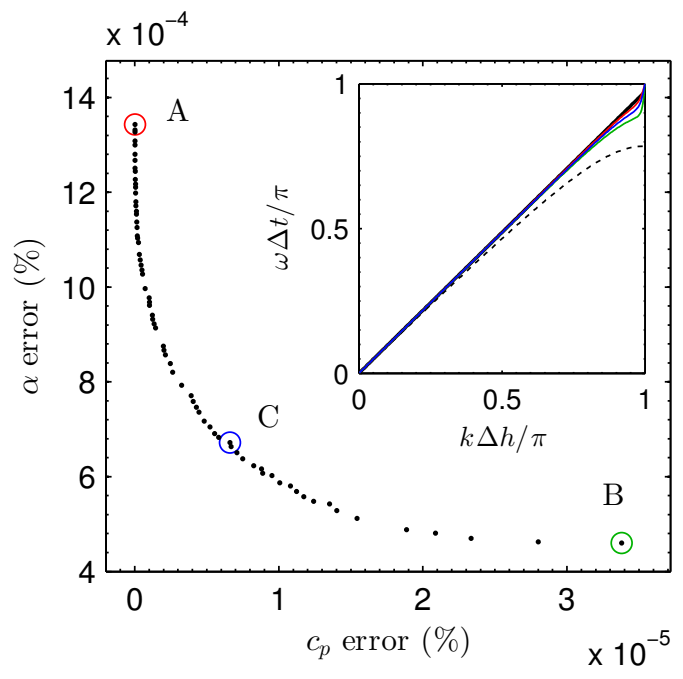

Figure 7.4: Pareto front retrieved by the multi-objective genetic optimization. Red marker (A) is the solution those relaxation parameters minimizes the numerical dispersion. The best fit in the attenuation are the parameters that provided the solution marked by green circle (B). A compromise between attenuation and dispersion is achieved at the individuals in the center of the Pareto front as the sample marked by blue circle (C). The inset shows the normalized dispersion relation retrieved by the individuals A-C. Dashed black line shows the numerical dispersion relation of the FDTD method for a Courant number of 0.94 without artificial relaxation. 

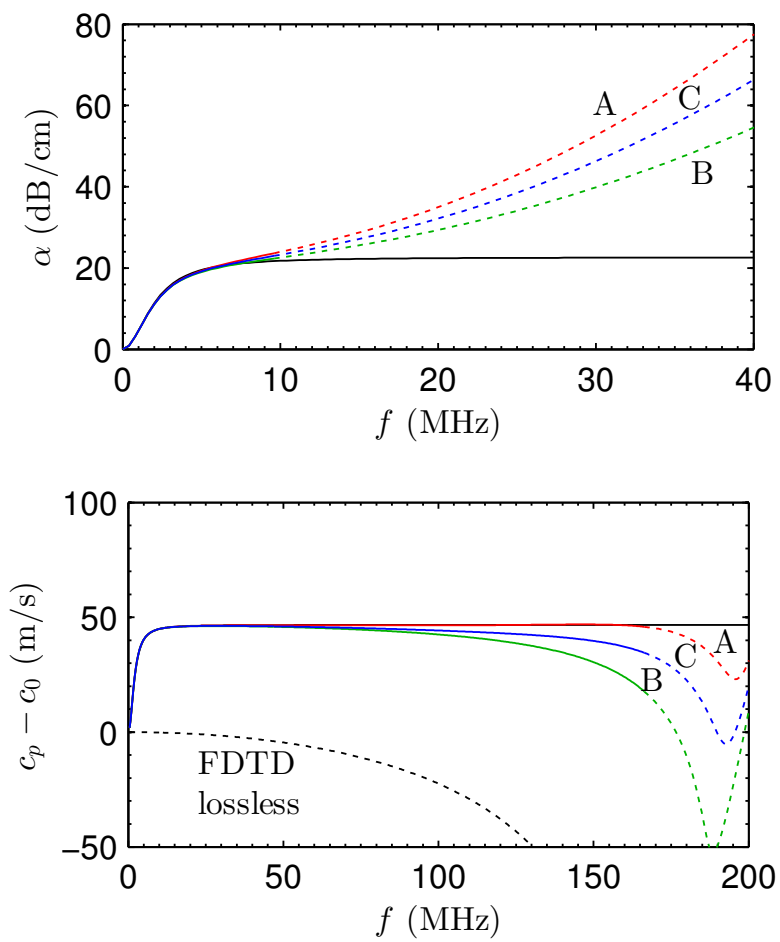

Figure 7.5: Retrieved attenuation (Up) and dispersion (Down) by the inclusion of artificial relaxation for the individuals $\mathrm{A}$ (red), B (green) and $\mathrm{C}$ (blue) of Fig. 7.4. Continuous lines represent the frequency range included by the optimization and dashed lines the not useful frequency range. As a consequence of correcting dispersion appears attenuation in the high frequency range that will be used as artificial attenuation for nonlinear stability.

spatial operators (Sparrow et al., 1991).

\subsubsection{Nonlinear steady solution for single relaxation process}

In order to validate the method in the nonlinear regime a full-wave simulation was developed in a mono-relaxing media using above parameters. Thus, the analytical (inverted) solution for the steady solution with $p=-p_{0}$ for $\tau=-\infty, p=p_{0}$ for $\tau=\infty$ and $p=0$ for $\tau=0$, for the retarded time $\tau=t-z / c_{0}$ reads (Hamilton et al., 1998b)

$$
\tau=\tau_{n} \ln \frac{\left(1+p / p_{0}\right)^{D-1}}{\left(1-p / p_{0}\right)^{D+1}}
$$

where $D=\eta_{n} \rho_{0} c_{0}^{2} / 2 \beta p_{0}$ measures the ratio of relaxation effects to nonlinear effects. 

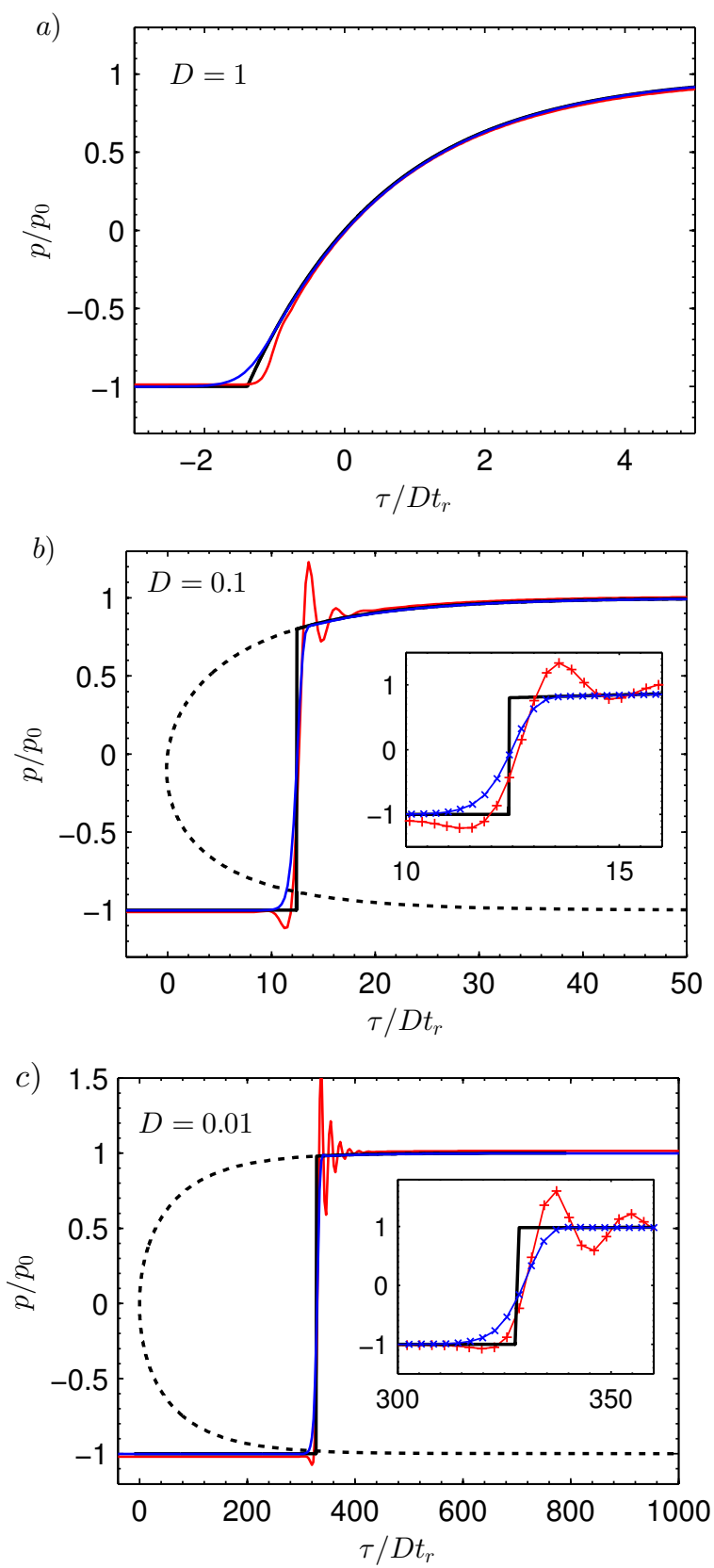

Figure 7.6: a) Analytical (gray line), numerical using artificial relaxation with corrected dispersion (blue line) and artificial viscosity (red) (Sparrow et al., 1991) for the nonlinear steady state solution for $D=1$. b) Nonlinear steady state solution for $D=0.1$. Inset shows detailed shock numerical solution for artificial relaxation (blue) and artificial viscosity (red). c) Nonlinear steady state solution for $D=0.01$. 
For $D>1$, where no shock is present, the solution retrieved by FDTD algorithm shows good agreement with analytical and no artificial relaxation is needed. However, for $D<1$ a discontinuity is present in the solution and convergence is only possible with the inclusion of extra numerical techniques.

Thus, Fig. 7.6 (a-c) shows the analytical and numerical solutions including artificial relaxation and artificial viscosity, where excellent agreement is achieved in all cases. In the case of artificial viscosity, higher harmonics are strongly attenuated and by reducing grid step convergence can be achieved. However, as long the phase speed of the higher spatial frequencies present in the shock is modified due to numerical dispersion, oscillations appears in the tail of the discontinuity, leading to the appearance of non-physical solutions. On the other hand, the artificial attenuation by relaxation also limits the harmonic growing so a smooth version of the shock appears. Moreover, artificial relaxation also corrects phase velocity so all the spatial frequencies travels at same speed and no oscillatory tail appears. The case of $D=0.01$ is shown in Fig. $7.6 \mathrm{c}$ ), where nonlinear effects strongly dominates over attenuation. In this case, tail oscillations provided by artificial viscosity increases in amplitude. In contrast, by including artificial relaxation a smoothed version of the shock is captured and accuracy is maintained.

\subsection{Results}

\subsubsection{Frequency power law attenuation}

Using methodology described above, the optimal relaxation parameters were obtained in order to fit the multiple-relaxation numerical attenuation to frequency power law attenuation in the form

$$
\alpha(\omega)=\alpha_{0} \omega^{\gamma}
$$

where $\gamma$ is the power law exponent and $\alpha_{0}$ the power law coefficient in $\mathrm{Np}(\mathrm{rad} / \mathrm{s})^{\gamma} \mathrm{m}^{-1}$. Moreover, the numerical dispersion was corrected by means of artificial relaxation in order to fit the corresponding frequency power law dispersion, where its analytical form satisfying causality can be expressed as (Waters et al., 2005)

$$
\frac{1}{c_{p}(\omega)}=\frac{1}{c\left(\omega_{0}\right)}+\alpha_{0} \tan \left(\frac{\pi \gamma}{2}\right)\left(|\omega|^{\gamma-1}-\left|\omega_{0}\right|^{\gamma-1}\right)
$$

This expression is valid for $0<\gamma<3$ with $\gamma \neq 1$, and an alternate equation can be found (Waters et al., 2005) in the limit for $\gamma=1$. Here, simulation parameters were $c_{0}=1500 \mathrm{~m} / \mathrm{s}, \rho_{0}=1000 \mathrm{~kg} / \mathrm{m}^{3}, B / A=5, \eta=8.90 \cdot 10^{-4} \mathrm{~Pa} \cdot \mathrm{s}, f_{0}=1$ $\mathrm{MHz}, \Delta r=\Delta z=1.3 \cdot 10^{-5} \mathrm{~m}, \Delta t=5.4 \cdot 10^{-9} \mathrm{~s}$; that leads to 26 elements per wavelength and a CFL number of 0.9. Only two independent relaxation processes were employed in this section to obtain the target frequency power laws. 


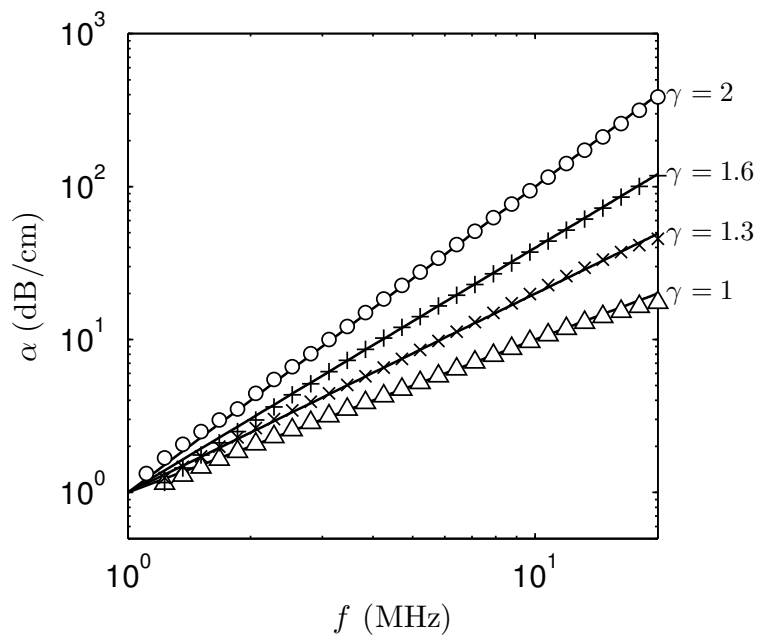

Figure 7.7: Attenuation retrieved by the numerical algorithm (markers) and target frequency power law attenuation (gray lines). By using the optimization algorithm the relaxation times and modulus were optimized for minimize the relative error between the target power laws of $\gamma=[1,1.3,1.6,2]$ and the attenuation retrieved.

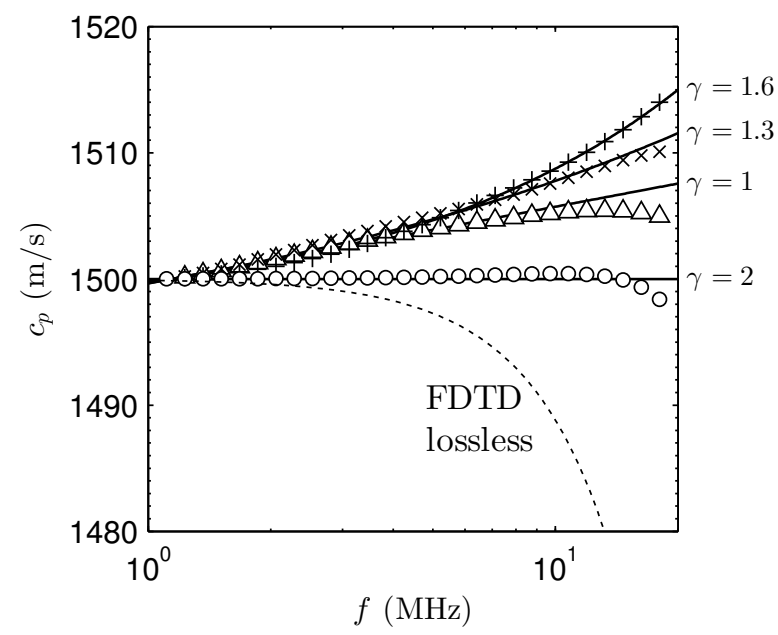

Figure 7.8: Phase speed retrieved by the numerical algorithm (markers) and target frequency power law attenuation (gray lines) for $\gamma=[1,1.3,1.6,2]$.

Following the above procedure, the relaxation times $\tau_{n}$ and relaxation modulus $\eta_{n}$ were optimized for different frequency power laws covering the range of that observed in tissues $\gamma=[1,1.3,1.6,2]$. The attenuation coefficient $\alpha_{0}$ was chosen for each power law to present an attenuation $\alpha=1 \mathrm{~dB} / \mathrm{cm} / \mathrm{MHz}^{\gamma}$. The fitting was developed over the typical frequency range for medical ultrasound applications, 


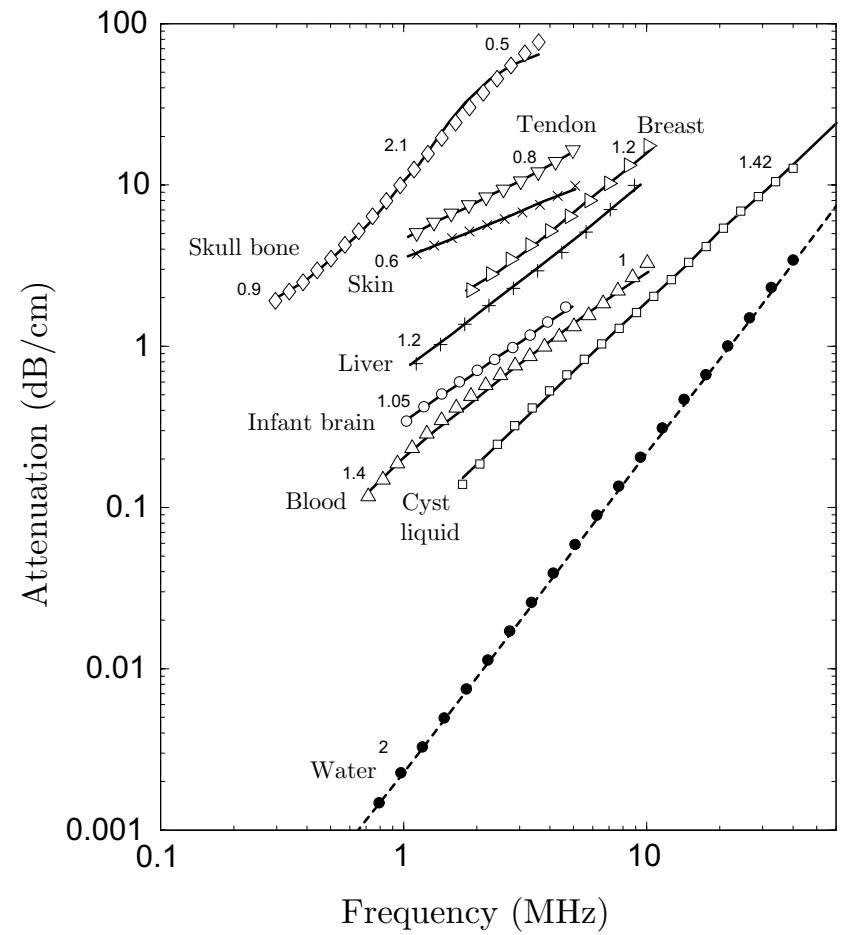

Figure 7.9: Experimental attenuation data for some tissues adapted from (Hill et al., 2004) (lines), and obtained by the numerical method (markers) by fitting the parameters of 2 relaxing processes. The numbers above the curves show the coefficient of the frequency power law $\gamma$ for each frequency region (i.e. the slope of the curve).

i. e. 1 to $20 \mathrm{MHz}$ for both attenuation and phase speed. The results for the attenuation and phase speed curves are plotted in Fig. 7.7 and Fig. 7.8, where the theoretical and the numerical predictions agree over the frequency range used for the fitting.

\subsubsection{Fitting attenuation for tissue experimental data}

Although a frequency power law dependence can describe the ultrasound attenuation over a finite frequency range, the attenuation data of some particular examples shows variation of the exponent over the entire frequency range (Hill et al., 2004). Thus, Fig. 7.9 shows experimental attenuation data curves for some tissues where the local slope of the power law changes over the measured frequency range. This behavior can be modeled by a sum of relaxation processes by optimizing the relaxation parameters as described above. Thus, the results show that most tissues 
Table 7.1: Error of the optimized attenuation response relative to the experimental data for $N$ relaxation processes.

\begin{tabular}{lccccc}
\hline Tissue & $\begin{array}{c}\text { Power law } \\
\text { (local slope) }\end{array}$ & $\begin{array}{c}N=1 \\
\varepsilon(\%)\end{array}$ & $\begin{array}{c}N=2 \\
\varepsilon(\%)\end{array}$ & $\begin{array}{c}N=3 \\
\varepsilon(\%)\end{array}$ & $\begin{array}{c}N=4 \\
\text { Skin }\end{array}$ \\
$f^{0.6}$ & 6.67 & 0.167 & 0.136 & 0.120 \\
Liver & $f^{1.2}$ & 7.62 & 0.517 & 0.404 & 0.165 \\
Blood & $f^{1.4}, f^{1}$ & 8.34 & 0.349 & 0.330 & 0.310 \\
Breast & $f^{0.9}, f^{1.2}$ & 5.20 & 0.216 & 0.209 & 0.205 \\
Skull bone & $f^{0.9}, f^{2.1}, f^{0.5}$ & 10.60 & 10.54 & 8.628 & 5.189 \\
\hline
\end{tabular}

with locally variable $\gamma$ can be fitted by only a pair of relaxation processes, as the same way that for constant-slope frequency power law attenuation (Cleveland et al., 1996).

In this way, Table 7.1 shows the error of the numerical attenuation relative to the experimental data. The percent relative error was computed as

$$
\varepsilon=\frac{100}{f_{2}-f_{1}} \int_{f_{1}}^{f_{2}} \frac{\left|\alpha_{e}(f)-\alpha(f)\right|}{\alpha_{e}(f)} \mathrm{d} f,
$$

where $\alpha_{e}(f)$ is the experimental attenuation data, $f_{1}$ and $f_{2}$ define the frequency range of the measurement.

As expected, the goodness of fit grows as the number of relaxation processes included increases. However, only two processes are enough to obtain relative errors below $1 \%$ for tissues with $\gamma<2$. In the case of tissues where a local value of $\gamma>2$ has been observed, the fitting procedure fails, like in the skull bone in the $2 \mathrm{MHz}$ range (Hill et al., 2004). The maximum slope achieved by single relaxation and thermo-viscous losses is $\gamma=2$ for any frequency, so a tissue showing that slope cannot be accurately modeled in this frequency region with the method proposed in this work. Another point of view of is from dispersion: Eq. (7.30 states that frequency power law medium with $2<\gamma<3$ presents standard dispersion (Waters et al., 2005), opposite to anomalous dispersion for media falling in the range $0<\gamma<2$. Therefore, the dispersion relation of media with $2<\gamma<3$ cannot be modeled by a sum of relaxation processes as long relaxation includes anomalous dispersion.

Using Kramers-Kronig relationships (O'Donnell et al., 1981), the variations of sound speed $\Delta c$ can be predicted by the frequency dependent attenuation. Table 7.2 shows the variation of sound speed observed in the numerical solution over the fitted frequency range. The magnitude of these variations are of the order of magnitude of those measured experimentally in this frequency range, and the frequency dependence observed for the variation is roughly linear as observed in real 
Table 7.2: Variation of sound speed $(\Delta c)$ observed numerically for the modeled tissues by means of two relaxation processes and analytical using the Kramers-Kronig relations.

\begin{tabular}{lcc}
\hline Tissue & $\begin{array}{c}\text { Numerical } \Delta c \\
(\mathrm{~m} / \mathrm{s})\end{array}$ & $\begin{array}{c}\text { Analytical } \Delta c \\
(\mathrm{~m} / \mathrm{s})\end{array}$ \\
\hline Skull bone & 80.737 & 70.720 \\
Skin & 10.148 & 2.460 \\
Breast & 2.323 & 2.455 \\
Liver & 3.118 & 2.339 \\
Blood & 0.865 & 0.907 \\
\hline
\end{tabular}

tissue (Hill et al., 2004). As expected from the relations between dispersion and absorption (O'Donnell et al., 1981), the magnitude of the variation in sound speed increases as the total variation of the absorption increases for a given frequency range.

\subsubsection{Nonlinear one-dimensional propagation in tissue-like media}

\section{Non-dispersive media}

In order to study the convergence of the numerical calculations to an analytical solution of the model in the nonlinear regime, a medium with frequency squared dependence attenuation is implemented using the adequate relaxation times and relaxation modulus as explained above. The solution for the frequency squared absorption is compared with the analytical solution for a plane wave traveling through a thermo-viscous fluid proposed by Mendousse (Pierce, 1989):

$$
\frac{p}{p_{0}}=\frac{\frac{4}{\Gamma} \sum_{n=1}^{\infty}(-1)^{n+1} I_{n}\left(\frac{\Gamma}{2}\right) n \mathrm{e}^{-n^{2} \sigma / \Gamma} \sin \left(n \omega t^{\prime}\right)}{I_{0}\left(\frac{\Gamma}{2}\right)+2 \sum_{n=1}^{\infty}(-1)^{n} I_{n}\left(\frac{\Gamma}{2}\right) n \mathrm{e}^{-n^{2} \sigma / \Gamma} \cos \left(n \omega t^{\prime}\right)}
$$

where $\Gamma$ is the Gol'dberg number, defined for power law media as $\Gamma=x_{a} / x_{s}$, with absorption length $x_{a}=1 / \alpha_{0} \omega^{\gamma}$, shock formation distance $x_{s}=1 / \beta \varepsilon k$ and normalized distance $\sigma=x / x_{s}$; with the parameter of nonlinearity $\beta=1+B / 2 A$ and the acoustic Mach number $\varepsilon=v / c_{0}$, with $v$ is the source particle velocity and $k$ the wavenumber.

Figure 7.11 (top) present the simulated waveforms at $\sigma=1$ and $\sigma=3$. The wave steepening due to nonlinear processes in the absence of dispersion are well resolved by the numerical method presented here. In order to study the accuracy of the algorithm, the amplitude of the first ten harmonics has been extracted for numerical and analytic solutions and plotted versus $\sigma$ in Fig. 7.11 (bottom). The 
observed relative error of the computational method decreases due to grid coarsening by a square law (i.e. the numerical scheme is second order accuracy). The magnitude of the error mainly depends on the number of elements per wavelength but, due to not ideal dispersion, also on the traveled propagated distance. Including the correction of dispersion by artificial relaxation, for a path length of $100 \lambda$, a grid of 26 elements per wavelength was needed to obtain a relative error below $1 \%$ for the third harmonic. Obviously, the relative error of the first and second harmonics will be always lower, i.e. the fundamental component error was 0.072 $\%$.

In addition, the solution was compared also to the obtained by a $k$-space method applied to the constitutive relations by using the $k$-wave algorithm (Treeby et al., 2012). This method was selected due to the low numerical dispersion and the possibility of including frequency power law attenuation. The result of both computational methods and Eq. (7.32) agree over all the spectral components analyzed, showing convergence to the analytic solution.

\section{Dispersive media}

In the case of frequency power law attenuation media with $\gamma \approx 1$ no general analytic solution exist in nonlinear regime for monochromatic progressive waves. Thus, in order to study convergence in this regime, the proposed FDTD solution was compared with the solution obtained by $k$-space methods (Treeby et al., 2012). By using same physical and grid parameters in both methods, the solutions agrees for different power laws. Thus, Fig. 7.12 (top) shows the good agreement for the waveforms measured at $\sigma=1$ and $\sigma=3$ obtained for $\gamma=1.6$, where the characteristic asymmetry of media with anomalous dispersion (e.g. relaxing, boundary layer effects) (Hamilton et al., 1998b) is observed: the shock front after the rarefaction phase is followed by a rounded positive compression profile. The spatial distribution for each harmonic is shown in Fig. 7.13 (bottom), where it is observed that the proposed FDTD solution with optimized attenuation and dispersion converges to the obtained by pseudo-spectral methods up to ten harmonics. As in the case of frequency squared media, grid refinement numerical tests have reported a second order accuracy of the FDTD method in nonlinear regime. 

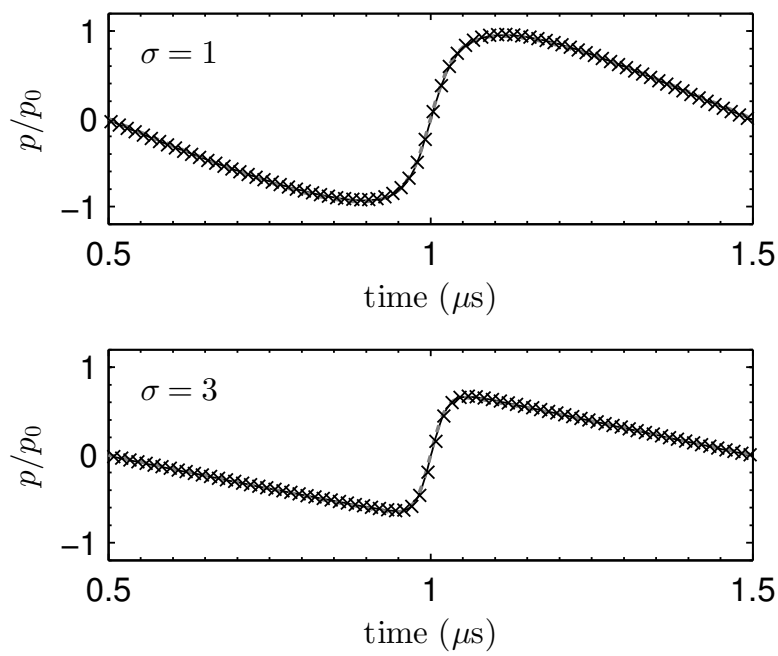

Figure 7.10: Waveforms for $\sigma=1$ and $\sigma=3$ in a media with frequency squared power law. Mendousse analytical solution (blue line), $k$-space numerical solution (gray line) and FDTD numerical solution (markers).

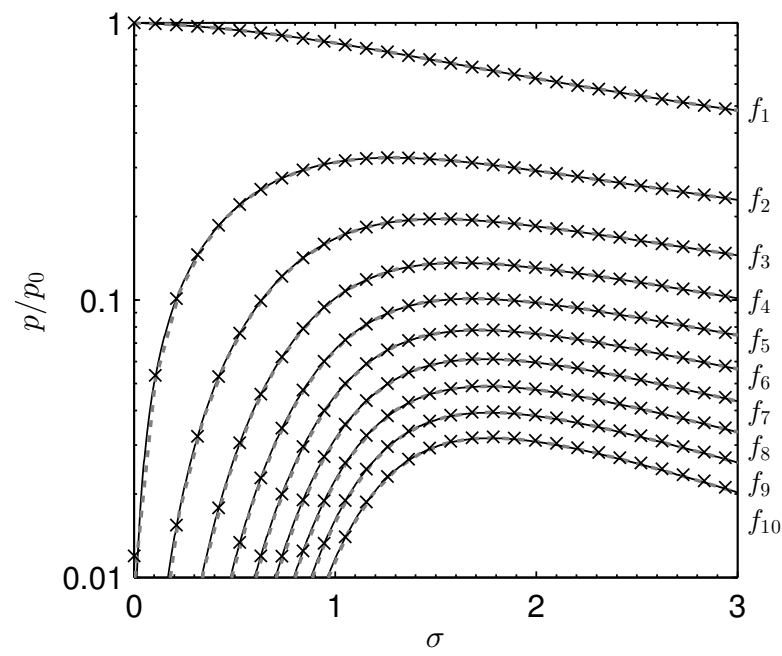

Figure 7.11: Spatial distribution of the first ten harmonics for $\gamma=2$. Mendousse analytical solution (blue line), $k$-space numerical solution (gray line) and FDTD numerical solution (markers). 

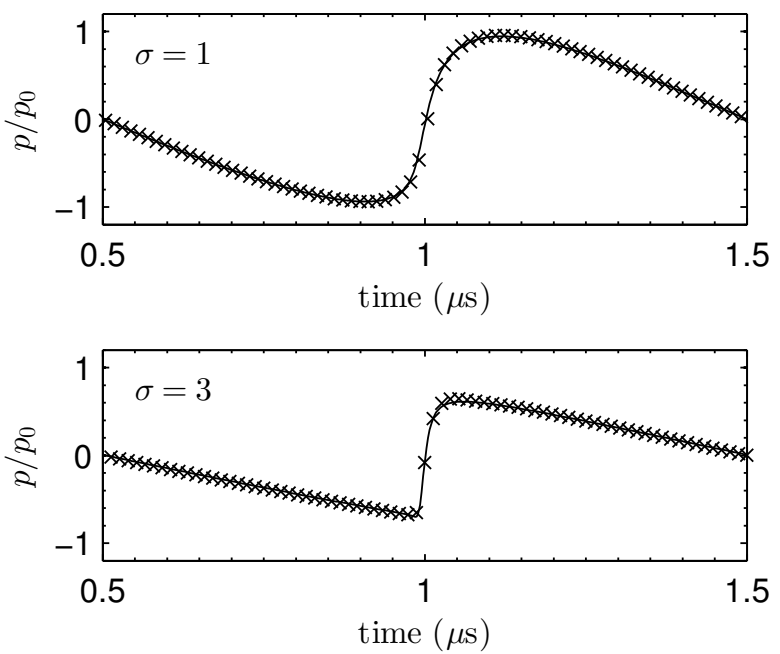

Figure 7.12: Waveforms for $\sigma=1$ and $\sigma=3$ in a media with frequency power law $(\gamma=1.6)$. $k$-space numerical solution (gray line) and FDTD numerical solution (markers).

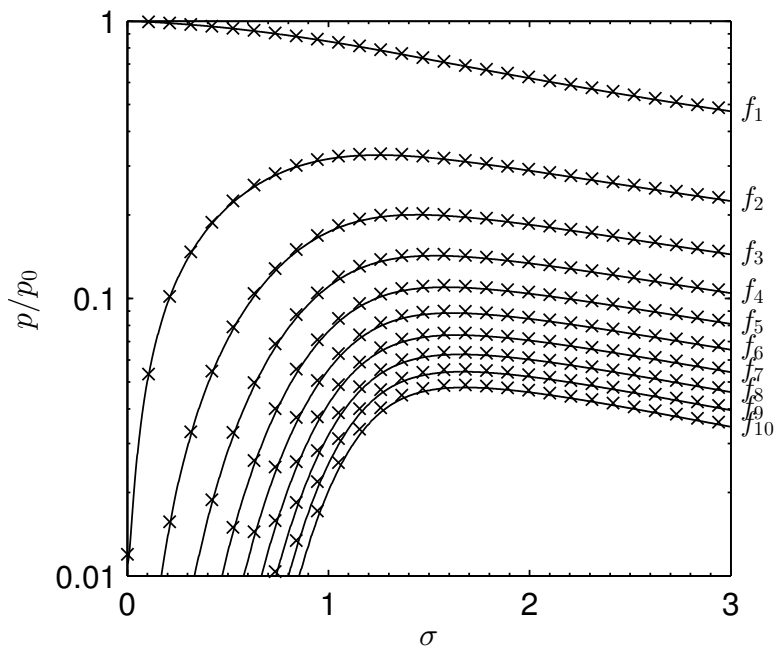

Figure 7.13: Spatial distribution of the first ten harmonics for $\gamma=1.6 . \quad k$-space numerical solution (gray line) and FDTD numerical solution (markers). 


\subsubsection{Nonlinear propagation in tissue-like media including diffraction}

\section{Experimental validation}

An experiment was designed to test the validity of the algorithm for intense beams in frequency power law attenuation media. The source was formed by a plane single element piezoceramic crystal (PZ 26, Ferroperm Piezoceramics, Denmark) mounted in a custom designed steel housing and a Polymethyl methacrylate (PMMA) focusing lens with aperture $A=50 \mathrm{~mm}$ and radius of curvature $R=50$ mm. The source was driven with a sinusoidal pulse burst of frequency $f_{0}=1.112$ $\mathrm{MHz}$ and $n=50$ cycles using a function generator (14 bits, $100 \mathrm{MS} / \mathrm{s}$, model PXI5412, National Instruments) and a linear RF amplifier (ENI 1040L, 400W, $55 \mathrm{~dB}, \mathrm{ENI}$, Rochester, NY). The pressure waveforms were acquired with a HNR $500 \mu \mathrm{m}$ needle PVDF hydrophone (Onda Corp, CA), and a digitizer (64 MS/s, model PXI5620, National Instruments) were used. A three-axis micropositioning system (OWIS GmbH, Germany) was used to move the hydrophone in three orthogonal directions with an accuracy of $10 \mu \mathrm{m}$ and a National Instruments PXITechnology controller NI8176 was used to control all the devices. The amplitude frequency response of the hydrophone was compensated in post-processing but not in phase due to the absence of phase calibration for this equipment. A scheme of the measurement setup is shown in Fig. 7.14.

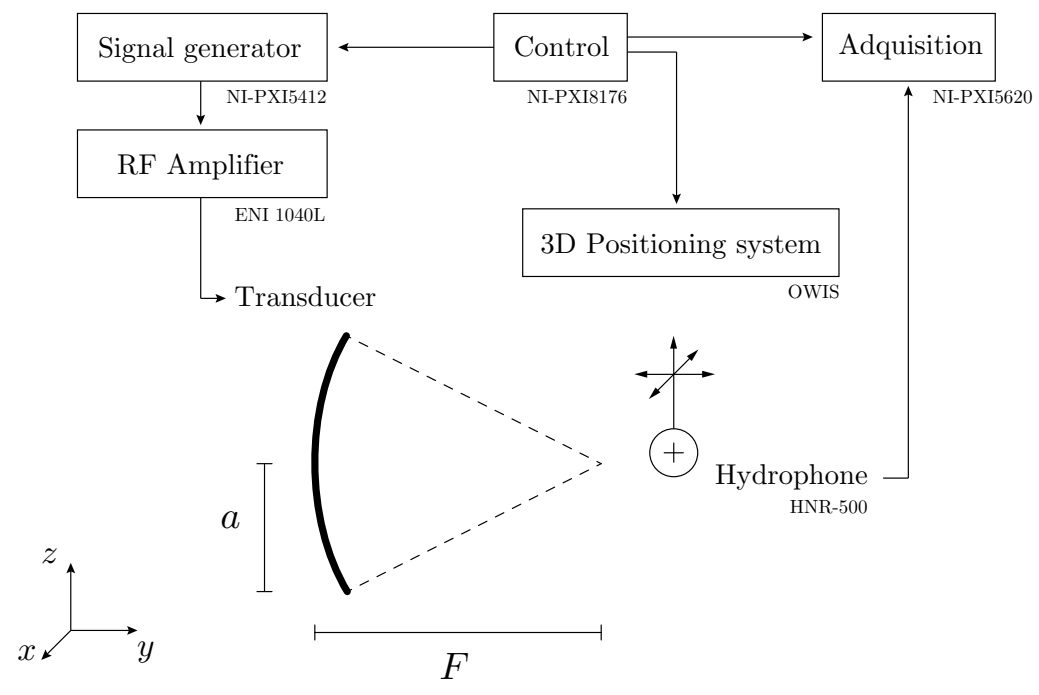

Figure 7.14: Scheme of the measurement system 

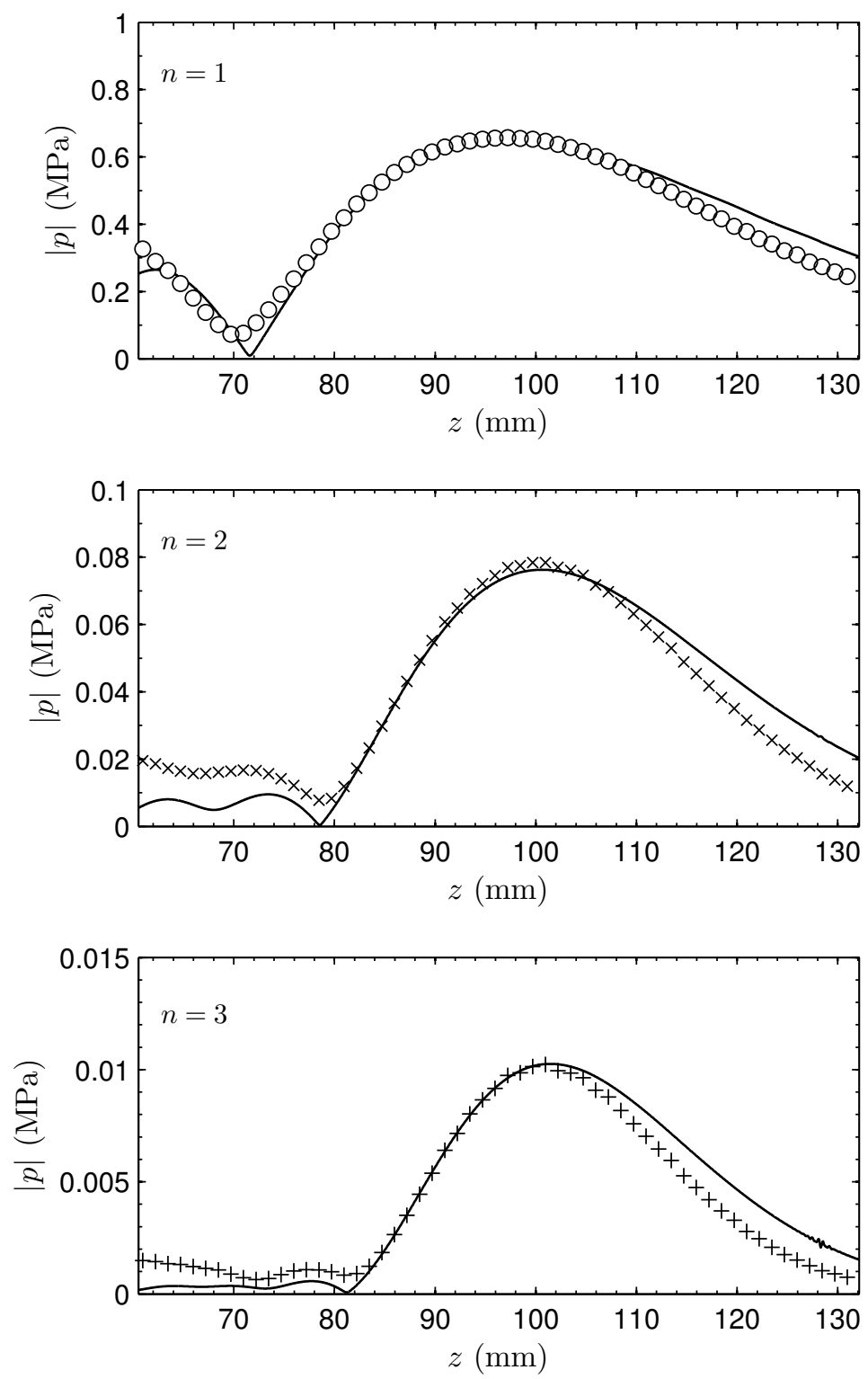

Figure 7.15: Spatial distribution of the fundamental $(o)$, second $(\times)$ and third $(+)$ harmonics obtained by the numerical (continuous line) and experimental methods (markers) for a focused transducer immersed in castor oil. 
The source was completely immersed in a castor oil tank $(350 \times 350 \times 350 \mathrm{~mm})$. We select this frequency power law attenuation media due to the low variability of its acoustic properties along existent literature (Liebler et al., 2004; Treeby et al., 2009). Using a sound speed inside the bulk of the lens $c_{l}=2711 \mathrm{~m} / \mathrm{s}$, and a sound speed of the castor oil of $c_{0}=1480 \mathrm{~m} / \mathrm{s}$ (at $26^{\circ} \mathrm{C}$ room temperature), the effective lens geometrical focal is estimated as $F=R /\left(1-c_{0} / c_{l}\right)=110.1 \mathrm{~mm}$, leading to a linear lossless gain of $G=13.4$.

On the other hand, a nonlinear simulation including diffraction and frequency power law attenuation with same parameters was carried out in a workstation (20 cores Intel Xeon E5-2680 CPU, 2.8GHz with 256 GB RAM). The boundary conditions were implemented for a spherical focused ultrasound source. The castor oil parameters at $26^{\circ} \mathrm{C}$ room temperature (Treeby et al., 2009), were $c_{0}=1480$ $\mathrm{m} / \mathrm{s}, \rho_{0}=961 \mathrm{~kg} / \mathrm{m}^{3}, \alpha=0.4 \mathrm{~dB} / \mathrm{cm} / \mathrm{MHz}^{\gamma}, \gamma=1.69, B / A=12.0$. The grid parameters were $\Delta r=\Delta z=29.6 \mu \mathrm{m}$ and $\Delta t=13.6 \mathrm{~ns}$, leading to a CFL number $S=0.95$ and $N_{\lambda}=50$ elements per wavelength at fundamental frequency, note that this grid leads to $N_{\lambda}=16$ for third harmonic.

The balance between nonlinear effects and power law attenuation can be estimated by using the Gol'dberg ratio, $\Gamma=x_{a} / x_{s}$ where $x_{s}$ is the shock formation distance and $x_{a}$ the media attenuation characteristic length. Thus, the amplitude of the source were selected to obtain a Gol'dberg ratio of $\Gamma=0.25$ in order to let the frequency power law attenuation effects slightly dominate over nonlinear effects. On the other hand, the ratio between diffraction effects and nonlinear effects can be described by the so called Khokhlov number $N=x_{d} / x_{s}$, where $x_{d}=k a^{2} / 2$ is the diffraction length and $a$ source radius. For the proposed test a Khokhlov number of $N=0.49$ was selected to let the nonlinear effect slightly dominate over diffraction effects. The selected excitation pressure amplitude was $p_{0}=87.7 \mathrm{kPa}$.

The results are summarized in Fig. 7.15, where axial pressure distribution for the fundamental, second and third harmonic are presented. A good agreement is found between simulations and the experimental test. Only far to the focal point the amplitude there exist differences between computations and experiments, that can be caused by nonuniform vibration of the source (Canney et al., 2008), boundary effects of the PMMA lens, or miss-alignment of the source axis and micropositioning system orthogonal directions along the $100 \mathrm{~mm}$ axial measurement.

The maximum amplitudes of the first harmonic were $p_{e 1}=0.6539 \mathrm{MPa}$ for the experiment and $p_{n 1}=0.6576$ for the numeric. The second harmonic peak pressure was $p_{e 2}=78.368 \mathrm{kPa}$ and $p_{n 2}=76.272 \mathrm{kPa}$, and the third harmonic peak pressure $p_{e 3}=10.146$ and $p_{n 3}=10.252 \mathrm{kPa}$ for the experimental and numeric respectively. The relative errors between numerical and experimental results are $0.56 \%$ for the fundamental frequency, $2.67 \%$ for the second harmonic and $1.04 \%$ for the third. No error estimation was done for the peak pressure due to the absence of a phase calibration of the hydrophone. 


\section{Highly focused beam}

In order to test the algorithm in the very high nonlinear regime with realistic tissue parameters a focused bowl of geometrical focal $F=50 \mathrm{~mm}$ and aperture $A=50$ mm, driven at $f_{0}=1 \mathrm{MHz}$ was numerically tested. These parameters leads to a source gain $G=26.5$ and a $f$-number $=1$, showing that source is beyond the paraxial limit. The media consist in two layers. The first, where the source was located, was water at $20^{\circ}$ with parameters $c_{1}=1482 \mathrm{~m} / \mathrm{s}, \rho_{1}=1000 \mathrm{~kg} / \mathrm{m}^{3}$, $B / A_{1}=5, \alpha_{1}=2.17 \times 10^{-3} \mathrm{~dB} / \mathrm{cm} \mathrm{MHz}^{\gamma}, \gamma_{1}=2$. At a distance $z_{l}=0.7 / F=35$ $\mathrm{mm}$, a layer of human liver tissue was placed, therefore the focal spot is located inside tissue at a depth of $15 \mathrm{~mm}$. Liver tissue parameters (Hill et al., 2004) were $c_{2}=1597, \rho_{2}=1050 \mathrm{~kg} / \mathrm{m}^{3}, B / A_{2}=7.9, \alpha_{2}=.75 \mathrm{~dB} / \mathrm{cm} \mathrm{MHz}^{\gamma}, \gamma_{2}=1.5$. In this case, the numerical grid parameters were $\Delta r=\Delta z=29.6 \mu \mathrm{m}$ and $\Delta t=11.9$ ns, leading to a CFL number $S=0.9$ and $N_{\lambda}=50$ elements per wavelength at fundamental frequency $\left(N_{\lambda}=16\right.$ for third harmonic).

Figure 7.16 shows the spatial peak pressure profiles for different excitation amplitudes. Thus, in Fig. 7.16 (top) the pressure of the source was $p_{0}=0.18 \mathrm{MPa}$, while in the bottom figure was increased to $p_{0}=0.94 \mathrm{MPa}$. Thus, for the selected parameters, Fig. 7.16 (top) present results for $\Gamma=0.16$ and $N=0.55$, so the attenuation effects dominates over nonlinear effects and nonlinearity dominates over diffraction effects. In this way, low asymmetry is observed between the positive compression peak, $p^{+}$, and the minimum rarefaction pressure distribution, $p^{-}$. The measured waveform at $z=F$, shown in the inset of Fig. 7.16 (top), is weakly distorted. However, there exist differences between its normalized peak amplitude $p^{+} / p_{0}=25.7$ and $p^{-} / p_{0}=21.05$, and the source characteristic linear gain, $G=26.5$. There are caused, in one hand by the attenuation effects, where the value of the lossy linear gain observed was $G_{\alpha}=p^{+} / p_{0}=p^{-} / p_{0}=23.1$ measured at $\mathrm{z}=\mathrm{F}$, i.e. the amplitude at the focal was reduced to $87.2 \%$ of the lossless amplitude. On the other hand, the differences due to the asymmetry between compression and rarefaction cycles are caused by the combined effect of nonlinearity and focusing.

If source amplitude is increased to $p_{0}=0.94 \mathrm{MPa}$, as shown in Fig. 7.16 (bottom), the ratio between attenuation and nonlinear effects is increased to $\Gamma=0.79$. In this regime, nonlinear effects are almost of the same order of attenuation effects. On the other hand, increasing source amplitude while keeping same transducer parameters implies also the Khoklov number changes to $N=0.11$, so the nonlinear effects clearly dominates over diffraction. In this regime, highly asymmetric pressure distribution is observed, where the values at focal point are $p^{+}=49.05 \mathrm{MPa}$ and $p^{-}=-14.91 \mathrm{MPa}$.

Other typical nonlinear phenomena characteristic of high intensity focused sources can also be observed: formation of sharp shock front and its corresponding harmonic generation, or, as Fig. (7.17) shows, narrowing of the beam for $p^{+}$ and broadening for $p^{-}$pressure distributions. In addition, nonlinear focal shift, 

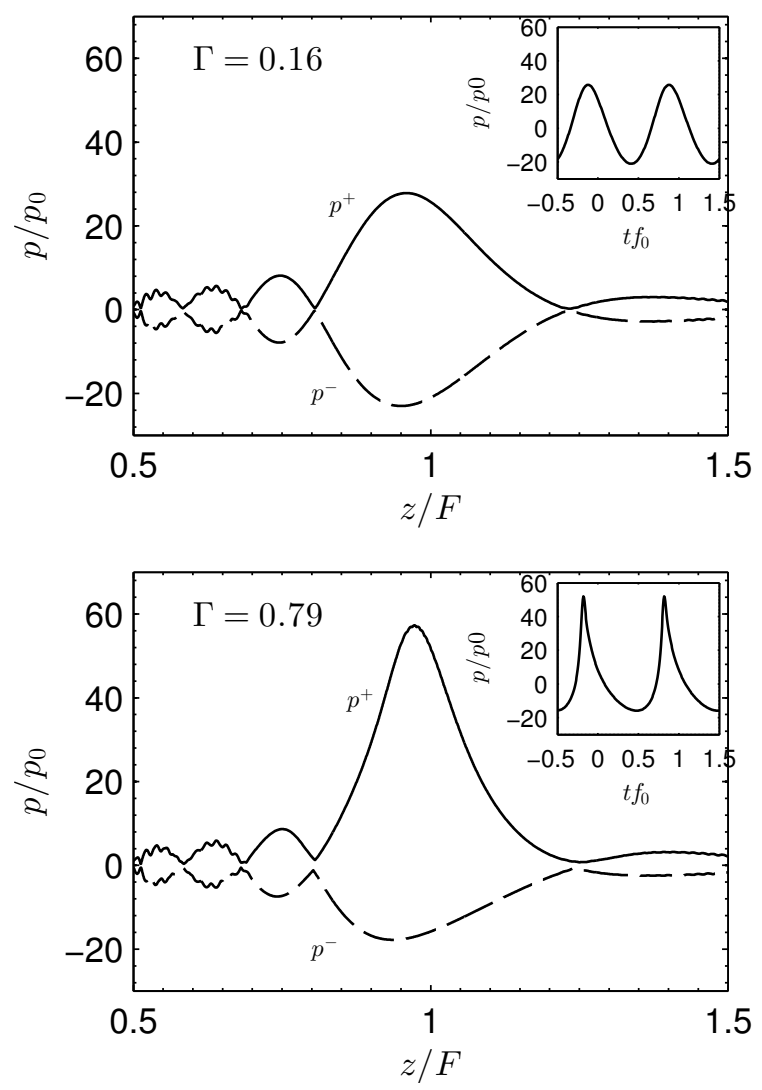

Figure 7.16: Axial spatial distribution of the peak compression $\left(p^{+}\right)$and minimum rarefaction $\left(p^{-}\right)$pressure for a focused transducer thought a liver tissue layer propagation. (Top) weakly nonlinear propagation $(\Gamma=0.16)$ and (bottom) strong nonlinear effects $(\Gamma=0.79)$. The insets show the waveforms recorded at the geometrical focal.

i.e. displacement of the peak pressure relative to the position of the liner peak pressure can be also predicted for tissue propagation. In the case of $\Gamma=0.79$ the nonlinear focal shift was $\Delta F^{+}=+1.05 \mathrm{~mm}$ and $\Delta F^{-}=-1.03 \mathrm{~mm}$ for the $p^{+}$ and $p^{-}$pressure distribution respectively.

\subsection{Summary}

A model for nonlinear acoustic waves in relaxing media is presented in a timedomain formulation which does not require convolutional operators. A numerical solution by means of finite-differences in time-domain have been obtained, showing that the theoretical attenuation and dispersion due to relaxation processes can be 

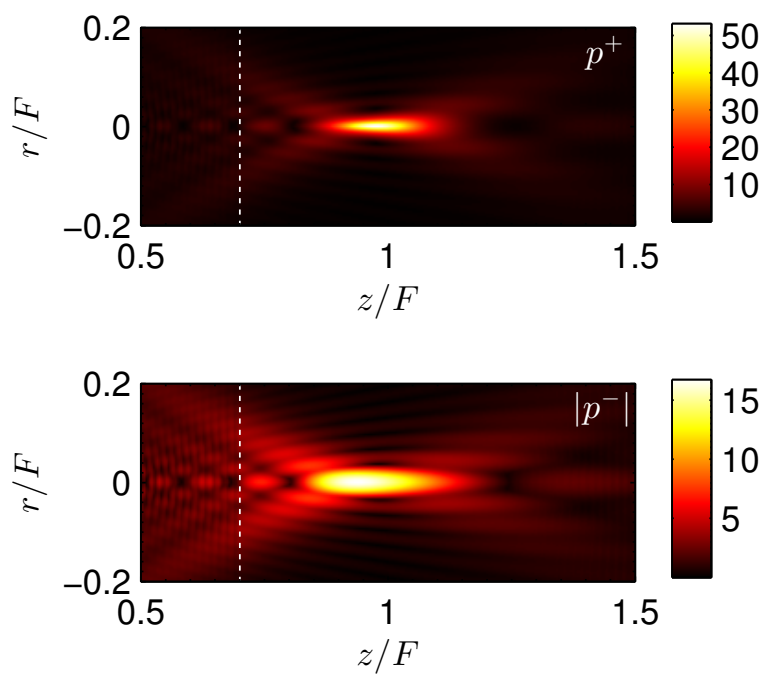

Figure 7.17: Spatial distribution of the peak compression $\left(p^{+}\right)$and minimum rarefaction $\left(p^{-}\right)$ pressure for a focused transducer thought a liver tissue layer propagation (boundary marked with dashed line) for $\Gamma=0.79$. Colorbars are in $p / p_{0}$ units.

achieved by the numerical method with accuracy. These result can be used to model typical relaxation process (e. g. the processes observed in air, associated to the molecules of oxygen and nitrogen, or in seawater, associated to the relaxation of boric acid and magnesium sulfate).

Moreover, a method for modeling a frequency power law on attenuation by means of multiple relaxation has been described. The proposed method can describe local variations of the exponent of the frequency power law, so an arbitrary attenuation curve in the range $0<\gamma<2$ can be modeled by means of the proper optimization of the relaxation coefficients. This feature of the presented method is an advantage when compared with most fractional derivatives methods, where the attenuation follows an exact but unique frequency power law over the entire frequency range. A broad range of human tissues have been modeled and the goodness of the fit using from two to four relaxation processes has been discussed.

Furthermore, a computational technique that exploits the anomalous dispersion of the relaxation processes is employed to mitigate the numerical dispersion of the finite-differences scheme. Thus, while phase speed is corrected by including artificial relaxation processes, its corresponding artificial attenuation is used to improve stability in the nonlinear regime. In this way, a smooth version of shock waves have been obtained and compared with analytic solutions. Furthermore, the validity of the algorithm including diffraction have been tested with experimental 
measurements of a focused beam in castor oil.

Due to the model is developed from the constitutive relation for nonlinear acoustics, most wave phenomena is captured. As a difference from the one-way models the proposed model implicitly includes multiple wave direction, and, due to the Lagrangian density of acoustic energy is implicitly included in the computation, multiple scattering and strong resonance effects are accurately described. Moreover, unlike KZK and other parabolic approximations, the proposed model captures the diffraction exactly, so for simulation of acoustic beams the field is not approximated only to the beam axis, but also in the near field and far to the beam axis and thus high focusing devices can be simulated.

The code has shown to be particularly appropriate if the problem to simulate presents axisymmetry, because the constitutive relations for nonlinear acoustics are solved in a computational 2D domain, while standard $k$-space methods need to employ full 3D domains due to the poor convergence of the Fourier series at discontinuities $(r=0)$. This is the case, for example, of the focused transducer simulated in section 7.5.4, where a full $3 \mathrm{D}$ solution will require a huge computational resources and time. Finally, the particle velocity vector is solved implicitly by the code and can be used to estimate other relevant magnitudes as the full nonlinear intensity vector, the nonlinear radiation force field in the absorbing media or the acoustic streaming in frequency power law attenuation fluids.

\section{References}

Aanonsen, Sigurd Ivar, Tor Barkve, Jacqueline Naze Tjo, et al. (1984). "Distortion and harmonic generation in the nearfield of a finite amplitude sound beam". In: J. Acoust. Soc. Am. 75.3, pp. $749-768$.

Albin, Nathan, Oscar P. Bruno, Theresa Y. Cheung, and Robin O. Cleveland (2012). "Fourier continuation methods for high-fidelity simulation of nonlinear acoustic beams". In: J. Acoust. Soc. Am. 132.4, pp. 2371-2387.

Blackstock, David T (1985). "Generalized Burgers equation for plane waves". In: J. Acoust. Soc. Am. 77.6, pp. 2050-2053.

Botteldooren, D. (1996). "Numerical model for moderately nonlinear sound propagation in threedimensional structures". In: J. Acoust. Soc. Am. 100.3, pp. 1357-1367.

Canney, Michael S, Michael R Bailey, Lawrence A Crum, Vera A Khokhlova, and Oleg A Sapozhnikov (2008). "Acoustic characterization of high intensity focused ultrasound fields: A combined measurement and modeling approach". In: The Journal of the Acoustical Society of America 124.4, pp. 2406-2420.

Caputo, M. (1967). "Linear models of dissipation whose Q is almost frequency independent II". In: Geophys. J. R. Astron. Soc. 13, pp. 529-539.

Carstensen, EL, WK Law, ND McKay, and TG Muir (1980). "Demonstration of nonlinear acoustical effects at biomedical frequencies and intensities". In: Ultrasound Med. Biol. 6.4, pp. 359368 .

Chen, W and S Holm (2003). "Modified Szabos wave equation models for lossy media obeying frequency power law". In: J. Acoust. Soc. Am. 114.5, pp. 2570-2574.

Chen, W and S Holm (2004). "Fractional Laplacian time-space models for linear and nonlinear lossy media exhibiting arbitrary frequency power-law dependency". In: J. Acoust. Soc. Am. 115.4, pp. 1424-1430. 
Christopher, P Ted and Kevin J Parker (1991). "New approaches to nonlinear diffractive field propagation". In: J. Acoust. Soc. Am. 90.1, pp. 488-499.

Cleveland, Robin O, Mark F Hamilton, and David T Blackstock (1996). "Time-domain modeling of finite-amplitude sound in relaxing fluids". In: J. Acoust. Soc. Am. 99.6, pp. 3312-3318.

Demi, L, KWA Van Dongen, and MD Verweij (2011). "A contrast source method for nonlinear acoustic wave fields in media with spatially inhomogeneous attenuation". In: J. Acoust. Soc. Am. 129.3, pp. 1221-1230.

Duck, Francis A. (1990). Physical Properties of Tissue: A Comprehensive Reference Book. San Diego, CA 92101, USA: Academic Press Inc. San Diego, p. 346.

Ginter, Siegfried, Marko Liebler, Eckard Steiger, Thomas Dreyer, and Rainer E. Riedlinger (2002). "Full-wave modeling of therapeutic ultrasound: nonlinear ultrasound propagation in ideal fluids." In: J. Acoust. Soc. Am. 111.5, pp. 2049-2059.

Goss, S. A., L. A. Frizzell, and F. Dunn (1979). "Ultrasonic absorption and attenuation in mammalian tissues". In: Ultrasound Med. Biol. 5 (2), pp. 181-186.

Hallaj, Ibrahim M and Robin O Cleveland (1999). "FDTD simulation of finite-amplitude pressure and temperature fields for biomedical ultrasound". In: J. Acoust. Soc. Am. 105.5, pp. L7L12.

Hallaj, Ibrahim M, Robin O Cleveland, and Kullervo Hynynen (2001). "Simulations of the thermo-acoustic lens effect during focused ultrasound surgery". In: The Journal of the Acoustical Society of America 109.5, pp. 2245-2253.

Hamilton, M.F. and D.T. Blackstock (1998b). Nonlinear Acoustics. Academic Press.

Hill, C.R., J. C. Bamber, and G.R. ter Haar (2004). Physical Principles of Medical Ultrasonics, 2nd ed. John Wiley \& Sons Ltd, Chichester, England.

Huijssen, Jacobus and Martin D Verweij (2010). "An iterative method for the computation of nonlinear, wide-angle, pulsed acoustic fields of medical diagnostic transducers". In: J. Acoust. Soc. Am. 127.1, pp. 33-44.

Jing, Yuan and Robin O Cleveland (2007). "Modeling the propagation of nonlinear threedimensional acoustic beams in inhomogeneous media". In: J. Acoust. Soc. Am. 122.3, pp. 13521364.

Jing, Yun, Du Shen, and Greg T Clement (2011). "Verification of the Westervelt equation for focused transducers". In: Ultrasonics, Ferroelectrics and Frequency Control, IEEE Transactions on 58.5, pp. 1097-1101.

Jing, Yun, Tianren Wang, and Greg T Clement (2012). "A k-space method for moderately nonlinear wave propagation". In: Ultrasonics, Ferroelectrics and Frequency Control, IEEE Transactions on 59.8, pp. 1664-1673.

Jongen, HAH, JM Thijssen, M Van Den Aarssen, and WA Verhoef (1986). "A general model for the absorption of ultrasound by biological tissues and experimental verification". In: The Journal of the Acoustical Society of America 79.2, pp. 535-540.

Kellya, James F. and Robert J. McGough (2009). "Fractal ladder models and power law wave equations". In: J. Acoust. Soc. Am. 126.4, pp. 2072-2081.

Khokhlova, VA, AE Ponomarev, MA Averkiou, and LA Crum (2006). "Nonlinear pulsed ultrasound beams radiated by rectangular focused diagnostic transducers". In: Acoustical Physics 52.4, pp. 481-489.

Lee, Yang-Sub and Mark F Hamilton (1995). "Time-domain modeling of pulsed finite-amplitude sound beams". In: J. Acoust. Soc. Am. 97.2, pp. 906-917.

LeVeque, R.J. (1992). Numerical Methods for Conservation Laws. Ed. by Lectures in Mathematics. ETH Zurich. Birkhauser Verlag.

Liebler, Marko, Siegfried Ginter, Thomas Dreyer, and Rainer E. Riedlinger (2004). "Full wave modeling of therapeutic ultrasound: efficient time-domain implementation of the frequency power-law attenuation." In: J. Acoust. Soc. Am. 116.5, pp. 2742-2750.

Liu, Q. H. (1999). "Perfectly matched layers for elastic waves in cylindrical and spherical coordinates". In: J. Acoust. Soc. Am. 105.4, pp. 2075-2084.

Liu, Qing-Huo and Jianping Tao (1997). "The perfectly matched layer for acoustic waves in absorptive media". In: J. Acoust. Soc. Am. 102.4, pp. 2072-2082. 
Lobanova, E. G., S. V. Lobanov, and V. A. Khokhlova (2014). "Counterpropagation of waves with shock fronts in a nonlinear tissue-like medium". In: Acoustical Physics 60.4, pp. 387397.

Manry, Charles W and Shira L Broschat (1996). "FDTD simulations for ultrasound propagation in a 2-D breast model". In: Ultrasonic imaging 18.1, pp. 25-34.

Mast, T Douglas, Laura M Hinkelman, Michael J Orr, Victor W Sparrow, and Robert C Waag (1997). "Simulation of ultrasonic pulse propagation through the abdominal wall". In: $J$. Acoust. Soc. Am. 102.2, pp. 1177-1190.

Mast, T Douglas, Laurent P Souriau, D-LD Liu, Makoto Tabei, Adrian I Nachman, and Robert C Waag (2001). "A k-space method for large-scale models of wave propagation in tissue". In: Ultrasonics, Ferroelectrics and Frequency Control, IEEE Transactions on 48.2, pp. 341-354.

Muir, TG and EL Carstensen (1980). "Prediction of nonlinear acoustic effects at biomedical frequencies and intensities". In: Ultrasound Med. Biol. 6.4, pp. 345-357.

Nachman, Adrian I, James F Smith III, and Robert C Waag (1990). "An equation for acoustic propagation in inhomogeneous media with relaxation losses". In: J. Acoust. Soc. Am. 88, p. 1584 .

Nasholm, Sven Peter and Sverre Holm (2011). "Linking multiple relaxation, power-law attenuation, and fractional wave equations". In: J. Acoust. Soc. Am. 130.5, pp. 3038-3045.

Naugolnykh, K. and L. Ostrovsky (1998). Nonlinear Wave Processes in Acoustics. Cambridge Texts in Applied Mathematics. Cambridge University Press.

Ochmann, Martin and Sergey Makarov (1993). "Representation of the absorption of nonlinear waves by fractional derivatives". In: The Journal of the Acoustical Society of America 94.6, pp. 3392-3399.

O'Donnell, M., E. T. Jaynes, and J. G. Miller (1981). "Kramers-Kronig relationship between ultrasonic attenuation and phase velocity". In: J. Acoust. Soc. Am. 69.3, pp. 696-701.

Pierce, A.D. (1989). Acoustics: An Introduction to Its Physical Principles and Applications. Acoustical Society of America.

Pinton, Gianmarco F, Jeremy Dahl, Stephen Rosenzweig, and Gregg E Trahey (2009). "A heterogeneous nonlinear attenuating full-wave model of ultrasound". In: Ultrasonics, Ferroelectrics and Frequency Control, IEEE Transactions on 56.3, pp. 474-488.

Prieur, Fabrice and Sverre Holm (2011). "Nonlinear acoustic wave equations with fractional loss operators". In: J. Acoust. Soc. Am. 130.3, pp. 1125-1132.

Rudenko, O.V. and S.I. Soluian (1977). Theoretical foundations of nonlinear acoustics. Studies in Soviet science. New York, NY 10011, USA: Consultants Bureau, p. 274.

Shahbazi, Khosro, Nathan Albin, Oscar P. Bruno, and Jan S. Hesthaven (2011). "Multi-domain Fourier-continuation/WENO hybrid solver for conservation laws". In: J. Comput. Phys. 230.24 , pp. $8779-8796$.

Soneson, Joshua E and Matthew R Myers (2007). "Gaussian representation of high-intensity focused ultrasound beams". In: The Journal of the Acoustical Society of America 122.5, pp. 2526-2531.

Sparrow, Victor W and Richard Raspet (1991). "A numerical method for general finite amplitude wave propagation in two dimensions and its application to spark pulses". In: J. Acoust. Soc. Am. 90.5, pp. 2683-2691.

Szabo, Thomas L (1994). "Time domain wave equations for lossy media obeying a frequency power law". In: J. Acoust. Soc. Am. 96.1, pp. 491-500.

Tavakkoli, Jahangir, Dominique Cathignol, Rémi Souchon, and Oleg A Sapozhnikov (1998). "Modeling of pulsed finite-amplitude focused sound beams in time domain". In: The Journal of the Acoustical Society of America 104.4, pp. 2061-2072.

Treeby, Bradley E, Benjamin T Cox, Edward Z Zhang, Sarah K Patch, and Paul C Beard (2009). "Measurement of broadband temperature-dependent ultrasonic attenuation and dispersion using photoacoustics". In: Ultrasonics, Ferroelectrics, and Frequency Control, IEEE Transactions on 56.8 , pp. $1666-1676$.

Treeby, Bradley E and BT Cox (2010). "Modeling power law absorption and dispersion for acoustic propagation using the fractional Laplacian". In: J. Acoust. Soc. Am. 127.5, pp. 27412748. 
Treeby, Bradley E, Jiri Jaros, Alistair P Rendell, and BT Cox (2012). "Modeling nonlinear ultrasound propagation in heterogeneous media with power law absorption using a k-space pseudospectral method". In: J. Acoust. Soc. Am. 131.6, pp. 4324-4336.

Varslot, Trond and Gunnar Taraldsen (2005). "Computer simulation of forward wave propagation in soft tissue". In: Ultrasonics, Ferroelectrics and Frequency Control, IEEE Transactions on 52.9, pp. 1473-1482.

Verweij, Martin D, Libertario Demi, and Koen WA van Dongen (2013). "Computation of nonlinear ultrasound fields using a linearized contrast source method". In: J. Acoust. Soc. Am. 134.2, pp. 1442-1453.

Waters, Kendall R, Joel Mobley, and James G Miller (2005). "Causality-imposed (KramersKronig) relationships between attenuation and dispersion". In: Ultrasonics, Ferroelectrics and Frequency Control, IEEE Transactions on 52.5, pp. 822-823.

Wismer, M. G. (2006). "Finite element analysis of broadband acoustic pulses through inhomogeneous media with power law attenuation". In: J. Acoust. Soc. Am. 120, pp. 3493-3502.

Wismer, Margaret G and Reinhold Ludwig (1995). "An explicit numerical time domain formulation to simulate pulsed pressure waves in viscous fluids exhibiting arbitrary frequency power law attenuation". In: Ultrasonics, Ferroelectrics and Frequency Control, IEEE Transactions on 42.6 , pp. 1040-1049.

Yang, Xinmai and Robin O Cleveland (2005). "Time domain simulation of nonlinear acoustic beams generated by rectangular pistons with application to harmonic imaging". In: J. Acoust. Soc. Am. 117.1, pp. 113-123.

Yuan, Xiaojuen, D. Borup, J. Wiskin, M. Berggren, and S. A. Johnson (1999). "Simulation of acoustic wave propagation in dispersive media with relaxation losses by using FDTD method with PML absorbing boundary condition". In: IEEE Transactions on Ultrasonics, Ferroelectrics, and Frequency Control 46.1, pp. 14-23.

Yuldashev, PV and VA Khokhlova (2011). "Simulation of three-dimensional nonlinear fields of ultrasound therapeutic arrays". In: Acoustical physics 57.3, pp. 334-343.

Zemp, Roger J, Jahangir Tavakkoli, and Richard SC Cobbold (2003). "Modeling of nonlinear ultrasound propagation in tissue from array transducers". In: J. Acoust. Soc. Am. 113.1, pp. 139-152.

\section{Publications}

The contents of this chapter have been presented in the following publications:

\section{Journal papers}

- Jiménez, N.; Camarena, F.; Redondo, J.; Sánchez-Morcillo, V.; Hou Y. and Konofagou E. E. "Time-domain simulation of ultrasound propagation in tissue-like medium based on the resolution of the nonlinear acoustic constitutive relations". The Journal of Acoustical Society of America, In preparation.

\section{Conference proceedings}

- Jiménez, N., F. Camarena, V. Sanchez-Morcillo, J. Redondo and E.E. Konofagou. "Time-domain simulation of constitutive relations for nonlinear acoustics including relaxation for frequency power law attenuation media modelling". ISNA 2015, 
International Symposium of Nonlinear Acoustics. June 29th - July 3rd 2015, Lyon, France (2015).

- Jiménez, N.; Redondo, J.; Sánchez-Morcillo, V. and Camarena, F. "Nonlinear ultrasound simulations including complex frequency dependent attenuation". 43rd Annual UIA Symposium, Madrid, Spain. 23 - 25 April. Physics Procedia, Volume 63, 2015, Pages 108-113, (2014)

- Jiménez, N.; Redondo, J.; Sanchez-Morcillo, V. and Camarena, F. "Ondas acústicas de amplitud finita en medios con relajación: aplicación a la modelización de tejidos biológicos". $44^{\circ}$ Tecniacústica'13, Valladolid, October, 1632-1637 (2013)

And the numerical method method have been applied for obtaining the following results

\section{Journal papers}

- Jiménez, N.; Redondo, J.; Sanchez-Morcillo, V. and Camarena, F. "Numerical Study of Nonlinear, Transcranial Focused Ultrasound Wave Propagation for BloodBrain Barrier Opening". IEEE Ultrasonics, Ferroelectrics and Frequency Control Society, In preparation.

- Jiménez, N.; Marquet, F.; Camarena, F.; Konofagou, E. E.; Redondo, J.; Roig, B. and Picó, R. "Estudio de la propagación no lineal de haces de ultrasonidos focalizados a través del cráneo para la apertura de la barrera hematoencefálica". Revista de Acústica, 43, 5-11 (2012)

\section{Conference proceedings}

- Ordoñez-Cebrián, P; Jiménez, N.; Espinosa, V.; Puig-Pons, V. and SoliveresGonzález, E. "Efficiency of parametric ultrasound generation in relaxing media for very shallow-water echo sounders". 43rd Annual UIA Symposium. Madrid, Spain. 23-25 April, (2014)

- Iglesias, P. C.; Jiménez, N.; Konofagou, E.; Camarena, F. and Redondo, J. "Transcranial Propagation with an Ultrasonic Mono-Element Focused Transducer". 43rd Annual UIA Symposium, Madrid, Spain. 23-25 April. Physics Procedia Volume 63, 2015, Pages 103-107, (2014)

- Iglesias, P. C.; Jimenez, N.; Redondo, J.; Konofagou, E. E. and Camarena, F. "Parametric study of transcranial focused ultrasound propagation for blood-brain barrier opening". European Symposium on Ultrasonic Characterization of Bone. Granada, 7-10 May, (2013)

- Jiménez, N.; Roig, B.; Redondo, J.; Pico, R.; Marquet, F.; Konofagou, E. E. and Camarena, F. "Numerical study of nonlinear, transcranial focused ultrasound wave propagation for blood-brain barrier (BBB) opening". ISTU 2011: International Society for Therapeutic Ultrasound Symposium, New York, EEUU, (2011) 


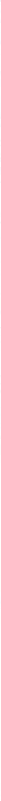

\title{
Chapter 8
}

\section{Nonlinear Plane Waves in Frequency Power Law Attenuation Media}

\begin{abstract}
In this chapter we revise fundamental aspects of nonlinear acoustic plane wave propagation through frequency power law attenuation media, with special focusing in the implications for finite amplitude ultrasound in biological media. The basic relationships between nonlinearity, absorption and dispersion are explored in detail, among with its implications for soft-tissue modeling in biomedical ultrasound applications. A derivation of simple expressions for quantify the role of the exponent of the power law in the balance between nonlinearity, dispersion and attenuation is presented. Detailed numerical calculations of the harmonic generation are also provided, where the efficiency in the harmonic generation and its relation with the weak frequency power law dispersion is studied.
\end{abstract}




\subsection{Introduction}

When modeling longitudinal acoustic wave propagation in biological soft-tissue media it is critical to account for the correct attenuation and phase speed. When dealing with linear monochromatic propagation, the values of media attenuation and phase speed for a given frequency can be explicitly included in the model equations from those obtained from experimental tests. A common practice is to adopt a sound speed value from the experimentally measured phase speed as $c_{0}=c_{p}\left(\omega_{0}\right)$, and to match a value for the fluid viscosity, $\mu$, that match the attenuation value at the desired frequency. However, if finite amplitude propagation is considered, the attenuation and phase velocity of each spectral component must be explicitly included in the model. In the case of biological media, where frequency power law attenuation is experimentally observed, there it is impossible to model these losses with only viscosity as long viscous losses present a frequency squared dependent attenuation and no dispersion. Thus, the inclusion of a causal-time domain operator that models frequency power law attenuation is necessary to accurately model the losses of each spectral component. Moreover, the inclusion of a frequency power law leads to the inevitable inclusion of dispersion that must be accurately modeled. Thus, for a frequency dependent attenuation in the form

$$
\alpha(\omega)=\alpha_{0} \omega^{\gamma}
$$

and by applying the Kramers-Kronig relationships (O'Donnell et al., 1981), the frequency dependent phase speed can be obtained as (Waters et al., 2005):

$$
\frac{1}{c_{p}(\omega)}=\frac{1}{c_{0}}+\alpha_{0} \tan \left(\frac{\pi \gamma}{2}\right)\left(|\omega|^{\gamma-1}-\left|\omega_{0}\right|^{\gamma-1}\right) .
$$

This expression is valid in the range $0<\gamma<3$ with $\gamma \neq 1$ and includes causality. In the limit $\gamma \rightarrow 1$ Eq. 8.2 reduces to

$$
\left.\frac{1}{c_{p}(\omega)}\right|_{\gamma=1}=\frac{1}{c_{0}}-\frac{2}{\pi} \alpha_{0} \log \left|\frac{\omega}{\omega_{0}}\right| .
$$

On the other hand, the complex wave number $\tilde{k}$ can be written as

$$
\tilde{k}(\omega)=\frac{\omega}{c_{p}(\omega)}-i \alpha(\omega)
$$



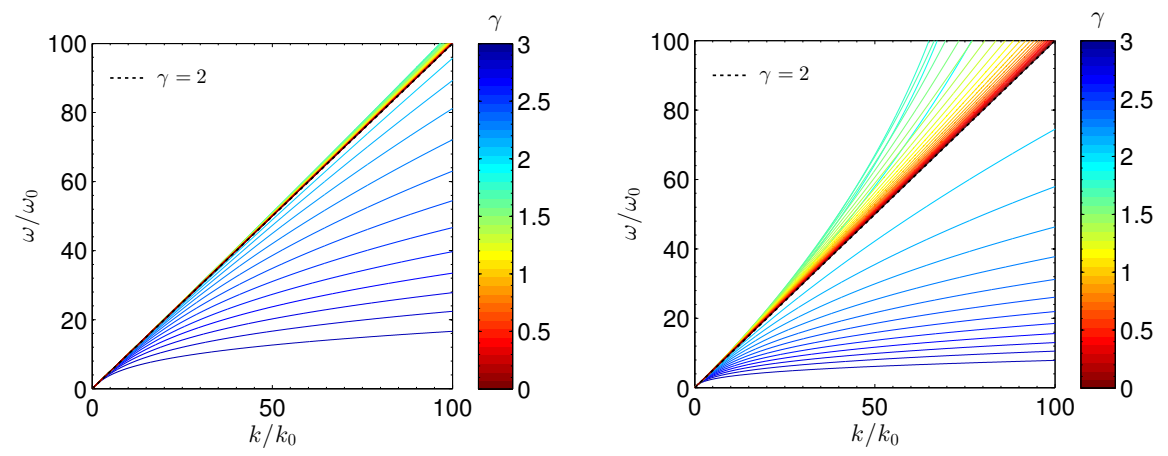

Figure 8.1: Dispersion relations for different frequency power laws for (a) $\alpha_{0}=10 \mathrm{~dB} / \mathrm{cm}$ and (b) $\alpha_{0}=1 \mathrm{~dB} / \mathrm{cm}$ measured at $1 \mathrm{MHz}$.

where $\operatorname{Im}(\tilde{k})$ is defined by the frequency power law attenuation and $\operatorname{Re}(\tilde{k})$ defines the phase speed. Combining Eq. 8.2, 8.1 and 8.4 the dispersion relations for a frequency power law media read

$$
\tilde{k}(\omega)=\frac{\omega}{c_{0}}+\alpha_{0} \omega \tan \left(\frac{\pi \gamma}{2}\right)\left(|\omega|^{\gamma-1}-\left|\omega_{0}\right|^{\gamma-1}\right)-i \alpha_{0} \omega^{\gamma}
$$

for $0<\gamma<1$ and $1<\gamma<3$, and

$$
\left.\tilde{k}(\omega)\right|_{\gamma=1}=\frac{\omega}{c_{0}}-\frac{2 \alpha_{0} \omega}{\pi} \log \left|\frac{\omega}{\omega_{0}}\right|-i \alpha_{0} \omega
$$

for $\gamma=1$.

Thus, as shown in Fig. 8.1, the dispersion modeled by a frequency power law shows three different behavior depending on the power law exponent. First, for frequency independent losses, $\gamma=0$, the media exhibit no dispersion as long $c_{p}=c_{0}$. This behavior is equivalent to the other simple physical attenuation models, e.g. the damped pendulum. On the other hand, this dispersionless regime is achieved also for $\gamma=2$, as it is observed in acoustics for bulk thermo-viscous losses in pure water. Second, for $0<\gamma<2$ the real part of the wavenumber reduces in magnitude as long the frequency increases, so phase speed is increased in the higher frequency range, leading to the so called "anomalous dispersion" (Hill et al., 2004) (e.g. as observed also in water capillary waves). Finally, for media obeying frequency power law attenuation with $2<\gamma<3$ the phase speed decreases with increasing frequency, so "normal" dispersion is achieved as observed in other common physical systems as water surface gravity waves.

The frequency power law attenuation obtained for a soft-tissue media with an attenuation of $1 \mathrm{~dB} / \mathrm{cm}$ and for $10 \mathrm{~dB} / \mathrm{cm}$ measured at $1 \mathrm{MHz}$ is shown in 
Fig. 8.2. In the same way, Fig. 8.3 shows the frequency dependent phase speed from Eq. $(8.2,8.3)$, where for a frequency range from 0.1 to $10 \mathrm{MHz}$ variations of about $\pm 9 \mathrm{~m} / \mathrm{s}$ are obtained in the case of $1 \mathrm{~dB} / \mathrm{cm}$ at $1 \mathrm{MHz}$, and $\approx \pm 90$ $\mathrm{m} / \mathrm{s}$ for the case of $10 \mathrm{~dB} / \mathrm{cm}$ for a linear power law. Thus, the magnitude of the dispersion of the system increases linearly with the attenuation magnitude. This behavior is in agreement with dispersion measurements in weakly (Szabo, 1995; Waters et al., 2000) and strongly (Fry et al., 1978) lossy media obeying frequency power law attenuation, and shows the importance of modeling correctly not only the attenuation but the dispersion when dealing with high damped media as biological tissues.
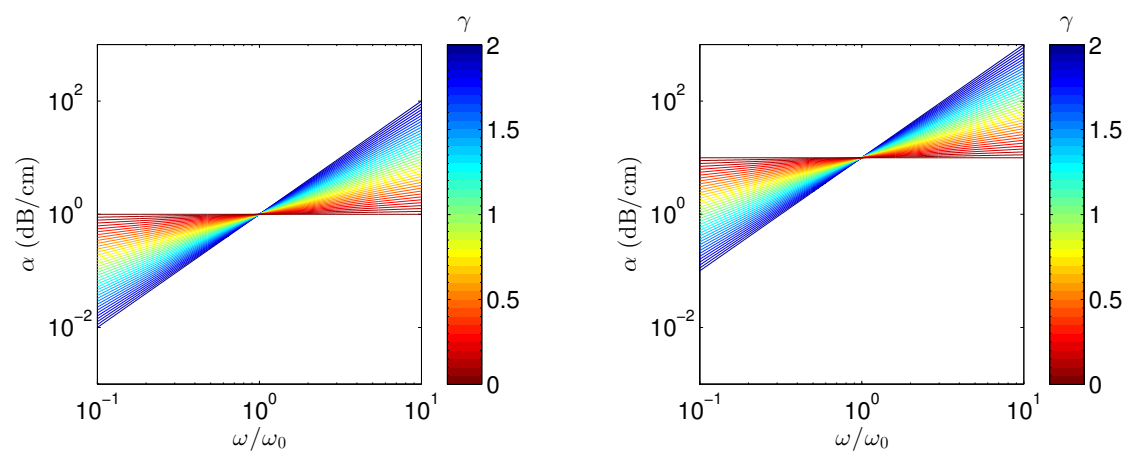

Figure 8.2: Frequency dependent attenuation obtained for different frequency power laws for (a) $\alpha_{0}=10 \mathrm{~dB} / \mathrm{cm}$ and (b) $\alpha_{0}=1 \mathrm{~dB} / \mathrm{cm}$ measured at $1 \mathrm{MHz}$.
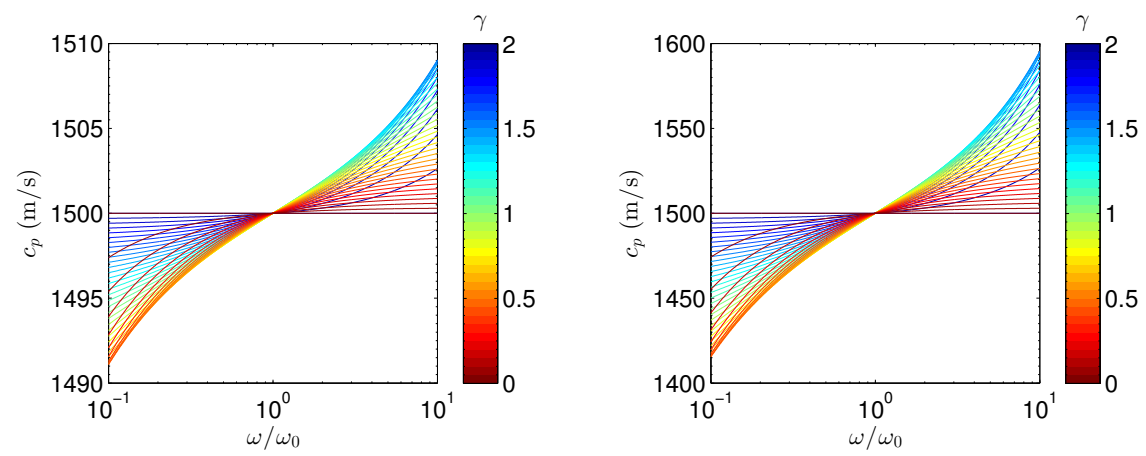

Figure 8.3: Phase velocity obtained for different frequency power laws for (a) $\alpha_{0}=20 \mathrm{~dB} / \mathrm{cm}$ and (b) $\alpha_{0}=2$ measured at $1 \mathrm{MHz}$. 


\subsection{Nonlinear coherence length}

As introduced in Chapter 2, the coherence length magnitude is very helpfully in quantifying the nonlinear acoustic effects in dispersive media. Thus, the efficiency of energy transfer from fundamental to second harmonic component is dependent on the phase matching condition:

$$
k(2 \omega)=2 k(\omega) .
$$

In general, for dispersive media this condition is not fulfilled, so the second harmonic characteristic linear growth develops the well known beating effect (Rudenko et al., 1977), where the beating spatial frequency for the second harmonic is

$$
\Delta k_{2}(\omega)=|2 k(\omega)-k(2 \omega)|
$$

and the coherence length for the second harmonic is defined as

$$
x_{c}(\omega)=\frac{\pi}{\Delta k_{2}(\omega)} .
$$

In the case study, i.e. in frequency power law attenuation media, using Eqs. (8.5, 8.6), Eq. (8.8) reduces to

$$
\Delta k_{2}(\omega)=\left(2^{\gamma}-2\right) \alpha_{0} \omega^{\gamma} \tan \left(\frac{\pi \gamma}{2}\right)
$$

for $0<\gamma<3$ with $\gamma \neq 1$, and

$$
\left.\Delta k_{2}(\omega)\right|_{\gamma=1}=-\frac{4 \log (2) \alpha_{0} \omega^{\gamma}}{\pi}
$$

for $\gamma=1$. Then, combining Eq. (8.9) and Eq. (8.10), the characteristic nonlinear coherence length (for second harmonic) can be calculated as

$$
x_{c}(\omega)=\frac{\pi \cot \left(\frac{\pi \gamma}{2}\right)}{\left(2^{\gamma}-2\right) \alpha_{0} \omega^{\gamma}}
$$




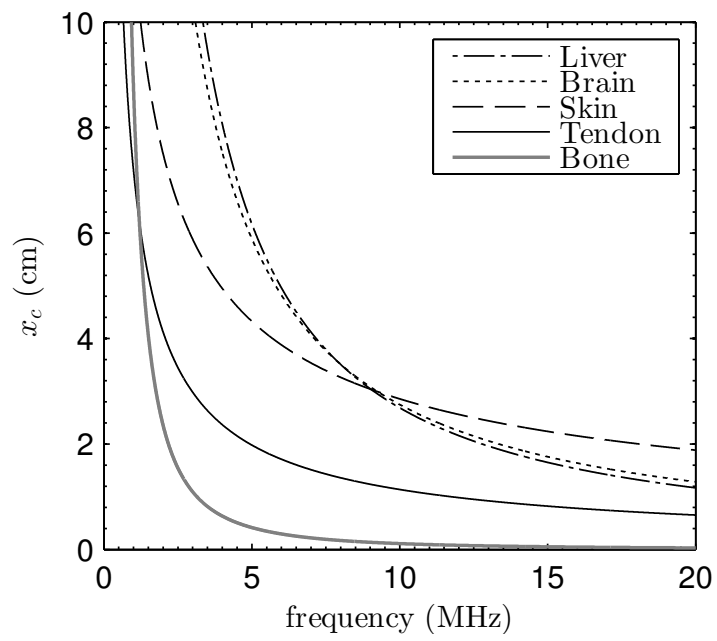

Figure 8.4: Coherence length as a function of frequency for a variety of tissues, where it has been assumed the following power laws: $\alpha_{\text {Liver }}=0.7 \mathrm{~dB} / \mathrm{cm} / \mathrm{MHz}^{1.2}, \alpha_{\text {Brain }}=0.87 \mathrm{~dB} / \mathrm{cm} / \mathrm{MHz}^{1.1}$, $\alpha_{\text {Skin }}=3.6 \mathrm{~dB} / \mathrm{cm} / \mathrm{MHz}^{0.6}, \alpha_{\text {Tendon }}=4.77 \mathrm{~dB} / \mathrm{cm} / \mathrm{MHz}^{0.8}, \alpha_{\text {Bone }}=10.5 \mathrm{~dB} / \mathrm{cm} / \mathrm{MHz}^{1.89}$ ' Typical values extracted from Ref. Hill et al. (2004).

for $0<\gamma<3$ and $\gamma \neq 1$, and

$$
\left.x_{c}(\omega)\right|_{\gamma=1}=\frac{\pi^{2}}{4 \log (2) \alpha_{0} \omega}
$$

for $\gamma=1$. It is worth noting here that the coherence length, $x_{c}$, is inversely proportional to attenuation magnitude. In this way, in frequency power law attenuation media with strong attenuation values and $\gamma \approx 1$, e.g. in bones, tendons(Fry et al., 1978) where strong dispersion is observed, and also for $\gamma \approx 3$, the efficiency of nonlinear harmonic generation is expected to be modified due to phase mismatching. The frequency dependence for different power laws of real tissues is shown in Fig. 8.4. Here, we show that the magnitude of coherence length is on the order of the size of the internal structures of the human body for ultrasound applications, i.e. $x \lesssim 10 \mathrm{~cm}$. Thus, dispersion effects, in addition to attenuation and nonlinear effects can also be important in the nonlinear full solution. However, in order to evaluate the relevance of each effect in the full-wave propagation (attenuation, dispersion and nonlinearity), we shall define the ratio of attenuation to dispersion effects.

Thus, the amplitude of each harmonic will be damped due to the increase in attenuation, following the corresponding power law. Thus, we shall define the second harmonic characteristic attenuation distance for the frequency power law 
attenuation media as

$$
x_{a}=\frac{1}{\alpha_{0} \omega^{\gamma}} .
$$

In this way, using Eq. $(8.12,8.14)$ we define the quantity $N_{c}$ as the ratio of attenuation and dispersion effects for power law media as

$$
N_{c}=\frac{x_{c}}{x_{a}}=\left|\frac{\pi \cot \left(\frac{\gamma \pi}{2}\right)}{2^{\gamma}-2}\right| .
$$

In the limit of $\gamma \rightarrow 1 \mathrm{Eq}$. (8.15) reduces to

$$
N_{c}=\frac{\pi^{2}}{4 \log 2} .
$$

One important remark is the ratio of attenuation to dispersion effects in a frequency power law media is frequency independent: both, the amount of attenuation and second harmonic phase mismatching magnitude are proportional to the quantity $\alpha_{0} \omega^{\gamma}$. Furthermore, an increase in the attenuation coefficient, $\alpha_{0}$, will increase in equal magnitude dispersion and attenuation effects. In this way, as Eq. (8.15) shows, the balance between attenuation and dispersion only depends on the exponent of the frequency power law media.

Figure 8.5 shows the ratio $N_{c}$ for different power laws. For frequency power laws of $0<\gamma<2$, i.e. those that model biological media, the ratio fall in the range $3.43 \lesssim N_{c}<\infty$. In this way, $N_{c} \rightarrow \infty$ for dispersionless media, i.e. for even exponent power $\gamma=0$ and $\gamma=2$. The higher dispersive regime is obtained for nearly linear frequency dependent absorption, where a local minimum is numerically found at

$$
\gamma \approx 1.2053
$$

In the range $0.5<\gamma<1.5$, the ratio of attenuation to dispersion effects, $N_{c}$, present variations of $+44 \%$ from the minimum value $\left(N_{c}=3.43\right)$.

On the other hand, as Fig. 8.5 shows, for materials with $2<\gamma<3$ the dispersive effects can be much more important than attenuation effects. In addition, using Eq. (8.15), we obtain $\left.N_{c}\right|_{\gamma=3}=0$, indicating that dispersive effects strongly 


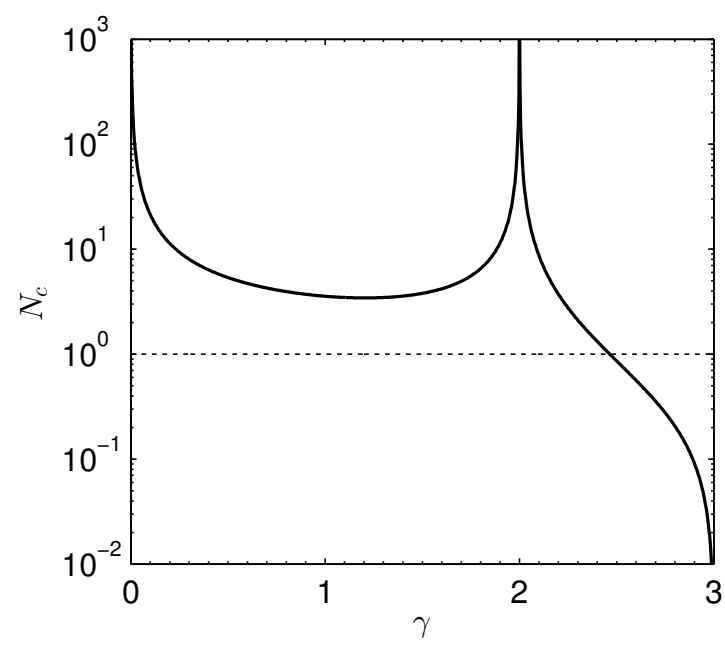

Figure 8.5: Ratio of attenuation versus dispersion, $N_{c}$, versus power law exponent $\gamma$.

dominates over attenuation effects. However, there exists few materials that falls in this range (Szabo, 1995). For the case of soft tissue media, where $1<\gamma<2$, the results arising from this section indicates that attenuation dominates over dispersion effects, but the phase mismatching between forced and propagating second harmonic wave is not negligible for nearly linear frequency dependent attenuation media. However, due to the absorption distance excess in about of one order magnitude the coherence distance, it is not possible to observe a complete beating. Instead, the effect of the weak dispersion in frequency power law media with $1<\gamma<2$ is a decreasing in the efficiency of energy transfer from the fundamental to higher harmonics. This aspect will be explored numerically in the next section.

\subsection{Competition between nonlinearity and power law attenuation and dispersion}

For a monochromatic plane wave in the absence of dissipation, either viscous or relaxation losses, the shock formation distance can be expressed as

$$
x_{s}=\frac{1}{\beta \varepsilon k},
$$

with $\varepsilon=v_{0} / c_{0}$ as the acoustic Mach number, $v_{0}$ the source amplitude, and $\beta=1+B / 2 A$ the parameter of nonlinearity that accounts for material $(B / A)$ and mass advection nonlinearity (unity factor). 
In order to measure the relative relevance of nonlinear and attenuation effects, we shall define the so called Gol'dberg number for frequency power law attenuation media as the ratio of characteristic absorption length, $x_{a}$, and shock distance, $x_{s}$ :

$$
\Gamma_{\gamma}=\frac{x_{a}}{x_{s}}=\frac{\beta p_{0}}{c_{0}^{3} \rho_{0} \alpha_{0} \omega^{(\gamma-1)}} .
$$

Thus, in the same sense of the classical definition of the Gol'dberg number for thermo-viscous fluids, for $\Gamma_{\gamma} \gg 1$ the shock distance is too short compared to the characteristic attenuation length and the nonlinear effects dominates over attenuation. In this way, for the range $0<\gamma<2$ where the dispersion remains weak, the nonlinear harmonic cascade can be developed and shock waves can be observed. In the limit $\Gamma_{\gamma} \rightarrow \infty$ model equations reduces to lossless nonlinear acoustic and analytical solutions can be obtained for a variety of boundary conditions (e.g. in the case of monochromatic plane wave propagation the well known Fubini-Blackstock-Fay solution describes the harmonic evolution). On the other hand, in the regime $\Gamma_{\gamma} \ll 1$ dissipation effects dominates over nonlinear effects. Although energy transfer from fundamental to higher harmonics could be observed, absorption prevents the development of shock waves. In the limit of $\Gamma_{\gamma} \rightarrow 0$, linear-damped propagation is achieved and no harmonic is generated.

However, in many practical cases $\Gamma_{\gamma} \approx 1$ and an analytical treatment of the problem is not available (with the exception of $\gamma=2$ where the so called Mendousse, Eq. 7.32, solution could be obtained). Due to plane-harmonic nonlinear wave propagation in a mono-relaxing media is still unsolved, the prospective is fair in order to find an exact analytical solution for multiple relaxation media or for a continuum of relaxation processes. In addition, no analytical solution is available for fractional derivatives nonlinear wave equations (Szabo, 1994) for the case of monochromatic plane wave. Thus, in this section we will explore numerical solutions of Eqs. $(7.1,7.2,7.10,7.8)$ by using the methods described in Chapter 7 in order to study the harmonic generation in frequency power law attenuation media.

In addition, one important remark arising from Eq. (8.19) for tissues with exact $\gamma=1$ is that the ratio between nonlinearities and attenuation effects does not depend on frequency. In this way, Eq. (8.19) predicts that given media parameters, $\left(\beta, c_{0}, \rho_{0}\right.$ and $\left.\alpha_{0}\right)$, a wave with fixed initial amplitude will develop exact nonlinear processes with independence of the wave frequency. In this way, the amplitude can be selected and fixed in order to produce shock waves as

$$
p_{0}>c_{0}^{3} \rho_{0} \alpha_{0} / \beta \text {. }
$$

If the frequency is increased the shock formation distance is moved towards the source, but according to Eq. (8.19) the Gol'dberg ratio remains constant and therefore the harmonic balance remains the same but stretched in space. 
As explained above, for $\Gamma_{\gamma} \approx 1$ the nonlinear and attenuation effects may play a role of similar importance in the full solution. Thus, there exist a competition between nonlinearity and attenuation effects that, at first order, leads the final amplitude for each harmonic. In one hand, harmonic generation is governed by nonlinearity, where second and higher harmonics are generated by cascade processes due to material nonlinearity and mass convection processes. On the other hand, each generated harmonic will be attenuated during propagation by its specific attenuation coefficient, provided by the frequency power law evaluated at each harmonic frequency.

Consider here media with equal attenuation at fundamental frequency, but different power law. In these media, it is obvious that for higher values of the power law exponent, $\gamma$, the attenuation of higher harmonics will be in any case higher than the attenuation for media with lower power law. Thus, in principle, the exponent has a great influence in the harmonic balance of the traveling nonlinear wave due to differences in attenuation, and these differences will be higher for higher harmonics.

However, as long frequency power law attenuation introduces dispersion, see Eq. (8.2), the phase matching conditions are in general no longer fulfilled. Therefore, dispersion modifies the efficiency on harmonic generation, together with the interplay between power law attenuation and nonlinear harmonic growing. Thus, from Eqs. $(8.10,8.11)$, phase matching conditions, i.e. $\Delta k_{2}=0$, are fulfilled for even powers, e.g. $\gamma=0,2, \ldots$. In these cases, propagation is nondispersive: the nonlinear efficiency is maximum and therefore the energy transference from fundamental to higher harmonics is maximized. On the other hand, for $\gamma \approx 1$, dispersive propagation is predicted and the nonlinear efficiency will be modified. Due to these effects cannot be isolated, we study the modification of the nonlinear efficiency numerically.

\subsection{Plane wave simulations in power law media}

As shown Chapter 7, the propagation of nonlinear acoustic waves in frequency power law attenuation can be modeled by the use of multiple relaxation processes. Thus, the set of Eq. (7.1, 7.2, 7.10 and 7.8) can be expressed for a plane wave travelling in $x$ direction as

$$
\begin{gathered}
\frac{\partial \rho}{\partial t}=-\frac{\partial\left(\rho v_{x}\right)}{\partial x} \\
\frac{\partial v_{x}}{\partial t}=-\frac{1}{\rho} \frac{\partial p}{\partial x}-\frac{1}{2} \frac{\partial v_{x}^{2}}{\partial x}+\frac{1}{\rho}\left(\frac{4}{3} \eta+\zeta\right) \frac{\partial^{2} v_{x}}{\partial x^{2}},
\end{gathered}
$$




$$
\begin{gathered}
p=c_{\infty}^{2} \rho^{\prime}+\frac{c_{0}^{2}}{\rho_{0}} \frac{B}{2 A} \rho^{\prime 2}-\sum_{n=1}^{N} S_{n}, \\
\frac{\partial S_{n}}{\partial t}=-\frac{1}{\tau_{n}} S_{n}+\frac{\eta_{n} c_{0}^{2}}{\tau_{n}} \rho^{\prime},
\end{gathered}
$$

where $v_{x}$ is the $x$ component of particle velocity vector.

In order to study the changes induced by power law attenuation and dispersion into the nonlinear efficiency, we consider media with equal attenuation coefficient at fundamental frequency, $\omega_{0}$, as shown in Fig. 8.2. Thus, by varying power law exponent from realistic values for biological media, $\gamma=[0.5,2]$, dispersion is introduced while the attenuation of the fundamental wave remains constant. As Eq. (8.15) shows, the balance between attenuation and dispersion for power law media $\left(N_{c}\right)$ only depends on the power law exponent. In this way, by increasing the attenuation magnitude both dispersion and attenuation effects will by modified in same amplitude. On the other hand, by modifying the attenuation magnitude the Gol'dberg number for power law media is also modified following Eq. (8.19). In summary, for media with equal attenuation coefficient at fundamental frequency, the balance between nonlinearity and attenuation effects, $\Gamma_{\gamma}$ is governed by the attenuation coefficient, while the balance between attenuation and dispersion effects, $N_{c}$, is governed by the exponent of the power law.

Numerical results for the spatial distribution of fundamental wave are shown in Fig. 8.6, while the spatial distribution for second and third are plotted in Fig. 8.7. The amplitude and fundamental wave of the excitation was maintained constant, thus, by changing attenuation magnitude the ratio between attenuation and nonlinearity is modified and therefore the ratio between dispersion and nonlinearity also is modified.

Here, the analytical nonlinear solution for lossless media (Bessel-Fubini's solution) is plotted in dotted line. Thus, for low attenuation media, i.e. when $\Gamma_{\gamma} \rightarrow \infty$, nonlinear effects dominate over attenuation and dispersion and therefore the numerical solution for frequency power law media converges to the lossless Bessel-Fubini's solution, see Fig. 8.6 and Fig. 8.7 for $\Gamma_{\gamma}=57.9$. In these cases, the fundamental wave decay is mainly due to energy transference to higher harmonics, that grow with propagation. Here, harmonic amplitude is independent on the exponent of the power law, showing that both dispersion and attenuation effects are much less smaller compared with nonlinear effects during propagation and can be neglected for $\Gamma_{\gamma} \rightarrow \infty$.

In contrast, as Figs. 8.6, 8.7 show for $\Gamma_{\gamma}=0.74$, for high damped media and weak nonlinearity, attenuation effects are comparable to nonlinear harmonic pumping. The fundamental wave not only decays for energy transfer but also due to high attenuation. 

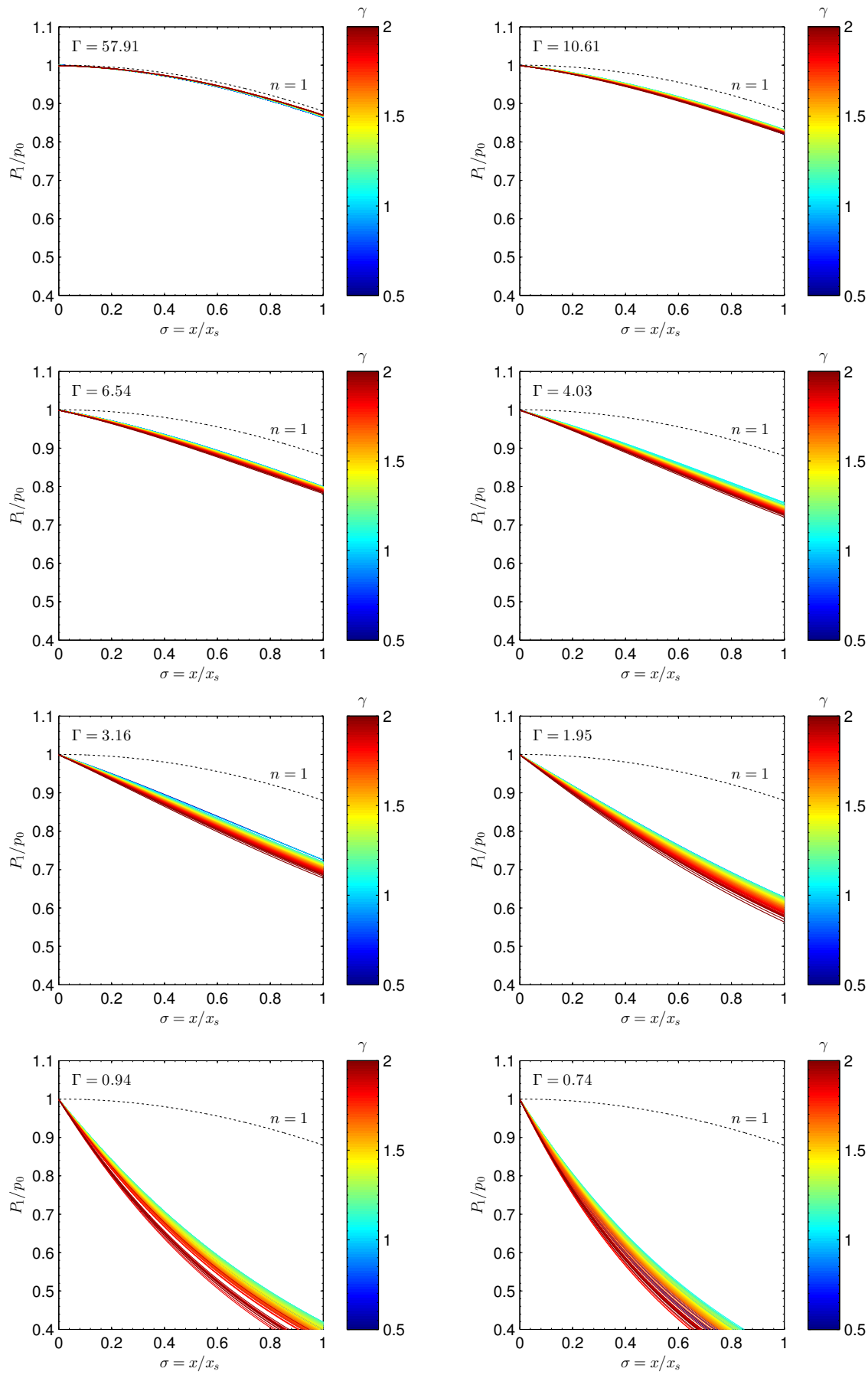

Figure 8.6: First harmonic normalized-amplitude spatial distribution for different frequency power laws. For each subplot, the ratio of nonlinear to power law attenuation effects, $\Gamma_{\gamma}$, is increased 

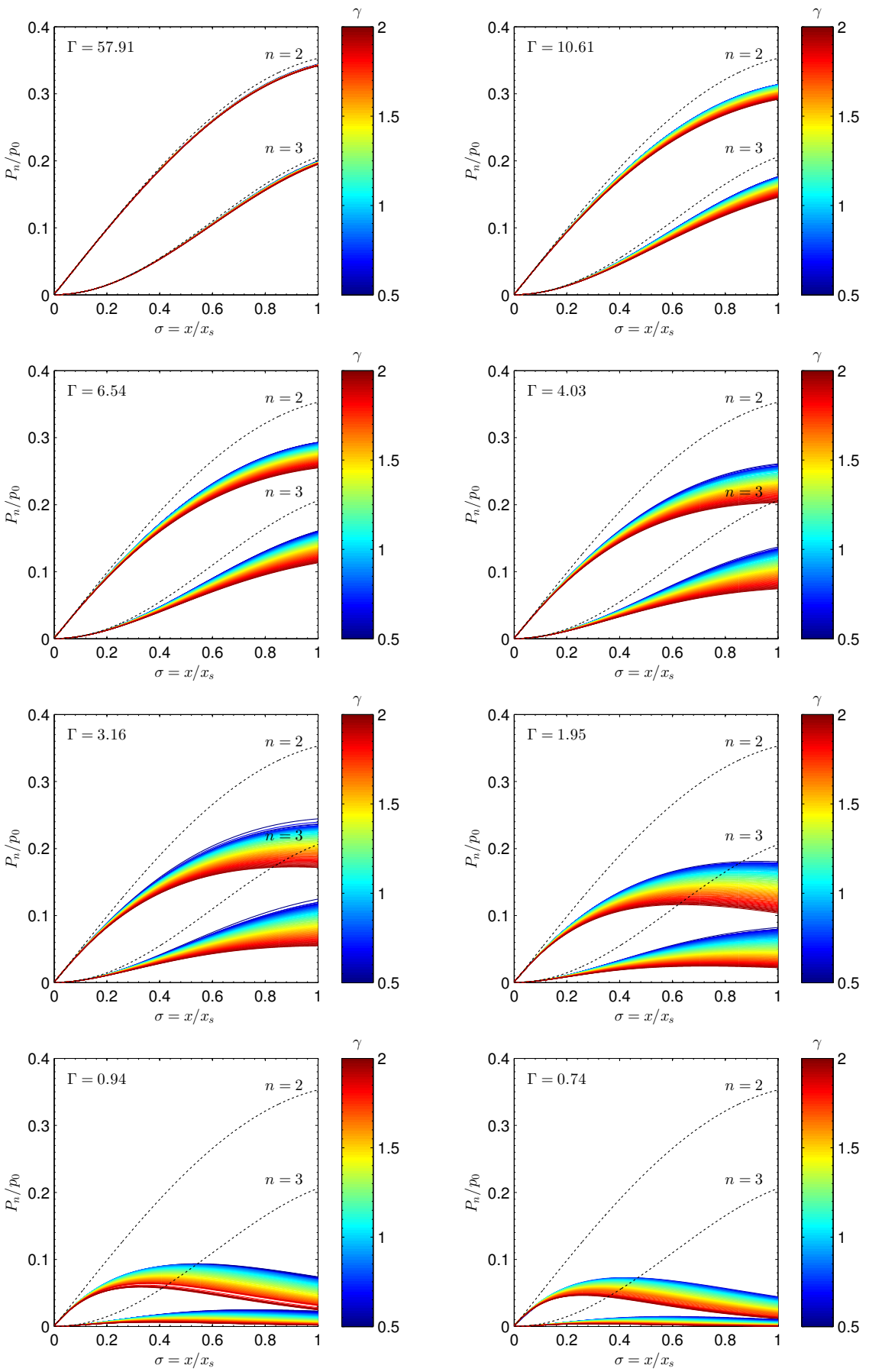

Figure 8.7: Second and third harmonic normalized-amplitude distribution for different frequency power laws. For each subplot, the ratio of nonlinear to power law attenuation effects, $\Gamma_{\gamma}$, is increased 
Thus, at distance $\sigma \approx 0.5$ fundamental wave have loss approximately half of its initial amplitude and therefore nonlinear energy transfer processes to second-andhigher harmonics also diminishes. On the other hand, second and third harmonics are mainly generated near the source, where fundamental wave is still have enough amplitude. However, attenuation of higher harmonics is in any case higher than for fundamental wave and therefore they are quickly damped by the power law lossy media. Due to the harmonic generation is strongly decreased far away from the source due to fundamental wave energy loss, second and higher harmonics are restricted near the source and its maxima value is produced for $\sigma<1$, in contrast with lossless media where the maxima of the harmonics is produced $\sigma \approx \pi / 2$.

It is worth noting here that the harmonic evolution is strongly dependent on the exponent of the power law. Thus, due to differences in attenuation coefficient for each harmonic (see Fig 8.2 for $\omega / \omega_{0}=2$ ), second-and-higher harmonic amplitude is damped by different magnitude, where obviously higher attenuation and therefore lower harmonic amplitude is observed for the cases of $\gamma \rightarrow 2$.

However, although the fundamental wave attenuation magnitude is the same, with independence of the power law exponent, the amplitude of the fundamental wave strongly varies. We recall here to Eq. (8.15), that shows the ratio between dispersion and attenuation. By varying the power law exponent dispersion is modified. Thus, the harmonic efficiency is only maximized for even powers of $\gamma$, where for $\gamma \approx 1$ dispersion effects are not negligible. This is the reason because more energy remains in the fundamental wave for $\gamma \approx 1$, dispersion is modifying the phase matching conditions and therefore the energy transfer from fundamental to second-and-higher harmonics is decreased.

\subsection{Nonlinear efficiency}

As explained above, the nonlinear efficiency can be strongly dependent not only on the magnitude of the attenuation, but also on the dispersion due to frequency power law attenuation. These effects have been shown previously for tissue like media in the absence of dispersion (Haran et al., 1983) and including power law dispersion (Kashcheeva et al., 2000; Wallace et al., 2001) for selected media. A closer view of these phenomena can be studied by showing the harmonic amplitude at a given distance, that for reference we choose $\sigma=1$. Thus, Fig. 8.8 shows the amplitude of the fundamental wave for different power law media and its dependence on the power law. As commented above, if the balance between nonlinear and attenuation effects is constant, the maximum amplitude of the fundamental component is observed for power laws where dispersion is maximized, i.e. for $\gamma \approx 1.2$. Furthermore, there exist differences between different Gol'dberg numbers: the balance between nonlinearity and dispersion is also coupled to the attenuation, so for high Gol'dberg numbers dispersion effects also dominate over nonlinear effects, leading to a decreasing in the nonlinear efficiency and therefore 


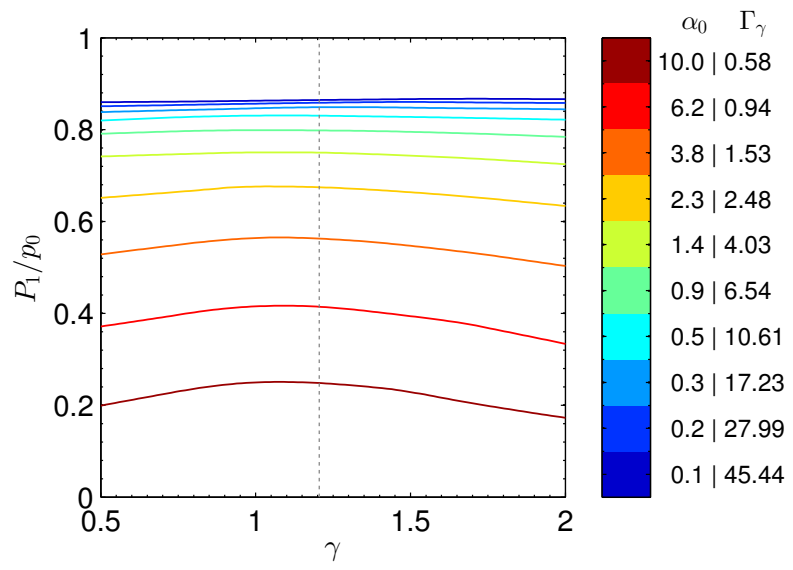

Figure 8.8: Amplitude of the fist harmonic for different Gol'dberg number $\left(\Gamma_{\gamma}\right)$ in function of the exponent of the frequency power law $(\gamma)$, measured at shock formation distance, $\sigma=1$. The attenuation of the media is selected for achieve a constant value at first harmonic frequency, so for this plot the amount of media attenuation is independent of the power law.

more energy remains in the fundamental component of the nonlinear wave.

A knowledge of the role of the power law dispersion into the second-and-higher harmonic components is not so evident. If dispersion is modified, i.e. we force a change in the power law exponent, the attenuation magnitude at second harmonic frequency will be also modified. On the other hand, is obvious that if attenuation is matched for second harmonic, e.g. being the source frequency $\omega_{0} / 2$ in Fig. 8.2, the attenuation magnitude at fundamental wave will be different.

Left side of Fig 8.9 shows the harmonic amplitude in function of the power law exponent at distance $\sigma=1$ for different Gol'dberg ratios. Observing the harmonic amplitude is not obvious that, as stated, more energy is transferred for nondispersive power law media $(\gamma \rightarrow 0,2, \ldots)$. Thus, as attenuation is strongly increased for second harmonic in function of the power law exponent, the harmonic amplitude is therefore more attenuated for the non-dispersive cases, masking the effect of nonlinear efficiency.

In this way, we introduce in the right side of Fig 8.9 a compensation of the second harmonic amplitude by the attenuation coefficient at this frequency. In this plot, it is appreciable that for nondispersive cases, $\gamma=2$, the harmonic amplitude is higher due to the phase matched generation, while for the dispersive cases there exist a reduction in the efficiency for second harmonic generation. However, this compensation does not take into account that harmonics are generated during propagation. Thus, it serves as an eye guide for illustration purposes but lacks of exact physical fidelity.

Similar plots, shown in Fig. 8.10, are generated for third harmonic at shock 

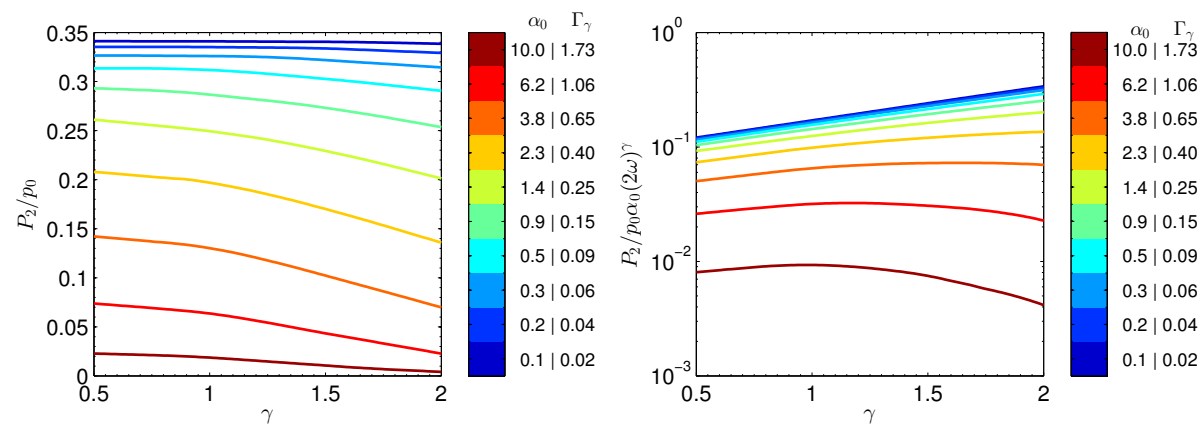

Figure 8.9: Left: Amplitude of the second harmonic for different Gol'dberg number $\left(\Gamma_{\gamma}\right)$ in function of the exponent of the frequency power law $(\gamma)$, measured at shock formation distance, $\sigma=1$. Right: Same curves compensated by the increment of attenuation of second harmonic, which is different for each power law.
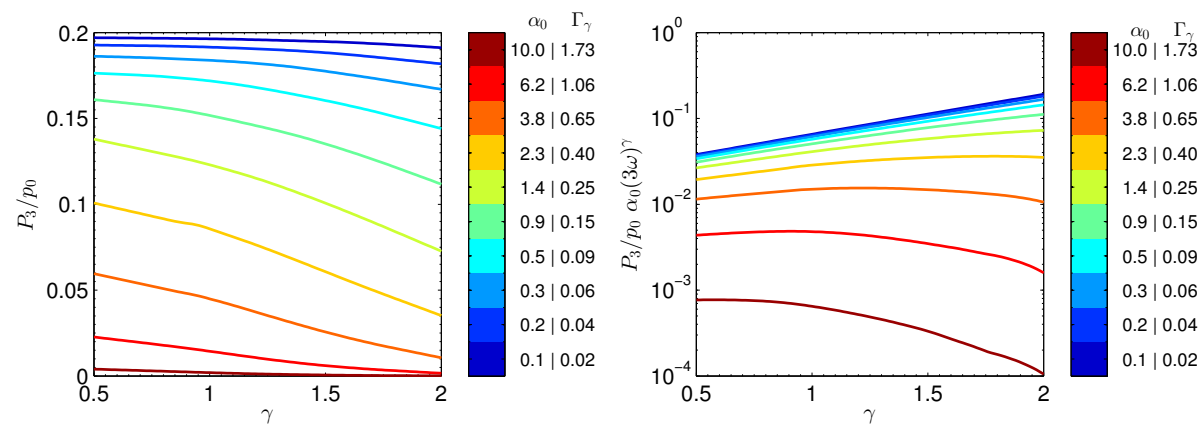

Figure 8.10: Left: Amplitude of the third harmonic for different Gol'dberg number $\left(\Gamma_{\gamma}\right)$ in function of the exponent of the frequency power law $(\gamma)$, measured at shock formation distance, $\sigma=1$. Right: Same curves compensated by the increment of attenuation of third harmonic, which is different for each power law.

formation distance. Here, the amplitude is lower compared to second harmonic due to nonlinear generation. In this case, for a given Gol'dberg ratio the differences in the harmonic amplitude between different power laws increases, mainly due to the increasing in the attenuation coefficient at third harmonic frequency. Moreover, if the attenuation of each harmonic amplitude is compensated, as shown in right hand side of Fig. 8.10, the behavior is the similar than the observed to the second harmonic efficiency.

\subsection{Phase changes induced by dispersion}

From the dispersion relations for power law media, showed in Fig.8.1, it is appreciable that second harmonic component propagates, for $0<\gamma<2$, faster than 

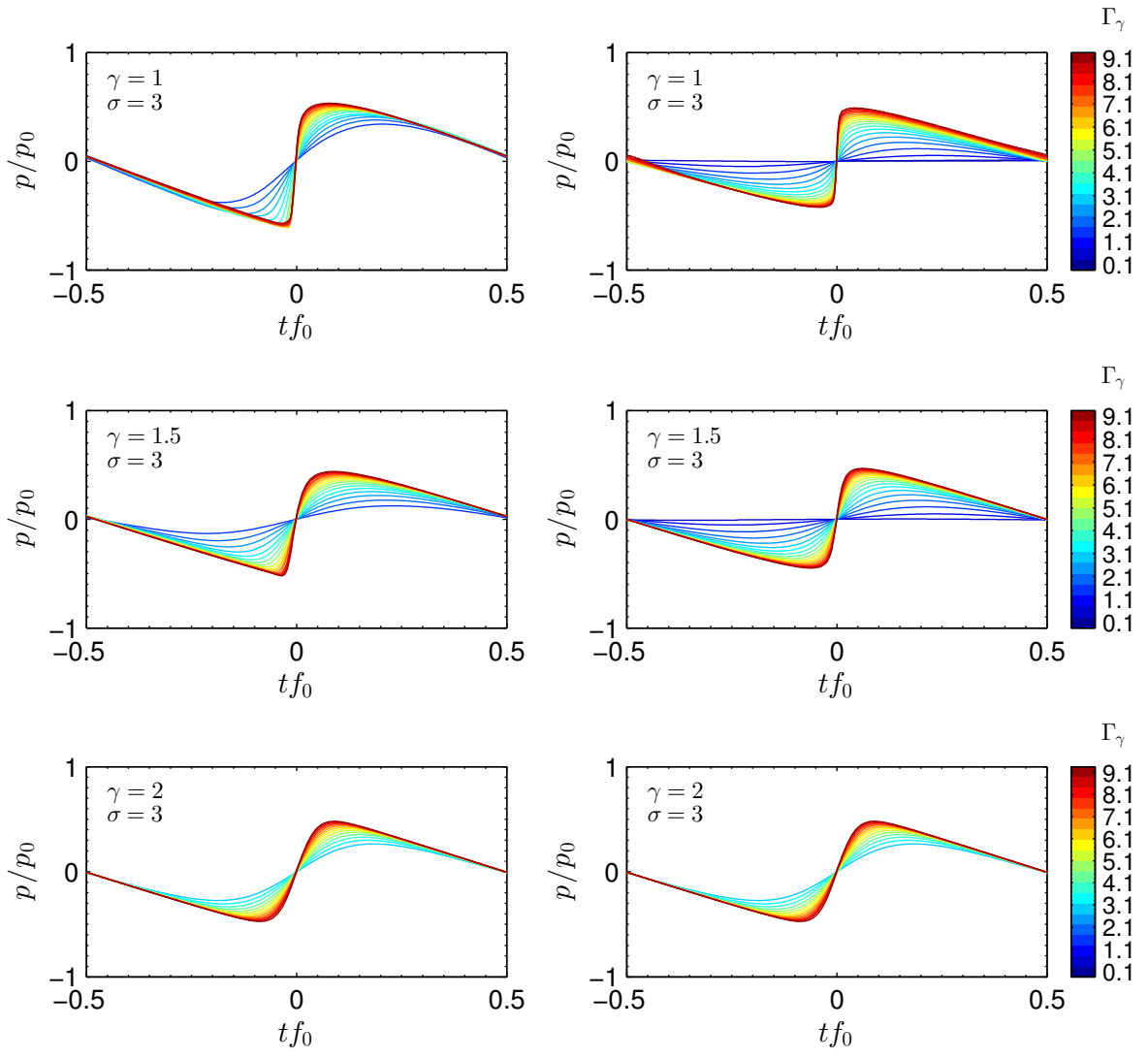

Figure 8.11: Waveforms calculated for (left) dispersive power law media at distance $\sigma=3$ and (right) artificial dispersionless only taken into account power law absorption. From up to down the power law exponents ranges are $\gamma=[1,1.5,2]$

fundamental wave. The second harmonic that is generated near the source, after propagation, will sum with the harmonics at higher distances. Due to the locally generated second harmonic waves are in phase with fundamental wave, there exist a phase difference between the forced (locally generated) and the background propagation second harmonic wave. This phase shift can be predicted from dispersion relations, as is dependent to phase matching conditions (Eq.8.8). In the case of second harmonic, a complete phase shift between propagating and forced waves occurs every $x=2 x_{c}$. In this way, as explained in Section 8.5, these processes modify the efficiency of the nonlinear generation in frequency power law attenuation media.

In addition, these phase changes also modify the shape of the nonlinear waveforms in frequency power law media. The shape of a nonlinear waveform is determined by its specific harmonic balance, but also the relative phase between 

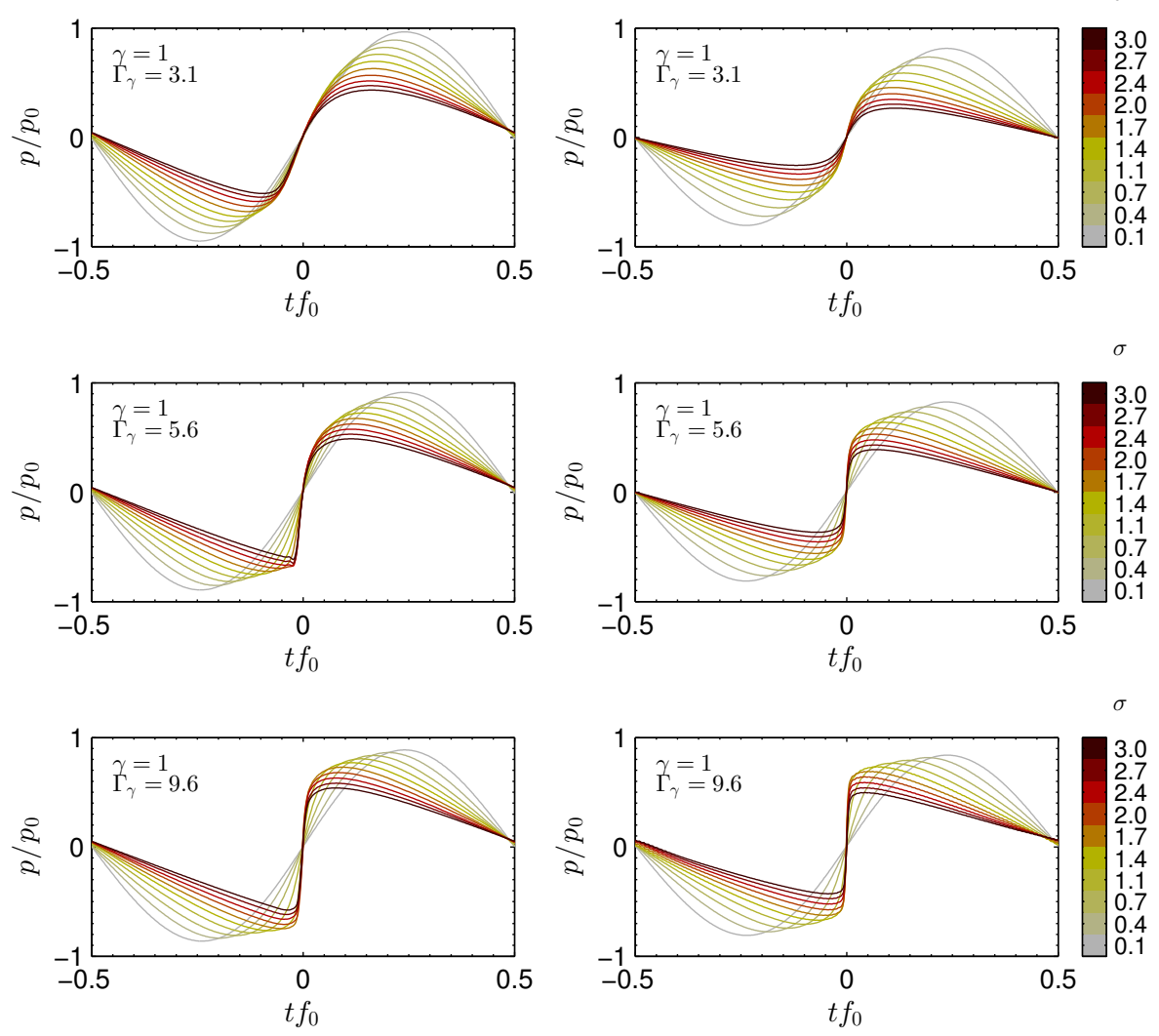

Figure 8.12: Waveform evolution calculated for linear power law $(\gamma=1)$. (Left) dispersive propagation. (Right) nondispersive simulation for comparison using artificial dispersionless power law absorption. From up to bottom the ratio between nonlinear and attenuation $\left(\Gamma_{\gamma}\right)$ effects is increased.

spectral components. In nondispersive media, the harmonic balance is a competition between nonlinearity and absorption processes. As seen in this Chapter, dispersion of power law media modifies this balance. Moreover, the relative phase between spectral components will be also modified due to dispersion and it will change the shape of the waveform.

To study these effects, we use a $k$-space pseudospectral method (Treeby et al., 2010; Treeby et al., 2012) to evaluate one dimensional nonlinear propagation. This method, through the spectral representation of the field, provides an efficient way to evaluate the linear integro-differential operator based fractional Laplacian that introduces frequency power law attenuation. This also provides a way to eliminate dispersion and evaluate only a nondispersive version of the frequency power law operator. 
Thus, Fig. 8.11 shows the nonlinear waveforms measured at distance $\sigma=3$ including dispersion and an artificial nondispersive version of the frequency power law attenuation operator in the $k$-space. As can be seen, the specific shape of the waveform depends on the exponent of power law: it provides a balance in the harmonic magnitude through attenuation processes and the above described changes on the efficiency through dispersion processes.

However, if the power law attenuation is maintained but dispersion is artificially not included in the calculations, there can be seen that the waveforms shape presents several changes. Thus, as Fig. 8.11 shows, dispersion breaks the symmetry of the nonlinear waveforms. In the case of nondispersive propagation the waveforms for $\Gamma_{\gamma} \gg 1$ converge to the well-known sawtooth waves as predicted by the Fubini-Blackstock-Fay solution (Hamilton et al., 1998a). For intermediate $\Gamma_{\gamma}$ values, due to different balance in harmonic attenuation during propagation, the shape of the final waveforms is also slightly changed. However, in this case of artificially nondispersive propagation, the shape of the waveforms conserve the symmetry between compression and rarefaction cycles typical of sawtooth waves.

In contrast, this symmetry is broken for the waveforms generated including dispersion, in the same sense as in other dispersive systems as mono-relaxing fluids (Hamilton et al., 1998a). A more detailed frame for $\gamma=1$ is presented in Fig. 8.12, where the complete evolution of the waveforms is represented in each frame. Here it can be seen how the shock front is smoothed in the compression phase, while remains sharp in the negative rarefaction phase.

\subsection{Conclusions}

In this chapter we have revised the fundamental problem of the competition between nonlinear and attenuation effects in frequency power law attenuation media, with special emphasis in the effects of the weak dispersion. We have derived simple expressions in order to quantify the role of the exponent of the power law in the balance between nonlinearity, dispersion and attenuation. The results show that the dispersion effects present more relevance in the nonlinear propagation for media obeying $\gamma \approx 1.2$, where the ratio between nonlinear characteristic distance and weak dispersion characteristic distance present its minimum. Detailed numerical examples are given in agreement with this predictions. This situation is of special interest due to most soft tissues typically present values of the power law exponents around this critical value.

The efficiency in the harmonic generation is studied numerically, showing that power law dispersion modifies the cumulative energy transfer to higher harmonics. Thus, considering a media with same attenuation value at the fundamental component, more energy remains in the fundamental harmonic component in the cases of nearly linear power law. On the other hand, in frequency power law 
media attenuation effects are of higher importance in the nonlinear propagation than weak dispersion effects. However, we have shown that the waveform shape also strongly depends on the weak dispersion, where the characteristic asymmetric profile is observed for dispersive frequency power law attenuation.

In real situations, where other phenomena must to be included (beam diffraction, tissue scattering and inhomogeneities, ...), it becomes far more complicated to study the role of each effect in the nonlinear propagation. Thus, plane wave propagation offers the possibility to isolate some of the physical mechanisms that governs nonlinear propagation (in this case attenuation and dispersion) and to understand the balance between them. The results presented in this chapter, far from present completely new physics, offers detailed calculations and a broad view of this fundamental problem.

\section{References}

Fry, FJ and JE Barger (1978). "Acoustical properties of the human skull". In: The Journal of the Acoustical Society of America 63.5, pp. 1576-1590.

Hamilton, Mark F, David T Blackstock, et al. (1998a). Nonlinear acoustics. Vol. 237. Academic press San Diego.

Haran, Michael E and Bill D Cook (1983). "Distortion of finite amplitude ultrasound in lossy media". In: The Journal of the Acoustical Society of America 73.3, pp. 774-779.

Hill, C.R., J. C. Bamber, and G.R. ter Haar (2004). Physical Principles of Medical Ultrasonics, 2nd ed. John Wiley \& Sons Ltd, Chichester, England.

Kashcheeva, S.S., O.A. Sapozhnikov, V.A. Khokhlova, M.A. Averkiou, and L.A. Crum (2000). "Nonlinear distortion and attenuation of intense acoustic waves in lossy media obeying a frequency power law". In: Acoustical Physics 46, pp. 170-177.

O'Donnell, M., E. T. Jaynes, and J. G. Miller (1981). "Kramers-Kronig relationship between ultrasonic attenuation and phase velocity". In: J. Acoust. Soc. Am. 69.3, pp. 696-701.

Rudenko, O.V. and S.I. Soluian (1977). Theoretical foundations of nonlinear acoustics. Studies in Soviet science. New York, NY 10011, USA: Consultants Bureau, p. 274.

Szabo, Thomas L (1994). "Time domain wave equations for lossy media obeying a frequency power law". In: J. Acoust. Soc. Am. 96.1, pp. 491-500.

Szabo, Thomas L (1995). "Causal theories and data for acoustic attenuation obeying a frequency power law". In: J. Acoust. Soc. Am. 97.1, pp. 14-24.

Treeby, Bradley E and BT Cox (2010). "Modeling power law absorption and dispersion for acoustic propagation using the fractional Laplacian". In: J. Acoust. Soc. Am. 127.5, pp. 27412748 .

Treeby, Bradley E, Jiri Jaros, Alistair P Rendell, and BT Cox (2012). "Modeling nonlinear ultrasound propagation in heterogeneous media with power law absorption using a k-space pseudospectral method". In: J. Acoust. Soc. Am. 131.6, pp. 4324-4336.

Wallace, Kirk D, Mark R Holland, and James G Miller (2001). "Improved description of shock wave evolution in media with frequency power law dependent attenuation". In: The Journal of the Acoustical Society of America 109.5, pp. 2263-2265.

Waters, Kendall R, Michael S Hughes, Joel Mobley, Gary H Brandenburge, and James G Miller (2000). "On the applicability of Kramers-Krönig relations for ultrasonic attenuation obeying a frequency power law". In: The Journal of the Acoustical Society of America 108.2, pp. 556563.

Waters, Kendall R, Joel Mobley, and James G Miller (2005). "Causality-imposed (KramersKronig) relationships between attenuation and dispersion". In: Ultrasonics, Ferroelectrics and Frequency Control, IEEE Transactions on 52.5, pp. 822-823. 


\section{Publications}

The contents of this chapter have been presented in the following publications:

\section{Conference proceedings}

- Jiménez, N.; Redondo, J.; Sánchez-Morcillo, V.; Iglesias, P. C. and Camarena, F. "On the Nonlinear Effects in Focused Ultrasound Beams with Frequency Power Law Attenuation" 43rd Annual UIA Symposium, Madrid, Spain. 23 - 25 April. In: Physics Procedia, Volume 63, 2015, Pages 47-53, (2014) 
Chapter 8. Nonlinear plane waves in frequency power law attenuation media. 


\title{
Chapter 9
}

\section{Nonlinear Acoustic Radiation Forces in Biological Media}

\begin{abstract}
In this Chapter we present the finite-amplitude dependence of the acoustic radiation force (ARF) in a soft tissue-like media. The acoustic radiation force is calculated in media with different exponent of the frequency power law attenuation. Numerical results are obtained for one dimensional propagation, where the spatial distribution of the force field is studied. The results obtained show that the nonlinear dependence of the acoustic radiation force sharply depends on the tissue attenuation model for both, weakly and strong nonlinear regime. We also show the underestimation of the momentum transfer in the nonlinear regime by calculating the ARF by using the wave intensity. Considerations due to nonlinear absorption are included and the relation between the momentum transfer and energy deposition is underlined.
\end{abstract}




\subsection{Introduction}

Waves carry energy and momentum. Propagation trough lossless homogeneous media implies the absence of interaction between the medium and the wave. However, if scattering or absorption processes are present, the energy and momentum can be transferred from the wave to the medium. In this sense, the radiation force is an universal wave phenomenon related to the momentum transfer from a wave to the medium. It has been demonstrated to exist for electromagnetic (Lebedew, 1901), acoustic waves (Faraday, 1831; Rayleigh, 1902), and also by phonons in crystal lattices (Sorbello, 1972) as those presented in Chapter 1.

In the case of acoustic waves, acoustic radiation forces (ARF) appear when a gradient in the acoustic energy density is produced by scattering or absorption processes. We refer to the critical review of Sarvazyan et al. (2010) for a broad historical references and a clear description of the ARF in the acoustic context. The acoustic radiation force is a time-average second-order process. In the case of a continuous monochromatic wave, steady state acoustic radiation forces appear when the wave is scattered by an object, reflected by a rigid surface or an interface between two media, or absorption is produced in the propagation. In all these situations, some momentum is transferred from the wave to the medium, and the consequence is that the acoustic radiation tensor Beyer (1978) is no longer spatially constant. In these cases, the bulk of the medium is time average stressed. The radiation forces are only one of the consequences of this stresses. In fluids, the acoustic radiation stresses induce time average movements or streams. In solids and elastic media in general, time average stresses induce constant strains, being the material deformed in the direction of the wave but also in the transversal direction. In fluids with inclusions, the scatterers can be statically compressed, deformed, pushed, and also rotated by the induced acoustic radiation stress.

Therefore, is no surprising the huge amount of practical applications that exploit the phenomena related acoustic radiation stresses due to the extremely broad possibilities for interacting with the media using acoustic waves.

In the case of biological media and medical ultrasound applications, the use of acoustic radiation force includes the power calibration of High Intensity Focused Ultrasound (HIFU) devices by radiation force balances (Wood et al., 1927); acoustical tweezers and levitation for particle and cell manipulation (Wu, 1991; Lee et al., 2006; Hultström et al., 2007); stimulation of sensory receptors by ultrasound radiation force or improving targeted drug and gene delivery (Sarvazyan et al., 2010). On the other hand, acoustic streaming can be used for stirring and mixing liquids (Sarvazyan et al., 2009), remote assessment of biological fluids (Nightingale et al., 1995) and microfluidics applications (Laurell et al., 2007; Hultström et al., 2007). It is worth noting here that in some of these techniques the effects of the acoustic radiation force and streaming are closely linked.

One important remark is that the time averaging of the acoustic radiation 
stresses can be modulated by a slowly varying time function. If the wave amplitude presents a low frequency modulation (compared to the acoustic variations), the radiation stresses are also modulated in time, leading to oscillating radiation forces inside the media. In elastic media, these stresses dynamically deform the bulk of the medium in axial and also in transversal direction. Thus, time varying shear deformations induce shear waves at the modulation frequency, that propagates transversely to the direction of the wave.

A huge amount of ultrasound medical diagnostic techniques have been developed in order to asset the media elasticity by measuring the mechanical response of the tissues under the action of acoustic radiation stresses. In general, the elasticity can be evaluated by measuring in some way the strains induced by the ultrasound field. These techniques include vibroacoustography (VA) (Fatemi et al., 1998; Urban et al., 2011), shear wave elasticity imaging (SWEI) (Sarvazyan et al., 1998), acoustic radiation force impulse imaging (ARFI) (Nightingale et al., 2001; Trahey et al., 2004), supersonic shear imaging (SSI) (Bercoff et al., 2004), harmonic motion imaging (HMI) (Konofagou et al., 2003; Vappou et al., 2009) and real time monitoring of lesion formation in HIFU therapy (Maleke et al., 2008). Also, the induced tissue deformations can be tracked with other imaging techniques as magnetic resonances (Fowlkes et al., 1995; Manduca et al., 2001). Acoustic streaming can be used to assessment and detecting fluid-filled lesions or cysts (Nightingale et al., 1995; Nightingale et al., 1999) or monitoring blood coagulation by sonorheometry (Viola et al., 2004). We refer to the reviews (Sarvazyan et al., 2010; Palmeri et al., 2011; Doherty et al., 2013) and references therein for further details.

Human soft tissues show strong acoustic attenuation processes, but also internal scattering due to micro-inhomogeneity. It is commonly accepted that the contribution to the acoustic radiation force in the bulk of soft tissues is mainly caused by absorption rather than scattering processes (Doherty et al., 2013). In order to obtain high amplitude acoustic radiation force fields and induce detectable deformations, the amplitude of the ultrasonic excitation is commonly high enough to excite nonlinear effects in some degree (Carstensen et al., 1980), even in diagnostic techniques (Sarvazyan et al., 2010). On the other hand, although radiation forces are observed also for small amplitude waves, the acoustic radiation force is a second order process and therefore requires solutions to the nonlinear model equations.

At ultrasound frequencies, soft tissues do not effectively support shear wave propagation. The typical shear wave speed in soft tissue is around two orders of magnitude lower that the longitudinal acoustic wave speed. Shear waves generated by low frequency modulated acoustic radiation forces, present different spatiotemporal scale to the longitudinal waves. The common approach is to model first the acoustic radiation force generated by the ultrasonic longitudinal waves. Then, shear waves and the corresponding tissue strain are treated as uncoupled problem: the time-varying acoustic radiation force field is used as an excitation of some elastic model, commonly a linear elastic or viscoelastic solid model. Therefore, the 
constitutive equations for nonlinear acoustics are accurate to describe the nonlinear generation of acoustic radiation force, provided that the proper attenuation and dispersion are included in the model as a frequency power law.

The aim of this Chapter is to study the acoustic radiation forces induced by intense waves in soft tissue. Thus, we present the nonlinear dependence of the acoustic radiation force by solving numerically the constitutive equations for nonlinear acoustics in a broad range of frequency power law attenuation media. We show special emphasis in the effects of the power law frequency attenuation.

\subsection{One dimensional acoustic radiation force}

The problem of accurately solve the acoustic radiation force is associated to the knowledge of the full acoustic field variables: pressure $p$, density $\rho$ and particle velocity vector $\mathbf{v}$. For plane waves in a lossless homogeneous media, the relation between these quantities is well-known, but in this case no acoustic radiation force appears. The inclusion of some frequency dependent attenuation and its corresponding dispersion induce a phase shift between the acoustic variables and its relationship becomes more complicated.

For the sake of simplicity, we start by review the acoustic radiation force generated by plane waves. We first expand in series the acoustic magnitudes, $p, \rho, v$ as in Refs (Sarvazyan et al., 2010; Doherty et al., 2013). Thus, each term includes a high-order correction to to the steady-state solutions $p_{0}, \rho_{0}, v_{0}$. For a quiescent homogeneous fluid, $v_{0}=0$ and $p_{0}$ and $\rho_{0}$ are time independent, $p_{1}, \rho_{1}$ and $v_{1}$ are the first order approximations to the acoustic field, and $p_{2}, \rho_{2}$ and $v_{2}$ are the second order corrections. Thus, the field can be expressed as

$$
\begin{aligned}
p & =p_{0}+p_{1}+p_{2}+\cdots \\
\rho & =\rho_{0}+\rho_{1}+\rho_{2}+\cdots \\
v & =0+v_{1}+v_{2}+\cdots
\end{aligned}
$$

assuming that each term is small that the preceding one.

The problem of modeling the acoustic radiation force $F^{V}$ (per unit volume), reduces to obtain the time-average of the change in momentum for a fluid as

$$
F^{V}=-\left\langle\rho \frac{\mathrm{D} v}{\mathrm{D} t}\right\rangle
$$

It is important to note that the period average (indicated as \langle\rangle ) of the first order magnitudes $\left(p_{1}, \rho_{1}, v_{1}\right)$ is zero. However, the time average of the momentum 
does not vanish due to the nonlinearity in material derivative of the momentum Eq.(9.4). Substitution of Eqs.(9.1-9.3) into the momentum equation, and keeping up to second order terms leads to (Doherty et al., 2013)

$$
F^{V}=-\left\langle\rho \frac{\mathrm{D} v}{\mathrm{D} t}\right\rangle=-\left\langle\rho_{0} \frac{\partial v_{2}}{\partial t}+\frac{\partial \rho_{1} v_{1}}{\partial t}+\rho_{0}\left(v_{1} \nabla \cdot v_{1}+v_{1} \cdot \nabla v_{1}\right)\right\rangle .
$$

The period average of the first two terms of the right hand side is zero, but the last 2 terms, that account for the nonlinearity of the material derivative, does not vanish. Thus, for a plane wave propagating in the $x$ direction, the acoustic radiation force per unit volume reads

$$
F_{x}^{V}=-\left\langle\rho_{0} \frac{\partial v_{1}^{2}}{\partial x}\right\rangle
$$

Then the volume radiation force for a decaying plane harmonic wave with instantaneous intensity $I^{\prime}=p_{1} v_{1}=\rho_{0} c_{0} v_{1}^{2}$, traveling in an homogeneous-lossy media can be expressed as

$$
F_{x}^{V}=-\frac{\partial}{\partial x}\left\langle\frac{I^{\prime}}{c_{0}}\right\rangle
$$

or in terms of the time-average intensity $I$ in a period $T=2 \pi / \omega$

$$
I=\frac{1}{T} \int_{t}^{t+T} I^{\prime} \mathrm{d} t=\left\langle I^{\prime}\right\rangle
$$

the volume radiation force is proportional to the gradient of the intensity divided by the sound speed

$$
F_{x}^{V}=-\frac{\partial}{\partial x}\left(\frac{I}{c_{0}}\right)
$$

Here we shall recognize that the quantity $I / c_{0}$ can be expressed as

$$
\frac{I}{c_{0}}=\frac{1}{2} \rho_{0} v^{2}+\frac{1}{2} \frac{p^{2}}{\rho_{0} c_{0}^{2}}
$$

where it is evident that is the total energy density for a plane wave. Thus, is clear that Eq. (9.9) essentially states that an acoustic radiation force must appears when a spatial change in the energy density distribution is produced. The derivation of this equation was developed for homogeneous media, thus, strictly speaking a change of the energy density can only be produced by absorption processes. However, it is well-known that acoustic radiation forces also appear when a change of 
the energy density is produced by reflection between media with different acoustic properties. Here, we ignore these contributions and assume a homogeneous absorbing soft tissue media, where the contribution of the tissue attenuation to the absorption of the wave is dominant compared to the internal scattering processes.

\section{Small amplitude waves}

Thus, if considering an absorbing-media with an attenuation coefficient $\alpha$, a monochromatic plane wave traveling will present a mean intensity as

$$
I=\rho_{0} c_{0} V_{0}^{2} \exp (2 \alpha x) / 2,
$$

with $V_{0}$ the excitation particle velocity.

Substituting the intensity of Eq. (9.11) in Eq. (9.9), the time average force per unit volume reads

$$
F_{x, \operatorname{lin}}^{V}=2 \alpha \frac{I}{c_{0}}=\alpha \frac{p^{\prime 2}}{\rho c_{0}^{2}},
$$

that is the most common approach to calculate the acoustic radiation force in absorbing media. We shall note some peculiarities of this simple expression. It can be observed that the resulting force is proportional to the wave intensity and the attenuation coefficient. For diagnostic ultrasound frequencies the absorption coefficient is typically high, around $1 \mathrm{~dB} / \mathrm{cm}$ or even higher, and for the commonly used intensities the magnitude of the acoustic radiation force is weak. Therefore, the induced displacements are had to detect $(<1 \mu \mathrm{m})$ (Doherty et al., 2013). Thus, for induce detectable displacements (typically from 1 to $10 \mu \mathrm{m}$ ) two basic mechanisms are used. First is focusing. By increasing the gain of the source the intensity can be increased locally inducing higher ARF and displacements. On the other hand, the intensity of the field can be increased directly by increasing the amplitude of the excitation. However, for both approaches the calculation of the radiation force becomes complex.

In the first place, Eq. (9.12) was derived for plane waves and therefore, the calculation of the acoustic radiation force for more complex fields by using this expression is not accurate. For directional beams this expression is valid for low focused fields and only at the focal, where the wavefront can be considered quasiplane. However, for strongly focused fields the assumption of planes waves no longer holds. For an accurate description of the acoustic radiation force the spatial features of the beam must be included. In the case of paraxial beams analytic approaches can be obtained for intense waves (Rudenko et al., 1996; Ostrovsky et al., 2007; Ostrovsky, 2008), but for strongly focused sources a better theoretical foundation is needed. Approaches include calculating the full radiation stress tensors numerically or by indirect measurement of the acoustic radiation forces, e.g. by estimating streaming in frequency power law absorbing fluids. 


\section{Nonlinear regime}

Secondly, if the source amplitude is increased, nonlinear effects can be produced. In the case of weakly nonlinear effects, the absorption of each harmonic is different following the tissue frequency power law attenuation. If Eq. (9.12) is used the force can be calculated by the sum of the contributions of each harmonic separately. However, strictly speaking this equation is not exact in nonlinear regime. The main reason is that in nonlinear regime the intensity distribution of the wave is not exactly the given by Eq. (9.11). As long the energy is transferred to the higher harmonics, and the higher spectral components present higher attenuation, the intensity decay is not exponential, leading to the so-called nonlinear absorption. Thus, in the nonlinear regime these processes leads to a increasing rate of momentum transfer from the wave to the media, and therefore the acoustic radiation force is increased.

If the losses are caused by thermo-viscous effects, alternatively the nonlinear acoustic radiation force can be calculated directly using the expression (Rudenko et al., 1996)

$$
F_{x, \text { thermo }}^{V}=\frac{b\left(\beta^{\prime}+1\right)}{c_{0}^{5} \rho_{0}^{3}}\left\langle\left(\frac{\partial p^{\prime}}{\partial t}\right)^{2}\right\rangle,
$$

where $\beta^{\prime}$ is the nonlinear compressibility (often neglected) and the dissipation coefficient, $b$, is given by

$$
b=\frac{4}{3} \mu+\mu_{B}+\kappa\left(\frac{1}{C_{V}}-\frac{1}{C_{p}}\right),
$$

that accounts for the quadratic frequency attenuation caused sound thermo-viscous diffusion processes, being $\mu$ the shear viscosity, $\mu_{B}$ the bulk viscosity, $\kappa$ the thermal conductivity and $C_{V}$ and $C_{p}$ the specific heats at constant volume and pressure respectively.

Equation (9.13) is valid in both, linear and nonlinear regimes. In the first case, substitution of a monochromatic wave in Eq. (9.13) gives Eq. (9.12). On the other hand, Eq. (9.13) states that for complex nonlinear acoustic fields with sharp waveforms, as those containing shock waves, the squared time-derivative of the pressure will have a non negligible period average value. Therefore, the nonlinear acoustic radiation force is increased when sharp waveforms are present in the domain.

\section{Sawtooth regime}

If the nonlinear processes are strong enough to produce very sharp wave steepening, the wave can be described by the a sawtooth waveform. This regime occurs at 
distances $\sigma>3$ and Gol'dberg ratios $\Gamma>>1$. Thus, an analytic expression can be obtained for waveforms containing shock waves by substituting a sawtooth waveform in Eq. (9.13). The substitution of a sawtooth profile into Eq. (9.13) gives (Pishchalnikov et al., 2002; Rudenko et al., 2004)

$$
F_{x, \text { saw }}^{V}=\frac{2 \pi^{2}}{3}\left(\beta^{\prime}+1\right)^{2} \omega \frac{\rho_{0}}{c_{0}^{2}} u_{0}^{3}(x),
$$

where $u_{0}(x)$ is the amplitude of the particle velocity in one period of the sawtooth. It is important to note that in the sawtooth regime the acoustic radiation force does not depend on the absorption of the media. This effect is due to the nonlinear intensity absorption dominates over the tissue attenuation in the sawtooth regime. On the other hand, the cubic amplitude dependence, instead of the quadratic one for the linear estimation, evidences the great potential of the sharp waveform to enhance acoustic radiation force in the nonlinear regime.

Thus, the acoustic radiation force for plane waves is well understood in the case of thermo-viscous losses, where the magnitude of the force varies between to limits (1) the small amplitude regime, where the force decays exponentially with distance, and (2) the nonlinear absorption regime, where the ARF is proportional to the cube of the sawtooth peak particle vibration.

\subsection{Nonlinear radiation force in tissue-like media}

In the case of frequency power law absorption media, the acoustic radiation force for small amplitude waves is equivalent by selecting the appropriate attenuation coefficient and the intensity of each spectral component. However, Eq. (9.13) is no longer valid for media with non-quadratic frequency dependent attenuation, and therefore the calculation of the ARF in the nonlinear regime becomes complex. Here, we calculate the ARF using the mean change in momentum by solving numerically the full constitutive relation of nonlinear acoustics by using the method described in Chapter 7.

The aim of our work is to understand the role of the frequency dependent attenuation in the nonlinear generation of acoustic radiation forces.

We start presenting the intensity of a plane wave in two absorbing media, for thermo-viscous absorption, where the exponent of the frequency power law is $\gamma=2$ and for tissue absorption, $\gamma=1$. Figure 9.1 shows the intensity distribution for these two attenuation models, where each curve represents a different Gol'dberg ratio: for $\Gamma>>1$ the nonlinear effects dominate over attenuation and the propagation can be considered lossless, and for $\Gamma \rightarrow 0$ the nonlinear effects are negligible and the propagation can be assumed linear. The analytical intensity for a lossless media is also presented (Hamilton et al., 1998a), were it can be appreciated the 

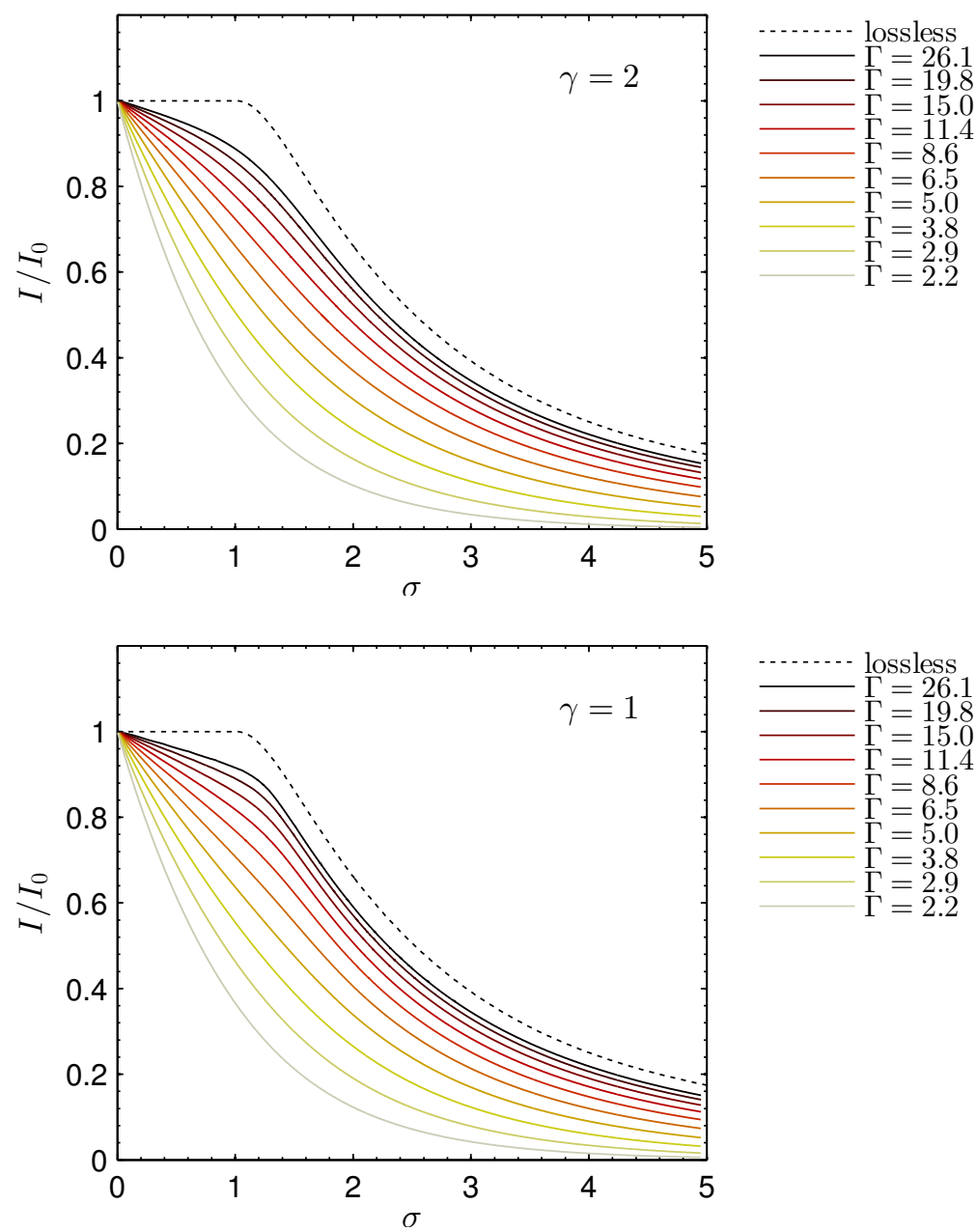

Figure 9.1: Spatial distribution of the nonlinear intensity in (top) viscous media and (bottom) tissue for increasing ratio between nonlinear and attenuation effects ( $\Gamma$ is the Gol'dberg ratio).

well-known distribution: in the shock-free region the intensity is constant and beyond $\sigma>1$ the nonlinear absorption activates and the intensity drops, even for lossless media.

For lower Gold'berg ratios the intensity decays exponentially in both lossy media. However, for higher wave amplitudes, when $\Gamma>>1$ the intensity does not decays exponentially and appreciable differences can be observed between thermoviscous and tissue model. Only for the very high nonlinear regimes the intensity distribution in both models converges to the lossless case. In these cases the nonlinear effects develop fast and the attenuation have no appreciable effect. Also, 

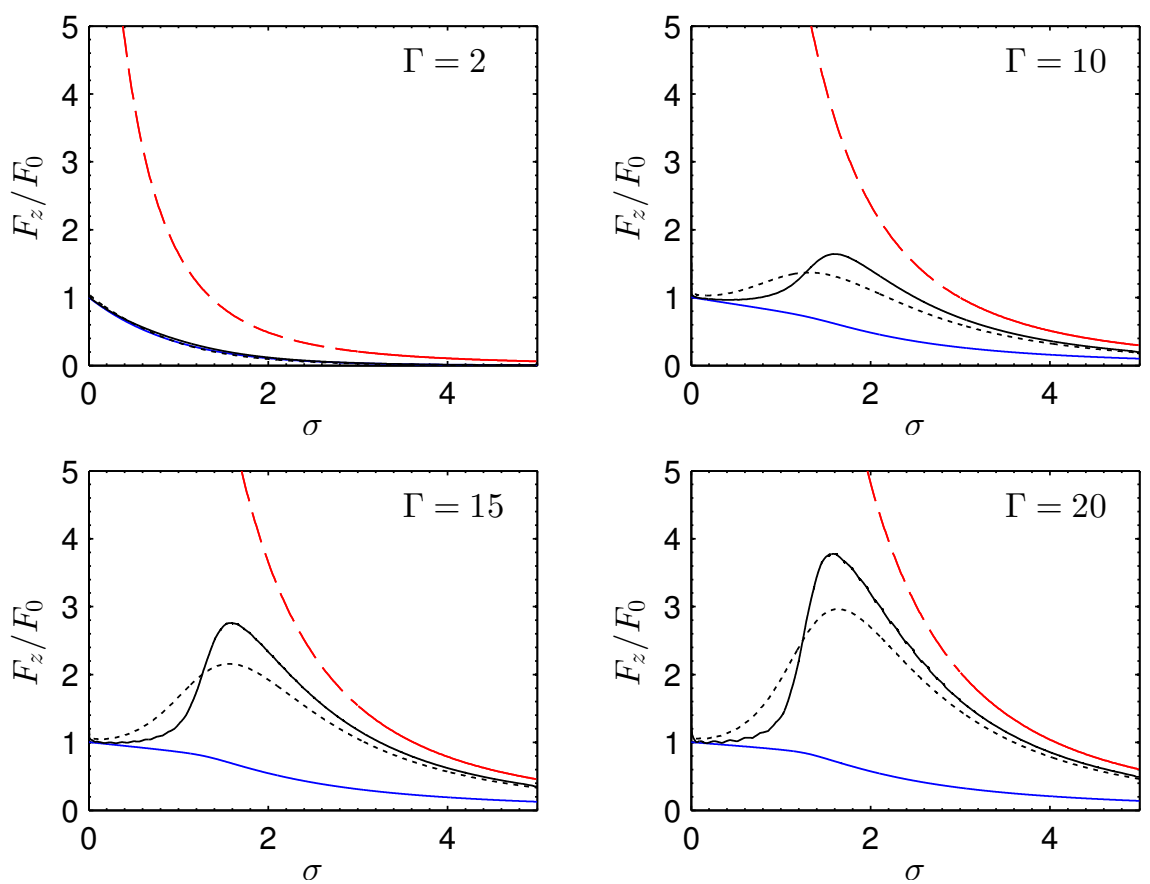

Figure 9.2: Spatial distribution of the normalized acoustic radiation force (ARF) for different Gold'berg ratios $(\Gamma)$. The distribution of the ARF is calculated for (black continuous line) soft-tissue and for (black dotted line) thermo-viscous media. The limiting case of linear ARF calculation using Eq. (9.12) is shown in blue line, while the sawtooth regime using Eq. (9.15 (valid for $\sigma>3$ ) is shown in red lines.

in all cases it can be seen that the intensity distribution have different shape in the shock-free region and beyond the shock formation distance.

Thus, as long the media is homogeneous and sound speed is constant, is evident that there exist differences in the gradient of the energy density. Therefore the resulting acoustic radiation force is amplitude dependent, and also different between attenuation models.

A close view can be observed in Fig. 9.2. There, the acoustic radiation force is shown for four selected amplitudes, being $\Gamma=(2,10,15,20)$ respectively. The limiting cases of linearized calculation using Eq. (9.12), and the sawtooth regime using Eq. (9.15) are shown in blue and red lines respectively. First, for $\Gamma=2$, the propagation is nonlinear, but the attenuation processes still comparable to the nonlinear processes. In this case, the intensity decay is almost exponentially, and therefore the ARF is proportional to the attenuation coefficient and wave intensity. The ARF in this case decays also almost exponentially and the expression (9.12), although not exact, is accurate to describe the ARF generation for both, thermoviscous and absorbing media. 

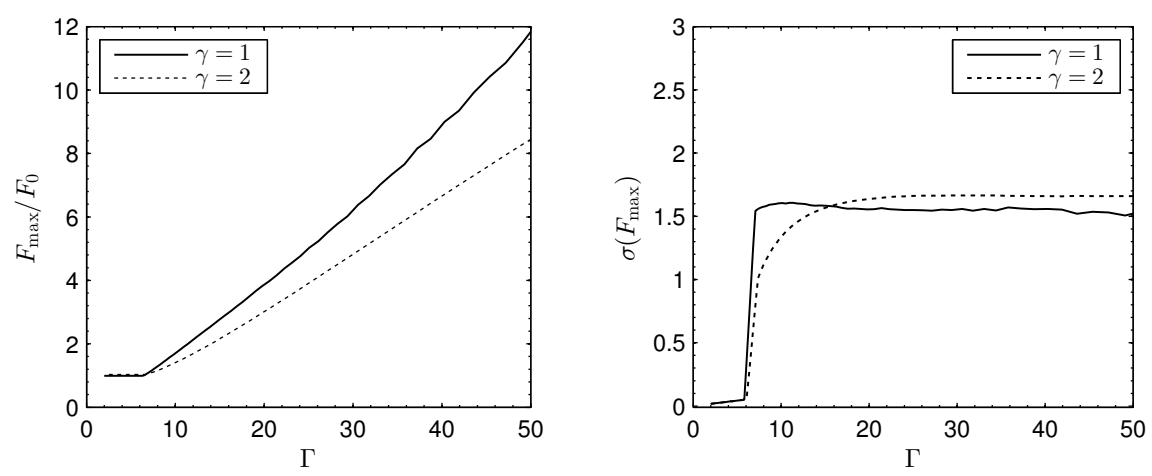

Figure 9.3: Peak acoustic radiation force value and its location in $\sigma$ coordinate as a function of the Gol'dberg ratio for (continuous line) soft-tissue model and (dotted line) thermo-viscous lossy medium.

However, if wave amplitude is increased, i.e. for $\Gamma=(10,15,20)$ in Fig. 9.2, the $\mathrm{ARF}$ distribution is no longer accurately described by Eq. (9.12). Moreover, there exist remarkable differences between tissue and thermo-viscous models. First, we set the same attenuation coefficient at the fundamental frequency for both models. Therefore, due to the attenuation is given by $\alpha=\alpha_{0} \omega^{\gamma}$, in the case of thermoviscous model the high spectral components present high overall attenuation than in the tissue model. Thus, in the nonlinear regime high spectral components are generated in both models, but in the thermo-viscous model they are attenuated faster and close to the source. The momentum transfer is increased near the source. By contrast, in the tissue model the attenuation of higher spectral components is lower, and therefore the momentum transfer is weaker near the source.

On the other hand, at distances near the shock formation distance this situation is inverted. At these distances, the amplitude of the harmonics in the tissue model is higher than in the thermo-viscous model due to the weaker high-frequency absorption. Thus, when nonlinear absorption activates and strongly dominates over tissue absorption, the momentum transfer of the tissue model is remarkable higher than in thermo-viscous lossy media.

Finally, for higher distances, and very strong nonlinearity, the ARF observed in both models converge to the sawtooth profile given by Eq. (9.15). This fact evidences that also in tissue model, the momentum transfer for waves including shocks is governed by the nonlinear absorption processes and almost independent of the tissue properties: the attenuation value and the exponent of the frequency power law attenuation. However, we remark that for intermediate distances at which there exists shocks, but the sawtooth profile is not yet developed, let say $1<\sigma<3$, the specific tissue power law strongly affects the acoustic radiation force generation.

The maximum value of the acoustic radiation force and its location is presented 
in Fig. 9.3. First, it can be seen that for low Gol'dberg ratios the acoustic radiation force maximum in located at the source and the value is proportional to the source intensity $F_{0}=2 \alpha I_{0} / c_{0}$. In these situations the media attenuation is stronger than the nonlinear absorption, and the ARF decays from its initial value. However, for higher Gol'dberg ratios the strong nonlinear effects dominates over absorption. As explained above the momentum transfer is increased due to nonlinear absorption processes and the acoustic radiation force is strongly enhanced. For these plane wave simulations, even without focusing, the nonlinearity increases the acoustic radiation force a order of magnitude relative to the acoustic radiation force at the source. On the other hand, an interesting feature is observed: due to the maximum of the acoustic radiation force is produced before the sawtooth regime, the maximum enhancing depends on the power law exponent.

Thus, this results evidences the importance of including soft tissue losses for estimate acoustic radiation forces, even in situations with strong nonlinearities. On the other hand, the maximum momentum transfer is located around $\sigma=\pi / 2$, where shocks are fully developed and the shock amplitude (the pressure jump at the discontinuity) is maximum. It can be observed that in Eq. (9.13), the maximum possible value of the time-derivative will be produced by a sharp time waveform. Thus, the maximum value is obtained at the location where the discontinuity jump reaches its maximum, for lossless propagation occurs exactly at $\sigma=\pi / 2$.

\subsection{Power law dependence}

We have shown the nonlinear generation of the acoustic radiation force for the case of linear frequency power law and for thermo-viscous losses. Here we extend the analysis for intermediate values of the exponent of the frequency power law. Thus, Fig. (9.4) presents an overview, where it is shown the acoustic radiation force generated as a function of the distance and as a function of the exponent of the power law. In first place, Fig. (9.4) (top) presents a case where nonlinear effects are of the order of the attenuation processes. Here, the ARF decays almost exponentially and there does not exist remarkable differences between the frequency power law attenuation models. The ARF is slightly greater for the higher exponents of the power law due to the absorption of the generated high spectral components is greater. Note in the linear regime the effects of the exponent of the power law are negligible for monochromatic plane waves.

However, for high nonlinear regimes, there can be observed a high (an nontrivial) dependence in the ARF and the exponent of the power law. There can be seen that the maximum ARF are generated in the case of linear power law. For thermo-viscous losses $(\gamma=2)$ the ARF is generated at higher rate near the source and the peak value is lower than for the linear power law case. But for intermediate values, there exist a trade-off between these effects: in one hand, the specific power law increases the attenuation in the high frequency limit, the absorption of the 
spectral components is increased rising the ARF near the source. On the other hand, when the power law exponent is increased, the nonlinear intensity absorption is increased near the source and therefore the intensity of the wave when shocks are produced is reduced. Thus, around $\sigma=\pi / 2$ the wave amplitude strongly depends on the attenuation produced before, near the source. Therefore the enhancement of the ARF generated by the sharp waveforms beyond the shock formation distance strongly depends on the trade-off between media absorption and the specific wave amplitude due to cumulative nonlinear absorption processes.

Figure 9.5 shows the ARF at four distances as a function of the exponent, where the above described effects can be observed. Near the source $(\sigma=0.5)$, the momentum transfer is governed by the media attenuation. Higher the exponent higher the momentum transfer for the superior harmonics and therefore, higher the nonlinear acoustic radiation force. This behavior holds until the existence of shocks in the solution, at $\sigma=1$. For longer distances, the situation is inverted. At the location where the discontinuity jump is maximum $(\sigma=\pi / 2)$ the maximum of the ARF is achieved for linear frequency power laws. For higher exponents, the value of the ARF is reduced. However the minimum is not located for the thermo-viscous case, but slightly before, around $\gamma=1.8$.

Here, we need to add another effect that we have omitted, that is the weak dispersion of the frequency power law attenuation media. As seen in Chapter 8, the efficiency in the harmonic generation is also dependent of the exponent of the power law. Essentially, the energy transfer from fundamental to higher harmonics is maximized for nondispersive case $(\gamma=2)$, and being reduced for nearly linear power laws due to weak dispersion. Thus, the harmonic energy transfer is increased for thermo-viscous losses, and due to its higher attenuation of the higher spectral components the ARF is slightly increased. The competition between nonlinear generation, attenuation and dispersion effects, and the nonlinear intensity absorption leads to the specific curves obtained numerically, where it is evident the critical effect of the power law absorption to accurately describe the acoustic radiation force. Differences between absorption models can lead to differences of the ARF values (about 25\%) and also strong differences in the spatial distribution.

\subsection{Relation of nonlinear absorption and tissue heating rate}

\section{Nonlinear absorption in tissue}

One of the factors that are closely related to the nonlinear behavior of the acoustic radiation force is the nonlinear intensity absorption. It is clear from Eq. (9.9), that the acoustic radiation force is closely dependent on the intensity distribution. On 

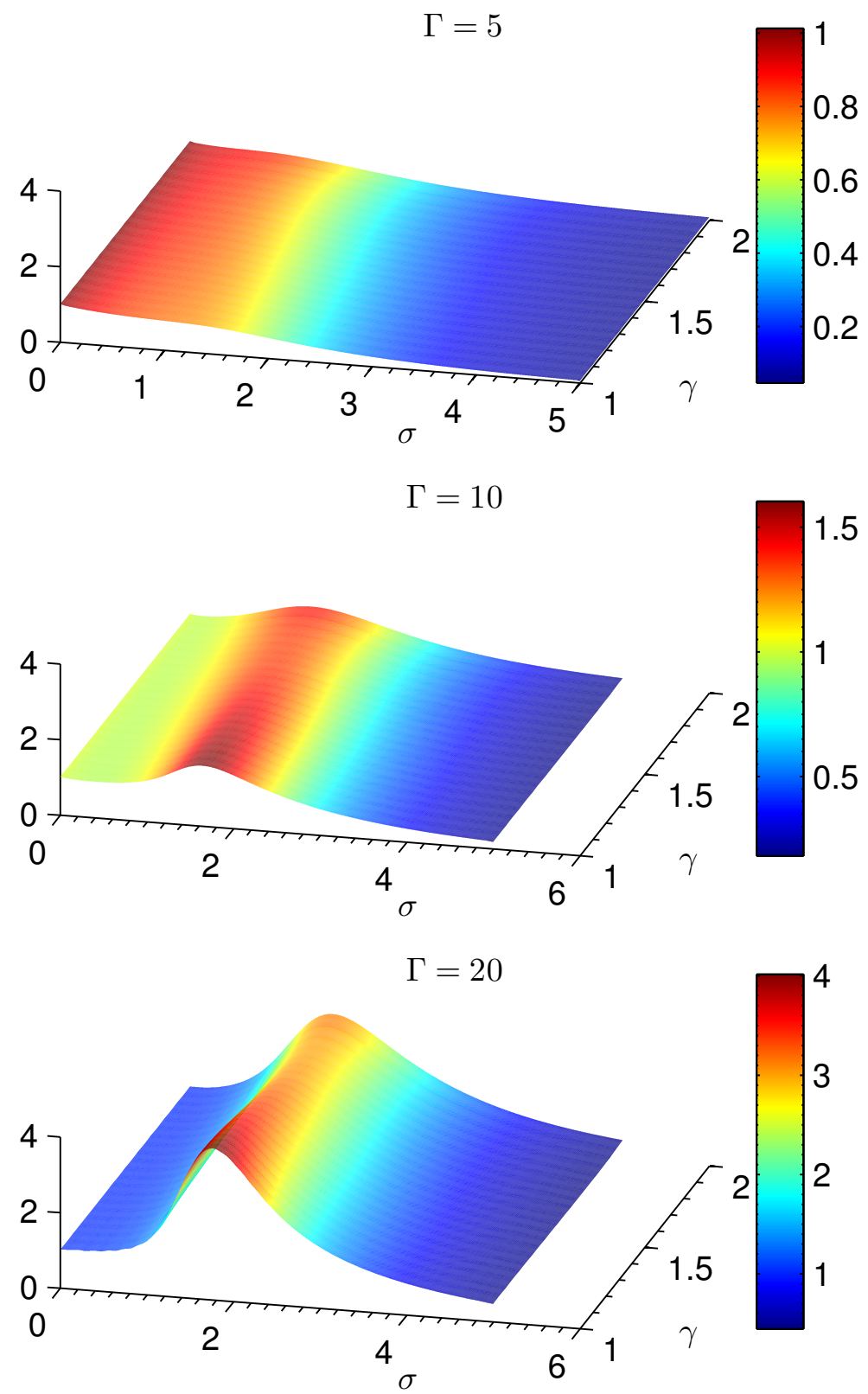

Figure 9.4: Spatial distribution of the acoustic radiation force for $\Gamma=(5,10,20)$ as a function of the exponent of the frequency power law attenuation, $\gamma$ and the propagation distance. Colorbars in $F / F_{0}$ units where $F_{0}$ is the ARF at the source. 

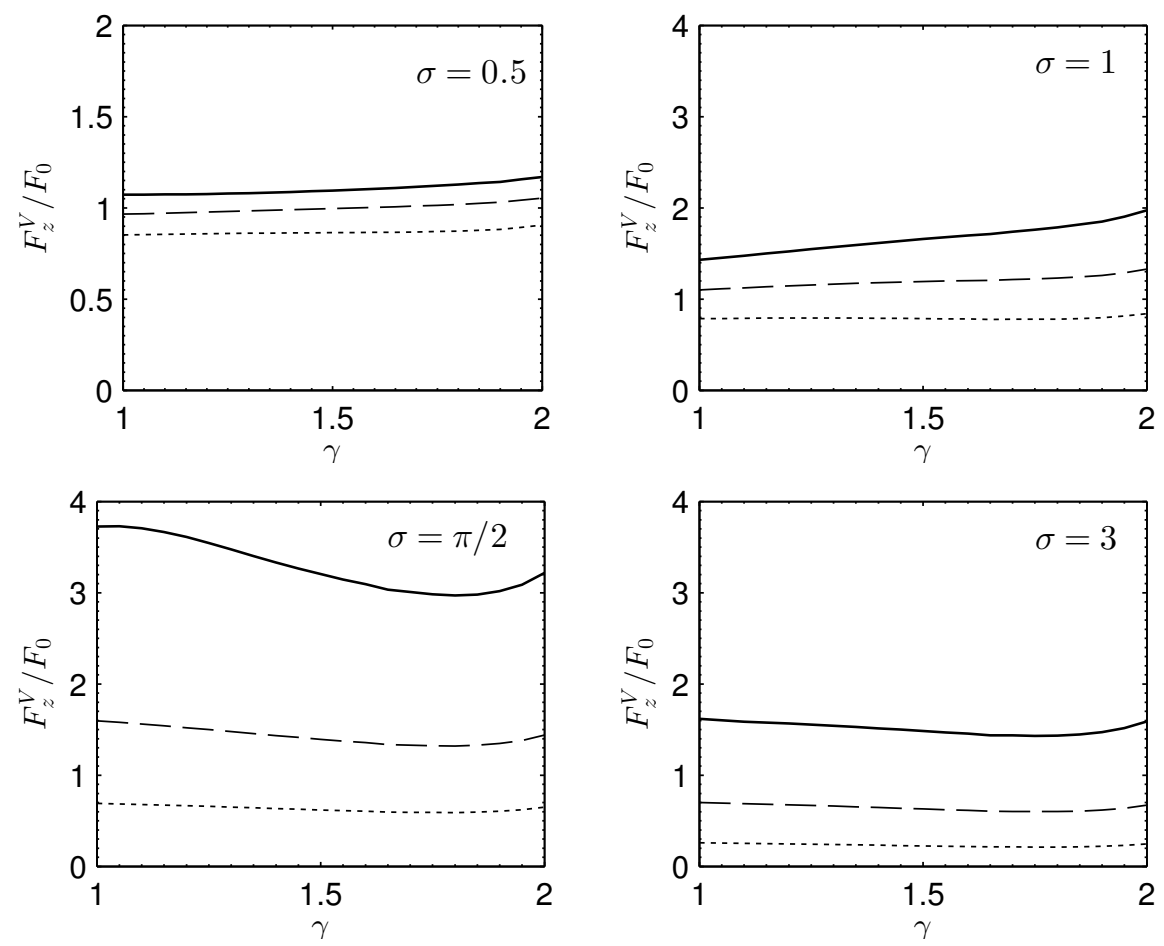

Figure 9.5: Acoustic radiation force (ARF) as a function of the power law exponent measured at distances $\sigma=(0.5,1, \pi / 2,3)$. Dotted lines are ARF for a Gol'dberg ratio $\Gamma=5$, dashed lines for $\Gamma=10$ and continuous lines for $\Gamma=20$.

the other hand, by the definition of the absorption we get

$$
\alpha_{f}=-\frac{\nabla \cdot I}{2 I}
$$

For small amplitude plane wave with $I=I_{0} \exp (-2 \alpha x)$, it reduces to

$$
\alpha_{f}=-\frac{1}{2 I} \frac{\partial I}{\partial x}=\alpha
$$

that is the linear absorption coefficient. However, for finite amplitude waves the effective intensity absorption is increased. For the lossless case, (equivalent to $\Gamma \rightarrow \infty)$, the nonlinear absorption is a function of the shock amplitude, $P_{\mathrm{sh}}$, (Hamilton et al., 1998a) as:

$$
\alpha_{f}=-\frac{\frac{2}{3} P_{\mathrm{sh}}^{3}}{\pi-\sigma P_{\mathrm{sh}}+P_{\mathrm{sh}} \cos \sigma P_{\mathrm{sh}}+\frac{2}{3} \sigma P_{\mathrm{sh}}^{3}} .
$$



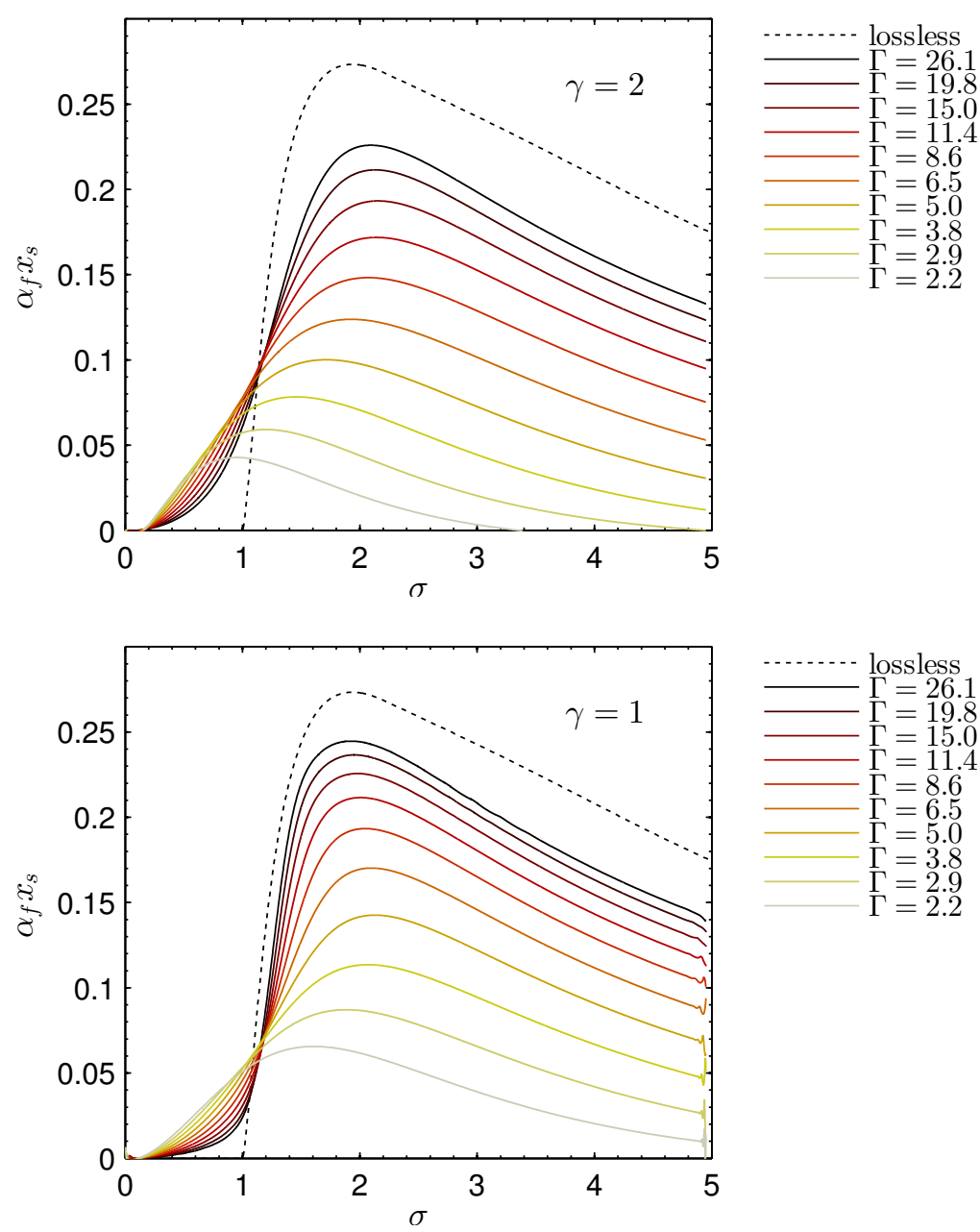

Figure 9.6: Nonlinear absorption for different Gol'dberg ratios. (Top) thermo-viscous absorption model, $\gamma=2$, and (bottom) tissue absorption model, $\gamma=1$.

In the case of thermo-viscous absorption the intensity can be obtained analytically from th Mendousse solution (Hamilton et al., 1998a), and the nonlinear absorption calculated using Eq. (9.16). In the case of other tissue model, the nonlinear absorption is calculated by obtaining numerically the intensity and then apply Eq. (9.16).

Thus, Fig. 9.6 shows the nonlinear absorption obtained for thermo-viscous and tissue models. These results have been partially shown in the literature previously, see e.g. the work of Kashcheeva et al. (2000), we review here for convenience. We can see that the nonlinear absorption is present in all simulations. Near the source, 

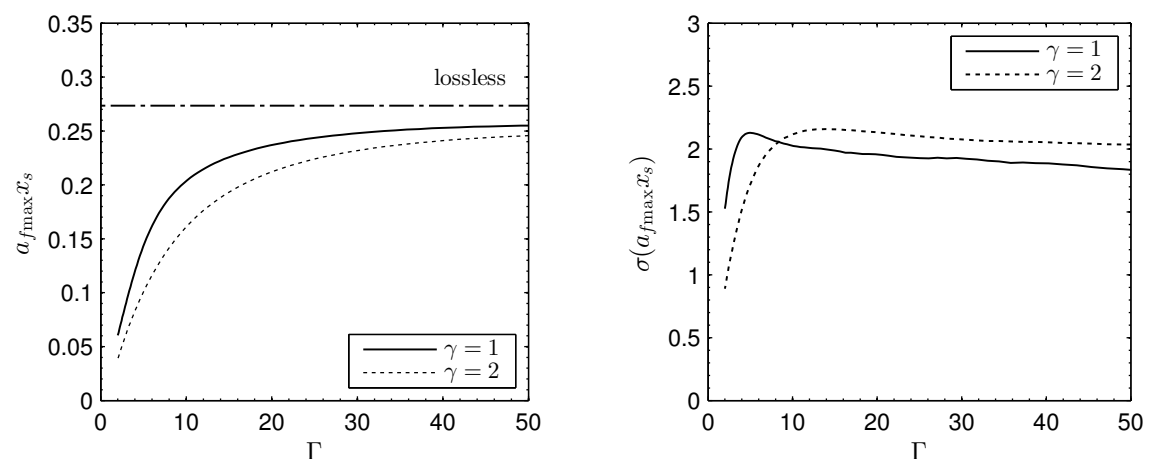

Figure 9.7: (Left) Maximum value of the nonlinear absorption as a function of the Gol'dberg ratio for (continuous line) tissue and (dotted line) thermo-viscous media. (Right) Location in the $\sigma$ coordinate as a function of the Gol'dberg ratio.

in the case of thermo-viscous losses the effective intensity absorption is higher than in the linear power law due to strong attenuation at the higher spectral components and when shocks are present, as explained above, the nonlinear absorption is higher for linear power law. The nonlinear intensity converges to the lossless estimation for very strong nonlinearities. In the case of linear power law the convergence is faster due to the low effective attenuation near the source.

These features are consistent to the acoustic radiation force, higher the effective attenuation, higher the ARF value. However, the maximum of the acoustic radiation force does not necessary coincide with the maximum of the nonlinear absorption, even in plane waves. By comparing Eq. (9.9) and Eq. (9.16), it can be appreciated the difference: the acoustic radiation force is proportional to the change of the energy density, while the nonlinear absorption is proportional to the change of the energy density normalized to the energy density. Note that in the linear regime, the acoustic radiation force decreases exponentially while the effective absorption is constant in space. For weak nonlinear effects, as shown by the lower curves in Fig. 9.6, the acoustic radiation force decays almost exponentially and the maximum is located at the source, while the maximum of the nonlinear absorption is always not at the source, but around $\pi / 2$. See also Figs. 9.7 and compare to Figs. 9.3. Both magnitudes are closely related though the momentum transfer, energy deposition, and intensity reduction, but are not the same.

\section{Nonlinear tissue heating rate}

As studied above, the result of the momentum transfer from the wave to the medium due to absorption processes is the appearance of a radiation force. On the other hand, the result of the dissipation of the acoustic energy of the wave is that the medium is heated. The heating rate per unit volume, $Q_{v}$, at which the 
temperature $T$ is increased is:

$$
\frac{\mathrm{d} T}{\mathrm{~d} t}=\frac{Q_{v}}{\rho_{0} C_{p}}
$$

where $C_{p}$ is the specific heat capacity at constant pressure (per unit mass) of the medium. On the other hand, the heating rate can be related to the wave intensity as (Hamilton et al., 1998a):

$$
\frac{\mathrm{d} T}{\mathrm{~d} t}=-\frac{1}{\rho_{0} C_{p}} \nabla \cdot I
$$

As can be seen by simple comparison with Eq. (9.9), for a plane wave the heating rate of the medium is proportional to the acoustic radiation force as

$$
\frac{\mathrm{d} T}{\mathrm{~d} t}=\frac{c_{0}}{\rho_{0} C_{p}} F_{x}^{V}
$$

Therefore, for a plane wave the heating rate coefficient, $Q_{v}$, is proportional to the acoustic radiation force:

$$
Q_{v}=c_{0} F_{x}^{V}
$$

For linear plane waves this relation reduces to the widely expression $Q_{v}=2 \alpha I$. However, as seen from the results of the preceding sections, for nonlinear waves both the amplitude and the spatial distribution of the heating rate calculated from Eq. (9.21) will not match the predictions using the linearized expression $Q_{v}=2 \alpha I$. The prediction of heating rate using the linearized expression will underestimate the temperature increasing in the nonlinear regime. Huge literature is devoted to the heat deposition by nonlinear fields, most of them for HIFU therapy applications and is well-known that the heat rate is increased by nonlinearity. However, most of the literature works still modeling the heating rate using the linearized expression.

On the other hand, once the medium is locally heated over the ambient temperature processes as diffusion, convection, conduction and radiation transfer heat from warmer to cooler regions. For tissues, the blood flowing trough capillaries and blood vessels redistributes heat. This process, known as tissue perfusion, and the heat diffusion due to thermal conductivity can be modeled by the Pennes bioheat transfer equation

$$
\frac{\mathrm{d} T}{\mathrm{~d} t}=\kappa \nabla^{2} T-\frac{\left(T-T_{0}\right)}{\tau}+\frac{Q_{v}}{\rho_{0} C_{p}},
$$

where $\kappa$ is thermal diffusivity, $\tau$ is the constant for perfusion and $T_{0}$ is the ambient temperature. It is worth noting here that due to the Laplacian term, temperature will spread strongly for sharp temperature distributions. Thus, the heat distribution will depend initially to the specific acoustic radiation force field (acting as 
heat source) and in second term (but coupled in time) to the diffusion/perfusion processes. As seen before, there exist remarkable differences on the (realistic) nonlinear estimation of the acoustic radiation force and its linearized approach (blue curves in Fig.9.2). These differences, not only in the value but in the distribution shape of the ARF, will lead to different heating patterns and must be included for realistic heating models if nonlinear effects are strong.

In the case of finite amplitude acoustic beams, it was shown previously that the peak intensity location does not necessary match the location of the ARF maximum, see e.g. (Camarena et al., 2013b). Thus, the heating pattern will be different using directly $Q_{v}=2 \alpha I$, or by estimating the energy deposition rate using the momentum transfer, i.e. the nonlinear acoustic radiation force distribution in the tissue.

\subsection{Conclusions}

In this Chapter we have calculated numerically the acoustic radiation force for intense ultrasound plane waves in biological media. By neglecting diffraction, the particular features of the acoustic radiation force in absorbing biological media can be studied and clearly understood. Thus, the relevance of the exponent of the frequency power law absorption is studied, showing that the correct tissue attenuation model is critical, even in the case of very strong nonlinearity. On the other hand, we have shown that in the nonlinear regime, accounting for the proper acoustic radiation force calculation is critical in order to do not underestimate the force value.

Furthermore, the relation of the acoustic radiation force to the effective or nonlinear absorption in tissue model is presented. Also, considerations on the effective heat deposition rate are also underlined, where it was shown that the nonlinear acoustic radiation force accurately describe the heating rate in the tissue.

However, more complete study is needed for realistically describe the acoustic radiation force in biomedical applications. In first place, in the case of acoustic beams, including diffraction not only implies the calculation of the acoustic radiation force in the direction of the beam, but also in the other directions. In other words, if diffraction is included, the nonlinear stress tensor must be calculated (Rudenko et al., 1996; Ostrovsky et al., 2007; Ostrovsky, 2008). Of special interest is the calculation of the nonlinear stress tensor for non-paraxial beams. Moreover, other interest related problems are the accurate modeling of the shear waves generated by nonlinear acoustic radiation force in tissue, and the acoustic radiation force generated due to tissue internal reflections, heating patterns with realistic blood streaming due to high intensity fields, or acoustic radiation force interaction with microbubbles inside capillaries. 


\section{References}

Bercoff, Jérémy, Mickaël Tanter, and Mathias Fink (2004). "Supersonic shear imaging: a new technique for soft tissue elasticity mapping". In: Ultrasonics, Ferroelectrics, and Frequency Control, IEEE Transactions on 51.4, pp. 396-409.

Beyer, Robert T (1978). "Radiation pressure-the history of a mislabeled tensor". In: The Journal of the Acoustical Society of America 63.4, pp. 1025-1030.

Camarena, Francisco, Silvia Adrián-Martínez, Noé Jiménez, and Víctor Sánchez-Morcillo (2013b). "Nonlinear focal shift beyond the geometrical focus in moderately focused acoustic beams". In: The Journal of the Acoustical Society of America 134.2, pp. 1463-1472.

Carstensen, EL, WK Law, ND McKay, and TG Muir (1980). "Demonstration of nonlinear acoustical effects at biomedical frequencies and intensities". In: Ultrasound Med. Biol. 6.4, pp. 359368.

Doherty, Joshua R, Gregg E Trahey, Kathryn R Nightingale, and Mark L Palmeri (2013). "Acoustic radiation force elasticity imaging in diagnostic ultrasound". In: Ultrasonics, Ferroelectrics and Frequency Control, IEEE Transactions on 60.4, pp. 685-701.

Faraday, M. (1831). "On a Peculiar Class of Acoustical Figures; and on Certain Forms Assumed by Groups of Particles upon Vibrating Elastic Surfaces". English. In: Philosophical Transactions of the Royal Society of London 121, pp. 299-340.

Fatemi, Mostafa and James F Greenleaf (1998). "Ultrasound-stimulated vibro-acoustic spectrography". In: Science 280.5360, pp. 82-85.

Fowlkes, JB, SY Emelianov, JG Pipe, AR Skovoroda, PL Carson, RS Adler, and AP Sarvazyan (1995). "Magnetic-resonance imaging techniques for detection of elasticity variation". In: Medical physics 22.11, pp. 1771-1778.

Hamilton, Mark F, David T Blackstock, et al. (1998a). Nonlinear acoustics. Vol. 237. Academic press San Diego.

Hultström, Jessica, Otto Manneberg, Katja Dopf, Hans M Hertz, Hjalmar Brismar, and Martin Wiklund (2007). "Proliferation and viability of adherent cells manipulated by standing-wave ultrasound in a microfluidic chip". In: Ultrasound in medicine E biology 33.1, pp. 145-151.

Kashcheeva, S.S., O.A. Sapozhnikov, V.A. Khokhlova, M.A. Averkiou, and L.A. Crum (2000). "Nonlinear distortion and attenuation of intense acoustic waves in lossy media obeying a frequency power law". In: Acoustical Physics 46, pp. 170-177.

Konofagou, Elisa E and Kullervo Hynynen (2003). "Localized harmonic motion imaging: theory, simulations and experiments". In: Ultrasound in medicine E biology 29.10, pp. 1405-1413.

Laurell, Thomas, Filip Petersson, and Andreas Nilsson (2007). "Chip integrated strategies for acoustic separation and manipulation of cells and particles". In: Chemical Society Reviews 36.3, pp. $492-506$.

Lebedew, Peter (1901). "Untersuchungen über die Druckkräfte des Lichtes". In: Annalen der Physik 311.11, pp. 433-458.

Lee, Jungwoo and K Kirk Shung (2006). "Effect of ultrasonic attenuation on the feasibility of acoustic tweezers". In: Ultrasound in medicine \& biology 32.10, pp. 1575-1583.

Maleke, Caroline and Elisa E Konofagou (2008). "Harmonic motion imaging for focused ultrasound (HMIFU): a fully integrated technique for sonication and monitoring of thermal ablation in tissues". In: Physics in medicine and biology 53.6, p. 1773.

Manduca, A., T.E. Oliphant, M.A. Dresner, J.L. Mahowald, S.A. Kruse, E. Amromin, J.P. Felmlee, J.F. Greenleaf, and R.L. Ehman (2001). "Magnetic resonance elastography: Non-invasive mapping of tissue elasticity". In: Medical Image Analysis 5.4, pp. 237-254.

Nightingale, Kathryn R, Phyllis J Kornguth, William F Walker, Bruce A McDermott, and Gregg E Trahey (1995). "A novel ultrasonic technique for differentiating cysts from solid lesions: preliminary results in the breast". In: Ultrasound in medicine 65 biology 21.6, pp. 745-751.

Nightingale, Kathryn R, Phyllis J Kornguth, and Gregg E Trahey (1999). "The use of acoustic streaming in breast lesion diagnosis: a clinical study". In: Ultrasound in medicine 6 biology 25.1 , pp. $75-87$. 
Nightingale, Kathryn R, Mark L Palmeri, Roger W Nightingale, and Gregg E Trahey (2001). "On the feasibility of remote palpation using acoustic radiation force". In: The Journal of the Acoustical Society of America 110.1, pp. 625-634.

Ostrovsky, LA (2008). "Radiation force in nonlinear, focused beams". In: The Journal of the Acoustical Society of America 124.3, pp. 1404-1407.

Ostrovsky, Lev, Alexander Sutin, Yuri Il'Inskii, Oleg Rudenko, and Armen Sarvazyan (2007). "Radiation force and shear motions in inhomogeneous media." In: The Journal of the Acoustical Society of America 121.3, pp. 1324-1331.

Palmeri, Mark L and Kathryn R Nightingale (2011). "Acoustic radiation force-based elasticity imaging methods". In: Interface Focus, rsfs20110023.

Pishchalnikov, Yu A, OA Sapozhnikov, and TV Sinilo (2002). "Increase in the efficiency of the shear wave generation in gelatin due to the nonlinear absorption of a focused ultrasonic beam". In: Acoustical Physics 48.2, pp. 214-219.

Rayleigh, Lord (1902). "On the pressure of vibrations". In: The London, Edinburgh, and Dublin Philosophical Magazine and Journal of Science 3.15, pp. 338-346.

Rudenko, Oleg V and Oleg A Sapozhnikov (2004). "Self-action effects for wave beams containing shock fronts". In: Physics-Uspekhi 47.9, p. 907.

Rudenko, OV, AP Sarvazyan, and S Yu Emelianov (1996). "Acoustic radiation force and streaming induced by focused nonlinear ultrasound in a dissipative medium". In: The Journal of the Acoustical Society of America 99.5, pp. 2791-2798.

Sarvazyan, Armen and Lev Ostrovsky (2009). "Stirring and mixing of liquids using acoustic radiation force". In: The Journal of the Acoustical Society of America 125.6, pp. 3548-3554.

Sarvazyan, Armen P, Oleg V Rudenko, Scott D Swanson, J Brian Fowlkes, and Stanislav Y Emelianov (1998). "Shear wave elasticity imaging: a new ultrasonic technology of medical diagnostics". In: Ultrasound in medicine \& biology 24.9, pp. 1419-1435.

Sarvazyan, Armen P, Oleg V Rudenko, and Wesley L Nyborg (2010). "Biomedical applications of radiation force of ultrasound: historical roots and physical basis". In: Ultrasound in medicine \& biology 36.9, pp. 1379-1394.

Sorbello, Richard S (1972). "Phonon-Radiation Force in Defect Crystal Lattices". In: Physical Review B 6.12, p. 4757.

Trahey, Gregg E, Mark L Palmeri, Rex C Bentley, and Kathryn R Nightingale (2004). "Acoustic radiation force impulse imaging of the mechanical properties of arteries: in vivo and ex vivo results". In: Ultrasound in medicine E biology 30.9, pp. 1163-1171.

Urban, Matthew W, Azra Alizad, Wilkins Aquino, James F Greenleaf, and Mostafa Fatemi (2011). "A review of vibro-acoustography and its applications in medicine". In: Current medical imaging reviews 7.4 , p. 350.

Vappou, Jonathan, Caroline Maleke, and Elisa E Konofagou (2009). "Quantitative viscoelastic parameters measured by harmonic motion imaging". In: Physics in medicine and biology 54.11 , p. 3579.

Viola, Francesco, Margaret D Kramer, Michael B Lawrence, James P Oberhauser, and William F Walker (2004). "Sonorheometry: a noncontact method for the dynamic assessment of thrombosis". In: Annals of biomedical engineering 32.5, pp. 696-705.

Wood, Robert Williams and Alfred L Loomis (1927). "The physical and biological effects of high-frequency sound-waves of great intensity". In: The London, Edinburgh, and Dublin Philosophical Magazine and Journal of Science 4.22, pp. 417-436.

Wu, Junru (1991). "Acoustical tweezers". In: The Journal of the Acoustical Society of America 89.5, pp. $2140-2143$. 


\section{Index of terms}

\section{Symbols}

$k$-space method, 162, 196, 246

\section{A}

Acoustic radiation force, 183, 252

Advection, 51

Angular momentum transfer, 183

Anomalous dispersion, 213, 231

Archimedean spiral, 158

Artificial viscosity, 208

Axisymmetric grating, 123, 137

\section{B}

Babinet's principle, 161

Band structure, 111

Band-gap, 49, 69

Beatings, 66

Bessel beam, 106, 122, 159

Bloch waves, 75, 113

Breathers, 6

Brillouin zone, 111

Burguers equation, 193

\section{C}

Castor oil, 218

Chirped lattice, 82

Coherence length, 66, 111, 233

Constitutive relations, 50, 195

Continuity equation, 50

Coulomb interaction, 8

Crowdion, 28

Cubic-like medium, 81, 90

\section{D}

Detuning, 66

Diffraction order, 141

Discrete wave equation, 9

Dispersion management of solitons, 99

Dispersion relation, 47, 231
Double-kink, 35

E

Equation of motion, 50

Evanescent waves, 70

Extremally linear media, 88

F

FDTD, 55, 114, 124, 139, 201

Fermat's spiral, 158

Fermi-Pasta-Ulam lattice, 8

Fluorescence, 82

Focusing, 135

Fourier continuation method, 196

Fractional Laplacian, 196, 246

Free and forced waves, 65

Frequency conversion mirror, 85

Frequency power law attenuation, 193, 230,262

Fresnel zone plates, 135

Fubini solution, 60

G

GaAs/AlAs lattice, 47

Gol'dberg ratio, 214, 260

Granular media, 47

$\mathbf{H}$

Harmonic enhancement, 79

Heat diffusion, 268

Heating rate, 268

Helix field, 176

High Intensity Focused Ultrasound (HIFU), 221

High order Bessel beam (HOBB), 159

I

Interstitial defects, 40 


\section{K}

KdV soliton, 91

Khokhlov number, 220

Kinks, 6

Kramers-Kronig relationships, 214, 230

KZK equation, 194

\section{L}

Lagrangian energy density, 54, 194

Lituus, 158

Localized nonlinear excitations, 4

\section{M}

Magic wave number, 16, 19

Mendousse solution, 214

Mica muscovite, 4

Multi-objective optimization, 205

Multilayered, 47

Multiple relaxation, 198

$\mathrm{N}$

Neighbours interaction, 23

Nonlinear absorption, 265

Nonlinear coupling of Bloch modes, 113

Nonlinear focal shift, 149, 222

Nonlinear focusing, 148, 221

Nonlinear tissue heating rate, 268

Numerical dispersion, 204

Numerical stability, 204

$\mathrm{O}$

One-way nonlinear models, 194

Optimization, 205

\section{$\mathbf{P}$}

Pareto front, 207

Peierls-Nabarro potential, 37

Pennes bioheat transfer equation, 268

Perfectly matched layers (PML), 203

Periodicity, 46

Perturbation method, 65

Phase matching, 63, 66, 111, 233

Phonon dispersion, 9

Phonons, 46

Plane wave expansion method, 110
Q

Quodons, 4

R

Radiation, 34

Rayleigh-Sommerfeld integral, 161

Relaxation, 197

Resonance conditions, 63

Rotating wave approximation, 17

Runge-Kutta method, 202

Rytov formula, 48

\section{S}

Saser, 47

Sawtooth wave, 258

Second order nonlinear equation, 52

Second order wave eqaution, 54

Self-collimation, 106, 111

Shock amplitude, 265

Shock waves, 210

Short range potential, 26

Soft-tissue, 213, 232

Solid state nuclear track detectors, 4

Soliton, 91, 97

Sonic crystals, 46, 107

Spiral grating, 159, 170

State equation, 51

Strain, 14

Substrate potential, 30

Superlattices, 46

Supersonic ultra-discrete kinks, 13

$\mathrm{T}$

Tail analisis, 10

Thermalization, 37

Topological charge, 183

Triangular waveform, 19, 22

V

Vortex, 163

W

Westervelt equation, 54, 194 